\title{
Vitamin C and Human Health
}

\author{
Edited by
}

Anitra C. Carr and Margreet M.C. Vissers

Printed Edition of the Special Issue Published in Nutrients 
Anitra C. Carr and Margreet M.C. Vissers (Eds.)

\section{Vitamin C and Human Health}

MDPI 
This book is a reprint of the special issue that appeared in the online open access journal Nutrients (ISSN 2072-6643) in 2013 (available at:

http://www.mdpi.com/journal/nutrients/special_issues/vitamin_C_and_human_health).

\section{Guest Editors}

Anitra C. Carr

Research Fellow \& Centre Co-ordinator, Centre for Free Radical Research, Department of

Pathology, University of Otago

Christchurch 8140, New Zealand

Margreet C. M. Vissers

Professor \& Associate Dean (Research), Centre for Free Radical Research, Department of Pathology, University of Otago

Christchurch 8140, New Zealand

\section{Editorial Office}

MDPI AG

Klybeckstrasse 64

Basel, Switzerland

\section{Publisher}

Shu-Kun Lin

Production Editor

Martyn Rittman

\section{Edition 2014}

MDPI • Basel • Beijing

ISBN 978-3-906980-62-1

(C) 2014 by the authors; licensee MDPI, Basel, Switzerland. All articles in this volume are Open Access distributed under the Creative Commons Attribution 3.0 license (http://creativecommons.org/licenses/by/3.0/), which allows users to download, copy and build upon published articles even for commercial purposes, as long as the author and publisher are properly credited, which ensures maximum dissemination and a wider impact of our publications. However, the dissemination and distribution of copies of this book as a whole is restricted to MDPI, Basel, Switzerland. 


\section{Table of Contents}

Anitra C. Carr and Margreet M.C. Vissers

Preface Guest Editors. V

\section{Methodology Overview}

Alexander J. Michels and Balz Frei

Myths, Artifacts, and Fatal Flaws: Identifying Limitations and Opportunities in Vitamin C Research

Nutrients 2013, 5(12), 5161-5192.

http://www.mdpi.com/2072-6643/5/12/5161

\section{Cancer \& Inflammation}

\section{Seyeon Park}

The Effects of High Concentrations of Vitamin C on Cancer Cells

Nutrients 2013, 5(9), 3496-3505

http://www.mdpi.com/2072-6643/5/9/3496

Amaya Azqueta, Solange Costa, Yolanda Lorenzo, Nasser E. Bastani and Andrew R. Collins

Vitamin C in Cultured Human (HeLa) Cells: Lack of Effect on DNA Protection and Repair Nutrients 2013, 5(4), 1200-1217

http://www.mdpi.com/2072-6643/5/4/1200

Bassem M. Mohammed, Bernard J. Fisher, Donatas Kraskauskas, Daniela Farkas, Donald F. Brophy, Alpha A. Fowler and Ramesh Natarajan

Vitamin C: A Novel Regulator of Neutrophil Extracellular Trap Formation Nutrients 2013, 5(8), 3131-3150 61

http://www.mdpi.com/2072-6643/5/8/3131

\section{Neurological Effects}

Fiona E. Harrison, Gene L. Bowman and Maria Cristina Polidori

Ascorbic Acid and the Brain: Rationale for the Use against Cognitive Decline

Nutrients 2014, 6(4), 1752-1781 


\section{Naohiro Iwata, Mari Okazaki, Meiyan Xuan, Shinya Kamiuchi, Hirokazu}

Matsuzaki and Yasuhide Hibino

Orally Administrated Ascorbic Acid Suppresses Neuronal Damage and Modifies Expression of SVCT2 and GLUT1 in the Brain of Diabetic Rats with Cerebral Ischemia-Reperfusion Nutrients 2014, 6(4), 1554-1577...

http://www.mdpi.com/2072-6643/6/4/1554

\section{Bioavailability}

Anitra C. Carr and Margreet C. M. Vissers

Synthetic or Food-Derived Vitamin C-Are They Equally Bioavailable?

Nutrients 2013, 5(11), 4284-4304.

http://www.mdpi.com/2072-6643/5/11/4284

Anitra C. Carr, Stephanie M. Bozonet, Juliet M. Pullar, Jeremy W. Simcock and

Margreet C. M. Vissers

A Randomized Steady-State Bioavailability Study of Synthetic versus Natural (KiwifruitDerived) Vitamin C

Nutrients 2013, 5(9), 3684-3695

http://www.mdpi.com/2072-6643/5/9/3684

Anitra C. Carr, Stephanie M. Bozonet and Margreet C. M. Vissers

A Randomised Cross-Over Pharmacokinetic Bioavailability Study of Synthetic versus Kiwifruit-Derived Vitamin C

Nutrients 2013, 5(11), 4451-4461 166

http://www.mdpi.com/2072-6643/5/11/4451

\section{Human Requirements}

Joris R. Delanghe, Marc L. De Buyzere, Marijn M. Speeckaert and Michel R. Langlois Genetic Aspects of Scurvy and the European Famine of 1845-1848

Nutrients 2013, 5(9), 3582-3588. 177

http://www.mdpi.com/2072-6643/5/9/3582

Maiken Lindblad, Pernille Tveden-Nyborg and Jens Lykkesfeldt

Regulation of Vitamin C Homeostasis during Deficiency

Nutrients 2013, 5(8), 2860-2879

http://www.mdpi.com/2072-6643/5/8/2860

\section{Daniel R. Gallie}

Increasing Vitamin C Content in Plant Foods to Improve Their Nutritional Value-Successes and Challenges

Nutrients 2013, 5(9), 3424-3446. 203

http://www.mdpi.com/2072-6643/5/9/3424 


\section{Preface}

Ascorbic acid is a small, simple, water soluble molecule, synthesised by most plants and animals, with the exception of humans and some animal species due to mutations in the gene encoding the terminal enzyme in the biosynthetic pathway. For humans, it is thus a vitamin (vitamin C) that must be obtained from the diet, with complete deficiency resulting in the fatal disease scurvy. Many functions have been attributed to this fascinating molecule and, despite nearly 90 years of research since its discovery, new roles are still being uncovered, including recent discoveries that it acts as a regulator of epigenetic marks and transcription factors ${ }^{(1)}$. In this volume we begin with a review by Michels and Frei on specific factors that need to be taken into consideration when carrying out vitamin $\mathrm{C}$ research. Translational research normally comprises a progression from in vitro/cell culture studies to animal models and finally to clinical trials. At each of these stages, there are requirements specific to vitamin $\mathrm{C}$ research that need to be integrated into study designs and this review describes these in detail.

Although normal vitamin $\mathrm{C}$ intake in humans is via ingestion, in the past decades there has been a surge of interest in the effects of intravenous administration of supra-physiological doses of vitamin C. This is particularly common in the treatment of cancer, and is an area of great controversy ${ }^{(2)}$, most of which can be attributed to the lack of an agreed mechanism of action and numerous issues around study design, including a lack of understanding of vitamin C pharmacokinetics ${ }^{(3)}$. Vitamin C administered intravenously bypasses the regulated intestinal uptake mechanism and results in significantly higher plasma concentrations than are obtained through oral intake. It is proposed that, at these high doses, vitamin $\mathrm{C}$ acts as a prodrug via metal ion-dependent generation of cytotoxic hydrogen peroxide, although other potential anticancer mechanisms are also possible ${ }^{(1)}$. In the contribution by Park, pharmacologic studies of the effects of high dose vitamin $\mathrm{C}$ on cancer cells are reviewed. In addition, Azqueta et al. have investigated the pro- and anti-oxidant effects of vitamin $\mathrm{C}$ on DNA damage and repair in cultured cervical cancer cells. High dose vitamin $\mathrm{C}$ has also been shown to improve the outcomes of patients with sepsis ${ }^{(4)}$. In their contribution, Mohammed et al. used a knockout murine model to investigate the effect of parenteral vitamin $\mathrm{C}$ on neutrophil extracellular trap formation, autophagy and apoptosis, in experimentally induced sepsis.

The highest levels of vitamin $\mathrm{C}$ in the body are found in the brain and neuroendocrine tissue. The brain is also relatively resistant to vitamin $\mathrm{C}$ depletion, indicating a vital role for the vitamin. Vitamin $\mathrm{C}$ likely has many functions in the brain ${ }^{(5)}$, including acting as a cofactor for monooxygenase-dependent synthesis of neurotransmitters and neuropeptide hormones, as well as recycling of the enzyme cofactor tetrahydrobiopterin. In their contribution, Harrison et al. review the role for vitamin $\mathrm{C}$ in the aging brain, covering vitamin $\mathrm{C}$ transport, animal studies, and human studies that suggest potential usefulness of vitamin $\mathrm{C}$ against cognitive decline. Iwata et al. have investigated the anti-inflammatory and antiapoptotic effects of vitamin $\mathrm{C}$ in the brains of diabetic rats with cerebral ischemia-reperfusion. They also reported vitamin $\mathrm{C}$-dependent regulation of the vitamin $\mathrm{C}$ transporter SVCT2, the transporter isoform responsible for vitamin $\mathrm{C}$ uptake in neuronal tissue ${ }^{(5)}$.

Early vitamin $\mathrm{C}$ research, particularly in animal models, indicated that food-derived vitamin $\mathrm{C}$ may have enhanced bioavailability compared with synthetic vitamin $\mathrm{C}$. This was attributed to the presence of plant-derived bio-flavonoids. We recently carried out a comparative bioavailability study in the gulonolactone oxidase knockout mouse and found significantly enhanced uptake of fruit-derived vitamin $\mathrm{C}^{(6)}$. In this volume, we present two translational comparative bioavailability studies carried out in non-smoking males, one a 
steady state study and the other a pharmacokinetic study. Vitamin C was assessed in plasma, urine, leukocytes, and skeletal muscle, and showed no differences in bioavailability between synthetic and fruit-derived vitamin $\mathrm{C}$. A review of the literature indicated that comparative differences were more likely to be observed in animal models than human studies. This is possibly due to differential expression of the vitamin C transporter SVCT1 in the intestines of humans compared with rodents, as the latter normally synthesise vitamin C endogenously and thus do not need to obtain it through their diet.

Human requirements for vitamin $\mathrm{C}$ can vary greatly depending on a number of physiological and lifestyle factors ${ }^{(7)}$. Genetic variants which affect vitamin $\mathrm{C}$ uptake and metabolism are associated with decreased plasma vitamin $\mathrm{C}$ status ${ }^{\left({ }^{8}\right)}$. In their contribution, Delanghe et al. correlate haptoglobin variant Hp2-2 and hereditary hemochromatosis, both associated with enhanced free iron and decreased vitamin $\mathrm{C}$ stability, with the incidence of scurvy observed in the European famine of the 1840s. Lindblad et al. provide a review of the transport and distribution of vitamin $\mathrm{C}$ in the body, and discuss its regulation during deficiency in cell culture studies and animal models. Plant foods are the major source of vitamin $\mathrm{C}$ in the diet, and, in his contribution to this volume, Gallie reviews strategies to increase the vitamin $\mathrm{C}$ content of food plants through increased synthesis and recycling of the vitamin.

We would like to acknowledge the authors who contributed to this volume, the reviewers of the original manuscripts published in the journal Nutrients, and the editorial staff at MDPI who contributed to the production of this volume on Vitamin C and Human Health.

Anitra C. Carr and Margreet M.C. Vissers

Guest Editors

\section{References}

1. Du J, Cullen JJ, Buettner GR. Ascorbic acid: Chemistry, biology and the treatment of cancer. Biochim Biophys Acta. 2012;1826(2):443-57.

2. Wilson MK, Baguley BC, Wall C, Jameson MB, Findlay MP. Review of high-dose intravenous vitamin C as an anticancer agent. Asia Pac J Clin Oncol. 2014;10(1):22-37.

3. Parrow NL, Leshin JA, Levine M. Parenteral ascorbate as a cancer therapeutic: a reassessment based on pharmacokinetics. Antioxid Redox Signal. 2013;19(17):2141-56.

4. Fowler AA, 3rd, Syed AA, Knowlson S, Sculthorpe R, Farthing D, DeWilde C, Farthing CA, Larus TL, Martin E, Brophy DF, Gupta S, Fisher BJ, Natarajan R. Phase I safety trial of intravenous ascorbic acid in patients with severe sepsis. J Transl Med. 2014;12:32.

5. Harrison FE, May JM. Vitamin C function in the brain: vital role of the ascorbate transporter SVCT2. Free Radic Biol Med. 2009;46(6):719-30.

6. Vissers MCM, Bozonet SM, Pearson JF, Braithwaite LJ. Dietary ascorbate affects steady state tissue levels in vitamin C-deficient mice: tissue deficiency after sub-optimal intake and superior bioavailability from a food source (kiwifruit). Am J Clin Nutr. 2011;93(2):292-301.

7. Frei B, Birlouez-Aragon I, Lykkesfeldt J. What is the Optimum Intake of Vitamin C in Humans? Crit Rev Food Sci Nutr. 2012;52(9):815-29.

8. Michels AJ, Hagen TM, Frei B. Human Genetic Variation Influences Vitamin C Homeostasis by Altering Vitamin C Transport and Antioxidant Enzyme Function. Annu Rev Nutr. 2013. 
Reprinted from Nutrients. Cite as: Michels, A.J.; Frei, B. Myths, Artifacts, and Fatal Flaws: Identifying Limitations and Opportunities in Vitamin C Research. Nutrients 2013, 5, 5161-5192.

Review

\title{
Myths, Artifacts, and Fatal Flaws: Identifying Limitations and Opportunities in Vitamin C Research
}

\author{
Alexander J. Michels * and Balz Frei
}

Linus Pauling Institute, 307 Linus Pauling Science Center, Oregon State University, Corvallis, OR 97331, USA; E-Mail: balz.frei@oregonstate.edu

* Author to whom correspondence should be addressed; E-Mail: michelsa@onid.orst.edu; Tel.: +1-541-737-5085; Fax: +1-541-737-5075.

Received: 11 October 2013; in revised form: 23 November 2013 / Accepted: 27 November 2013 / Published: 16 December 2013

\begin{abstract}
Research progress to understand the role of vitamin $\mathrm{C}$ (ascorbic acid) in human health has been slow in coming. This is predominantly the result of several flawed approaches to study design, often lacking a full appreciation of the redox chemistry and biology of ascorbic acid. In this review, we summarize our knowledge surrounding the limitations of common approaches used in vitamin $\mathrm{C}$ research. In human cell culture, the primary issues are the high oxygen environment, presence of redox-active transition metal ions in culture media, and the use of immortalized cell lines grown in the absence of supplemental ascorbic acid. Studies in animal models are also limited due to the presence of endogenous ascorbic acid synthesis. Despite the use of genetically altered rodent strains lacking synthesis capacity, there are additional concerns that these models do not adequately recapitulate the effects of vitamin $\mathrm{C}$ deprivation and supplementation observed in humans. Lastly, several flaws in study design endemic to randomized controlled trials and other human studies greatly limit their conclusions and impact. There also is anecdotal evidence of positive and negative health effects of vitamin $\mathrm{C}$ that are widely accepted but have not been substantiated. Only with careful attention to study design and experimental detail can we further our understanding of the possible roles of vitamin $\mathrm{C}$ in promoting human health and preventing or treating disease.
\end{abstract}

Keywords: vitamin C; ascorbic acid; cell culture; animals; human; study design 


\section{Introduction}

Ascorbic acid, the reduced form of vitamin $\mathrm{C}$, is an essential component of the human diet. Small amounts of ascorbic acid can prevent the deficiency disease, scurvy, while accumulation of high levels of ascorbate in plasma and tissues may protect against oxidative damage and limit inflammation. However, ascorbic acid is unlike many other vitamins owing in part to its unique redox chemistry. In addition, the "tight control" of vitamin C status and metabolism in the body, along with biological effects of supplementation that may differ between animals that can synthesize ascorbate versus species that cannot synthesize ascorbate, like humans, set it apart in the micronutrient field [1-3]. Thus, many common research practices that are sufficient for the study of other vitamins and minerals are often inadequate for the study of vitamin $\mathrm{C}$, leaving the specific challenges to the design and execution of experiments utilizing ascorbic acid underappreciated. Indeed, there are many examples of supplementation studies making poor assumptions and drawing mistaken conclusions that have persisted in the vitamin $\mathrm{C}$ literature. Although several landmark discoveries have broadened our understanding of vitamin C's role in human biology, the research is still plagued by a host of myths, artifacts, and flawed scientific reasoning that undermines efforts to determine the roles that vitamin $\mathrm{C}$ may play in human health and disease.

As we continue performing vitamin $\mathrm{C}$ research in the future, it is worthwhile to periodically review the literature for experimental approaches that may no longer be valid based on contemporary knowledge. The purpose of this article is to closely examine studies where vitamin $\mathrm{C}$ research has "failed" due to methodological, experimental, or design flaws and learn from these errors to help improve future studies, rather than to review studies that have found beneficial effects of vitamin $\mathrm{C}$ in human health, as has been done previously [1,3-5]. In so doing, we will re-evaluate two common models, cultured cells and experimental animals, and highlight aspects of each system that may contribute to erroneous conclusions. In addition, we will evaluate human research, continuing from previous reviews of the subject [1,2], including an examination of the design and execution of randomized controlled trials (RCTs). Finally, we will explore some of the technical aspects of vitamin $\mathrm{C}$ research, in order to promote better awareness of sample handling issues and analytical techniques that are critical for the proper interpretation of study outcomes.

\section{Review of Studies Using Ascorbic Acid}

\subsection{Ascorbic Acid in Human Cell Culture}

The conditions found in a typical cell culture environment promote the oxidation and subsequent degradation of ascorbic acid. Therefore, ascorbate is not usually added to cell culture media, as it often leads to the production of deleterious free radicals and reactive oxygen species (ROS). However, such conditions are non-physiological with respect to vitamin $\mathrm{C}$, which is found in all extra- and intracellular, aqueous solutions in vivo. The consequences of such "a-scorbic"—or scorbutic_cell culture environment are not fully understood, but it is obvious that any "ascorbate-dependent" enzymatic reactions, the cells' redox "milieu", and antioxidant network must be severely impaired. Furthermore, reintroducing vitamin $\mathrm{C}$ to such a cell culture system may give rise to additional artifacts. In this section, we examine the factors that lead to ascorbate oxidation in cell culture media, 
the artifacts related to the absence of ascorbate in cultured cells or its addition under normal cell culture conditions, and methods that are currently being developed to allow safe addition of ascorbate to cells for physiologically relevant research. Overall, studying vitamin $\mathrm{C}$ in cell culture is fraught with many pitfalls and results need to be approached and interpreted with care.

\subsubsection{Ascorbate Stability in Cell Culture}

The primary concern with the use of ascorbic acid in cell culture is the stability of the molecule under typical incubation conditions. Cell culture incubators use approximately $90 \%-95 \%$ air and $5 \%-10 \% \mathrm{CO}_{2}$, resulting in oxygen levels approximately $10-100$ times greater than those found in the circulation and in tissues. Increased oxygen tension can promote a pro-oxidant environment in cell culture media [6], possibly generating a wide variety of ROS that can react with, and hence deplete, ascorbate [7]. However, high oxygen levels alone are not sufficient to cause substantial ascorbic acid oxidation. Although the reduction potential of the reduced form of vitamin $\mathrm{C}$, the ascorbate mono-anion $\left(\mathrm{AscH}^{-}\right)$, is sufficient to reduce molecular oxygen to superoxide radicals (Equation 1), the reaction kinetics make this process very slow (estimated second-order rate constant, $\mathrm{k}_{2} \approx 10^{-4} \mathrm{M}^{-1} \mathrm{~s}^{-1}$ ) [8].

$$
\mathrm{AscH}^{-}+\mathrm{O}_{2} \rightarrow \mathrm{Asc}^{\cdot-}+\mathrm{O}_{2}^{\cdot-}
$$

The stability of the ascorbate mono-anion is apparent in deionized water or simple salt solutions: ascorbate added to phosphate-buffered saline (PBS) shows minimal oxidation over a six-h time period in a cell culture incubator (Figure 1, PBS) or when exposed to ambient air at room temperature (data not shown). In contrast, ascorbate incubated in cell culture media under standard conditions is rapidly oxidized [9,10] (Figure 1, RPMI). The composition of the cell culture media plays an important role in the rate of ascorbate oxidation: in serum-free RPMI medium the half-life of ascorbate is about $1.5 \mathrm{~h}$ (Figure 1); however, a more rapid loss of ascorbate has been noted in other cell culture media formulations such as MEM or Williams E media (data not shown), including more complex solutions containing serum $[6,11,12]$.

Figure 1. Ascorbate oxidation in buffer or cell culture medium. Ascorbate $(100 \mu \mathrm{M})$ was added to RPMI 1640 or phosphate-buffered saline (PBS) and monitored over time. Chelated RPMI was used after overnight treatment with Chelex 100 resin and the addition of diethylenetriaminepentaacetic acid (DTPA, $1 \mathrm{mM}$ ) as described in Methods. RPMI RT represents media not incubated under $5 \% \mathrm{CO}_{2}$ but under ambient air at room temperature.

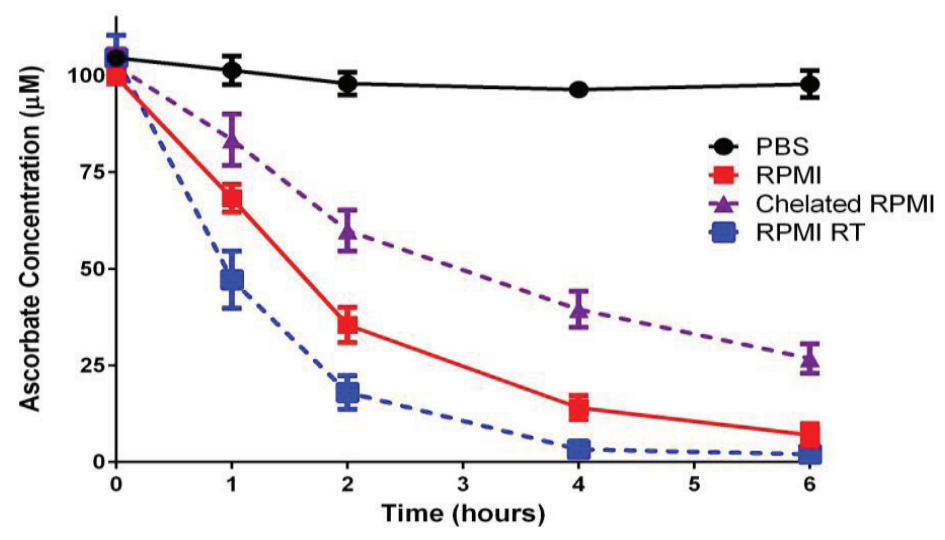


Iron and copper are present in cell culture media, either added as part of the media formulation or appearing fortuitously, as they are required for normal cell growth and function [13,14]. However, the reduced forms of iron (ferrous iron, $\mathrm{Fe}^{2+}$ ) and copper (cuprous copper, $\mathrm{Cu}^{+}$) are able to reduce molecular oxygen to superoxide and, hence, participate in the production of ROS in cell culture systems. The Haber-Weiss reaction (also known as the superoxide-driven Fenton reaction) involves the reduction of the oxidized forms of iron (ferric iron, $\mathrm{Fe}^{3+}$ ) or copper (cupric copper, $\mathrm{Cu}^{2+}$ ) by superoxide (Equation 2) and the subsequent conversion of hydrogen peroxide (formed, e.g., by dismutation of superoxide radicals) to hydroxyl radicals and hydroxide by the reduced metal ions (Fenton reaction, Equation 3) (Scheme 1). Hence, the metal ions act as catalysts and are required only in trace amounts for the Haber-Weiss reaction (Equation 4).

Scheme 1. Metal-dependent and metal-independent production of reactive oxygen species by ascorbate in cell culture media.

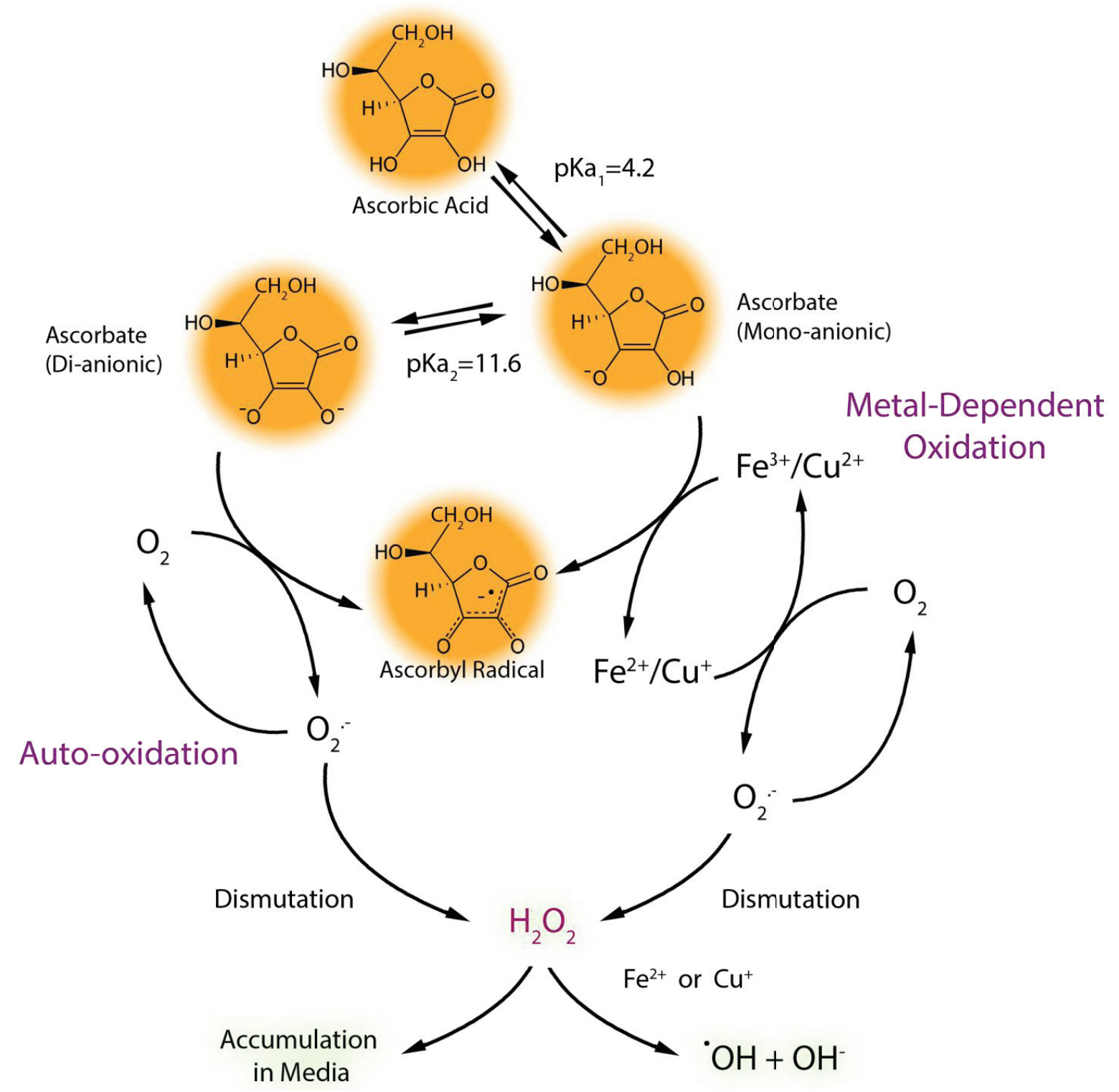


One of the most important biological functions of ascorbate is the reduction of $\mathrm{Fe}^{3+}$ or $\mathrm{Cu}^{2+}$ in the active site of enzymes, providing electrons used either in the hydroxylation of the enzymes' substrates or the maintenance of the active-site metal ion in the reduced state [3]. In the case of enzyme-bound metals, the transfer of electrons occurs in a controlled manner, which minimizes deleterious side reactions. However, in a solution of non-protein bound iron or copper, added ascorbate will reduce these metal ions (Equation 5); replacing superoxide in Equation 2, leading to superoxide production and facilitating the flow of electrons into the Haber-Weiss reaction (Equation 4). Even if media formulations are carefully controlled, trace amounts of these transition metals may be found on cell culture glassware, plastic dishes, and reagents, fueling ascorbate oxidation and ROS production $[8,15]$.

$$
\begin{aligned}
& \mathrm{Fe}^{3+} / \mathrm{Cu}^{2+}+\mathrm{O}_{2}{ }^{--} \rightarrow \mathrm{Fe}^{2+} / \mathrm{Cu}^{+}+\mathrm{O}_{2} \\
& \mathrm{Fe}^{2+} / \mathrm{Cu}^{+}+\mathrm{H}_{2} \mathrm{O}_{2} \rightarrow \mathrm{Fe}^{3+} / \mathrm{Cu}^{2+}+\mathrm{OH}^{\bullet}+\mathrm{OH}^{-} \\
& \text {Sum: } \mathrm{O}_{2}^{--}+\mathrm{H}_{2} \mathrm{O}_{2} \stackrel{\mathrm{Fe} / \mathrm{O}_{2}^{u}}{\mathrm{u}}+\mathrm{OH}^{\bullet}+\mathrm{OH}^{-} \\
& \mathrm{AscH}^{-}+\mathrm{Fe}^{3+} / \mathrm{Cu}^{2+} \rightarrow \mathrm{Asc}^{--}+\mathrm{Fe}^{2+} / \mathrm{Cu}^{+}+\mathrm{H}^{+}
\end{aligned}
$$

In biological fluids and inside cells in vivo, a combination of low oxygen levels and protein-bound metal ions greatly reduce ascorbate-mediated pro-oxidant effects [16-18]. Consequently, ascorbate in human plasma does not get readily oxidized [19]. By contrast, ascorbate addition to cell culture media results in the production of ROS, including superoxide radicals, hydrogen peroxide (from the dismutation of superoxide radicals), and hydroxyl radicals (Scheme 1) [6,15]. For this reason, ascorbic acid is often mistaken for inducing a pro-oxidant environment in cell culture systems [17,20], although the effects of hydrogen peroxide production specifically may be masked by other media components, such as serum proteins, pyruvate, or $\alpha$-ketoglutarate $[6,11,21,22]$. A more accurate description may be that ascorbate unmasks the presence of catalytic transition metals in the cell culture environment [15]. Treating cell culture media with metal chelating agents slows the rate of ascorbate oxidation (Figure 1, Chelated RPMI), increasing the half-life from approximately $1.5 \mathrm{~h}$ in standard media (RPMI) to about $2.7 \mathrm{~h}$. Interestingly, metal chelation does not completely prevent ascorbate oxidation (Figure 1), suggesting that other media components also contribute to ascorbate loss [6].

One such contributing factor may be ascorbate auto-oxidation: the direct reaction of ascorbate with molecular oxygen [8]. As stated above, the direct interaction between the ascorbate mono-anion and oxygen is highly unfavorable; however, the ascorbate di-anion ( $\mathrm{Asc}^{2-}$ ) rapidly reacts with oxygen (Equation 6). At $\mathrm{pH} 7.0$, the concentration of $\mathrm{Asc}^{2-}$ is low, but its contribution to the rate of ascorbate oxidation can become considerable under conditions when $\mathrm{pH}$ rises or with increasing concentrations of added ascorbate (Scheme 1) [23]. Cell culture media pH is often stabilized by the addition of sodium bicarbonate to offset the acidic effects of the high $\mathrm{CO}_{2}$ environment. However, when this media is exposed to air outside the incubator, the $\mathrm{pH}$ value can rise rapidly to 8.0 or higher [11]. RPMI media equilibrated to ambient air accelerates ascorbate oxidation when compared to media in a cell culture incubator (Figure 1, RPMI RT versus RPMI), decreasing the half-life from about $1.5 \mathrm{~h}$ to less than one hour. As the $\mathrm{Asc}^{2-}$ present in solution reacts with oxygen to form dehydroascorbic acid, additional $\mathrm{Asc}^{2-}$ ions are generated to re-establish the equilibrium driven 
by the $\mathrm{pH}$ (Scheme 1 and Equation 7). This continuous production of $\mathrm{Asc}^{2-}$ would fuel auto-oxidation, which is prevalent when supraphysiological levels of ascorbate are added to cell culture media, since the $\mathrm{Asc}^{2-}$ concentration would increase proportionally and greatly enhance oxidation effects. Although concentrated ascorbic acid will lower the $\mathrm{pH}$ of cell culture media, investigators usually offset this by the addition of sodium hydroxide to limit the impact of the acidic $\mathrm{pH}$ on cells. However, any shift toward a neutral or slightly alkaline environment will increase the metal-independent, $\mathrm{pH}$-driven pro-oxidant effects of ascorbate, which may explain the limited effect of metal chelators to reduce the cytotoxicity of millimolar concentrations of ascorbate towards cancer cells [24].

$$
\begin{gathered}
\mathrm{Asc}^{2-}+\mathrm{O}_{2} \rightarrow \mathrm{Asc}^{\cdot-}+\mathrm{O}_{2}^{{ }^{--}} \\
\mathrm{AscH}_{2} \leftrightarrow \mathrm{AscH}^{-}+\mathrm{H}^{+} \leftrightarrow \mathrm{Asc}^{2-}+2 \mathrm{H}^{+}
\end{gathered}
$$

Ascorbate levels can be maintained in cell culture media by frequent addition of vitamin C [9], but the persistent oxidation will continuously generate dehydroascorbic acid [25] and breakdown products, such as oxalate and threonate [26]. Extracellular concentrations of dehydroascorbic acid in excess of 1-2 $\mu \mathrm{M}$ are considered non-physiological, given its short half-life [25] and rapid uptake and by cells [27-29]. Although cells can reduce the dehydroascorbic acid to ascorbic acid, it is currently unclear what effects constant exposure of cells to high levels of dehydroascorbic acid or its breakdown products may have. For example, dehydroascorbic acid exposure has induced stress signaling and cytotoxicity in some cell types, probably due to the loss of NADPH or glutathione needed for dehydroascorbic acid reduction [30]. Oxalate has been shown to exert cytotoxic effects [31], and threonate can impact cell signaling pathways [32]. If anything, these exposures are more likely to generate artifacts as a consequence of the cell culture environment.

\subsection{2. "Cellular Scurvy"}

Even under conditions of severe vitamin $\mathrm{C}$ deficiency, i.e., scurvy, some ascorbate is still present in cells and tissues of humans in vivo. As discussed above, cell culture media are not usually supplemented with ascorbic acid, due to its inherent instability in these media. As a consequence, many researchers have reported that cells in culture are devoid of any detectible amounts of ascorbate, even with the use of extremely sensitive HPLC techniques [24,33-38]. Similarly, many complete cell culture media containing fetal bovine serum (FBS) have no detectable amounts of ascorbate [33,34,36,38], and our own analysis of various commercial sources of media and FBS has shown identical results (unpublished observations). The effects of these ascorbate-free conditions are not well defined or understood. However, it should be recognized that many immortalized cell lines likely have been maintained under scorbutic conditions for generations. In this manner, a whole host of cell culture artifacts may be expected when ascorbate is reintroduced into the system.

Cells in culture can be maintained without ascorbic acid because it is not essential to cell growth and division. The biological functions of ascorbate as an electron donor in enzymatic synthesis pathways do not have an absolute requirement for ascorbate $[3,39,40]$. These enzymes can use other reducing substrates as sources of electrons [39,41], and enzyme activity can still occur in the absence of ascorbate, albeit at a far decreased rate [42]. In particular, the $\alpha$-ketoglutarate-dependent 
dioxygenases, such as those involved in collagen synthesis and regulation of hypoxia-inducible factor $1 \alpha$ (HIF-1 $\alpha$ ), do not require ascorbate as part of the normal catalytic cycle; ascorbate is only needed to rescue the enzyme should an uncoupled enzymatic reaction occur [3]. It has also been suggested that ascorbate may function to maintain intracellular iron in the ferrous state, making it available to replenish or replace ferric iron in the active site of these enzymes [43]. It is possible that cells in culture adapt by increasing ferrous iron uptake and turn-over of iron-containing proteins, partially circumventing the need for ascorbic acid. Regardless of the mechanism, it is evident from cell culture studies that "ascorbate-requiring" enzymes, such as those involved in collagen synthesis [44], degradation of HIF-1 $\alpha$ [43], norepinephrine and $\alpha$-amidated peptide synthesis [45], and histone and DNA demethylase activity [46], still exhibit some residual activity in the absence of ascorbate. However, these enzymes have diverse effects in different tissues, and their activity in an ascorbate-free environment may not be reflective of their roles in vivo.

On the other hand, normal physiological functioning of cells can be recapitulated when ascorbate is provided. Ascorbate appears to play an important role in the normal function of cultured endothelial cells, raising antioxidant protection, reducing oxidative stress and damage, and increasing eNOS activity when compared to cells devoid of vitamin C [33,38]. These effects on eNOS, at least, appear dependent on the ability of ascorbate to enhance the stability of tetrahydrobiopterin [34] and influence AMP-activated kinase (AMPK) activity [47]. In addition, ascorbate supplementation of cultured endothelial cells tightens cell-to-cell junctions that are critical for maintaining an endothelial barrier in vivo [48] and regulates NADPH oxidase activity [49], a critical component of the inflammatory response.

It is important to note that the effect of ascorbate supplementation may also greatly vary by cell type. Many of the aforementioned effects of ascorbate are observed in primary cell lines. Although propagated in the absence of ascorbate, the response to ascorbate supplementation in these cells reflects responses seen in vivo. Cancer cell lines and other immortalized cells, however, often show cytotoxic effects in response to ascorbate addition that are not observed in primary cell lines [24]. This may be the result of adaptations that have accumulated in these cells due to the "culture shock" that alters the normal physiological responses to stimuli [6], possibly involving iron dysregulation or aberrant cell signaling responses.

\subsubsection{Proper Use of Ascorbate in Cell Culture}

Cell culture study designs may have a large impact on the results obtained with ascorbic acid. To minimize artifacts, cell culture experiments should replicate in vivo conditions as closely as possible. Ascorbate levels in media should be maintained within the physiological range of human plasma (about 5-100 $\mu \mathrm{M}$ ), and the use of supraphysiological concentrations should be avoided, unless conditions of intravenous vitamin $\mathrm{C}$ infusion are being mimicked [24]. When ascorbic acid is added to cell culture, the loss of ascorbate in the media competes with the intracellular accumulation of ascorbate. Although cells may accumulate ascorbate, once the media is depleted of ascorbic acid, intracellular ascorbate levels decline slowly through oxidation or efflux [33-35,38], once again returning cells to a depleted state. Meanwhile, degradation products may accumulate in the media or cells, which would normally be removed under physiological conditions. In addition, cells without 
ascorbic acid are not a proper control for ascorbate treatment, as some level of ascorbate is always present in all cells of the human body.

Ascorbate should be added to culture media in a way that limits the rate of ascorbate oxidation and the effects of ROS that may be formed. The use of serum-free media that has been supplemented with transferrin to control iron or copper redox chemistry shows great promise in stem cell studies [46]. The stability of ascorbate can be enhanced by low oxygen growth conditions and the use of stabilized derivatives of ascorbate such as ascorbate-2-phosphate (AAP) that cannot participate in redox chemistry outside the cell yet can maintain physiological intracellular ascorbate levels [9]. Furthermore, pyruvate or $\alpha$-ketoglutarate in cell culture media can be used to blunt the effects of any hydrogen peroxide formed $[21,22]$, although they will not prevent the loss of ascorbic acid.

Due to the inherent instability of the molecule, there is an absolute necessity for monitoring ascorbic acid levels in media and cells during cell culture experiments. As with animal and human studies described below, this is the only method currently available to assess the vitamin $\mathrm{C}$ status of cells, and is a valuable tool for understanding the mechanisms of ascorbate's biological actions. Unfortunately, ascorbate levels are rarely measured in cell culture, animal, or human studies, which severely limits their validity and any conclusions that can be drawn.

Despite precautions, conventional cell culture conditions will promote an environment in which ascorbate artifacts are commonplace. Culturing cells with vitamin $\mathrm{C}$ requires control over many aspects of the media and culture conditions that has heretofore been lacking. Monitoring ascorbate levels and limiting oxidation may not be sufficient to fully recapitulate the physiological roles of vitamin C. While redesigning cell culture systems to support biologically relevant reactions of ascorbic acid and eliminate artifacts may limit the practicality of experimental designs, these changes are necessary for cell culture models to have continued use in vitamin $\mathrm{C}$ research.

\subsection{Animal Studies Involving Ascorbic Acid}

Since it can be synthesized de novo and is not an essential nutrient for most animals, ascorbate should not be referred to as a vitamin in these animals. Conceptually, this questions the use of most experimental animal models, in particular rats and mice, to study the role of ascorbic acid in human health and disease [50]. This echoes recent studies that have cast doubt on the pervasive use of rodent models to mimic human inflammatory responses [51]. Although genetically variant strains incapable of synthesizing ascorbic acid have been established, none of these models fully recapitulate vitamin $\mathrm{C}$ transport functions or the effects of vitamin $\mathrm{C}$ depletion and repletion observed in humans or guinea pigs [50]. Indeed, there is evidence that regulation of vitamin $\mathrm{C}$ transport was altered during the evolutionary loss of L-gulonolactone oxidase (GULO) expression, which does not occur in genetic knockouts. Here, we examine the current issues related to the use of animal models in ascorbate research. Overall, if animals are to be used, the limitations imposed by the model should be well understood. Animal experiments should be avoided if comparable studies can be performed in human subjects. 


\subsubsection{When a Vitamin in not a Vitamin}

Most animal species express a functional copy of L-gulonolactone oxidase, an enzyme with the synthesis of L-ascorbic acid as its only known function. In these animals, the regulation of GULO activity appears to depend primarily on substrate availability, namely the production of gulonolactone as a branch product from glucuronate synthesis [26] derived from UDP-glucose, a product of glycogen breakdown [52,53]. Thus, agents that stimulate glycogenolysis also stimulate ascorbate synthesis when an animal is in a fed state (i.e., when glycogen is present); in contrast, prolonged fasting causes ascorbate synthesis to decline [54]. UDP-glucuronate is also needed for glucuronidation of xenobiotics, and there is a correlation between ascorbate synthesis activity and xenobiotic metabolism [26].

In each of the few mammalian species that do not synthesize ascorbic acid, such as guinea pigs, fruit bats, and primates, the loss of GULO has occurred at a genetic level. Although the mutations accumulated in this gene differ [55], the result is essentially the same: a loss of GULO activity. These species have adapted to the loss of de novo synthesis by consuming primarily plant sources of ascorbate. While all non-synthesizing animal species are at risk of developing scurvy and may die when vitamin $\mathrm{C}$ intake ceases for an extended period of time, this is a condition that does not occur normally in other animals, including most commonly used experimental animal models, such as mice and rats. Thus, in contrast to ascorbic acid-synthesizing species, in non-synthesizing species, including humans, vitamin $\mathrm{C}$ absorption is required to prevent deficiency and maintain health. This represents a fundamental shift from a perspective of diet-health interactions, as the absorption-derived versus glycogen-derived source of ascorbic acid may represent differences in ascorbate transport and carbohydrate metabolism that exist in humans and synthesizing animals.

Although evidence in the literature is limited, both rats and mice appear to poorly absorb ascorbate from the diet. Early studies on the small intestine in rats revealed that ascorbate uptake is a passive process, resulting mainly in intestinal mucosa accumulation but not transport to the circulation [56]. Several studies have demonstrated a profound difference in absorption between the rat and guinea pig small intestine [57-59], the latter displaying a robust transepithelial transport system that is sodium-dependent [59-61], similar to the human ileum [61,62]. A recent study in rats monitored the absorption of a single oral dose of ascorbic acid or dehydroascorbic acid given by gavage. The administration of $12 \mathrm{mg}$ of dehydroascorbic acid led to a significant increase in plasma ascorbic acid concentration, but administration of $12 \mathrm{mg}$ of ascorbic acid did not [63]. An inability to efficiently transport vitamin $\mathrm{C}$ is also seen in feeding studies, where mice fully capable of synthesizing ascorbate required at least $45 \mathrm{mg}$ of ascorbate per day in their diet to show any significant increases in plasma ascorbate concentration [64]. Although it is difficult to extrapolate these doses to humans, allometric scaling based on calorie consumption suggests that a dose of $45 \mathrm{mg}$ in a $20 \mathrm{-g}$ mouse is equivalent to about $3 \mathrm{~g}$ in a 70-kg person [65].

Studies on the bioavailability of different forms of ascorbic acid supplements also support the notion that ascorbate is poorly absorbed by rodent models. For example, Ester-C, a calcium ascorbate-threonate mixture, is reported to be more bioavailable in ODS rats than an equivalent dose of ascorbic acid [66]. However, the same comparison of supplements shows that Ester-C has a lower rate of absorption - and certainly no enhanced bioavailability — in human volunteers [67]. 
Furthermore, work in GULO knockout mice suggested an enhanced bioavailability of vitamin C contained in a kiwifruit puree compared to a pure ascorbic acid supplement gel [68]. However, more recent human data suggest that vitamin $\mathrm{C}$ from kiwifruit or a supplement is equally bioavailable [69].

Overall, these data support a species-specific route of ascorbate absorption. While indirect absorption of ascorbate may occur in rats and mice-likely mediated by ascorbic acid oxidation and transient formation and transport of dehydroascorbic acid - there is clear evidence of an active, sodium-dependent transport of vitamin $\mathrm{C}$ in guinea pigs and humans. Therefore, the use of rats or mice as a model of human vitamin $\mathrm{C}$ absorption and metabolism is ill advised. As indicated above, such studies with experimental animals should be avoided if they can be performed in humans, unless the purpose of the study is to better understand ascorbic acid absorption and metabolism in rodents. More importantly, no conclusions should and can be drawn from such studies for human vitamin $\mathrm{C}$ transport or metabolism.

Poor uptake of dietary ascorbate, or complete lack of it, is expected in animals that synthesize ascorbate, as they do not have a need for dietary ascorbate. In addition, high levels of ascorbate in the intestine would likely cause down-regulation of tissue ascorbate synthesis. However, the relationship between absorption and synthesis does not appear to be this simple. Genetically altered rat and mouse models lacking ascorbate synthesis have low tissue levels of ascorbate without supplementation $[64,68,70,71]$. Although these animals absorb dietary ascorbate, the levels needed to prevent scurvy or saturate tissues are relatively high compared to guinea pigs and humans on a body-weight basis (Table 1). These levels are especially high when contrasted to food sources of ascorbate. As an example, to saturate all tissues, GULO knockout mice need to be supplemented with $3.3 \mathrm{~g} / \mathrm{L}$ of ascorbate in the drinking water, resulting in an intake of approximately $16.5 \mathrm{mg}$ per day [68,72]. Based on allometric scaling, this dose in mice corresponds to about $1 \mathrm{~g}$ per day in a 70-kg person [65]. Although the metabolic rate of these animals likely contributes to high ascorbate requirements, this does not sufficiently explain data supporting an excessive inefficiency of intestinal absorption.

\subsubsection{Transporter Troubles}

Based on the evidence presented above, animals unable to synthesize vitamin $\mathrm{C}$ appear to display an active transport system in the intestine that is both sensitive to varying levels of ascorbic acid and sodium-dependent. This implicates a role of the sodium-dependent vitamin $\mathrm{C}$ transport (SVCT) proteins. SVCT1 has been implicated in dietary ascorbate absorption in human enterocytes, as it is found primarily on the luminal side of intestinal cells and involved in trans-epithelial ascorbate transport [73-75]. However, mice genetically modified to remove functional expression of the SVCT1 gene (Slc23 $\mathrm{a}^{-1-}$ ) show similar intestinal ascorbate absorption as wild type mice [76], suggesting SVCT1 is not involved in this process in these animals. Indeed, the concentration of ascorbic acid needed in the drinking water of GULO knockout mice to maintain tissue saturation $(3.3 \mathrm{~g} / \mathrm{L}$ or $18.75 \mathrm{mM})$ far exceeds the transport capacity of SVCT 1 , which has a measured $K_{\mathrm{m}}$ below $250 \mu \mathrm{M}$ [77]. On the other hand, the ability of rats to absorb dehydroascorbic acid has been linked to the intestinal expression of glucose transport proteins (GLUTs), namely GLUT2 and GLUT8 that show $\mathrm{K}_{\mathrm{m}}$ values of approximately $2-3 \mathrm{mM}$ [63]. 


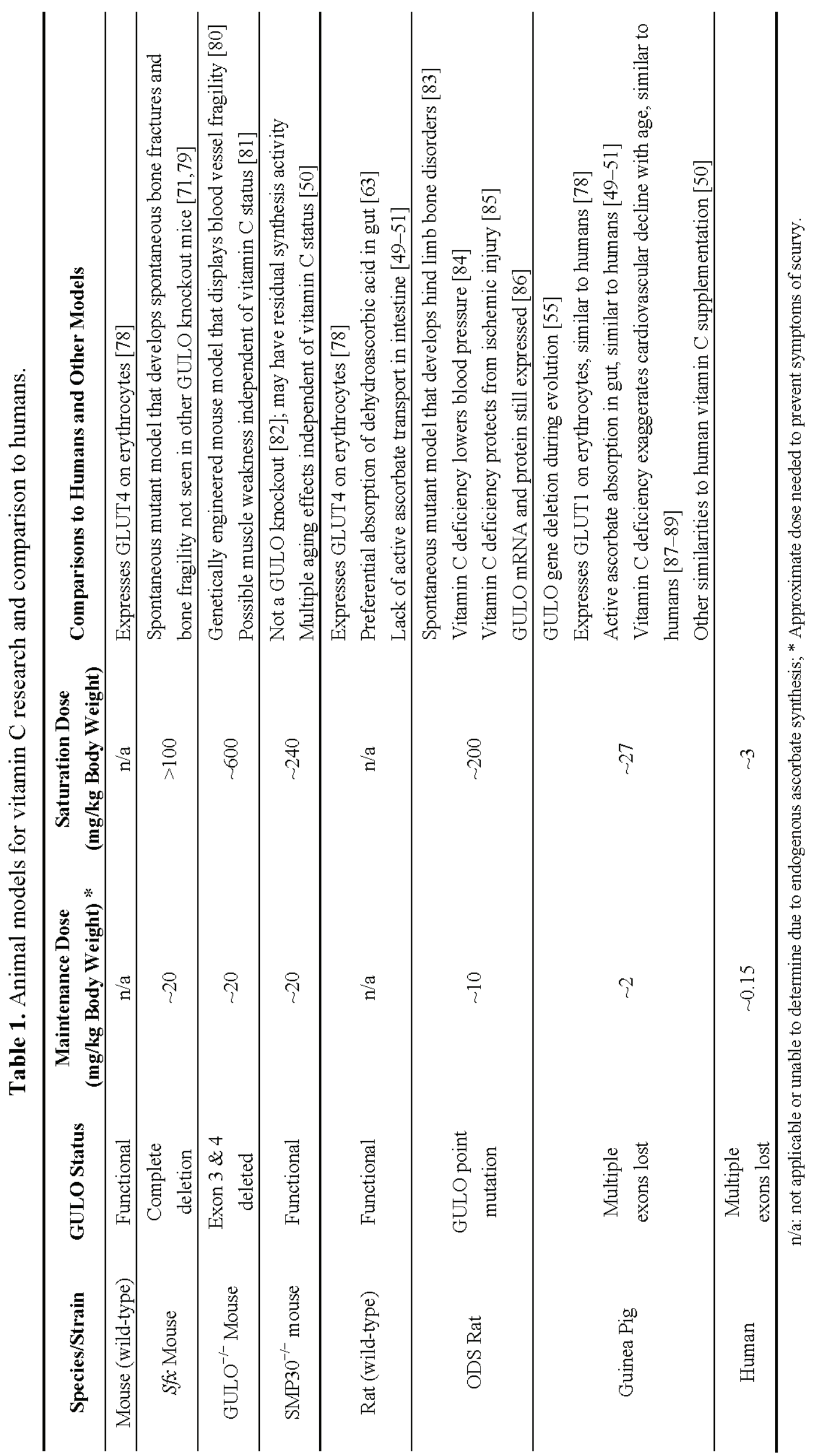


Unfortunately, little is known about differences in expression or regulation of the SVCTs between humans, guinea pigs, rats, and mice. Comparison of the amino acid sequences of mouse, rat, and human SVCT1 shows that seven amino acid residues are missing from the human SVCT1 sequence [90]. This deletion creates a potential protein kinase C (PKC)-binding site in the human sequence not found in rats or mice. Stimulation of PKC has been implicated in membrane trafficking of human SVCT1 [91]. Additionally, the $C$-terminal sequence of rat and mouse SVCT1 has a one-amino acid change in a critical four-amino acid sequence required for apical targeting of the transport protein [92]. SVCT2, responsible for uptake of ascorbate from the blood stream, contains an additional 56 amino acids in the $N$-terminal region of the human protein sequence that are not found in rats and mice [90]. The only study to date on a species difference in SVCT regulation found that buthionine sulfoximine (BSO), a glutathione synthesis inhibitor, reduced the expression of both SVCT1 and SVCT2 in rat liver cell lines, a phenomenon not observed in human hepatoma cells [93].

Although the absorption of dehydroascorbic acid is not considered a major pathway for the maintenance of whole body ascorbic acid levels, it is considered an important scavenger pathway to maintain cellular ascorbate levels if extracellular ascorbate is oxidized to dehydroascorbic acid [94]. Since human erythrocytes do not express SVCT proteins [95], ascorbic acid transport across the plasma membrane is facilitated by dehydroascorbic acid uptake mediated through GLUTs.

Recent evidence suggests that GLUT1 is responsible for dehydroascorbic acid uptake in human red blood cells, enhanced by the co-expression of a protein called stomatin during erythropoiesis [78]. By contrast, mice lose GLUT1 during maturation, and GLUT4 is the predominant glucose transporter expressed in adult mouse erythrocytes [96]. GLUT4, by contrast, has diminished capacity to transport dehydroascorbic acid [97], which is reflected in the transport capacity of murine erythrocytes [78]. Interestingly, GLUT1 expression and the associated dehydroascorbic acid transport in red blood cells are found only in species unable to synthesize ascorbate-lacking from every animal with endogenous ascorbate-synthesis capacity, even in closely related species such as chinchilla and guinea pigs, or lemurs and margot monkeys [78]. Furthermore, this remarkable switch in glucose transport proteins may be indicative of differences in global gene expression patterns between ascorbate synthesizing versus non-synthesizing animals.

\subsubsection{Choose Models with Care}

Although wild-type rat and mice models are still being employed for ascorbate research, the presence of endogenous synthesis alone would suggest that these animals represent a poor model for understanding the role of vitamin $\mathrm{C}$ in human health and disease. At the very least, animal models without endogenous ascorbate synthesis should be used. Five rodent models exist, although each with their own limitations (Table 1). Only one of these models, the GULO knockout mouse, was specifically engineered to disable ascorbic acid synthesis [80]. The other mouse and rat models are not genetically engineered and display individual characteristics that poorly recapitulate the effects of human vitamin C deficiency [50]. On the other hand, guinea pigs have lost GULO activity during evolution and likely display compensatory genetic adaptations similar to humans relating to the loss of ascorbate synthesis, such as GLUT1 activity on erythrocytes (see above). Although guinea pigs are currently being employed by some research groups, the lack of molecular and genetic tools for this animal model will likely drive the continued use of rats and mice models instead. 
Regardless of the species, experimental conditions, or route of ascorbate administration, it needs to be stressed that measuring ascorbate levels is absolutely necessary for any animal experiment. One of the most frequent assumptions is that dietary ascorbate will always result in a change in tissue and plasma ascorbate levels. However, the relative contributions of de novo ascorbate synthesis (if present), absorption of dietary ascorbate, and ascorbate distribution throughout the body are not inherently predictable and must be directly measured. Studies using oral ascorbate administration can be complicated by animals with poor absorptive capacity, and these animals may rely on the production of dehydroascorbic acid in the intestine. Furthermore, stable ascorbate derivatives used in animal diets, such as ascorbate phosphate or ascorbate palmitate, are not well studied and may not distribute throughout the body as ascorbate would if provided by itself. It is also not recommended to rely on intravenous, intraperitoneal, or subcutaneous ascorbate injections, since they may cause the production of hydrogen peroxide in the extracellular space [98].

Since ascorbate is a dietary factor in humans versus a product of carbohydrate metabolism in mice and rats, and there are differences in SVCT and GLUT regulation between species, the results of most animal studies with ascorbic acid cannot be extrapolated to humans. Continued evaluation of rodents as relevant models to study ascorbic acid in human health and disease depends on a thorough understanding of the differences between rodents and humans with respect to ascorbate metabolism, regulation, and biological functions. However, it seems apparent that, aside from the guinea pig, animal studies should be avoided as much as possible, with continued focus placed on conducting relevant human studies instead.

\subsection{Human Studies with Vitamin C}

Randomized controlled trials are considered the "gold standard" by the Institute of Medicine's Food and Nutrition Board for determining efficacy of micronutrients, including vitamin $\mathrm{C}$, in promoting human health and preventing or treating disease [1,2]. However, RCT study designs have serious limitations and pitfalls, and require careful scrutiny to avoid misinterpretation of results and erroneous conclusions. The drive to establish correlations in prospective cohort studies or show a treatment effect in RCTs often ignores assessment of intermediary biomarkers or other biological measures that could provide insights into mechanisms and help establish causation. Indeed, many vitamin $C$ supplementation studies are performed in combination with other supplements, most often "antioxidant vitamins" E and $\beta$-carotene, and fail to assess the subjects' vitamin $\mathrm{C}$ status at baseline and following supplementation or biomarkers related to vitamin C's proposed mechanism of action, e.g., antioxidant or anti-inflammatory effects [2]. Understanding the study population, limitations of study design, and the nuances of the ascorbic acid chemistry is necessary to avoid many of these pitfalls and artifacts. Furthermore, myths about the health effects of vitamin $\mathrm{C}$ supplementation plague the entire body of research and may prompt the spread of misinformation. In this section, we explore the limitations to human research with vitamin $\mathrm{C}$ and review the myths surrounding vitamin $\mathrm{C}$ supplementation. In the future, it will be necessary to promote a new approach to conducting vitamin $\mathrm{C}$ research in humans. 


\subsubsection{Vitamin C RCTs: Failures in Design}

Many, but not all, prospective cohort studies have observed inverse associations between vitamin $\mathrm{C}$ intake or plasma levels and the incidence of chronic diseases, including coronary heart disease, ischemic stroke, hypertension, and certain types of cancer $[1,15]$. However, several large RCTs have shown no benefit of vitamin $\mathrm{C}$ supplementation when taken alone or in combination with other micronutrients [28,51]. This apparent failure of vitamin C supplements to affect human health can be attributed to many factors related to study design. The most predominant is the use of the standard RCT study design, which is intended to test the safety and efficacy of a pharmaceutical drug in individuals that are at high risk or are suffering from a condition or illness. By contrast, enrollees in vitamin C supplementation studies, and diet-related RCTs in general, are usually health-conscious individuals who are likely to consume an above-average diet and maintain a healthy body weight [99]. As a consequence, these individuals have a lower disease incidence and a better nutritional status, including vitamin $\mathrm{C}$, than the general population - both of which negatively affect the statistical power of the study. Statistical power is further compromised by the fact that there is no true placebo group in these studies, as even the non-supplemented subjects continue to obtain vitamin $\mathrm{C}$ from their diet throughout the duration. These and other serious flaws in study design, including lack of a single supplement (vitamin $\mathrm{C}$ only), quality of the methodology employed, and lack of discrimination by genetic polymorphisms, have led some to the unfortunate conclusion that very few well-designed, well-controlled trials of supplemental vitamin $\mathrm{C}$ have ever been conducted [2].

One reason previous studies have failed to show health benefits of vitamin $\mathrm{C}$ may be the assumption that an individual's plasma or body ascorbate status directly reflects their dietary or supplemental intake of vitamin $\mathrm{C}$. To the contrary, analysis of food frequency questionnaires has revealed that there is little correlation between assessed vitamin $\mathrm{C}$ intake and plasma ascorbate levels [100], likely due to inaccuracies in dietary assessment methodology using food frequency questionnaires or food diaries, inaccuracies in the USDA nutrient database, loss of ascorbate during storage, cooking or processing, and large inter-individual differences in vitamin $\mathrm{C}$ absorption and metabolism. An example of the latter is the lower plasma ascorbate levels observed in the elderly when compared to younger adults consuming equivalent amounts of vitamin $\mathrm{C}$ [101], suggesting changes in absorptive capacity with age. In addition, smoking, chronic aspirin use, high alcohol consumption, high BMI, and low socioeconomic status [102] are all factors that have been associated with lower plasma vitamin C levels. Furthermore, genetic variation in SVCTs, haptoglobin, and glutathione $S$-transferases also may lead to altered plasma ascorbate levels depending on the various single nucleotide polymorphisms involved [103]. In each of these cases, the exact relationship of plasma vitamin $\mathrm{C}$ status with vitamin $\mathrm{C}$ consumption is unclear. However, this explains why food frequency questionnaires have little predictive value for evaluating the effect vitamin $\mathrm{C}$ consumption on disease risk, while plasma ascorbate levels display clear inverse relationship [104]. Therefore, the use of dietary analysis in studies pertaining to vitamin $\mathrm{C}$ should be only a secondary measure of vitamin $\mathrm{C}$ status, at best. The gold standard must be measurement of plasma ascorbate levels.

Human pharmacokinetic data show that there is a sigmoidal dose-response relationship between plasma ascorbate levels and vitamin C dose for both men [105] and women [106]. Those with frank deficiency have plasma ascorbate values below $11 \mu \mathrm{M}$ and are at risk for scurvy because 
corresponding tissue levels are low. Marginal deficiency $(<23 \mu \mathrm{M})$ and suboptimal concentrations $(<50 \mu \mathrm{M})$ are levels that exist on the steep part of the dose-response curve, thought to be indicative of increasing levels of ascorbate in most tissues, based on correlations with ascorbate levels in circulating leukocytes. Plasma concentrations start leveling off at doses above $200 \mathrm{mg} /$ day and approach maximal levels in the range of 60-90 $\mu \mathrm{M}$, when the threshold levels for renal reabsorption are reached and leukocytes also are saturated with vitamin $\mathrm{C}$.

There are limitations to these pharmacokinetic data that must be recognized. First, the studies were performed in a small number of young, healthy individuals and, hence, are limited in their statistical power. As described above, many factors can influence the relationship between plasma and dietary ascorbate, including age and disease status, which may affect vitamin $\mathrm{C}$ transport and metabolism. Therefore, vitamin $\mathrm{C}$ pharmacokinetics may be substantially different in old or diseased individuals compared to young, healthy subjects. Second, we cannot assume that tissue saturation occurs in every organ along the same continuum of plasma ascorbate levels. Studies in animals show preferential uptake and retention of ascorbate in organs that have high requirements for the vitamin $[68,70,107]$. Thus, the brain may saturate at lower ascorbate intake and plasma levels than other organs, such as liver or circulating cells. Data in human volunteers suggest that in skeletal muscle ascorbate is more responsive to changes in plasma ascorbate status than in neutrophils or mononuclear cells [108], suggesting different routes of vitamin $C$ transport and levels of tissue saturation. One study showed a continued uptake and no apparent saturation of ascorbate in the human eye lens with increasing plasma ascorbate levels [109]. Thus, the implication here is that transport rate and saturation point in various cells and tissues of the body are variable and may not be directly extrapolated from plasma ascorbate levels.

From the above considerations, three critical issues emerge in relation to RCT design. First, individuals recruited for a research study should have low plasma ascorbate levels at baseline to increase the likelihood of affecting changes in ascorbate status in tissues through vitamin $\mathrm{C}$ supplementation. Subjects already consuming enough vitamin $\mathrm{C}$ to provide near-maximal or saturating plasma and tissue levels of ascorbate are highly unlikely to demonstrate any further biological or health effects upon vitamin $\mathrm{C}$ supplementation. Second, the intervention must be proven effective, demonstrating - at the very least - an elevation in plasma ascorbate steady-state levels. Again, if a research subject's vitamin C status does not change, no changes in health or disease outcomes can be expected unless it can be supported by an alternate mechanism. In many cases, no biological effect can be expected of increasing vitamin $\mathrm{C}$ levels if no functional deficit is present. For instance, although studies support the use of vitamin $\mathrm{C}$ in improving vascular function and reducing blood pressure [5], continued supplementation of vitamin $\mathrm{C}$ when plasma levels are already at saturation will not yield additional vasodilation, and changes in blood pressure are not expected if a subject is already within a healthy blood pressure range. Lastly, in the absence of tissue ascorbate measurements, the study design and endpoints must relate to our knowledge about the distribution of vitamin $\mathrm{C}$ in the body. For example, if brain ascorbate levels are near saturation at low vitamin $\mathrm{C}$ intake and plasma levels, it is unreasonable to expect an effect on brain function over a wide range of intake and plasma ascorbate levels.

Along with attention to these issues, there needs to be a push toward measurement of mechanism-based endpoints and clinically-relevant, intermediary biomarkers to assess the effects of 
vitamin $\mathrm{C}$ supplementation. As mentioned above, in biological systems vitamin $\mathrm{C}$ always acts as a reductant, which may be expressed as antioxidant, anti-inflammatory, enzyme cofactor, or pro-oxidant activity, depending on the specific context. Commonly in clinical trials, vitamin $\mathrm{C}$ is assumed to act as an antioxidant or an anti-inflammatory without actual measurements to support such a role, which limits the evaluation of the trial as a successful intervention and the interpretation of the data. Measures of oxidative stress, such as $\mathrm{F}_{2}$-isoprostanes [110], serve as clinically relevant markers suggesting an antioxidant effect, while changes in circulating levels of inflammatory markers such as C-reactive protein or soluble cellular adhesion molecules can support an anti-inflammatory effect [111]. Without these corroborating data, results from RCTs will continue to be limited in impact and relevance.

With correct study design, it is generally believed that quality research data can be obtained from human subjects. However, the costs of large, tightly controlled RCTs with vitamin C alone are likely to be prohibitive to the implementation of such studies. Therefore, it is likely that smaller, high-quality intervention trials will have to suffice in the future.

\subsubsection{Technical Issues of Human Studies}

Vitamin C research is heavily dependent on accurate assessment of ascorbate in biological samples. Unfortunately, there is no standard method for measuring vitamin $\mathrm{C}$ that has been applied across the field. It is generally acceptable practice to preserve samples in acid or methanol after sample extraction. Furthermore, it is preferred that a direct measure of ascorbic acid be made, such as HPLC separation followed by electrochemical detection (ECD). Other chromatographic methods are generally avoided, especially those that require oxidation of the sample followed by derivatization as these procedures can generate erroneous results. The detection of dehydroascorbic acid cannot be achieved with ECD unless the sample is first treated with a reductant, obtaining total ascorbate levels from which dehydroascorbic acid levels can be inferred [112,113]. However, the value and interpretation of dehydroascorbic acid measurements in biological samples is questionable (see below).

The labile nature of ascorbic acid outside the body underscores the need for controlled conditions during collection, processing, and storage of biological samples. In many human studies, poor standards in obtaining blood or tissues specifically for vitamin $\mathrm{C}$ analysis are complicated by a lack of controlled sample handling in many clinical settings. Phlebotomy is common practice, but many factors can contribute to the instability of ascorbic acid in biological samples. Although the use of vacutainers for plasma samples is generally acceptable for vitamin $\mathrm{C}$ analysis, the choice of vacutainer type and anticoagulants can influence the results obtained [114]. As an example, plasma ascorbate levels were determined from five individuals using various anticoagulants or no anticoagulant (serum) as a control. Despite individual variability in plasma ascorbate levels reflected in the standard error, the data (Figure 2) suggest that the use of $\mathrm{K}_{2}$ EDTA vacutainers results in a significant loss of ascorbate compared to both sodium and lithium heparin containers. Consistent with these data, EDTA has been shown to accelerate the oxidation of ascorbate in whole blood and plasma $[115,116]$ and be unable to prevent the loss of ascorbate in the presence of iron or copper [8]. In addition, it has been suggested that fluoride and serum vacutainers should be avoided for vitamin C analysis [114]. 
Figure 2. Vacutainer effects on plasma ascorbate levels. Plasma ascorbate concentrations were determined from different anticoagulant-containing or untreated vacutainers as described in Materials and Methods. Plasma ascorbate means are the average and standard error from five different subjects. ANOVA analysis shows a significant $(p<0.05)$ decline in plasma ascorbate levels in $\mathrm{K}_{2}$ EDTA vacutainers when compared to lithium or sodium heparin vacutainers as denoted by asterisk (*).

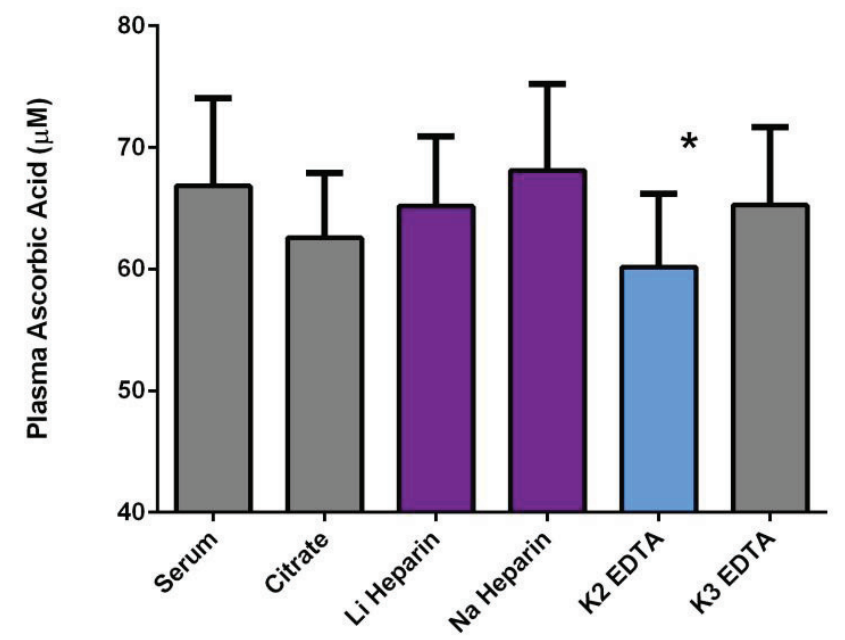

The oxidation of vitamin $\mathrm{C}$ in plasma is accelerated by heat, light, and elevated $\mathrm{pH}$, similar to cell culture media as described above. Sample mishandling can cause the aberrant generation of dehydroascorbic acid in the sample and, over time, will cause a loss of total ascorbate. For instance, by careful preparation of the sample under nitrogen and limited exposure to heat and light, dehydroascorbic acid levels can be minimized (Figure 3). More reasonable, standard preparation methods with brief exposures to air, light, and heat result in little change in plasma ascorbate levels (Figure 3b). On the other hand, exposing samples to room temperature for hours not only can result in a significant decline in (reduced) ascorbic acid, but a loss of total ascorbic acid as well (Figure 3a). Not only does this result in an inaccurate estimate of dehydroascorbic acid levels in the exposed sample (Figure 3c) but the loss of total ascorbate suggests degradation of dehydroascorbic acid has also occurred.

The implication of these data (Figure 3) and others [114-116] is that care in sample handling with concern for ascorbate oxidation is crucial for accurate ascorbate analysis. Study designs must incorporate specific handling conditions of samples intended for ascorbate analysis (ideally by immediate plasma isolation, rapid acidification, and freezing below $-20{ }^{\circ} \mathrm{C}$ ) to avoid misinterpretations compounded by the use of poorly preserved samples. Furthermore, it also suggests that the presence of dehydroascorbic acid in clinical samples is more a measure of sample handling than a biologically relevant marker of in vivo oxidative stress. 
Figure 3. Effects of sample handling on plasma ascorbate and dehydroascorbic acid concentrations. Vacutainers with blood samples were prepared under oxidation controlled, standard and exposed conditions as described in Materials and Methods. Plasma total ascorbate (a) and reduced ascorbate (b) levels are highest in controlled samples, showing declines under standard and exposed preparations that are reflected in calculated dehydroascorbic acid levels (c). Significant changes were observed in the exposed group when compared to controlled or standard samples as determined by ANOVA, and denoted with an asterisk (*).

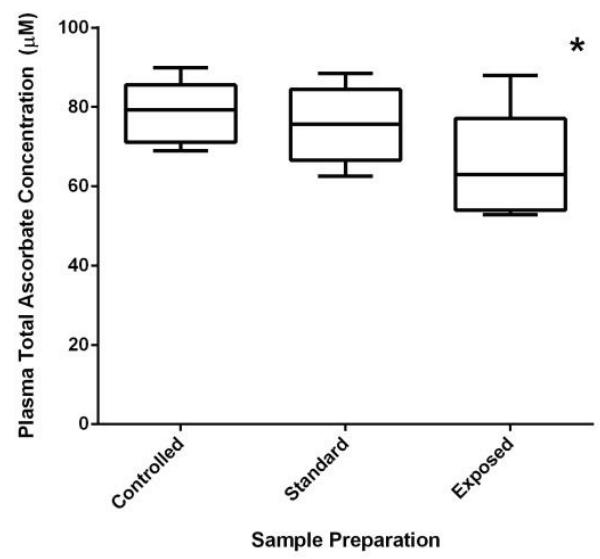

(a)

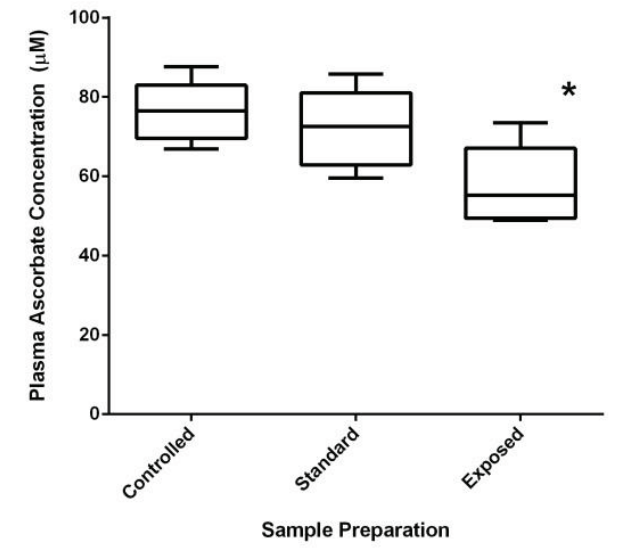

(b)

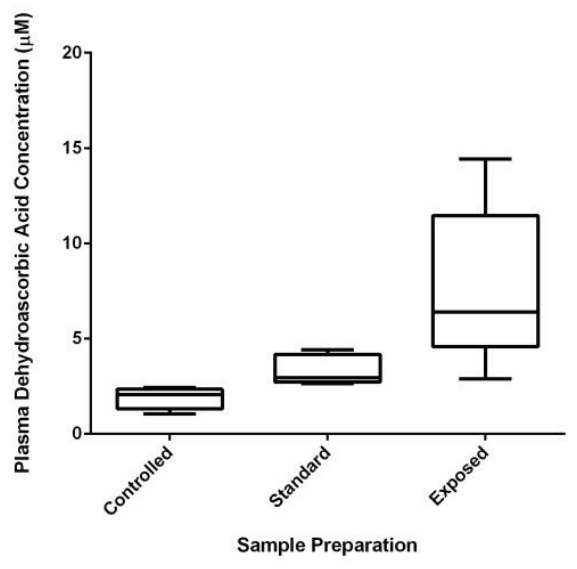

(c)

\subsubsection{Health Effects of Vitamin C: Reality versus Mythology}

Many excellent evidence-based reviews have summarized the health effects of vitamin $\mathrm{C}$, focusing on vitamin C's possible role in the prevention or treatment of cardiovascular disease, cancer, diabetes, and other diseases [1,2,4,117]. However, there are a number of health effects attributed to vitamin $\mathrm{C}$ supplementation that are not supported by controlled trials with fundamental, mechanism-based endpoints. Opponents of vitamin C supplementation have sensationalized studies that have suggested deleterious health claims, most of which are of little consequence to the general population. For example, since vitamin C promotes iron absorption [118], this led to claims that vitamin C supplements were detrimental in individuals suffering from iron overload and hemochromatosis. On the contrary, in animals and humans with iron overload, high plasma ascorbate 
levels are protective against oxidative damage induced by excess iron [18,19]. Indeed, the recommendation for these individuals is to not avoid ascorbate, but instead limit dietary iron intake.

Another commonly cited risk of ascorbate supplementation is the formation of kidney stones, as oxalate is a product of dehydroascorbic acid breakdown. The evidence linking ascorbate supplement use and incidence of kidney stones in otherwise healthy individuals is mixed [119-121]. Large amounts of oxalate derived from ascorbate would require excessive vitamin $\mathrm{C}$ oxidation, but the mechanism underlying this oxidation has not been explored. Studies that suggest an increased risk observed it in individuals taking more than $1000 \mathrm{mg}$ of vitamin C per day, in far excess of the amount that can be obtained from food sources. Without further study, it is prudent to caution individuals with kidney disease or a history of kidney stones against taking large amounts of vitamin C supplements. Unfortunately, this has also resulted in practitioners to advise individuals undergoing dialysis to severely restrict vitamin $\mathrm{C}$ consumption, leading to deficiencies [122].

The consumption of large doses of vitamin $\mathrm{C}$ supplements has also been occasionally associated with skin rash, heartburn, nausea, and diarrhea. These are usually the result of the formulation of the vitamin $\mathrm{C}$ tablets but may also be caused by excessive consumption of vitamin $\mathrm{C}$ in a short period of time. Large doses of vitamin $\mathrm{C}$ have been anecdotally associated with vitamin $\mathrm{B}_{12}$ deficiency and systemic conditioning (also known as "rebound scurvy"), conditions that have never been documented clinically [123]. Patients with glucose-6-phosphate dehydrogenase (G6PD) deficiency have also been cautioned against taking vitamin $\mathrm{C}$ supplements, due to reports of hemolytic anemia that have also not been substantiated [124].

One of the most persistent health claims for vitamin $\mathrm{C}$ supplementation is the prevention and treatment of the common cold. Recent meta-analyses of the data from 70 years of placebo-controlled trials demonstrated an overabundance of poorly controlled trials with no apparent focus on the mechanism and biological relevance of vitamin $C$ supplementation $[125,126]$. Despite the large number of research studies, the evidence supporting the effects of vitamin $\mathrm{C}$ supplements on cold incidence and duration has been relatively weak, with the exception of marathon runners, skiers, soldiers in subarctic conditions [125], or individuals with chronic gastritis [127]. A tendency to include older data is a common pitfall for the analysis of vitamin $\mathrm{C}$ research, performed when our understanding of vitamin $\mathrm{C}$ in biology was limited, and thus allowing for a bias toward poorer designed studies. Our understanding of vitamin C's role in biology has improved over time, leaving a re-analysis of these studies unable to provide any substantial conclusions with respect to the common cold and other proposed health effects of vitamin $\mathrm{C}$ supplementation. To resolve the controversies, a modern approach of evaluating rigorously designed, mechanism-based studies is necessary.

\subsubsection{Supplementing C: From Formulation to Dose}

Vitamin C formulations are as diverse as the recommendations and health claims made in support various marketed supplements. However, there is no data to suggest that any formulation containing ascorbate, alone or in combination with bioflavonoids, liposomes, vitamin $\mathrm{C}$ breakdown products, or

minerals, has any measurable effect on vitamin $\mathrm{C}$ bioavailability in humans. While there are claims that vitamin $\mathrm{C}$ from natural versus synthetic sources, there is little evidence to support this in human subjects [128]. 
Pharmacokinetic studies suggest the consumption of at least $200 \mathrm{mg} /$ day of vitamin C in healthy young men and women results in plasma concentrations greater than $50 \mu \mathrm{M}[105,106]$. At this dose, nearly complete oral bioavailability is obtained, leukocyte saturation is achieved, and urinary excretion is minimized, suggesting a saturation point has been reached for vitamin $\mathrm{C}$ transport. A dose of $200 \mathrm{mg}$ /day can be achieved through a diet rich in fresh fruit and vegetables. Although the limitations of extrapolating this data have been discussed, it has been suggested that $200 \mathrm{mg} / \mathrm{day}$ of vitamin $\mathrm{C}$ should be set as an optimal intake level for the general population [1]. As mentioned above, it is possible that increased intake of vitamin $\mathrm{C}$ is necessary for plasma saturation in some individuals, such as the elderly [101] or individuals with various genetic polymorphisms [103], which would likely necessitate the use of supplements.

Can a case be made for "megadose" levels of vitamin C, or those in excess of $1 \mathrm{~g} /$ day? As much as different authors have put forth their own perspectives on the subject, there are few RCTs examining the effect of large daily oral doses of vitamin C. Thus, it is difficult to speculate on the beneficial effect of such doses. Pharmacokinetic data show that daily oral doses of vitamin $\mathrm{C}$ up to $2.5 \mathrm{~g}$ /day result in higher plasma ascorbate levels than a $200 \mathrm{mg}$ /day dose, despite decreased bioavailability and increased urinary excretion of vitamin C [105,106]; however, the differences are small and biologically most likely of little consequence. It has been speculated that high plasma ascorbate levels can be maintained by frequent dosing with ascorbic acid [129], but it unclear what health effect would be achieved by this regimen. To date, no papers have shown a benefit of achieving greater-than-saturation plasma levels in the long term, and this mechanism would need to be delineated before a recommendation could be made.

One possible effect of megadose levels of vitamin $\mathrm{C}$ may be in the gastrointestinal tract, where it may prevent the formation of carcinogenic $N$-nitroso compounds [130] and highly reactive electrophilic compounds from the diet, such as acrolein [131] or lipid peroxidation products [132]. An intriguing idea for a biological role of vitamin $\mathrm{C}$ in the digestive tract is an exploration of beneficial changes to microbiota populations in the gut. However, in all of these cases, studies have yet to be performed, currently making it premature to draw any conclusions about the beneficial effects of oral supplementation with high-dose vitamin C. Large doses of intravenous ascorbate are associated with anti-cancer effects [133] and represent a promising area of research in the future that should be a subject of future review.

\section{Methods}

\subsection{Ascorbate in Cell Culture Media}

Phosphate Buffered Saline (PBS) solution was prepared from PBS tabs (Sigma Aldrich, St. Louis, MO, USA) dissolved in double deionized, filter sterilized water. Hyclone RPMI 1640 (Thermo Fisher Scientific, Waltam, MA, USA) was either used alone or after overnight chelation with Chelex 100 resin and the addition of $1 \mathrm{mM}$ diethylenetriaminepentaacetic acid (DTPA). PBS or media was placed in 6-well culture dishes in a cell culture incubator $\left(5 \% / 95 \%\right.$ : $\mathrm{CO}_{2} /$ air; $\left.37{ }^{\circ} \mathrm{C}\right)$ or at room temperature. Low trace metal ascorbic acid (Sigma Aldrich, St. Louis, MO, USA) was dissolved at a concentration of $10 \mathrm{mM}$ in deionized water before addition to culture dishes. At specified times, aliquots were removed and acidified in 15\% perchloric acid (PCA) containing 
$5 \mathrm{mM}$ DTPA for vitamin C analysis as described below. Cell culture media containing 10\% FBS from different commercial sources (Hyclone, Thermo Fisher, Sigma or ATCC) or aliquots of FBS alone were also acidified with an equal amount of PCA, as described above for the analysis of ascorbate content. However, no ascorbate was detected unless ascorbate was added to these solutions.

\subsection{Human Subjects and Blood Collection}

Five subjects were recruited for blood collection as part of a larger study on vitamin C metabolism approved by the Institutional Review Board for the Protection of Human Subjects at Oregon State University. Subjects were healthy non-smokers taking no prescription or non-prescription drugs that might influence vitamin $\mathrm{C}$ metabolism. For the study comparing anticoagulants, blood was collected after an overnight fast in vacutainers containing no additives or sodium heparin, lithium heparin, K2 EDTA, K3 EDTA, or sodium citrate (all from Becton Dickinson, Franklin Lakes, NJ, USA). Immediately after collection, aliquots of blood were collected from vacutainers and centrifuged at $16,000 \times \mathrm{g}$ for $1 \mathrm{~min}$ for the separation of plasma from red blood cells. Aliquots of plasma were immediately acidified in $15 \%$ PCA containing $5 \mathrm{mM}$ DTPA and placed at $4{ }^{\circ} \mathrm{C}$ to limit ascorbate oxidation until analysis, performed immediately, as described below.

For samples collected for the analysis of vitamin C oxidation in plasma, blood was collected in sodium heparin vacutainers from subjects after an overnight fast. Vacutainers were centrifuged in an Allegra X-15R (Beckman Coulter, Brea, CA, USA) at $4000 \times g$ at $4{ }^{\circ} \mathrm{C}$ for the separation of plasma. Vacutainers were then treated in one of three protocols: Oxidation control samples ("Control") were kept on ice with limited light exposure until the vacutainer was opened in a chamber previously purged with nitrogen gas. Plasma was removed and acidified in PCA with DTPA. Vacutainers prepared with the standard protocol ("Standard") were kept at room temperature and opened in ambient air before plasma samples were acidified with PCA. Vacutainers exposed to the environment ("Exposed") were opened under ambient air at room temperature and plasma placed in a clear $2 \mathrm{~mL}$ tube. Tubes containing plasma were then incubated at room temperature for two hours before acidification.

\subsection{Vitamin C and Urate Analysis}

Plasma, media, and FBS samples were prepared for ascorbate analysis using HPLC as previously described [134]. Briefly, acid extracts were diluted in sodium acetate/methanol/water mobile phase $(0.3 \% / 7.5 \% / 92 \% \mathrm{w} / \mathrm{v})$ containing Q12 ion pairing reagent and $\mathrm{pH}$-adjusted with $2.58 \mathrm{M} \mathrm{KH}_{2} \mathrm{PO}_{4}$ buffer (pH 9.8). Samples were separated on Watters 2695 using an LC-8 column (Supelco) under an applied potential of $600 \mathrm{mV}$ using an electrochemical detector (BSA). Under these conditions, both urate and ascorbate are resolved and quantified by comparisons to authentic standards with a detection limit of approximately $1 \mathrm{nM}$. For human plasma samples, urate values are used to normalize ascorbate values to minimize any variations within subject samples and to control for volume differences in vacutainer analyses. To obtain total ascorbate, samples were reduced with the addition of tris-2-carboxyethyl phosphine (TCEP) as described elsewhere [113]. Dehydroascorbic acid content is estimated by the difference between TCEP-reduced and standard preparations. 


\subsection{Statistics}

Statistical analysis was performed by GraphPad Prism software, version 6 (GraphPad Software, Inc., La Jolla, CA, USA). Statistical differences between ascorbate samples were determined by two tailed ANOVA using a Tukey post-hoc analysis, with a $p$-value below 0.05 considered significant. Non-linear regression analysis was used to determine the half-life of ascorbate in PBS or media.

\section{Conclusions}

Performing successful research on vitamin $\mathrm{C}$, especially in the context of human health, requires specific focus and attention to detail. Although this review touched on many of the aspects of cell culture, animal, and human studies that are complicated by the use of ascorbic acid, it is not a complete guide to performing vitamin $\mathrm{C}$ research. Each study design is unique, so it must be critically analyzed with respect to ascorbate chemistry and biology to best understand whether and how it will make a contribution in the overall field. As described above, the effects of ascorbic acid within a biological system are potentially multifold, requiring extreme caution in implementation and interpretation.

As we change our view of vitamin $\mathrm{C}$ research, we must first address the most prevalent issues in frequently used model systems. The combination of high oxygen tension and the presence of reactive transition metals lead to a rapid oxidation of ascorbic acid in cell culture. The rapid loss of ascorbate in culture media promotes a pro-oxidant environment with the generation of superoxide radicals, hydrogen peroxide, and hydroxyl radicals, as described above. Moreover, depriving cells of ascorbic acid for countless generations produces a cell culture environment that is no longer relevant to human health. Overall, steps must be taken to control ascorbate oxidation if vitamin $\mathrm{C}$ research is to continue in cell culture. The use of stable ascorbate derivatives, metal chelation, and low oxygen culture conditions show promise, but much additional work is needed. Understanding the effects of prolonged ascorbate deprivation of cells is also necessary to determine if certain cell lines must be eliminated from use with ascorbic acid.

The continued use of ascorbate-synthesizing animal models also complicates all studies on ascorbic acid in vivo. Currently, mouse and rat models that lack ascorbic acid synthesis are available, but show considerable differences from guinea pigs and humans in terms of ascorbic acid bioavailability and metabolism. For example, the apparent lack of intestinal absorption of ascorbic acid in rodent models does not appear related to the synthesis capacity, and must be considered in supplementation studies. Furthermore, the effects of vitamin $\mathrm{C}$ deprivation appear to have effects in one model system that are not recapitulated in others, although we do not know if strain differences or dietary factors may have influenced these observations. The use of animal models is especially troubling in light of genetic alterations in the regulation and expression of vitamin $\mathrm{C}$ transport proteins in animals without functional ascorbic acid synthesis. Further studies are required to determine if other genetic adaptations have occurred - specifically in primate evolution - that may determine the difference in response of animals and humans to vitamin $\mathrm{C}$ supplementation.

Currently, studies involving vitamin $\mathrm{C}$ consumption in human subjects are not held to a rigorous standard. Randomized controlled trials, normally considered the benchmark for determining the impact of a compound on human health, have been poorly designed in regard to the chemistry and 
biology of ascorbic acid. While remaining aware of the effects that different study populations may have on the outcome of vitamin $\mathrm{C}$ supplementation, there is a need to determine vitamin $\mathrm{C}$ status in subjects before, during, and after a supplementation trial. Evidence shows that methods to record vitamin $\mathrm{C}$ intake by using food frequency questionnaires or food diaries are insufficient means of achieving this goal. Therefore study design needs to include assessments of vitamin $\mathrm{C}$ status using measurements of plasma, and possibly tissue, ascorbic acid levels. Only with these more precise determinations can we begin to speculate on mechanisms of action and eliminate speculations surrounding the health effects of vitamin $\mathrm{C}$ supplementation. Furthermore, eliminating the technical issues surrounding the measurement of vitamin $\mathrm{C}$ in clinical settings are additional steps needed to preserve data integrity.

While all study designs deserve higher scrutiny, a note of caution must also be placed in the interpretation of vitamin $\mathrm{C}$ research. It is all too common for vitamin $\mathrm{C}$ to be assigned a role (as an antioxidant or a pro-oxidant, for instance) without any supporting evidence to validate those claims. It cannot be overemphasized that the primary role of ascorbic acid in biological systems is that of a reductant, and the most established health effects of this reductive power are related to ascorbate's role as an electron-donating enzyme cofactor used, e.g., for pro-collagen, carnitine, and catecholamine biosynthesis [3]. Ascorbic acid has many additional roles in the body beyond these enzyme functions, for example antioxidant protection, and likely more roles will be elucidated in the future. With a critical evaluation of all potential roles of ascorbic acid, the borders of vitamin $\mathrm{C}$ research can advance with balanced, evidence-based approaches. Already, emerging roles for vitamin $\mathrm{C}$ in various hydroxylase enzymes have recently placed a focus on HIF-1 $\alpha$-dependent gene expression [41,43] and changes in histone and DNA methylation [135,136], suggesting vitamin C may regulate global changes in gene expression. Further innovation has been demonstrated in studies on vitamin C bioavailability [69,108], metabolomics of vitamin C deficiency [137], genetic variation of the vitamin $\mathrm{C}$ transporters [103], and intravenous vitamin $\mathrm{C}$ infusions in cancer therapy [138,139].

As vitamin $\mathrm{C}$ research progresses into the 21 st century, it has become clear that much more work still lies ahead of us. The experimental faults, artifacts, and myths currently afflicting vitamin $\mathrm{C}$ research limit the impact of many studies, making their contributions to general knowledge of the biological roles of ascorbic acid unremarkable at best, and confusing and detracting from the real issues at worst. This can also stretch beyond the realm of laboratory research, as the persistence of poorly controlled studies within this field often undermines efforts in the medical community to recommend vitamin $\mathrm{C}$ as a safe, effective means of promoting health. If nothing else, it weakens efforts to fund additional, well-designed RCTs necessary to establish definitive health claims that are desperately needed. In light of these issues, we must increase scrutiny of vitamin C studies in the past as well as the present, holding them to a higher standard based on the evidence discussed here if we are to make a lasting contribution to our understanding of vitamin C's impact on human health.

\section{Conflicts of Interest}

The authors declare no conflict of interest. 


\section{References}

1. Frei, B.; Birlouez-Aragon, I.; Lykkesfeldt, J. Authors' perspective: What is the optimum intake of vitamin C in humans? Crit. Rev. Food Sci. Nutr. 2012, 52, 815-829.

2. Lykkesfeldt, J.; Poulsen, H.E. Is vitamin C supplementation beneficial? Lessons learned from randomised controlled trials. Br. J. Nutr. 2010, 103, 1251-1259.

3. Michels, A.J.; Frei, B.B. Vitamin C. In Biochemical, Physiological, and Molecular Aspects of Human Nutrition, 3rd ed.; Stipanuk, M.H., Caudill, M.A., Eds.; Elsevier/Saunders: St. Louis, MO, USA, 2012; pp. 626-654.

4. Carr, A.C.; Frei, B. Toward a new recommended dietary allowance for vitamin $\mathrm{C}$ based on antioxidant and health effects in humans. Am. J. Clin. Nutr. 1999, 69, 1086-1107.

5. Juraschek, S.P.; Guallar, E.; Appel, L.J.; Miller, E.R., III. Effects of vitamin C supplementation on blood pressure: A meta-analysis of randomized controlled trials. Am. J. Clin. Nutr. 2012, 95, 1079-1088.

6. Halliwell, B. Oxidative stress in cell culture: An under-appreciated problem? FEBS Lett. 2003, 540, 3-6.

7. Buettner, G.R. The pecking order of free radicals and antioxidants: Lipid peroxidation, $\alpha$-tocopherol, and ascorbate. Arch. Biochem. Biophys. 1993, 300, 535-543.

8. Buettner, G.R.; Jurkiewicz, B.A. Catalytic metals, ascorbate and free radicals: Combinations to avoid. Radiat. Res. 1996, 145, 532-541.

9. Frikke-Schmidt, H.; Lykkesfeldt, J. Keeping the intracellular vitamin C at a physiologically relevant level in endothelial cell culture. Anal. Biochem. 2010, 397, 135-137.

10. Chepda, T.; Cadau, M.; Girin, P.; Frey, J.; Chamson, A. Monitoring of ascorbate at a constant rate in cell culture: Effect on cell growth. In Vitro Cell. Dev. Biol. Anim. 2001, 37, 26-30.

11. Wee, L.M.; Long, L.H.; Whiteman, M.; Halliwell, B. Factors affecting the ascorbate- and phenolic-dependent generation of hydrogen peroxide in Dulbecco's Modified Eagles Medium. Free Radic. Res. 2003, 37, 1123-1130.

12. Clement, M.V.; Ramalingam, J.; Long, L.H.; Halliwell, B. The in vitro cytotoxicity of ascorbate depends on the culture medium used to perform the assay and involves hydrogen peroxide. Antioxid. Redox Signal. 2001, 3, 157-163.

13. Cazzola, M.; Bergamaschi, G.; Dezza, L.; Arosio, P. Manipulations of cellular iron metabolism for modulating normal and malignant cell proliferation: Achievements and prospects. Blood 1990, 75, 1903-1919.

14. Halliwell, B.; Gutteridge, J.M.C. Free Radicals in Biology and Medicine, 4th ed.; Oxford University Press: Oxford, UK, 2007.

15. Buettner, G.R. In the absence of catalytic metals ascorbate does not autoxidize at $\mathrm{pH} 7$ : Ascorbate as a test for catalytic metals. J. Biochem. Biophys. Methods 1988, 16, 27-40.

16. Minetti, M.; Forte, T.; Soriani, M.; Quaresima, V.; Menditto, A.; Ferrari, M. Iron-induced ascorbate oxidation in plasma as monitored by ascorbate free radical formation. No spin-trapping evidence for the hydroxyl radical in iron-overloaded plasma. Biochem. J. 1992, 282, 459-465. 
17. Carr, A.; Frei, B. Does vitamin $\mathrm{C}$ act as a pro-oxidant under physiological conditions? FASEB J. 1999, 13, 1007-1024.

18. Chen, K.; Suh, J.; Carr, A.C.; Morrow, J.D.; Zeind, J.; Frei, B. Vitamin C suppresses oxidative lipid damage in vivo, even in the presence of iron overload. Am. J. Physiol. Endocrinol. Metab. 2000, 279, E1406-E1412.

19. Berger, T.M.; Polidori, M.C.; Dabbagh, A.; Evans, P.J.; Halliwell, B.; Morrow, J.D.; Roberts, L.J., II; Frei, B. Antioxidant activity of vitamin C in iron-overloaded human plasma. J. Biol. Chem. 1997, 272, 15656-15660.

20. Halliwell, B. Vitamin C: Antioxidant or pro-oxidant in vivo? Free Radic. Res. 1996, 25, 439-454.

21. Long, L.H.; Halliwell, B. Artefacts in cell culture: Pyruvate as a scavenger of hydrogen peroxide generated by ascorbate or epigallocatechin gallate in cell culture media. Biochem. Biophys. Res. Commun. 2009, 388, 700-704.

22. Long, L.H.; Halliwell, B. Artefacts in cell culture: $\alpha$-Ketoglutarate can scavenge hydrogen peroxide generated by ascorbate and epigallocatechin gallate in cell culture media. Biochem. Biophys. Res. Commun. 2011, 406, 20-24.

23. Buettner, G.R.; Jurkiewicz, B.A. Ascorbate free radical as a marker of oxidative stress: An EPR study. Free Radic. Biol. Med. 1993, 14, 49-55.

24. Chen, Q.; Espey, M.G.; Krishna, M.C.; Mitchell, J.B.; Corpe, C.P.; Buettner, G.R.; Shacter, E.; Levine, M. Pharmacologic ascorbic acid concentrations selectively kill cancer cells: Action as a pro-drug to deliver hydrogen peroxide to tissues. Proc. Natl. Acad. Sci. USA 2005, 102, 13604-13609.

25. Bode, A.M.; Cunningham, L.; Rose, R.C. Spontaneous decay of oxidized ascorbic acid (dehydro-L-ascorbic acid) evaluated by high-pressure liquid chromatography. Clin. Chem. 1990, 36, 1807-1809.

26. Linster, C.L.; van Schaftingen, E. Vitamin C. Biosynthesis, recycling and degradation in mammals. FEBS J. 2007, 274, 1-22.

27. Washko, P.W.; Wang, Y.; Levine, M. Ascorbic acid recycling in human neutrophils. J. Biol. Chem. 1993, 268, 15531-15535.

28. Rumsey, S.C.; Kwon, O.; Xu, G.W.; Burant, C.F.; Simpson, I.; Levine, M. Glucose transporter isoforms GLUT1 and GLUT3 transport dehydroascorbic acid. J. Biol. Chem. 1997, 272, 18982-18989.

29. Vera, J.C.; Rivas, C.I.; Fischbarg, J.; Golde, D.W. Mammalian facilitative hexose transporters mediate the transport of dehydroascorbic acid. Nature 1993, 364, 79-82.

30. Wilson, J.X. The physiological role of dehydroascorbic acid. FEBS Lett. 2002, 527, 5-9.

31. Pedraza, C.E.; Chien, Y.C.; McKee, M.D. Calcium oxalate crystals in fetal bovine serum: Implications for cell culture, phagocytosis and biomineralization studies in vitro. J. Cell. Biochem. 2008, 103, 1379-1393.

32. Kwack, M.H.; Ahn, J.S.; Kim, M.K.; Kim, J.C.; Sung, Y.K. Preventable effect of L-threonate, an ascorbate metabolite, on androgen-driven balding via repression of dihydrotestosterone-induced dickkopf-1 expression in human hair dermal papilla cells. BMB Rep. 2010, 43, 688-692. 
33. Smith, A.R.; Visioli, F.; Hagen, T.M. Vitamin C matters: Increased oxidative stress in cultured human aortic endothelial cells without supplemental ascorbic acid. FASEB J. 2002, 16, 1102-1104.

34. Huang, A.; Vita, J.A.; Venema, R.C.; Keaney, J.F., Jr. Ascorbic acid enhances endothelial nitric-oxide synthase activity by increasing intracellular tetrahydrobiopterin. J. Biol. Chem. 2000, 275, 17399-17406.

35. Bergsten, P.; Amitai, G.; Kehrl, J.; Dhariwal, K.R.; Klein, H.G.; Levine, M. Millimolar concentrations of ascorbic acid in purified human mononuclear leukocytes. Depletion and reaccumulation. J. Biol. Chem. 1990, 265, 2584-2587.

36. Martin, A.; Frei, B. Both intracellular and extracellular vitamin C inhibit atherogenic modification of LDL by human vascular endothelial cells. Arterioscler. Thromb. Vas. Biol. 1997, 17, 1583-1590.

37. May, J.M.; Qu, Z.C.; Meredith, M.E. Mechanisms of ascorbic acid stimulation of norepinephrine synthesis in neuronal cells. Biochem. Biophys. Res. Commun. 2012, 426, 148-152.

38. Visioli, F.; Smith, A.; Zhang, W.; Keaney, J.F., Jr.; Hagen, T.; Frei, B. Lipoic acid and vitamin $\mathrm{C}$ potentiate nitric oxide synthesis in human aortic endothelial cells independently of cellular glutathione status. Redox Rep. 2002, 7, 223-227.

39. Englard, S.; Seifter, S. The biochemical functions of ascorbic acid. Annu. Rev. Nutr. 1986, 6, 365-406.

40. Bruick, R.K.; McKnight, S.L. A conserved family of prolyl-4-hydroxylases that modify HIF. Science 2001, 294, 1337-1340.

41. Flashman, E.; Davies, S.L.; Yeoh, K.K.; Schofield, C.J. Investigating the dependence of the hypoxia-inducible factor hydroxylases (factor inhibiting HIF and prolyl hydroxylase domain 2) on ascorbate and other reducing agents. Biochem. J. 2010, 427, 135-142.

42. Myllyharju, J. Prolyl 4-hydroxylases, the key enzymes of collagen biosynthesis. Matrix Biol. 2003, 22, 15-24.

43. Knowles, H.J.; Raval, R.R.; Harris, A.L.; Ratcliffe, P.J. Effect of ascorbate on the activity of hypoxia-inducible factor in cancer cells. Cancer Res. 2003, 63, 1764-1768.

44. Murad, S.; Grove, D.; Lindberg, K.A.; Reynolds, G.; Sivarajah, A.; Pinnell, S.R. Regulation of collagen synthesis by ascorbic acid. Proc. Natl. Acad. Sci. USA 1981, 78, 2879-2882.

45. Diliberto, E.J., Jr.; Daniels, A.J.; Viveros, O.H. Multicompartmental secretion of ascorbate and its dual role in dopamine $\beta$-hydroxylation. Am. J. Clin. Nutr. 1991, 54, 1163S-1172S.

46. Monfort, A.; Wutz, A. Breathing-in epigenetic change with vitamin C. EMBO Rep. 2013, 14, 337-346.

47. Ladurner, A.; Schmitt, C.A.; Schachner, D.; Atanasov, A.G.; Werner, E.R.; Dirsch, V.M.; Heiss, E.H. Ascorbate stimulates endothelial nitric oxide synthase enzyme activity by rapid modulation of its phosphorylation status. Free Radic. Biol. Med. 2012, 52, 2082-2090.

48. May, J.M.; Qu, Z.C.; Qiao, H. Transfer of ascorbic acid across the vascular endothelium: Mechanism and self-regulation. Am. J. Physiol. Cell Physiol. 2009, 297, C169-C178.

49. Wu, F.; Schuster, D.P.; Tyml, K.; Wilson, J.X. Ascorbate inhibits NADPH oxidase subunit p47phox expression in microvascular endothelial cells. Free Radic. Biol. Med. 2007, 42, 124-131. 
50. Yu, R.; Schellhorn, H.E. Recent applications of engineered animal antioxidant deficiency models in human nutrition and chronic disease. J. Nutr. 2013, 143, 1-11.

51. Seok, J.; Warren, H.S.; Cuenca, A.G.; Mindrinos, M.N.; Baker, H.V.; Xu, W.; Richards, D.R.; McDonald-Smith, G.P.; Gao, H.; Hennessy, L.; et al. Genomic responses in mouse models poorly mimic human inflammatory diseases. Proc. Natl. Acad. Sci. USA 2013, 110, 3507-3512.

52. Braun, L.; Csala, M.; Poussu, A.; Garzo, T.; Mandl, J.; Banhegyi, G. Glutathione depletion induces glycogenolysis dependent ascorbate synthesis in isolated murine hepatocytes. FEBS Lett. 1996, 388, 173-176.

53. Chan, T.S.; Wilson, J.X.; O’Brien, P.J. Glycogenolysis is directed towards ascorbate synthesis by glutathione conjugation. Biochem. Biophys. Res. Commun. 2004, 317, 149-156.

54. Banhegyi, G.; Braun, L.; Csala, M.; Puskas, F.; Mandl, J. Ascorbate metabolism and its regulation in animals. Free Radic. Biol. Med. 1997, 23, 793-803.

55. Yang, H. Conserved or lost: Molecular evolution of the key gene GULO in vertebrate vitamin C biosynthesis. Biochem. Genet. 2013, 51, 413-425.

56. Spencer, R.P.; Purdy, S.; Hoeldtke, R.; Bow, T.M.; Markulis, M.A. Studies on intestinal absorption of L-ascorbic acid-1-C-14. Gastroenterology 1963, 44, 768-773.

57. Hornig, D.; Weber, F.; Wiss, O. Site of intestinal absorption of ascorbic acid in guinea pigs and rats. Biochem. Biophys. Res. Commun. 1973, 52, 168-172.

58. Hornig, D. Distribution of ascorbic acid, metabolites and analogues in man and animals. Ann. N. Y. Acad. Sci. 1975, 258, 103-118.

59. Stevenson, N.R.; Brush, M.K. Existence and characteristics of Na positive-dependent active transport of ascorbic acid in guinea pig. Am. J. Clin. Nutr. 1969, 22, 318-326.

60. Bianchi, J.; Wilson, F.A.; Rose, R.C. Dehydroascorbic acid and ascorbic acid transport systems in the guinea pig ileum. Am. J. Physiol. 1986, 250, G461-G468.

61. Mellors, A.J.; Nahrwold, D.L.; Rose, R.C. Ascorbic acid flux across mucosal border of guinea pig and human ileum. Am. J. Physiol. 1977, 233, E374-E379.

62. Stevenson, N.R. Active transport of L-ascorbic acid in the human ileum. Gastroenterology 1974, 67, 952-956.

63. Corpe, C.P.; Eck, P.; Wang, J.; Al-Hasani, H.; Levine, M. Intestinal dehydroascorbic acid (DHA) transport mediated by the facilitative sugar transporters, GLUT2 and GLUT8. J. Biol. Chem. 2013, 288, 9092-9101.

64. Tsao, C.S.; Leung, P.Y.; Young, M. Effect of dietary ascorbic acid intake on tissue vitamin C in mice. J. Nutr. 1987, 117, 291-297.

65. Rucker, R.B. Allometric scaling, metabolic body size and interspecies comparisons of basal nutritional requirements. J. Anim. Physiol. Anim. Nutr. 2007, 91, 148-156.

66. Bush, M.J.; Verlangieri, A.J. An acute study on the relative gastro-intestinal absorption of a novel form of calcium ascorbate. Res. Commun. Chem. Pathol. Pharmacol. 1987, 57, 137-140.

67. Johnston, C.S.; Luo, B. Comparison of the absorption and excretion of three commercially available sources of vitamin C. J. Am. Diet. Assoc. 1994, 94, 779-781. 
68. Vissers, M.C.; Bozonet, S.M.; Pearson, J.F.; Braithwaite, L.J. Dietary ascorbate intake affects steady state tissue concentrations in vitamin C-deficient mice: Tissue deficiency after suboptimal intake and superior bioavailability from a food source (kiwifruit). Am. J. Clin. Nutr. 2011, 93, 292-301.

69. Carr, A.C.; Bozonet, S.M.; Pullar, J.M.; Simcock, J.W.; Vissers, M.C. A randomized steady-state bioavailability study of synthetic versus natural (Kiwifruit-Derived) vitamin C. Nutrients 2013, 5, 3684-3695.

70. Iwama, M.; Shimokado, K.; Maruyama, N.; Ishigami, A. Time course of vitamin C distribution and absorption after oral administration in SMP30/GNL knockout mice. Nutrition 2011, 27, 471-478.

71. Mohan, S.; Kapoor, A.; Singgih, A.; Zhang, Z.; Taylor, T.; Yu, H.; Chadwick, R.B.; Chung, Y.S.; Donahue, L.R.; Rosen, C.; et al. Spontaneous fractures in the mouse mutant sfx are caused by deletion of the gulonolactone oxidase gene, causing vitamin $\mathrm{C}$ deficiency. J. Bone Miner. Res. 2005, 20, 1597-1610.

72. Meredith, M.E.; Harrison, F.E.; May, J.M. Differential regulation of the ascorbic acid transporter SVCT2 during development and in response to ascorbic acid depletion. Biochem. Biophys. Res. Commun. 2011, 414, 737-742.

73. Boyer, J.C.; Campbell, C.E.; Sigurdson, W.J.; Kuo, S.M. Polarized localization of vitamin C transporters, SVCT1 and SVCT2, in epithelial cells. Biochem. Biophys. Res. Commun. 2005, $334,150-156$.

74. Maulen, N.P.; Henriquez, E.A.; Kempe, S.; Carcamo, J.G.; Schmid-Kotsas, A.; Bachem, M.; Grunert, A.; Bustamante, M.E.; Nualart, F.; Vera, J.C. Up-regulation and polarized expression of the sodium-ascorbic acid transporter SVCT1 in post-confluent differentiated CaCo-2 cells. J. Biol. Chem. 2003, 278, 9035-9041.

75. MacDonald, L.; Thumser, A.E.; Sharp, P. Decreased expression of the vitamin C transporter SVCT1 by ascorbic acid in a human intestinal epithelial cell line. Br. J. Nutr. 2002, 87, 97-100.

76. Corpe, C.P.; Tu, H.; Eck, P.; Wang, J.; Faulhaber-Walter, R.; Schnermann, J.; Margolis, S.; Padayatty, S.; Sun, H.; Wang, Y.; et al. Vitamin C transporter Slc23a1 links renal reabsorption, vitamin $\mathrm{C}$ tissue accumulation, and perinatal survival in mice. J. Clin. Investig. 2010, 120, 1069-1083.

77. Savini, I.; Rossi, A.; Pierro, C.; Avigliano, L.; Catani, M.V. SVCT1 and SVCT2: Key proteins for vitamin C uptake. Amino Acids 2008, 34, 347-355.

78. Montel-Hagen, A.; Kinet, S.; Manel, N.; Mongellaz, C.; Prohaska, R.; Battini, J.L.; Delaunay, J.; Sitbon, M.; Taylor, N. Erythrocyte Glut1 triggers dehydroascorbic acid uptake in mammals unable to synthesize vitamin C. Cell 2008, 132, 1039-1048.

79. Beamer, W.G.; Rosen, C.J.; Bronson, R.T.; Gu, W.; Donahue, L.R.; Baylink, D.J.; Richardson, C.C.; Crawford, G.C.; Barker, J.E. Spontaneous fracture (sfx): A mouse genetic model of defective peripubertal bone formation. Bone 2000, 27, 619-626.

80. Maeda, N.; Hagihara, H.; Nakata, Y.; Hiller, S.; Wilder, J.; Reddick, R. Aortic wall damage in mice unable to synthesize ascorbic acid. Proc. Natl. Acad. Sci. USA 2000, 97, 841-846.

81. Ward, M.S.; Lamb, J.; May, J.M.; Harrison, F.E. Behavioral and monoamine changes following severe vitamin C deficiency. J. Neurochem. 2013, 124, 363-375. 
82. Kondo, Y.; Inai, Y.; Sato, Y.; Handa, S.; Kubo, S.; Shimokado, K.; Goto, S.; Nishikimi, M.; Maruyama, N.; Ishigami, A. Senescence marker protein 30 functions as gluconolactonase in L-ascorbic acid biosynthesis, and its knockout mice are prone to scurvy. Proc. Natl. Acad. Sci. USA 2006, 103, 5723-5728.

83. Horio, F.; Ozaki, K.; Yoshida, A.; Makino, S.; Hayashi, Y. Requirement for ascorbic acid in a rat mutant unable to synthesize ascorbic acid. J. Nutr. 1985, 115, 1630-1640.

84. Horio, F.; Hayashi, K.; Mishima, T.; Takemori, K.; Oshima, I.; Makino, S.; Kakinuma, A.; Ito, H. A newly established strain of spontaneously hypertensive rat with a defect of ascorbic acid biosynthesis. Life Sci. 2001, 69, 1879-1890.

85. Vergely, C.; Perrin, C.; Laubriet, A.; Oudot, A.; Zeller, M.; Guilland, J.C.; Rochette, L. Postischemic myocardial recovery and oxidative stress status of vitamin $\mathrm{C}$ deficient rat hearts. Cardiovasc. Res. 2001, 51, 89-99.

86. Nishikimi, M.; Koshizaka, T.; Kondo, K.; Ozawa, T.; Yagi, K. Expression of the mutant gene for L-gulono-gamma-lactone oxidase in scurvy-prone rats. Experientia 1989, 45, 126-129.

87. Fernandez, M.L.; McNamar, D.J. Dietary fat-mediated changes in hepatic apoprotein B/E receptor in the guinea pig: Effect of polyunsaturated, monounsaturated, and saturated fat. Metabolism 1989, 38, 1094-1102.

88. Bell, J.P.; Mosfer, S.I.; Lang, D.; Donaldson, F.; Lewis, M.J. Vitamin C and quinapril abrogate LVH and endothelial dysfunction in aortic-banded guinea pigs. Am. J. Physiol. Heart Circ. Physiol. 2001, 281, H1704-H1710.

89. Wolkart, G.; Wenzl, M.V.; Beretta, M.; Stessel, H.; Schmidt, K.; Mayer, B. Vascular tolerance to nitroglycerin in ascorbate deficiency. Cardiovasc. Res. 2008, 79, 304-312.

90. Liang, W.J.; Johnson, D.; Jarvis, S.M. Vitamin C transport systems of mammalian cells. Mol. Membr. Biol. 2001, 18, 87-95.

91. Liang, W.J.; Johnson, D.; Ma, L.S.; Jarvis, S.M.; Wei, J.L. Regulation of the human vitamin C transporters expressed in COS-1 cells by protein kinase C [corrected]. Am. J. Physiol. Cell Physiol. 2002, 283, C1696-C1704.

92. Subramanian, V.S.; Marchant, J.S.; Boulware, M.J.; Said, H.M. A C-terminal region dictates the apical plasma membrane targeting of the human sodium-dependent vitamin $\mathrm{C}$ transporter-1 in polarized epithelia. J. Biol. Chem. 2004, 279, 27719-27728.

93. Mardones, L.; Zuniga, F.A.; Villagran, M.; Sotomayor, K.; Mendoza, P.; Escobar, D.; Gonzalez, M.; Ormazabal, V.; Maldonado, M.; Onate, G.; et al. Essential role of intracellular glutathione in controlling ascorbic acid transporter expression and function in rat hepatocytes and hepatoma cells. Free Radic. Biol. Med. 2012, 52, 1874-1887.

94. Nualart, F.J.; Rivas, C.I.; Montecinos, V.P.; Godoy, A.S.; Guaiquil, V.H.; Golde, D.W.; Vera, J.C. Recycling of vitamin C by a bystander effect. J. Biol. Chem. 2003, 278, 10128-10133.

95. May, J.M.; Qu, Z.C.; Qiao, H.; Koury, M.J. Maturational loss of the vitamin C transporter in erythrocytes. Biochem. Biophys. Res. Commun. 2007, 360, 295-298.

96. Montel-Hagen, A.; Blanc, L.; Boyer-Clavel, M.; Jacquet, C.; Vidal, M.; Sitbon, M.; Taylor, N. The Glut1 and Glut4 glucose transporters are differentially expressed during perinatal and postnatal erythropoiesis. Blood 2008, 112, 4729-4738. 
97. Rumsey, S.C.; Daruwala, R.; Al-Hasani, H.; Zarnowski, M.J.; Simpson, I.A.; Levine, M. Dehydroascorbic acid transport by GLUT4 in Xenopus oocytes and isolated rat adipocytes. J. Biol. Chem. 2000, 275, 28246-28253.

98. Chen, Q.; Espey, M.G.; Sun, A.Y.; Pooput, C.; Kirk, K.L.; Krishna, M.C.; Khosh, D.B.; Drisko, J.; Levine, M. Pharmacologic doses of ascorbate act as a prooxidant and decrease growth of aggressive tumor xenografts in mice. Proc. Natl. Acad. Sci. USA 2008, 105, 11105-11109.

99. Roberts, L.J., II; Traber, M.G.; Frei, B. Vitamins E and C in the prevention of cardiovascular disease and cancer in men. Free Radic. Biol. Med. 2009, 46, 1558.

100. Dehghan, M.; Akhtar-Danesh, N.; McMillan, C.R.; Thabane, L. Is plasma vitamin C an appropriate biomarker of vitamin $\mathrm{C}$ intake? A systematic review and meta-analysis. Nutr. J. 2007, 6, 41.

101. Brubacher, D.; Moser, U.; Jordan, P. Vitamin C concentrations in plasma as a function of intake: A meta-analysis. Int. J. Vitam. Nutr. Res. 2000, 70, 226-237.

102. Schleicher, R.L.; Carroll, M.D.; Ford, E.S.; Lacher, D.A. Serum vitamin C and the prevalence of vitamin C deficiency in the United States: 2003-2004 National Health and Nutrition Examination Survey (NHANES). Am. J. Clin. Nutr. 2009, 90, 1252-1263.

103. Michels, A.J.; Hagen, T.M.; Frei, B. Human genetic variation influences vitamin C homeostasis by altering vitamin $\mathrm{C}$ transport and antioxidant enzyme function. Annu. Rev. Nutr. 2013, 33, 45-70.

104. Harding, A.H.; Wareham, N.J.; Bingham, S.A.; Khaw, K.; Luben, R.; Welch, A.; Forouhi, N.G. Plasma vitamin C level, fruit and vegetable consumption, and the risk of new-onset type 2 diabetes mellitus: The European prospective investigation of cancer-Norfolk prospective study. Arch. Intern. Med. 2008, 168, 1493-1499.

105. Levine, M.; Conry-Cantilena, C.; Wang, Y.; Welch, R.W.; Washko, P.W.; Dhariwal, K.R.; Park, J.B.; Lazarev, A.; Graumlich, J.F.; King, J.; et al. Vitamin C pharmacokinetics in healthy volunteers: Evidence for a recommended dietary allowance. Proc. Natl. Acad. Sci. USA 1996, 93, 3704-3709.

106. Levine, M.; Wang, Y.; Padayatty, S.J.; Morrow, J. A new recommended dietary allowance of vitamin C for healthy young women. Proc. Natl. Acad. Sci. USA 2001, 98, 9842-9846.

107. Kim, H.; Bae, S.; Yu, Y.; Kim, Y.; Kim, H.R.; Hwang, Y.I.; Kang, J.S.; Lee, W.J. The analysis of vitamin $\mathrm{C}$ concentration in organs of gulo ${ }^{-1-}$ mice upon vitamin $\mathrm{C}$ withdrawal. Immune Netw. 2012, 12, 18-26.

108. Carr, A.C.; Bozonet, S.M.; Pullar, J.M.; Simcock, J.W.; Vissers, M.C. Human skeletal muscle ascorbate is highly responsive to changes in vitamin $\mathrm{C}$ intake and plasma concentrations. Am. J. Clin. Nutr. 2013, 97, 800-807.

109. Taylor, A.; Jacques, P.F.; Nowell, T.; Perrone, G.; Blumberg, J.; Handelman, G.; Jozwiak, B.; Nadler, D. Vitamin C in human and guinea pig aqueous, lens and plasma in relation to intake. Curr. Eye Res. 1997, 16, 857-864. 
110. Dietrich, M.; Block, G.; Benowitz, N.L.; Morrow, J.D.; Hudes, M.; Jacob, P., III; Norkus, E.P.; Packer, L. Vitamin C supplementation decreases oxidative stress biomarker f2-isoprostanes in plasma of nonsmokers exposed to environmental tobacco smoke. Nutr. Cancer 2003, 45, 176-184.

111. Block, G.; Jensen, C.D.; Dalvi, T.B.; Norkus, E.P.; Hudes, M.; Crawford, P.B.; Holland, N.; Fung, E.B.; Schumacher, L.; Harmatz, P. Vitamin C treatment reduces elevated C-reactive protein. Free Radic. Biol. Med. 2009, 46, 70-77.

112. Dhariwal, K.R.; Washko, P.W.; Levine, M. Determination of dehydroascorbic acid using high-performance liquid chromatography with coulometric electrochemical detection. Anal. Biochem. 1990, 189, 18-23.

113. Lykkesfeldt, J. Determination of ascorbic acid and dehydroascorbic acid in biological samples by high-performance liquid chromatography using subtraction methods: Reliable reduction with tris[2-carboxyethyl]phosphine hydrochloride. Anal. Biochem. 2000, 282, 89-93.

114. Lykkesfeldt, J. Ascorbate and dehydroascorbic acid as biomarkers of oxidative stress: Validity of clinical data depends on vacutainer system used. Nutr. Res. 2012, 32, 66-69.

115. Karlsen, A.; Blomhoff, R.; Gundersen, T.E. Stability of whole blood and plasma ascorbic acid. Eur. J. Clin. Nutr. 2007, 61, 1233-1236.

116. Chung, W.Y.; Chung, J.K.; Szeto, Y.T.; Tomlinson, B.; Benzie, I.F. Plasma ascorbic acid: Measurement, stability and clinical utility revisited. Clin. Biochem. 2001, 34, 623-627.

117. Tveden-Nyborg, P.; Lykkesfeldt, J. Does Vitamin C deficiency increase lifestyle-associated vascular disease progression? Evidence based on experimental and clinical studies. Antioxid. Redox Signal. 2013, doi:10.1089/ars.2013.5382.

118. Cook, J.D.; Reddy, M.B. Effect of ascorbic acid intake on nonheme-iron absorption from a complete diet. Am. J. Clin. Nutr. 2001, 73, 93-98.

119. Taylor, E.N.; Stampfer, M.J.; Curhan, G.C. Dietary factors and the risk of incident kidney stones in men: New insights after 14 years of follow-up. J. Am. Soc. Nephrol. 2004, 15, 3225-3232.

120. Thomas, L.D.; Elinder, C.G.; Tiselius, H.G.; Wolk, A.; Akesson, A. Ascorbic acid supplements and kidney stone incidence among men: A prospective study. JAMA Intern. Med. 2013, 173, 386-388.

121. Taylor, E.N.; Fung, T.T.; Curhan, G.C. DASH-style diet associates with reduced risk for kidney stones. J. Am. Soc. Nephrol. 2009, 20, 2253-2259.

122. Singer, R.F. Vitamin C supplementation in kidney failure: Effect on uraemic symptoms. Nephrol. Dial. Transpl. 2011, 26, 614-620.

123. Hathcock, J.N.; Azzi, A.; Blumberg, J.; Bray, T.; Dickinson, A.; Frei, B.; Jialal, I.; Johnston, C.S.; Kelly, F.J.; Kraemer, K.; et al. Vitamins E and C are safe across a broad range of intakes. Am. J. Clin. Nutr. 2005, 81, 736-745.

124. Rees, D.C.; Kelsey, H.; Richards, J.D. Acute haemolysis induced by high dose ascorbic acid in glucose-6-phosphate dehydrogenase deficiency. BMJ 1993, 306, 841-842.

125. Hemila, H.; Chalker, E. Vitamin C for preventing and treating the common cold. Cochrane Database Syst. Rev. 2013, 1, CD000980. 
126. Douglas, R.M.; Hemila, H. Vitamin C for preventing and treating the common cold. PLoS Med. 2005, 2, e168.

127. Sasazuki, S.; Sasaki, S.; Tsubono, Y.; Okubo, S.; Hayashi, M.; Tsugane, S. Effect of vitamin C on common cold: Randomized controlled trial. Eur. J. Clin. Nutr. 2006, 60, 9-17.

128. Carr, A.C.; Vissers, M.C. Synthetic or food-derived vitamin C-Are they equally bioavailable? Nutrients 2013, 5, 4284-4304.

129. Padayatty, S.J.; Sun, H.; Wang, Y.; Riordan, H.D.; Hewitt, S.M.; Katz, A.; Wesley, R.A.; Levine, M. Vitamin C pharmacokinetics: Implications for oral and intravenous use. Ann. Intern. Med. 2004, 140, 533-537.

130. Tannenbaum, S.R. Preventive action of vitamin C on nitrosamine formation. Int. J. Vitam. Nutr. Res. Suppl. 1989, 30, 109-113.

131. Kesinger, N.G.; Stevens, J.F. Covalent interaction of ascorbic acid with natural products. Phytochemistry 2009, 70, 1930-1939.

132. Miranda, C.L.; Reed, R.L.; Kuiper, H.C.; Alber, S.; Stevens, J.F. Ascorbic acid promotes detoxification and elimination of 4-hydroxy-2(E)-nonenal in human monocytic THP-1 cells. Chem. Res. Toxicol. 2009, 22, 863-874.

133. Du, J.; Cullen, J.J.; Buettner, G.R. Ascorbic acid: Chemistry, biology and the treatment of cancer. Biochim. Biophys. Acta 2012, 1826, 443-457.

134. Frei, B.; England, L.; Ames, B.N. Ascorbate is an outstanding antioxidant in human blood plasma. Proc. Natl. Acad. Sci. USA 1989, 86, 6377-6381.

135. Yin, R.; Mao, S.Q.; Zhao, B.; Chong, Z.; Yang, Y.; Zhao, C.; Zhang, D.; Huang, H.; Gao, J.; Li, Z.; et al. Ascorbic acid enhances Tet-mediated 5-methylcytosine oxidation and promotes DNA demethylation in mammals. J. Am. Chem. Soc. 2013, 135, 10396-10403.

136. Chung, T.L.; Brena, R.M.; Kolle, G.; Grimmond, S.M.; Berman, B.P.; Laird, P.W.; Pera, M.F.; Wolvetang, E.J. Vitamin C promotes widespread yet specific DNA demethylation of the epigenome in human embryonic stem cells. Stem Cells 2010, 28, 1848-1855.

137. Kirkwood, J.S.; Lebold, K.M.; Miranda, C.L.; Wright, C.L.; Miller, G.W.; Tanguay, R.L.; Barton, C.L.; Traber, M.G.; Stevens, J.F. Vitamin C deficiency activates the purine nucleotide cycle in zebrafish. J. Biol. Chem. 2012, 287, 3833-3841.

138. Monti, D.A.; Mitchell, E.; Bazzan, A.J.; Littman, S.; Zabrecky, G.; Yeo, C.J.; Pillai, M.V.; Newberg, A.B.; Deshmukh, S.; Levine, M. Phase I evaluation of intravenous ascorbic acid in combination with gemcitabine and erlotinib in patients with metastatic pancreatic cancer. PLoS One 2012, 7, e29794.

139. Welsh, J.L.; Wagner, B.A.; van't Erve, T.J.; Zehr, P.S.; Berg, D.J.; Halfdanarson, T.R.; Yee, N.S.; Bodeker, K.L.; Du, J.; Roberts, L.J., II; et al. Pharmacological ascorbate with gemcitabine for the control of metastatic and node-positive pancreatic cancer (PACMAN): Results from a phase I clinical trial. Cancer Chemother. Pharmacol. 2013, 71, 765-775. 
Reprinted from Nutrients. Cite as: Park, S. The Effects of High Concentrations of Vitamin C on Cancer Cells. Nutrients 2013, 5, 3496-3505.

Review

\title{
The Effects of High Concentrations of Vitamin $\mathrm{C}$ on Cancer Cells
}

\section{Seyeon Park}

Department of Applied Chemistry, Dongduk Women's University, 23-1 Wolgok-dong, Sungbuk-ku, Seoul 136-714, Korea; E-Mail: sypark21@dongduk.ac.kr or seypark21@hotmail.com; Tel.: +82-2-940-4514; Fax: +82-2-940-4193

Received: 8 July 2013; in revised form: 22 August 2013 / Accepted: 23 August 2013 /

Published: 9 September 2013

\begin{abstract}
The effect of high doses of vitamin C for the treatment of cancer has been controversial. Our previous studies, and studies by others, have reported that vitamin $\mathrm{C}$ at concentrations of $0.25-1.0 \mathrm{mM}$ induced a dose- and time-dependent inhibition of proliferation in acute myeloid leukemia (AML) cell lines and in leukemic cells from peripheral blood specimens obtained from patients with AML. Treatment of cells with high doses of vitamin $\mathrm{C}$ resulted in an immediate increase in intracellular total glutathione content and glutathione-S transferase activity that was accompanied by the uptake of cysteine. These results suggest a new role for high concentrations of vitamin $\mathrm{C}$ in modulation of intracellular sulfur containing compounds, such as glutathione and cysteine. This review, discussing biochemical pharmacologic studies, including pharmacogenomic and pharmacoproteomic studies, presents the different pharmacological effects of vitamin $\mathrm{C}$ currently under investigation.
\end{abstract}

Keywords: high concentrations of vitamin C; pharmacogenomics; pharmacoproteomics

\section{Introduction}

There is increasing evidence that vitamin C (ascorbate) is selectively toxic to some types of tumor cells, functioning as a pro-oxidant [1-3]. Vitamin $C$ at concentrations of $10 \mathrm{nM}-1 \mathrm{mM}$ induced apoptosis in neuroblastoma and melanoma cells [4] and was shown to be an important modulator for the growth of mouse myeloma cells in an in vitro colony assay [5]. Studies have established that the growth of leukemic progenitor cells from patients with acute myeloid leukemia (AML) and myelodysplastic syndromes (MDS) can be significantly modulated by vitamin $\mathrm{C}$ [6,7]. Intravenous (i.v.) administration of sodium 5,6-benzylidene-1-ascorbate (SBA) to inoperable cancer patients 
induced a significant reduction in tumor volume without any adverse side effects [8]. Furthermore, recent clinical studies have reported that manipulation of vitamin $\mathrm{C}$ levels in vivo can result in clinical benefit for patients with AML and solid tumors [9].

Recent pharmacokinetic studies reported that $10 \mathrm{~g}$ of ascorbate given by i.v. produced plasma concentrations of nearly $6 \mathrm{mM}$, which were 25 -fold higher than concentrations resulting from the same oral dose [10-12]. Depending on the dose, as much as a 70-fold difference in plasma concentration was found between oral and i.v. administration [13]. Complementary and alternative medicine practitioners worldwide currently use ascorbate i.v. in patients, in part because there are no apparent harmful effects [14-16]. Physiological concentration of vitamin C is under $0.1 \mathrm{mM}$, while plasma vitamin $\mathrm{C}$ concentrations that cause toxicity to cancer cells in vitro (1 $\mathrm{mM}-10 \mathrm{mM}$, depending on cell lines) can be achieved clinically by intravenous administration, which means a high dose of vitamin $\mathrm{C}$.

In this review, in vitro and in vivo studies are summarized, showing that ascorbate killed cancer cells (Table 1). In addition, the mechanisms and physiologic relevance under investigation are also described. The results suggest that doses of vitamin $\mathrm{C}$ induce oxidative stress in cancer cells. Our previous results indicated that treatment of malignant lymphocytic cell lines with vitamin $\mathrm{C}$ $(0.25-1 \mathrm{mM})$ for $24 \mathrm{~h}$ led to a marked dose-dependent decrease of cell proliferation [17]. The responsive cell lines were human myeloid leukemia cell line HL-60, retinoic acid (RA)-sensitive acute promyelocytic leukemia (APL) cell line NB4 and RA-resistant APL cell line NB4-R1. Different types of leukemia cells, such as K562 (chronic myelogenous leukemia cell line) [17] and KG1 (myeloblast cell line) [18], were also responsive to vitamin C. A similar result was obtained with cells containing over $90 \%$ of blasts from patients with AML. In these cell lines, induction of apoptosis by vitamin $\mathrm{C}$ demonstrated a dose-dependent effect. In addition, vitamin C weakly induced apoptosis in ovarian cell lines, including SK-OV-3, OVCAR-3 and 2774 [17]. For many of the cancer cell lines, ascorbate concentrations caused a 50\% decrease in cell survival. The half maximal concentration $\left(\mathrm{IC}_{50}\right)$ values were less than $5 \mathrm{mM}$, and all tested normal cells were insensitive to $20 \mathrm{mM}$ ascorbate [13].

Table 1. Effects of vitamin C treatment on cell survival $[13,17,18]$.

\begin{tabular}{cc}
\hline Cell Line & IC $_{\mathbf{5 0}}(\mathbf{m M})$ \\
\hline HL-60 & $0.33 \pm 0.18^{*}$ \\
NB4 & $0.76 \pm 0.14^{*}$ \\
NB4-R1 & $0.45 \pm 0.24^{*}$ \\
NB4-R2 & $0.75 \pm 0.3^{*}$ \\
KG1 & $0.79 \pm 0.22^{*}$ \\
K562 & $0.5 \pm 0.11^{*}$ \\
U937 & $0.3 \pm 0.16^{*}$ \\
Normal Bone Marrow & $1 \pm 0.3^{*}$ \\
Patient with AML & $0.84 \pm 0.16^{*}$ \\
OVCAR & $>10 *$ \\
SK-OV3 & $>10 *$ \\
JLP119 & $<1$ \\
\hline
\end{tabular}


Table 1. Cont.

\begin{tabular}{cc}
\hline MCF7 & 2 \\
MB231 & 7 \\
Hs587T & 20 \\
KLN205 & $<1$ \\
RAG & $<2$ \\
CT26 & 4 \\
B16 & 7 \\
LL/2 & 11 \\
Hs587Bst & $>20$ \\
CCD34SK & $>20$ \\
Human normal lymphocyte & $>20$ \\
Human normal monocyte & $>20$ \\
\hline
\end{tabular}

The $\mathrm{IC}_{50}$ values are means \pm standard deviations from triplicate experiments. HL-60, human myeloid leukemia; NB4, NB4-R1, NB4-R2, human acute promyelocytic leukemia (APL); KG1, human myeloblast; K562, human chronic myelogenous leukemia; U937, human histiocytic lymphoma; OVCAR, SK-OV3, ovarian cancer; JLP119, human lymphoma; MCF7, MB231, Hs587t, human breast cancer; KLN205, mouse lung cancer; RAG, mouse kidney cancer; CT26, mouse colon cancer; B16, mouse melanoma; LL/2, mouse lung cancer; Hs587Bst, human normal breast cells; CCD34SK, human normal fibroblast cells. * $\mathrm{IC}_{50}$ value was determined using $\mathrm{H}^{3}$ incorporation proliferation assay for $24 \mathrm{~h}$.

\section{Molecular Mechanisms Induced by Vitamin C}

In our previous study, vitamin $\mathrm{C}$ at concentrations of $0.25-2.0 \mathrm{mM}$ significantly induced apoptosis in AML cell lines. Vitamin $\mathrm{C}$ induced oxidation of glutathione (GSH) to its oxidized form (GSSG). As a result, $\mathrm{H}_{2} \mathrm{O}_{2}$ accumulated in a concentration-dependent manner, in parallel with the induction of apoptosis. The direct role of $\mathrm{H}_{2} \mathrm{O}_{2}$ in the induction of apoptosis in AML cells was demonstrated by the finding that catalase could completely abrogate vitamin $\mathrm{C}$-induced apoptosis [17].

A 30-min incubation of NB4 cells with $0-10 \mathrm{mM}$ vitamin $\mathrm{C}$ resulted in its uptake in a concentration-dependent manner [17,19]. In accordance with its proposed pro-oxidant activity, vitamin $\mathrm{C}$ treatment reduced the GSH/GSSG ratio, which correlated with increased intracellular $\mathrm{H}_{2} \mathrm{O}_{2}$ in the NB4 cell line. Levine et al. suggested that the effect was due only to extracellular and not intracellular ascorbate, and that ascorbate-mediated cell death was probably due to protein-dependent extracellular $\mathrm{H}_{2} \mathrm{O}_{2}$ generation, via ascorbate radical formation from ascorbate as the electron donor [13].

Although $\mathrm{H}_{2} \mathrm{O}_{2}$ induced by ascorbate was first generated extracellularly, it is possible that it could diffuse across the plasma membrane into the intracellular space. Although studies in yeast and bacteria have shown that diffusion of $\mathrm{H}_{2} \mathrm{O}_{2}$ across membranes is limited, some reports have suggested that selected aquaporin homologues from plants and mammals can channel $\mathrm{H}_{2} \mathrm{O}_{2}$ across these membranes [20-25]. The susceptibility of cancer cells to ascorbate treatment might therefore be related to the permeability of hydrogen peroxide.

In our studies, vitamin $\mathrm{C}$ dramatically increased intracellular GSH oxidation and reactive oxygen species (ROS) levels within $3 \mathrm{~h}$ in a concentration-dependent manner. However, this GSH oxidation and the ROS accumulation did not last for a long period of time. After $3 \mathrm{~h}$, the increase in GSH has 
been observed and hypothesized to be a defense mechanism [18]. No additional ROS accumulation resulted from the change in GSH. However, based upon our studies, the dramatic changes of intracellular oxidation state within $3 \mathrm{~h}$ seem to be enough to induce intracellular signaling. The studies showed that treatment with $1 \mathrm{mM}$ vitamin $\mathrm{C}$ for only 30-60 min, followed by removal when replacing media, could induce apoptosis in both HL-60 and NB4 cells. This observation is consistent with initiation of apoptosis, resulting from generation of $\mathrm{H}_{2} \mathrm{O}_{2}$ after treatment with ascorbate. However, treatment with $\mathrm{As}_{2} \mathrm{O}_{3}$ resulted in less ROS accumulation than with vitamin $\mathrm{C}$, and it was not in a concentration-dependent manner. However, ROS accumulation increased up to $24 \mathrm{~h}$, with a long-lasting effect. Treatment with vitamin $\mathrm{C}$ combined with $\mathrm{As}_{2} \mathrm{O}_{3}$ increased $\mathrm{ROS}$ accumulation, as well as sustained the effect for up to $24 \mathrm{~h}$. These results are consistent with cellular data showing that apoptosis induced by $\mathrm{As}_{2} \mathrm{O}_{3}$ is evident even at three days [26,27].

\section{Proteomics Studies}

Proteomics provides important qualitative information on post-translational modifications to proteins and quantitative data on protein expression in response to a particular stimulus. This information is particularly important when it provides data on early cellular events, such as the stimulus and signaling cascades triggered independently of protein neosynthesis. In accordance with its proposed pro-oxidant activity, vitamin C-mediated reduction in the GSH/GSSG ratio correlated with an intracellular $\mathrm{H}_{2} \mathrm{O}_{2}$ increase in the NB4 cell line [17,19]. This type of change in regional oxidation state could cause changes in the cellular milieu that could result in changes in protein structure. This is especially true of the oxidation state of cysteine sulfur, which is important for determining the tertiary structure of proteins. The immediate effects of cell stimuli are associated with protein post-translational modifications, such as phosphorylation, glutathionylation and cysteine oxidation. To study these early modifications, NB4 human leukemia cells were treated with $0.5 \mathrm{mM}$ vitamin $\mathrm{C}$ and then analyzed by two-dimensional analysis. Approximately 240 different spots that were focused in a $\mathrm{pH}$ range of 4-7 were detected per sample.

After exposing cells to vitamin $\mathrm{C}$, we observed one new spot, three intensified spots and five attenuated spots [19]. Each of these spots were excised, digested with trypsin and analyzed by matrix-assisted laser desorption/ionization-time of flight (MALDI-TOF). Peptide mass fingerprint analysis and non-redundant sequence database matching allowed the unambiguous identification of all of the analyzed species [19]. An important protein identified from the proteomic analysis was a thiol/disulfide exchange catalyst, protein disulfide isomerase (PDI), which was a marker of the effect of vitamin C on NB4 cells [19]. PDI belongs to the superfamily of thiol/disulfide exchange catalysts, which act as protein-thiol-oxidoreductase enzymes, sharing sequence homology with thioredoxin [28]. PDI is composed of four domains, which have similarities with thioredoxin folds (i.e., a-b-b'-a') [29]. In our study [19], the intensity of the spot corresponding to the PDI $b$ subunit was decreased in vitamin C-treated cells as compared with control cells. These results demonstrated that thiol/disulfide exchange proteins are regulated in NB4 cells after vitamin C exposure. This is consistent with our study showing that intracellular GSH/GSSG exchange occurs shortly after vitamin C exposure [17].

When we measured cysteine uptake in leukemia cell lines exposed to vitamin $\mathrm{C}$ by using

${ }^{35} \mathrm{~S}$-labeled-L-Cys in the media, the time-dependent rate of cysteine uptake in the cell culture 
increased significantly. The rate of uptake, determined under conditions without vitamin C, was very low. The glutathione synthesis inhibitor, buthionine sulfoximine, potently inhibited this increase, suggesting that incorporation of cysteine that corresponded to the amount of increased glutathione was mediated by glutathione synthesis. Overall, our results indicated that vitamin $\mathrm{C}$-induced glutathione synthesis was accompanied by intracellular cysteine uptake.

Glutathione-S transferases (GSTs) are enzymes that catalyze the conjugation of electrophilic substitution to GSH, which protects cells by removing reactive oxygen species and regenerating $S$-thiolated proteins [30]. Intracellular total GSH levels in AML cells incubated with vitamin C peaked around $3 \mathrm{~h}$, then declined, while the increase in incorporated $\left[{ }^{35} \mathrm{~S}\right]$-L-Cys peaked at $3 \mathrm{~h}$ and remained high. These results showed that $\left[{ }^{35} \mathrm{~S}\right]$-L-Cys transported into cells through cysteine uptake was incorporated and transferred intracellularly, strongly suggesting that the sulfhydryl transfer system is affected by vitamin C [30].

We therefore hypothesize that the biochemical pathway leading to thiol/disulfide redox regulation could be activated by vitamin C. Interestingly, of the proteins whose expressions changed by vitamin $\mathrm{C}$ treatment, immunoglobulin heavy chain binding protein (BiP, identical to Hsp70 chaperone) [19], like PDI, is also a multi-domain chaperone. BiP associated with the $\alpha$-subunit of prolyl 4-hydroxylase (P4-H) by a disulfide bond [31]. P4-H consists of two distinct polypeptides, the catalytically more important $\alpha$-subunit and the $\beta$-subunit, which is identical to the multifunctional enzyme, PDI [31]. Thus, BiP associated with the $\alpha$-subunit of P4-H, which is another partner of PDI. The interaction of PDI with its substrates was due to a change in disulfide bonds, indicating that intrachain disulfide bonds between domains and substrates had been reduced [19]. Taken together, these results suggested that vitamin $\mathrm{C}$ oxidizes intracellular-reduced glutathione and affects disulfide bond formation in proteins [30].

Tropomyosin was also identified as a marker of the oxidative effect of vitamin C in NB4 cells. The spot corresponding to tropomyosin was positioned at an isoelectric point (pI) of approximately 5.0 and was attenuated in vitamin C-treated cells. In addition, a new spot having almost the same molecular weight was detected, which was positioned at a $\mathrm{pI}$ of 4.9 [19]. This new spot was also identified as tropomyosin, suggesting that post-translational chemical modification had affected its pI value. This result is consistent with previous data showing that the extracellular signal-regulated kinase (ERK)-mediated phosphorylation of tropomyosin-1 promoted cytoskeleton remodeling in response to oxidative stress [32]. The acidic shift of the spot with pI 5.0 to the phosphorylated tropomyosin spot by treatment with vitamin $\mathrm{C}$ was found to be abrogated by co-treatment with PD98059 [19], demonstrating that phosphorylation of tropomyosin was responsible for the observed acidic shift.

The significance of this observation may be related to differences in the regulation of the actomyosin contractile system in non-muscle cells as compared with that present in muscle cells. In addition, proteins that specifically reacted with sera from chronic myeloid leukemia patients included structural proteins, such as $\beta$-tubulin and tropomyosin isoforms [33]. Although the function of these proteins in myeloid leukemia needs further investigation, tropomyosin may have value as a leukemia-associated antigen and as a molecular target in antigen-based immunotherapy. In this regard, it is important to note that vitamin $\mathrm{C}$ causes a tropomyosin isoform to be modified during the immediate early response. 


\section{In Vivo Studies}

Levine et al. reported that reaction products obtained from ascorbate in vitro are also found in vivo [34]. They showed that after i.v. injection, ascorbate baseline concentrations of 50-100 $\mu \mathrm{M}$ in blood and extracellular fluids peaked to $>8 \mathrm{mM}$. After intraperitoneal injection, peaks approached $3 \mathrm{mM}$ in both fluids. They hypothesized that in vivo, ascorbate was a prodrug for selective delivery of ascorbate radical and $\mathrm{H}_{2} \mathrm{O}_{2}$ to the extracellular space. Moreover, a regimen of daily pharmacologic ascorbate treatment significantly decreased growth rates of ovarian $(p<0.005)$, pancreatic $(p<0.05)$ and glioblastoma $(p<0.001)$ tumors established in mice [35]. In addition to inducing oxidative stress, high concentrations of ascorbic acid inhibited cell migration and the gap filling capacity of endothelial progenitor cells (EPCs) [36], and it has been reported that ascorbic acid inhibited corneal neovascularization in a rat model [37].

High concentrations of ascorbic acid also inhibited tumor growth in BALB/C mice implanted with sarcoma 180 cancer cells [38]. The survival rate increased by $20 \%$ in the group that received high doses of ascorbic acid, compared to controls. Gene expression studies from biopsy and wound healing analysis in vivo and in vitro suggested that the carcinostatic effect induced by high doses of ascorbic acid were related to inhibition of angiogenesis [39]. In addition, intraperitoneal administration of high doses of ascorbic acid quantitatively upregulated Raf kinase inhibitory protein (RKIP) and annexin A5 expression in a group of BALB/C mice implanted with S-180 sarcoma cancer cells. The increase in RKIP protein levels suggested that these proteins are involved in the ascorbic acid-mediated suppression of tumor formation [39]. Moreover, high doses of ascorbic acid ( 1 $\mathrm{mM})$ enhanced the apoptosis of cancer cells during co-treatment with paclitaxel, and the combinational treatment of paclitaxel and ascorbic acid ameliorated the side effects caused by paclitaxel in BALB/c mice implanted with or without sarcoma 180 cancer cells [40].

\section{Clinical Studies}

Cases of apparent responses of malignancies to intravenous vitamin $\mathrm{C}$ therapy have been reported, although they were reported without sufficient detail [15,41-46]. A recent study showed that oral administration of the maximum tolerated dose of vitamin $\mathrm{C}(18 \mathrm{~g} /$ day $)$ produced peak plasma concentrations of only $0.22 \mathrm{mM}$, whereas intravenous administration of the same dose produced plasma concentrations approximately 25-fold higher. Larger doses (50-100 g) given intravenously resulted in plasma concentrations of approximately $14 \mathrm{mM}$ [41].

Some case reports stated that high dose i.v. vitamin $\mathrm{C}$ has been used by Complementary and Alternate Medicine (CAM) practitioners [47]. Phase I evaluation of intravenous vitamin C in combination with gemcitabine and erlotinib in patients with metastatic pancreatic cancer data did not reveal increased toxicity with the addition of ascorbic acid [48]. No side effects were reported for most patients, while 59 were reported to have lethargy or fatigue out of 11,233 patients that received intravenous vitamin C in 2006 and 8876 in 2008 [47]. Overall, it was reported that high dose intravenous vitamin $\mathrm{C}$ did not appear to cause serious side effects in patients.

Another clinical study reported the depletion of L-ascorbic acid alternating with its supplementation in the treatment of patients with acute myeloid leukemia or myelodysplastic syndromes [49]. During the supplementation phase, patients received daily i.v. administration of 
vitamin C. A pre-therapy in vitro assay was performed for vitamin C sensitivity of leukemic cells from individual patients. Of the nine patients with the in vitro assay indicating their leukemic cells were sensitive to vitamin $\mathrm{C}$, seven exhibited a clinical response, compared with none of the six patients who were insensitive to vitamin $\mathrm{C}$.

\section{Conclusions}

Previous studies have shown that vitamin $\mathrm{C}$ is involved in the mechanism of action of the intracellular microenvironment (oxidation) state changes that improve therapeutic potential, including apoptosis and necrosis. Although it is difficult to postulate precise vitamin $\mathrm{C}$-specific mechanisms at this time, identification of genes or proteins that are specifically regulated by vitamin $\mathrm{C}$ in certain cellular phenotypes could improve the efficacy of therapies.

\section{Conflicts of Interest}

The author declares no conflicts of interest.

\section{References}

1. Bram, S.; Froussard, P.; Guichard, M.; Jasmin, C.; Auqery, Y.; Sinoussi-Barre, F.; Wray, W. Ascorbic acid preferential toxicity for malignant melanoma cells. Nature 1980, 284, 629-631.

2. Bruchelt, G.; Baader, L.; Reith, A.G.; Holger, N.L.; Gebhardt, S.; Niethammer, D. Rationale for the Use of Ascorbic Acid in Neuroblastoma Therapy. In Human Neuroblastoma; Schwab, M., Ed.; Harwood Academic Publishers: Newark, NJ, USA, 1993; pp. 34-40.

3. Fujinaga, S.; Sakagami, H.; Kuribayashi, N.; Takahashi, H.; Amano, Y.; Sakagami, T.; Takeda, M. Possible role of hydrogen peroxide in apoptosis induction by ascorbic acid in human myelogenous leukemic cell lines. Showa Univ. Med. Sci. 1994, 6, 135-144.

4. De Laurenzi, V.; Melino, G.; Savini, I.; Annicchiarico-Petruzzelli, M.; Finazzi-Agro, A.; Avigliano, L. Cell death by oxidative stress and ascorbic acid regeneration in human neuroectodermal cell lines. Eur. J. Cancer 1995, 31, 463-466.

5. Park, C.H.; Bergsagel, D.E.; McCulloch, E.A. Ascorbic acid: A culture requirement for colony formation by mouse plasmacytoma cells. Science 1971, 174, 720-722.

6. Park, C.H. The biological nature of the effect of ascorbic acids on the growth of human leukemic cells. Cancer Res. 1985, 45, 3969-3973.

7. Park, C.H.; Kimler, B.F.; Bodensteiner, D.; Lynch, S.R.; Hassanein, R.S. In vitro growth modulation by L-ascorbic acid of colony-forming cells from bone marrow of patients with myelodysplastic syndromes. Cancer Res. 1992, 52, 4458-4466.

8. Sakagami, H.; Asano, K.; Fukuchi, K.; Gomi, K.; Ota, H.; Kazama, K.; Tanuma, S.; Kochi, M. Induction of tumor degeneration by sodium benzylideneascorbate. Anticancer Res. 1991, 11, $1533-1538$.

9. Park, C.H.; Kim, W.S.; Park, C.; Lee, M.H.; Boo, Y.C.; Yoon, S.S. Clinical disease suppression and reduction in acute myeloid leukemia and solid tumors by very high dose of L-ascorbic acid: A new concept and in search of molecular targets. Clin. Cancer Res. 1999, 5, 3784s. 
10. Padayatty, S.J.; Sun, H.; Wang, Y.; Riordan, H.D.; Hewitt, S.M.; Katz, A.; Wesley, R.A.; Levine, M. Vitamin C Pharmacokinetics: Implications for Oral and Intravenous Use. Ann. Intern. Med. 2004, 140, 533-537.

11. Levine, M.; Conry-Cantilena, C.; Wang, Y.; Welch, R.W.; Washko, P.W.; Dhariwal, K.R.; Park, J.B.; Lazarev, A.; Graumlich, J.; King, J.; et al. Vitamin C Pharmacokinetics in healthy volunteers: Evidence for a recommended dietary allowance. Proc. Natl. Acad. Sci. USA 1996, 93, 3704-3709.

12. Levine, M.; Wang, Y.; Padayatty, S.J.; Morrow, J.D. A new recommended dietary allowance of vitamin C for healthy young women. Proc. Natl. Acad. Sci. USA 2001, 98, 9842-9846.

13. Chen, Q.; Espey, M.G.; Cherukuri, M.K.; Mitchell, J.B.; Corpe, C.P.; Buettner, G.R.; Shacter, E.; Levine, M. Ascorbic acid at Pharmacologic concentrations selectively kill cancer cells: Ascorbic acid as a pro-drug to deliver hydrogen peroxide to tissues. Proc. Natl. Acad. Sci. USA 2005, 102, 13604-13609.

14. Levine, M.; Rumsey, S.C.; Daruwala, R.C.; Park, J.B.; Wang, Y. Criteria and recommendation for vitamin C intake. J. Am. Med. 1999, 281, 1415-1423.

15. Riordan, N.H.; Riordan, H.D.; Casciari, J.J. Clinicla and experimental experiences with intravenous vitamin C. J. Orthomol. Med. 2000, 15, 201-203.

16. Riordan, H.D.; Hunninghake, R.E.; Riordan, N.H.; Jackson, J.J.; Meng, X.; Taylor, P.; Casciari, J.J.; Gonzalez, M.J.; Miranda-Massari, J.R.; Mora, E.M.; et al. Intravenous Ascorbic Acid: Protocol for its Application and Use. P. R. Health Sci. J. 2003, 22, 287-290.

17. Park, S.; Han, S.S.; Park, C.H.; Hahm, E.R.; Lee, S.J.; Park, H.K.; Lee, S.H.; Kim, W.S.; Jung, C.W.; Park, K.; et al. L-Ascorbic acid induces apoptosis in acute myeloid leukemia cells via hydrogen peroxide-mediated mechanisms. Int. J. Biochem. Cell Biol. 2004, 36, 2180-2195.

18. Park, S. Samsung Medical Center, Seoul, Korea. Unpublished work, 2004.

19. Park, S.; Lee, J.; Yeom, C.H. A proteomic approach to the identification of early molecular targets changed by L-ascorbic acid in NB4 human leukemia cells. J. Cell. Biochem. 2006, 99, 1628-1641.

20. Bienert, G.P.; Schjoerring, J.K.; Jahn, T.P. Membrane transport of hydrogen peroxide. Biochim. Biophys. Acta 2006, 1758, 994-1003.

21. Seaver, L.C.; Imlay, A.J. Hydrogen peroxide fluxes and compartmentalization inside growing Escherichia coli. J. Bacteriol. 2001, 183, 7182-7189.

22. Antunes, F.; Cadenas, E. Estimations of $\mathrm{H}_{2} \mathrm{O}_{2}$ gradients across biomembranes. FEBS Lett. 2000, 475, 121-126.

23. Makino, N.; Sasaki, K.; Hashida, K.; Sakakura, Y. A metabolic model describing the $\mathrm{H}_{2} \mathrm{O}_{2}$ elimination by mammalian cells including $\mathrm{H}_{2} \mathrm{O}_{2}$ permeation through cytoplasmatic and peroxisomal membranes: comparison with experimental data. Biochim. Biophys. Acta 2004, 1673, 149-159.

24. Sousa-Lopes, A.; Antunes, F.; Cyrne, L.; Marinho, H.S. Decreased cellular permeability to $\mathrm{H}_{2} \mathrm{O}_{2}$ protects Saccharomyces cerevisiaein stationary phase against oxidative stress. FEBS Lett. 2004, 578, 152-156.

25. Mathai, J.C.; Sitaramam, V. Strech sensitivity of transmembrane mobility of hydrogen peroxide through voids in the bilayer. J. Biol. Chem. 1994, 269, 17784-17793. 
26. Zhu, X.H.; Shen, Y.L.; Jing, Y.K.; Cai, X.; Jia, P.M.; Huang, Y.; Tang, W.; Shi, G.Y.; Sun, Y.P.; Dai, J.; et al. Apoptosis and Growth Inhibition in Malignant Lymphocytes After Treatment With Arsenic Trioxide at Clinically Achievable Concentrations. J. Natl. Cancer Inst. 1999, 91, $772-778$.

27. Dai, J.; Weinberg, R.S.; Waxman, S.; Jing, Y. Malignant cells can be sensitized to undergo growth inhibition and apoptosis by arsenic trioxide through modulation of the glutathione redox system. Blood 1999, 93, 268-277.

28. Noiva, R. Protein disulfide isomerase: The multifunctional redox chaperone of the endoplasmic reticulum. Semin. Cell Dev. Biol. 1999, 10, 481-493.

29. Kemmink, J.; Darby, N.J.; Dijkstra, K.; Nilges, M.; Creighton, T.E. The folding catalyst protein disulfide isomerase is constructed of active and inactive thioredoxin modules. Curr. Biol. 1997, 7, 239-245.

30. Park, S. Apoptosis of Leukemia Cells Induced by L-Ascorbic Acid and Arsenic Trioxide: The Effect of Oxidative Stress and Glutathione homeostasis.Albina. In Cell Apoptosis and Cancer; Taylor, A.W., Ed.; Nova Science Publishers, Inc.: New York, NY, USA, 2007; pp. 87-111.

31. John, D.C.; Bulleid, N.J. Intracellular dissociation and reassembly of prolyl 4-hydroxylase: The alpha-subunits associated with the immunoglobulin-heavy-chain binding protein (BiP) allowing reassembly with the $\beta$-subunit. Biochem. J. 1996, 317, 659-665.

32. Houle, F.; Rousseau, S.; Morrice, N.; Luc, M.; Mongrain, S.; Turner, C.E.; Tanaka, S.; Moreau, P.; Huot, J. Extracellular signal-regulated kinase mediates phosphorylation of tropomyosin-1 to promote cytoskeleton remodeling in response to oxidative stress: Impact on membrane blebbing. Mol. Biol. Cell 2003, 14, 1418-1432.

33. Zou, L.; Wu, Y.; Pei, L.; Zhong, D.; Gen, M.; Zhao, T.; Wu, J.; Ni, B.; Mou, Z.; Han, J.; et al. Identification of leukemia-associated antigens in chronic myeloid leukemia by proteomic analysis. Leuk. Res. 2005, 29, 1387-1391.

34. Chen, Q.; Espey, M.G.; Sun, A.Y.; Lee, J.H.; Krishna, M.C.; Shacter, E.; Choyke, P.L.; Pooput, C.; Kirk, K.L.; Buettner, G.R.; et al. Ascorbate in pharmacologic concentrations selectively generates ascorbate radical and hydrogen peroxide in extracellular fluid in vivo. Proc. Natl. Acad. Sci. USA 2007, 104, 8749-8754.

35. Chen, Q.; Espey, M.G.; Sun, A.Y.; Pooput, C.; Kirk, K.L.; Krishna, M.C.; Khosh, D.B.; Drisko, J.; Levin, M. Pharmacologic doses of ascorbate act as a prooxidant and decrease growth of aggressive tumor xenografts in mice. Proc. Natl. Acad. Sci. USA 2008, 105, 11105-11109.

36. Mikirova, N.A.; Ichim, T.E.; Riordan, N.H. Anti-angiogenic effect ofhigh doses of ascorbic acid. J. Transl. Med. 2008, 6, doi:10.1186/1479-5876-6-50.

37. Peyman, G.A.; Kivilcim, M.; Morales, A.M.; DellaCroce, J.T.; Conway, M.D. Inhibition of corneal angiogenesis by ascorbic acid in the ratmodel. Graefe's Arch. Clin. Exp. Ophthalmol. 2007, 245, 1461-1467.

38. Yeom, C.H.; Lee, G.; Park, J.; Yu, J.; Park, S.; Yi, S.Y.; Lee, H.; Hong, Y.; Yang, J.; Lee, S. High dose concentration administration of ascorbic acid inhibits tumor growth in BLAB/C mice implanted with sarcoma 180 cancer cells via the restriction of angiogenesis. J. Transl. Med. 2009, 7, doi:10.1186/1479-5876-7-70. 
39. Park, S.; Ahn, E.S.; Lee, S.; Jung, M.; Park, J.H.; Yi, S.Y.; Yeom, C.H. Proteomicanalysis reveals upregulation of RKIP in S-180 implanted BALB/C mouse after treatment with ascorbic acid. J. Cell. Biochem. 2009, 106, 1136-1145.

40. Park, J.H.; Davis, K.R.; Lee, G.; Jung, M.; Jung, Y.; Park, J.; Yi, S.Y.; Lee, M.A.; Lee, S.; Yeom, C.H.; et al. Ascorbic acid alleviates toxicity of paclitaxel without interfering with the anticancer efficacy in mice. Nutr. Res. 2012, 32, 873-883.

41. Padayatty, S.J.; Riordan, H.D.; Hewitt, S.M.; Katz, A.; Hoffer, L.J.; Levine, M. Intravenously administered vitamin $\mathrm{C}$ as cancer therapy: Three cases. CMAJ 2006, 174, 937-942.

42. Riordan, H.D.; Jackson, J.A.; Riordan, N.H.; Schultz, M. High-dose intravenous vitamin C in the treatment of a patient with renal cell carcinoma of the kidney. J. Orthomol. Med. 1998, 13, $72-73$.

43. Riordan, H.D.; Riordan, N.H.; Jackson, J.A.; Casciari, J.J.; Hunninghake, R.; Gonzalez, M.J.; Mora, E.M.; Miranda-Massari, J.R.; Rosario, N.; Rivera, A. Intravenous vitamin C as a chemotherapy agent: a report on clinical cases. P. R. Health Sci. J. 2004, 23, 115-118.

44. Riordan, H.D.; Jackson, J.A.; Schultz, M. Case study: High-dose intravenous vitamin C in the treatment of a patient with adenocarcinoma of the kidney. J. Orthomol. Med. 1990, 5, 5-7.

45. Jackson, J.A.; Riordan, H.D.; Hunninghake, R.E.; Riordan, N. High-dose intravenous vitamin C and long-time survival of a patient with cancer of the head of the pancreas. J. Orthomol. Med. 1995, 10, 87-88.

46. Riordan, N.H.; Jackson, J.A.; Riordan, H.D. Intravenous vitamin C in a terminal cancer patient. J. Orthomol. Med. 1996, 11, 80-82.

47. Padayatty, S.J.; Sun, A.Y.; Chen, Q.; Espey, M.G.; Drislo, J.; Levine, M. Vitamin C: Intravenous use by complementary and alternative medicine practitioners and adverse effects. PLoS One 2010, 5, e11411.

48. Monti, D.A.; Mitchell, E.; Bazzan, A.J.; Littman, S.; Zabrecky, G.; Yeo, C.J.; Pillai, M.V.; Newberg, A.B.; Deshmukh, S.; Levine, M. Phase I evaluation of intravenous ascorbic acid in combination with gemcitabine and erlotinib in patients with metastatic pancreatic cancer. PLoS One 2012, 7, e29794.

49. Park, C.H.; Kimler, B.F.; Yi, S.Y.; Park, S.H.; Kim, K.; Jung, C.W.; Kim, S.H.; Lee, E.R.; Rha, M.; Kim, S.; et al. Depletion of L-ascorbic acid alternating with its supplementation in the treatment of patients with acute myeloid leukemia or myelodysplastic syndromes. Eur. J. Haematol. 2009, 83, 108-118. 
Reprinted from Nutrients. Cite as: Azqueta, A.; Costa, S.; Lorenzo, Y.; Bastani, N.E.; Collins, A.R. Vitamin $\mathrm{C}$ in Cultured Human (HeLa) Cells: Lack of Effect on DNA Protection and Repair. Nutrients 2013, 5, 1200-1217.

Article

\title{
Vitamin C in Cultured Human (HeLa) Cells: Lack of Effect on DNA Protection and Repair
}

\author{
Amaya Azqueta ${ }^{1,2, *}$, Solange Costa ${ }^{2,3}$, Yolanda Lorenzo ${ }^{2}$, Nasser E. Bastani ${ }^{2}$ and \\ Andrew R. Collins ${ }^{2}$ \\ 1 Department of Pharmacology and Toxicology, University of Navarra, C/Irunlarrea 1, \\ 31009 Pamplona, Spain \\ 2 Department of Nutrition, Institute of Basic Medical Sciences, University of Oslo, \\ PB 1046 Blindern, 0316 Oslo, Norway; E-Mails: solange.costa2@gmail.com (S.C.); \\ y.c.lorenzo@medisin.uio.no (Y.L.); n.e.bastani@medisin.uio.no (N.E.B.); \\ a.r.collins@medisin.uio.no (A.R.C.) \\ 3 Environmental Health Department, National Institute of Health, Rua Alexandre Herculano 321, \\ 4000-055 Porto, Portugal \\ * Author to whom correspondence should be addressed; E-Mail: amazqueta@unav.es; \\ Tel.: +34-948-425-653 (ext. 806574); Fax: +34-948-425-652.
}

Received: 19 February 2013; in revised form: 18 March 2013 / Accepted: 20 March 2013 / Published: 9 April 2013

\begin{abstract}
Aims: Dietary antioxidants, including vitamin C, may be in part responsible for the cancer-preventive effects of fruits and vegetables. Human intervention trials with clinical endpoints have failed to confirm their protective effects, and mechanistic studies have given inconsistent results. Our aim was to investigate antioxidant/pro-oxidant effects of vitamin $\mathrm{C}$ at the cellular level. Experimental approach: We have used the comet assay to investigate effects of vitamin $\mathrm{C}$ on DNA damage, antioxidant status, and DNA repair, in HeLa (human tumor) cells, and HPLC to measure uptake of vitamin C into cells. Results: Even at concentrations in the medium as high as $200 \mu \mathrm{M}$, vitamin $\mathrm{C}$ did not increase the background level of strand breaks or of oxidized purines in nuclear DNA. Vitamin $\mathrm{C}$ is taken up by HeLa cells and accumulates to $\mathrm{mM}$ levels. Preincubation of cells with vitamin $\mathrm{C}$ did not render them resistant to strand breakage induced by $\mathrm{H}_{2} \mathrm{O}_{2}$ or to purine oxidation by photosensitizer plus light. Vitamin $\mathrm{C}$ had no effect on the rate of repair of strand breaks or oxidized bases by HeLa cells. However, vitamin $\mathrm{C}$ at a concentration of less than $1 \mu \mathrm{M}$, or extract from cells preincubated for $6 \mathrm{~h}$ with vitamin $\mathrm{C}$, was able to induce damage (strand breaks) in lysed, histone-depleted nuclei
\end{abstract}


(nucleoids). Conclusion: In these cultured human cells, vitamin $\mathrm{C}$ displays neither antioxidant nor pro-oxidant properties; nor does it affect DNA strand break or base excision repair.

Keywords: DNA damage; DNA protection; DNA repair; vitamin C

\section{Introduction}

Vitamin C (ascorbic acid) is one of the best known and most studied dietary antioxidants. Its antioxidant credentials are well-established in vitro, since it can readily be demonstrated to prevent oxidation of lipids, DNA and other biological molecules. However, it is also possible for vitamin C to act as a pro-oxidant, through its ability to reduce transition metal ions, thus promoting the Fenton reaction which, acting on peroxides, produces highly reactive hydroxyl radicals [1]. The effect of vitamin $\mathrm{C}$ in vivo has been investigated in human trials with DNA oxidation as the measured endpoint. They include single dose intervention and longer trials, typically of a few weeks with daily supplementation. The first single-dose study was carried out by Green et al. [2], who administered $35 \mathrm{mg} / \mathrm{kg}$ of vitamin C to six healthy volunteers after an overnight fast and found a protection against $\gamma$ radiation-induced DNA breaks in white blood cells, using the comet assay (described below). Since then, more than 20 intervention studies with vitamin $\mathrm{C}$ have been carried out, and they have been critically reviewed by Duarte and Lunec [3] who find that most of the studies show either a decrease in DNA oxidation or no effect, while there are some that show an increase in DNA lesions. Møller and Loft [4] also describe conflicting results with vitamin C, concluding that more studies with better designs to avoid bias should be made in order to understand the role of vitamin $\mathrm{C}$ in protecting against DNA oxidation. In a subsequent supplementation trial, Møller et al. [5] found that a slow release formulation of vitamin $\mathrm{C}$ had a more pronounced and sustained protective effect on the steady state level of DNA base oxidation than did a normal release formulation.

Herbert et al. [6] carried out a double-blind placebo-controlled trial giving different doses of vitamin $\mathrm{C}(0,80,200$ and $400 \mathrm{mg} /$ day $)$ to four groups of 40 healthy volunteers over a period of 15 weeks (plus a 10 week washout period). They showed that vitamin $\mathrm{C}$ did not affect the intracellular level of 8-oxodGuo measured by HPLC. (This challenged an earlier report that vitamin C caused oxidation of bases in DNA [7]).

Sram et al. [8] recently reviewed human studies based on measurement of a variety of biomarkers of genetic damage - including some trials aimed at specific groups with occupational/environmental exposure to genotoxins. Generally either a protective effect or no effect was seen, depending - they suggest - on factors such as individual diet-derived vitamin $C$ concentrations, levels of exposure to xenobiotics, and oxidative stress.

Bjelakovic et al. [9] published a meta-analysis of randomized trials of antioxidant supplements aimed at primary and secondary prevention of various diseases, with mortality as the endpoint. They concluded that antioxidant supplements have no beneficial effects on mortality, though in the case of vitamin $\mathrm{C}$ the number of individuals sampled is still relatively small and a definitive statement cannot be made. 
Reviews of this subject conclude that we still need more studies to clarify the effect of vitamin C in humans $[1,8-10]$. We considered that it would be useful to investigate effects of vitamin $\mathrm{C}$ on genetic stability in cell culture, and so have examined, in the human tumor cell line HeLa, potential pro-oxidant, DNA-damaging effects of the vitamin; protection against DNA breakage caused by $\mathrm{H}_{2} \mathrm{O}_{2}$; and protection against base oxidation induced by photosensitizer plus visible light. In addition, we investigate the possibility that vitamin $\mathrm{C}$ might influence the capacity of cells for DNA repair.

We applied the comet assay, as used in many human biomonitoring studies, to the measurement of DNA damage, both strand breaks (SBs) and oxidized bases (employing the enzyme formamidopyrimidine DNA glycosylase (FPG) which converts 8-oxoGua and breakdown products of damaged purines to SBs). We used two approaches to measure repair of DNA damage [11]. If cells are subjected to damage (SBs or base oxidation), and then incubated to allow cellular repair, the residual lesions can be measured at intervals to show the kinetics of damage removal. Alternatively, in an in vitro assay, a cell extract is incubated with substrate DNA containing specific damage. The ability of the extract to introduce DNA breaks in the substrate cells reflects the activity of the enzymes responsible for the initial steps of repair, i.e., removing the lesion.

\section{Materials and Methods}

\subsection{Cell Culture}

HeLa cells (derived from human cervical cancer) were grown in DMEM supplemented with 10\% fetal bovine serum and antibiotics ( $100 \mathrm{U} / \mathrm{mL}$ penicillin and $100 \mu \mathrm{g} / \mathrm{mL}$ streptomycin). According to the formulation for DMEM it does not contain vitamin C. Vitamin C was not detectable in the batch of serum used. Cells were maintained as monolayer cultures at $37{ }^{\circ} \mathrm{C}$ in a humidified atmosphere with $5 \% \mathrm{CO}_{2}$ and routinely passaged by trypsinization when nearly confluent.

\subsection{Cell Treatment for DNA Damage and Protection}

HeLa cells in culture medium were incubated with $0,5,25,50,100$ or $200 \mu \mathrm{M}$ vitamin $\mathrm{C}$

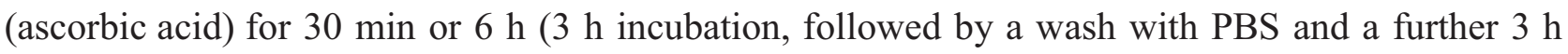
incubation with vitamin $\mathrm{C}$ at the same concentration) at $37{ }^{\circ} \mathrm{C}$ in the dark. The two consecutive $3 \mathrm{~h}$ treatments were designed to allow for the possible instability of vitamin $\mathrm{C}$ in solution. Vitamin $\mathrm{C}$ was dissolved in PBS in the dark just before use in each experiment. After treatment the comet assay was performed as described below. To check for DNA protection, after vitamin $\mathrm{C}$ treatment, cells were washed with PBS and then treated on ice with $25 \mu \mathrm{M} \mathrm{H}_{2} \mathrm{O}_{2}$ for 5 min to induce SBs, or with $1 \mu \mathrm{M}$ of the photosensitizer Ro (Ro-19-8022, from F. Hoffmann-La Roche) plus 1.5 min visible light (500 W tungsten halogen lamp, at $33 \mathrm{~cm}$ on ice) to induce oxidized purines, mostly 8-oxoGua. After treatment the comet assay was performed (see below). Three independent experiments were carried out.

\subsection{Measuring Uptake of Vitamin C}

Near-confluent cultures of HeLa cells in $60 \mathrm{~mm}$ dishes were incubated for $6 \mathrm{~h}(3 \mathrm{~h}+$ wash $+3 \mathrm{~h}$, as above) with vitamin $\mathrm{C}$ at 50 or $200 \mu \mathrm{M}$. At the end of this incubation, or after a further $24 \mathrm{~h}$ 
incubation in fresh medium without vitamin $\mathrm{C}$, cells were washed with PBS and scraped with a silicone rubber scraper into suspension in PBS. A cell count was carried out. The suspension was centrifuged ( $400 \times \mathrm{g}, 5 \mathrm{~min})$ and the pellet resuspended in $250 \mu \mathrm{L}$ of PBS to which an equal volume of $10 \%$ metaphosphoric acid was added before storing at $-20{ }^{\circ} \mathrm{C}$.

The frozen, acidified samples were thawed, and centrifuged $\left(3500 \times \mathrm{g}\right.$ at $4{ }^{\circ} \mathrm{C}$ for $\left.10 \mathrm{~min}\right)$. A volume of $100 \mu \mathrm{L}$ clear supernatant was mixed with $400 \mu \mathrm{L}$ of the mobile phase $(2 \%$ acetonitrile in $2.5 \mathrm{mM} \mathrm{NaH} 2 \mathrm{PO}_{4}, 2.5 \mathrm{mM}$ dodecyltrimethyl ammonium chloride and $1.25 \mathrm{mM} \mathrm{Na} 2$ EDTA in Milli-Q water) for direct determination of vitamin $\mathrm{C}$ by high-performance liquid chromatography (HPLC). For separation of vitamin $\mathrm{C}$ from interfering sample constituents, a Chromolith Performance RP18-e, $4.6 \mathrm{~mm} \times 100 \mathrm{~mm}$ column was used, with a Chromolith Performance RP18-e, $4.6 \mathrm{~mm} \times$ $10 \mathrm{~mm}$ guard column (Phenomenex, Torrance, USA). The injection volume was $5 \mu \mathrm{L}$ and the flow rate was $6.0 \mathrm{~mL} / \mathrm{min}$. A variable wavelength ultraviolet (UV) detector was used at $264 \mathrm{~nm}$.

\subsection{Treatment of Cells for Cellular Repair Assay}

Repair of DNA damage can be studied by treating cells with a DNA-damaging agent, incubating, and measuring the damage remaining at intervals. Using this cellular repair assay we investigated the kinetics of DNA SB rejoining and base excision repair (BER). To check the influence of vitamin $\mathrm{C}$ on the repair of SBs, HeLa cells were pre-incubated with 0,50 or $100 \mu \mathrm{M}$ vitamin $\mathrm{C}$ overnight. Then cells were washed with PBS, treated with 0 or $100 \mu \mathrm{M} \mathrm{H}_{2} \mathrm{O}_{2}$ for $5 \mathrm{~min}$ on ice, and incubated for 10, 30 or $60 \mathrm{~min}$ in culture medium. To check the influence of vitamin $\mathrm{C}$ on the repair of oxidized bases, pre-incubation overnight with 0,50 or $100 \mu \mathrm{M}$ vitamin $\mathrm{C}$ was followed by a wash with PBS, treatment with 0 or $1 \mu \mathrm{M}$ Ro plus $5 \mathrm{~min}$ of light, and incubation for 1, 2, 4, 6, 8, or $24 \mathrm{~h}$ in culture medium. In another experimental design, HeLa cells in culture medium were pre-incubated with a higher concentration $(200 \mu \mathrm{M})$ of vitamin $\mathrm{C}$ but for only $30 \mathrm{~min}$. Then cells were washed with PBS, treated with $1 \mu \mathrm{M}$ Ro plus $2.5 \mathrm{~min}$ of light, and incubated for $3 \mathrm{~h}$ in culture medium including 0 or $200 \mu \mathrm{M}$ vitamin $\mathrm{C}$. They were then washed with PBS and incubated again in culture medium with the same concentration of vitamin $\mathrm{C}$ for a further $3 \mathrm{~h}$. The comet assay was performed after each time of incubation. The concentration of vitamin $\mathrm{C}$ used in these experiments was not genotoxic. Three independent experiments were performed.

\subsection{Comet Assay}

Just after treatment (or after incubation for repair), HeLa cells were trypsinized and resupended in PBS $\left(1 \times 10^{6}\right.$ cells $\left./ \mathrm{mL}\right)$. Thirty $\mu \mathrm{L}$ of each cell suspension were mixed with $140 \mu \mathrm{L}$ of $1 \%$ low melting point agarose, and two drops of $70 \mu \mathrm{L}$ were spread onto microscope slides precoated with $1 \%$ of normal melting point agarose. Glass cover slips were placed on the drops of agarose, which were allowed to set at $4{ }^{\circ} \mathrm{C}$. Then the cover slips were removed and the cells embedded in agarose were lysed for $1 \mathrm{~h}$ by immersion in $2.5 \mathrm{M} \mathrm{NaCl}, 0.1 \mathrm{M} \mathrm{Na}_{2}$ EDTA, $0.1 \mathrm{M}$ Tris base, $\mathrm{pH} 10$ and 1\% Triton X-100 at $4{ }^{\circ} \mathrm{C}$ (lysis solution). For measurement of SBs, the slides were then placed in a horizontal gel electrophoresis tank and the DNA was allowed to unwind for $40 \mathrm{~min}$ in freshly prepared alkaline electrophoresis solution (0.3 $\mathrm{M} \mathrm{NaOH}$ and $\left.1 \mathrm{mM} \mathrm{Na}_{2} \mathrm{EDTA}, \mathrm{pH}>13\right)$. 
For measurement of oxidized purines, after lysis, slides were washed three times (5 min each time) with buffer $\mathrm{F}(0.1 \mathrm{M} \mathrm{KCl}, 0.5 \mathrm{mM} \mathrm{Na} 2$ EDTA, $40 \mathrm{mM}$ HEPES, $0.2 \mathrm{mg} / \mathrm{mL} \mathrm{BSA}, \mathrm{pH} 8.0)$ and incubated for $30 \mathrm{~min}$ at $37{ }^{\circ} \mathrm{C}$ with FPG in buffer $\mathrm{F}$, or with buffer $\mathrm{F}$ alone, in a moist box. After incubation the slides were placed in the electrophoresis solution.

Electrophoresis was carried out in the alkaline solution for $30 \mathrm{~min}$ at $1.1 \mathrm{~V} / \mathrm{cm}$ and approximately $300 \mathrm{~mA}$ at $4{ }^{\circ} \mathrm{C}$. The slides were washed in $0.4 \mathrm{M}$ Tris base $(\mathrm{pH} 7.5)$ for $10 \mathrm{~min}$ at $4{ }^{\circ} \mathrm{C}$ to neutralize the excess alkali and $10 \mathrm{~min}$ in water at $4{ }^{\circ} \mathrm{C}$. Then they were left to dry overnight.

Gels were stained with $25 \mu \mathrm{L}$ of DAPI (4',6-diamidine-2-phenylindol dihydrochloride, $1 \mu \mathrm{g} / \mathrm{mL}$ ), covered with a cover slip and coded before microscopic analysis. DAPI-stained nuclei were evaluated with a Nikon Eclipse TS-100 fluorescence microscope. A total of 50 comets on each gel were visually scored as belonging to one of five classes according to the tail intensity. Each comet class was given a value between 0 and $4:(0)=$ undamaged and (4) = maximum damage [12]. The total score in arbitrary units (AU) was calculated by the following equation:

(percentage of cells in class $0 \times 0)+($ percentage of cells in class $1 \times 1)+($ percentage of cells in class $2 \times 2)+($ percentage of cells in class $3 \times 3)+($ percentage of cells in class $4 \times 4)$.

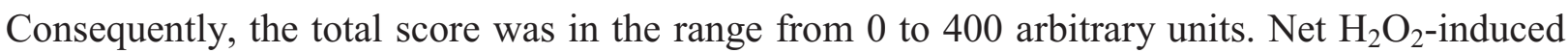
damage was calculated by subtracting the comet score for non- $\mathrm{H}_{2} \mathrm{O}_{2}$-treated cells from the score + $\mathrm{H}_{2} \mathrm{O}_{2}$. Net FPG-sensitive sites were calculated by subtracting the score for the slide incubated with buffer from the score for the slide incubated with enzyme. Net Ro-induced damage was calculated as the difference in net FPG-sensitive sites between Ro-treated cells and cells not treated with Ro.

\subsection{In Vitro Assay for DNA Repair}

The in vitro repair assay is used to check the ability of cell extracts to carry out the first steps of BER. Gel-embedded nucleoids from HeLa cells carrying oxidized bases (substrate) were incubated with extracts from HeLa cells pre-incubated with/without vitamin $\mathrm{C}$ and with different concentrations of vitamin $\mathrm{C}$ directly.

\subsubsection{Preparation of Substrate Cells}

HeLa cells were grown to $80 \%$ confluence, washed with PBS and treated with $1 \mu \mathrm{M}$ Ro plus 4 min of light. Then they were washed with cold PBS, detached by trypsinization, centrifuged for 5 min at $800 \times g$ at $4{ }^{\circ} \mathrm{C}$ and resuspended in freezing medium (DMEM supplemented with $20 \%$ fetal bovine serum, and $10 \% \mathrm{DMSO}$ ) at a concentration of $1 \times 10^{6}$ cells per mL. Aliquots of $0.7 \mathrm{~mL}$ were frozen slowly and stored at $-80^{\circ} \mathrm{C}$.

\subsubsection{Preparation of Cell Extracts}

Extracts were prepared from HeLa cells incubated with $0,50,100$ or $200 \mu \mathrm{M}$ vitamin $\mathrm{C}$ for $6 \mathrm{~h}$ $(3 \mathrm{~h}+$ wash $+3 \mathrm{~h})$. They were washed with cold PBS, trypsinized, centrifuged at $800 \times g$ for $5 \mathrm{~min}$ at $4{ }^{\circ} \mathrm{C}$ and resuspended in cold PBS at a concentration of $1.25 \times 10^{6}$ cells per $\mathrm{mL}$. Then the cell suspension was split into aliquots of $1 \mathrm{~mL}$ and centrifuged at $14,000 \times \mathrm{g}$ for $5 \mathrm{~min}$ at $4{ }^{\circ} \mathrm{C}$. The supernatant of each aliquot was completely discarded and the dry pellets were frozen in liquid 
nitrogen and stored at $-80{ }^{\circ} \mathrm{C}$. After thawing the pellets were resuspended in $32 \mu \mathrm{L}$ of extraction buffer A (45 mM HEPES, 0.4 M KCl, $1 \mathrm{mM}$ EDTA, $0.1 \mathrm{mM}$ dithiothreitol and 10\% glycerol, $\mathrm{pH} 7.8$ ) containing $0.25 \%$ of Triton $\mathrm{X}-100$, mixed using vortex at high speed for $5-10 \mathrm{~s}$, incubated for $5 \mathrm{~min}$ on ice and centrifuged at $14,000 \times \mathrm{g}$ for $5 \mathrm{~min}$ at $4{ }^{\circ} \mathrm{C}$. Then $27 \mu \mathrm{L}$ of the supernatant was mixed with $110 \mu \mathrm{L}$ of cold reaction buffer $\mathrm{F}$.

\subsubsection{The Reaction}

The substrate cells were thawed, diluted in cold PBS, centrifuged at $800 \times g$ for 5 min at $4{ }^{\circ} \mathrm{C}$ and resuspended in PBS at a concentration of $1 \times 10^{6}$ cells per $\mathrm{mL}$. As described in section 2.4 gels were made, put in lysis for $1 \mathrm{~h}$ and washed 3 times ( 5 min each time) with buffer F. Then $30 \mu \mathrm{L}$ of extract (experimental treatment), FPG (positive control), or buffer F (negative control) were placed on each gel (two gels per condition) and covered with a cover slip. Slides were placed in a moist box and incubated for 10 and $20 \mathrm{~min}$. Afterwards the rest of the comet assay was performed as described above.

\subsubsection{Treatment of Nucleoids with Vitamin C}

In order to check the direct effect of vitamin $\mathrm{C}$ on DNA, non-damaged HeLa cell nucleoids, or substrate nucleoids prepared as described in Section 2.6.1, were treated for 10 or 20 min with vitamin $\mathrm{C}$ dissolved in buffer $\mathrm{A}$ at $0,1,5$ or $25 \mu \mathrm{M}$ and diluted with buffer $\mathrm{F}$, as described in Section 2.6.2. The reaction was carried out as described in Section 2.6.3. The final concentrations of vitamin $\mathrm{C}$ were therefore $0,0.25,1.25$, and $6.25 \mu \mathrm{M}$.

\subsection{Statistical Analysis}

The statistical analysis was performed by using the software SPSS 11.0. Data are presented with descriptive analysis (mean $\pm \mathrm{SD}$ for 3 independent experiments). The comparisons between total comet scores of the different groups were performed by the non-parametric Kruskal-Wallis test followed by Mann-Whitney U test when the first one showed the presence of differences. Probability $p \leq 0.05$ was accepted as the level of significance.

\section{Results}

\subsection{Direct Induction of DNA Damage}

We evaluated DNA damage, i.e., SBs and oxidized bases, just after incubating HeLa cells with different concentrations of vitamin $\mathrm{C}$ for $30 \mathrm{~min}$ or $6 \mathrm{~h}$, using the comet assay with and without FPG. Treatment with different concentrations of vitamin $\mathrm{C}(5-200 \mu \mathrm{M})$ did not cause or decrease SBs or base oxidation in HeLa cells (Figure 1A,B). 
Figure 1. Effect of different concentrations of vitamin C on DNA integrity (SBs and net formamidopyrimidine DNA glycosylase (FPG)-sensitive sites) of HeLa cells treated for $30 \mathrm{~min}(\mathbf{A})$ and $6 \mathrm{~h}(\mathbf{B})$ and measured using the comet assay. Bars: SD calculated from the results of 3 independent experiments. No significant effects were seen.

\section{A. 30 min incubation}

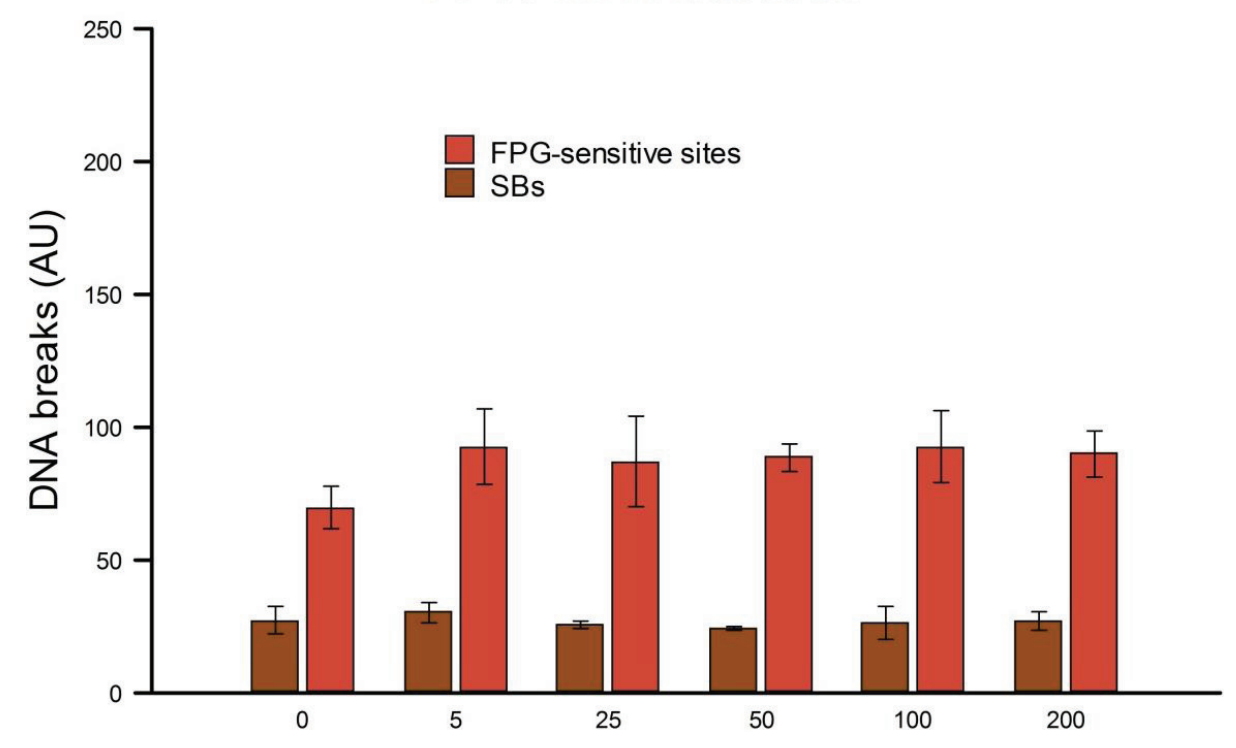

Concentration of vitamin $\mathrm{C}(\mu \mathrm{M})$

B. 6 h incubation

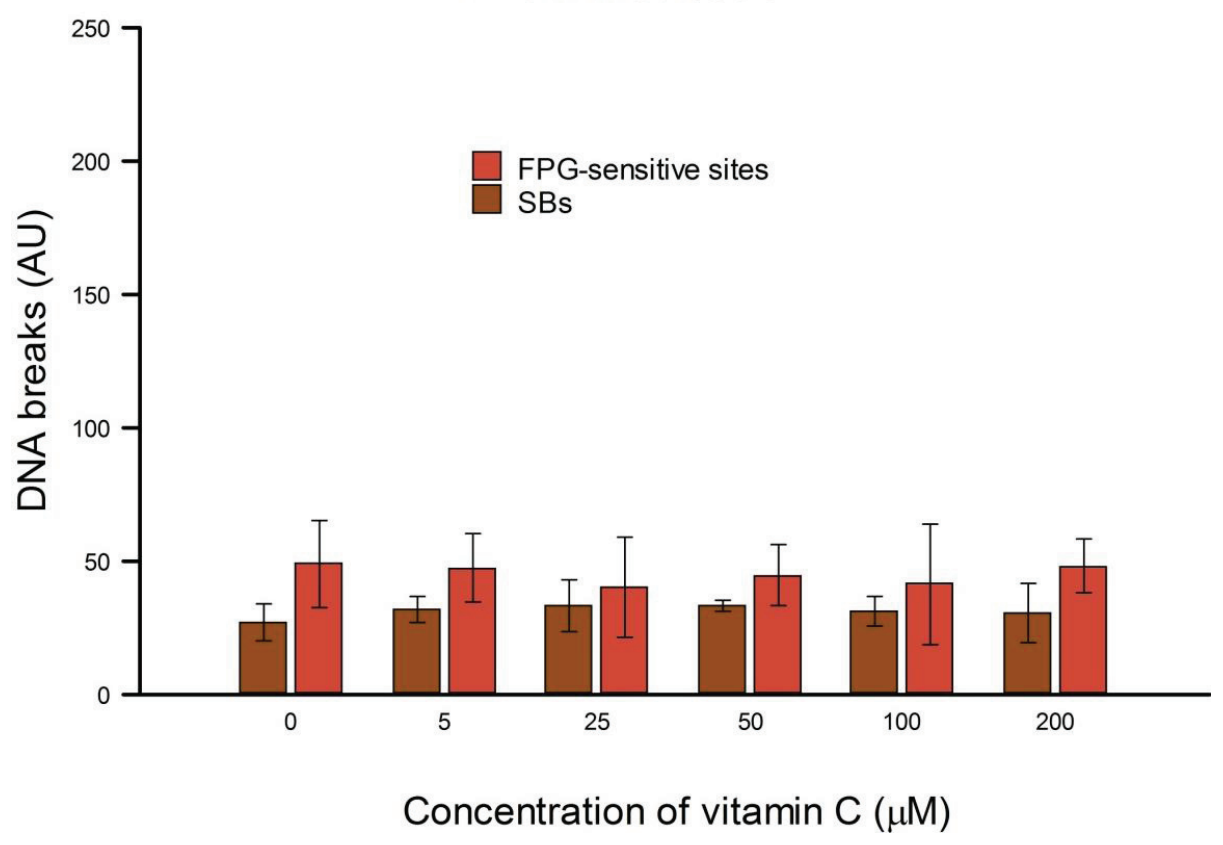

\subsection{Protection against $\mathrm{H}_{2} \mathrm{O}_{2-}$ and Ro-Induced DNA Damage}

Using the comet assay we investigated the possible protective effect of vitamin $\mathrm{C}$ against DNA damage caused by $\mathrm{H}_{2} \mathrm{O}_{2}$ or Ro plus light after pre-incubating HeLa cells with vitamin $\mathrm{C}(5-200 \mu \mathrm{M})$ for $30 \mathrm{~min}$ or $6 \mathrm{~h}$. In HeLa cells the yield of damage, net $\mathrm{H}_{2} \mathrm{O}_{2}$-induced breaks or net Ro-induced FPG-sensitive sites, was unchanged by the vitamin $\mathrm{C}$ incubation (Figures 2A,B and 3A,B). 
Figure 2. Effect of pre-incubation of HeLa cells with different concentrations of vitamin $\mathrm{C}$ for $30 \mathrm{~min}(\mathbf{A})$ or $6 \mathrm{~h}(\mathbf{B})$ on damage induced by $\mathrm{H}_{2} \mathrm{O}_{2}$. Net $\mathrm{H}_{2} \mathrm{O}_{2}$-induced damage was calculated by subtracting comet score without $\mathrm{H}_{2} \mathrm{O}_{2}$ (as in Figure 1) from the total comet score with $\mathrm{H}_{2} \mathrm{O}_{2}$. Bars: SD calculated from the results of 3 independent experiments. No significant effects were seen.

\section{A. 30 min incubation}

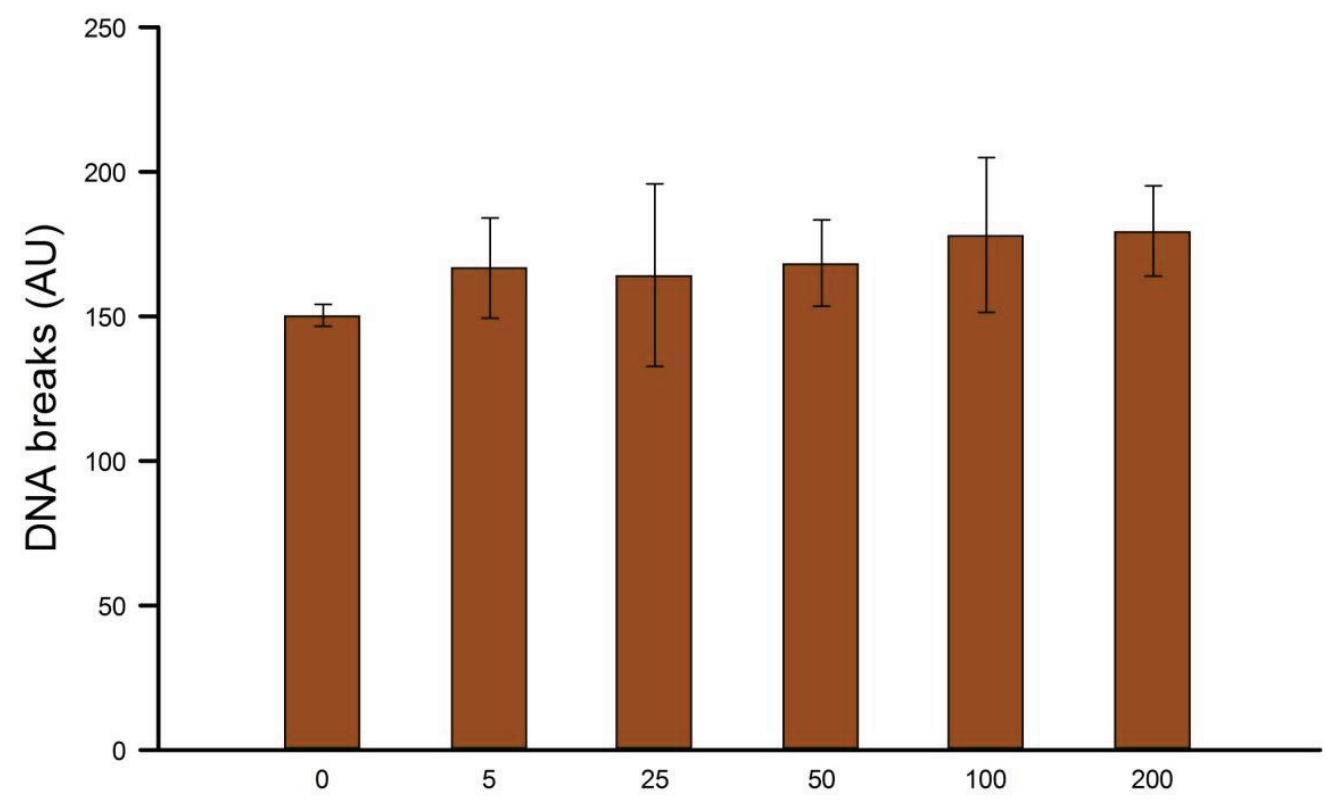

Concentration of vitamin $\mathrm{C}(\mu \mathrm{M})$

B. 6 h incubation

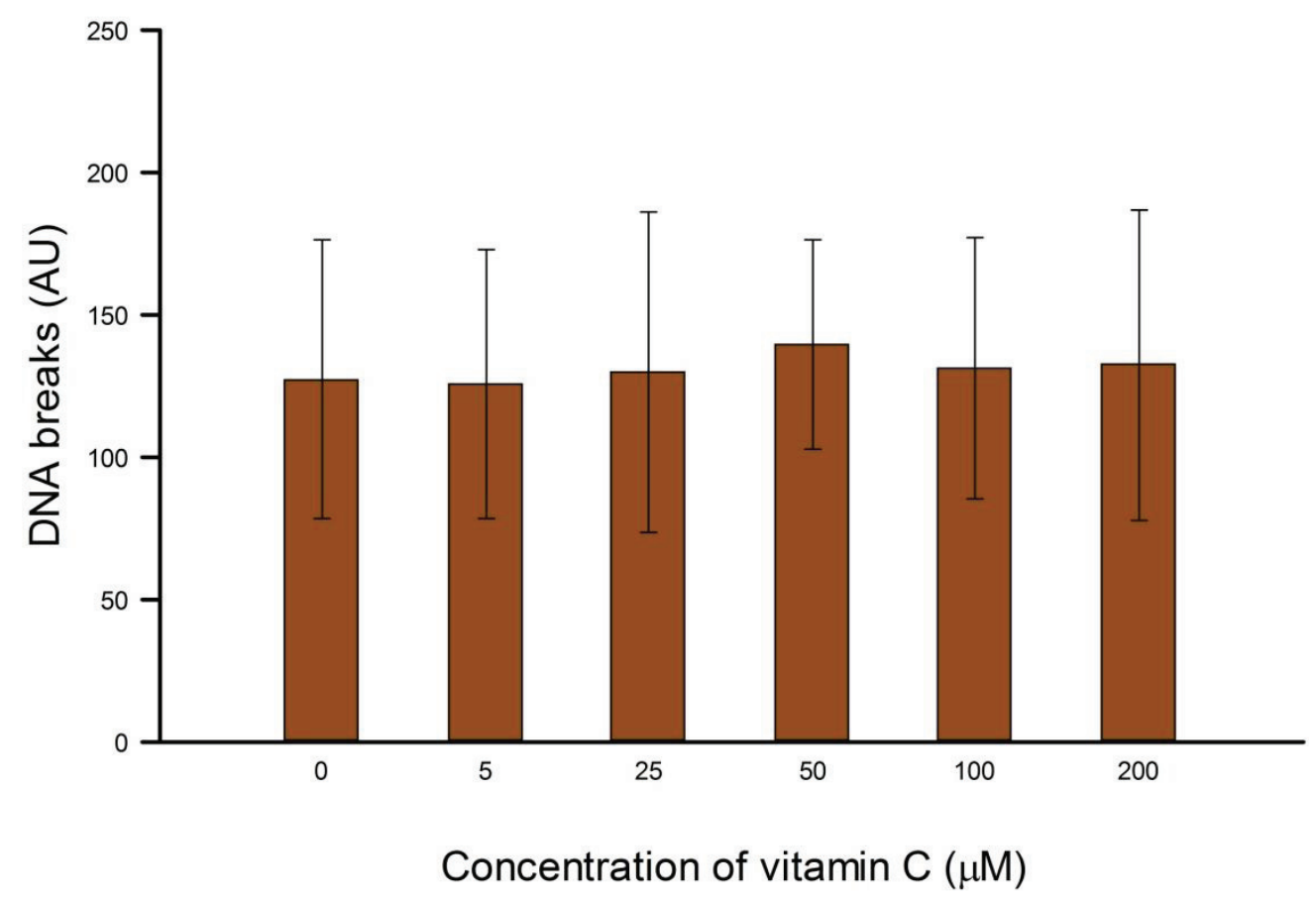


Figure 3. Effect of pre-incubation of HeLa cells with different concentrations of vitamin $\mathrm{C}$ for $30 \mathrm{~min}(\mathbf{A})$ or $6 \mathrm{~h}$ (B) on FPG-sensitive sites induced by Ro plus light. Net Ro-induced damage was calculated by subtracting the FPG-sensitive sites without Ro plus light (as in Figure 1) from the FPG-sensitive sites with Ro plus light. Bars: SD calculated from the results of 3 independent experiments. No significant effects were seen.

\section{A. 30 min incubation}

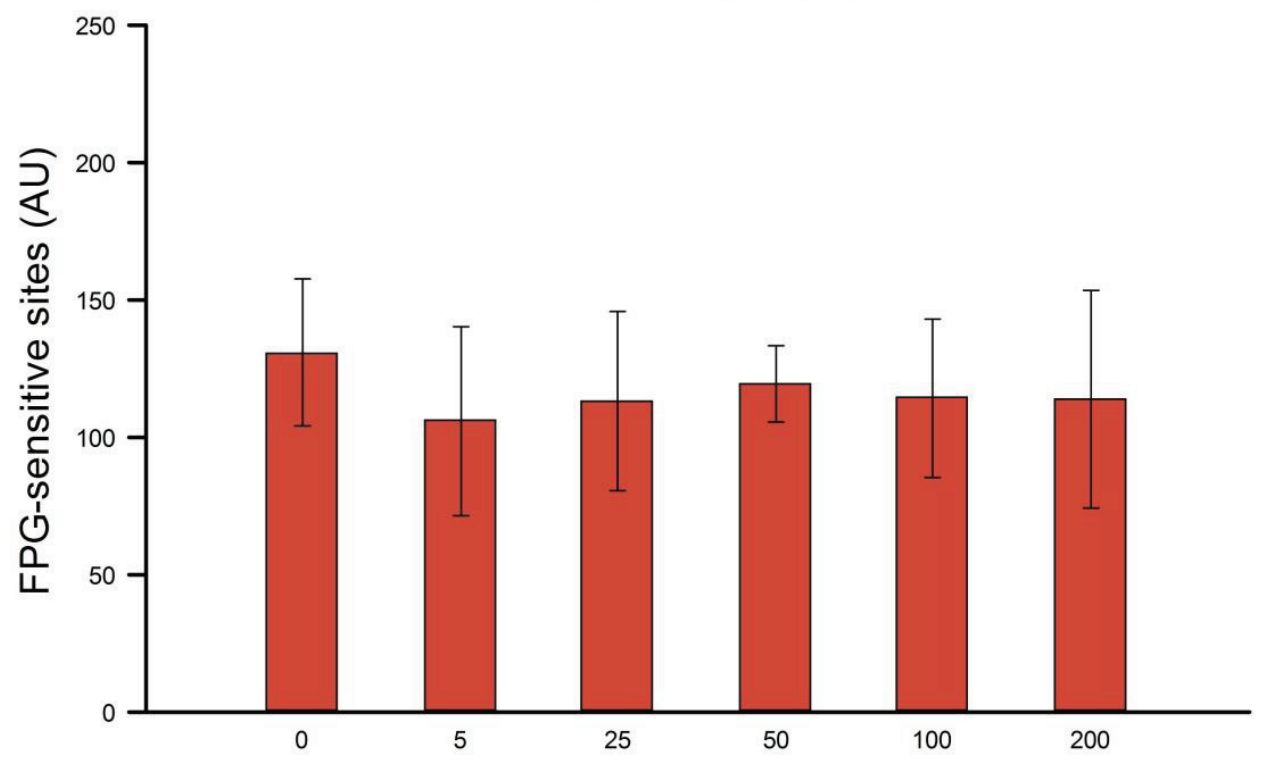

Concentration of vitamin $\mathrm{C}(\mu \mathrm{M})$

B. 6 h incubation

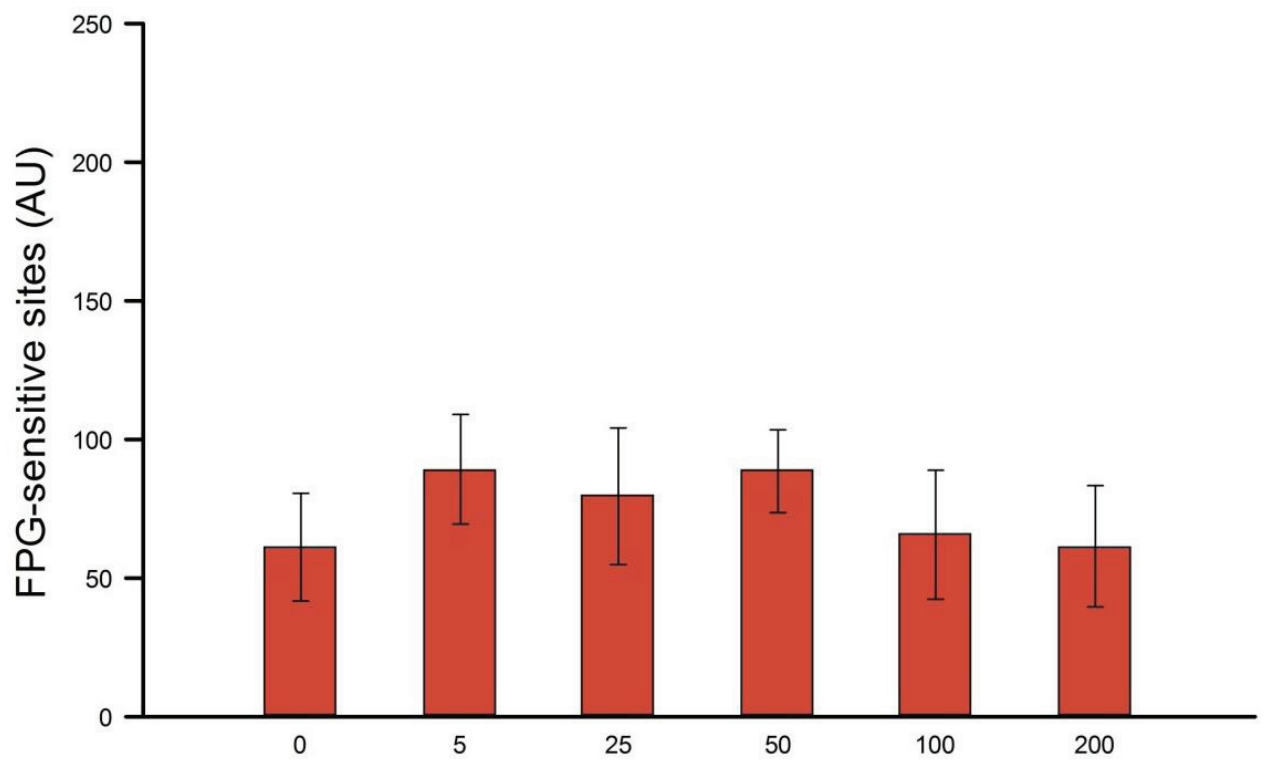

Concentration of vitamin $\mathrm{C}(\mu \mathrm{M})$

\subsection{Cellular Repair of $S B S$}

We studied the effect of vitamin $\mathrm{C}$ on the kinetics of repair of SBs induced by $\mathrm{H}_{2} \mathrm{O}_{2}$ after pre-incubating HeLa cells with different concentrations of vitamin $\mathrm{C}$ overnight. After $\mathrm{H}_{2} \mathrm{O}_{2}$ 
treatment cells were incubated and SBs remaining were measured at intervals. As is seen in Figure 4, rejoining of SBs is rapid, with a $t_{1 / 2}$ of about $20 \mathrm{~min}$ and complete repair by $60 \mathrm{~min}$. Pre-incubation of cells with 50 or $100 \mu \mathrm{M}$ vitamin C overnight did not affect the repair of the SBs induced by $\mathrm{H}_{2} \mathrm{O}_{2}$.

Figure 4. Effect of overnight pre-incubation of HeLa cells with different concentrations of vitamin $\mathrm{C}$ on the kinetics of repair of SBs: residual SBs were measured after different times of incubation after $\mathrm{H}_{2} \mathrm{O}_{2}$ treatment. C-: control cells without any kind of treatment. Bars: SD calculated from the results of 3 independent experiments. No significant effects were seen.

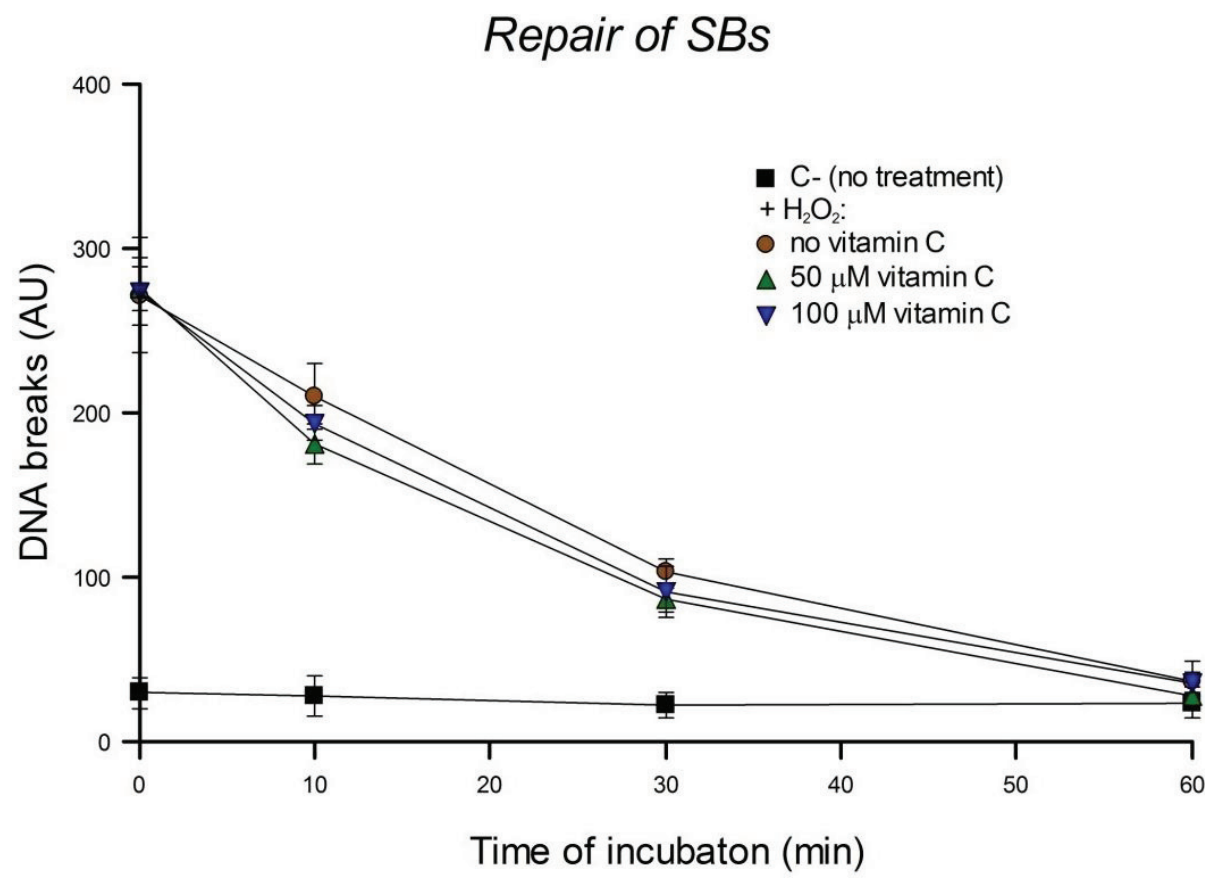

\subsection{Cellular Repair of Oxidized Purines}

We next investigated the effect of vitamin $\mathrm{C}$ on the kinetics of BER after pre-incubating $\mathrm{HeLa}$ cells with different concentrations of vitamin $\mathrm{C}$ overnight. We induced oxidized purines, mostly 8-oxoGua, using Ro plus light. The comet assay was performed after different times of incubation of the treated cells up to $6 \mathrm{~h}$. The pre-incubation of cells with 50 or $100 \mu \mathrm{M}$ vitamin C overnight did not affect the removal of the FPG-sensitive sites induced by Ro plus light (data not shown). We modified the protocol by pre-incubating HeLa cells with $200 \mu \mathrm{M}$ of vitamin $\mathrm{C}$ for $30 \mathrm{~min}$, and adding the same concentration of vitamin $\mathrm{C}$ also during the $6 \mathrm{~h}$ incubation period after inducing the damage. Figure 5 shows that the repair of oxidized bases is much slower than SB rejoining, and the presence of vitamin $\mathrm{C}$ during the incubation period after damaging the cells still did not influence the repair of the FPG-sensitive sites induced by Ro plus light. 
Figure 5. Effect of incubation of HeLa cells with $200 \mu \mathrm{M}$ of vitamin $\mathrm{C}$ before and during the repair of oxidized bases induced by Ro plus light; residual FPG-sensitive sites were measured at different time points. Bars: SD calculated from the results of 3 independent experiments. No significant effects were seen.

\section{Repair of oxidised bases}

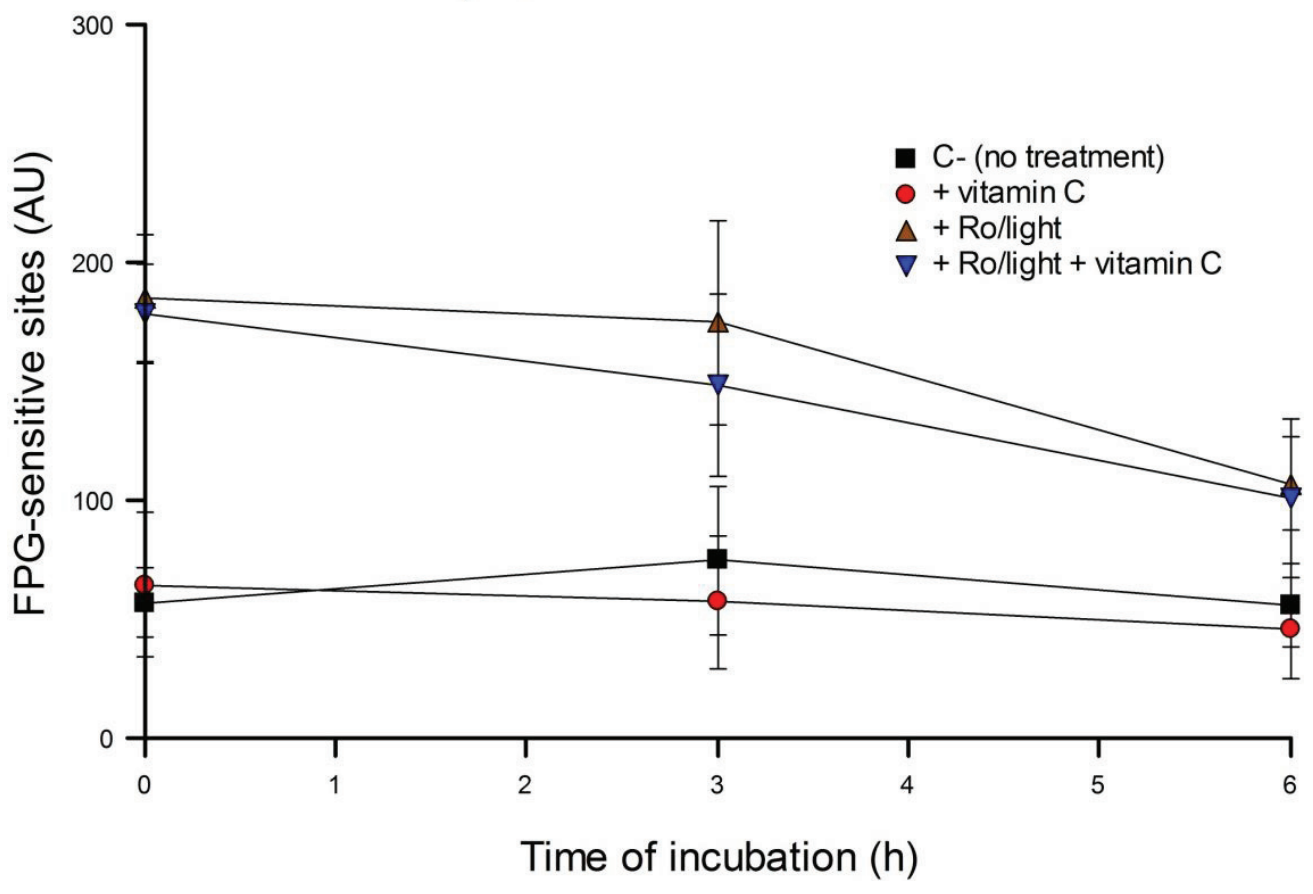

\subsection{In Vitro Repair Assay}

As a final check as to whether vitamin $\mathrm{C}$ has any effect on DNA repair activity we used an in vitro approach, measuring the ability of extracts of vitamin $\mathrm{C}$-preincubated cells to recognize and incise oxidized bases in substrate nucleoids. HeLa cells were treated with $200 \mu \mathrm{M}$ vitamin $\mathrm{C}$ for $6 \mathrm{~h}$. Extracts were prepared and incubated for 10 or 20 min with gel-embedded HeLa nucleoid DNA containing oxidized purines. (Previous experiments [13] have shown no significant breaks induced in undamaged substrate.) Then the comet assay was performed to measure the breaks induced by the extracts, as an indicator of repair activity. Extracts from HeLa cells preincubated with $200 \mu \mathrm{M}$ vitamin C produced a significant increase in SBs in substrate nucleoids (compared with extracts from non-vitamin C-treated cells) at both reaction incubation times (Figure 6A). Lower concentrations of vitamin $\mathrm{C}(50$ or $100 \mu \mathrm{M})$ did not have any effect. However, when we checked the possibility that vitamin $\mathrm{C}$ was having a direct effect on the DNA, we found that breaks were in fact induced in HeLa nucleoid DNA containing oxidized purines or in undamaged DNA (Figure 6B,C). A significant effect is seen at a concentration as low as $1 \mu \mathrm{M}$. This concentration relates to the concentration as prepared in buffer A, before $5 \times$ dilution with buffer F. Therefore the concentration capable of damaging DNA is actually as low as $0.2 \mu \mathrm{M}$. 
Figure 6. (A) Effect of extracts from HeLa cells pre-treated with 0 and $200 \mu \mathrm{M}$ of vitamin $\mathrm{C}$ for $6 \mathrm{~h}$ on a DNA substrate containing oxidized purines. (B) Effect of different concentrations of vitamin $\mathrm{C}$ on a DNA substrate containing oxidized purines. (C) Effect of different concentrations of vitamin $C$ on a non-damaged DNA substrate. In (A), Buffer F was used as a negative control. In (A) and (B), where nucleoids contained oxidized bases, FPG was employed as a positive control. Concentrations of vitamin $\mathrm{C}$ displayed in the box on panels $(\mathbf{B})$ and $(\mathbf{C})$ refer to concentrations in buffer A: final concentrations were $4 \times$ less. Bars: SD calculated from the results of 3 independent experiments. Statistical comparisons $(* p<0.05)$ : vitamin $\mathrm{C}$ (different concentrations) $v s .0 \mu \mathrm{M}$.

\section{A. Extracts plus damaged substrate}

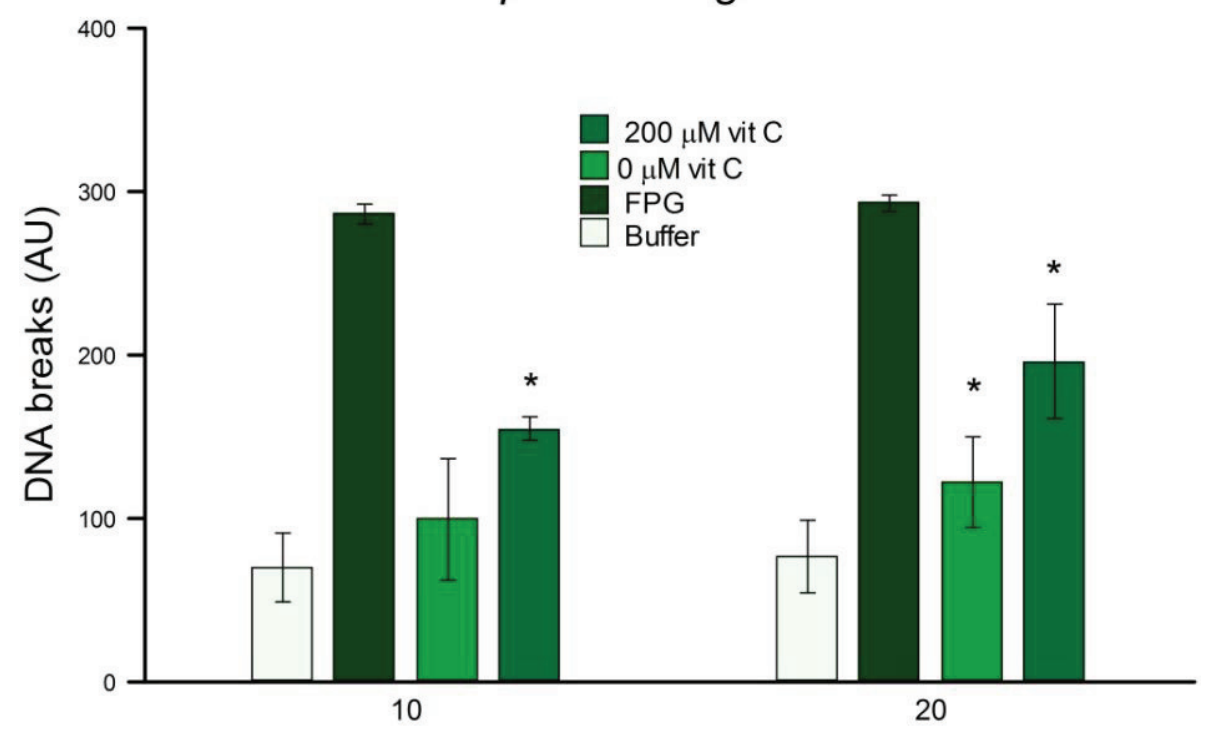

Time of incubation ( $\mathrm{min}$ )

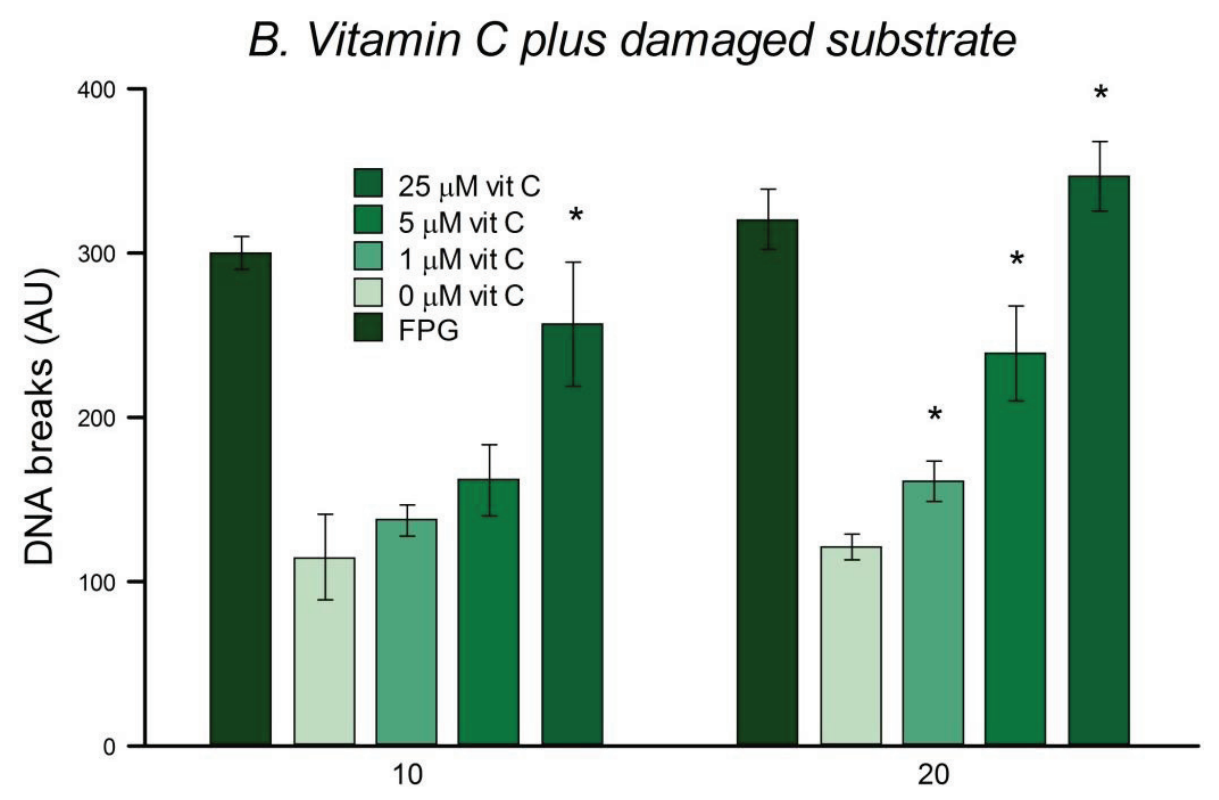

Time of incubation (min) 
Figure 6. Cont.

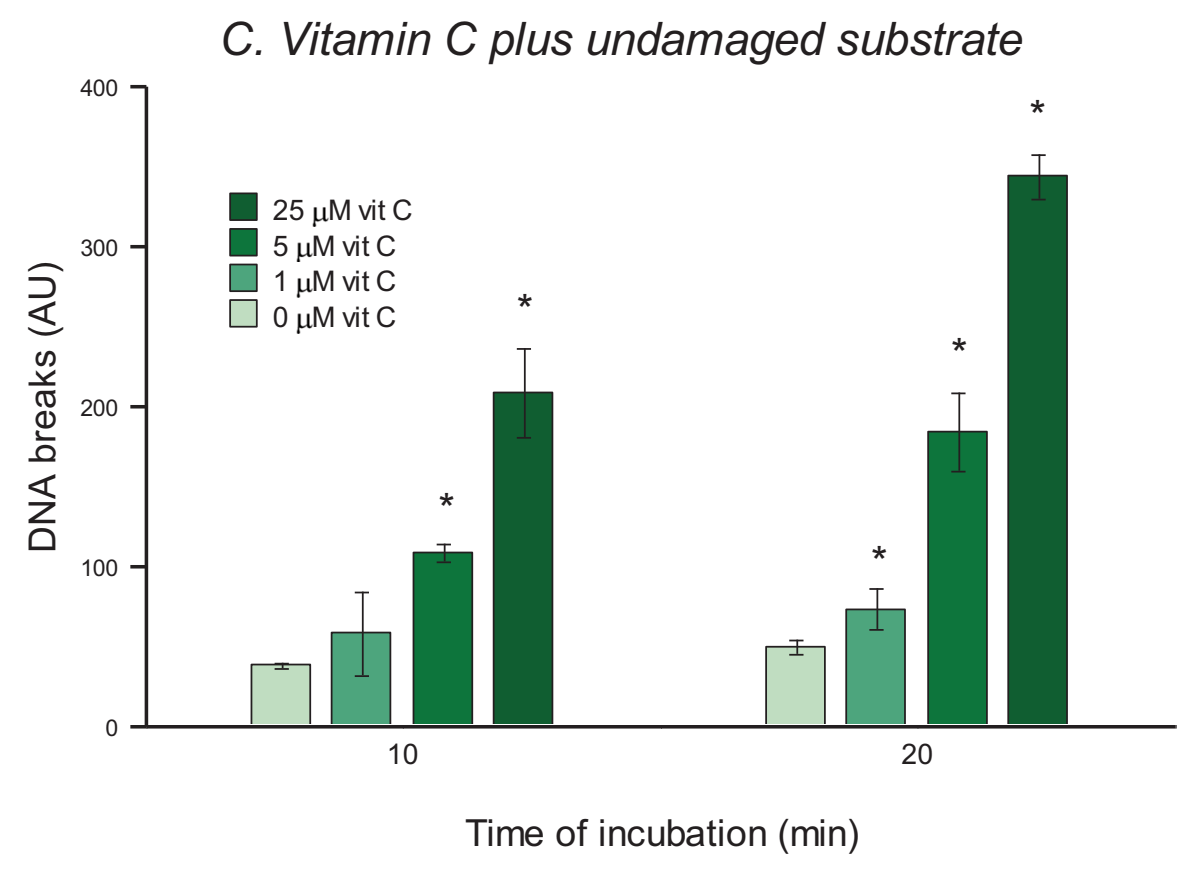

\subsection{Uptake of Vitamin $C$}

Concentrations of vitamin C measured in cell pellets after $6 \mathrm{~h}$ or $24 \mathrm{~h}$ incubation are shown in Figure 7. The concentration within the cells is estimated on the basis of a volume of $5 \mu \mathrm{L}$ per $10^{6}$ cells. It is clear that vitamin $\mathrm{C}$ is taken up by HeLa cells and accumulates to a concentration far higher than that provided in the medium.

Figure 7. Uptake of vitamin $\mathrm{C}$ by HeLa cells. Cells were incubated for $6 \mathrm{~h}$ in medium containing vitamin $\mathrm{C}$ at $50 \mu \mathrm{M}$ (light shading) or $200 \mu \mathrm{M}$ (dark shading). Intracellular vitamin $\mathrm{C}$ was measured by HPLC at the end of this incubation or after a further $24 \mathrm{~h}$ incubation.

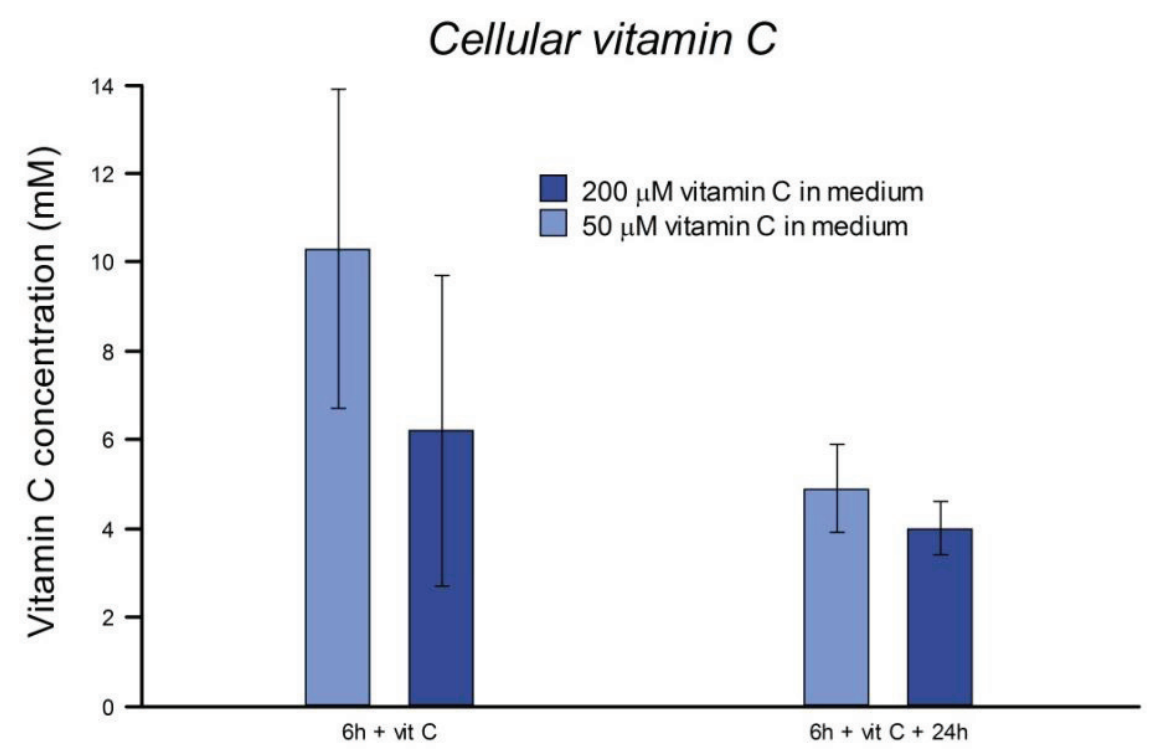




\section{Discussion}

In vitro, vitamin $\mathrm{C}$ is capable of acting as a pro-oxidant, particularly in combination with metal ions [14], and there are concerns that it might lead to excessive DNA oxidation in humans. There are reports of increases in oxidized bases in the DNA of white blood cells after supplementation of normal subjects with vitamin $C$ [7] or with vitamin $C$ plus ferrous sulfate [15]. However, these studies were carried out using GC-MS to measure oxidized bases - a technique now recognized as being particularly prone to artifact and therefore unsuitable for determining low level base oxidation [16].

The saturation plasma level for vitamin $\mathrm{C}$ in humans is around $80 \mu \mathrm{M}$, and this concentration is approached with daily intake of $200 \mathrm{mg} /$ day [17] — an intake that can feasibly be attained by a recommended intake of several portions of fruits and vegetables each day. The justification for exceeding in our experiments the maximum vitamin $\mathrm{C}$ concentration found in plasma is that vitamin $\mathrm{C}$ is reportedly not stable in cell culture medium; $40 \%$ depletion was detected after $2 \mathrm{~h}$ of incubation [18], and only about $30 \%$ remained at $6 \mathrm{~h}$.

In vivo, in subjects supplemented with $200 \mathrm{mg}$ of vitamin $\mathrm{C}$ per day, active accumulation by lymphocytes results in intracellular levels saturating at $3.5 \mathrm{mM} \mathrm{[17].} \mathrm{Welch} \mathrm{et} \mathrm{al.} \mathrm{[19]} \mathrm{reported} \mathrm{a}$ linear accumulation of vitamin $\mathrm{C}$ up to about $0.5 \mathrm{mM}$ in $4 \mathrm{~h}$ in human fibroblasts incubated in $50 \mu \mathrm{M}$ vitamin $\mathrm{C}$. We found that the concentration of vitamin $\mathrm{C}$ inside HeLa cells after culture in medium with the vitamin was in the mM range, and was still high after $24 \mathrm{~h}$. Although they have undergone many changes in their several decades of continuous culture from their origin in tumor tissue, HeLa cells evidently retain the active transport mechanisms responsible in normal cells for uptake of vitamin C [19], and so we regard them as suitable for an investigation of intracellular effects of vitamin $\mathrm{C}$.

In the present study, in spite of the high intracellular concentration of vitamin $\mathrm{C}$, there was no increase in SBs or FPG-sensitive sites. Others have reported no increase in SBs and/or oxidized bases in various cell types after incubation with vitamin $\mathrm{C}$ - in human HepG2 liver-derived cells with 10 $\mu \mathrm{M}$ vitamin $\mathrm{C}$ [20], in primary human fibroblasts incubated with $100 \mu \mathrm{M}$ vitamin $\mathrm{C}$ [21], and most recently in HL-60 cells (human leukemic cell line) incubated with concentrations of 50-250 $\mu \mathrm{M}$ vitamin $\mathrm{C}[22,23]$.

In our experiments, preincubation with up to $200 \mu \mathrm{M}$ vitamin $\mathrm{C}$ had no significant effect on the level of $\mathrm{H}_{2} \mathrm{O}_{2}$-induced breaks or 8-oxoGua induced by treatment with Ro plus light. There are mixed reports on the effect of pre-incubation with vitamin $\mathrm{C}$ on the DNA damage inflicted by genotoxic agents. Vitamin C pre-incubation (30 min, 10 or $50 \mu \mathrm{M}$ ) decreased the SBs caused by streptozotocin in lymphocytes or HeLa cells [24], and protected against the effect of $\mathrm{NiCl}_{2}$ in lymphocytes [25]. Duarte and Jones [21] preincubated primary human fibroblasts for $12 \mathrm{~h}$ with 20,100 or $500 \mu \mathrm{M}$ vitamin $\mathrm{C}$, and-also using the comet assay-found an increase in $\mathrm{H}_{2} \mathrm{O}_{2}$-induced damage. Carbofuran-induced damage to human lymphocyte DNA was decreased by preincubation with $30 \mu \mathrm{M}$ vitamin $\mathrm{C}$ [26]. Similar protection was afforded by $10-50 \mu \mathrm{M}$ vitamin $\mathrm{C}$ pretreatment against damage induced by 2-hydroxyethyl methacrylate and urethane dimethacrylate (used in dental treatment) [27,28].

When vitamin $\mathrm{C}$ is present simultaneously with a genotoxic agent (rather than during a preincubation), potentiation of the damaging effect of the latter may be seen - even when the effect 
of preincubation is a protective one. Such a dual effect was reported by Wozniak and Blasiak [25] for $\mathrm{NiCl}_{2}$. Vanadyl sulfate led to more DNA breaks in HeLa cells and lymphocytes when they were co-incubated with vitamin $\mathrm{C}$ for $1 \mathrm{~h}$ [29], perhaps as a result of a metal ion-vitamin $\mathrm{C}$ interaction. Both DNA breaks and oxidized purines were induced when HL-60 cells were incubated with vitamin $\mathrm{C}$ and copper (II) sulfate, but no such damage was caused by vitamin $\mathrm{C}$ alone [23]. Vitamin $\mathrm{C}$ was unable to protect human submandibular gland and oral epithelial cells against DNA damage induced by bracken fern, and in fact showed a synergistic effect on DNA breakage [30]. In contrast, co-incubation of HepG2 cells with both vitamin $\mathrm{C}$ and $N$-nitrosamines resulted in fewer DNA breaks/oxidized bases [20] - perhaps an effect of vitamin $\mathrm{C}$ mediated through xenobiotic metabolizing enzymes.

To summarize our results relating to DNA damage, in HeLa cells - which clearly are able to take up and accumulate vitamin $\mathrm{C}$-we find no sign of either pro- or anti-oxidant effects.

There are some human intervention studies that suggest a possible regulatory role for vitamin $\mathrm{C}$ in the repair of oxidized DNA - a topic highlighted by Sram et al. in their recent review [8] as deserving further attention. Cooke et al. [31] measured 8-oxodGuo in lymphocyte DNA, serum and urine from 30 healthy volunteers involved in a 6 week placebo and 6 week vitamin $\mathrm{C}(500 \mathrm{mg} /$ day $)$ supplementation study (plus a washout period). They found a decrease in the levels of 8-oxodGuo in DNA and an increase in urine and serum. In addition to reservations about the less than ideal study design and the likely errors in the measurement of 8-oxodGuo, the interpretation of the results is questionable, since the presence of 8-oxodGuo in serum or urine does not directly reflect OGG activity; the base, not the nucleoside, is excised during BER. Tarng et al. [32] supplemented chronic hemodialysis patients with $300 \mathrm{mg}$ of vitamin $\mathrm{C}$, and found a significant upregulation of hOGG1 mRNA at $24 \mathrm{~h}$ after vitamin $\mathrm{C}$ administration. Astley et al. [33] took lymphocytes from human volunteers after 3 weeks of supplementation with the modest dose of $60 \mathrm{mg}$ vitamin $\mathrm{C}$ per 2 days. There was no significant effect on plasma vitamin $\mathrm{C}$, and no change in repair capacity (detected as repair synthesis on an oxidatively damaged plasmid template).

Supplementation for 3 weeks with green kiwifruits led to a significant increase in plasma vitamin $\mathrm{C}$, decreases in endogenous base oxidation and $\mathrm{H}_{2} \mathrm{O}_{2}$-induced damage ex vivo, and an enhancement of lymphocyte BER on a substrate containing 8-oxoGua [34]; however, golden kiwifruits modestly increased plasma vitamin $\mathrm{C}$ and showed protection against DNA oxidation but no effect on DNA repair [35]. Guarnieri et al. [36] supplemented volunteers with $0.5 \mathrm{~g} /$ day vitamin $\mathrm{C}$ in normal or slow release form (together with vitamin E); only the slow release form stimulated BER capacity in lymphocytes.

Reports in the literature on effects of vitamin C on DNA repair in cells in culture are sparse. Konopacka et al. [37] irradiated mouse lymphocytes with 2 Gy of $\gamma$-rays, and then incubated them with or without vitamin $\mathrm{C}$, vitamin $\mathrm{E}$ and $\beta$-carotene; rejoining of strand breaks was faster in the presence of antioxidants.

We report here that vitamin $\mathrm{C}$ has no effect on cellular repair of SBs or oxidized bases in HeLa cells. The in vitro test for 8-oxoGua DNA glycosylase (OGG) activity could not be carried out, since it was evident that vitamin $\mathrm{C}$ itself can cause damage to nucleoid DNA (Figure 6), presumably acting as a pro-oxidant (as mentioned in the Introduction). This cleavage could be mediated via production of hydroxyl radicals in the presence of DNA-bound transition metal such as copper [14]. 
The reason for native nuclear DNA being resistant to attack by vitamin $\mathrm{C}$, while nucleoid DNA is susceptible, is not clear, though it may be that this apparently pro-oxidant effect of vitamin $\mathrm{C}$ is prevented by the high concentrations of the thiol-rich antioxidant glutathione present in the intact nucleus. Bergstrom et al. $[22,23]$ report the oxidation of dGuo in vitro by vitamin $\mathrm{C}$. To summarize, we have found no evidence in HeLa cells for pro-oxidant effects of vitamin $\mathrm{C}$ at extracellular concentrations up to $200 \mu \mathrm{M}$, but also no evidence for antioxidant protection. Nor did we see any effect on the repair of SBs or oxidized bases. Vitamin $\mathrm{C}$ at low concentration is, however, capable of damaging nucleoid DNA. The same strategy was used in our laboratory to study the antioxidant/pro-oxidant effects of $\beta$-cryptoxanthin [13]. Our results showed that this carotenoid does not cause DNA damage but, in contrast to vitamin $\mathrm{C}$, it protects HeLa cells from damage induced by $\mathrm{H}_{2} \mathrm{O}_{2}$ or photosensitizer plus light. In addition, it enhances DNA repair, as measured with the same assays as were used in the present report.

\section{Acknowledgments}

We thank F. Hoffmann-La Roche for the gift of Ro 19-8022, and the Research Council of Norway and the Throne Holstfond for financial support. AA thanks the Ministerio de Educación y Ciencia ("Juan de la Cierva" programme, 2009) of the Spanish Government for personal support. We confirm that there are no conflicts of interest. AA and ARC conceived the project and wrote the paper. AA also carried out experiments, with SC, YL and NEB; all authors contributed to analysis and interpretation of results.

\section{References}

1. Carr, A.; Frei, B. Does vitamin $\mathrm{C}$ act as a pro-oxidant under physiological conditions? FASEB J. 1999, 13, 1007-1024.

2. Green, M.H. L.; Lowe, J.E.; Waugh, A.P.W.; Aldridge, K.E. Effect of diet and vitamin C on DNA strand breakage in freshly-isolated human white blood cells. Mutat. Res. 1994, 316, 91-102.

3. Duarte, T.L.; Lunec, J. Review: Part of the series: From dietary antioxidants to regulators in cellular signalling and gene expression review: When is an antioxidant not an antioxidant? A review of novel actions and reactions of vitamin C. Free Radic. Res. 2005, 39, 671-686.

4. Moller, P.; Loft, S. Oxidative DNA damage in human white blood cells in dietary antioxidant intervention studies. Am. J. Clin. Nutr. 2002, 76, 303-310.

5. Møller, P.; Viscovich, M.; Lykkesfeldt, J.; Loft, S.; Jensen, A.; Poulsen, H.E. Vitamin C supplementation decreases oxidative DNA damage in mononuclear blood cells of smokers. Eur. J. Nutr. 2004, 43, 267-274.

6. Herbert, K.; Fletcher, S.; Chauhan, D.; Ladapo, A.; Nirwan, J.; Munson, S.; Mistry, P. Dietary supplementation with different vitamin C doses: No effect on oxidative DNA damage in healthy people. Eur. J. Nutr. 2006, 45, 97-104.

7. Podmore, I.D.; Griffiths, H.R.; Herbert, K.E.; Mistry, N.; Mistry, P.; Lunec, J. Vitamin C exhibits pro-oxidant properties. Nature 1998, 392, 559.

8. Sram, R.J.; Binkova, B.; Rossner, J. Vitamin C for DNA damage prevention. Mutat. Res. 2012, 733, 39-49. 
9. Bjelakovic, G.; Nikolova, D.; Gluud, L.L.; Simonetti, R.G.; Gluud, C. Mortality in randomized trials of antioxidant supplements for primary and secondary prevention: Systematic review and meta-analysis. JAMA 2007, 297, 842-857.

10. McCall, M.; Frei, B. Can antioxidant vitamins materially reduce oxidative damage in humans? Free Radic. Biol. Med. 1999, 26, 1034-1053.

11. Azqueta, A.; Shaposhnikov, S.; Collins, A.R. DNA Repair Measured by the Comet Assay. In DNA Repair; Kruman, I., Ed.; InTech: Rijeka, Croatia, 2011.

12. Collins, A.R.; Azqueta, A.; Brunborg, G.; Gaivão, I.; Giovannelli, L.; Kruszewski, M.; Smith, C.C.; Stetina, R. The comet assay: Topical issues. Mutagenesis 2008, 23, 143-151.

13. Lorenzo, Y.; Azqueta, A.; Luna, L.; Bonilla, F.; Domínguez, G.; Collins, A.R. The carotenoid B-cryptoxanthin stimulates the repair of DNA oxidation damage in addition to acting as an antioxidant in human cells. Carcinogenesis 2009, 30, 308-314.

14. Halliwell, B. Vitamin C: Antioxidant or pro-oxidant in vivo? Free Radic. Res. 1996, 25, 439-454.

15. Rehman, A.; Collis, C.S.; Yang, M.; Kelly, M.; Diplock, A.T.; Halliwell, B.; Rice-Evans, C. The effects of iron and vitamin $\mathrm{C}$ co-supplementation on oxidative damage to DNA in healthy volunteers. Biochem. Biophys. Res. Commun. 1998, 246, 293-298.

16. ESCODD; Gedik, C.M.; Collins, A. Establishing the background level of base oxidation in human lymphocyte DNA: Results of an interlaboratory validation study. FASEB J. 2005, 19, $82-84$.

17. Levine, M.; Conry-Cantilena, C.; Wang, Y.; Welch, R.W.; Washko, P.W.; Dhariwal, K.R.; Park, J.B.; Lazarev, A.; Graumlich, J.F.; King, J.; Cantilena, L.R. Vitamin C pharmacokinetics in healthy volunteers: Evidence for a recommended dietary allowance. Proc. Natl. Acad. Sci. USA 1996, 93, 3704-3709.

18. Duarte, T. Effects of Vitamin C on DNA Damage, Iron Homeostasis and Gene Expression Profiling in Human Fibroblasts. Ph.D. Dissertation, University of Leicester, Leicester, UK, 2006.

19. Welch, R.W.; Bergsten, P.; Butler, J.D.; Levine, M. Ascorbic acid accumulation and transport in human fibroblasts. Biochem. J. 1993, 294, 505-510.

20. Arranz, N.; Haza, A.I.; Garcia, A.; Rafter, J.; Morales, P. Protective effect of vitamin C towards $\mathrm{N}$-nitrosamine-induced DNA damage in the single-cell gel electrophoresis (SCGE)/HepG2 assay. Toxicol. In Vitro 2007, 21, 1311-1317.

21. Duarte, T.L.; Jones, G.D. Vitamin C modulation of $\mathrm{H}_{2} \mathrm{O}_{2}$-induced damage and iron homeostasis in human cells. Free Radic. Biol. Med. 2007, 43, 1165-1175.

22. Bergström, T.; Bergman, J.; Möller, L. Vitamin A and C compounds permitted in supplements differ in their abilities to affect cell viability, DNA and the DNA nucleoside deoxyguanosine. Mutagenesis 2011, 26, 735-744.

23. Bergström, T.; Ersson, C.; Bergman, J.; Möller, L. Vitamins at physiological levels cause oxidation to the DNA nucleoside deoxyguanosine and to DNA - alone or in synergism with metals. Mutagenesis 2012, 27, 511-517.

24. Blasiak, J.; Sikora, A.; Wozniak, K.; Drzewoski, J. Genotoxicity of streptozotocin in normal and cancer cells and its modulation by free radical scavengers. Cell Biol. Toxicol. 2004, 20, 83-96.

25. Wozniak, K.; Blasiak, J. Free radicals-mediated induction of oxidized DNA bases and DNA-protein cross-links by nickel chloride. Mutat. Res. 2002, 514, 233-243. 
26. Sharma, R.K.; Sharma, B. In-vitro carbofuran induced genotoxicity in human lymphocytes and its mitigation by vitamins C and E. Dis. Markers 2012, 32, 153-163.

27. Pawlowska, E.; Poplawski, T.; Ksiazek, D.; Szczepanska, J.; Blasiak, J. Genotoxicity and cytotoxicity of 2-hydroxyethyl methacrylate. Mutat. Res. Genet. Toxicol. Environ. Mutagen. 2010, 696, 122-129.

28. Poplawski, T.; Loba, K.; Pawlowska, E.; Szczepanska, J.; Blasiak, J. Genotoxicity of urethane dimethacrylate, a tooth restoration component. Toxicol. In Vitro 2010, 24, 854-862.

29. Wozniak, K.; Blasiak, J. Vanadyl sulfate can differentially damage DNA in human lymphocytes and HeLa cells. Arch. Toxicol. 2004, 78, 7-15.

30. Campos-da-Paz, M.; Pereira, L.O.; Bicalho, L.S.; Dórea, J.G.; Poças-Fonseca, M.J.; Santos Mde, F. Interaction of bracken-fern extract with vitamin $\mathrm{C}$ in human submandibular gland and oral epithelium cell lines. Mutat. Res. 2008, 652, 158-163.

31. Cooke, M.S.; Evans, M.D.; Podmore, I.D.; Herbert, K.E.; Mistry, N.; Mistry, P.; Hickenbotham, P.T.; Hussieni, A.; Griffiths, H.R.; Lunec, J. Novel repair action of vitamin C upon in vivo oxidative DNA damage. FEBS Lett. 1998, 439, 363-367.

32. Tarng, D.C.; Liu, T.Y.; Huang, T.P. Protective effect of vitamin C on 8-hydroxy-2(prime)-deoxyguanosine level in peripheral blood lymphocytes of chronic hemodialysis patients. Kidney Int. 2004, 66, 820-831.

33. Astley, S.; Elliott, R.M.; Archer, D.B.; Southon, S. Evidence that dietary supplementation with carotenoids and carotenoid-rich foods modulates the DNA damage:repair balance in human lymphocytes. Br. J. Nutr. 2004, 91, 63-72.

34. Collins, A.R.; Harrington, V.; Drew, J.; Melvin, R. Nutritional modulation of DNA repair in a human intervention study. Carcinogenesis 2003, 24, 511-515.

35. Brevik, A.; Gaivao, I.; Medin, T.; Jorgenesen, A.; Piasek, A.; Elilasson, J.; Karlsen, A.; Blomhoff, R.; Veggan, T.; Duttaroy, A.; Collins, A. Supplementation of a western diet with golden kiwifruits (Actinidia chinensis var.'Hort 16A':) effects on biomarkers of oxidation damage and antioxidant protection. Nutr. J. 2011, 10, 54.

36. Guarnieri, S.; Loft, S.; Riso, P.; Porrini, M.; Risom, L.; Poulsen, H.; Dragsted, L.O.; Møller, P. DNA repair phenotype and dietary antioxidant supplementation. Br. J. Nutr. 2008, 99, 1018-1024.

37. Konopacka, M.; Widel, M.; Rzeszowka-Wolny, J. Modifying effect of vitamins C, E and beta-carotene against gamma-ray-induced DNA damage in mouse cells. Mutat. Res. 1998, 417, 85-94. 
Reprinted from Nutrients. Cite as: Mohammed, B.M.; Fisher, B.J.; Kraskauskas, D.; Farkas, D.; Brophy, D.F.; Fowler, A.A., III; Natarajan, R. Vitamin C: A Novel Regulator of Neutrophil Extracellular Trap Formation. Nutrients 2013, 5, 3131-3150.

Article

\section{Vitamin C: A Novel Regulator of Neutrophil Extracellular Trap Formation}

Bassem M. Mohammed ${ }^{1,2}$, Bernard J. Fisher ${ }^{3}$, Donatas Kraskauskas ${ }^{3}$, Daniela Farkas ${ }^{3}$, Donald F. Brophy ${ }^{1}$, Alpha A. Fowler III ${ }^{3}$ and Ramesh Natarajan ${ }^{3, *}$

1 Department of Pharmacotherapy and Outcomes Science, Virginia Commonwealth University, Richmond, VA 23298, USA; E-Mails: mohammedbm@vcu.edu (B.M.M.); dbrophy@vcu.edu (D.F.B.)

2 Department of Clinical Pharmacy, Faculty of Pharmacy, Cairo University, Cairo 11562, Egypt

3 Division of Pulmonary Disease and Critical Care Medicine, Virginia Commonwealth University, Richmond, VA 23298, USA; E-Mails: bjfisher@vcu.edu (B.J.F.); dkraskauskas@vcu.edu (D.K.); dfarkas3@vcu.edu (D.F.); afowler@mcvh-vcu.edu (A.A.F.)

* Author to whom correspondence should be addressed; E-Mail: rnataraj@vcu.edu; Tel.: +1-804-827-1013; Fax: +1-804-628-0325.

Received: 13 May 2013; in revised form: 30 July 2013 / Accepted: 5 August 2013 /

Published: 9 August 2013

Abstract: Introduction: Neutrophil extracellular trap (NET) formation was recently identified as a novel mechanism to kill pathogens. However, excessive NET formation in sepsis can injure host tissues. We have recently shown that parenteral vitamin $\mathrm{C}(\mathrm{VitC})$ is protective in sepsis. Whether VitC alters NETosis is unknown. Methods: We used Gulo $^{-/-}$mice as they lack the ability to synthesize VitC. Sepsis was induced by intraperitoneal infusion of a fecal stem solution (abdominal peritonitis, FIP). Some VitC deficient $\mathrm{Gulo}^{-/-}$mice received an infusion of ascorbic acid (AscA, $200 \mathrm{mg} / \mathrm{kg}$ ) $30 \mathrm{~min}$ after induction of FIP. NETosis was assessed histologically and by quantification for circulating free DNA (cf-DNA) in serum. Autophagy, histone citrullination, endoplasmic reticulum (ER) stress, NFKB activation and apoptosis were investigated in peritoneal PMNs. Results: Sepsis produced significant NETs in the lungs of VitC deficient Gulo ${ }^{-/}$ mice and increased circulating cf-DNA. This was attenuated in the VitC sufficient $\mathrm{Gulo}^{-/-}$mice and in VitC deficient Gulo ${ }^{-/-}$mice infused with AscA. Polymorphonuclear neutrophils (PMNs) from VitC deficient $\mathrm{Gulo}^{-/-}$mice demonstrated increased activation of ER stress, autophagy, histone citrullination, and NFאB activation, while apoptosis was inhibited. VitC also significantly attenuated PMA induced NETosis in PMNs from 
healthy human volunteers. Conclusions: Our in vitro and in vivo findings identify VitC as a novel regulator of NET formation in sepsis. This study complements the notion that VitC is protective in sepsis settings.

Keywords: vitamin C; sepsis; neutrophils; NETosis; cell free DNA; nuclear factor $\kappa \mathrm{B}$

\section{Introduction}

Polymorphonuclear neutrophils (PMN) play key roles in the host response to pathogens by regulating innate host defenses and modulating inflammation. PMN combat pathogens by multiple mechanisms including phagocytosis, followed by exposure to reactive oxygen intermediates (short-lived and long-lived) in phagolysosomes [1], degranulation, which involves release of anti-bacterial peptides and proteases to kill pathogens [2], as well as production of cytokines and other inflammatory mediators. Aside from these traditional mechanisms, another mechanism for pathogen killing, the formation of neutrophil extracellular traps (NETs) by NETosis, a novel cell death pathway different from apoptosis and necrosis, was recently identified [3,4]. Although NETosis plays a crucial role in host defense during local infection by trapping and killing pathogens, excessive NET formation during systemic infections becomes self-defeating by promoting tissue injury and organ damage [5].

Sepsis, a leading cause of death with high mortality rates, is characterized by excessive inflammation and exuberant immune responses that lead to increased circulating PMN levels and extensive PMN sequestration in the lung. This massive influx of PMNs to the lungs often leads to acute lung injury (ALI) [6]. One postulated mechanism by which PMNs cause ALI is NETosis [7]. In sepsis, NETs are formed in response to pro-inflammatory stimuli such as lipopolysaccharide (LPS) and interleukin-8 (IL-8) [8,9] by expulsion of genomic DNA into web-like extracellular structures that display antimicrobial proteins such as histones, neutrophil elastase, and myeloperoxidase [10]. During NETosis, various signaling pathways lead to dissolution of nuclear envelope, thus allowing the mixing of nuclear chromatin with granular antimicrobial proteins from cytoplasmic granules, and then, by releasing the DNA into lattice-like structures, NETs concentrate proteases and antimicrobial proteins in the vicinity of trapped pathogens. However, in sepsis, exposure to NETs also produces organ injury. Indeed, Dwivedi et al. recently showed that NETosis, as determined by the circulating cell free DNA (cf-DNA) content, could predict ICU mortality in severe sepsis better than existing severity of illness or organ dysfunction scoring systems and was also better than IL-6, thrombin, and protein $C$ [11]. While effective targeting or inhibition of NET structures has been suggested as therapy to benefit sepsis [8], identification of agents with the potential to alter NET formation remains elusive.

Vitamin C (VitC) is an essential vitamin for humans. While its role as an endogenous antioxidant is well recognized, our recent research suggests that VitC beneficially impacts multiple pathways associated with sepsis [12]. Its pleiotropic mechanisms including attenuation of the pro-inflammatory response, enhancement of epithelial barrier function, increasing alveolar fluid clearance, and prevention of coagulation abnormalities constitute a primary line of defense that is protective in sepsis syndromes [13]. Intracellular levels of VitC in various tissues differ significantly 
from circulating plasma levels with high cellular concentrations considered to be indicative of essential metabolic function [14]. In particular, VitC accumulates in millimolar quantities in PMNs where it regulates neutrophil apoptosis $[14,15]$. We recently showed that VitC attenuated neutrophiliccapillaritis and improved survival in murine sepsis models [12,13]. However, whether VitC alters NETosis in sepsis settings remains unknown. Humans lack functional L-gulono- $\gamma$-lactone oxidase (Gulo), the final enzyme in the biosynthesis of VitC [16]. In contrast, mice express functional Gulo, resulting in tissues generally maintaining high levels of VitC. In order to translate data from VitC studies in mice to humans we have examined NETosis in septic mice lacking Gulo $\left(\mathrm{Gulo}^{-/}\right.$). Our studies show that VitC sufficiency attenuated NETosis in septic mice. Importantly, at a cellular level, we show that VitC deficient PMN were more susceptible to undergo NETosis through increased activation of endoplasmic reticulum (ER) stress and autophagy, processes considered vital for NETosis [17]. VitC deficient PMNs displayed increased expression of peptidylargininedeiminase 4 (PAD4), the key enzyme required for hypercitrullination of histones and chromatin decondensation [18]. Moreover, our studies show that the pro-survival transcription factor nuclear factor kappa B (NFkB) was augmented in the VitC deficient PMNs while apoptosis was suppressed. The inhibitory effect of VitC on NETosis was recapitulated in phorbolmyristate acetate (PMA) activated human PMN.

\section{Experimental Section}

\subsection{Animals}

$\mathrm{Gulo}^{-/-}$mice were bred in-house from an established homozygous colony as previously described [19]. Vitamin C sufficient mice were fed ad libitum with regular chow and water supplemented with vitamin $\mathrm{C}(0.330 \mathrm{~g} / \mathrm{L})$ renewed twice per week. Vitamin $\mathrm{C}$ deficient mice were generated by reducing vitamin $\mathrm{C}$ supplementation $(0.033 \mathrm{~g} / \mathrm{L})$ for one week, followed by complete removal of dietary vitamin $\mathrm{C}$ for an additional two weeks. Others have shown that this reduced supplementation significantly decreases the concentration of $\mathrm{VitC}$ in immune cells, plasma and organs $[20,21]$.

\subsubsection{Feces Induced Peritonitis}

Polymicrobial sepsis (peritonitis) was induced by intraperitoneal (i.p.) introduction of fecal stem solution into the peritoneum as described previously $[13,19]$. Thirty minutes after fecal challenge $(45 \mathrm{mg} / \mathrm{mL})$, some mice received i.p. injection of $\mathrm{VitC}$ as ascorbic acid (200 mg/kg in saline). Untreated mice received i.p. saline instead of VitC. Blood was collected $16 \mathrm{~h}$ later by cardiac puncture, and lungs harvested. Blood was allowed to coagulate, spun to separate serum, and stored at $-80{ }^{\circ} \mathrm{C}$ for batch analysis of cell-free DNA (see below).

All animal studies were performed in accordance to the Virginia Commonwealth University Animal Care and Use Committee's approved protocols (Protocol \# AM10100, approved 15 March, 2011).

\subsection{2. $\mathrm{Gulo}^{-/-}$Mice Were Divided into Five Groups}

(1) (+): vitamin C sufficient mice received saline alone (0.4 mL, i.p.) 
(2) FIP(+): vitamin C sufficient mice received fecal stem solution $(0.4 \mathrm{~mL}$, i.p.) followed $30 \mathrm{~min}$ later by saline $(0.1 \mathrm{~mL}$, i.p. $)$

(3) (-): vitamin C deficient mice received saline alone (0.4 mL, i.p.)

(4) FIP(-): vitamin C deficient mice received fecal stem solution ( $0.4 \mathrm{~mL}$, i.p.) followed 30 min later by saline $(0.1 \mathrm{~mL}$, i.p. $)$

(5) FIP(-) + AscA: vitamin C deficient mice received fecal stem solution (0.4 mL, i.p.) followed $30 \mathrm{~min}$ later by ascorbic acid $(0.1 \mathrm{~mL}$, i.p. $)$

\subsection{Isolation of Mouse Peritoneal Neutrophils}

Induction of an enriched exudate of leukocytes in the peritoneal cavity of mice was performed by i.p. injection of $1 \mathrm{~mL}$ of aged, sterile 3\% thioglycollate solution [16]. After $16 \mathrm{~h}$, mice were euthanized, and the peritoneal cavity was flushed with $5 \mathrm{~mL}$ sterile Hanks' balanced salt solution containing 1\% BSA (HBSS). The leukocyte pellet containing $\sim 80 \%$ neutrophils and $\sim 20 \%$ macrophages was washed with HBSS and resuspended in RPMI-1640 medium. Total cell counts were determined with a hemacytometer. Leukocyte viability was assessed by trypan blue exclusion ( $>99 \%)$. PMNs were then purified by adherence to a plastic dish as described by Tsurubuchi et al. [22]. Briefly, cells from peritoneal exudate were plated into a $35-\mathrm{mm}$ plastic dish and incubated at $37{ }^{\circ} \mathrm{C}$ in $5 \% \mathrm{CO}_{2}$ in air for $10 \mathrm{~min}$ in HBSS. The cells were washed twice with fresh HBSS to remove non-adherent cells. Although there was loss of some PMNs, which did not adhere to the dish, this procedure eliminated most of the macrophages. Cytochemical staining of adherent cells using HARLECO ${ }^{\circledR}$ Hemacolor ${ }^{\circledR}$ Solution (EMD Millipore, EMD Millipore) revealed that $>95 \%$ of the adherent cells were PMNs.

\subsection{Immunofluorescence and Differential Interference Contrast Imaging of Lung NETs}

Formalin fixed paraffin embedded mouse lung sections $(3 \mu \mathrm{m})$ were rehydrated and heat induced antigen retrieval performed in $0.01 \mathrm{M}$ citrate buffer $\mathrm{pH} 6.0$ for $20 \mathrm{~min}$. Sections were blocked with $1 \%$ normal swine serum (NSS, DAKO, Carpinteria, CA, USA) and incubated with primary antibody \#1, rat anti-mouse CD41 (MWReg30, ab33661, Abcam, Cambridge, MA, USA), 1:10 diluted in $1 \% \mathrm{NSS} / \mathrm{PBS}$ overnight at $4{ }^{\circ} \mathrm{C}$. Sections were then incubated with goat anti-rat Alexa Fluor $^{\circledR} 488$ 1:50 (Abcam) in PBS for $4 \mathrm{~h}$ followed by incubation with primary antibody \#2, rabbit anti-myeloperoxidase (ab45977, Abcam) 1:10 diluted in PBS overnight at $4{ }^{\circ} \mathrm{C}$. Sections were then incubated with chicken anti-rabbit Alexa Fluor ${ }^{\circledR} 647$ (Invitrogen, Life Technologies, Grand Island, NY, USA) 1:50 in PBS for $4 \mathrm{~h}$ followed by incubation with primary antibody \#3, mouse anti-histone H2A (L88A6, Cell Signaling, Danvers, MA, USA) 1:200 in PBS overnight at $4{ }^{\circ} \mathrm{C}$, and then finally incubated with goat anti-mouse IgG1 Alexa Fluor ${ }^{\circledR} 594$ (Invitrogen) 1:50 in PBS for $4 \mathrm{~h}$. Nuclear counterstain was performed with DAPI (Invitrogen) 1:500 for 5 min and sections mounted with Slow Fade Gold (Invitrogen). Negative controls were run in parallel with nonspecific IgG or specific isotype. Confocal microscopy was performed with a Leica TCS SP2 laser scanning confocal microscopy system of the VCU Department of Anatomy and Neurobiology Microscope Facility. Separate images of optical sections were acquired with filters for Alexa Fluor ${ }^{\circledR}$ (AF) 488, 594, 647 and DAPI. Images were assembled with ImageJ software. 


\subsection{RNA Isolation and Real-Time Quantitative PCR (QPCR) Analysis}

Isolation of total RNA and real-time QPCR analyses were performed as described previously [12]. Primers used for QPCR are listed in Table 1.

Table 1. Murine primers used for Quantitative PCR (QPCR).

\begin{tabular}{cc}
\hline Name & Sequence 5' to 3' \\
\hline ATF4 forward & CCTAGGTCTCTTAGATGACTATCTGGAGG \\
ATF4 reverse & CCAGGTCATCCATTCGAAACAGAGCATCG \\
BiP forward & GTGCAGCAGGACATCAAGTTCTGCC \\
BiP reverse & TTCCCAAATACGCCTCAGCAGTCTCC \\
CHOP forward & CACCTATATCTCATCCCCAGGAAACG \\
CHOP reverse & TTCCTTGCTCTTCCTCCTCTTCCTCC \\
EDEM forward & GCCCTTTGGTGACATGACAATTGAGG \\
EDEM reverse & TCATTATTGCTGTCAGGAGGAACACC \\
XBP-1s forward & TGAGTCCGCAGCAGGTGC \\
XBP-1s reverse & CAACTTGTCCAGAATGCCCAAAAGG \\
XBP-1 un forward & AAGAACACGCTTGGGAATGGACACGC \\
XBP-1un reverse & ACCTGCTGCAGAGGTGCACATAGTC \\
PAD4 forward & ACAGGTGAAAGCAGCCAGC \\
PAD4 reverse & AGTGATGTAGATCAGGGCTTGG \\
ATG3 forward & CACCACTGTCCAACATGGC \\
ATG3 reverse & GTTTACACCGCTTGTAGCATGG \\
ATG5 forward & ACAAGCAGCTCTGGATGGG \\
ATG5 reverse & GGAGGATATTCCATGAGTTTCCG \\
ATG6 forward & CACGAGCTTCAAGATCCTGG \\
ATG6 reverse & TCCTGAGTTAGCCTCTTCCTCC \\
ATG7 forward & ACGATGACGACACTGTTCTGG \\
ATG7 reverse & AGGTTACAGGGATCGTACACACC \\
ATG8 forward & ACAAAGAGTGGAAGATGTCCG \\
ATG8 reverse & GGAACTTGGTCTTGTCCAGG \\
TNF $\alpha$ forward & GATGAGAAGTTCCCAAATGGC \\
TNF $\alpha$ reverse & TTGGTGGTTGCTACGACG \\
IL-1 $\beta$ forward & CAGAACTCAACTGTGAAATGCC \\
IL-1 $\beta$ reverse & GATAGCTCTTTCTCGATTCCG \\
18S forward & AGAGTCTCGTTCGTTATCGG \\
18 reverse & \\
& \\
\hline
\end{tabular}

\subsection{Western Blot Analysis}

Neutrophil whole-cell and nuclear extracts were isolated for Western blot analysis as described previously [13]. Nuclear extracts were isolated using the NE-PER kit (Pierce Biotechnology, Rockford, IL, USA). Proteins were resolved by SDS polyacrylamide gel electrophoresis (4\%-20\%) and electrophoretically transferred to polyvinylidene fluoride membranes $(0.2 \mu \mathrm{m}$ pore size $)$. Immunodetection was performed using chemiluminescent detection with the Renaissance Western Blot Chemiluminescence Reagent Plus (Perkin Elmer Life Sciences Inc., Boston, MA, USA). Blots 
were stripped using the Restore ${ }^{\mathrm{TM}}$ Western Blot Stripping Buffer (Pierce Biotechnology Inc., Rockford, IL, USA) as described by the manufacturer. Purified rabbit polyclonal antibodies to LC3B (L7543, Sigma-Aldrich), cleaved caspase-3 (\#9661, Cell Signaling), caspase-3 (\#9662, Cell Signaling), p62/SQSTM1 (NBP1-48320, Novus Biologicals), NFкB p65 (sc-109, Santa Cruz Biotechnology), Lamin B (sc-6216, Santa Cruz Biotechnology), and actin (sc-1616, Santa Cruz Biotechnology) were used in this study. Optical densities of antibody-specific bands were determined using Quantity One acquisition and analysis software (Bio-Rad, Hercules, CA, USA).

\subsection{Isolation of Human Neutrophils and NETs Release}

Human neutrophils were isolated by density gradient centrifugation and hypotonic lysis [23]. Cells were adjusted to $2 \times 10^{6} / \mathrm{mL}$ in RPMI-1640, seeded onto 8 -well IbiTreat $\mu$-slides (Ibidi \#80826), $0.3 \mathrm{~mL}$ per well, and allowed to adhere for $15 \mathrm{~min}$. PMNs were VitC loaded by incubating for $1 \mathrm{~h}$ with $0.3 \mathrm{mM}$ or $3 \mathrm{mM}$ buffered ascorbic acid (Mylan Institutional LLC, Rockville, IL, USA). Neutrophils were stimulated with $50 \mathrm{nM}$ PMA for three hours at $37^{\circ} \mathrm{C}$. Neutrophil conditioned media were centrifuged at $400 \times \mathrm{g}$ for $5 \mathrm{~min}$ and the supernatants used for quantification of cf-DNA [24].

\subsection{Immunofluorescence Staining of Human NETs}

PMNs were fixed with 4\% paraformaldehyde, permeabilized with $0.15 \%$ Triton X-100 in PBS, and blocked with 5\% normal chicken serum (Sigma) in PBS. To stain NETs, slides were incubated with a mouse monoclonal anti-myeloperoxidase antibody (1:200; Santa Cruz sc-52707) and a secondary Alexa Fluor ${ }^{\circledR}$ 488-conjugated chicken anti-mouse IgG antibody (1:200; Molecular Probes A-21200). After staining of DNA with DAPI, neutrophil-derived NET formation was visualized by immunofluorescence microscopy performed on an Olympus model IX70 inverted microscope outfitted with an IX-FLA fluorescence observation system equipped with a FITC and DAPI filter cubes (Chroma Technology, Brattleboro, VT, USA) through Uplan FI objectives $(20 \times, 60 \times)$. Images were captured by an Olympus XM10 digital camera using CellSens imaging software (Olympus America, Melville, NY, USA).

\subsection{Quantification of Cell Free DNA}

The levels of cf-DNA in human neutrophil supernatants and mouse serum were quantified using the Invitrogen Quant-iTPicoGreendsDNA assay kit according to the manufacturer's instructions (Life Technologies, Grand Island, NY, USA). Fluorescence intensity was measured on a SpectraMax Gemini XPS microplate reader with excitation at $490 \mathrm{~nm}$ and emission at $525 \mathrm{~nm}$, with a $515 \mathrm{~nm}$ emission cutoff filter (Molecular Devices, Sunnyvale, CA, USA).

\subsection{Statistical Analysis}

Statistical analysis was performed using SAS 9.3 and GraphPad Prism 6.0 (GraphPad Software, San Diego, CA, USA). Data are expressed as mean \pm SE. Results were compared using Student-Newman-Keuls test or one-way ANOVA and the post hoc Tukey test to identify specific differences between groups. Statistical significance was confirmed at a $p$ value of $<0.05$. 
Figure 1. Vitamin $\mathrm{C}$ sufficient $\mathrm{Gulo}^{-/-}$mice demonstrate reduced lung NETs and lower cf-DNA following peritonitis-induced sepsis. Representative immunofluorescence and differential interference contrast imaging of lung NETs (A-E): (A) VitC sufficient Gulo $^{-/-}$mice $(+)$received saline alone $(0.4 \mathrm{~mL}$, i.p.); (B) FIP exposed VitC sufficient Gulo $^{-/-}$mice $[\mathbf{F I P}(+)]$ received fecal stem solution $(45 \mathrm{mg} / \mathrm{mL}$, i.p. $)$ followed $30 \mathrm{~min}$ later by saline $\left(0.1 \mathrm{~mL}\right.$, i.p.); (C) VitC deficient $\mathrm{Gulo}^{-/-}$mice $(-)$received saline alone (0.4 mL, i.p.); (D) FIP exposed VitC deficient Gulo ${ }^{-/-}$mice $[$FIP(-)] mice received fecal stem solution $(45 \mathrm{mg} / \mathrm{mL}$, i.p.) followed $30 \mathrm{~min}$ later by saline $(0.1 \mathrm{~mL}$, i.p.). (E) AscA treated FIP exposed VitC deficient Gulo ${ }^{-/-}$mice $[$FIP $(-)+$AscA $]$mice received fecal stem solution (45 mg/mL, i.p.) followed $30 \mathrm{~min}$ later by AscA (200 mg/kg, i.p.). Platelet CD-41 (green), histones (red), and myeloperoxidase (grey) are seen in the merged images. Arrowheads indicate NET formation shown by co-staining for platelet CD-41 (green), histones (red), myeloperoxidase (grey), and DAPI (blue) in the vascular and alveolar spaces. Arrows indicate extensive extra-nuclear histones (red); (10 $\times$ magnification, $N=3$ for each group). (F) Serum levels of cf-DNA were quantified using the Quant-iTPicoGreen dsDNA assay kit $(N=5-11$ for each group, $p<0.05)$.

$(+)$
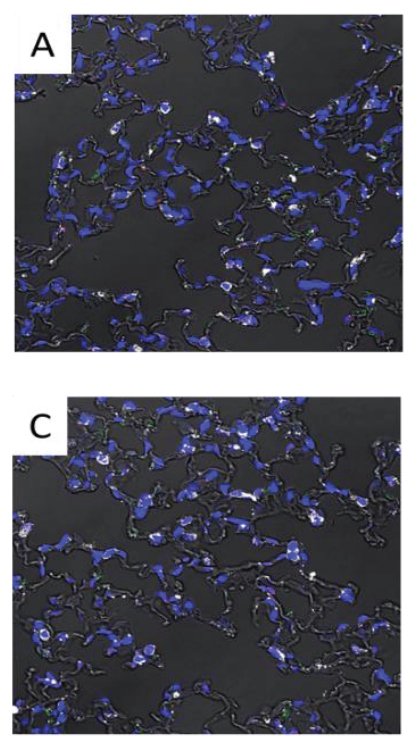

$(-)$
FIP (+)
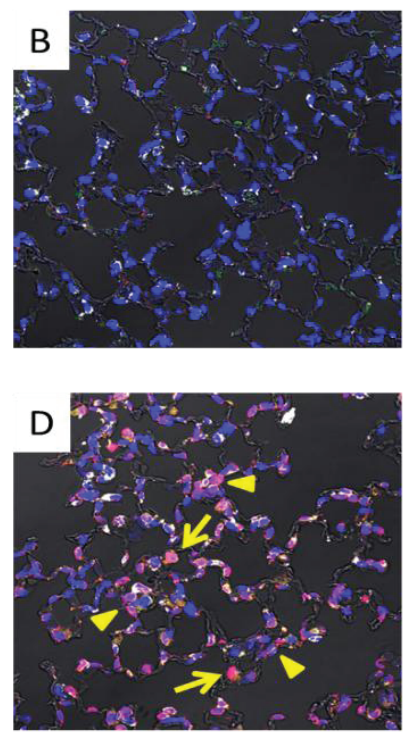

FIP (-)

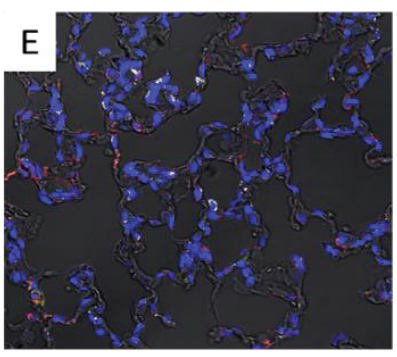

FIP (-)+AscA

\section{$\mathbf{F}$}

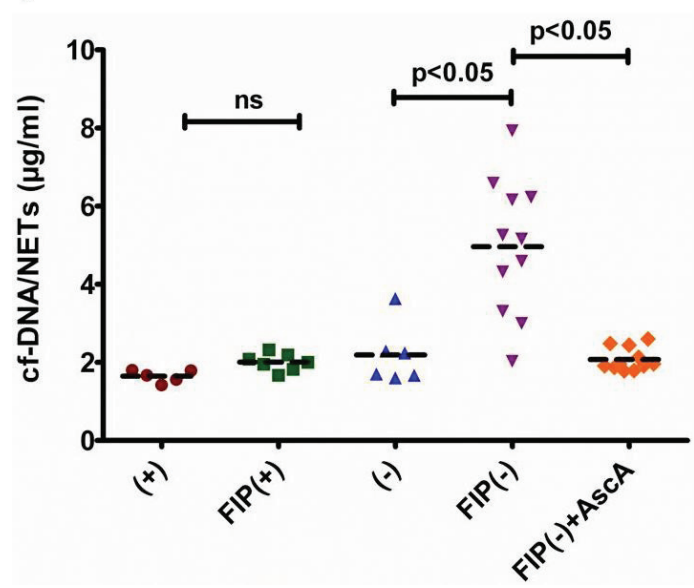




\section{Results}

\subsection{Vitamin C Sufficient Mice Demonstrate Reduced Lung NETs and Lower cf-DNA Following} Peritonitis-Induced Sepsis

We have previously shown that fecal peritonitis promotes PMN infiltration of the lungs in VitC deficient mice [19]. Here we used immunofluorescence staining and DIC microscopy to examine the extent of NETs in lungs of mice following FIP induced sepsis. Immuno-positive staining for platelet CD-41 (green), nuclear histones (red), and myeloperoxidase (grey) are visible in the lungs of saline exposed mice (Figure 1A). No appreciable immuno-positive staining differences were seen in the lungs of saline exposed VitC deficient mice (Figure 1C). FIP induced a mild increase in CD-41 immuno-positivity as well as some cytosolic histone staining (Figure 1B). However, no significant histological changes were evident in the VitC sufficient septic mice. In contrast, FIP induced significant NETs in VitC deficient mice as evidenced by dramatically increased co-staining for platelet CD-41 (green), histones (red), and myeloperoxidase (grey) in the vascular and alveolar spaces of septic mice (arrowheads, Figure 1D). Moreover, extensive extra-nuclear staining of histones (arrows) is also evident in this representative section along with thickened alveolar walls. Importantly, FIP exposed vitamin $\mathrm{C}$ deficient mice treated with ascorbic acid exhibited significant attenuation of NETs (Figure 1E).

In order to quantify NETs we determined levels of cf-DNA in the serum of VitC sufficient and deficient mice $16 \mathrm{~h}$ after sham treatment or FIP. Levels of serum cf-DNA were significantly elevated in the FIP exposed VitC deficient mice (Figure 1F, 5-fold, $p<0.05$ ). Treatment of septic VitC deficient mice with ascorbic acid significantly lowered the cf-DNA values to control levels $(p<0.05)$. In addition peritoneal neutrophils from vitamin $\mathrm{C}$ deficient mice were more susceptible to NETosis than those from vitamin C deficient mice (Supplementary Material, Figure S1).

\subsection{Vitamin C Deficient Neutrophils Show Increased PAD4 mRNA}

Unlike apoptosis, rapid intracellular decondensation of nuclear chromatin is a hallmark of NETosis $[19,25]$. Decondensation of nuclear chromatin requires the removal of positively charged arginine residues on histones by deimination or citrullination, which is carried out by a family of peptidylargininedeiminases (PAD). Of these, only PAD4 is expressed by neutrophils [26] and possesses a classical nuclear localization signal [27]. Importantly Wang et al. have shown that PAD4 is indispensable for NETosis [18]. Therefore, we examined mRNA expression of PAD4 in PMNs from VitC sufficient and deficient mice. As seen in Figure 2, PAD4 mRNA expression was significantly higher in thioglycollate elicited peritoneal PMNs from VitC deficient mice $(p<0.05)$.

\subsection{Autophagy Signaling Is Induced in Vitamin C Deficient Neutrophils}

Autophagy is a vital process for the catabolism of cytosolic proteins and organelles, but has also been shown to be required for NETosis [17,28]. To examine whether VitC regulates autophagy in PMNs we assessed the expression of several autophagy genes in thioglycollate elicited PMNs from VitC sufficient and deficient mice. As seen in Figure 3A, the expression of autophagy related signaling molecules (except for ATG6) were significantly elevated in the VitC deficient PMNs $(p<0.05)$. 
Figure 2. Vitamin $C$ deficient neutrophils show increased PAD4 mRNA. Real time QPCR for PAD4 shows two-fold increase in mRNA expression from peritoneal PMNs of VitC deficient Gulo ${ }^{-/-}$mice when compared to PMNs from VitC sufficient Gulo ${ }^{-/-}$mice $\left(N=6\right.$ for each group, $\left.{ }^{*} p<0.05\right)$.

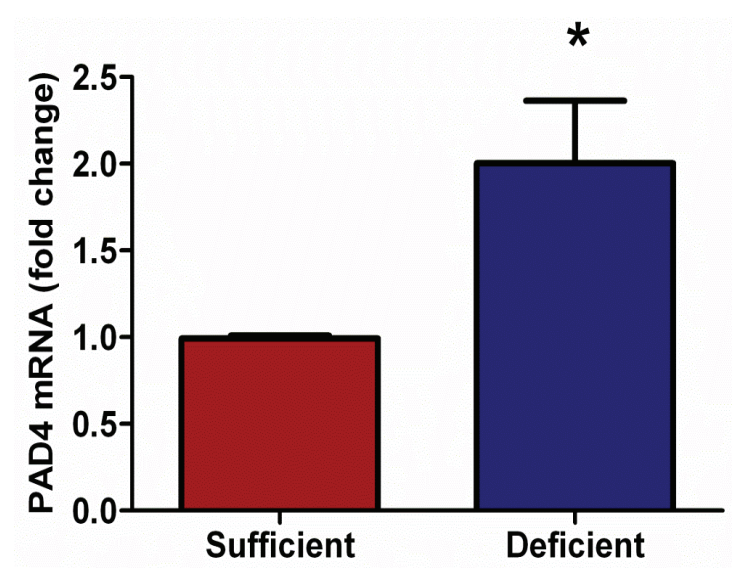

Activation of the autophagic process causes lipidation of ATG8/LC3B (LC3B-I to LC3B-II conversion) and the lipid-modified LC3B-II translocates to autophagosomes. This LC3B-I to LC3B-II conversion is considered a critical marker of autophagy activation [29]. We observed significantly enhanced LC3B-I to LC3B-II conversion in cell lysates of VitC deficient PMNs by immunoblotting (Figure 3B, $p<0.05$ ).

Figure 3. Autophagy signaling is induced in Vitamin $C$ deficient neutrophils. (A) Real time QPCR for ATG3, ATG5, ATG6, ATG7, and ATG8 mRNA from peritoneal PMNs of VitC sufficient and deficient Gulo ${ }^{-1-}$ mice, $\left(N=6\right.$ for each group, $\left.{ }^{*} p<0.05\right)$. (B) Representative Western blot for expression of LC3B-I and LC3B-II from peritoneal PMNs of VitC sufficient and deficient Gulo ${ }^{-/-}$mice. Densitometry of LC3B-II/actin from peritoneal PMNs of VitC sufficient and deficient Gulo ${ }^{-/-}$mice $(N=6$ for each group, ${ }^{*} p<0.05$ ). (C) Representative Western blot for expression of $\mathrm{p} 62$ and actin from peritoneal PMNs of VitC sufficient and deficient $\mathrm{Gulo}^{-/}$mice. Densitometry of normalized p62 expression from peritoneal PMNs of VitC sufficient and deficient $\mathrm{Gulo}^{-/-}$mice $(N=6$ for each group, ns $p=0.3)$.

A

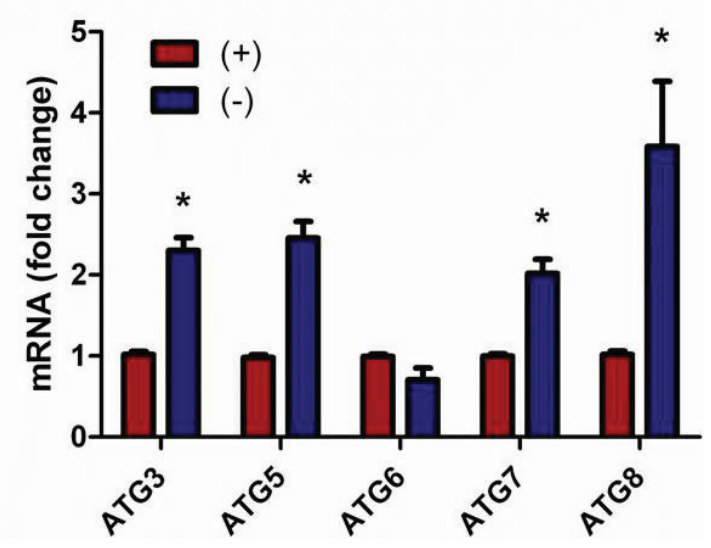


Figure 3. Cont.

B
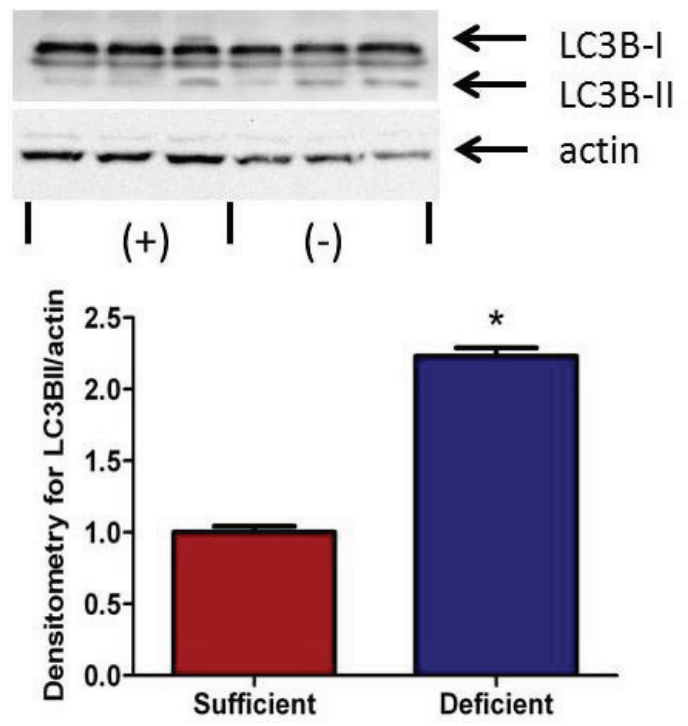

C
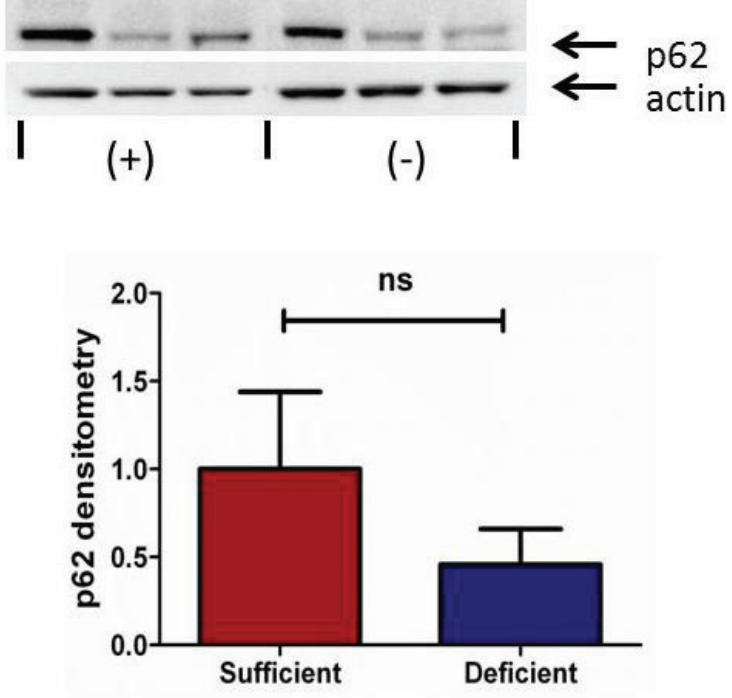

To further investigate the regulation of autophagy signaling by VitC in PMNs we examined the accumulation of p62/sequestosome I in these cell lysates. The loss of p62 in cells is typically indicative of increased autophagic activity [30]. Detection of p62 by immunoblotting showed a trend towards decreases p62 levels in the VitC deficient PMNs (Figure 3C). However this decline did not reach statistical significance $(p=0.3)$.

Figure 4. Endoplasmic reticulum stress associated gene expression in up-regulated in vitamin C deficient neutrophils. Real time QPCR for activating transcription factor 4 (ATF4), glucose-regulated protein 78 (Grp78, BiP), C/EBP homologous protein (CHOP), ER degradation-enhancing $\alpha$-mannosidase-like protein (EDEM), X-box binding protein-1 spliced (XBP-1s), and unspliced (XBP-1un) mRNA from peritoneal PMNs of VitC sufficient and deficient Gulo ${ }^{-/}$mice, $\left(N=6\right.$ for each group, $\left.{ }^{*} p<0.05\right)$.

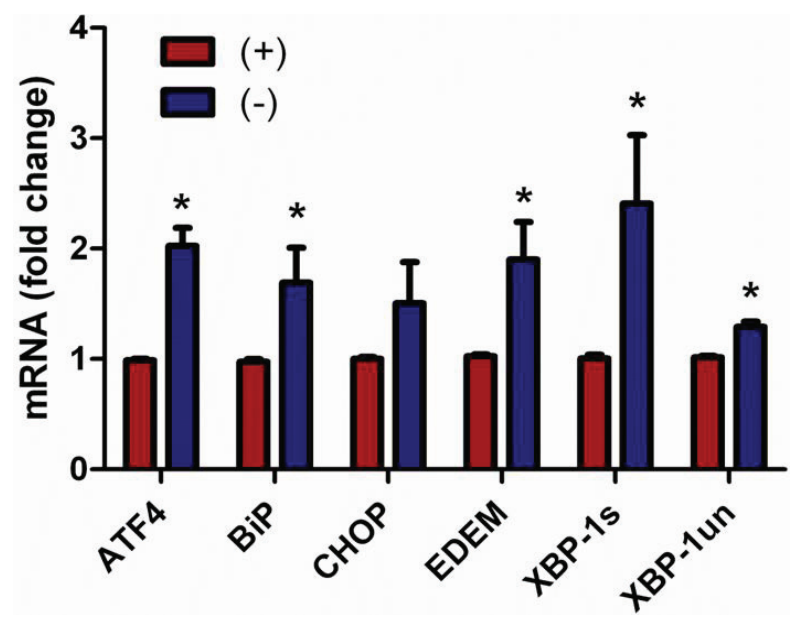




\subsection{Endoplasmic Reticulum Stress Associated Gene Expression Is Up-Regulated in Vitamin C} Deficient Neutrophils

Signaling initiated by the ER stress response (unfolded protein response, UPR) actively participates in autophagy and ultimately contributes to the cell fate decision [31]. Since autophagy signaling was induced in the VitC deficient PMNs, we next examined ER stress gene expression in the PMNs. As seen in Figure 4, all the UPR genes examined except for CHOP were significantly up-regulated in PMNs from VitC deficient mice $(p<0.05)$.

\subsection{Vitamin C Deficient Neutrophils Undergo Attenuated Apoptosis}

To determine the extent of apoptosis in peritoneal PMNs from VitC sufficient and VitC deficient mice, we examined a well characterized marker of apoptosis, cleaved caspase-3, by immunoblotting of PMN cell lysates. As seen in Figure 5, caspase-3 activation was significantly lower in VitC deficient PMNs $(p<0.05)$.

Figure 5. Vitamin C deficient neutrophils undergo attenuated apoptosis. Representative Western blot for expression of caspase- 3 and cleaved caspase- 3 from peritoneal PMNs of VitC sufficient and deficient Gulo ${ }^{-/-}$mice. Densitometry of cleaved caspase-3/caspase-3 from peritoneal PMNs of VitC sufficient and deficient $\mathrm{Gulo}^{-/-}$mice $(N=6$ for each group, $* p<0.05)$.

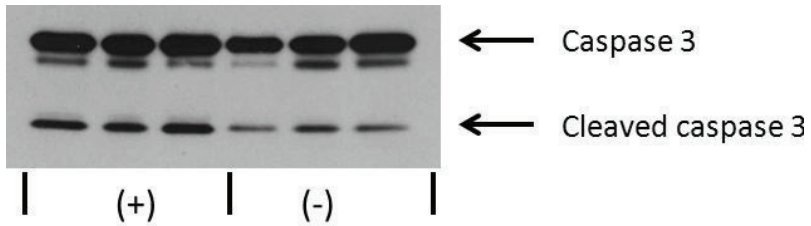

$(+) \quad \mid(-)$

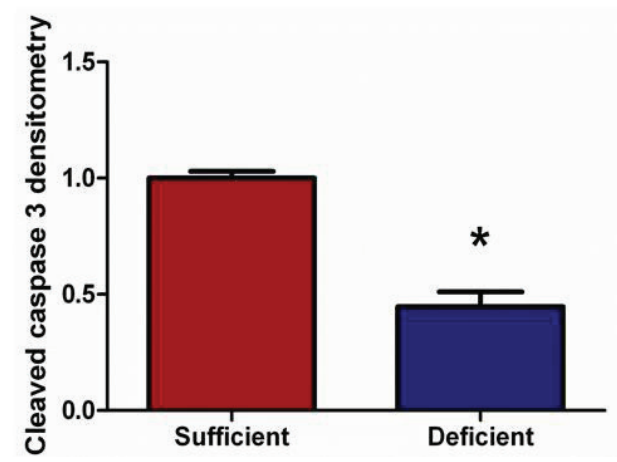

\subsection{Vitamin C Deficient Neutrophils Exhibit Increased NFKB Activation}

The transcription factor NFKB modulates the expression of many immuno-regulatory mediators in the acute inflammatory response in sepsis. Yang et al. found that diminished nuclear translocation of NFKB in peripheral PMNs was associated with less time on the ventilator and improved survival in critically ill patients [32]. NFKB activation is also associated with increased ROS production and endoplasmic reticulum stress signaling [33]. Therefore, we examined nuclear translocation of NFאB in peritoneal PMNs isolated from VitC sufficient and deficient mice. As seen in Figure 6A, significantly increased NFאB translocation was observed in nuclei of VitC deficient PMNs $(p<0.05)$. 
Increased nuclear $\mathrm{NF} \kappa \mathrm{B}$ translocation was associated with induction of the $\mathrm{NF} \kappa \mathrm{B}$ dependent pro-inflammatory genes for TNF $\alpha$ and IL-1 $\beta$ (Figure $6 \mathrm{~B}, p<0.05$ ).

Figure 6. Vitamin $\mathrm{C}$ deficient neutrophils exhibit increased $\mathrm{NF} \kappa \mathrm{B}$ activation. (A) Representative Western blot for nuclear expression of NFkBp65 and Lamin B from peritoneal PMNs of VitC sufficient and deficient $\mathrm{Gulo}^{-/}$mice. Densitometry of NFkBp65/Lamin B from peritoneal PMNs of VitC sufficient and deficient Gulo ${ }^{-/}$mice $\left(N=4\right.$ for each group, $\left.{ }^{*} p<0.05\right)$. (B) Real time QPCR for TNF $\alpha$ and IL-1 $\beta$ mRNA from peritoneal PMNs of VitC sufficient and deficient Gulo ${ }^{-/-}$mice, $(N=6$ for each group, $* p<0.05)$.

A
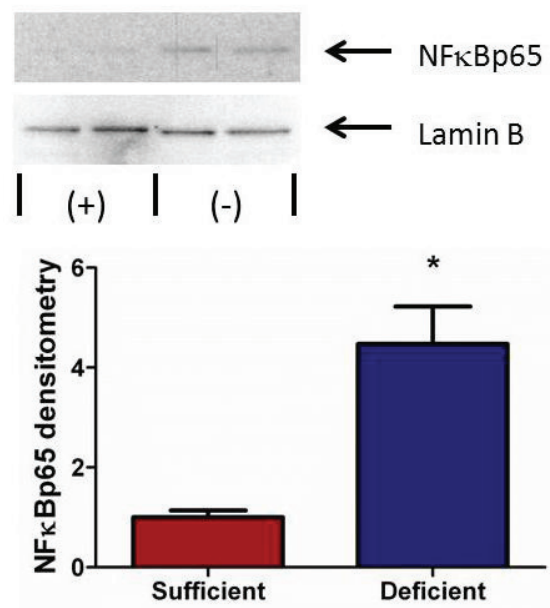

B

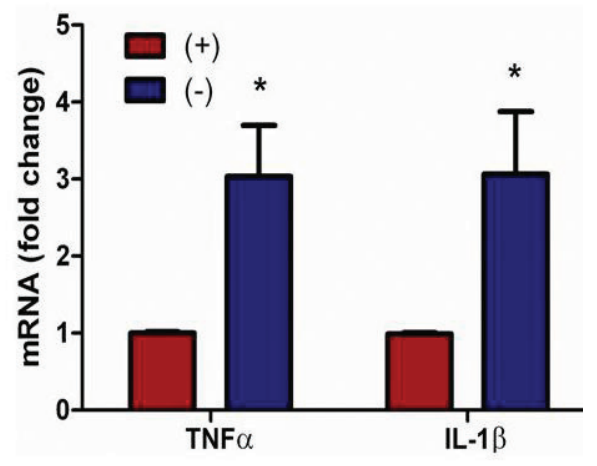

\subsection{Vitamin C Attenuates NET Formation in Activated Human Neutrophils}

Freshly isolated human PMNs formed NETs following activation by PMA (50 nM) for three hours as seen by immunofluorescence staining (Figure 7B,E). Loading the cells with VitC (3 mM) prior to PMA stimulation greatly reduced NET formation by human PMN (Figure 7C,F). Further, quantification of cf-DNA from the supernatants showed VitC ( $3 \mathrm{mM})$ loading significantly reduced NETs release from activated PMN (Figure 7G, $p<0.05$ ).

\section{Discussion}

In this study we show that VitC could play a critical role in regulating the ultimate fate of PMNs in sepsis. Activated PMNs undergo extensive NETosis in septic mice lungs, resulting in potential damage to lung alveolar and endothelial cells. This effect was predominant in PMNs from VitC deficient mice and could be rescued by VitC infusion after the onset of sepsis. In contradistinction, PMNs from VitC sufficient mice underwent attenuated NETosis. Importantly, at a molecular level, VitC deficient peritoneal PMNs were likely to be more pro-inflammatory, to resist apoptosis, and to preferentially undergo NETosis. 
Figure 7. Vitamin $\mathrm{C}$ attenuates NET formation in activated human neutrophils. Representative image of immunofluorescent staining for NETs in human neutrophils: DNA (blue); myeloperoxidase (green). Upper Panels: Control PMNs (A, 20×); PMNs exposed to PMA (50 nM) for $3 \mathrm{~h}(\mathbf{B}, 20 \times)$; PMNs loaded with VitC (3 mM) for $1 \mathrm{~h}$ and then exposed to PMA (50 nM) for $3 \mathrm{~h}(\mathbf{C}, 20 \times)$. Lower Panels: Control PMNs (D, 60×); PMNs exposed to PMA (50 nM) for $3 \mathrm{~h}(\mathbf{E}, 60 \times)$; PMNs loaded with VitC (3 mM) for $1 \mathrm{~h}$ and then exposed to PMA $(50 \mathrm{nM})$ for $3 \mathrm{~h}(\mathbf{F}, 60 \times) .(N=3$ for each group, Magnification: upper panel 20×, lower panel 60×). (G) Quantification of cf-DNA in the supernatants above $(N=3$ for each group, $p<0.05)$.
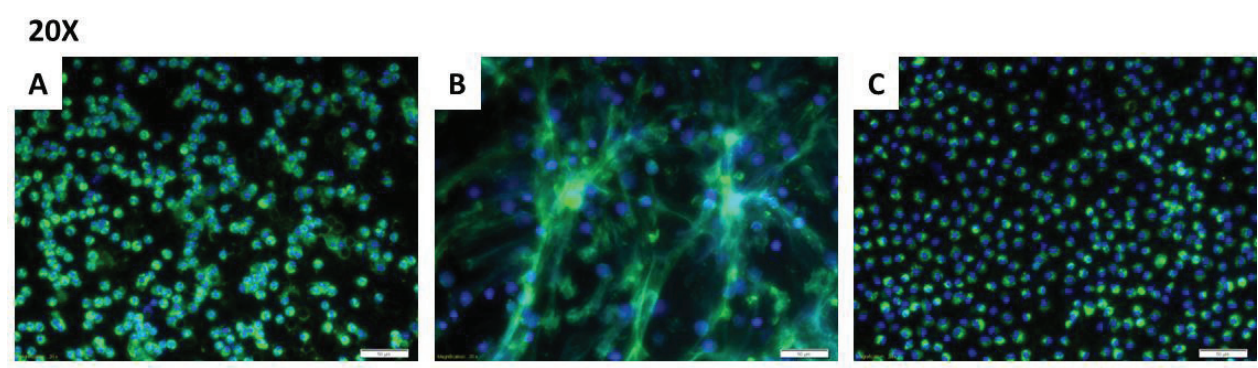

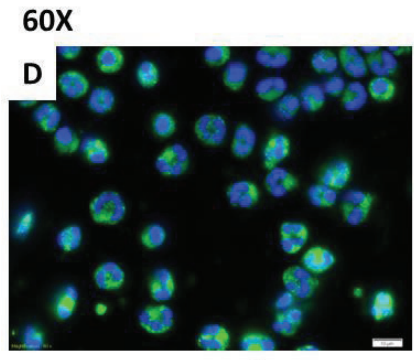

Control

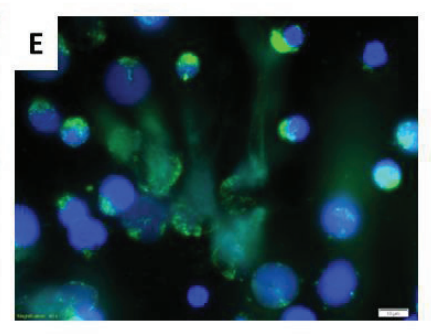

PMA

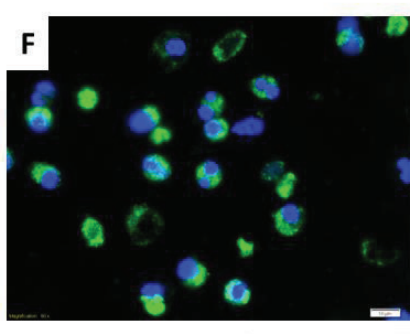

PMA+VitC

G

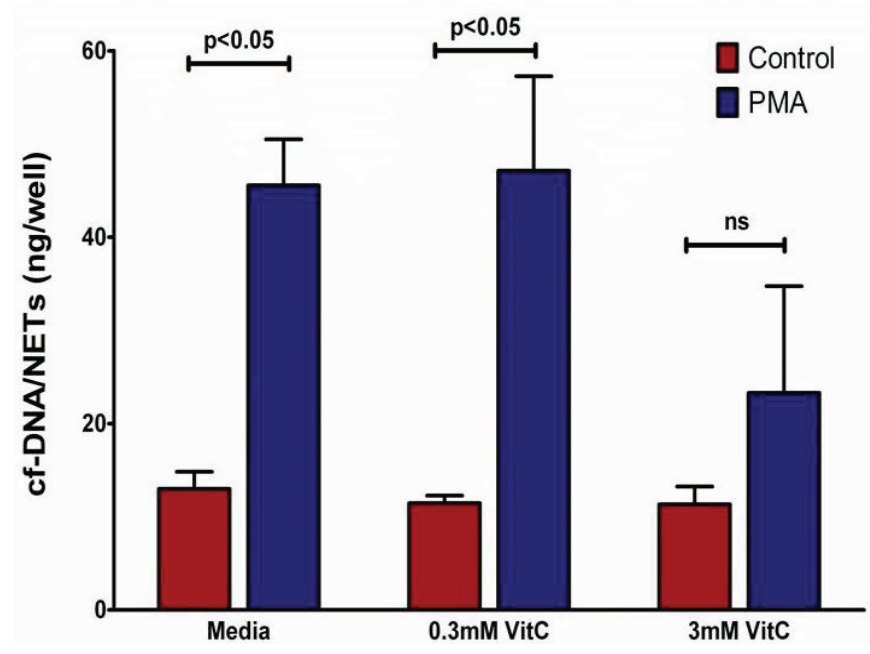

Although several signaling mechanisms responsible for NET formation have been reported, critical regulatory elements remain unidentified. This study advances our understanding of PMN function and NET biology by identifying a novel regulatory mechanism for NET formation in both murine and human PMNs. Using our previously well-characterized model of abdominal peritonitis induced sepsis we show that sepsis promotes NET formation in lungs of VitC deficient mice (Figure 1). NETosis in this model was accompanied by increased circulating cf-DNA (Figure 1F). VitC 
sufficiency or infusion of VitC after initiation of sepsis significantly decreased NETosis in lungs and circulating cf-DNA content (Figure 1). NET formation in VitC deficient peritoneal PMNs required activation of well characterized signaling pathways including ROS generation (data not shown), activation of the peptidylargininedeiminase PAD4 (Figure 2), autophagy (Figure 3), endoplasmic reticulum stress (Figure 4), and inhibition of apoptosis (Figure 5). NFkB, a pro-inflammatory, pro-survival transcription factor was activated in the VitC deficient peritoneal PMNs (Figure 6). VitC sufficiency or treatment with VitC attenuated these signaling pathways in PMNs.

Intracellular chromatin decondensation is essential for NET formation. Chromatin decondensation is brought about by peptidylargininedeiminase 4 (PAD4), a nuclear enzyme that deiminates arginine residues on histone tails thereby converting positively charged arginines to uncharged citrullines $[17,18]$. The importance of PAD4 is that many NET forming stimuli including PMA, LPS, and IL-8 as well as various bacterial and fungal species converge to its activation. While PAD4 is expressed in PMNs and is localized to the nucleus [26,27], little is known about its mechanism of action or its transcriptional regulation. Ying et al. have shown that PAD1, which belongs to the same family of enzymes as PAD4, is transcriptionally regulated by $\mathrm{NF \kappa B} \mathrm{[34].} \mathrm{We}$ have previously shown that VitC blocks NFkB activation in septic mouse lungs [12]. Cárcamo et al.

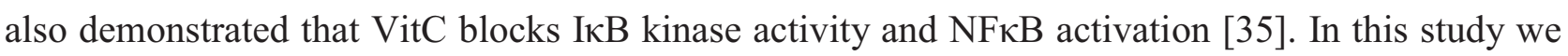
observed that nuclear NFאB levels were higher in the VitC deficient PMNs (Figure 6A). Further, PAD4 mRNA expression was also significantly higher in PMNs from VitC deficient mice (Figure 2). Therefore, we hypothesize that VitC decreases PAD4 expression by suppressing NFkB activation in PMNs. Further, by decreasing PAD4 expression VitC could decrease histone citrullination activity and therefore chromatin decondensation in VitC sufficient PMNs.

Autophagy has been identified as a well-conserved, homeostatic mechanism that clears damaged organelles or proteins and plays an essential role in cell survival during periods of nutrient depletion [36]. Despite the view that it might not occur in neutrophils, autophagy was recently shown to occur both in murine and human PMNs [37,38]. While Mitroulis et al. reported that autophagy occurs in human PMNs in response to PMA activation [39]. Remijsen et al. have shown that autophagy is necessary for the induction of intracellular chromatin decondensation during PMA-induced NETosis [17]. In our study, we found increased expression of autophagy genes (Figure 3A) as well as significantly enhanced LC3B-I to LC3B-II conversion in VitC deficient PMNs (Figure 3B) indicative of the presence of more autophagosomes in VitC deficient PMNs. However, LC3B-I to LC3B-II conversion is a static measure of autophagosome number, and does not measure the actual activity of the pathway. The increased LC3B-II could be interpreted as either high autophagic activity or a downstream block in the system that results in an accumulation of LC3B-II protein, even though autophagic degradation itself is diminished. To supplement our observations we examined levels of p62/sequestosome I, a cytosolic chaperone protein with an LC3B binding domain [40]. The normal function of $\mathrm{p} 62$ protein is to carry polyubiquitinated proteins to the autphagolysosome where it binds to LC3B before getting degraded. Thus, the loss of $p 62$ protein is a measure of the flux of autophagy and indicative of increased autophagy [41]. In our studies we found a trend towards decreased p62 levels in the VitC deficient PMNs (Figure 3C). While this decline did not reach statistical significance, in combination with the increased autophagy gene expression and increased LC3B conversion, our data imply increased autophagy in VitC deficient PMNs. 
The unfolded protein response (UPR) and autophagic machinery have been shown to be critically linked to each other. It is well established that activation of the UPR genes transcriptionally up-regulates several autophagy related genes required for induction and construction of the autophagy machinery [42]. However, it is not known whether activation of the UPR drives autophagy and eventually leads to NET formation in PMNs. Our study shows that most of the UPR genes examined except for CHOP were significantly up-regulated in PMNs from VitC deficient mice (Figure 4). This implies that VitC deficient PMNs could be actively undergoing ER stress, which in turn could drive autophagy genes and increase their susceptibility to undergo NETosis.

The transcription factor NFKB is central to pro-inflammatory/pro-survival responses in sepsis. It is normally sequestered to $\mathrm{I} \kappa \mathrm{B}$ in the cytosol. Upon appropriate stimulation, I $\mathrm{I} B$ is degraded allowing NFKB to migrate to the nucleus and drives transcription of numerous genes that regulate the immune response in sepsis. Moine et al. have demonstrated increased NFKB translocation in the lungs of patients with ALI [43]. Yang et al. found that increased nuclear levels of NFkB in unstimulated neutrophils were associated with a worse clinical outcome [32]. As discussed above, NFkB likely drives expression of PAD4 in PMNs. NFkB activation also drives expression of pro-survival genes [44]. In this study we found that nuclear NFKB translocation was higher in VitC deficient PMNs (Figure 6A). Further, NFאB translocation in these VitC deficient PMNs increased expression of the pro-inflammatory genes TNF $\alpha$ and IL-1 $\beta$ (Figure 6). NFkB activation also inhibited apoptosis as seen by the reduced activation of caspase 3 in VitC deficient PMNs (Figure 5). These results suggest that $\mathrm{NF \kappa B}$ may play a critical role in modulating cell signaling pathways that eventually regulate the fate of PMNs. By activating PAD4 (chromatin decondensation), inducing ER stress and subsequent autophagy, and inhibiting apoptosis, $\mathrm{NF \kappa B}$ may drive the cellular machinery of VitC deficient PMNs towards NET formation (Figure 8). VitC sufficiency or infusion of VitC allows PMNs to increase intracellular levels of VitC and attenuate NFkB activation. This could dampen the pathways required for NETosis and may allow PMNs to undergo apoptosis instead. While the decreased apoptosis rate in VitC deficient PMNs may benefit the host by giving more time for PMNs to perform their innate immune functions, studies show that it could also be detrimental in sepsis due to the PMN-dependent inflammation and tissue damage that could be heightened by a prolonged lifespan. Recent reports in the literature have implicated NETs in transfusion-related acute lung injury (TRALI), the leading cause of death after transfusion therapy $[45,46]$. NETs were shown to be present during TRALI both in mice and humans and so it was suggested that targeting NET formation may be a new approach for the treatment of acute lung injury. While we did not examine TRALI in our studies, it is conceivable that VitC infusion could be a useful adjunct for the prevention/treatment of TRALI or other disease states involving exuberant formation of NETs particularly in the lungs.

Our study has several limitations: (1) It is possible that the PMNs isolated within the peritoneal cavity by thioglycollate could be partially activated; (2) We examined PMN function ex vivo. Further in vivo studies are needed to characterize the fate of PMNs; (3) others have performed studies with PMNs isolated from bone marrow instead of thioglycollate elicitation. These PMNs would be less "activated" when compared to thioglycollate elicited PMNs, but would also have a large component of immature PMNs which have been shown to behave somewhat differently from mature PMNs [47]. 
Figure 8. Schematic hypothesis of regulation of signaling pathways that leads to NETosis by VitC. Septic stimuli activate NFאB in PMNs with increased activation observed in VitC deficient PMNs. NFאB nuclear translocation drives expression of PAD4, ER stress and autophagy signaling genes while inhibiting caspase 3 in activated PMNs. This drives the fate of activated PMN away from apoptosis and enhances NETosis. VitC likely blocks up-regulation of PAD4, ER stress and autophagy signaling genes by decreasing NFאB activation. Activated PMNs now undergo apoptosis while NETosis is attenuated.

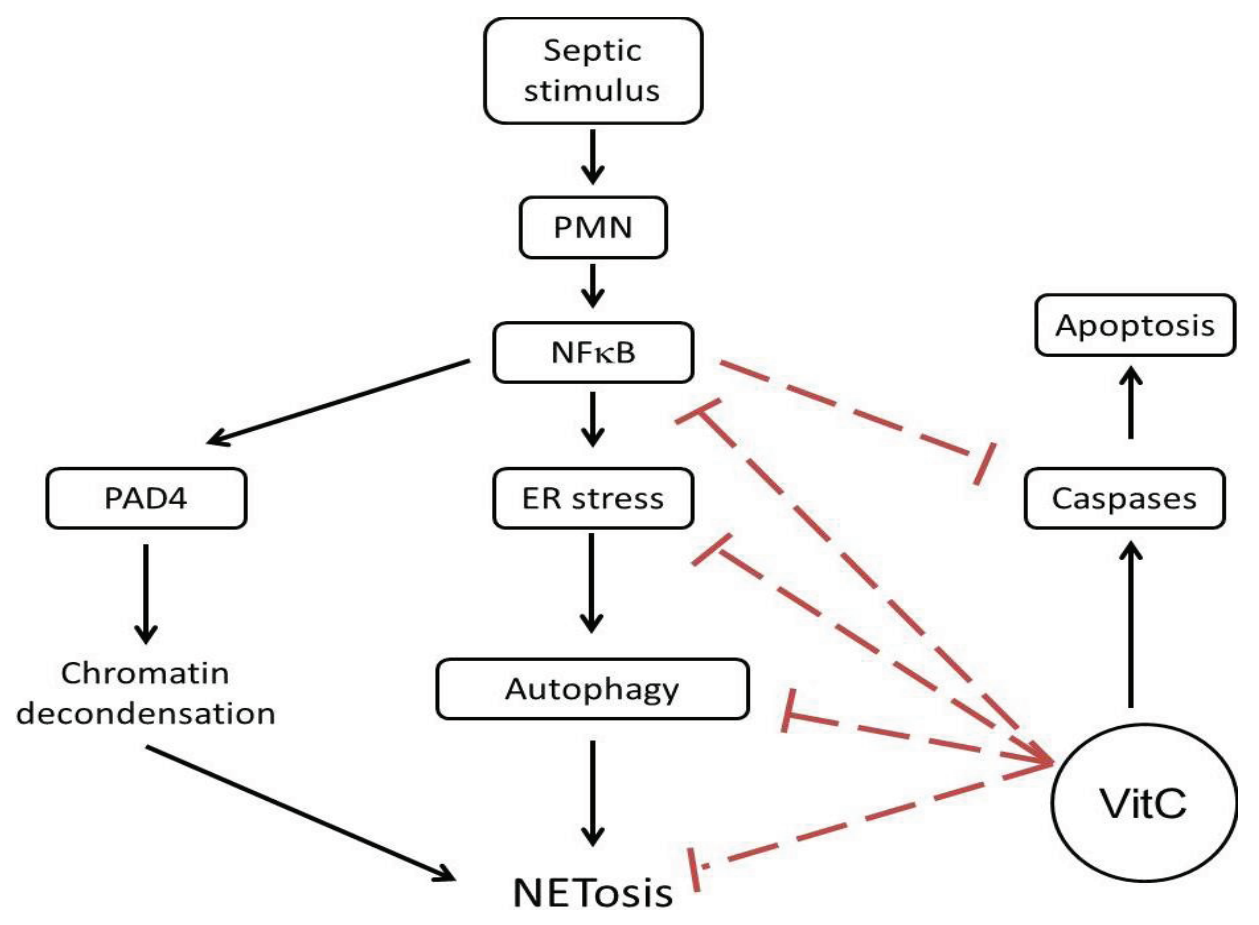

\section{Conclusions}

In the past few years circulating cf-DNA has been identified as a prognostic marker in severe sepsis $[11,48,49]$. Indeed cf-DNA was shown to have better discriminatory power than IL-6, thrombin or protein $\mathrm{C}$ to predict ICU mortality in sepsis [11]. The cellular origin of cf-DNA from host cells was shown by Dwivedi et al. [11] who confirmed that the release of cf-DNA was independent of the infecting organism and was likely mediated by inflammatory mediators generated during the exacerbated host immune response. Our study showed attenuated NET formation and reduced cf-DNA in the serum of septic VitC sufficient mice and in VitC deficient mice treated with ascorbic acid (Figure 1). Our study did not examine the origin of cf-DNA in the serum of these mice. It is possible that some of this DNA could be non-neutrophilic in origin since mast cells, eosinophils, and basophils have also been shown to expel their DNA in a manner similar to PMNs [50]. However, a detailed examination of the origin of cf-DNA in these septic mice is beyond the scope of this study. Nevertheless, data from our study implies that attenuation of NETs maybe crucial for resolution of sepsis in mice.

Overall, our in vitro and in vivo findings identify a novel regulatory mechanism that limits NET formation in sepsis. These findings implicate VitC as a previously unrecognized layer of regulation that prevents generation of excessive NETs. 


\section{Acknowledgments}

This research was supported by grants from the Aubery Sage Mac Farlane Endowment for Acute Lung Injury Research, the AD Williams Research Foundation (NR), the Jeffress Memorial Trust (NR) and the Victoria Johnson Center for Lung Research.

Microscopy was performed at the VCU Department of Anatomy and Neurobiology Microscopy Facility, supported in part with funding from NIH-NINDS Center core grant (5P30NS047463).

\section{Conflict of Interest}

The authors declare no conflict of interest.

\section{References}

1. Winterbourn, C.C.; Kettle, A.J. Redox reactions and microbial killing in the neutrophil phagosome. Antioxid. Redox Signal. 2013, 18, 642-660.

2. Borregaard, N. Neutrophils, from marrow to microbes. Immunity 2010, 33, 657-670.

3. Takei, H.; Araki, A.; Watanabe, H.; Ichinose, A.; Sendo, F. Rapid killing of human neutrophils by the potent activator phorbol 12-myristate 13-acetate (PMA) accompanied by changes different from typical apoptosis or necrosis. J. Leukoc. Biol. 1996, 59, 229-240.

4. Brinkmann, V.; Reichard, U.; Goosmann, C.; Fauler, B.; Uhlemann, Y.; Weiss, D.S.; Weinrauch, Y.; Zychlinsky, A. Neutrophil extracellular traps kill bacteria. Science 2004, 303, 1532-1535.

5. Narasaraju, T.; Yang, E.; Samy, R.P.; Ng, H.H.; Poh, W.P.; Liew, A.A.; Phoon, M.C.; van Rooijen, N.; Chow, V.T. Excessive neutrophils and neutrophil extracellular traps contribute to acute lung injury of influenza pneumonitis. Am. J. Pathol. 2011, 179, 199-210.

6. Grommes, J.; Soehnlein, O. Contribution of neutrophils to acute lung injury. Mol. Med. 2011, 17, 293-307.

7. Saffarzadeh, M.; Juenemann, C.; Queisser, M.A.; Lochnit, G.; Barreto, G.; Galuska, S.P.; Lohmeyer, J.; Preissner, K.T. Neutrophil extracellular traps directly induce epithelial and endothelial cell death: A predominant role of histones. PLoS One 2012, 7, e32366.

8. Douda D.N.; Jackson, R.; Grasemann, H.; Palaniyar, N. Innate immune collectin surfactant protein D simultaneously binds both neutrophil extracellular traps and carbohydrate ligands and promotes bacterial trapping. J. Immunol. 2011, 187, 1856-1865.

9. Gupta, A.K.; Hasler, P.; Holzgreve, W.; Gebhardt, S.; Hahn, S. Induction of neutrophil extracellular DNA lattices by placental microparticles and IL-8 and their presence in preeclampsia. Hum. Immunol. 2005, 66, 1146-1154.

10. Papayannopoulos, V.; Zychlinsky, A. NETs: A new strategy for using old weapons. Trends Immunol. 2009, 30, 513-521.

11. Dwivedi, D.J.; Toltl, L.J.; Swystun, L.J.; Pogue, J.; Liaw, K.L.; Weitz, J.I.; Cook, D.J.; Fox-Robichaud, A.E.; Liaw, P.C.; Canadian Critical Care Translational Biology Group. Prognostic utility and characterization of cell-free DNA in patients with severe sepsis. Crit. Care 2012, 16, R151. 
12. Fisher, B.J.; Seropian, I.M.; Kraskauskas, D.; Thakkar, J.N.; Voelkel, N.F.; Fowler, A.A.; Natarajan, R. Ascorbic acid attenuates lipopolysaccharide-induced acute lung injury. Crit. Care Med. 2011, 39, 1454-1460.

13. Fisher, B.J.; Kraskauskas, D.; Martin, E.J.; Farkas, D.; Wegelin, J.A.; Brophy, D.; Ward, K.R.; Voelkel, N.F.; Fowler, A.A.; Natarajan, R. Mechanisms of attenuation of abdominal sepsis induced acute lung injury by ascorbic acid. Am. J. Physiol. Lung Cell. Mol. Physiol. 2012, 303, L20-L32.

14. Padayatty, S.J.; Katz, A.; Wang, Y.; Eck, P.; Kwon, O.; Lee, J.H.; Chen, S.; Corpe, C.; Dutta, A.; Dutta, S.K.; et al. Vitamin C as an antioxidant: evaluation of its role in disease prevention. J. Am. Coll. Nutr. 2003, 22, 18-35.

15. Vissers, M.C.; Wilkie, R.P. Ascorbate deficiency results in impaired neutrophil apoptosis and

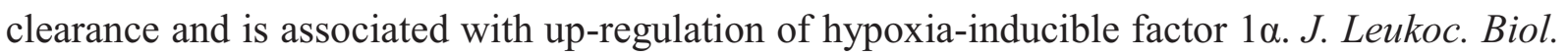
2007, 81, 1236-1244.

16. Nishikimi, M.; Fukuyama, R.; Minoshima, S.; Shimizu, N.; Yagi, K. Cloning and chromosomal mapping of the human nonfunctional gene for L-gulono- $\gamma$-lactone oxidase, the enzyme for L-ascorbic acid biosynthesis missing in man. J. Biol. Chem. 1994, 269, 13685-13688.

17. Remijsen, Q.; Vanden Berghe, T.; Wirawan, E.; Asselbergh, B.; Parthoens, E.; de Rycke, R.; Noppen, S.; Delforge, M.; Willems, J.; Vandenabeele, P. Neutrophil extracellular trap cell death requires both autophagy and superoxide generation. Cell Res. 2011, 21, 290-304.

18. Wang, Y.; Li, M.; Stadler, S.; Correll, S.; Li, P.; Wang, D.; Hayama, R.; Leonelli, L.; Han, H.; Grigoryev, S.A.; et al. Histone hypercitrullination mediates chromatin decondensation and neutrophil extracellular trap formation. J. Cell Biol. 2009, 184, 205-213.

19. Fisher, B.J.; Kraskauskas, D.; Martin, E.J.; Farkas, D.; Puri, P.; Massey, H.D.; Idowu, M.O.; Brophy, D.; Voelkel, N.F.; Fowler, A.A.; et al. Attenuation of sepsis induced organ injury by vitamin C. J. Parenter. Enter. Nutr. 2013, in press.

20. Kim, H.; Bae, S.; Yu, Y.; Kim, Y.; Kim, H.R.; Hwang, Y.I.; Kang, J.S.; Lee, W.J. The analysis of vitamin $\mathrm{C}$ concentration in organs of gulo $\left({ }^{--}\right)$mice upon vitamin $\mathrm{C}$ withdrawal. Immune Netw. 2012, 12, 18-26.

21. Vissers, M.C.; Bozonet, S.M.; Pearson, J.F.; Braithwaite, L.J. Dietary ascorbate intake affects steady state tissue concentrations in vitamin $\mathrm{C}$-deficient mice: Tissue deficiency after suboptimal intake and superior bioavailability from a food source (kiwifruit). Am. J. Clin. Nutr. 2011, 93, 292-301.

22. Tsurubuchi, T.; Aratani, Y.; Maeda, N.; Koyama, H. Retardation of early-onset PMA-induced apoptosis in mouse neutrophils deficient in myeloperoxidase. J. Leukoc. Biol. 2001, 70, 52-58.

23. Fowler, A.A.; Fisher, B.J.; Centor, R.M.; Carchman R.A. Development of the adult respiratory distress syndrome: Progressive alteration of neutrophil chemotactic and secretory processes. Am. J. Pathol. 1984, 116, 427-435.

24. Meng, W.; Paunel-Görgülü, A.; Flohé, S.; Witte, I.; Schädel-Höpfner, M.; Windolf, J.; Lögters T.T. Deoxyribonuclease is a potential counter regulator of aberrant neutrophil extracellular traps formation after major trauma. Mediat. Inflamm. 2012, 2012, doi:10.1155/2012/149560. 
25. Fuchs, T.A.; Abed, U.; Goosmann, C.; Hurwitz, R.; Schulze, I.; Wahn, V.; Weinrauch, Y.; Brinkmann, V.; Zychlinsky, A. Novel cell death program leads to neutrophil extracellular traps. J. Cell Biol. 2007, 176, 231-241.

26. Asaga, H.; Nakashima, K.; Senshu, T.; Ishigami, A.; Yamada, M. Immunocytochemical localization of peptidylargininedeiminase in human eosinophils and neutrophils. J. Leukoc. Biol. 2001, 70, 46-51.

27. Mastronardi, F.G.; Wood, D.D.; Mei, J.; Raijmakers, R.; Tseveleki, V.; Dosch, H.M.; Probert, L.; Casaccia-Bonnefil, P.; Moscarello, M.A. Increased citrullination of histone H3 in multiple sclerosis brain and animal models of demyelination: A role for tumor necrosis factor-induced peptidylargininedeiminase 4 translocation. J. Neurosci. 2006, 26, 11387-11396.

28. Cheng, O.Z.; Palaniyar, N. NET balancing: A problem in inflammatory lung diseases. Front. Immunol. 2013, 4, 1-13.

29. Klionsky, D.J.; Abeliovich, H.; Agostinis, P.; Agrawal, D.K.; Aliev, G.; Askew, D.S.; Baba, M.; Baehrecke, E.H.; Bahr, B.A.; Ballabio, A.; et al. Guidelines for the use and interpretation of assays for monitoring autophagy in higher eukaryotes. Autophagy 2008, 4, 151-175.

30. Bjorkoy, G.; Lamark, T.; Pankiv, S.; Overvatn, A.; Brech, A.; Johansen, T. Monitoring autophagic degradation of p62/SQSTM1. Methods Enzymol. 2009, 452, 181-197.

31. Deegan, S.; Saveljeva, S.; Gorman, A.M.; Samali, A. Stress-induced self-cannibalism: On the regulation of autophagy by endoplasmic reticulum stress. Cell. Mol. Life Sci. 2013, 70, 2425-2441.

32. Yang, K.Y.; Arcaroli, J.J.; Abraham, E. Early alterations in neutrophil activation are associated with outcome in acute lung injury. Am. J. Respir. Crit. Care Med. 2003, 167, 1567-1574.

33. Clarke, R.; Cook, K.L.; Hu, R.; Facey, C.O.; Tavassoly, I.; Schwartz, J.L.; Baumann, W.T.; Tyson, J.J.; Xuan, J.; Wang, Y.; et al. Endoplasmic reticulum stress, the unfolded protein response, autophagy, and the integrated regulation of breast cancer cell fate. Cancer Res. 2012, 72, 1321-1331.

34. Ying, S.; Kojima, T.; Kawada, A.; Nachat, R.; Serre, G.; Simon, M.; Takahara, H. An intronic enhancer driven by NF- $\mathrm{KB}$ contributes to transcriptional regulation of peptidylargininedeiminase type I gene in human keratinocytes. J. Investig. Dermatol. 2010, 130, 2543-2552.

35. Cárcamo, J.M.; Pedraza, A.; Bórquez-Ojeda, O.; Golde, D.W. Vitamin C suppresses

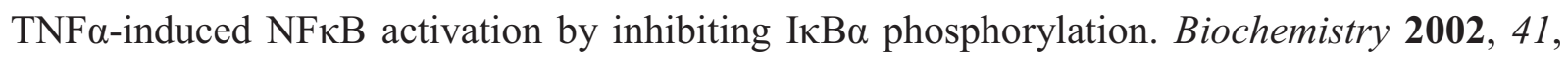
12995-13002.

36. Klionsky, D.J.; Emr, S.D. Autophagy as a regulated pathway of cellular degradation. Science 2000, 290, 1717-1721.

37. Beertsen, W.; Willenborg, M.; Everts, V.; Zirogianni, A.; Podschun, R.; Schröder, B.; Eskelinen, E.L.; Saftig, P. Impaired phagosomal maturation in neutrophils leads to periodontitis in lysosomal-associated membrane protein-2 knockout mice. J. Immunol. 2008, 180, 475-482.

38. Huang, J.; Canadien, V.; Lam, G.Y.; Steinberg, B.E.; Dinauer, M.C.; Magalhaes, M.A.; Glogauer, M.; Grinstein, S.; Brumell, J.H. Activation of antibacterial autophagy by NADPH oxidases. Proc. Natl. Acad. Sci. USA 2009, 106, 6226-6231. 
39. Mitroulis, I.; Kourtzelis, I.; Kambas, K.; Rafail, S.; Chrysanthopoulou, A.; Speletas, M.; Ritis, K. Regulation of the autophagic machinery in human neutrophils. Eur. J. Immunol. 2010, 40, $1461-1472$.

40. Pankiv, S.; Clausen, T.H.; Lamark, T.; Brech, A.; Bruun, J.A.; Outzen, H.; Overvatn, A.; Bjorkoy, G.; Johansen, T. P62/SQSTM1 binds directly to Atg8/LC3 to facilitate degradation of ubiquitinated protein aggregates by autophagy. J. Biol. Chem. 2007, 282, 24131-24145.

41. Patel, A.S.; Morse, D.; Choi, A.M. Regulation and functional significance of autophagy in respiratory cell biology and disease. Am. J. Respir. Cell Mol. Biol. 2013, 48, 1-9.

42. Rouschop, K.M.; van den Beucken, T.; Dubois, L.; Niessen, H.; Bussink, J.; Savelkouls, K.; Keulers, T.; Mujcic, H.; Landuyt, W.; Voncken, J.W.; et al. The unfolded protein response protects human tumor cells during hypoxia through regulation of the autophagy genes MAP1LC3B and ATG5. J. Clin. Investig. 2012, 120, 127-141.

43. Moine, P.; McIntyre, R.; Schwartz, M.D.; Kaneko, D.; Shenkar, R.; Le Tulzo, Y.; Moore, E.E.; Abraham, E. NF- $\mathrm{BB}$ regulatory mechanisms in alveolar macrophages from patients with acute respiratory distress syndrome. Shock 2000, 13, 85-91.

44. Burstein, E.; Duckett, C.S. Dying for NF-кB? Control of cell death by transcriptional regulation of the apoptotic machinery. Curr. Opin. Cell Biol. 2003, 15, 732-737.

45. Caudrillier, A.; Kessenbrock, K.; Gilliss, B.M.; Nguyen, J.X.; Marques, M.B.; Monestier, M.; Toy, P.; Werb, Z.; Looney, M.R. Platelets induce neutrophil extracellular traps in transfusion-related acute lung injury. J. Clin. Investig. 2012, 122, 2661-2671.

46. Thomas, G.M.; Carbo, C.; Curtis, B.R.; Martinod, K.; Mazo, I.B.; Schatzberg, D.; Cifuni, S.M.; Fuchs, T.A.; von Andrian, U.H.; Hartwig, J.H.; et al. Extracellular DNA traps are associated with the pathogenesis of TRALI in humans and mice. Blood 2012, 119, 6335-6343.

47. Drifte, G.; Dunn-Siegrist, I.; Tissières, P.; Pugin, J. Innate immune functions of immature neutrophils in patients with sepsis and severe systemic inflammatory response syndrome. Crit. Care Med. 2013, 41, 820-832.

48. Rhodes, A.; Wort, S.J.; Thomas, H.; Collinson, P.; Bennett, E.D. Plasma DNA concentration as a predictor of mortality and sepsis in critically ill patients. Crit. Care 2006, 10, R60.

49. Saukkonen, K.; Lakkisto, P.; Pettila, V.; Varpula, M.; Karlsson, S.; Ruokonen, E.; Pulkki, K. Cell-free plasma DNA as a predictor of outcome in severe sepsis and septic shock. Clin. Chem. 2008, 54, 1000-1007.

50. Guimarães-Costa, A.B.; Nascimento, M.T.; Wardini, A.B.; Pinto-da-Silva, L.H.; Saraiva, E.M. ETosis: A Microbicidal Mechanism beyond Cell Death. J. Parasitol. Res. 2012, 2012, 929743. 
Reprinted from Nutrients. Cite as: Harrison, F.E.; Bowman, G.L.; Polidori, M.C. Ascorbic Acid and the Brain: Rationale for the Use against Cognitive Decline. Nutrients 2014, 6, 1752-1781.

Review

\title{
Ascorbic Acid and the Brain: Rationale for the Use against Cognitive Decline
}

\section{Fiona E. Harrison ${ }^{1}$, Gene L. Bowman ${ }^{2}$ and Maria Cristina Polidori ${ }^{3, *}$}

1 Division of Diabetes, Endocrinology and Metabolism, Department of Medicine, Vanderbilt University Medical Center, Nashville, TN 37232, USA;

E-Mail: fiona.harrison@vanderbilt.edu

2 Brain Institute, Department of Neurology, NIA-Aging and Alzheimer's Disease Center Oregon Health and Science University, Portland, OR 97239, USA; E-Mail: bowmang@ohsu.edu

3 Geriatrics Department, University of Cologne Medical Faculty, Cologne 50937, Germany

* Author to whom correspondence should be addressed;

E-Mail: maria.polidori-nelles@uk-koeln.de; Tel.: +49-221-4788-6704;

Fax: +49-221-4788-6710.

Received: 25 October 2013; in revised form: 24 March 2014 / Accepted: 10 April 2014 /

Published: 24 April 2014

\begin{abstract}
This review is focused upon the role of ascorbic acid (AA, vitamin C) in the promotion of healthy brain aging. Particular attention is attributed to the biochemistry and neuronal metabolism interface, transport across tissues, animal models that are useful for this area of research, and the human studies that implicate AA in the continuum between normal cognitive aging and age-related cognitive decline up to Alzheimer's disease. Vascular risk factors and comorbidity relationships with cognitive decline and AA are discussed to facilitate strategies for advancing AA research in the area of brain health and neurodegeneration.
\end{abstract}

Keywords: ascorbic acid; vitamin C; brain; cognitive function; alzheimer's disease; dementia; aging; elderly; endothelial function; blood-brain barrier; SVCT (sodium-dependent vitamin $\mathrm{C}$ transporter)

\section{Introduction}

Ascorbic acid (AA; Vitamin C) is a remarkable water-soluble antioxidant concentrated predominately in citrus fruits, strawberries and vegetables (e.g., spinach and broccoli) and found in 
many supplement formulations (LPI Micronutrient Information Center). The adequate functioning of the human organism in general, and of the brain in particular, is highly dependent on AA, but humans are completely dependent on dietary sources due to the evolutionary process leaving us a gene incapable of producing the enzyme gulonolactone oxidase needed to yield AA from glucose [1]. Thus, humans require a constant stream of AA from the diet and rely on robust "carrier" transport and "barrier" integrity mechanisms to meet the brain's demand. AA is the most powerful water-soluble antioxidant of the organism, and key to preventing oxidative lipid damage in biological systems [2]. It forms the first line of antioxidant defence under many types of oxidizing conditions. It can rapidly intercept free radicals in the aqueous phase before they attack lipids [3]. As an antioxidant, AA also provides protection against oxidative stress-induced cellular damage by neutralization of lipid hydroperoxyl (LHP) radicals and by protecting proteins from alkylation by electrophilic lipid peroxidation activity [4].

Several pathological processes can involve the production of free radicals, antioxidant depletion, oxidative and nitrosative stress including vascular disease and cognitive impairments seen in aging older adults (Dementia of Alzheimer's type). Dementia, with its most prevalent form, Alzheimer's disease (AD), is characterized by an insidious progressive nature that usually begins with memory deficits followed by disturbances in other cognitive domains that eventually reach a level that impacts functions of daily life. Age-related dementias also disrupt the family unit, which must often scramble to meet day-to-day care requirements to compensate for loss of independence. The economic conditions associated with this phenomenon of disability and dependence is an enormous and pressing threat to public health. The major risk factor for dementia and AD remains to be advanced age; therefore the projected impact of $\mathrm{AD}$ can be estimated by frequency in strata of the population. For example, in the US about $14 \%$ of the older adult population age 65 and older carry a diagnosis of dementia and age 85 and older is about $47 \%$. In either case, these cohort effects will stress the value of anti-AD strategies while general practitioners, geriatricians, neurologists and health care professionals all around the globe are projected to face over 115 million people with dementia from all causes by 2050 [5].

The pathological hallmarks of $\mathrm{AD}$ include a loss in synaptic function and accumulation of extracellular amyloid- $\beta$ plaques and intraneuronal neurofibrillary tangles. Oxidative stress displays chronological primacy in the onset of $\mathrm{AD}$, and in its prephase, mild cognitive impairment (MCI) [6-9]. Thus, preventive therapy that is safe and effective for reducing oxidative damage seen early in neurodegenerative disorders and applied before the onset of dementia is a public health priority.

This overview will focus on the biological rationale for the avoidance of AA deficiency in the promotion of healthy brain aging. We include discussion on the biochemistry and transport of AA, much of which has been clarified through basic research using genetically modified mouse models, and also the clinical studies designed to better understand the influence over cognitive health in our aging populations.

\section{Ascorbic Acid Biochemistry and Transport}

AA concentration is higher in the brain than almost all other organs, and in fact may only be equaled in concentration in the adrenal glands. Scurvy, the classical clinical syndrome of AA deficiency, is rare, although not unheard of, in developed populations, but sub-clinical deficiency is 
still widespread, particularly in at-risk populations such as the elderly, hospitalized and those with poor access to good nutrition. One or more symptoms of scurvy were observed in 18 out of 145 (12\%) of elderly patients on admission to hospital, and all but one of this group were classed as being AA deficient as confirmed from plasma levels [10]. Interestingly, 7 of the 23 (30\%) controls (no signs of clinical scurvy) also had AA deficiency according to plasma levels, and all of the patients included in the study were classed as having depleted serum AA. Animal studies have shown that brain preferentially retains $\mathrm{AA}$ at the expense of other organs with cerebellar, hippocampal and cortical areas appearing to retain AA the most effectively under conditions of depletion which may have a bearing on diseases that target specific areas of the brain [11]. However, the brain is unable to hold maximal or optimal or even sufficient levels in the face of chronic insufficiency, which may have important effects on pathological aging and neurodegenerative diseases. Some populations, such as the elderly and smokers likely require even higher intakes of AA [12]. Roles for AA are widespread and include its vital role as an antioxidant as well as action as a cofactor for a number of dioxygenase enzymes involved in the synthesis of carnitine, collagen, and neurotransmitters including dopamine, norepinephrine and serotonin (reviewed [13]). AA can inhibit LDL oxidation and increase resistance of LDL to oxidation (for review, see $[4,14]$ ). AA also plays a role in the function of endothelial nitric oxide synthase (eNOS) by recycling the eNOS cofactor, tetrahydrobiopterin, which is relevant for arterial elasticity and blood pressure regulation $[4,15]$. Each of these roles plus atherogenic factors may contribute to the preventative role of AA in the development of cognitive impairment.

Evidence of altered glutamate transport (e.g., changes in EAAT2 and EAAT3 transporters) is seen in human AD postmortem samples, particularly in patients with hippocampal sclerosis [16]. AA is released from astrocytes as glutamate is taken up, and this relationship is termed a hetero-exchange although this does not fully represent how the two processes are tethered [17,18]. It is presumed that AA moderates the oxidative stress induced by glutamate [19] and so is protective against overstimulation and cell death. This relationship has been more closely investigated in relation to Huntington's disease [20-22], which also involves cell death. GLT-1 is a high affinity transporter that relies on Na-dependent transport across an electrochemical gradient for rapid removal of glutamate from the synapse. It is sensitive to oxidative stress, and disruption of the transporter leads to glutamate accumulation and hyperstimulation of receptors. Memantine is the first of a new class of drugs for $\mathrm{AD}$ that blocks NMDA receptors and its efficacy suggests that further research into glutamatergic signaling and $\mathrm{AD}$ is warranted. Therefore, as the role of glutamate transport and NMDA receptors in $\mathrm{AD}$ becomes clearer, this may be revealed as another key area where high intracellular AA levels are critical for brain health.

Further excitement has recently been generated in the field of epigenetics with a potential new answer for why AA is concentrated so strongly in CSF and brain parenchyma (reviewed in [23]). Nutrition is perhaps the quintessential example of environmental modification of the genome, and recent work has highlighted a direct role for AA that cannot be replicated with other antioxidants. 5-mc (5-methylcytosine) is oxidized to 5-hmc (5-hydroxymethylcytosine) as part of dynamic DNA demethylation. This reaction, and further oxidation of 5-hmc, are both catalyzed by the activity of TET (ten-eleven translocation) dioxygenase enzymes, for which AA is a critical co-factor (needed for the reduction of iron $\mathrm{Fe}^{3+}$ to its active form $\mathrm{Fe}^{2+}$ ) [24-26]. Thus AA is vital for neuronal repair as 
well as new cell generation and here may play a direct role in the transcription and expression of hundreds of different genes. 5-hmc and Tet proteins are abundant in brain and knockout of Tet1 in mice indicated that it may be involved in synaptic plasticity and memory extinction in addition to DNA methylation [27]. The specific relation to $\mathrm{AD}$ and other degenerative disorders is not yet clear, but this exciting field may provide new clues.

AA is a one-electron donor that readily reacts with a range of reactive oxygen species (ROS) to neutralize or decrease their reactivity. Loss of the electron leads to formation of the ascorbate free radical, which can be efficiently recycled to ascorbic acid through enzymatic means (summarized in Figure 1). Ascorbate radicals react preferentially with themselves forming ascorbic acid and dehydroascorbate. This oxidized form of AA can also be recycled back to ascorbic acid, although in some cases it undergoes irreversible ring opening and may be lost. AA also supports the regeneration of other antioxidants, such as vitamin E and glutathione (GSH), in biological tissues, thus combatting oxidative stress through various pathways. This close relationship between GSH and AA is such that AA has often been observed to "take the first hit" for GSH in response to oxidative stressors, and GSH is involved in reduction of dehydroascorbate to ascorbate [28].

Figure 1. Summary of ascorbic acid oxidation and recycling. Adapted from [13].

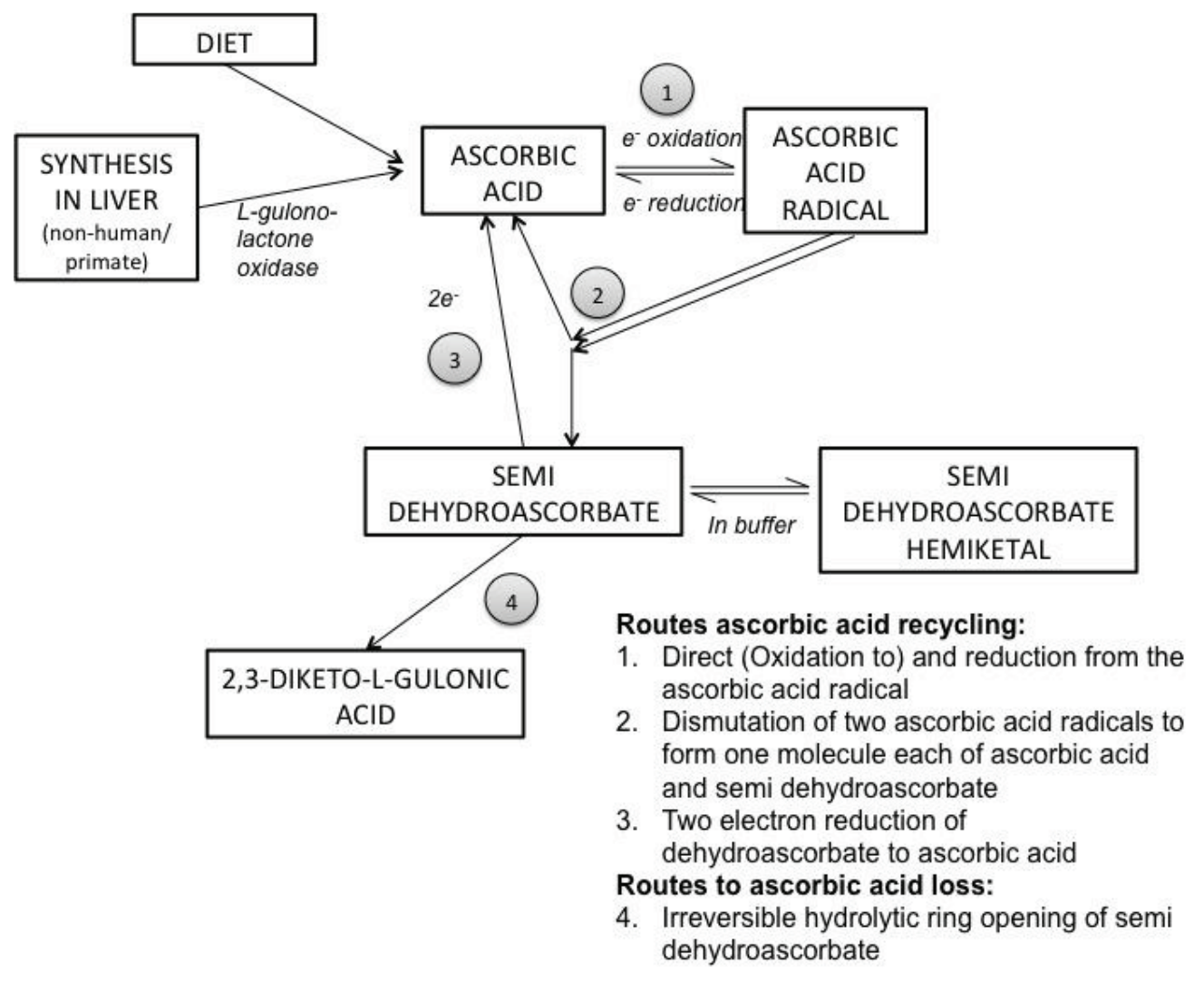

It should also be considered that AA and some of its degradation products may be involved in some potentially damaging functions [29]. For example, the Maillard reaction, most commonly studied in relation to food, is also a step in the formation of advanced glycation end products (AGEs). Evidence from a mouse model that selectively over-expressed the vitamin C transporter SVCT2 in the eye [30] implicated AA in age-related damage to crystalline proteins in the lens. There is also some debate as to the potential pro-oxidant role of AA via the Fenton reaction. AA reacts with metal 
ions in enzymes (e.g., hydroxylases, oxygenases) that require them to be in a reduced state for optimal enzyme function. In vitro, reactions with these ions can lead to the production of hydroxyl radicals and other reactive molecules. In vivo, catalytic metal ions are less available as their levels are typically kept in check in healthy individuals by metal binding proteins (e.g., ferritin, transferrin). Thus, in vivo, the evidence typically supports the antioxidant roles of AA (reviewed in detail [31,32]). One exception may be in the case of iron overload. A limited pro-oxidant effect of increased dietary AA, seen as elevated liver malondialdehyde in combination with decreased glutathione peroxidase, was found in mice fed supplemental dietary iron (although only in the lower and not the high iron diet group) [33]. It should be noted though that this study was performed under conditions of AA sufficiency, in wild-type mice that synthesize their own AA. Such an effect of pro-oxidation reactions was not found in plasma from adults or pre-term infants [3], or in AA- and iron-supplemented guinea pigs [34] and in general AA is not considered a risk factor even in patients with hereditary hemochromatosis [35]. High iron is thought to be a risk factor for AD [36] although the data are equivocal. In a study of $116 \mathrm{AD}$ patients compared to 89 healthy controls, dysregulated iron homeostasis (lower serum iron, ferritin and transferrin, combined with several genetic markers for altered iron metabolism) was associated with $\mathrm{AD}$ [37]. The full relationship between AA, iron and $\mathrm{AD}$ may warrant further investigation. A further indication of the complexity of the relationships between AA, amyloid and oxidative damage is that in isolated rat brain mitochondria, and in the presence of AA and iron, amyloid- $\beta_{1-42}$ was actually found to have an antioxidant effect and prevent formation of hydrogen peroxide, presumably through metal chelation [38]. Although aggregated amyloid- $\beta_{1-42}$ cannot be argued to be healthy in brain tissue, this finding certainly raises interest for researchers in this field who have typically considered amyloid- $\beta$ to be solely detrimental and an inducer of ROS.

Details of the complexity of the transport of AA within the central nervous system have been understood for almost half a century. Seminal work by Hammarström [39] using radio-labeled AA in guinea pigs first showed that peripherally-administered AA did indeed reach the brain, but that it appeared to travel via the choroid plexus and not traverse the blood brain barrier directly. Reports that AA levels in the CSF exceeded those found in blood, and were less affected by variations in plasma AA, suggested the existence of an active and saturable transporter for AA in the choroid plexus, which was then determined to be the case [40]. The sodium dependent vitamin $\mathrm{C}$ transporter 2 (SVCT2) itself was not described until much later but is responsible for transport both at the choroid plexus and in the neurons. There are two sodium-dependent vitamin C transporters, SVCT1 and SVCT2, which are responsible for transport of AA. Distribution of the two transporters varies across organs [41-43], but SVCT2 is the only transporter expressed in the brain. Regulation of the active transport of AA by these transporters may also vary across organs and in particular disease states (reviewed [44]). It allows for accumulation of AA in cells against the concentration gradient in SVCT2-dependent tissues. Following characterization of SVCT1 [43,45] single nucleotide polymorphisms (SNPs) were identified in both SVCT1 and SVCT2 and some studies are now being performed to ascertain how they alter AA status and whether they confer additional risk for diseases [46,47]. Several SNPs have been identified in the SLC23A1 gene coding for SVCT1, and it is argued that this may be less constrained than the $S L C 23 A 2$ gene $[45,48]$. Altered function of the SVCT1 would have important effects on AA absorption and excretion and so would impact 
nutritional requirements. One synonymous and three nonsynonymous SNPs resulted in diminished vitamin C uptake in Xenopus laevis oocytes [49]. One of these SNPs led to an $80 \%$ decrease in uptake, and was found to have a relative occurrence of 6\%-17\% in African-Americans [49]. These studies are of interest to brain research because it is presumed that such SNPs could cause chronically lower circulating AA levels, which would also potentially affect brain levels. Although fewer SVCT2 SNPs have been identified, these could have an even greater effect on brain AA levels and impact disease progression.

Expression of SVCT2 mRNA and protein varies in brain and other organs during development, presumably directly linked to very high, and changing AA levels during this critical period Nevertheless developmental regulation appears to be particularly specialized and AA regulation of SVCT2 is not common to all cell types, nor to all ages. Thus, SVCT1 and SVCT2 expression, including in brain, is regulated by several disease states associated with oxidative stress (e.g., streptozotocin-induced diabetes and middle cerebral artery occlusion) [50,51] supporting the idea of potential for change in neurodegenerative disorders such as AD. Brain levels of AA in humans are dependent on the SVCT2 (see Figure 2) but also on dietary intake and intestinal absorption via SVCT1. Given that AA can also mediate the permeability of endothelial cell layers in culture $[52,53]$, it can be seen that individual variability in either SVCT1 or SVCT2 transporter function could directly impact brain levels and vasculature in a similar manner to dietary deficiency. One particularly interesting question that may yet be answered with animal studies is the question of how SVCT2 transporter function may change with age and disease status and what impact this would have on AA levels. SVCT2 mRNA and protein both vary during development showing an inverse correlation with brain AA levels, whereas no such changes were seen in brain during AA deficiency in gulo-/- mice indicating that adult brain is unable to respond to long periods of deficiency by altering transport [54-56]. Changes in transporter function have not, to our knowledge, been studied in normally aging or AD mouse models, but if transporter function varied among groups, this could lower both CSF and intraneuronal AA levels independently of dietary intake and circulating AA levels.

There are several animals that, like humans, do not synthesize their own AA including guinea pigs, primates and some fish [57]. Nevertheless, transport is conserved across species and study of AA transporters in situ can be conducted in most animal models. Cell lines and primary culture techniques are available to study different organs and tissues, which have yielded useful information on expression, membrane location and transport kinetics of SVCT1 and SVCT2 in, for example, intestinal CaCo-2 cells, epithelial cells, endothelial cells, hepatocytes, muscle, intervertebral discs, Schwann cells and others [58-65]. An important consideration is that SVCT2 is regulated to some degree both by AA levels and by oxidative stress and can therefore develop in culture, as seen in astrocytes [66,67], which renders this approach potentially problematic. Other weaknesses of culture systems include the difficulties of maintaining a constant AA level in the media because it is so readily oxidized to dehydroascorbate. Dehydroascorbate can be lost reasonably quickly and can be transported into cells via glucose transporters and then recycled back to ascorbate within the cell. HPLC methods for measuring AA are very accurate if care is taken with sample preparation to minimize loss, and radiolabeled AA is available for assays for quantification in culture. Nevertheless to accurately model human in vivo situations, particularly in reference to specific diseases such as $\mathrm{AD}$, animal models are also needed. 
Different AA levels across brain regions have been reported in human and rodent brains $[11,68,69]$. Figure 2 shows a schematic representation of the distribution of SVCT2 (red, solid line) in the brain, and SVCT1 in intestines (green, dotted line). SVCT2 is highly, but not necessarily regularly, distributed throughout the brain. Figure components are not drawn to scale.

Figure 2. Location and distribution of SVCT1 and SVCT2 transporters and their importance in maintaining optimal brain ascorbic acid levels.

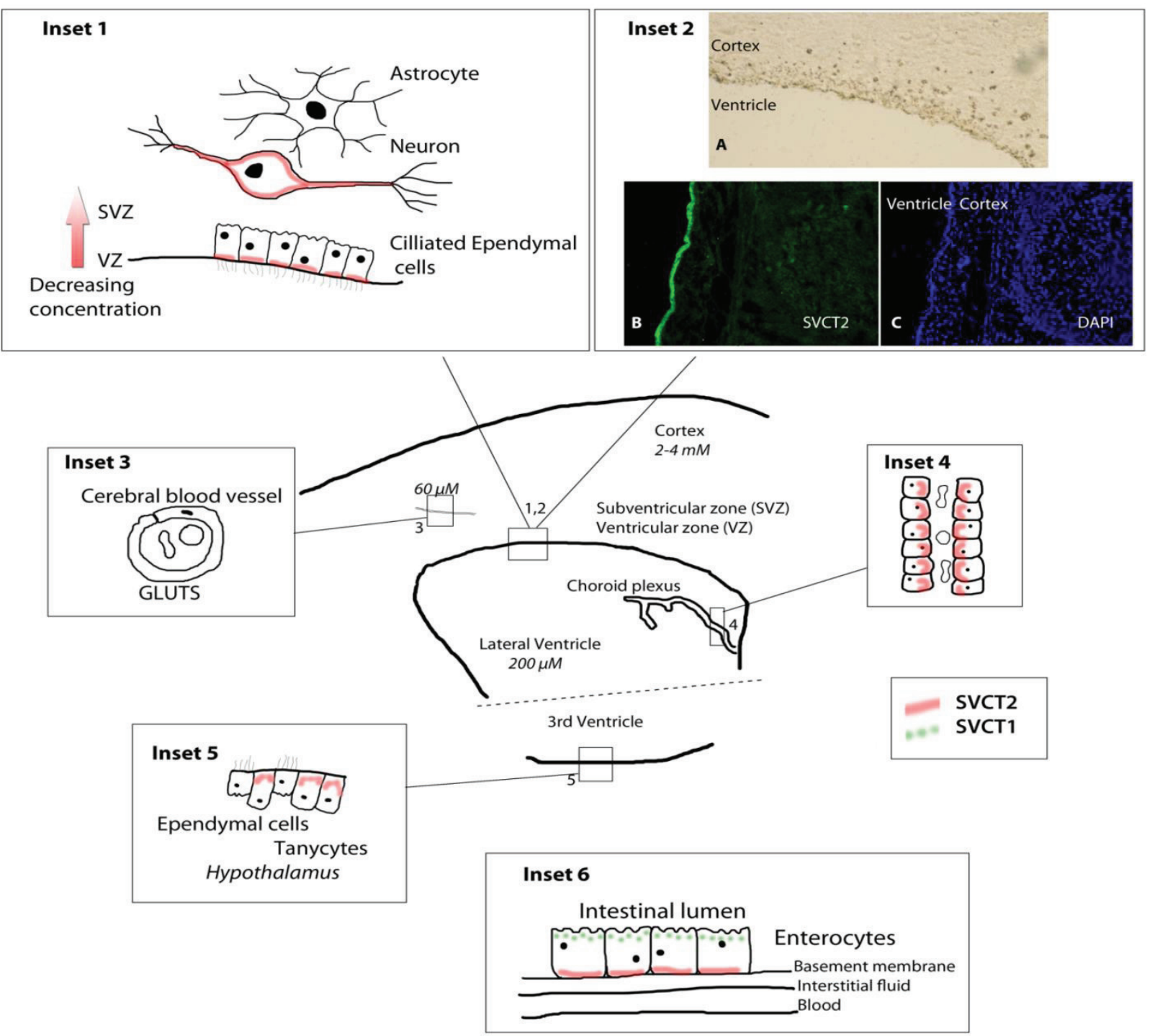

Inset 1. Cortex and lining of lateral ventricle. It was originally proposed that AA might follow a pattern of decreasing concentration with increasing distance from the ventricles following a pattern of diffusion [68]. It has since been shown that SVCT2 and AA distribution (indicated by red coloring) depend on cell type and correlate with neuronal density [70,71]. In situ hybridization in adult rat showed SVCT2 distribution throughout the brain, with highest expression in olfactory bulbs, hippocampus, cerebellum, cortex and choroid plexus [42]. High SVCT2 expression is seen in the ependymal cells lining the ventricular walls, with decreasing concentration in more external areas of the cortex by in situ hybridization and immunohistochemistry techniques [71]; Neurons. Primary culture of embryonic (E17) neurons shows SVCT2 in soma and cell processes [71]. Primary culture of mouse hippocampal cultures (E16-17) showed punctate staining of SVCT2 in axons [72]. Using immunohistochemical techniques in hippocampus, SVCT2 was observed in cell bodies but not processes by immunohistochemistry [73]; Astrocytes take up 
dehydroascorbate via glucose transporters (GLUTS) and recycle it to ascorbic acid, which can be taken up by neurons. SVCT2 was not observed in astrocytes by in situ hybridization in adult rat brain, even following excitotoxic stimulation with quinolinic acid, although SVCT2 was observed through Northern blot technique in cultured astrocytes from rat embryo [66,74]; Inset 2. Cortex and lining of lateral ventricle. Panels A-C show cortex and ventricle. In panel A, AA is stained with silver stain [75] and can be seen at its most concentrated closest to the ventricle lining. In panel B, fluorescent stain for the S19 antibody staining for SVCT2 shows high expression in cells along the ventricle lining. Panel $\mathrm{C}$ shows DAPI stain for cell bodies. (Images courtesy of J.M. May); Inset 3. Cross-section of Cerebral blood vessel. There is no SVCT2 present in blood vessels in the brain. Glucose transporters (GLUTS) are found in endothelial cells and may transport dehydroascorbate. This contributes a negligible amount to brain AA stores under normal circumstances because there is typically competitive inhibition by glucose. An exception to this state may be after transient ischemia in which case SVCT2 has been shown to develop in mice [76]; Inset 4. Choroid plexus. SVCT2 is located throughout the choroid plexus for transfer of AA from blood to CSF. High expression of SVCT2 in choroid plexus cells has been shown by in situ hybridization in several studies [42,71,74]; Inset 5. Tanycytes associated with the hypothalamus in the wall of the third ventricle. Tanycytes are highly specialized hypothalamic glial cells that are capable of forming tight junctions and participate in the formation of the barrier between CSF and brain tissue. SVCT2 has been shown by in situ hybridization and immunohistochemistry in different subtypes of tanycytes in mouse brain [75]; Inset 6. SVCT1 and SVCT2 expression in the intestine. Expression of both transporters allows for uptake of AA from food and distribution around the body (and to brain). SVCT1 is located on the apical side of the enterocytes and is responsible for taking up AA as it passes through the intestinal lumen. SVCT2 is located on the basolateral side of cells. This is thought to be more important in animals that can synthesize their own AA, but do not consume an AA rich diet, and must still supply enterocytes with AA from blood [60]. Similar localization of SVCT1 on the apical membrane with SVCT2 found on the basolateral membrane is also seen in kidney cells. How AA passes out of the cells into the interstitial fluid and blood supply is still not confirmed, and is a conundrum given the typical direction of the SVCT2 for uptake into cells.

\section{Ascorbic Acid Transport and Synthesis: Animal Models}

Early work on AA and the effects of deficiency was accomplished using guinea pigs that are among the few non-primate mammals that naturally lack a functional gulo gene [77]. These studies on the course and effects of scurvy were conducted even before the identification of ascorbic acid as vitamin C [78,79] with inclusion of "the antiscorbutic factor" [80], (such as in fresh raw cabbage). Guinea pigs are particularly sensitive to AA (and other) deficiencies $[81,82]$, and are still a highly utilized model for a number of human disease states, for example in relation to dyslipidemia (reviewed in [83]). The recent (since 2000) increased focus on mouse models results from the ability to use genetically altered mouse lines to impact both transport and synthesis in the same animals. Much more is known about the mouse genome than guinea pig and in many cases the models have been also bred to the same background strain making experiments directly comparable. Data can thus be interpreted, and experiments planned, with reference to the huge literature of biomedical and pharmaceutical research that has already taken place in mice making disease and treatment-relevant findings much more likely. Brief descriptions of the relevant mouse models, including gulo knockout mice, and SVCT1/2 knockout models and SVCT2 transgenic mice are reported hereafter.

Gulo knockout mice: Gulo knockout mice carry an inactivated form of the gene L-gulono- $\gamma$-lactone oxidase and are thus completely dependent on dietary AA [84]. These mice, when maintained on 
sub-optimal supplementation levels, are the closest to the human state of lifetime AA dietary deficiency. Knockout mice fed low levels of AA have decreased antioxidant capacity and also altered cholesterol metabolism and exhibit profound changes in vasculature. Oxidative stress has since been confirmed in these mice in adults and during development $[11,85,86]$. These mice also have an aberrant behavioral phenotype including poorer sensorimotor skills, and altered response to activity-inducing effects of cholinergic and dopaminergic compounds scopolamine and methamphetamine even when adequately supplemented as adults [86-88]. It is proposed that these changes result from low AA during some part of the developmental process. AA deficient gulo mice have been the subject of metabolomics profiling which has identified metabolic shifts as a response to oxidative stress of AA deficiency (e.g., upregulation of glutathione production, decreased carnitine production) [89]. Neutrophils from AA deficient gulo mice are more likely to undergo necrosis than normal apoptosis. They are not recognized by macrophages and thus clearance is also affected [90]. Such responses are important in any disease where inflammation is a factor, such as AD. Senescence marker protein 30 (SMP30) was first identified as a protein that decreased with age. Sequencing and creation of an SMP30 knockout mouse later identified this protein as the gulo enzyme [91,92]. These mice are likewise dependent on dietary AA and under conditions of deficiency their brains are susceptible to oxidative stress (generation of superoxide) [93,94]. A further mouse line exhibiting spontaneous bone fracture derived from in-bred balb/c mice [95] does not synthesize AA [96]. These mice die at an early age if not supplemented, and have been used for studies of AA level and gene transcription [97], however, this line is much less well-described. Disruption to catecholamine levels in brain and adrenal glands has been shown in each of these mouse lines [98,99]. Such data that follow a consistent pattern across the different mouse models are a strong support for the role of AA in any particular area and also of the validity of the models themselves.

SVCT1 knockout mice: SVCT1 knockout mice are viable and fertile [49]. They excrete significantly more AA in the urine than wild type and heterozygous littermates and have lower circulating blood levels. In addition to this loss of renal reabsorption of AA, uptake into liver was also dramatically decreased. Also of interest is the lower brain levels in these mice, presumably linked to lower circulating levels because SVCT1 is not expressed in brain and so should not have a direct effect (Figure 3).

SVCT2 knockout mice: The first report of the SVCT2 knockout mouse described homozygous mice that did not survive past birth (newborns never take a first breath) and had almost undetectable AA levels in all organs measured (brain, liver, adrenal glands, kidney, pituitary glands, pancreas and muscle) [100]. Subsequent studies found that AA levels were also decreased in placenta and lung as well as brain and other organs reported previously [101]. These low AA levels were associated with increased oxidative stress and cell death. Hemorrhaging, initially only reported in cortical areas [100] was also seen in the brain stem area which may contribute to the lack of breathing in the newborn mice, particularly because no obvious problems were identified in the lung in the earlier study. Primary cultured cells from hippocampi of embryonic SVCT2 knockout mice showed decreased neuronal activity, stunted neurite outgrowth and were much more sensitive to exogenously administered oxidative stress [72]. Investigation into catecholamine systems in these mice has yielded mixed data. One report showed large decreases (50\%) of norepinephrine in the adrenal glands with no change in dopamine levels, whereas smaller changes in the brain (decrease of 20\%-25\%) were not significant 
for either dopamine or norepinephrine in SVCT2 knockout mice [102]. Additionally, morphological differences in the chromaffin cells of the adrenal glands were also noted in this study. Other reports have shown significant alteration to dopamine and norepinephrine systems, including metabolites, in embryonic SVCT2 knockout and SVCT2 transgenic (described below) brains [103].

Figure 3. Direct comparison of cortex levels of ascorbic acid in different mouse lines run concurrently. All mice used were male, aged 9-12 months (retired breeders).

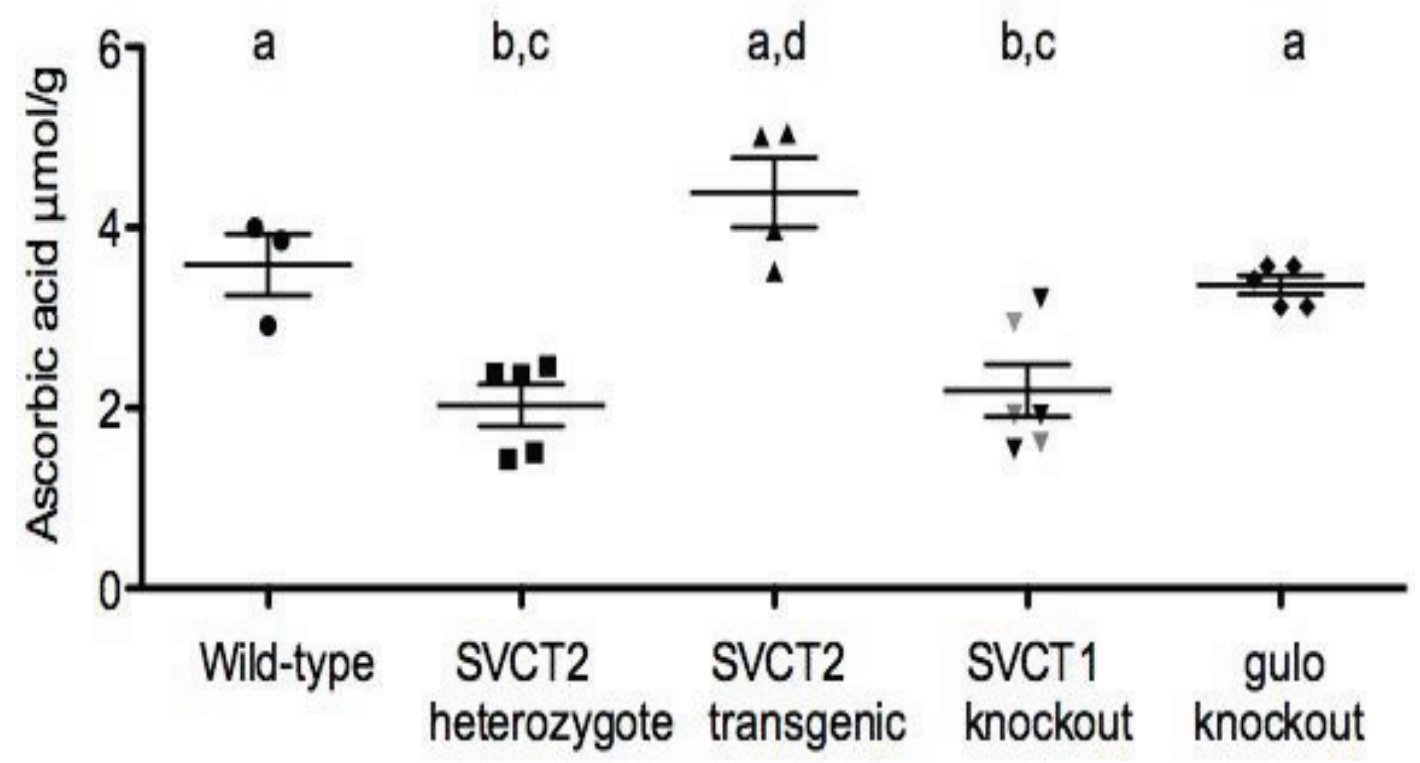

Increase in AA in SVCT2-Tg mice is dependent on level of over-expression, which varies among mice and in this small sample was only 1.3-2.0-fold compared to wild-type (not shown). Brain levels did not differ between supplemented (black triangles) and non-supplemented (grey triangles) SVCT1 knockout mice so combined average is shown. Normal brain levels can be achieved in gulo knockout mice with supplementation of at least $0.33 \mathrm{~g} / \mathrm{L}$, (shown here at supplementation with $1.0 \mathrm{~g} / \mathrm{L}$ ). Mean AA levels that share a superscript do not differ. Differing superscripts indicate significant difference ${ }^{\text {(a, b) }} p<0.05$, (c, d) $p<0.001$ by Univariate ANOVA with Tukey post-hoc comparisons (overall group effect $\mathrm{F}(4,18)=13.0$, $p<0.001)$.

SVCT2 transgenic mice: Recently, a new mouse line has been created that expresses additional copies of the SVCT2 [104]. This mouse has increased SVCT2 mRNA expression in all organs measured and a related increase in AA level in all organs except the liver, with up to two-fold increases in the brain, depending on mRNA expression. These mice were otherwise phenotypically indistinguishable from wild type but were more resistant to a severe oxidative stress in lung induced by paraquat. Although not designed to represent an attainable human situation, these mice may be able to inform on SVCT2 regulation and the role of vitamin C in high oxidative stress situations such as neurodegenerative disease.

\section{Relation of Mice Models to Human Studies}

A number of shortcomings exist to studies of AA in humans, such as accurate assessment of intake and determination of tissue levels of AA (discussed below). Thus, information from animal models can be critical to understanding changes in the brains of humans under AA deficiency. The 
gulo and SVCT2 knockout models have already been used to model long-term AA deficiency in disease states such as Alzheimer's disease, diabetes and atherosclerosis [88,105-107] by crossing them with other mouse models of disease (e.g., APP/PSEN1, ApoE). These models can even be crossed together to create in vivo models of extreme deficiency [108], although these may be less directly applicable to the human case. One key advantage to such models is the ability to investigate numerous biochemical correlates to disease and nutritional deficiency, including behavioral and cognition changes. Of course there are weaknesses to any animal model, particularly when genetic changes are artificially manufactured, and follow an all-or-none approach rather than the gradations of functional change from mutations seen in the general population. Nevertheless, the availability of models to study all aspects of transport and synthesis together provides a very strong future for pre-clinical AA research. It is important to note, however, that the changes reported in these models, particularly oxidative damage, or changes in neurotransmitter function, are not specific to Alzheimer's disease, and in fact are relevant to many types of neurodegenerative diseases, and to an extent in normal as well as pathological aging. For example, none of these mouse models exhibits robust cognitive deficits from AA deficiency alone. Future research must identify exact roles for AA in disease pathology, and how this can be applied specifically to Alzheimer's disease process as well as other disease states where applicable. This includes studying the impact of chronic deficiency resulting in sub-optimal brain levels, without clinical signs associated with scurvy. Knowledge of how AA transport and accumulation is regulated in specific brain areas, and how this may be affected by specific SNPs or disease states may permit better understanding of when and how best to intervene to correct levels, or how to identify populations that may be more at risk of deficiency.

\section{Dietary Intake of Ascorbic Acid, Cognition and Alzheimer's Disease}

Associations between risk for $\mathrm{AD}$ and $\mathrm{AA}$ intake have been investigated in several large population studies, both in the US and also in one large European sample. One early study appeared very promising when data was reported from the Chicago Healthy Aging Project (CHAP) showing that none of the $633>65$ years old, dementia-free participants that supplemented with AA, developed AD over the follow up period (mean 4 years) [109]. Study of dietary intakes in the same cohort did not support the same protective effect of AA [110]. It may be that even the dietary levels ingested were insufficient for the protective effect and that supplements are necessary to maintain optimal levels. In the earlier study only supplementation was considered, as single nutrient, multi-vitamin or no supplement, without comment as to dose. In the latter study, while the median all-source intake was $124.7 \mathrm{mg} /$ day, with $16.1 \%$ of population taking supplements of some kind, the intake ranges were very large. In the lowest quintile intake from food and supplements was estimated at below $93 \mathrm{mg} /$ day and the highest quintile was between 310 and $2530 \mathrm{mg} / \mathrm{day}$. In neither case are blood AA levels reported and thus it is difficult to accurately determine AA status in the different populations. The differences between dietary and synthetic AA intake, and their comparable bioavailability in humans, are discussed in this issue [111]. Self-reported AA intakes (alone or in combination with vitamin $\mathrm{E}$ ) were not predictive of $\mathrm{AD}$ diagnosis in a cohort of nearly 5,000 participants aged 65 and older over the course of 5 years [112]. Nor were beneficial effects of AA intake observed in a study of 980 dementia-free men and women of the Washington Heights-Inwood Columbia Aging Project [113]. 
A more complex pattern of effects was reported in the Honolulu Asia Aging Study which comprised men aged 71 to 93 years [114]. In cognitively intact individuals, AA intake was associated with a higher likelihood for enhanced cognitive function. High AA and vitamin E intake were associated with lower likelihood for vascular dementia. In contrast, there was no relationship between AD diagnoses and AA intake. Findings from the Cache County Study [115] suggest that AA and vitamin $\mathrm{E}$ supplementation may have some synergistic effects in reducing risk for $\mathrm{AD}$, but $\mathrm{AA}$ alone did not decrease risk for AD. Self reported intake questionnaires and telephone assessments of cognitive ability were used in the very large cohort of nearly 15,000 women in the Nurses health study. No consistent associations were found between AA and cognitive ability in this group [116,117].

Perhaps one of the most interesting results came from the Rotterdam Scan Study [118]. This large study of over 5000 participants reported an $18 \%$ reduced relative risk for AD with higher AA intake. Most revealing was that the most dramatic protective effects were seen in smokers. Smoking leads to rapid depletion of AA in plasma in addition to additional ROS generated following inhalation of smoke. It therefore seems likely that rescuing AA deficiency in this group may have been more useful than supplementing AA on top of dietary in healthier individuals.

If AA deficiency really is a key factor in the development of $\mathrm{AD}$, then it might be expected that populations with poorer intake would be more prone to developing dementia, or follow a faster path of deterioration once diagnosed. For the most part these studies draw strength from the large sample sizes used, numbering in the thousands. On the other hand, reliance on dietary intake questionnaires may be problematic, particularly in a study of cognitive ability where reliability may be acutely affected by even mild changes in recall ability [119]. Current intake may not reflect lifetime dietary habits and given data that suggest that amyloid plaque burden begins to form well before middle age [120], intakes during younger adulthood may be equally as important as supplements taken by older adults, perhaps contributing to a biological buffer against disease pathogenesis. Overall, the two promising positive results are outweighed by the seven studies that did not confirm a link between AA and AD. Ideally, reliable biomarkers of diet should be employed instead, or preferably in addition to study of dietary intake [121]. Measurement of AA levels, preferably over several time points, permits determination of whether participants have deficient or depleted AA levels, and more importantly allow grouping according to AA level. In this way dietary versus supplemental intake is not as important as the resulting AA levels, and high level supplements, which, due to the limitations of the SVCT1 transporter in the intestines, may not functionally provide more AA than lower supplements or good diet, are not weighted more heavily and biasing results.

\section{Blood Ascorbic Acid, Cognition and Alzheimer's Disease}

For studies that have made measurements of biological AA levels in blood and/or CSF, the sample sizes are typically much lower than the population based studies described above (see Table 1). The logistics and cost of so many measurements may be prohibitive, and even in studies that have a prospective aspect, true control groups are not possible. It would be unethical to maintain one group of participants at low AA levels, and it is also not possible to control for lifetime dietary habits in these studies. Nevertheless, these cross sectional studies can certainly provide an accurate and useful picture of typical nutrition profiles in various populations, alongside cognitive analyses. 


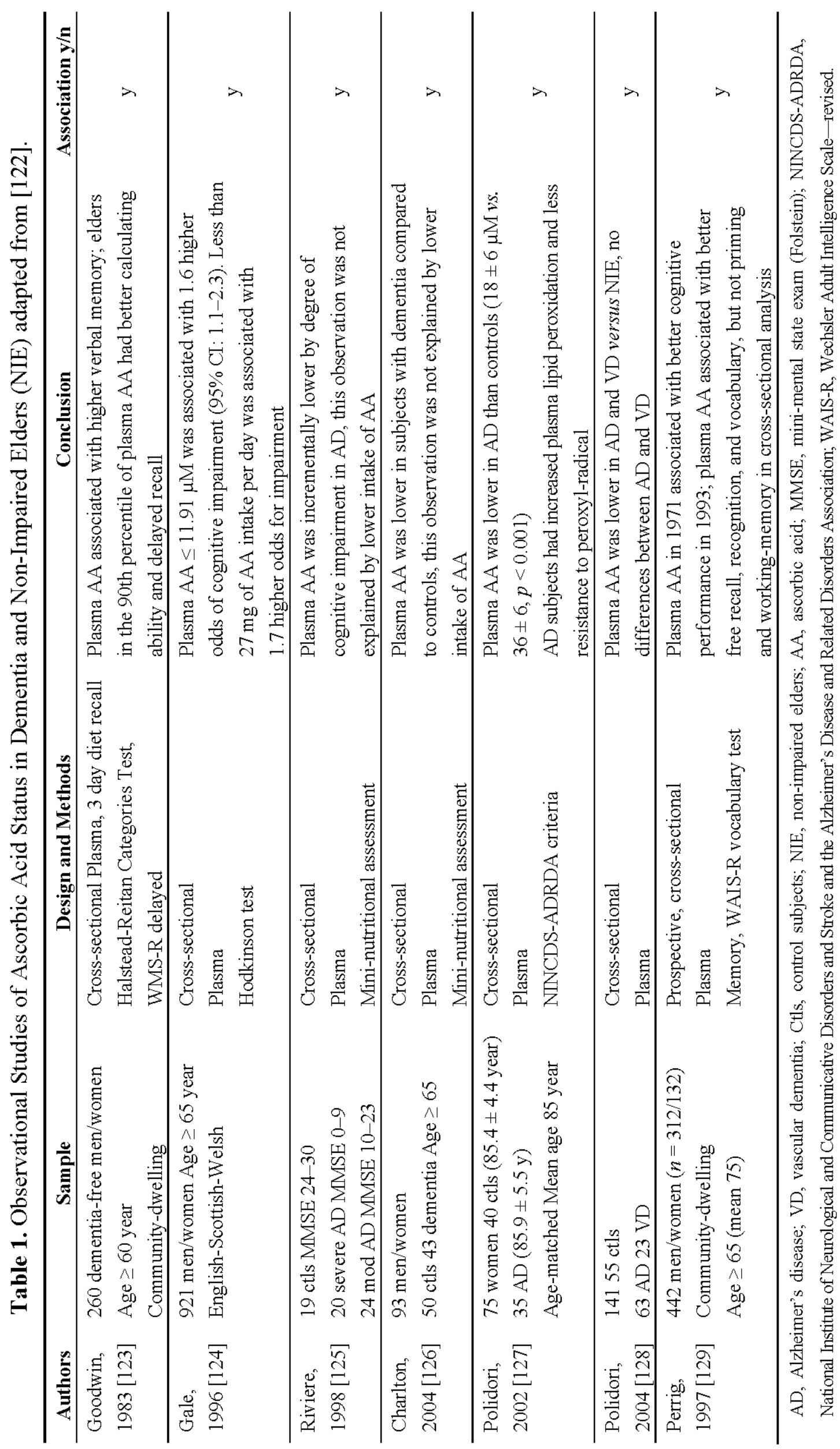


An early study by Goodwin et al. [123] used the revealing method of dividing participants into percentiles according to AA status. Using these methods verbal memory recall and calculations were indeed associated with higher plasma AA. In contrast, and in support of the idea that deficiency can impact development of AD and other dementias Gale et al. [124] report a significantly increased risk for cognitive impairment in those with depleted $(<12 \mu \mathrm{M})$ plasma AA or very low intake ( $<28 \mathrm{mg}$ /day). Higher plasma AA was associated with better free recall, recognition and vocabulary, but not working memory in a prospective sample of older men ( $>65$ years) [129]. In general, plasma levels of AA in the above studies have been consistently observed to be around $20 \mu \mathrm{M}$ in patients with $\mathrm{AD}$, i.e., about the half of those measured in controls [7,125,127]. In agreement with studies showing that plasma AA levels are depleted in $\mathrm{AD}$ independent of dietary intake, peripheral AA depletion in $\mathrm{AD}$ patients with respect to controls has been repeatedly confirmed after correction for age, gender, fruit and vegetable intake, and comorbidities [7,125,127]. Another element strongly suggestive of low AA levels as a co-causal factor for neurodegeneration and AD rather than epiphenomenon of $\mathrm{AD}$ is the observation of similarly depleted plasma AA levels in both MCI patients and $\mathrm{AD}$ patients compared to controls [7]. Comparison of all these studies where effects are on particular subtypes of memory of cognition, versus disease risk, may indicate that avoiding deficiency, and optimally supplementing with AA may benefit different facets of cognitive health. Nevertheless, the directions of effect seem to be in the same direction.

\section{Cerebrospinal Fluid Ascorbic Acid, Cognition, and Alzheimer's Disease}

Examination of the AA in the CSF reflects nutrient content with direct access to the brain parenchyma. This proximal representation should be considered the gold standard of brain nutrition in living subjects [130]. Presumably owing to the more intrusive nature of the testing far fewer studies have reported CSF AA (Table 2).

Paraskevas et al. [131] report high variation in plasma AA levels in hospitalized groups of $17 \mathrm{AD}$, 19 amyotrophic lateral sclerosis and 15 control patients, but reasonably stable CSF levels. They conclude that maintenance of the plasma:CSF ratio must be due to appropriate action of the SVCT2 at the choroid plexus. Lack of data on dietary intakes and case classifications limit the full utility of this study. Quinn et al. [132] examined the cross-sectional differences in CSF and plasma AA between 10 AD cases versus 10 healthy controls. Another cross-sectional study conducted by Glaso et al. [133] examined the mean differences between plasma and CSF AA in women with and without dementia of AD type. In both of these studies mean plasma and CSF AA were less (although not significantly in [132]) and the CSF-to-plasma AA ratio was higher in AD versus controls. 


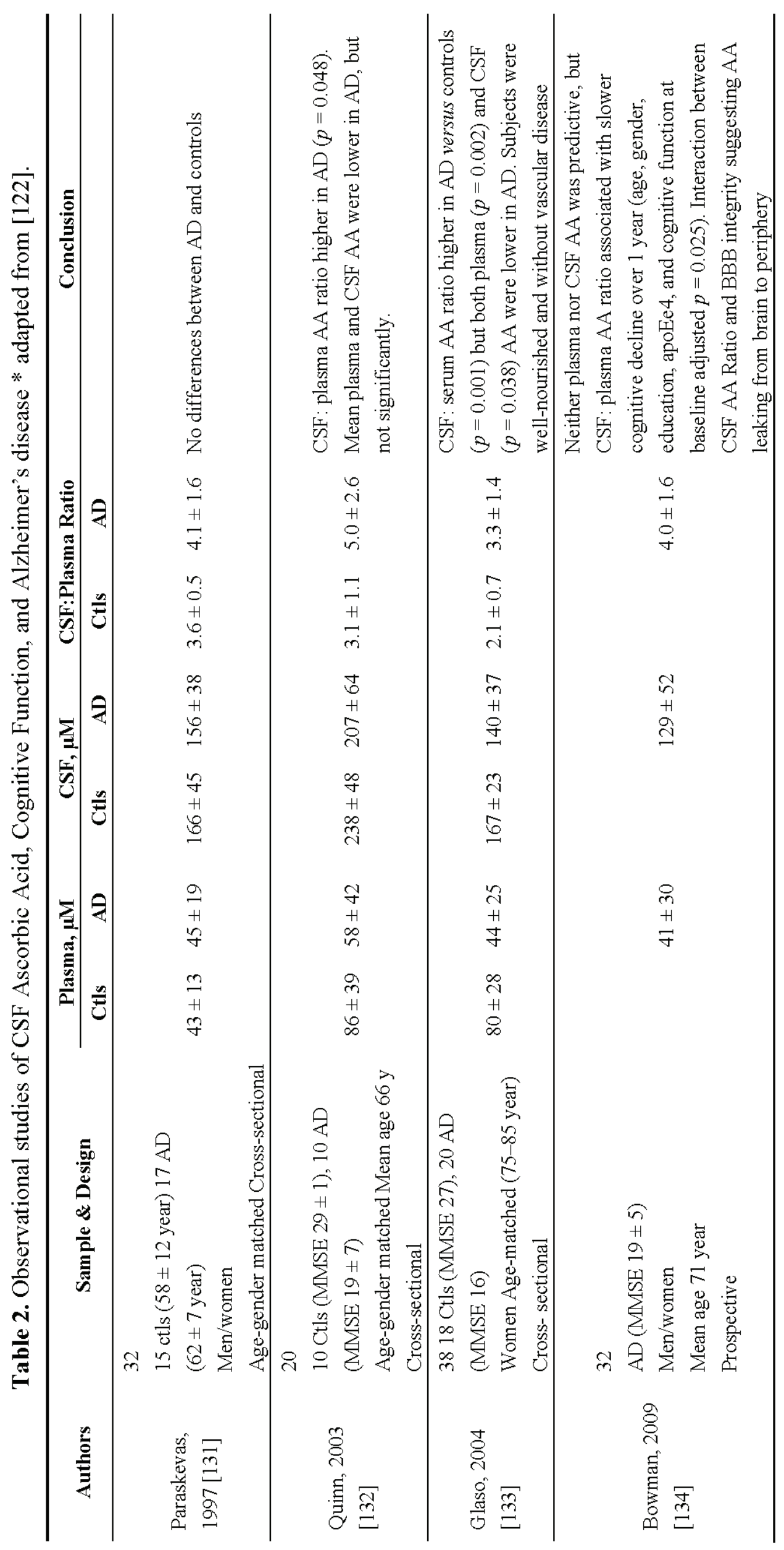




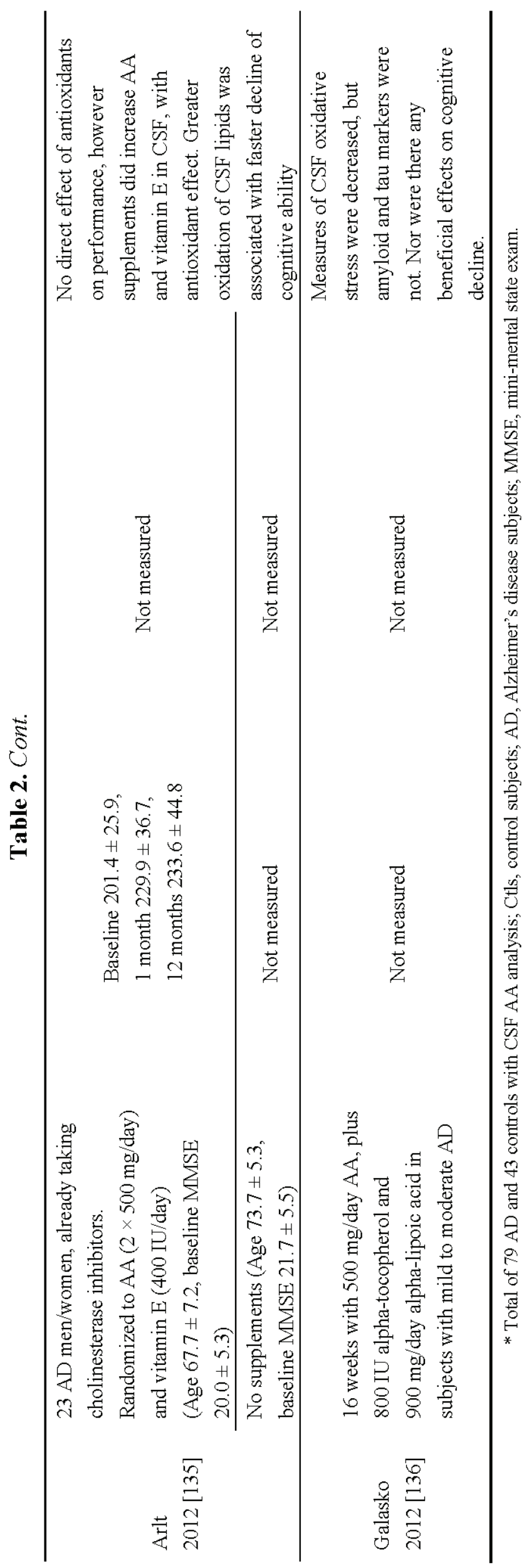


Ideally, such studies would be prospective, with baseline and later AA levels taken of both plasma and CSF AA across at least one year, in addition to cognitive assessment. One such prospective analysis also included CSF Albumin Index to reflect blood-brain barrier integrity in living probable mild to moderate AD patients over a year [134]. However, a higher CSF-to-plasma AA ratio was associated with a slower rate of decline although neither plasma nor CSF AA alone was predictive. The relationship between CSF AA ratio and rate of decline was attenuated when CSF Albumin Index (a validated measure of BBB integrity) was added to the regression model. These findings suggest that maintenance of high CSF-to-plasma AA ratio may be important to preventing cognitive decline in $\mathrm{AD}$ and that $\mathrm{BBB}$ impairment unfavorably alters this ratio. This study was unable to distinguish whether transport mechanisms for AA (i.e., SVCT2) were disturbed as well as the integrity of the BBB since the CSF albumin index reflects only barrier disturbances to our knowledge. How much of this "barrier" impairment is accompanied by "carrier" dysfunction is one area for future research interest.

A recent study on dietary supplements examined the antioxidant effect of $1000 \mathrm{mg}$ AA per day (two $\times 500 \mathrm{mg}$ ) plus $400 \mathrm{IU}$ vitamin $\mathrm{E}$ in mild to moderate $\mathrm{AD}$ patients who were also taking cholinesterase inhibitors [135]. In this relatively small, open label study, one year of supplements did not have a direct effect on cognition. Nevertheless, the authors were able to successfully demonstrate that supplements led to higher AA and vitamin $\mathrm{E}$ in the CSF and also decreased lipid peroxidation in the CSF. Greater levels of oxidation were also associated with faster cognitive decline. Unfortunately CSF was only measured in the supplement group, and dietary intake, or baseline group differences were not accounted for (although none of the subjects was taking supplements at the start of the study). In an additional study supplementation for 16 weeks with $500 \mathrm{mg} / \mathrm{d}$ AA with $800 \mathrm{IU} / \mathrm{d}$ vitamin $\mathrm{E}$ and $900 \mathrm{mg} /$ day alpha-lipoic acid in 24 mild to moderately affected AD patients screened to exclude cases of vascular disease, were compared to 18 controls [136]. The antioxidant mix was also found to decrease oxidative stress in the CSF ( $\mathrm{F}_{2}$-isoprostanes), however, no effects were seen on CSF $\mathrm{A} \beta_{1-42}$, tau or $p$-tau. No improvements were seen in cognition, in fact this group appeared to suffer faster cognitive decline. Critically, all subjects in the study were allowed to continue taking their own vitamin supplements up to $200 \mathrm{mg}$ /day AA, this included 52\% of the antioxidant group and $43 \%$ of controls. AA levels at baseline or following treatment are not reported, and thus it is not possible to assess whether groups truly differ; $200 \mathrm{mg} /$ day in a supplement plus a reasonable diet could permit AA repletion in the placebo group and mitigate the chance of seeing differences in cognition although the oxidative stress data clearly indicates benefits of the antioxidant cocktail. A recent review of several studies above concluded that CSF levels within normal range for AA (and folate and additional CSF proteins), despite lower plasma levels indicated preservation of choroid plexus function and AA transport into CSF [137]. However, they also discuss the lack of definitive data on potential for change in CSF volume or turnover.

\section{Ascorbic Acid and Vascular Cognitive Aging}

The primary focus of this review is on $\mathrm{AD}$, both with and without a vascular component. The main pathological hallmarks of AD (amyloid and tau deposits, neuronal death, neurotransmitter signaling, synaptic density) are shared across cases because the nature of AD is multifactorial. However, in the same way that many of these symptoms are also found in other cases of dementia, 
e.g., pure vascular dementia, frontotemporal dementia, dementia with lewy bodies, it can easily be argued that many of the findings described are related to neurodegeneration in general and are thus applicable to many dementias and even normal aging. Previous schools of thought have considered vascular- versus AD-type dementia cases as separate entities, however accumulating evidence suggests that vascular pathology plays a central role in AD onset and development [138-140]. In a community sample of older adults (75+ years) almost $50 \%$ of the clinically diagnosed AD cases showed a possible vascular component [141]. Similarly, the next most prevalent dementia form, vascular dementia, presents overlapping traditional hallmarks of $\mathrm{AD}$ including amyloid- $\beta$ accumulation. As a direct consequence of this complexity, a cure is difficult to find, and prevention becomes even more critical. The role of AA has recently been reviewed in relation to endothelial cell function, which may explain importance in its role in vascular health. In addition to the more well-known functions of AA such as synthesis and deposition of collagen in the basement membrane and antioxidant defense against ROS, other roles include stimulating endothelial proliferation, inhibiting apoptosis, and sparing endothelial cell-derived nitric oxide to help modulate blood flow [15].

The ability of AA to prevent age-associated cognitive decline and dementia risk may require a population with high vascular risk. AA has demonstrated some ability to reduce vascular risk factors and more recently vascular comorbidities are being acknowledged as important factors to reduce for prevention of age related dementias [138,139,142-147]. Some of the "vascular" mechanisms include: (1) reducing carotid intima-media-thickness [2,148]; (2) reducing lipid peroxidation [3,4,149-151]; and (3) reducing endothelial dysfunction [15].

We recently observed healthy elderly in the lowest plasma AA tertile at higher risk of carotid intima-media thickness $>1.2 \mathrm{~mm}$; a threshold established as pathologic by the European Society of Hypertension and the European Society of Cardiology 2007. It should be noted that this relationship was not appreciated with other antioxidants that include: uric acid, vitamins A and E, and enzymatic enzymes superoxide dismutase and glutathione oxidase activity. Another study of 8453 participants in NHANES II concluded that individuals with plasma $\mathrm{AA} \geq 45.4 \mu \mathrm{M}$ had a $21 \%-25 \%$ reduced risk for CVD-related death and a 25\%-29\% reduced risk of all-cause mortality compared to the participants with plasma AA $<23 \mu \mathrm{M}$ [152]. In the European Prospective Investigation into Cancer and Nutrition (EPIC)-Norfolk study [153] a 33\% lower risk of developing coronary heart disease was shown in subjects with the highest plasma AA compared to the lowest (mean, $27.6 \mu \mathrm{M}$ ) over six years of follow up. Long in advance to these results, the EPIC-Norfolk study had found that plasma AA concentrations in 8860 men and 10,636 women were inversely correlated to mortality from all causes and CVD. In this study, each $20 \mu \mathrm{mol} /$ liter increase in plasma AA was associated with a 20\%-30\% reduction in risk for all-cause and CVD mortality [154]. When 563 elderly men were randomly allocated to one of four treatment groups in a clinical trial that included dietary intervention, omega-3 supplementation, both or neither, carotid intima-media thickness progression over a three-year term was reduced in those undergoing dietary intervention that included daily AA intake [155]. Vitamin E [156] or combined antioxidants [157] in the dietary intervention groups were not successful.

These data suggest that AA has a role in modifying vascular risk factors and vascular disease, which could represent mechanisms by which AA might reduce dementia risk in people carrying this vascular risk profile [158]. 
Recent research has demonstrated that it is now possible to non-invasively measure AA (and GSH) levels in the human brain using MEGA-PRESS MRI (a type of spectroscopic MRI imaging) [159]. These techniques were used to compare AA, GSH and lactate in brain in 22 young (20 years) versus 22 normally aging (76.6 years) subjects [160]. The study reported decreased GSH and increased lactate with age, indicating oxidative damage, but no change in AA between the groups. The authors recruited candidates that ate less than five fruits and vegetables per day and did not take supplements, then provided food calculated to contain $30 \mathrm{mg} / 1000 \mathrm{kcal}$ in an attempt to provide all subjects with the recommended daily intake. Thus, potential group differences were already minimized, and at $\sim 60 \mathrm{mg}$ /day circulating and brain AA levels may still have been sub-optimal in all subjects, reflected by the estimated brain levels of $0.4-1.2 \mu \mathrm{mol} / \mathrm{g}$ (their Figure 3 [160]) tissue wet weight. It is reported that brain AA content was not associated with AA in blood, however these data are not recorded. So although this study does not provide a definitive statement of AA in brain in the elderly, development of this fascinating and ingenious technique to measure AA in brain may add a critical factor to future studies of the role of AA in the brain.

\section{Conclusion}

This review highlights several key points relating to the role of AA in healthy brain aging: (1) both human and animal studies demonstrate AA deficiency in association with oxidative stress markers, and oxidative stress is a consistent observation in AD; (2) there is inconsistency among the large observational studies relating dietary intake of AA to cognition. However, it remains unclear whether this inconsistency is methodological in nature (e.g., the subjective dietary surveys used to capture AA intake) since biomarkers of both AA (and oxidative stress) present more consistent results favouring an important role for AA in cognitive health; (3) there are genetic (SVCT1 and SVCT2 SNPs) and non-genetic (e.g., age) factors that modulate AA absorption and assimilation, which could indicate an increased demand for AA in subsets of the population such as the elderly and those with an $\mathrm{AD}$ diagnosis. Thus, we do not suggest that AA deficiency in isolation can explain AD neuro- and psycho-pathology, however, we do propose more research focused on investigating the specific role of AA in AD pathogenesis with meticulous attention to the study design (e.g., people with low AA and high vascular risk may be best suited for intervention). This activity should provide more conclusive data on this remarkable micronutrient highly concentrated in the brain.

\section{Acknowledgments}

FEH is supported by grant AG038739 from the NIA. GLB is supported by grant NIH/NCCAM AT004777 and NIH/NIA AG043398. The authors would like to thank Jack Petros for technical assistance in tissue collection and measurement of ascorbic acid levels.

\section{Conflicts of Interest}

The authors declare no conflict of interest. 


\section{References}

1. Nishikimi, M.; Fukuyama, R.; Minoshima, S.; Yagi, K. Cloning and chromosomal mapping of the human nonfunctional gene for L-gulono-gamma-lactone oxidase, the enzyme for L-ascorbic acid biosynthesis missing in man. J. Biol. Chem. 1994, 269, 13685-13688.

2. Frei, B.; England, L.; Ames, B.N. Ascorbate is an outstanding antioxidant in human blood plasma. Proc. Natl. Acad. Sci. USA 1989, 86, 6377-6381.

3. Berger, T.M.; Polidori, M.C.; Dabbagh, A.; Evans, P.J.; Halliwell, B.; Morrow, J.D.; Roberts, L.J., II; Frei, B. Antioxidant activity of vitamin C in iron-overloaded human plasma. J. Biol. Chem. 1997, 272, 15656-15660.

4. Traber, M.G.; Stevens, J.F. Vitamins C and E: Beneficial effects from a mechanistic perspective. Free Radic. Biol. Med. 1997, 51, 1000-1013.

5. Prince, M.; Bryce, R.; Albanese, E.; Wimo, A.; Ribeiro, W.; Ferri, C.P. The global prevalence of dementia: A systematic review and metaanalysis. Alzheimer's Dement. 2013, 9, 63-75 e62.

6. Pratico, D.; Clark, C.M.; Liun, F.; Rokach, J.; Lee, V.Y.; Trojanowski, J.Q. Increase of brain oxidative stress in mild cognitive impairment: A possible predictor of Alzheimer disease. Arch. Neurol. 2002, 59, 972-976.

7. Rinaldi, P.; Polidori, M.C.; Metastasio, A.; Mariani, E.; Mattioli, P.; Cherubini, A.; Catani, M.; Cecchetti, R.; Senin, U.; Mecocci, P. Plasma antioxidants are similarly depleted in mild cognitive impairment and in Alzheimer's disease. Neurobiol. Aging 2003, 24, 915-919.

8. Mecocci, P.; Polidori, M.C. Antioxidant clinical trials in mild cognitive impairment and Alzheimer's disease. Biochim. Biophys. Acta 2012, 1822, 631-638.

9. Mangialasche, F.; Kivipelto, M.; Mecocci, P.; Rizzuto, D.; Palmer, K.; Winblad, B.; Fratiglioni, L. High plasma levels of vitamin E forms and reduced Alzheimer's disease risk in advanced age. J. Alzheimer's Dis. (JAD) 2010, 20, 1029-1037.

10. Raynaud-Simon, A.; Cohen-Bittan, J.; Gouronnec, A.; Pautas, E.; Senet, P.; Verny, M.; Boddaert, J. Scurvy in hospitalized elderly patients. J. Nutr. Health Aging 2010, 14, 407-410.

11. Harrison, F.E.; Green, R.J.; Dawes, S.M.; May, J.M. Vitamin C distribution and retention in the mouse brain. Brain Res. 2010, 1348, 181-186.

12. Brubacher, D.; Moser, U.; Jordan, P. Vitamin C concentrations in plasma as a function of intake: A meta-analysis. Int. J. Vitam Nutr. Res. 2000, 70, 226-237.

13. Harrison, F.E.; May, J.M. Vitamin C function in the brain: vital role of the ascorbate transporter SVCT2. Free Radic Biol. Med. 2009, 46, 719-730.

14. Kaliora, A.C.; Dedoussis, G.V.; Schmidt, H. Dietary antioxidants in preventing atherogenesis. Atherosclerosis 2006, 187, 1-17.

15. May, J.M.; Harrison, F.E. Role of Vitamin C in the Function of the Vascular Endothelium. Antioxid. Redox Signal. 2013, 19, 2068-2083.

16. Proper, E.A.; Hoogland, G.; Kappen, S.M.; Jansen, G.H.; Rensen, M.G.; Schrama, L.H.; van Veelen, C.W.; van Rijen, P.C.; van Nieuwenhuizen, O.; Gispen, W.H.; et al. Distribution of glutamate transporters in the hippocampus of patients with pharmaco-resistant temporal lobe epilepsy. Brain 2002, 125, 32-43. 
17. Wilson, J.X.; Peters, C.E.; Sitar, S.M.; Daoust, P.; Gelb, A.W. Glutamate stimulates ascorbate transport by astrocytes. Brain Res. 2000, 858, 61-66.

18. Estrada-Sanchez, A.M.; Rebec, G.V. Corticostriatal dysfunction and glutamate transporter 1 (GLT1) in Huntington's disease: Interactions between neurons and astrocytes. Basal Ganglia 2012, 2, 57-66.

19. Ballaz, S.; Morales, I.; Rodriguez, M.; Obeso, J.A. Ascorbate prevents cell death from prolonged exposure to glutamate in an in vitro model of human dopaminergic neurons. J. Neurosci. Res. 2013, 91, 1609-1617.

20. Dorner, J.L.; Miller, B.R.; Klein, E.L.; Murphy-Nakhnikian, A.; Andrews, R.L.; Barton, S.J.; Rebec, G.V. Corticostriatal dysfunction underlies diminished striatal ascorbate release in the R6/2 mouse model of Huntington's disease. Brain Res. 2009, 1290, 111-120.

21. Rebec, G.V.; Pierce, R.C. A vitamin as neuromodulator: Ascorbate release into the extracellular fluid of the brain regulates dopaminergic and glutamatergic transmission. Prog. Neurobiol. 1994, 43, 537-565.

22. Rebec, G.V.; Barton, S.J.; Marseilles, A.M.; Collins, K. Ascorbate treatment attenuates the Huntington behavioral phenotype in mice. Neuroreport 2003, 14, 1263-1265.

23. Spector, R.; Johanson, C.E. The nexus of vitamin homeostasis and DNA synthesis and modification in mammalian brain. Mol. Brain 2014, 7, 3.

24. Blaschke, K.; Ebata, K.T.; Karimi, M.M.; Zepeda-Martinez, J.A.; Goyal, P.; Mahapatra, S.; Tam, A.; Laird, D.J.; Hirst, M.; Rao, A.; et al. Vitamin C induces Tet-dependent DNA demethylation and a blastocyst-like state in ES cells. Nature 2013, 500, 222-226.

25. Yin, R.; Mao, S.Q.; Zhao, B.; Chong, Z.; Yang, Y.; Zhao, C.; Zhang, D.; Huang, H.; Gao, J.; Li, Z.; et al. Ascorbic acid enhances Tet-mediated 5-methylcytosine oxidation and promotes DNA demethylation in mammals. J. Am. Chem. Soc. 2013, 135, 10396-10403.

26. Minor, E.A.; Court, B.L.; Young, J.I.; Wang, G. Ascorbate induces ten-eleven translocation (Tet) methylcytosine dioxygenase-mediated generation of 5-hydroxymethylcytosine. J. Biol. Chem. 2013, 288, 13669-13674.

27. Rudenko, A.; Dawlaty, M.M.; Seo, J.; Cheng, A.W.; Meng, J.; Le, T.; Faull, K.F.; Jaenisch, R.; Tsai, L.H. Tet1 is critical for neuronal activity-regulated gene expression and memory extinction. Neuron 2013, 79, 1109-1122.

28. Meister, A. Glutathione, ascorbate, and cellular protection. Cancer Res. 1994, 54, 1969s-1975s.

29. Smuda, D.G.M. Maillard degradation pathways of vitamin C. Angew. Chem. Int. Ed. 2013, 52, 4887-4891.

30. Fan, X.; Reneker, L.W.; Obrenovich, M.E.; Strauch, C.; Cheng, R.; Jarvis, S.M.; Ortwerth, B.J.; Monnier, V.M. Vitamin $\mathrm{C}$ mediates chemical aging of lens crystallins by the Maillard reaction in a humanized mouse model. Proc. Natl. Acad. Sci. USA 2006, 103, 16912-16917.

31. Carr, A.; Frei, B. Does vitamin $\mathrm{C}$ act as a pro-oxidant under physiological conditions? FASEB J. 1999, 13, 1007-1024.

32. Halliwell, B. Vitamin C: Antioxidant or pro-oxidant in vivo? Free Radic. Res. 1996, 25, 439-454.

33. Premkumar, K.; Bowlus, C.L. Ascorbic acid does not increase the oxidative stress induced by dietary iron in $\mathrm{C} 3 \mathrm{H}$ mice. J. Nutr. 2004, 134, 435-438. 
34. Collis, C.S.; Yang, M.; Diplock, A.T.; Hallinan T.; Rice-Evans, C.A. Effects of co-supplementation of iron with ascorbic acid on antioxidant-Pro-oxidant balance in the guinea pig. Free Radic. Res. 1997, 27, 113-121.

35. Gerster, H. High-dose vitamin C: A risk for persons with high iron stores? Int. J. Vitam Nutr. Res. 1999, 69, 67-82.

36. Loef, M.; Walach, H. Copper and iron in Alzheimer's disease: A systematic review and its dietary implications. Br. J. Nutr. 2012, 107, 7-19.

37. Crespo, A.C.; Silva, B.; Marques, L.; Marcelino, E.; Maruta, C.; Costa, S.;Timoteo, A.; Vilares, A.; Couto, F.S.; Faustino, P.; et al. Genetic and biochemical markers in patients with Alzheimer's disease support a concerted systemic iron homeostasis dysregulation. Neurobiol. Aging 2014, 35, 777-785.

38. Sinha, M.; Bhowmick, P.; Banerjee, A.; Chakrabarti, S. Antioxidant role of amyloid beta protein in cell-free and biological systems: Implication for the pathogenesis of Alzheimer disease. Free Radic. Biol. Med. 2013, 56, 184-192.

39. Hammarstrom, L. Autoradiographic studies on the distribution of C14-labelled ascorbic acid and dehydroascorbic acid. Acta Physiol. Scand. 1966, 70, 3-83.

40. Spector, R.; Lorenzo, A.V. Ascorbic acid homeostasis in the central nervous system. Am. J. Physiol. 1973, 225, 757-763.

41. Savini, I.; Rossi, A.; Pierro, C.; Avigliano, L.; Catani, M.V. SVCT1 and SVCT2: Key proteins for vitamin C uptake. Amino Acids 2008, 34, 347-355.

42. Tsukaguchi, H.; Tokui, T.; Mackenzie, B.; Berger, U.V.; Chen, X.Z.; Wang, Y.; Brubaker, R.F.; Hediger, M.A. A family of mammalian $\mathrm{Na}+$-dependent L-ascorbic acid transporters. Nature 1999, 399, 70-75.

43. Wang, Y.; Mackenzie, B.; Tsukaguchi, H.; Weremowicz, S.; Morton, C.C.; Hediger, M.A. Human vitamin C (L-ascorbic acid) transporter SVCT1. Biochem. Biophys. Res. Commun. 2000, 267, 488-494.

44. Lindblad, M.; Tveden-Nyborg, P.; Lykkesfeldt, J. Regulation of Vitamin C Homeostasis during Deficiency. Nutrients 2013, 5, 2860-2879.

45. Erichsen, H.C.; Eck, P.; Levine, M.; Chanock, S. Characterization of the genomic structure of the human vitamin C transporter SVCT1 (SLC23A2). J. Nutr. 2001, 131, 2623-2627.

46. Erichsen, H.C.; Peters, U.; Eck, P.; Welch, R.; Schoen, R.E.; Yeager, M.; Levine, M.; Hayes, R.B.; Chanock, S. Genetic variation in sodium-dependent vitamin C transporters SLC23A1 and SLC23A2 and risk of advanced colorectal adenoma. Nutr. Cancer 2008, 60, 652-659.

47. Michels, A.J.; Hagen, T.M.; Frei, B. Human genetic variation influences vitamin C homeostasis by altering vitamin $\mathrm{C}$ transport and antioxidant enzyme function. Annu. Rev. Nutr. 2013, 33, 45-70.

48. Eck, P.; Erichsen, H.C.; Taylor, J.G.; Corpe, C.; Chanock, S.J.; Levine, M. Genomic and functional analysis of the sodium-dependent vitamin C transporter SLC23A1-SVCT1. Genes Nutr. 2007, 2, 143-145. 
49. Corpe, C.P.; Tu, H.; Eck, P.; Wang, J.; Faulhaber-Walter, R.; Schnermann, J.; Margolis, S.; Padayatty, S.; Sun, H.; Wang, Y.; et al. Vitamin C transporter Slc23a1 links renal reabsorption, vitamin $\mathrm{C}$ tissue accumulation, and perinatal survival in mice. J. Clin. Investig. 2010, 120, 1069-1083.

50. Hierro, C.; Monte, M.J.; Lozano, E.; Gonzalez-Sanchez, E.; Marin, J.J.; Macias, R.I. Liver metabolic/oxidative stress induces hepatic and extrahepatic changes in the expression of the vitamin C transporters SVCT1 and SVCT2. Eur. J. Nutr. 2014, 53, 401-412.

51. Gess, B.; Sevimli, S.; Strecker, J.K.; Young, P.; Schabitz, W.R. Sodium-dependent vitamin C transporter 2 (SVCT2) expression and activity in brain capillary endothelial cells after transient ischemia in mice. PLoS One 2011, 6, e17139.

52. May, J.M.; Qu, Z.C. Ascorbic acid prevents increased endothelial permeability caused by oxidized low density lipoprotein. Free Radic. Res. 2010, 44, 1359-1368.

53. May, J.M.; Qu, Z.C. Ascorbic acid prevents oxidant-induced increases in endothelial permeability. Biofactors 2011, 37, 46-50.

54. Amano, A.; Aigaki, T.; Maruyama, N.; Ishigami, A. Ascorbic acid depletion enhances expression of the sodium-dependent vitamin C transporters, SVCT1 and SVCT2, and uptake of ascorbic acid in livers of SMP30/GNL knockout mice. Arch. Biochem. Biophys. 2010, 496, 38-44.

55. Meredith, M.E.; Harrison, F.E.; May, J.M. Differential regulation of the ascorbic acid transporter SVCT2 during development and in response to ascorbic acid depletion. Biochem. Biophys. Res. Commun. 2011, 414, 737-742.

56. Nualart, F.; Castro, T.; Low, M.; Henriquez, J.P.; Oyarce, K.; Cisternas, P.; Garcia, A.; Yanez, A.J.; Bertinat, R.; Montecinos, V.P.; et al. Dynamic expression of the sodium-vitamin C co-transporters, SVCT1 and SVCT2, during perinatal kidney development. Histochem. Cell Biol. 2013, 139, 233-247.

57. Jimenez-Fernandez, E.; Ponce, M.; Zuasti, E.; Fernandez-Diaz, C.; Manchado, M.; Infante, C. Molecular characterization and transcriptional regulation of the sodium-dependent vitamin $\mathrm{C}$ transporter genes (slc23a1 and slc23a2) in a teleost fish, the Senegalese sole (Solea senegalensis). Comp. Biochem. Physiol. Part B Biochem. Mol. Biol. 2012, 161, 208-218.

58. Gess, B.; Lohmann, C.; Halfter, H.; Young, P. Sodium-dependent vitamin C transporter 2 (SVCT2) is necessary for the uptake of L-ascorbic acid into Schwann cells. Glia 2010, 58, 287-299.

59. Maulen, N.P.; Henriquez, E.A.; Kempe, S.; Carcamo, J.G.; Schmid-Kotsas, A.; Bachem, M.; Grunert, A.; Bustamante, M.E.; Nualart, F.; Vera, J.C. Up-regulation and polarized expression of the sodium-ascorbic acid transporter SVCT1 in post-confluent differentiated CaCo-2 cells. J. Biol. Chem. 2003, 278, 9035-9041.

60. Boyer, J.C.; Campbell, C.E.; Sigurdson, W.J.; Kuo, S.M. Polarized localization of vitamin C transporters, SVCT1 and SVCT2, in epithelial cells. Biochem. Biophys. Res. Commun. 2005, 334, 150-156.

61. MacDonald, L.; Thumser, A.E.; Sharp, P. Decreased expression of the vitamin C transporter SVCT1 by ascorbic acid in a human intestinal epithelial cell line. Br. J. Nutr. 2002, 87, 97-100. 
62. Varma, S.; Sobey, K.; Campbell, C.E.; Kuo, S.M. Hierarchal contribution of N- and C-terminal sequences to the differential localization of homologous sodium-dependent vitamin $\mathrm{C}$ transporters, SVCT1 and SVCT2, in epithelial cells. Biochemistry 2009, 48, 2969-2980.

63. May, J.M.; Li, L.; Qu, Z.C. Oxidized LDL up-regulates the ascorbic acid transporter SVCT2 in endothelial cells. Mol. Cell. Biochem. 2010, 343, 217-222.

64. Qiao, H.; May, J.M. Development of ascorbate transporters in brain cortical capillary endothelial cells in culture. Brain Res. 2008, 1208, 79-86.

65. Chothe, P.P.; Chutkan, N.; Sangani, R.; Wenger, K.H.; Prasad, P.D.; Thangaraju, M.; Hamrick, M.W.; Isales, C.M.; Ganapathy, V.; Fulzele, S. Sodium-coupled vitamin C transporter (SVCT2): expression, function, and regulation in intervertebral disc cells. Spine J. 2013, 13, 549-557.

66. Berger, U.V.; Hediger, M.A. The vitamin C transporter SVCT2 is expressed by astrocytes in culture but not in situ. Neuroreport 2000, 11, 1395-1399.

67. Korcok, J.; Yan, R.; Siushansian, R.; Dixon, S.J.; Wilson, J.X. Sodium-ascorbate cotransport controls intracellular ascorbate concentration in primary astrocyte cultures expressing the SVCT2 transporter. Brain Res. 2000, 881, 144-151.

68. Oke, A.F.; May, L.; Adams, R.N. Ascorbic acid distribution patterns in human brain. A comparison with nonhuman mammalian species. Ann. N. Y. Acad. Sci. 1987, 498, 1-12.

69. Mefford, I.N.; Oke, A.F.; Adams, R.N. Regional distribution of ascorbate in human brain. Brain Res. 1981, 212, 223-226.

70. Rice, M.E.; Russo-Menna, I. Differential compartmentalization of brain ascorbate and glutathione between neurons and glia. Neuroscience 1998, 82, 1213-1223.

71. Caprile, T.; Salazar, K.; Astuya, A.; Cisternas, P.; Silva-Alvarez, C.; Montecinos, H.; Millan, C.; de Los Angeles Garcia, M.; Nualart, F. The Na+-dependent L-ascorbic acid transporter SVCT2 expressed in brainstem cells, neurons, and neuroblastoma cells is inhibited by flavonoids. J. Neurochem. 2009, 108, 563-577.

72. Qiu, S.; Li, L.; Weeber, E.J.; May, J.M. Ascorbate transport by primary cultured neurons and its role in neuronal function and protection against excitotoxicity. J. Neurosci. Res. 2007, 85, 1046-1056.

73. Mun, G.H.; Kim, M.J.; Lee, J.H.; Kim, H.J.; Chung, Y.H.; Chung, Y.B.; Kang, J.S.; Hwang, Y.I.; Oh, S.H.; Kim, J.G.; et al. Immunohistochemical study of the distribution of sodium-dependent vitamin C transporters in adult rat brain. J. Neurosci. Res. 2006, 83, 919-928.

74. Garcia Mde, L.; Salazar, K.; Millan, C.; Rodriguez, F.; Montecinos, H.; Caprile, T.; Silva, C.; Cortes, C.; Reinicke, K.; Vera, J.C.; et al. Sodium vitamin C cotransporter SVCT2 is expressed in hypothalamic glial cells. Glia 2005, 50, 32-47.

75. Chinoy, N.J.; Sanjeevan, A.G. On the specificity of alcoholic acidic silver nitrate reagent for the histochemical localization of ascorbic acid. A reappraisal. Histochemistry 1978, 56, 275-282.

76. Berger, U.V.; Lu, X.C.; Liu, W.; Tang, Z.; Slusher, B.S.; Hediger, M.A. Effect of middle cerebral artery occlusion on mRNA expression for the sodium-coupled vitamin $\mathrm{C}$ transporter SVCT2 in rat brain. J. Neurochem. 2003, 86, 896-906. 
77. McHenry, E.W.; Reedman, E.J.; Sheppard, M. The physiological properties of ascorbic acid: An effect upon the weights of guinea-pigs. Biochem. J. 1938, 32, 1302-1304.

78. Svirbely, J.L.; Szent-Gyorgyi, A. The chemical nature of vitamin C. Biochem. J. 1932, 26, 865-870.

79. Szent-Gyorgyi, A.H.W.N. "Hexuronic Acid" (Ascorbic Acid) as the Antiscorbutic Factor. Nature 1933, 131, 24.

80. Anderson WES, A.H. The effect of acute scurvy on the subsequent nutrition and growth of guinea pigs. J. Biol. Chem. 1924, 61, 181-191.

81. Burk, R.F.; Christensen, J.M.; Maguire, M.J.; Austin, L.M.; Whetsell, W.O., Jr.; May, J.M.; Hill, K.E.; Ebner, F.F. A combined deficiency of vitamins $\mathrm{E}$ and $\mathrm{C}$ causes severe central nervous system damage in guinea pigs. J. Nutr. 2006, 136, 1576-1581.

82. Hill, K.E.; Motley, A.K.; May, J.M.; Burk, R.F. Combined selenium and vitamin C deficiency causes cell death in guinea pig skeletal muscle. Nutr. Res. 2009, 29, 213-219.

83. Frikke-Schmidt, H.; Lykkesfeldt, J. Role of marginal vitamin C deficiency in atherogenesis: In vivo models and clinical studies. Basic Clin. Pharmacol. Toxicol. 2009, 104, 419-433.

84. Maeda, N.; Hagihara, H.; Nakata, Y.; Hiller, S.; Wilder, J.; Reddick, R. Aortic wall damage in mice unable to synthesize ascorbic acid. Proc. Natl. Acad. Sci. USA 2000, 97, 841-846.

85. Harrison, F.E.; Meredith, M.E.; Dawes, S.M.; Saskowski, J.L.; May, J.M. Low ascorbic acid and increased oxidative stress in gulo(-/-) mice during development. Brain Res. 2010, 1349, $143-152$.

86. Harrison, F.E.; Yu, S.S.; Van Den Bossche, K.L.; Li, L.; May, J.M.; McDonald, M.P. Elevated oxidative stress and sensorimotor deficits but normal cognition in mice that cannot synthesize ascorbic acid. J. Neurochem. 2008, 106, 1198-1208.

87. Chen, Y.; Curran, C.P.; Nebert, D.W.; Patel, K.V.; Williams, M.T.; Vorhees, C.V. Effect of vitamin $C$ deficiency during postnatal development on adult behavior: functional phenotype of Gulo(-/-) knockout mice. Genes Brain Behav. 2012, 11, 269-277.

88. Harrison, F.E.; May, J.M.; McDonald, M.P. Vitamin C deficiency increases basal exploratory activity but decreases scopolamine-induced activity in APP/PSEN1 transgenic mice. Pharmacol. Biochem. Behav. 2010, 94, 543-552.

89. Duggan, G.E.; Joan Miller, B.; Jirik, F.R.; Vogel, H.J. Metabolic profiling of vitamin C deficiency in Gulo-/- mice using proton NMR spectroscopy. J. Biomol. NMR 2011, 49, $165-173$.

90. Vissers, M.C.; Wilkie, R.P. Ascorbate deficiency results in impaired neutrophil apoptosis and clearance and is associated with up-regulation of hypoxia-inducible factor 1alpha. J. Leukoc. Biol. 2007, 81, 1236-1244.

91. Ishigami, A.; Fujita, T.; Handa, S.; Shirasawa, T.; Koseki, H.; Kitamura, T.; Enomoto, N.; Sato, N.; Shimosawa, T.; Maruyama, N. Senescence marker protein-30 knockout mouse liver is highly susceptible to tumor necrosis factor-alpha- and Fas-mediated apoptosis. Am. J. Pathol. 2002, 161, 1273-1281. 
92. Kondo, Y.; Inai, Y.; Sato, Y.; Handa, S.; Kubo, S.; Shimokado, K.; Goto, S.; Nishikimi, M.; Maruyama, N.; Ishigami, A. Senescence marker protein 30 functions as gluconolactonase in L-ascorbic acid biosynthesis, and its knockout mice are prone to scurvy. Proc. Natl. Acad. Sci. USA 2006, 103, 5723-5728.

93. Kondo, Y.; Sasaki, T.; Sato, Y.; Amano, A.; Aizawa, S.; Iwama, M.; Handa, S.; Shimada, N.; Fukuda, M.; Akita, M.; et al. Vitamin C depletion increases superoxide generation in brains of SMP30/GNL knockout mice. Biochem. Biophys. Res. Commun. 2008, 377, 291-296.

94. Son, T.G.; Zou, Y.; Jung, K.J.; Yu, B.P.; Ishigami, A.; Maruyama, N.; Lee, J. SMP30 deficiency causes increased oxidative stress in brain. Mech. Ageing Dev. 2006, 127, 451-457.

95. Beamer, W.G.; Rosen, C.J.; Bronson, R.T.; Gu, W.; Donahue, L.R.; Baylink, D.J.; Richardson, C.C.; Crawford, G.C.; Barker, J.E. Spontaneous fracture (sfx): A mouse genetic model of defective peripubertal bone formation. Bone 2000, 27, 619-626.

96. Mohan, S.; Kapoor, A.; Singgih, A.; Zhang, Z.; Taylor, T.; Yu, H.; Chadwick, R.B.; Chung, Y.S.; Donahue, L.R.; Rosen, C.; et al. Spontaneous fractures in the mouse mutant sfx are caused by deletion of the gulonolactone oxidase gene, causing vitamin $\mathrm{C}$ deficiency. J. Bone Miner. Res. 2005, 20, 1597-1610.

97. Jiao, Y.; Zhang, J.; Yan, J.; Stuart, J.; Gibson, G.; Lu, L.; Willaims, R.; Wang, Y.J.; Gu, W. Differential gene expression between wild-type and Gulo-deficient mice supplied with vitamin C. Genet. Mol. Biol. 2011, 34, 386-395.

98. Ward, M.S.; Lamb, J.; May, J.M.; Harrison, F.E. Behavioral and monoamine changes following severe vitamin C deficiency. J. Neurochem. 2013, 124, 363-375.

99. Amano, A.; Tsunoda, M.; Aigaki, T.; Maruyama, N.; Ishigami, A. Effect of ascorbic acid deficiency on catecholamine synthesis in adrenal glands of SMP30/GNL knockout mice. Eur. J. Nutr. 2014, 53, 177-185.

100. Sotiriou, S.; Gispert, S.; Cheng, J.; Wang, Y.; Chen, A.; Hoogstraten-Miller, S.; Miller, G.F.; Kwon, O.; Levine, M.; Guttentag, S.H.; et al. Ascorbic-acid transporter Slc23a1 is essential for vitamin $\mathrm{C}$ transport into the brain and for perinatal survival. Nat. Med. 2002, 8, 514-517.

101. Harrison, F.E.; Dawes, S.M.; Meredith, M.E.; Babaev, V.R.; Li, L.; May, J.M. Low vitamin C and increased oxidative stress and cell death in mice that lack the sodium-dependent vitamin $\mathrm{C}$ transporter SVCT2. Free Radic. Biol. Med. 2010, 49, 821-829.

102. Bornstein, S.R.; Yoshida-Hiroi, M.; Sotiriou, S.; Levine, M.; Hartwig, H.G.; Nussbaum, R.L.; Eisenhofer, G. Impaired adrenal catecholamine system function in mice with deficiency of the ascorbic acid transporter (SVCT2). FASEB J. 2003, 17, 1928-1930.

103. Meredith, M.E.; May, J.M. Regulation of embryonic neurotransmitter and tyrosine hydroxylase protein levels by ascorbic acid. Brain Res. 2013, 1539, 7-14.

104. Harrison, F.E.; Best, J.L.; Meredith, M.E.; Gamlin, C.R.; Borza, D.B.; May, J.M. Increased expression of SVCT2 in a new mouse model raises ascorbic acid in tissues and protects against paraquat-induced oxidative damage in lung. PLoS One 2012, 7, e35623.

105. Babaev, V.R.; Whitesell, R.R.; Li, L.; Linton, M.F.; Fazio, S.; May, J.M. Selective macrophage ascorbate deficiency suppresses early atherosclerosis. Free Radic. Biol. Med. 2011, 50, 27-36. 
106. Babaev, V.R.; Li, L.; Shah, S.; Fazio, S.; Linton, M.F.; May, J.M. Combined vitamin C and vitamin E deficiency worsens early atherosclerosis in apolipoprotein E-deficient mice. Arterioscler. Thromb. Vasc. Biol. 2010, 30, 1751-1757.

107. Kook, S.Y.; Lee, K.M.; Kim, Y.; Cha, M.Y.; Kang, S.; Baik, S.H.; Lee, H.; Park, R.; Mook-Jung, I. High-dose of vitamin C supplementation reduces amyloid plaque burden and ameliorates pathological changes in the brain of 5XFAD mice. Cell. Death Dis. 2014, 5, e1083.

108. Pierce, M.R.; Diasio, D.L.; Rodrigues, L.M.; Harrison, F.E.; May, J.M. Combined vitamin C and E deficiency induces motor defects in gulo(-/-)/SVCT2(+/-) mice. Nutr. Neurosci. 2013, $16,160-173$.

109. Morris, M.C.; Beckett, L.A.; Scherr, P.A.; Hebert, L.E.; Bennett, D.A.; Field, T.S.; Evans, D.A. Vitamin E and vitamin C supplement use and risk of incident Alzheimer disease. Alzheimer Dis. Assoc. Disord. 1998, 12, 121-126.

110. Morris, M.C.; Evans, D.A.; Bienias, J.L.; Tangney, C.C.; Bennett, D.A.; Aggarwal, N.; Wilson, R.S.; Scherr, P.A. Dietary intake of antioxidant nutrients and the risk of incident Alzheimer disease in a biracial community study. JAMA 2002, 287, 3230-3237.

111. Carr, A.C.; Bozonet, S.M.; Pullar, J.M.; Simcock, J.W.; Vissers, M.C. A Randomized Steady-State Bioavailability Study of Synthetic versus Natural (Kiwifruit-Derived) Vitamin C. Nutrients 2013, 5, 3684-3695.

112. Gray, S.L.; Anderson, M.L.; Crane, P.K.; Breitner, J.C.; McCormick, W.; Bowen, J.D.; Teri, L.; Larson, E. Antioxidant vitamin supplement use and risk of dementia or Alzheimer's disease in older adults. J. Am. Geriatr. Soc. 2008, 56, 291-295.

113. Luchsinger, J.A.; Tang, M.X.; Shea, S.; Mayeux, R. Antioxidant vitamin intake and risk of Alzheimer disease. Arch. Neurol. 2003, 60, 203-208.

114. Masaki, K.H.; Losonczy, K.G.; Izmirlian, G.; Foley, D.J.; Ross, G.W.; Petrovitch, H.; Havlik, R.; White, L.R. Association of vitamin E and C supplement use with cognitive function and dementia in elderly men. Neurology 2000, 54, 1265-1272.

115. Zandi, P.P.; Anthony, J.C.; Khachaturian, A.S.; Stone, S.V.; Gustafson, D.; Tschanz, J.T.; Norton, M.C.; Welsh-Bohmer, K.A.; Breitner, J.C. Reduced risk of Alzheimer disease in users of antioxidant vitamin supplements: the Cache County Study. Arch. Neurol. 2004, 61, 82-88.

116. Grodstein, F.; Chen, J.; Willett, W.C. High-dose antioxidant supplements and cognitive function in community-dwelling elderly women. Am. J. Clin. Nutr. 2003, 77, 975-984.

117. Devore, E.E.; Kang, J.H.; Stampfer, M.J.; Grodstein, F. The association of antioxidants and cognition in the Nurses' Health Study. Am. J. Epidemiol. 2013, 177, 33-41.

118. Engelhart, M.J.; Geerlings, M.I.; Ruitenberg, A.; van Swieten, J.C.; Hofman, A.; Witteman, J.C.; Breteler, M.M. Dietary intake of antioxidants and risk of Alzheimer disease. JAMA 2002, 287, 3223-3229.

119. Bowman, G.L.; Shannon, J.; Ho, E.; Traber, M.G.; Frei, B.; Oken, B.S.; Kaye, J.A.; Quinn, J.F. Reliability and validity of food frequency questionnaire and nutrient biomarkers in elders with and without mild cognitive impairment. Alzheimer Dis. Assoc. Disord. 2011, 25, 49-57.

120. Rodrigue, K.M.; Kennedy, K.M.; Devous, M.D., Sr.; Rieck, J.R.; Hebrank, A.C.; Diaz-Arrastia, R.; Mathews, D.; Park, D.C. beta-Amyloid burden in healthy aging: Regional distribution and cognitive consequences. Neurology 2012, 78, 387-395. 
121. Bowman, G.L.; Silbert, L.C.; Howieson, D.; Dodge, H.H.; Traber, M.G.; Frei, B.; Kaye, J.A.; Shannon, J.; Quinn, J.F. Nutrient biomarker patterns, cognitive function, and MRI measures of brain aging. Neurology 2012, 78, 241-249.

122. Bowman, G.L. Ascorbic acid, cognitive function, and Alzheimer's disease: A current review and future direction. Biofactors 2012, 38, 114-122.

123. Goodwin, J.S.; Goodwin, J.M.; Garry, P.J. Association between nutritional status and cognitive functioning in a healthy elderly population. JAMA 1983, 249, 2917-2921.

124. Gale, C.R.; Martyn, C.N.; Cooper, C. Cognitive impairment and mortality in a cohort of elderly people. BMJ 1996, 312, 608-611.

125. Riviere, S.; Birlouez-Aragon, I.; Nourhashemi, F.; Vellas, B. Low plasma vitamin C in Alzheimer patients despite an adequate diet. Int. J. Geriatr. Psychiatry 1998, 13, 749-754.

126. Charlton, K.E.; Rabinowitz, T.L.; Geffen, L.N.; Dhansay, M.A. Lowered plasma vitamin C, but not vitamin E, concentrations in dementia patients. J. Nutr. Health Aging 2004, 8, 99-107.

127. Polidori, M.C.; Mecocci, P. Plasma susceptibility to free radical-induced antioxidant consumption and lipid peroxidation is increased in very old subjects with Alzheimer disease. J. Alzheimer's Dis. (JAD) 2002, 4, 517-522.

128. Polidori, M.C.; Mattioli, P.; Aldred, S.; Cecchetti, R.; Stahl, W.; Griffiths, H.; Senin, U.; Sies, H.; Mecocci, P. Plasma antioxidant status, immunoglobulin g oxidation and lipid peroxidation in demented patients: relevance to Alzheimer disease and vascular dementia. Dement. Geriatr. Cogn. Disord. 2004, 18, 265-270.

129. Perrig, W.J.; Perrig, P.; Stahelin, H.B. The relation between antioxidants and memory performance in the old and very old. J. Am. Geriatr. Soc. 1997, 45, 718-724.

130. Spector, R. Nutrient transport systems in brain: 40 years of progress. J. Neurochem. 2009, 111, 315-320.

131. Paraskevas, G.P.; Kapaki, E.; Libitaki, G.; Zournas, C.; Segditsa, I.; Papageorgiou, C. Ascorbate in healthy subjects, amyotrophic lateral sclerosis and Alzheimer's disease. Acta Neurol. Scand. 1997, 96, 88-90.

132. Quinn, J.; Suh, J.; Moore, M.M.; Kaye, J.; Frei, B. Antioxidants in Alzheimer's disease-vitamin C delivery to a demanding brain. J. Alzheimers Dis. 2003, 5, 309-313.

133. Glaso, M.; Nordbo, G.; Diep, L.; Bohmer, T. Reduced concentrations of several vitamins in normal weight patients with late-onset dementia of the Alzheimer type without vascular disease. J. Nutr. Health Aging 2004, 8, 407-413.

134. Bowman, G.L.; Dodge, H.; Frei, B.; Calabrese, C.; Oken, B.S.; Kaye, J.A.; Quinn, J.F. Ascorbic acid and rates of cognitive decline in Alzheimer's disease. J. Alzheimers Dis. 2009, 16, 93-98.

135. Arlt, S.; Muller-Thomsen, T.; Beisiegel, U.K.; Ontush, A. Effect of One-Year Vitamin C- and E-Supplementation on Cerebrospinal Fluid Oxidation Parameters and Clinical Course in Alzheimer's Disease. Neurochem. Res. 2012, 37, 2706-2714.

136. Galasko, D.R.; Peskind, E.; Clark, C.M.; Quinn, J.F.; Ringman, J.M.; Jicha, G.A.; Cotman, C.; Cottrell, B.; Montine, T.J.; Thomas, R.G.; Aisen, P. Antioxidants for Alzheimer disease: A randomized clinical trial with cerebrospinal fluid biomarker measures. Arch. Neurol. 2012, $69,836-841$. 
137. Spector, R.; Johanson, C.E. Sustained choroid plexus function in human elderly and Alzheimer's disease patients. Fluids Barriers CNS 2013, 10, 28.

138. Polidori, M.C.; Pientka, L. Bridging the pathophysiology of Alzheimer's disease with vascular pathology: The feed-back, the feed-forward, and oxidative stress. J. Alzheimer's Dis. (JAD) 2012, 28, 1-9.

139. Polidori, M.C.; Pientka, L.; Mecocci, P. A review of the major vascular risk factors related to Alzheimer's disease. J. Alzheimer's Dis. (JAD) 2012, 32, 521-530.

140. de la Torre, J.C. Cerebral hemodynamics and vascular risk factors: setting the stage for Alzheimer's disease. J. Alzheimer's Dis. (JAD) 2012, 32, 553-567.

141. Aguero-Torres, H.; Kivipelto, M.; von Strauss, E. Rethinking the dementia diagnoses in a population-based study: What is Alzheimer's disease and what is vascular dementia? A study from the kungsholmen project. Dement. Geriatr. Cogn. Disord. 2006, 22, 244-249.

142. de la Torre, J.C.; Stefano, G.B. Evidence that Alzheimer's disease is a microvascular disorder: The role of constitutive nitric oxide. Brain Res. Brain Res. Rev. 2000, 34, 119-136.

143. Polidori, M.C.; Mecocci, P.; Frei, B. Plasma vitamin C levels are decreased and correlated with brain damage in patients with intracranial hemorrhage or head trauma. Stroke 2001, 32, 898-902.

144. Knopman, D.; Boland, L.L.; Mosley, T.; Howard, G.; Liao, D.; Szklo, M.; McGovern, P.; Folsom, A.R. Cardiovascular risk factors and cognitive decline in middle-aged adults. Neurology 2001, 56, 42-48.

145. Polidori, M.C.; Pratico, D.; Savino, K.; Rokach, J.; Stahl, W.; Mecocci, P. Increased F2 isoprostane plasma levels in patients with congestive heart failure are correlated with antioxidant status and disease severity. J. Card. Fail. 2004, 10, 334-338.

146. Polidori, M.C.; Pratico, D.; Ingegni, T.; Mariani, E.; Spazzafumo, L.; Del Sindaco, P.; Cecchetti, R.; Yao, Y.; Ricci, S.; Cherubini, A.; et al. Effects of vitamin C and aspirin in ischemic stroke-related lipid peroxidation: Results of the AVASAS (Aspirin Versus Ascorbic acid plus Aspirin in Stroke) Study. Biofactors 2005, 24, 265-274.

147. Wendell, C.R.; Zonderman, A.B.; Metter, E.J.; Najjar, S.S.; Waldstein, S.R. Carotid intimal medial thickness predicts cognitive decline among adults without clinical vascular disease. Stroke 2009, 40, 3180-3185.

148. Frei, B. To C or not to C, that is the question! J. Am. Coll. Cardiol. 2003, 42, 253-255.

149. Polidori, M.C.; Mecocci, P.; Reimann, A.; Cherubini, A.; Cecchetti, R.; Briviba, K.; Stahl, W.; Sies, H.; Senin, U. Plasma lipid peroxidation and vitamin C status in healthy centenarians. J. Am. Geriatr. Soc. 1999, 47, 1038-1039.

150. Hunter, D.C.; Skinner, M.A.; Wolber, F.M.; Booth, C.L.; Loh, J.M.; Wohlers, M.; Stevenson, L.M.; Kruger, M.C. Consumption of gold kiwifruit reduces severity and duration of selected upper respiratory tract infection symptoms and increases plasma vitamin C concentration in healthy older adults. Br. J. Nutr. 2012, 108, 1235-1245.

151. Moretti, M.; Colla, A.; de Oliveira Balen, G.; dos Santos, D.B.; Budni, J.; de Freitas, A.E.; Farina, M.; Severo Rodrigues, A.L. Ascorbic acid treatment, similarly to fluoxetine, reverses depressive-like behavior and brain oxidative damage induced by chronic unpredictable stress. J. Psychiatr. Res. 2012, 46, 331-340. 
152. Simon, J.A.; Hudes, E.S.; Tice, J.A. Relation of serum ascorbic acid to mortality among US adults. J. Am. Coll. Nutr. 2001, 20, 255-263.

153. Boekholdt, S.M.; Meuwese, M.C.; Day, N.E.; Luben, R.; Welch, A.; Wareham, N.J.; Khaw, K.T. Plasma concentrations of ascorbic acid and C-reactive protein, and risk of future coronary artery disease, in apparently healthy men and women: the EPIC-Norfolk prospective population study. Br. J. Nutr. 2006, 96, 516-522.

154. Khaw, K.T.; Bingham, S.; Welch, A.; Luben, R.; Wareham, N.; Oakes, S.; Day, N. Relation between plasma ascorbic acid and mortality in men and women in EPIC-Norfolk prospective study: A prospective population study. European Prospective Investigation into Cancer and Nutrition. Lancet 2001, 357, 657-663.

155. Ellingsen, I.; Seljeflot, I.; Arnesen, H.; Tonstad, S. Vitamin C consumption is associated with less progression in carotid intima media thickness in elderly men: A 3-year intervention study. Nutr. Metab. Cardiovasc. Dis. (NMCD) 2009, 19, 8-14.

156. Hodis, H.N.; Mack, W.J.; LaBree, L.; Mahrer, P.R.; Sevanian, A.; Liu, C.R.; Liu, C.H.; Hwang, J.; Selzer, R.H.; Azen, S.P. Alpha-tocopherol supplementation in healthy individuals reduces low-density lipoprotein oxidation but not atherosclerosis: The Vitamin E Atherosclerosis Prevention Study (VEAPS). Circulation 2002, 106, 1453-1459.

157. Zureik, M.; Galan, P.; Bertrais, S.; Mennen, L.; Czernichow, S.; Blacher, J.; Ducimetiere, P.; Hercberg, S. Effects of long-term daily low-dose supplementation with antioxidant vitamins and minerals on structure and function of large arteries. Arterioscler. Thromb. Vasc. Biol. 2004, 24, 1485-1491.

158. Luzzi, S.; Vella, L.; Bartolini, M.; Provinciali, L.; Silvestrini, M. Atherosclerosis in the evolution of Alzheimer's disease: Can treatment reduce cognitive decline? J. Alzheimer's Dis. (JAD) 2010, 20, 893-901.

159. Terpstra, M.; Marjanska, M.; Henry, P.G.; Tkac, I.; Gruetter, R. Detection of an antioxidant profile in the human brain in vivo via double editing with MEGA-PRESS. Magn. Reson. Med. 2006, 56, 1192-1199.

160. Emir, U.E.; Raatz, S.; McPherson, S.; Hodges, J.S.; Torkelson, C.; Tawfik, P.; White, T.; Terpstra, M. Noninvasive quantification of ascorbate and glutathione concentration in the elderly human brain. NMR Biomed. 2011, 24, 888-894. 
Reprinted from Nutrients. Cite as: Iwata, N.; Okazaki, M.; Xuan, M.; Kamiuchi, S.; Matsuzaki, H.; Hibino, Y. Orally Administrated Ascorbic Acid Suppresses Neuronal Damage and Modifies Expression of SVCT2 and GLUT1 in the Brain of Diabetic Rats with Cerebral Ischemia-Reperfusion. Nutrients 2014, 6, 1554-1577.

Article

\title{
Orally Administrated Ascorbic Acid Suppresses Neuronal Damage and Modifies Expression of SVCT2 and GLUT1 in the Brain of Diabetic Rats with Cerebral Ischemia-Reperfusion
}

\author{
Naohiro Iwata ${ }^{1}$, Mari Okazaki ${ }^{1,2, *}$, Meiyan Xuan ${ }^{1}$, Shinya Kamiuchi ${ }^{1}$, Hirokazu Matsuzaki ${ }^{2}$ \\ and Yasuhide Hibino ${ }^{1}$
}

1 Laboratory of Immunobiochemistry, Faculty of Pharmaceutical Sciences, Josai University, Saitama 350-0295, Japan; E-Mails: n-iwata@josai.ac.jp (N.I.); genbien@josai.ac.jp (M.X.); kamiuchi@josai.ac.jp (S.K.); seitaib@josai.ac.jp (Y.H.)

2 Laboratory of Pharmacology, Faculty of Pharmaceutical Sciences, Josai University, Saitama 350-0295, Japan; E-Mail: ma-tsu@josai.ac.jp

* Author to whom correspondence should be addressed; E-Mail: okazaki@josai.ac.jp; Tel./Fax: +81-49-271-7677.

Received: 30 December 2013; in revised form: 28 March 2014 / Accepted: 1 April 2014 /

Published: 15 April 2014

\begin{abstract}
Diabetes mellitus is known to exacerbate cerebral ischemic injury. In the present study, we investigated antiapoptotic and anti-inflammatory effects of oral supplementation of ascorbic acid (AA) on cerebral injury caused by middle cerebral artery occlusion and reperfusion $(\mathrm{MCAO} / \mathrm{Re})$ in rats with streptozotocin-induced diabetes. We also evaluated the effects of AA on expression of sodium-dependent vitamin C transporter 2 (SVCT2) and glucose transporter 1 (GLUT1) after MCAO/Re in the brain. The diabetic state markedly aggravated MCAO/Re-induced cerebral damage, as assessed by infarct volume and edema. Pretreatment with AA (100 mg/kg, p.o.) for two weeks significantly suppressed the exacerbation of damage in the brain of diabetic rats. AA also suppressed the production of superoxide radical, activation of caspase-3, and expression of proinflammatory cytokines (tumor necrosis factor- $\alpha$ and interleukin-1 $\beta$ ) in the ischemic penumbra. Immunohistochemical staining revealed that expression of SVCT2 was upregulated primarily in neurons and capillary endothelial cells after MCAO/Re in the nondiabetic cortex, accompanied by an increase in total AA $(\mathrm{AA}+$ dehydroascorbic acid) in the tissue, and that these responses were suppressed in
\end{abstract}


the diabetic rats. AA supplementation to the diabetic rats restored these responses to the levels of the nondiabetic rats. Furthermore, AA markedly upregulated the basal expression of GLUT1 in endothelial cells of nondiabetic and diabetic cortex, which did not affect total AA levels in the cortex. These results suggest that daily intake of AA attenuates the exacerbation of cerebral ischemic injury in a diabetic state, which may be attributed to anti-apoptotic and anti-inflammatory effects via the improvement of augmented oxidative stress in the brain. AA supplementation may protect endothelial function against the exacerbated ischemic oxidative injury in the diabetic state and improve AA transport through SVCT2 in the cortex.

Keywords: ascorbic acid; diabetes mellitus; oxidative stress; apoptosis; proinflammatory; cytokine; sodium-dependent vitamin C transporter 2 (SVCT2); glucose transporter 1 (GLUT1); rat; streptozotocin; middle cerebral artery occlusion and reperfusion

\section{Introduction}

Diabetes mellitus is a metabolic disorder associated with chronic hyperglycemia, which is known to enhance systemic oxidative stress, predisposing to diabetic complications. Diabetes is a major risk factor for atherosclerotic diseases such as acute brain ischemia [1,2]. Moreover, it increases the risks of morbidity and mortality after stroke $[3,4]$. Oxidative stress plays an essential role in the pathogenesis of transient cerebral ischemic injury [3-5]. In particular, reperfusion after a long period of vessel occlusion triggers the explosive generation of reactive oxygen species (ROS), such as superoxide radical $\left(\mathrm{O}_{2}^{-}\right)$, hydroxyl radical, hydrogen peroxide, etc., which causes apoptosis and delayed death of cells through oxidative damage to lipids, proteins, and DNA in the ischemic penumbral region [6-9]. In addition to apoptotic cell death, inflammatory neurodegeneration is another crucial process contributing to cerebral damage after ischemia and reperfusion [10]. ROS have been shown to activate nuclear factor- $\kappa \mathrm{B}$, which enhances the transcription of the genes encoding proinflammatory cytokines such as tumor necrosis factor- $\alpha$ (TNF- $\alpha)$ and interleukin- $1 \beta$ (IL-1 $\beta$ ), leading to inflammatory responses [11]. Myeloperoxidase (MPO) expressed by microglia, a histopathological marker of inflammation, generates cytotoxic ROS and leads to further inflammatory damage in the ischemic tissue [12]. Accumulating evidence indicates that hyperglycemia in diabetes is associated with a decrease in the antioxidant potential and an increase in ROS generation [13-15]. In addition, diabetes has been shown to be a proinflammatory state that increases the risk of vascular complications [16,17]. Thus, the enhanced oxidative stress and inflammatory responses in the diabetic state may substantially contribute to the aggravation of cerebral injury caused by transient ischemia and subsequent reperfusion.

L-ascorbic acid (AA) is an essential antioxidant for scavenging free radicals in the brain. AA participates not only in sustaining the normal function of the central nervous system (CNS) but in ameliorating the damage induced by pathological conditions that increase the generation of ROS [18]. The CNS maintains relatively high concentrations of AA, indicating a neuroprotective role for AA [19]. The transport of AA from the plasma to the CNS is mainly mediated by 
sodium-dependent vitamin C transporter 2 (SVCT2). In addition, glucose transporter 1 (GLUT1) is located in the endothelial cells of the blood-brain barrier (BBB) and transports oxidized AA ([dehydroascorbic acid (DHA)) as another source of AA to the brain. Upregulation of SVCT2 [20] and GLUT1 [21] expression has been demonstrated in rats with cerebral ischemic injury, which suggests that AA is necessary for protection against oxidative neuronal injury. The concentration of AA is considered to reflect oxidative stress in animal tissues sensitively [22]. Patients with ischemic stroke or diabetes have lower concentrations of AA in the plasma, suggesting that a systemic decrease in AA is a consequence of enhanced consumption of AA by elevated oxidative stress $[23,24]$. In a previous study, we demonstrated that chronic supplementation with AA attenuates oxidative stress in both the plasma and the brain and alleviates cerebral injury induced by middle cerebral artery occlusion and reperfusion $(\mathrm{MCAO} / \mathrm{Re})$ in rats with streptozotocin (STZ)-induced diabetes [25]. We showed that the activity of antioxidant enzymes (superoxide dismutase, catalase, and glutathione peroxidase) is decreased, and lipid peroxidation is accelerated in the brain of diabetic rats; those detrimental oxidative processes are inhibited by AA supplementation. These data suggest that the enhanced oxidative stress in the diabetic state causes the functional impairment of antioxidant enzymes, and the resulting diminution in antioxidative defense can cause further enhancement of the generation of ROS and subsequent neuronal apoptosis and inflammatory neurodegeneration in the ischemic brain. Nonetheless, there is a shortage of studies so far on the antiapoptotic and anti-inflammatory effects of AA in cerebral ischemia with diabetes.

In the present study, we evaluated the effects of chronic oral pretreatment with AA on the production of $\mathrm{O}_{2}{ }^{-}$and on apoptosis in the brain after MCAO/Re in rats with STZ-induced diabetes. To test whether AA suppresses inflammatory responses induced by $\mathrm{MCAO} / \mathrm{Re}$, we also examined the effects of AA on the expression of TNF- $\alpha$, IL-1 $\beta$, and MPO in the brain. Although the transport of AA to the CNS is an important factor for its neuroprotection, changes in expression of SVCT2 and GLUT1 proteins in response to AA supplementation and/or cerebral ischemia in diabetic state have not been reported. Therefore, we investigated the effects of AA supplementation on the expression of SVCT2 and GLUT1 after MCAO/Re in the brain of diabetic rats.

\section{Experimental Section}

\subsection{Experimental Animals}

Animal care and surgical procedures were performed in accordance with guidelines approved by the National Institutes of Health (Bethesda, MD, USA) and the Josai University Animal Research Committee. Male Sprague-Dawley rats (4 weeks old, weight 120-140 g) were purchased from Japan SLC (Shizuoka, Japan) and were housed under standard conditions with a temperature-controlled environment $\left(23^{\circ} \mathrm{C} \pm 0.5^{\circ} \mathrm{C}\right)$ and a $12 \mathrm{~h}$ light/dark cycle. The animals were allowed free access to rodent chow (CE-2, CLEA Japan, Tokyo, Japan) and water. Type 1 diabetes was induced in the rats (diabetic group) by a single intraperitoneal injection of STZ $(50 \mathrm{mg} / \mathrm{kg}$ of body weight) dissolved in $0.1 \mathrm{mM}$ sodium citrate, $\mathrm{pH} 4.5$, while the normal control rats (nondiabetic group) were injected with the buffer only [26]. Seven days after the injection of STZ, a blood sample was collected by tail vein paracentesis, following which plasma glucose was measured using a glucose analyzer (Ascensia, 
Bayer Yakuhin, Osaka, Japan). Diabetes was defined as a blood glucose level greater than $300 \mathrm{mg} / \mathrm{dL}$. Then, the diabetic and nondiabetic groups were divided into 2 groups and were housed for an additional 6 weeks until stroke was induced by MCAO/Re. AA (L-Ascorbic acid, Wako Pure Chemicals Industries, Osaka, Japan) (100 mg/kg; nondiabetic and diabetic AA-supplemented groups) or distilled water (nondiabetic and diabetic control groups) was orally administered through nasogastric tube once daily for the last 2 weeks. AA was stored at $+4{ }^{\circ} \mathrm{C}$, and dissolved in distilled water fresh each time just before administration.

\section{2. $M C A O / R e$}

The experimental MCAO/Re rat model was prepared as described previously [25]. The rats were anesthetized with halothane ( $4 \%$ for induction and $1.5 \%$ for maintenance) under spontaneous respiration. After a midline incision on the neck, the right common carotid artery was isolated under an operating microscope. All branches of the external carotid artery were ligated. The tip of a 4-0 surgical nylon monofilament rounded by flame heating was inserted through the internal carotid artery and advanced to occlude the origin of MCA. The rectal temperature was maintained at $37{ }^{\circ} \mathrm{C}$ with a heat lamp and a heating pad during the operation. After $2 \mathrm{~h}$ of occlusion, the filament was withdrawn to enable reperfusion. The distance from bifurcation of the common carotid artery to the tip of the suture was approximately $20 \mathrm{~mm}$ in all rats. Cerebral blood flow was detected using a Laser Doppler flowmetry (ATBF-LC1, Unique Medical, Tokyo, Japan), and approximately 50\% reduction of its baseline associated with MCAO was ascertained in the rats. Then, the rats were allowed to recover from anesthesia at room temperature and were killed after $24 \mathrm{~h}$ of reperfusion. Sham operation involved the same manipulations but insertion of the filament.

\subsection{Infarct and Edema Assessment}

After $24 \mathrm{~h}$ of reperfusion, the rats were subjected to general halothane anesthesia and decapitated. The brain was immediately removed and placed in ice-cold saline. Each brain was then cut into $2 \mathrm{~mm}$ coronal slices in a rat brain matrix. The brain slices were immediately immersed in 2\% 2,3,5-triphenyl tetrazolium chloride (TTC, Wako Pure Chemicals Industries, Osaka, Japan) at $37{ }^{\circ} \mathrm{C}$ for $15 \mathrm{~min}$ and then in $4 \%$ formaldehyde [26,27]. Infarct areas were identified using an image analysis system (Scion Image 1.62, Frederick, MD, USA) and were combined to obtain the infarct volumes per brain according to the following formula: corrected infarct volume $(\%)=($ left hemisphere volume $-($ right hemisphere volume - the infarct volume $)) \times$ 100/left hemisphere volume. Edema in the ischemic hemisphere was also calculated as follows: edema $(\%)=($ right hemisphere volume - the infarct volume $) /$ left hemisphere volume $\times 100$.

\subsection{Neurological Evaluation}

Postischemic neurological deficits were evaluated after $24 \mathrm{~h}$ of reperfusion on a 5-point scale as follows: grade 0 , no deficit; grade 1 , failure to fully extend the right forepaw; grade 2, spontaneous circling or walking to a contralateral side; grade 3, walking only when stimulated; grade 4, unresponsive to stimulation and a depressed level of consciousness; and grade 5, death [25,27]. Before MCAO, the neurological score was zero in all the rats. The rats that did not exhibit 
neurological deficits after MCAO/Re were excluded from the study. Grades of the neurological score were evaluated by an investigator blinded to the treatment protocol.

\subsection{Detection of $\mathrm{O}_{2}^{-}$Production in the Brain}

Detection of intracellular $\mathrm{O}_{2}{ }^{-}$production in the ischemic penumbral region of the cortex after $\mathrm{MCAO} / \mathrm{Re}$ was performed by histochemical staining of freshly frozen brain sections ( $8 \mu \mathrm{m}$ thick) with the fluorescent probe dihydroethidium (DHE). The brain sections were immediately incubated with DHE (10 $\mu \mathrm{mol} / \mathrm{L}$, Sigma-Aldrich Japan, Tokyo, Japan) in phosphate-buffered saline for $30 \mathrm{~min}$ at $37{ }^{\circ} \mathrm{C}[28,29]$. To determine the fluorescence intensity of oxidized DHE, 3 visual fields within the penumbral cortex regions of each hemisphere were photographed using a confocal laser scanning microscope (Fluoview FV1000, OLYMPUS, Tokyo, Japan) with excitation at $510 \mathrm{~nm}$ and emission at $580 \mathrm{~nm}$. Fluorescence intensity of oxidized DHE was quantified using imaging software (FV10-ASW 1.7, OLYMPUS, Tokyo, Japan). Analyses of immunohistochemistry were performed by an investigator blinded to the treatment protocol.

\subsection{Immunohistochemistry}

Immunohistochemical staining was performed as described previously [20,30,31]. The brain was fixed with $4 \%$ phosphate-buffered paraformaldehyde. Coronal brain sections ( $8 \mu \mathrm{m}$ thick) were incubated with $3 \%$ hydrogen peroxide for $40 \mathrm{~min}$ at room temperature to inhibit endogenous peroxidase and then incubated with blocking buffer (4\% Block Ace, Dainippon Sumitomo Pharma, Osaka, Japan) for $2 \mathrm{~h}$. Then, the slices were incubated with polyclonal rabbit anti-IL-1 $\beta$ antibody (1:300, Santa Cruz Biotechnology, Dallas, TX, USA), polyclonal rabbit anti-TNF- $\alpha$ antibody (1:200, Rabbit mAb, Hycult Biotech, Uden, The Netherlands), polyclonal rabbit anti-cleaved caspase-3 antibody (1:100, Cell Signaling Technology, Danvers, MA, USA), or monoclonal mouse anti-MPO (1:100, Hycult Biotech, Uden, The Netherlands), polyclonal rabbit anti-SVCT2 antibody (1:100, Santa Cruz Biotechnology, Dallas, TX, USA), polyclonal rabbit anti-GLUT1 antibody (1:100, Santa Cruz Biotechnology, Dallas, TX, USA) in $0.01 \mathrm{~mol} / \mathrm{L}$ phosphate-buffered saline overnight at $4{ }^{\circ} \mathrm{C}$. In a double-immunohistchemical study for determination of the cell types of expressing SVCT2 or GLUT1, monoclonal mouse anti-neuronal nuclei (NeuN) antibody (1:300, Millipore, Billerica, MA, USA) and monoclonal mouse anti-rat endothelial cell antigen (RECA1) antibody (1:200, Abcom, Cambridge, UK) were used. After washing with phosphate-buffered saline, the slices were incubated with either Cy3- or FITC-conjugated secondary antibody (1:200, Millipore, Billerica, MA, USA) for $2 \mathrm{~h}$ at room temperature. Finally, the sections were incubated with the nuclear stain TO-PRO-3 (1:10,000, Invitrogen, Carlsbad, CA, USA) in phosphate-buffered saline for $10 \mathrm{~min}$ at room temperature with gentle agitation. Immunofluorescence was visualized using the laser scanning confocal microscope and the intensity was measured using the imaging software. Three sections per rat and 3-4 rats per group were used for the analyses.

\subsection{Real-Time Polymerase Chain Reaction (PCR) Analysis}

The expression levels of SVCT2 (Slc23a2) and GLUT1 (Slc2al) mRNA were assessed by quantitative real-time polymerase chain reaction (PCR) as described previously [32]. The rats 


\section{6}

subjected to MCAO were killed after $24 \mathrm{~h}$ of reperfusion, and total RNA samples were prepared from the ischemic penumbral cortex of each rat. Total RNA was extracted using the RNeasy Mini Kit (QIAGEN, Hilden, Germany) according to the manufacturer's protocol. Total RNA (500 pg) from each sample was reverse-transcribed with oligo-dT and random hexamer primers using reverse transcriptase (PrimeScript RT Enzyme Mix I; Takara RNA PCR Kit, Takara Biomedicals, Shiga, Japan). Real-time PCR was performed with $10 \mathrm{ng}$ of cDNA and a pair of gene-specific primers (Takara Biomedicals, Shiga, Japan) that were added to the SYBR Premix EX Taq (Takara Biomedicals, Shiga, Japan) and subjected to PCR amplification on the iCycler iQ Real-Time Detection System (Bio-Rad Laboratories, Hercules, CA, USA) ( 1 cycle at $95{ }^{\circ} \mathrm{C}$ for $10 \mathrm{~s}$ and 50 cycles at $95{ }^{\circ} \mathrm{C}$ for $5 \mathrm{~s}$ and $60{ }^{\circ} \mathrm{C}$ for $34 \mathrm{~s}$ ). $\beta$-Actin expression was used to normalize the cDNA levels. The PCR products were analyzed using a melting curve to ascertain specificity of the amplification. The data were expressed as mean $\pm \mathrm{SD}$ relative to the sham-operated nondiabetic group.

\subsection{Measurement of Total AA Level}

Total AA (AA + DHA) levels in the plasma and cortex have been determined by spectrophotometric method using Vitamin C Assay kit (ROIK02, Shima Laboratories, Tokyo, Japan) which is based on 2,4-dinitrophenylhydrazine method [33]. Briefly, plasma was mixed with an equal volume of $10 \%$ metaphosphoric acid solution. Cortex tissue was mixed with 14 times volume of $5.4 \%$ metaphosphoric acid solution and homogenized. After centrifugation of the solutions at $10,000 \times \mathrm{g}$ for $15 \mathrm{~min}$ at $4{ }^{\circ} \mathrm{C}$, the supernatants were used to the assay.

\subsection{Statistical Analysis}

Two-way ANOVA, followed by post hoc Tukey's multiple-comparison test, was used for statistical analysis. Neurological deficit scores were analyzed using the Kruskal-Wallis test, followed by the Mann-Whitney $U$ test. In all cases, a $p$ value of $<0.05$ was assumed to denote statistical significance.

\section{Results}

\subsection{Blood Glucose and Body Weight}

Body weight and blood glucose data from the experimental rats were obtained throughout the study period (Table 1). Similar to our previous study [25], the diabetic control group of rats had a significantly decreased body weight and an increased blood glucose level compared with the nondiabetic control group. There were no significant differences in those parameters between the AA-supplemented groups and their controls.

Table 1. Effects of oral supplementation with AA on body weight and blood glucose levels in nondiabetic (non-DM) and diabetic (DM) groups of rats.

\begin{tabular}{ccc}
\hline Groups & Body Weight (g) & Blood Glucose (mg/dL) \\
\hline Non-DM & $335 \pm 16$ & $112 \pm 11$ \\
Non-DM + AA & $340 \pm 23$ & $135 \pm 9$ \\
DM & $255 \pm 32 *$ & $485 \pm 42 *$ \\
DM + AA & $269 \pm 36 *$ & $469 \pm 71 *$ \\
\hline
\end{tabular}

The data are shown as mean \pm SD. ${ }^{*} p<0.01 v s$. the nondiabetic group $(n=6-7)$. 


\subsection{Ischemic Brain Injury and Neurological Deficits}

Figure 1 shows MCAO/Re-induced brain injury in the nondiabetic control, nondiabetic + AA, diabetic control, and diabetic + AA groups of rats. Examples of TTC staining in the coronal brain sections at $24 \mathrm{~h}$ after MCAO/Re are shown in Figure 1A. The infarct developed in the corpus striatum and cortex of the nondiabetic control rats. In the diabetic rats, the infarction zone was remarkably enlarged and extended to a large part of the left striatum and cortex. In contrast, the infarcts in the AA-supplemented groups were smaller than those in their respective controls. Quantitative assays revealed that the infarct volume and edema in the diabetic control group were significantly increased by approximately 2.5 -fold and 2-fold, respectively, compared with those in the nondiabetic control group (Figure 1B,C). AA supplementation in the nondiabetic group significantly decreased infarction and edema. Furthermore, AA almost completely suppressed the exacerbation of brain damage by diabetes.

Figure 1. Effects of AA supplementation on infarction induced by $\mathrm{MCAO} / \mathrm{Re}$ in the brain of nondiabetic and diabetic rats. (A) Representative photographs of staining of coronal brain sections from the rats of the nondiabetic + sham operation group and from distilled water-administered (Control) or AA (100 mg/kg)-supplemented nondiabetic or diabetic groups with $\mathrm{MCAO}$ with reperfusion $(\mathrm{MCAO} / \mathrm{Re})$. AA or water was orally administered once daily for 2 weeks; (B) Infarct volume in ischemic hemispheres of the diabetic and nondiabetic groups after MCAO/Re by TTC staining; (C) Edema volume in ischemic hemispheres of the diabetic and nondiabetic groups after MCAO/Re. Data are presented as means $\pm \mathrm{SD}(n=6-7) . * * p<0.01$ compared with the nondiabetic control group. ${ }^{\# \#} p<0.01$ compared with the diabetic control group. DM in the figure denotes diabetic, while non-DM denotes nondiabetic.

\section{(A)}

\begin{tabular}{|c|c|c|c|c|}
\hline Sham & \multicolumn{4}{|c|}{ MCAO/Re } \\
\hline Control & & & & \\
\hline non-DM & non-DM & DM & non-DM & DM \\
\hline
\end{tabular}

(B)

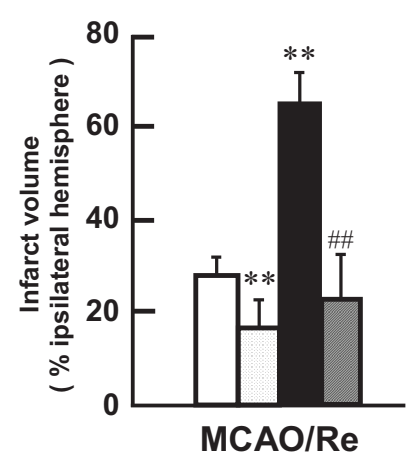

(C)

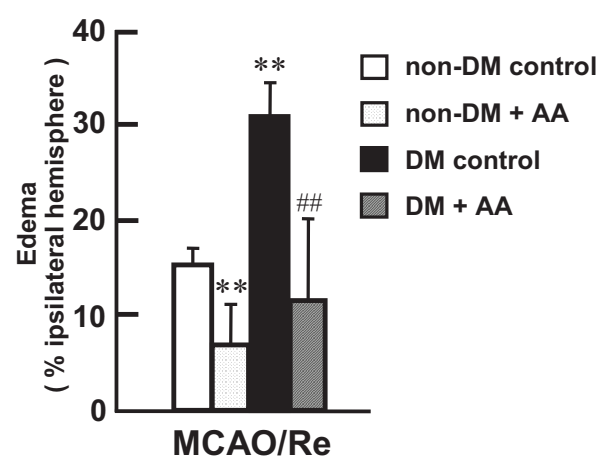


Consistent with the aforementioned results of the brain injury experiments, neurological deficits were exacerbated in the diabetic control group of rats (Figure 2). Compared with the diabetic control group, the diabetic + AA group showed significant alleviation of the neurological deficits.

Figure 2. Effects of AA supplementation on neurological deficits induced by $\mathrm{MCAO} / \mathrm{Re}$ in nondiabetic and diabetic rats. Postischemic neurological deficits were evaluated on a 5 -point scale at $24 \mathrm{~h}$ of reperfusion after $2 \mathrm{~h}$ of MCAO. Data are mean $\pm \mathrm{SD}$ of 6-7 rats per group. ${ }^{* *} p<0.01$ compared with the nondiabetic control group. ${ }^{\# \#} p<0.01$ compared with the diabetic control group. DM in the figure denotes diabetic, while non-DM denotes nondiabetic.

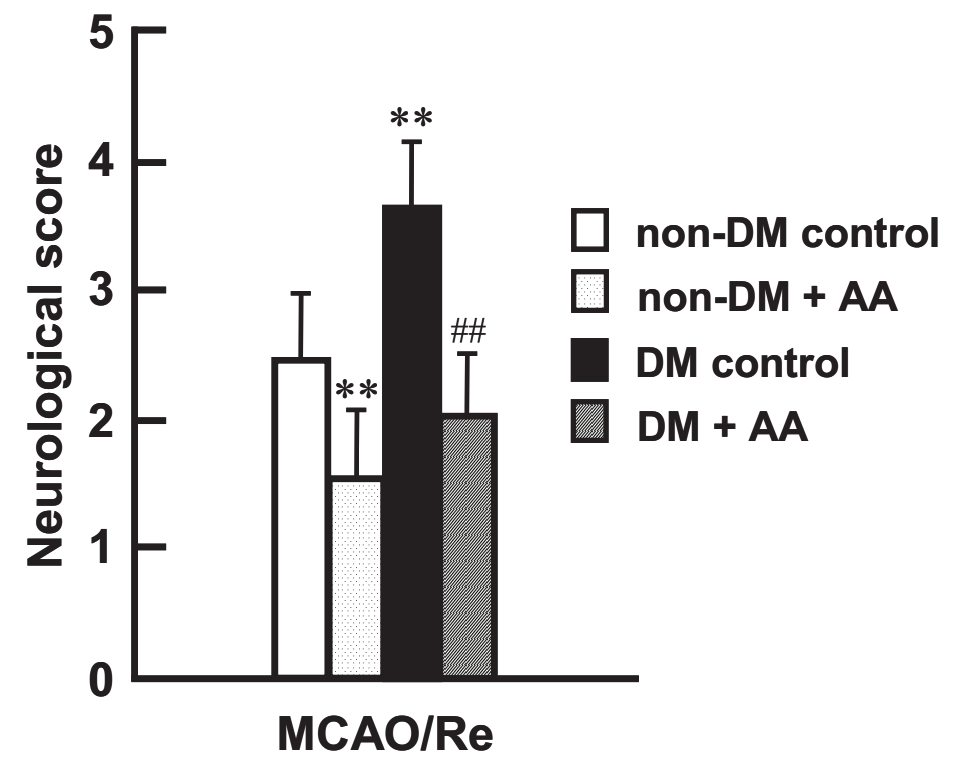

\section{3. $\mathrm{O}_{2}^{-}$Production after Ischemia with Reperfusion}

Figure 3 shows fluorescence intensity of DHE in the penumbral cortex, which depends on intracellular $\mathrm{O}_{2}^{-}$production. Representative histological images of DHE staining in the nondiabetic control, nondiabetic + AA, diabetic control, and diabetic + AA groups are shown in Figure 3A. DHE-positive cells with low fluorescence intensity were sparsely distributed in the cortex of sham-operated nondiabetic rats. In contrast, sham-operated diabetic rats had an increased number of DHE-positive cells with higher fluorescence intensity in the cortex, indicating basal augmentation of the generation of ROS in the brain of the diabetic rats. The intensity of DHE fluorescence was remarkably increased by $\mathrm{MCAO} / \mathrm{Re}$ in the nondiabetic rats and was further augmented in the diabetic rats, suggesting that the exacerbated injury can be attributed to enhanced generation of ROS in diabetes. AA supplementation significantly reduced the fluorescence of DHE in the cortex of the nondiabetic and diabetic rats compared with that in their respective controls. 
Figure 3. Effects of AA supplementation on production of $\mathrm{O}_{2}{ }^{-}$after $\mathrm{MCAO} / \mathrm{Re}$ in the brain of nondiabetic and diabetic rats. (A) Representative photographs of superoxide production detected by DHE staining in coronal sections of the cortex from the nondiabetic and diabetic rats; (B) Quantitative analysis of DHE fluorescence intensity in the cortex. The data are presented as mean $\pm \operatorname{SD}(n=3-4)$. $* p<0.05, * * p<0.01$ compared with the nondiabetic control group. ${ }^{\#} p<0.05,{ }^{\#} p<0.01$ compared with the diabetic control group. DM in the figure denotes diabetic, while non-DM denotes nondiabetic.

(A)

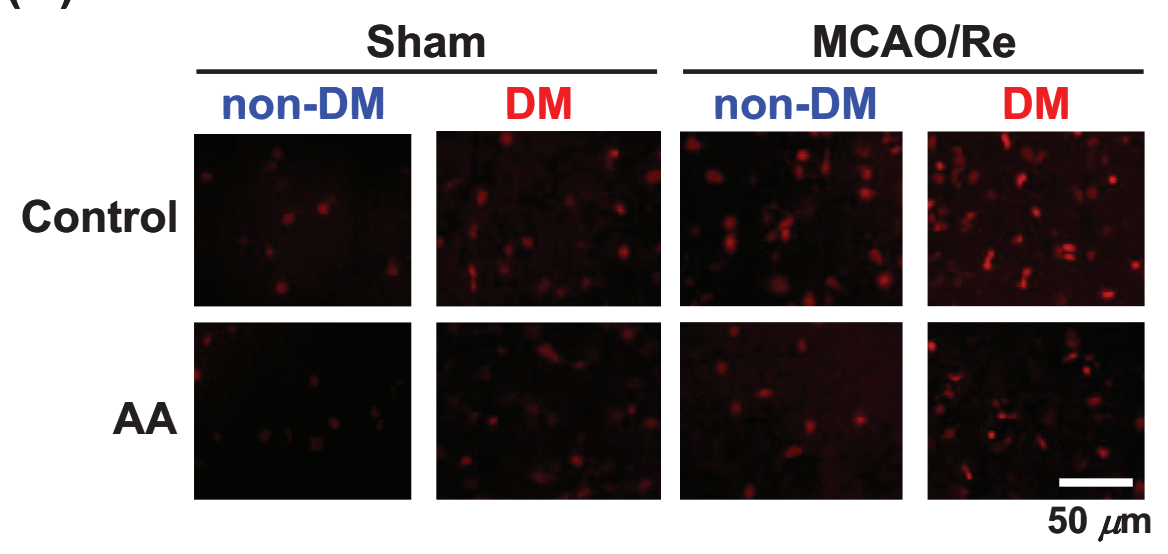

(B)

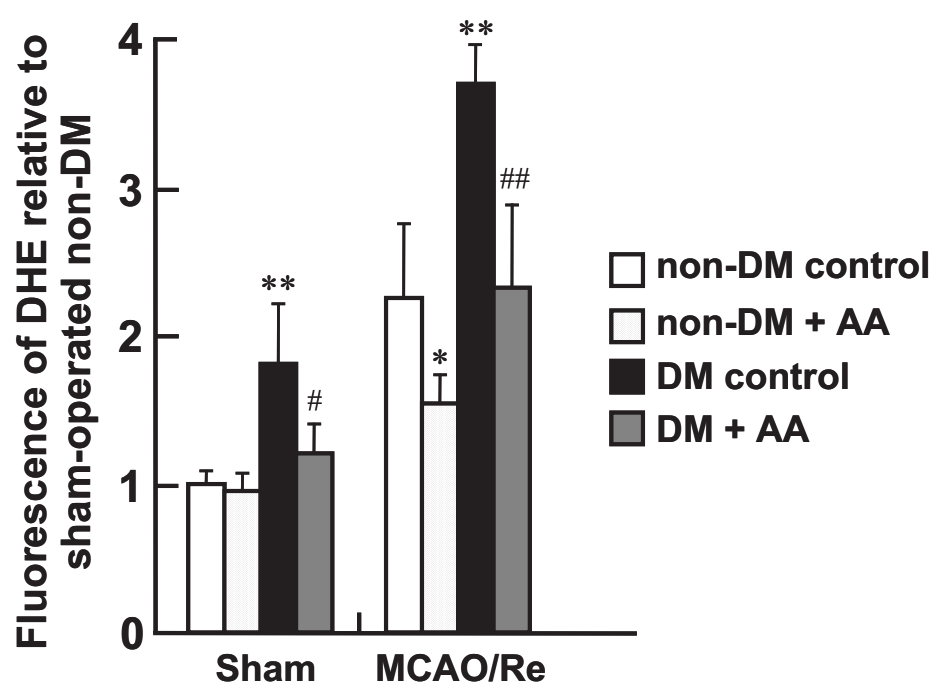

\subsection{Apoptosis Induced by Ischemia with Reperfusion}

Activation of caspase-3, a key mediator of the execution phase of apoptosis, was determined by immunostaining for cleaved caspase-3, which is an activated form of this enzyme, in the ischemic penumbral cortex of the four experimental groups after MCAO/Re (Figure 4). Compared with the nondiabetic control group, the number of cleaved caspase-3 positive cells was remarkably increased by $\mathrm{MCAO} / \mathrm{Re}$ in the diabetic control group. AA significantly attenuated the MCAO/Re-induced activation of caspase- 3 in the nondiabetic and diabetic groups. 
Figure 4. Effects of AA supplementation on cleaved caspase-3 after MCAO/Re in the brain of nondiabetic and diabetic rats. (A) Representative photographs of cleaved caspase-3 immunostaining in the cortex coronal sections of nondiabetic and diabetic rats; (B) Quantitative analysis of cleaved caspase-3 positive cells (fluorescence intensity in the cortex). The data are presented as mean $\pm \mathrm{SD}(n=3-4) .{ }^{*} p<0.05,{ }^{*} * p<0.01$ compared with the nondiabetic control group. ${ }^{\#} p<0.01$ compared with the diabetic control group. DM in the figure denotes diabetic, while non-DM denotes nondiabetic.

(A)

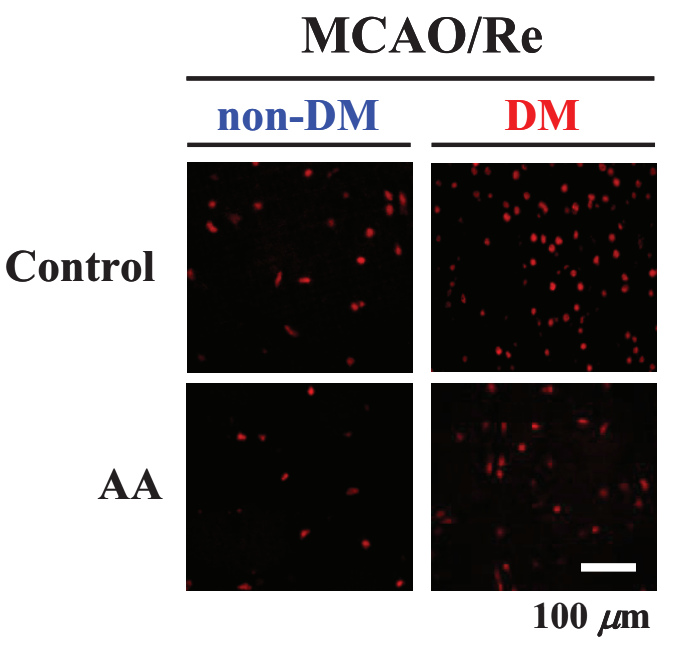

(B)

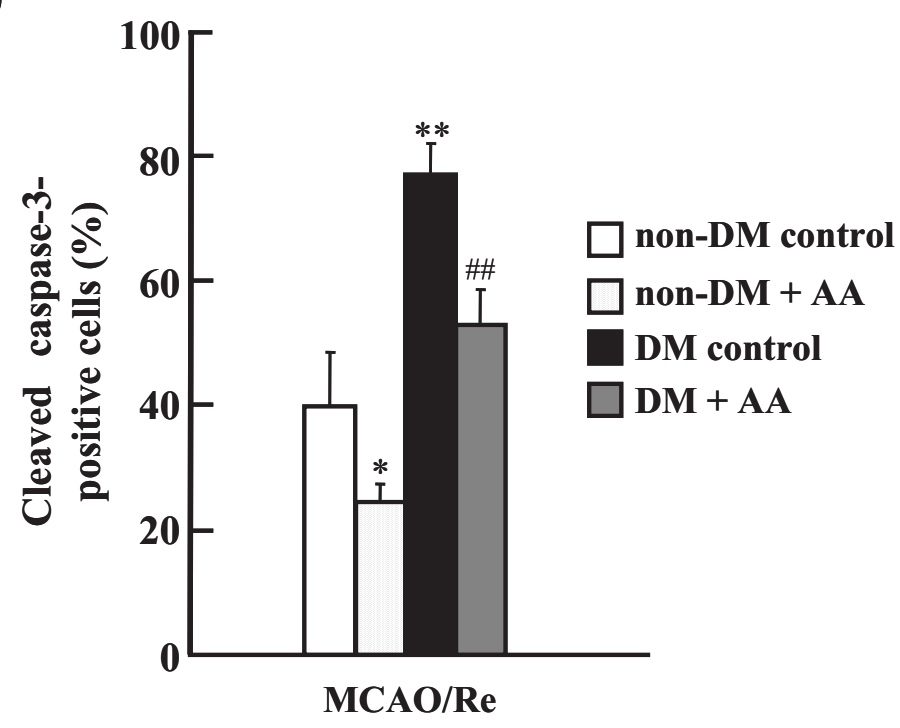

\subsection{Expression of IL-1 $\beta, T N F-\alpha$, and MPO in the Cortex}

To assess the effects of AA supplementation on the expression of proinflammatory cytokines, we performed immunohistochemical staining for IL- $1 \beta$ and TNF- $\alpha$. This experiment confirmed upregulation of the protein level of these cytokines as a result of $\mathrm{MCAO} / \mathrm{Re}$ and diabetes (Figures 5 and 6). Quantification of the immunostaining data showed that the sham-operated diabetic control group had a significant increase in IL- $1 \beta$ and TNF- $\alpha$ expression compared with the sham-operated nondiabetic control group, suggesting basal augmentation of the inflammatory response in the 
diabetic brain. MCAO/Re significantly increased the expression levels of those proinflammatory cytokines in the nondiabetic cortex. The ischemia-induced upregulation of those cytokines was markedly accelerated by diabetes: the diabetic control group showed a 15.9- and 21.0-fold increase in IL-1 $\beta$ and TNF- $\alpha$ expression, respectively, compared with the nondiabetic control group. AA supplementation significantly suppressed the basal and ischemia-enhanced expression of these cytokines in diabetic rats.

Figure 5. Effects of AA supplementation on expression of IL-1 $\beta$ in the penumbral cortex after $\mathrm{MCAO} / \mathrm{Re}$ in the brain of nondiabetic and diabetic rats. (A) Representative photographs of IL-1 $\beta$ immunostaining (red fluorescence) and staining of nuclei by TO-PRO-3 (blue fluorescence) in the cortex coronal sections of nondiabetic and diabetic rats; (B) Quantitative analysis of IL-1 $\beta$ fluorescence intensity in the cortex. The data are presented as mean $\pm \mathrm{SD}(n=3-4) .{ }^{* *} p<0.01$ compared with the nondiabetic control group. ${ }^{\#} p<0.05$ compared with the diabetic control group. DM in the figure denotes diabetic, while non-DM denotes nondiabetic.

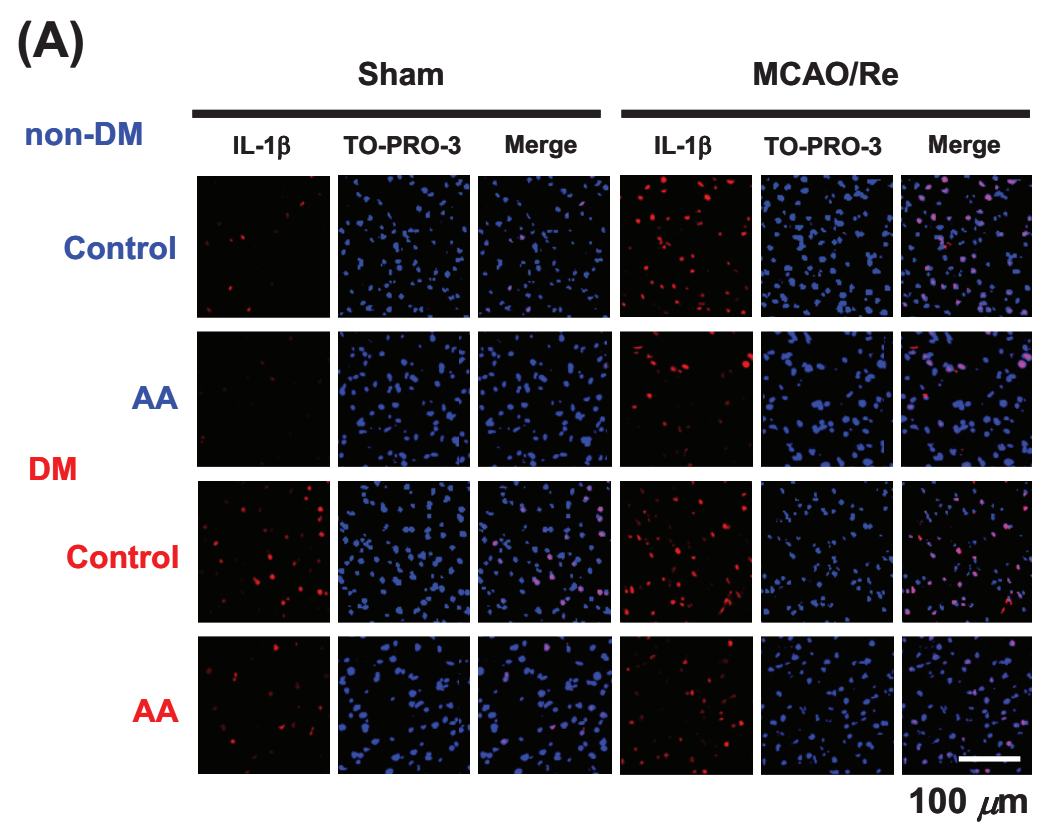

(B)

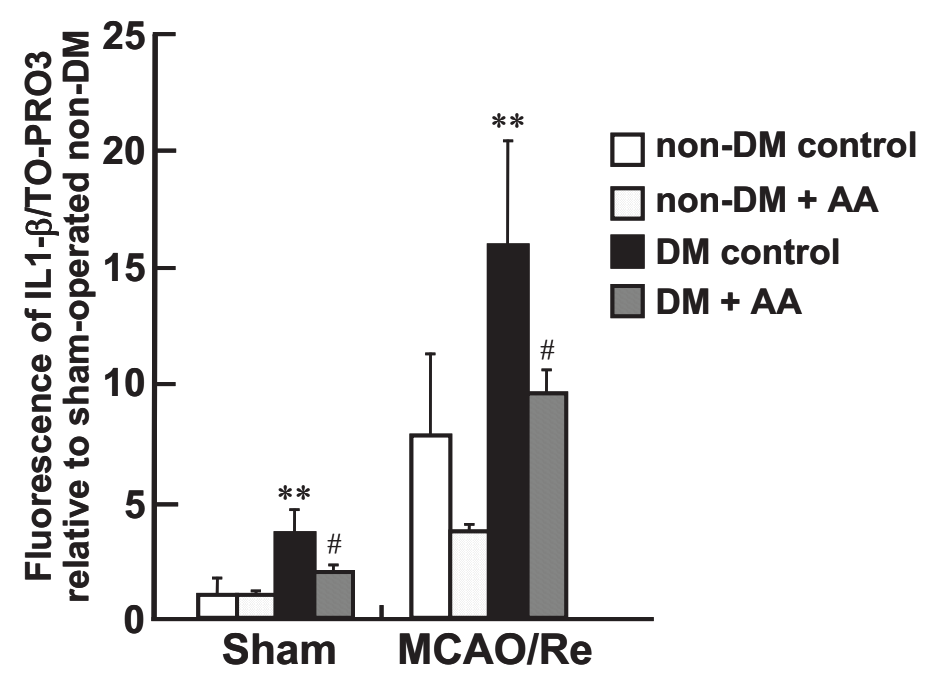


Figure 6. Effects of AA supplementation on expression of TNF- $\alpha$ in the penumbral cortex after $\mathrm{MCAO} / \mathrm{Re}$ in the brain of nondiabetic and diabetic rats. (A) Representative photographs of TNF- $\alpha$ immunostaining (red fluorescence) and staining of nuclei by TOPRO-3 (blue fluorescence) in the cortex coronal sections of nondiabetic and diabetic rats; (B) Quantitative analysis of TNF- $\alpha$ fluorescence intensity in the cortex. The data are presented as mean $\pm \mathrm{SD}(n=3-4)$. ${ }^{* *} p<0.01$ compared with the nondiabetic control group. ${ }^{\#} p<0.05$ compared with the diabetic control group. DM in the figure denotes diabetic, while non-DM denotes nondiabetic.

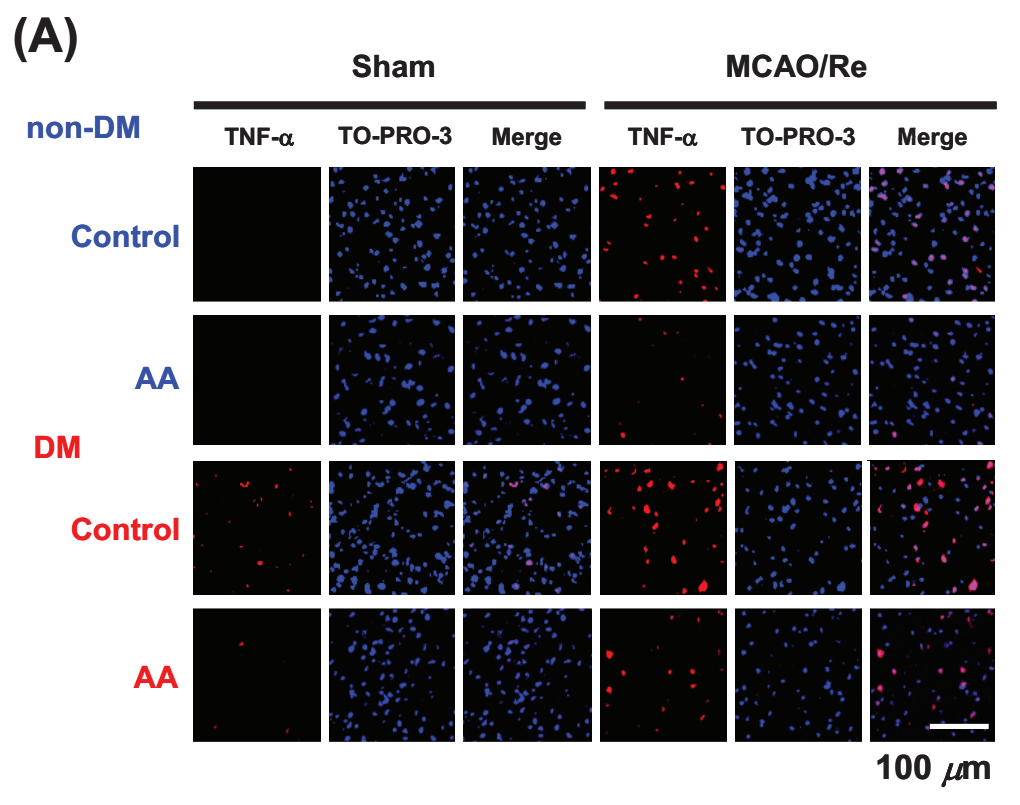

(B)

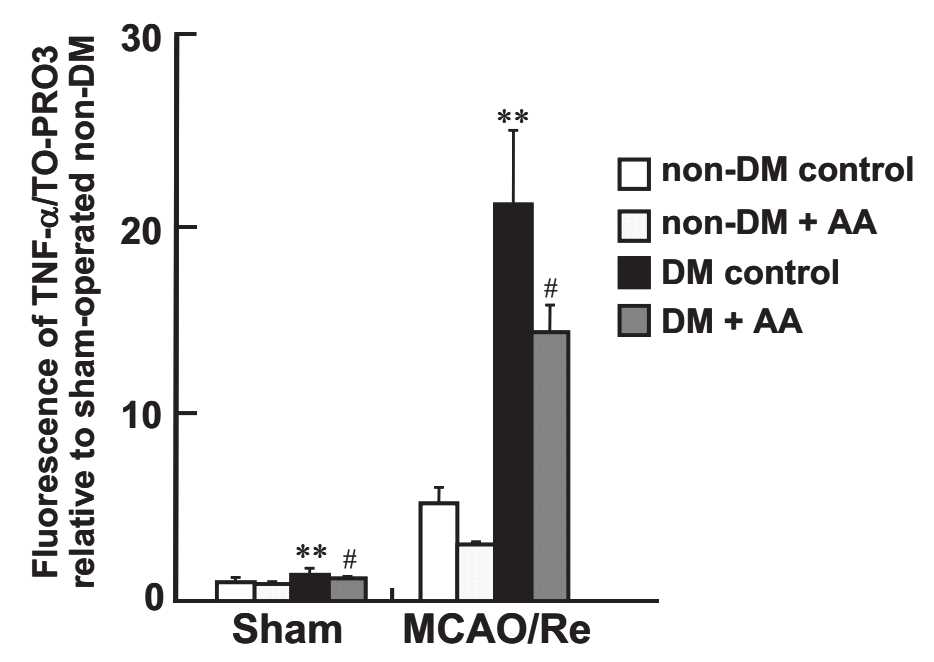

In the nondiabetic rat cortex, the protein expression of MPO was markedly upregulated after $\mathrm{MCAO} / \operatorname{Re}$ (Figure 7). AA supplementation abrogated the ischemia-induced increase in MPO expression. Compared with the nondiabetic rats, the basal level of MPO expression increased only slightly in the diabetic rats. MCAO/Re upregulated the expression of MPO to the level similar to that in the nondiabetic group. AA supplementation had no effect on the ischemia-induced increase in the MPO level in the diabetic rats. 
Figure 7. Effects of AA supplementation on MPO expression after MCAO/Re in the brain of nondiabetic and diabetic rats. (A) Representative photographs of MPO immunostaining in the cortex coronal sections of nondiabetic and diabetic rats; (B) Quantitative analysis of MPO fluorescence intensity in the cortex. The data are presented as mean $\pm \mathrm{SD}(n=3-4)$. ${ }^{*} p<0.05$ compared with the nondiabetic control group. DM in the figure denotes diabetic, while non-DM denotes nondiabetic.

(A)

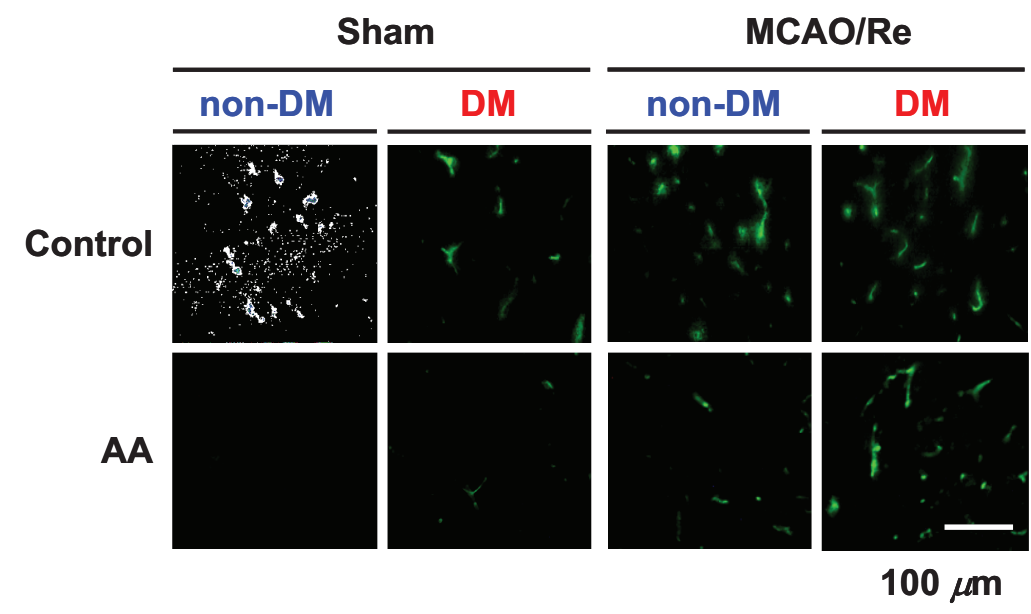

(B)

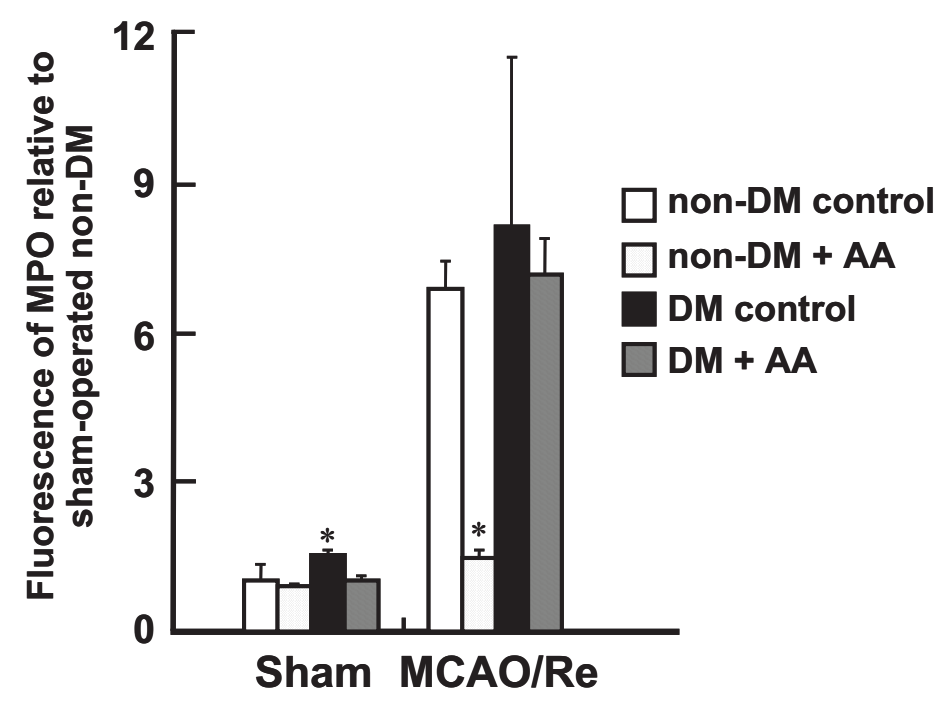

\subsection{Expression of SVCT2 and GLUT1 in the Cortex}

The expression and transport activity of SVCT2 as a specific transporter of AA in the CNS have been reported to be enhanced after $\mathrm{MCAO} / \mathrm{Re}$ in mice [20]. In addition, DHA is known to be transported across the BBB via GLUT1 [19]; thus, we investigated effects of AA supplementation on changes in the expression of SVCT2 and GLUT1 mRNA in diabetic animals with or without MCAO/Re (Figure 8). There was no significant difference in the basal expression levels of SVCT2 mRNA between the nondiabetic and diabetic groups or between each of them and their AA-supplemented groups. In the nondiabetic and nondiabetic + AA groups, the expression level of 
SVCT2 mRNA was equally upregulated at $24 \mathrm{~h}$ after MCAO/Re. In contrast, the upregulation of ischemia-induced SVCT2 mRNA expression was not observed in the diabetic control group, whereas it was observed in the diabetic + AA group. Compared with the nondiabetic control group, the diabetic state significantly downregulated the expression of GLUT1 mRNA in the cortex. AA supplementation increased the expression of GLUT1 in the nondiabetic and diabetic rats. In the nondiabetic and diabetic control groups, $\mathrm{MCAO} / \mathrm{Re}$ had a stimulating effect on the expression of GLUT1 mRNA that was similar to the effect of AA supplementation. Further enhancement by ischemia was not detected in the AA-supplemented groups.

Figure 8. Effects of AA supplementation on SVCT2 and GLUT1 mRNA expression in the ischemic penumbral cortex of nondiabetic and diabetic rats. Expression levels of SVCT2 mRNA (Slc23a2) (A) and GLUT1 mRNA (Slc2a1) (B) were assessed using real-time PCR analysis of the penumbral cortex of nondiabetic and diabetic rats after $\mathrm{MCAO} / \mathrm{Re}$. The data are presented as mean $\pm \mathrm{SD}(n=3-6) .{ }^{\dagger} p<0.05,{ }^{\dagger \dagger} p<0.01$ compared with the respective sham-operated controls. $* p<0.05, * * p<0.01$.

(A)

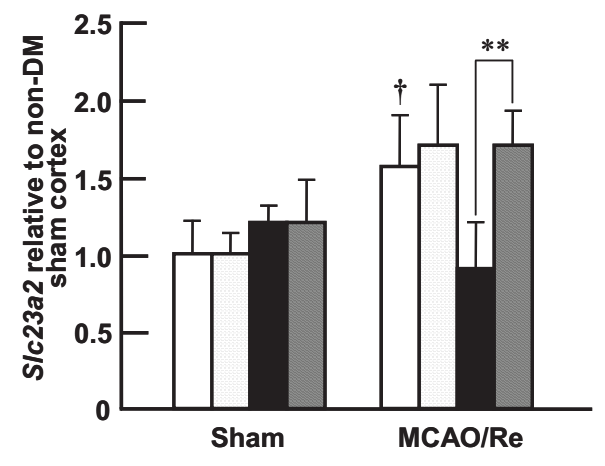

(B)

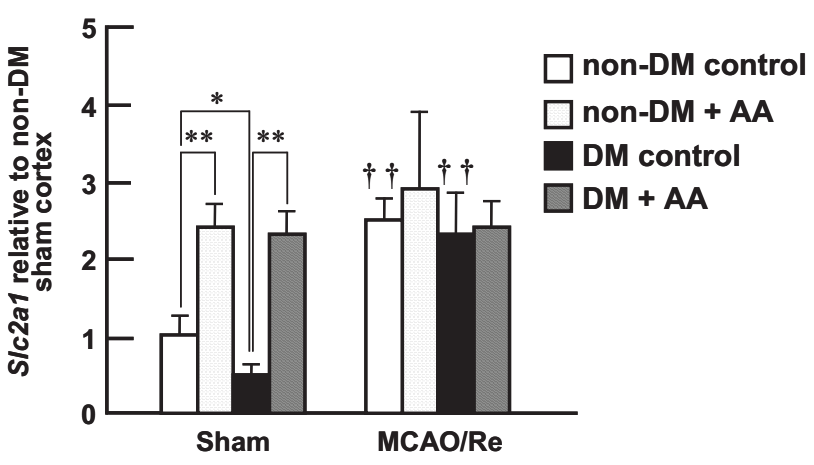

To assess the effects of AA supplementation on the expression of SVCT2 protein after $\mathrm{MCAO} / \mathrm{Re}$ in detail, the localization and the expression levels of SVCT2 in the penumbral cortex were examined by immunohistochemical staining (Figure 9A). Double immunofluorescence staining with antibodies of anti-SVCT2 and anti-NeuN, a biomarker for neurons, revealed that majority of the cells exhibiting SVCT2 expressed NeuN in the sham-operated nondiabetic and diabetic rat cortex (Figure 9A). In addition, the cells exhibiting the endothelial cell maker RECA1 showed a low level of SVCT2-immunoreactivity (Figure 9B). These expression levels in the neurons and capillary endothelial cells of SVCT2 were upregulated by $\mathrm{MCAO} / \mathrm{Re}$ in the nondiabetic rats, but not in the diabetic rats. In agreement with the results from the mRNA measurement, quantification of the immunofluorescence revealed that the expression levels of SVCT2 immunoreactivity were significantly increased in response to $\mathrm{MCAO} / \mathrm{Re}$ both in the nondiabetic control and AA-supplemented nondiabetic groups (Figure 9C). This reaction against $\mathrm{MCAO} / \mathrm{Re}$ was abrogated in the diabetic control group, whereas the AA-supplemented diabetic rats showed an increased expression of SVCT2 in the cortex after MCAO/Re. 
Figure 9. Effects of AA supplementation on SVCT2 and GLUT1 expression in the ischemic penumbral cortex of nondiabetic and diabetic rats. Expression levels of SVCT2 $(\mathbf{A}-\mathbf{C})$ and GLUT1 (D,E) were assessed using immunohistostaining of the penumbral cortex of nondiabetic and diabetic rats after MCAO/Re. Immunohistochemical expression of SVCT2 (red fluorescence) merged with NeuN (A) or with RECA1 (B) (green fluorescence); (C) Quantitative analysis of SVCT2 fluorescence intensity in the cortex. ${ }^{\#} p<0.05,{ }^{\#} p<0.01$. ${ }^{*} p<0.05, * * p<0.01$ compared with the respective sham-operated nondiabetic controls; (D) Immunohistochemical expression of GLUT1 (red fluorescence) merged with RECA1 (green fluorescence); (E) Quantitative analysis of SVCT2 fluorescence intensity in the cortex. ${ }^{*} p<0.05,{ }^{* *} p<0.01$ compared with the respective sham-operated nondiabetic controls. ${ }^{\dagger} p<0.05,{ }^{\dagger \dagger} p<0.01$ compared with the respective sham-operated diabetic controls.

\section{(A) SVCT2}

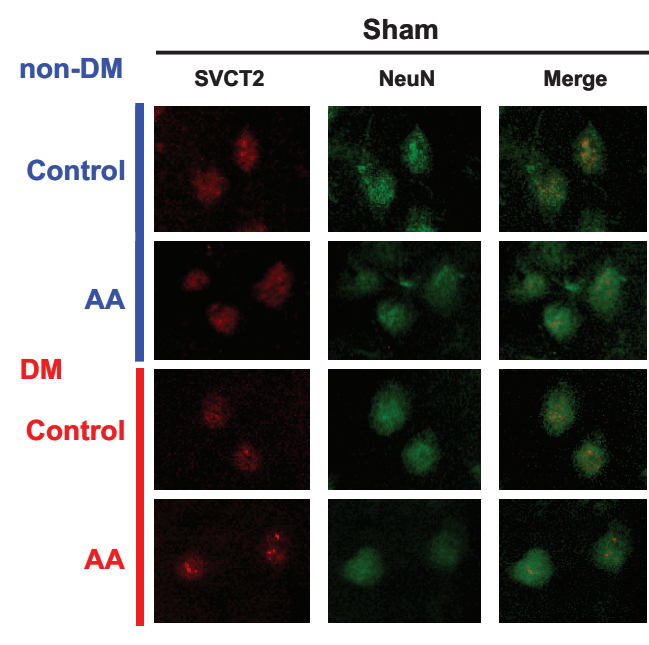

(B)

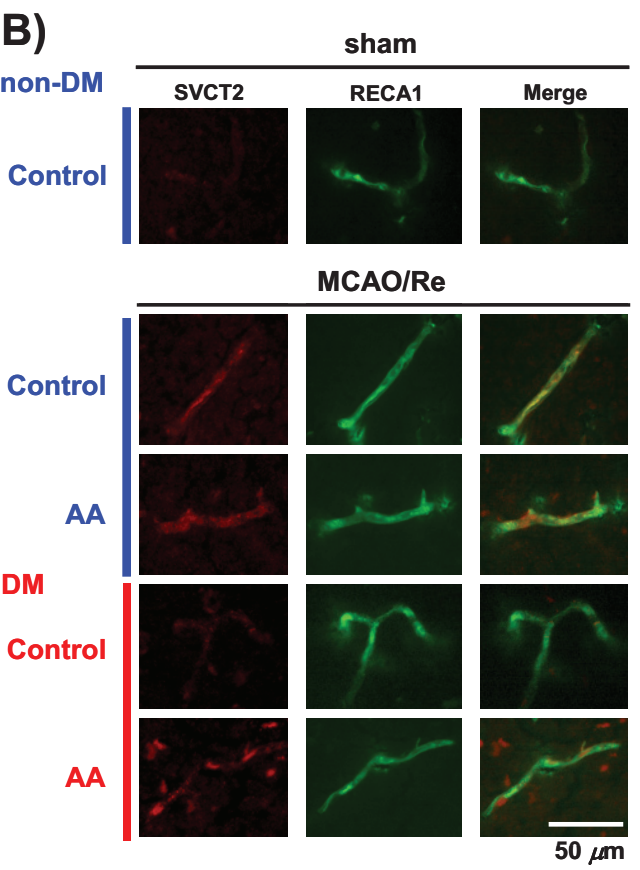

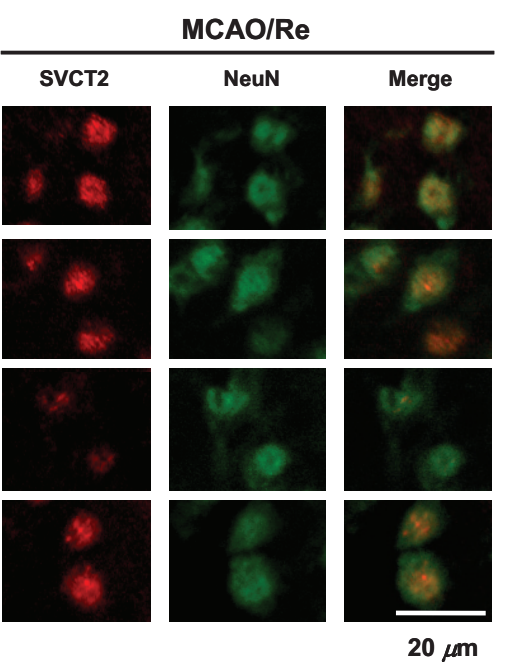

(C)
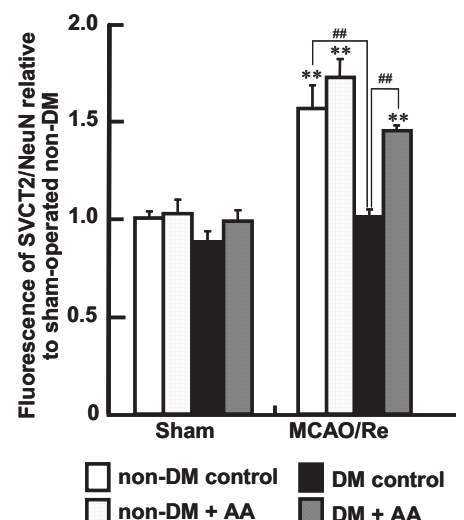
Figure 9. Cont.

\section{(D) GLUT1}

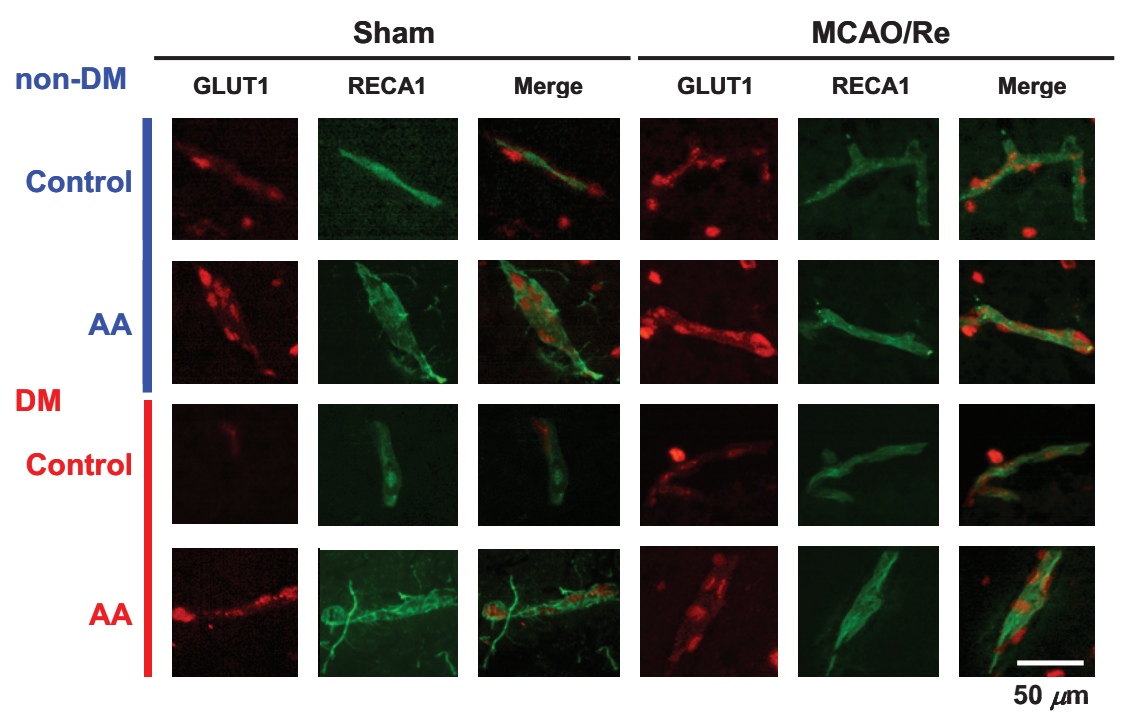

(E)

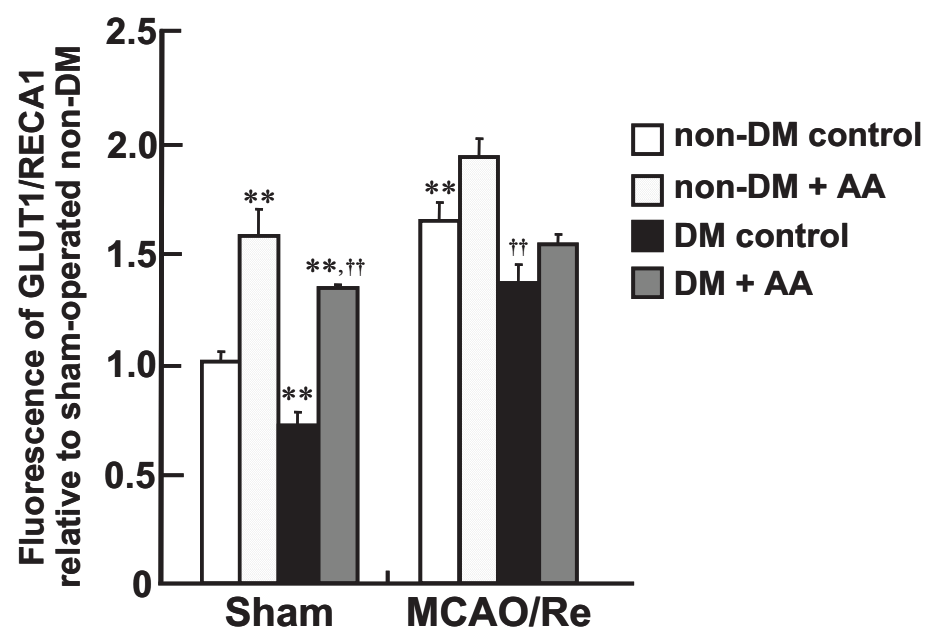

On the other hand, GLUT1 immunoreactivity was predominantly colocalized with RECA1 (Figure 9D). Some of the cells exhibiting NeuN showed a weak GLUT1-immunoreactivity (data not shown). In the sham-operated nondiabetic and diabetic groups with AA supplementation, the expression levels of GLUT1 in the capillary endothelial cells were remarkably upregulated. Additionally, MCAO/Re showed a stimulating effect on the GLUT1 expression both in the nondiabetic and diabetic cortex. Quantitative analyses of immunofluorescence in the sections revealed that the sham-operated diabetic control rat cortex had a lower expression level of GLUT1 compared with the nondiabetic rat cortex, and AA supplementation or $\mathrm{MCAO} / \mathrm{Re}$ caused a significant increase in the expression of GLUT1 (Figure 9E).

\subsection{Total AA Levels in the Plasma and Cortex}

To determine whether the changes in expression levels of SVCT2 and GLUT1 induced by AA supplementation or by MCAO/Re affect the concentrations of AA in the cortex, we measured total 
AA (AA + DHA) levels in the plasma and cortex of the experimental groups (Figure 10). It has been indicated that reduction of DHA to AA or decomposition of DHA to 2,3-diketo-1-gulonic acid is rapid in the plasma and brain, and that the concentrations of DHA in these tissues are $0 \%-2 \%$ of AA [19]. Therefore, total AA levels are considered to almost equal to the AA levels. The plasma of the sham-operated diabetic control rats had a significant smaller amount of total AA levels compared with the nondiabetic rats (Figure 10A). AA supplementation restored the decrement of the total AA levels in the diabetic rats to almost the same levels as those in the nondiabetic control rats. $\mathrm{MCAO} / \mathrm{Re}$ had little effect on the total AA levels in the plasma of all groups. In contrast, neither diabetic state nor AA supplementation affected the total AA levels in the cortex of sham-operated groups. MCAO/Re significantly decreased the total AA levels both in the nondiabetic and diabetic cortex. AA supplementation to the diabetic rats caused a significant increase in the total AA levels in the cortex in response to $\mathrm{MCAO} / \mathrm{Re}$.

Figure 10. Effects of AA supplementation on levels of total AA (AA + DHA) in the plasma and cortex of nondiabetic and diabetic rats. Total AA (AA + DHA) levels in the plasma (A) and cortex (B) measured in nondiabetic and diabetic rats after MCAO/Re. The data are presented as mean $\pm \operatorname{SD}(n=3-6) .{ }^{*} p<0.05, * * p<0.01$.

\section{(A) Plasma}

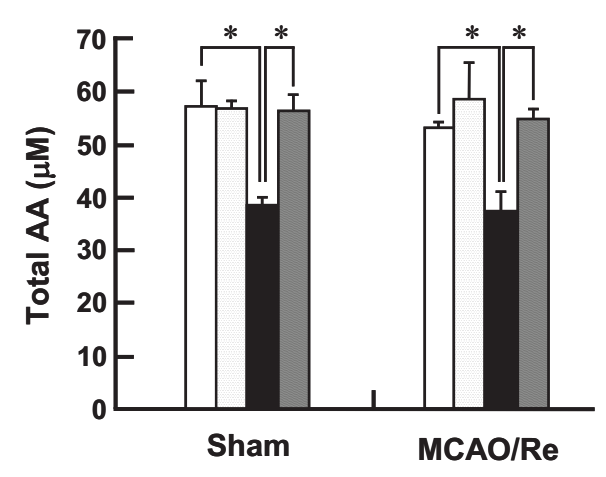

\section{(B) Cortex}

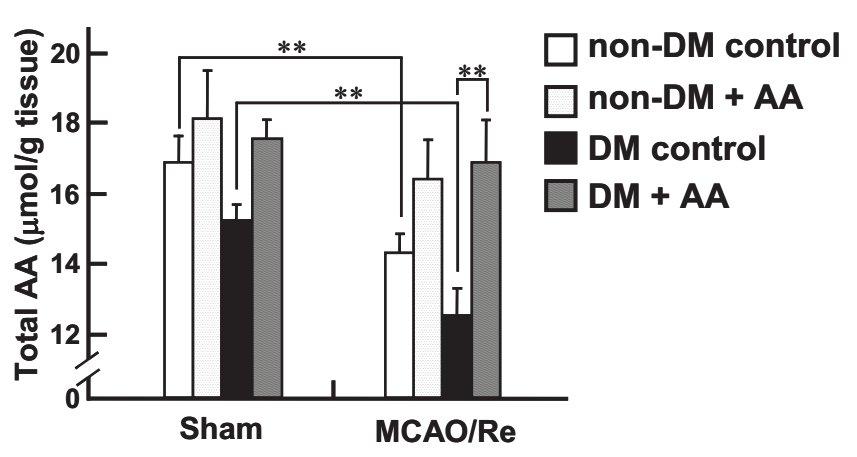

\section{Discussion}

The present study confirmed that the STZ-evoked diabetic state aggravates neuronal damage caused by a transient cerebral ischemia and subsequent reperfusion in rats. We found that diabetes enhances the production of $\mathrm{O}_{2}{ }^{-}$, activates caspase-3, and induces the expression of proinflammatory cytokines (TNF- $\alpha$ and IL-1 $\beta$ ) in the brain. These detrimental effects are markedly potentiated by cerebral ischemia and reperfusion, leading to greater infarct growth and aggravation of apoptosis and inflammation. Our results show that chronic supplementation with AA inhibits the apoptotic changes and proinflammatory responses, and attenuates the exacerbation of cerebral injury and neurological deficits in the diabetic state. These beneficial effects of AA could be attributed to its antioxidant and anti-inflammatory properties. We observed that the expression level of SVCT2 increases in response to $\mathrm{MCAO} / \mathrm{Re}$ in the nondiabetic cortex, which is accompanied by an increase in the total AA (AA + DHA) in the tissues, and that these responses are abolished in the diabetic rats. AA supplementation to the diabetic rats restored these responses to the levels of the nondiabetic rats. Therefore, supplementation with AA may enhance the transport of AA into 
neuronal cells, resulting in reinforcement of the antioxidant defense and alleviation of oxidative ischemic injury in the brain of diabetic rats.

Because rats can synthesize their own AA, unlike humans, they seem to hardly suffer from low levels of internal AA [19]. However, there is experimental evidence that STZ-induced type 1 diabetic rats have low levels of AA in the plasma, liver, kidney, and other tissues [23,34,35]. It is conceivable that the enhanced oxidative stress in diabetes consumes the circulating AA and that the resulting diminution in the AA concentration leads to further enhancement of the reactions mediated by free radicals. This study indicates that the diabetic rats have low levels of AA in the plasma, and that chronic oral pretreatment with $\mathrm{AA}$ in diabetic rats decreases the cerebral $\mathrm{O}_{2}{ }^{-}$ generation, apoptosis, and infarction induced by MCAO/Re; all of these effects could be a result of improved antioxidant status in the diabetic brain. Indeed, we observed that AA supplementation to the diabetic rats prevents the diminution of the total AA levels in the cortex associated with ischemia-reperfusion. On the other hand, we found that AA supplementation causes insufficient downregulation of proinflammatory cytokines and has no effect on MPO expression in diabetic rats after MCAO/Re. The relative low dose of AA (100 mg/kg) which we used in this study might not be efficacious against the severe inflammatory responses induced by the combination of stroke and diabetes.

Concentrations of AA in plasma and peripheral extracellular fluid after ingestion are strictly controlled by intestinal absorption, tissue transport, and renal excretion. Chen et al. [36] shows that orally administrated AA at doses $>200 \mathrm{mg} / \mathrm{kg}$ is declined to $\approx 60 \mu \mathrm{M}$ in plasma and extracellular fluid of femoral muscle in rats. In the brain, concentration of AA is relatively high compared with that in the plasma [24]. Two mechanisms of AA recruitment into the brain are known: the transport of AA through SVCT2, a specific transporter for AA, and the transport of DHA across the BBB through GLUT1 with subsequent immediate reduction in the brain. The gradation of AA concentrations between the plasma and the CNS is thought to be maintained by SVCT2, because AA is mainly present in the reduced form in the plasma. On the other hand, the cerebroprotective effects of AA supplementation remain controversial, whereas beneficial effects of DHA administration for neuronal oxidative stress and inflammation seem to be well established [37-39]. Previous studies do not support acute cerebroprotective effects of treatment with AA under normal conditions because AA is not transported into the brain rapidly, owing to the high affinity and low ability for AA transport by SVCT2. Ahn and colleagues [40] reported that chronic AA supplementation has little or no effect on elevated oxidative stress in STZ-induced diabetic brain, whereas it is efficacious in the liver and kidneys. This phenomenon can be attributed to a lack of SVCT2 expression in endothelial cells of the BBB [41,42]. Recently, the evidence indicating that AA supplementation suppresses diabetes or ischemia-induced oxidative damage in the hippocampus and cortex of experimental animals has been accumulated [43-45]. These regions are not only vulnerable to oxidative stress but abundant in SVCT2 compared to other regions in the brain, namely, there may be some kinds of differences in sensitivity for AA supplementation depending on brain regions. Therefore, it seems possible that previous experiments using whole-brain samples might have not been adequate to detect the cerebroprotective effects of AA supplementation.

The system of DHA transport through GLUT1 in the BBB may not substantially contribute to total brain AA concentrations under normal conditions, because DHA concentrations in the plasma 
are usually only $0 \%-2 \%$ of AA concentrations $[19,46]$. Nonetheless, during oxidative stress in the CNS such as stroke and/or treatment with DHA, this route could play a significant role in the recruitment of AA $[39,47]$. Our study demonstrated that diabetes downregulates the expression of GLUT1 in the cortex, in line with previous studies [21,25]. AA supplementation to the diabetic rats markedly upregulated the basal expression of GLUT1; however, it exerted no significant increase in the total AA levels in the cortex of the rats. Taken together, contribution of the DHA transport system through GLUT1 to the cerebroprotection seems to be limited in the diabetic rats. The upregulation of GLUT1 expression by AA supplementation was observed also in the cortex of nondiabetic sham-operated group, despite that there were little changes in the total AA levels in the plasma of the rats. These results may indicate that orally supplemented AA has no direct enhancing effect on the expression of GLUT1 in the nondiabetic rats. However, AA concentration in the plasma is considered to increase within several hours after oral administration and decline to the basal level $24 \mathrm{~h}$ after the administration by excretion of the excess of AA, at which we collected the plasma samples from the experimental animals. Therefore, one possibility is that intermittent increases in AA concentration in the plasma repeated for two weeks could stimulate expression of GLUT1 in the cortex. Future research needs to be done with regard to the detailed mechanism underlying the upregulation of GLUT1 expression by AA supplementation.

SVCT2 has been shown to be crucial for maintaining AA for protection against oxidative stress in the CNS. It has been shown that cultured cells lacking this protein are vulnerable to oxidative stress [18]. In the present study, diabetes itself caused no apparent changes in the expression of SVCT2 in the cortex. On the other hand, after MCAO/Re, the expression of SVCT2 in the penumbral cortex, of which the majority seems to be localized in neurons, is upregulated in the nondiabetic rats. Gess and colleagues [20] have demonstrated that SVCT2 is specifically expressed in brain capillary endothelial cells and transports AA across the BBB during the subacute phase after $\mathrm{MCAO} / \mathrm{Re}$ in mice. We also observed that expression levels of SVCT2 in capillary endothelial cells are increased after $\mathrm{MCAO} / \mathrm{Re}$ in the nondiabetic rat cortex. This has been proposed as one of the mechanisms that protect the brain against oxidative injury under pathological conditions such as stroke and neurodegeneration. In contrast, we found that the upregulation of SVCT2 expression was abrogated in the diabetic rats, suggesting that the cerebroprotective mechanism via AA recruitment may be disrupted in the diabetic state. The abnormality of endothelial cell function in STZ-diabetic rats has been indicated to be related to the ROS generation in microvascular walls [17]. Seno and coworkers [48] demonstrated that the inflammatory cytokines TNF- $\alpha$ and IL-1 $\beta$ suppress the transport of AA through SVCT2 in human umbilical vein endothelial cells. Indeed, we detected a robust increase in the expression these cytokines in the brain of diabetic rats after $\mathrm{MCAO} / \mathrm{Re}$, which may reverse the upregulation of SVCT2 by MCAO/Re itself. AA supplementation to the diabetic rats upregulated the expression of SVCT2 in the cortex and restored the total AA levels to the nondiabetic levels. The antioxidative properties of AA may protect endothelial functions including the transcriptional regulation of SVCT2 against the exacerbated ischemic oxidative injury and the enhanced proinflammatory responses in the diabetic state, and may improve AA transport through SVCT2 in the BBB. Further experiments using radiolabeled tracers or other rodent models such as $\mathrm{SVCT}^{+/-}$mice are needed to elucidate these cerebroprotective mechanisms of AA, including its effects on the specific transport of AA into the diabetic brain. 


\section{Conclusions}

In the present study, we found that chronic AA supplementation inhibits the apoptotic and proinflammatory changes and attenuates the exacerbation of cerebral injury and neurological deficits in the diabetic state. These phenomena could be attributed to the antioxidant activity and anti-inflammatory effects of AA. Diabetes repressed the enhancement of SVCT2 expression induced by ischemia-reperfusion in the neurons and capillary endothelial cells, whereas the downregulated expression of SVCT2 was restored to control levels by AA supplementation. Therefore, chronic AA supplementation may enhance and normalize the transport of AA into the CNS and may thus reinforce the antioxidant defense and alleviate oxidative ischemic injury in the brain of diabetic rats.

\section{Acknowledgments}

This study was supported by Grants-in-Aid for Scientific Research from the Japan Society for the Promotion of Science (No. 23790750 and 25460910).

\section{Conflicts of Interest}

The authors declare no conflict of interest.

\section{References}

1. Baynes, J.W. Role of oxidative stress in development of complications in diabetes. Diabetes 1991, 40, 405-412.

2. Stephens, J.W.; Khanolkar, M.P.; Bain, S.C. The biological relevance and measurement of plasma markers of oxidative stress in diabetes and cardiovascular disease. Atherosclerosis 2009, 202, 321-329.

3. Biller, J.; Love, B.B. Diabetes and stroke. Med. Clin. N. Am. 1994, 77, 95-110.

4. Vinik, A.; Flemmer, M.J. Diabetes and macrovascular disease. J. Diabetes Compl. 2002, 16, 235-245.

5. Niizuma, K.; Endo, H.; Chan, P.H. Oxidative stress and mitochondrial dysfunction as determinants of ischemic neuronal death and survival. J. Neurochem. 2009, 109, 133-138.

6. Nakka, V.P.; Gusain, A.; Mehta, S.L.; Raghubir, R. Molecular mechanisms of apoptosis in cerebral ischemia: Multiple neuroprotective opportunities. Mol. Neurobiol. 2008, 37, 7-38.

7. Niizuma, K.; Yoshioka, H.; Chen, H.; Kim, G.S.; Jung, J.E.; Katsu, M.; Okami, N.; Chan, P.H. Mitochondrial and apoptotic neuronal death signaling pathways in cerebral ischemia. Biochim. Biophys. Acta 2010, 1802, 92-99.

8. Warner, D.S.; Sheng, H.; Batinić-Haberle, I. Oxidants, antioxidants and the ischemic brain. J. Exp. Biol. 2004, 207, 3221-3231.

9. Rolo, A.P.; Palmeira, C.M. Diabetes and mitochondrial function: Role of hyperglycemia and oxidative stress. Toxicol. Appl. Pharmacol. 2006, 212, 167-178.

10. Brown, G.C.; Neher, J.J. Inflammatory neurodegeneration and mechanisms of microglial killing of neurons. Mol. Neurobiol. 2010, 41, 242-247. 
11. Saeed, S.A.; Shad, K.F.; Saleem, T.; Javed, F.; Khan, M.U. Some new prospects in the understanding of the molecular basis of the pathogenesis of stroke. Exp. Brain Res. 2007, 182, $1-10$.

12. Breckwoldt, M.O.; Chen, J.W.; Stangenberg, L.; Aikawa, E.; Rodriguez, E.; Qiu, S.; Moskowitz, M.A.; Weissleder, R. Tracking the inflammatory response in stroke in vivo by sensing the enzyme myeloperoxidase. Proc. Natl. Acad. Sci. USA 2008, 105, 18584-18589.

13. Jin, H.M.; Zhou, D.C.; Gu, H.F.; Qiao, Q.Y.; Fu, S.K.; Liu, X.L.; Pan, Y. Antioxidant $\mathrm{N}$-acetylcysteine protects pancreatic $\beta$-cells against aldosterone-induced oxidative stress and apoptosis in female db/db mice and insulin-producing MIN6 cells. Endocrinology 2013, 154, 4068-4077.

14. Bhattacharya, S.; Gachhui, R.; Sil, P.C. Effect of Kombucha, a fermented black tea in attenuating oxidative stress mediated tissue damage in alloxan induced diabetic rats. Food Chem. Toxicol. 2013, 60, 328-340.

15. Feng, B.; Yan, X.F.; Xue, J.L.; Xu, L.; Wang, H. The Protective effects of $\alpha$-lipoic acid on kidneys in type 2 diabetic goto-kakisaki rats via reducing oxidative stress. Int. J. Mol. Sci. 2013, 14, 6746-6756.

16. Yamamoto, Y.; Yamamoto, H. RAGE-mediated inflammation, type 2 diabetes, and diabetic vascular complication. Front. Endocrinol. 2013, 4, doi:10.3389/fendo.2013.00105.

17. Sridulyakul, P.; Wongeak-In, N.; Patumraj, S. Correlations between endothelial functions and ROS detection in diabetic microvascular wall: Early and late ascorbic acid supplementation. Int. J. Vasc. Med. 2012, 2012, doi:10.1155/2012/709695.

18. Qiu, S.; Li, L.; Weeber, E.J.; May, J.M. Ascorbate transport by primary cultured neurons and its role in neuronal function and protection against excitotoxicity. J. Neurosci. Res. 2007, 85, 1046-1056.

19. May, J.M. Vitamin C transport and its role in the central nervous system. Subcell. Biochem. 2012, 56, 85-103.

20. Gess, B.; Sevimli, S.; Strecker, J.K.; Young, P.; Schäbitz, W.R. Sodium-dependent vitamin C transporter 2 (SVCT2) expression and activity in brain capillary endothelial cells after transient ischemia in mice. PLoS One 2011, 6, e17139.

21. Zhang, W.W.; Zhang, L.; Hou, W.K.; Xu, Y.X.; Xu, H.; Lou, F.C.; Zhang, Y.; Wang, Q. Dynamic expression of glucose transporters 1 and 3 in the brain of diabetic rats with cerebral ischemia reperfusion. Chin. Med. J. 2009, 122, 1996-2001.

22. Attele, A.S.; Zhou, Y.P.; Xie, J.T.; Wu, J.A.; Zhang, L.; Dey, L.; Pugh, W.; Rue, P.A.; Polonsky, K.S.; Yuan, C.S. Antidiabetic effects of Panax ginseng berry extract and the identification of an effective component. Diabetes 2002, 51, 1851-1858.

23. Takahashi, N.; Morimoto, S.; Okigaki, M.; Seo, M.; Someya, K.; Morita, T.; Matsubara, H.; Sugiura, T.; Iwasaka, T. Decreased plasma level of vitamin C in chronic kidney disease: Comparison between diabetic and non-diabetic patients. Nephrol. Dial. Transplant. 2011, 26, $1252-1257$.

24. Harrison, F.E.; May, J.M. Vitamin C function in the brain: Vital role of the ascorbate transporter SVCT2. Free Radic. Biol. Med. 2009, 46, 719-730. 
25. Iwata, N.; Okazaki, M.; Kamiuchi, S.; Hibino, Y. Protective effects of oral administrated ascorbic acid against oxidative stress and neuronal damage after cerebral ischemia/reperfusion in diabetic rats. J. Health Sci. 2010, 56, 20-30.

26. Iwata, N.; Okazaki, M.; Kasahara, C.; Kamiuchi, S.; Suzuki, F.; Iizuka, H.; Hibino, Y. Protective effects of a water-soluble extract from culture medium of Ganoderma lucidum mycelia against neuronal damage after cerebral ischemia/reperfusion in diabetic rats. J. Jpn. Soc. Nutr. Food Sci. 2008, 61, 119-127.

27. Yamazaki, Y.; Harada, S.; Tokuyama, S. Post-ischemic hyperglycemia exacerbates the development of cerebral ischemic neuronal damage through the cerebral sodium-glucose transporter. Brain Res. 2012, 13, 113-120.

28. Muranyi, M.; Ding, C.; He, Q.; Lin, Y.; Li, P.A. Streptozotocin-induced diabetes causes astrocyte death after ischemia and reperfusion injury. Diabetes 2006, 55, 349-355.

29. Li, Z.; Iwai, M.; Wu, L.; Liu, H.W.; Chen, R.; Jinno, T.; Suzuki, J.; Tsuda, M.; Gao, X.Y.; Okumura, M.; et al. Fluvastatin enhances the inhibitory effects of a selective AT1 receptor blocker, valsartan, on atherosclerosis. Hypertension 2004, 44, 758-763.

30. Faraco, G.; Fossati, S.; Bianchi, M.E.; Patrone, M.; Pedrazzi, M.; Sparatore, B.; Moroni, F.; Chiarugi, A. High mobility group box 1 protein is released by neural cells upon different stresses and worsens ischemic neurodegeneration in vitro and in vivo. J. Neurochem. 2007, 103, 590-603.

31. Caprile, T.; Salazar, K.; Astuya, A.; Cisternas, P.; Silva-Alvarez, C.; Montecinos, H.; Millán, C.; de Los Angeles García, M.; Nualart, F. The $\mathrm{Na}^{+}$-dependent L-ascorbic acid transporter SVCT2 expressed in brainstem cells, neurons, and neuroblastoma cells is inhibited by flavonoids. J. Neurochem. 2009, 108, 563-577.

32. Liu, K.; Mori, S.; Takahashi, H.K.; Tomono, Y.; Wake, H.; Kanke, T.; Sato, Y.; Hiraga, N.; Adachi, N.; Yoshino, T.; et al. Anti-high mobility group box 1 monoclonal antibody ameliorates brain infarction induced by transient ischemia in rats. FASEB J. 2007, 21, 3904-3916.

33. Bradley, D.W.; Emery, G.; Maynard, J.E. Vitamin C in plasma: A comparative study of the vitamin stabilized with trichloroacetic acid or metaphosphoric acid and the effects of storage at $-70^{\circ},-20^{\circ}, 4^{\circ}$, and $25^{\circ}$ on the stabilized vitamin. Clin. Chim. Acta 1973, 44, 47-52.

34. Clarke, J.; Snelling, J.; Ioannides, C.; Flatt, P.R.; Barnett, C.R. Effect of vitamin C supplementation on hepatic cytochrome P450 mixed-function oxidase activity in streptozotocin-diabetic rats. Toxicol. Lett. 1996, 89, 249-256.

35. Kashiba, M.; Oka, J.; Ichikawa, R.; Kasahara, E.; Inayama, T.; Kageyama, A.; Kageyama, H.; Osaka, T.; Umegaki, K.; Matsumoto, A.; et al. Impaired ascorbic acid metabolism in streptozotocin-induced diabetic rats. Free Radic. Biol. Med. 2002, 33, 1221-1230.

36. Chen, Q.; Espey, M.G.; Sun, A.Y.; Lee, J.H.; Krishna, M.C.; Shacter, E.; Choyke, P.L.; Pooput, C.; Kirk, K.L.; Buettner, G.R.; et al. Ascorbate in pharmacologic concentrations selectively generates ascorbate radical and hydrogen peroxide in extracellular fluid in vivo. Proc. Natl. Acad. Sci. USA 2007, 104, 8749-8754. 
37. Huang, J.; Agus, D.B.; Winfree, C.J.; Kiss, S.; Mack, W.J.; McTaggart, R.A.; Choudhri, T.F.; Kim, L.J.; Mocco, J.; Pinsky, D.J.; et al. Dehydroascorbic acid, a blood-brain barrier transportable form of vitamin $\mathrm{C}$, mediates potent cerebroprotection in experimental stroke. Proc. Natl. Acad. Sci. USA 2001, 98, 10989-10991.

38. Mack, W.J.; Mocco, J.; Ducruet, A.F.; Laufer, I.; King, R.G.; Zhang, Y.; Guo, W.; Pinsky, D.J.; Connolly, E.S., Jr. A cerebroprotective dose of intravenous citrate/sorbitol-stabilized dehydroascorbic acid is correlated with increased cerebral ascorbic acid and inhibited lipid peroxidation after murine reperfused stroke. Neurosurgery 2006, 59, 383-388.

39. Bémeur, C.; Ste-Marie, L.; Desjardins, P.; Vachon, L.; Butterworth, R.F.; Hazell, A.S.; Montgomery, J. Dehydroascorbic acid normalizes several markers of oxidative stress and inflammation in acute hyperglycemic focal cerebral ischemia in the rat. Neurochem. Int. 2005, 46, 399-407.

40. Ahn, T.; Yun, C.H.; Oh, D.B. Tissue-specific effect of ascorbic acid supplementation on the expression of cytochrome P450 2E1 and oxidative stress in streptozotocin-induced diabetic rats. Toxicol. Lett. 2006, 166, 27-36.

41. García Mde, L.; Salazar, K.; Millán, C.; Rodríguez, F.; Montecinos, H.; Caprile, T.; Silva, C.; Cortes, C.; Reinicke, K.; Vera, J.C.; et al. Sodium vitamin C cotransporter SVCT2 is expressed in hypothalamic glial cells. Glia 2005, 50, 32-47.

42. Mun, G.H.; Kim, M.J.; Lee, J.H.; Kim, H.J.; Chung, Y.H.; Chung, Y.B.; Kang, J.S.; Hwang, Y.I.; Oh, S.H.; Kim, J.G.; et al. Immunohistochemical study of the distribution of sodium-dependent vitamin C transporters in adult rat brain. J. Neurosci. Res. 2006, 83, 919-928.

43. Bhutada, P.; Mundhada, Y.; Bansod, K.; Tawari, S.; Patil, S.; Dixit, P.; Umathe, S.; Mundhada, D. Protection of cholinergic and antioxidant system contributes to the effect of berberine ameliorating memory dysfunction in rat model of streptozotocin-induced diabetes. Behav. Brain Res. 2011, 220, 30-41.

44. Miura, S.; Ishida-Nakajima, W.; Ishida, A.; Kawamura, M.; Ohmura, A.; Oguma, R.; Sato, Y.; Takahashi, T. Ascorbic acid protects the newborn rat brain from hypoxic-ischemia. Brain Dev. 2009, 31, 307-317.

45. Jafari Anarkooli, I.; Sankian, M.; Vahedi, F.; Bonakdaran, S.; Varasteh, A.R.; Haghir, H. Evaluation of insulin and ascorbic acid effects on expression of Bcl-2 family proteins and caspase-3 activity in hippocampus of STZ-induced diabetic rats. Cell Mol. Neurobiol. 2009, 29, 133-140.

46. Dhariwal, K.R.; Hartzell, W.O.; Levine, M. Ascorbic acid and dehydroascorbic acid measurements in human plasma and serum. Am. J. Clin. Nutr. 1991, 54, 712-716.

47. Agus, D.B.; Gambhir, S.S.; Pardridge, W.M.; Spielholz, C.; Baselga, J.; Vera, J.C.; Golde, D.W. Vitamin C crosses the blood-brain barrier in the oxidized form through the glucose transporters. J. Clin. Investig. 1997, 100, 2842-2848.

48. Seno, T.; Inoue, N.; Matsui, K.; Ejiri, J.; Hirata, K.; Kawashima, S.; Yokoyama, M. Functional expression of sodium-dependent vitamin $\mathrm{C}$ transporter 2 in human endothelial cells. J. Vasc. Res. 2004, 41, 345-351. 
Reprinted from Nutrients. Cite as: Carr, A.C.; Vissers, M.C.M. Synthetic or Food-Derived Vitamin C-Are They Equally Bioavailable? Nutrients 2013, 5, 4284-4304.

Review

\title{
Synthetic or Food-Derived Vitamin C-Are They Equally Bioavailable?
}

\section{Anitra C. Carr * and Margreet C. M. Vissers}

Centre for Free Radical Research, Department of Pathology and Biomedical Science, University of Otago, Christchurch, P.O. Box 4345, Christchurch 8140, New Zealand;

E-Mail: margreet.vissers@otago.ac.nz

* Author to whom correspondence should be addressed; E-Mail: anitra.carr@otago.ac.nz; Tel.: +64-3-378-6498; Fax: +64-3-378-6540.

Received: 30 August 2013; in revised form: 22 September 2013 / Accepted: 14 October 2013 / Published: 28 October 2013

\begin{abstract}
Vitamin C (ascorbate) is an essential water-soluble micronutrient in humans and is obtained through the diet, primarily from fruits and vegetables. In vivo, vitamin C acts as a cofactor for numerous biosynthetic enzymes required for the synthesis of amino acid-derived macromolecules, neurotransmitters, and neuropeptide hormones, and is also a cofactor for various hydroxylases involved in the regulation of gene transcription and epigenetics. Vitamin C was first chemically synthesized in the early 1930s and since then researchers have been investigating the comparative bioavailability of synthetic versus natural, food-derived vitamin C. Although synthetic and food-derived vitamin $\mathrm{C}$ is chemically identical, fruit and vegetables are rich in numerous nutrients and phytochemicals which may influence its bioavailability. The physiological interactions of vitamin $\mathrm{C}$ with various bioflavonoids have been the most intensively studied to date. Here, we review animal and human studies, comprising both pharmacokinetic and steady-state designs, which have been carried out to investigate the comparative bioavailability of synthetic and food-derived vitamin $\mathrm{C}$, or vitamin $\mathrm{C}$ in the presence of isolated bioflavonoids. Overall, a majority of animal studies have shown differences in the comparative bioavailability of synthetic versus natural vitamin $\mathrm{C}$, although the results varied depending on the animal model, study design and body compartments measured. In contrast, all steady state comparative bioavailability studies in humans have shown no differences between synthetic and natural vitamin $\mathrm{C}$, regardless of the subject population, study design or intervention used. Some pharmacokinetic studies in humans have shown transient and small comparative differences between synthetic and natural
\end{abstract}


vitamin $\mathrm{C}$, although these differences are likely to have minimal physiological impact. Study design issues and future research directions are discussed.

Keywords: ascorbate; dietary vitamin C; bioavailability; animal studies; human studies; bioflavonoids

\section{Introduction}

Vitamin C (ascorbate) is an essential water-soluble micronutrient in humans and is obtained through the diet primarily from fruits and vegetables [1]. In vivo, it acts as a cofactor for numerous biosynthetic enzymes required for the synthesis of amino acid-derived macromolecules, neurotransmitters and neuropeptide hormones [2], and for various hydroxylases involved in the regulation of gene transcription and epigenetics [3,4]. Vitamin $\mathrm{C}$ is concentrated from the plasma into the body's organs and is found in particularly high concentrations in the pituitary and adrenal glands and in the corpus luteum [5], although skeletal muscle, brain, and liver comprise the largest body pools [6]. Most animals can synthesize vitamin $\mathrm{C}$ from glucose in the liver [7]; however, humans and a small selection of animal species have lost the ability to synthesize vitamin $\mathrm{C}$ due to mutations in the gene encoding L-gulono- $\gamma$-lactone oxidase, the terminal enzyme in the vitamin $\mathrm{C}$ biosynthetic pathway [8]. Therefore, an adequate and regular dietary intake is essential to prevent hypovitaminosis $\mathrm{C}$ and the potentially fatal deficiency disease, scurvy [9].

In the mid 1700s the Royal Navy surgeon James Lind carried out controlled dietary trials and determined that citrus fruit could cure individuals with scurvy (reviewed in [10]). However, it wasn't until the early 1900s that experimental scurvy was first produced in guinea pigs through dietary restriction and shown to be prevented by feeding the animals fresh fruits and vegetables. In the early 1930s vitamin C was isolated from fruit and vegetables and adrenal cortex and was named "hexuronic acid", which was shown to cure scurvy in guinea pigs and was subsequently renamed ascorbic acid to reflect its anti-scorbutic properties. Vitamin C was first chemically synthesized in 1933 [10] and, since the mid 1930s, the question of the comparative bioavailability of synthetic versus natural, food-derived vitamin $\mathrm{C}$ in animal models and human subjects has been a point of consideration.

The bioavailability of dietary vitamin $\mathrm{C}$ represents the proportion of the micronutrient that is absorbed by the intestines and is available for metabolic processes within the body. In vivo vitamin $\mathrm{C}$ levels are a function of uptake, metabolism, and excretion (see [11] for an excellent review of these processes). Vitamin $\mathrm{C}$ is actively transported into the body via two sodium-dependent vitamin $\mathrm{C}$ transporters, SVCT1 and SVCT2 [12,13]. These transporters exhibit different tissue distributions and uptake kinetics. SVCT1 is expressed in epithelial tissues and is primarily responsible for intestinal uptake and renal reabsorption of vitamin $\mathrm{C}$, the latter helping to maintain whole body homeostasis [13]. SVCT2 is expressed in specialized and metabolically active tissues and is required for delivery of vitamin $\mathrm{C}$ to tissues with a high demand for the vitamin either for enzymatic reactions [2] and/or to help protect these tissues from oxidative stress [13]. Both of these transporters show significantly more affinity for the L- versus D-isoform of vitamin C (Figure 1) [12,14], and this 
selectivity likely explains earlier observations of significantly lower tissue accumulation and anti-scorbutic activity of D-ascorbic acid in guinea pigs $[15,16]$. Although D-ascorbic acid is a commonly added food preservative [17], administration of D- and L-ascorbic acid together does not affect the bioavailability of the latter in humans [18].

Through its action as a reducing agent and antioxidant, ascorbate undergoes one and two electron oxidations to produce the ascorbyl radical and dehydroascorbic acid (DHA) (Figure 1). Recent research has shown that DHA can be taken up by the facilitative glucose transporters GLUT2 and GLUT8 in the small intestine [19]. Cells are also able to transport DHA via GLUT1 and GLUT3 [20,21], followed by intracellular reduction to ascorbate [22,23]. However, transport via the GLUTs is in competition with glucose which is at relatively high concentrations throughout the body and although different fruits and vegetables have been shown to contain relatively high amounts of DHA [24], the in vivo contribution of DHA is uncertain due to its minimal circulating and organ levels $[25,26]$ (although white blood cells may be an exception to this) $[27,28]$.

Figure 1. Vitamin $\mathrm{C}$ in its reduced form (ascorbic acid), shown as both its L- and D-isomers, and its two electron oxidation form (dehydroascorbic acid, DHA). DHA can be readily reduced back to ascorbic acid in vivo via both chemical and enzymatic pathways [23].

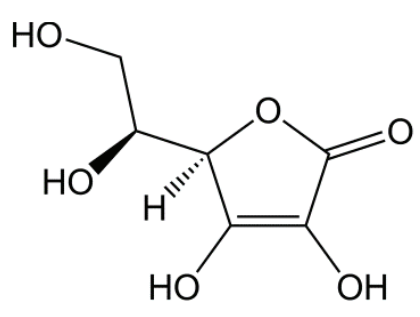

L-Ascorbic Acid

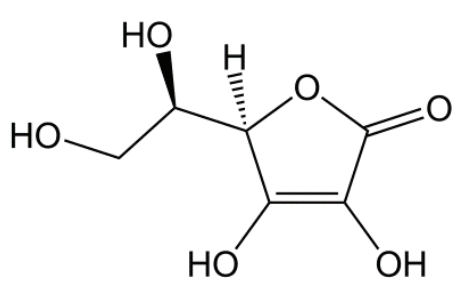

D-Ascorbic acid

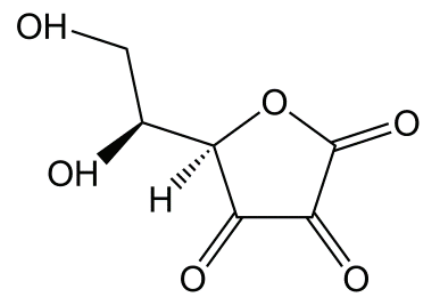

Dehvdroascorbic acid

Synthetic and food-derived vitamin $\mathrm{C}$ is chemically identical. However, fruit and vegetables are rich in numerous micronutrients (vitamins and minerals), dietary fiber, and phytochemicals (e.g., bioflavonoids), and the presence of some of these may affect the bioavailability of vitamin C. Vitamin $\mathrm{C}$ has long been known to interact with vitamin $\mathrm{E}$ by reducing the tocopheroxyl radical and regenerating native tocopherol [29]. Some fruit, such as kiwifruit, contain relatively high amounts of vitamin $\mathrm{E}$ and one animal study has indicated that vitamin $\mathrm{E}$ is able to preserve vitamin $\mathrm{C}$ in vivo [30]. Food-derived (and synthetic) vitamin $\mathrm{C}$ is well known to increase non-heme iron uptake and body status, likely via its ability to reduce iron from its ferric to ferrous state [31,32]. However, whether iron can affect vitamin C bioavailability is less clear [33-35]. Although iron has been shown to increase the uptake of vitamin $\mathrm{C}$ in cultured intestinal cells [33], human intervention studies have shown no effect of iron intake on vitamin $\mathrm{C}$ bioavailability [34,35]. One study has indicated that specific dietary fibers, such as hemicellulose and pectin, may affect the excretion of vitamin C [36], however, their influence on vitamin $\mathrm{C}$ uptake was not determined.

Plant-derived flavonoids have been of interest since the mid 1930s, when they were initially referred to as "vitamin P", primarily due to their effect on vascular permeability [37]. At the time, there was much debate in the literature regarding the role of "vitamin P" in experimental [38-42] 
and human scurvy [37,43-45]. Flavonoids can act as antioxidants via direct scavenging of free radicals [46,47] and/or chelation of redox-active metal ions [48,49]. As a result, it has been suggested that flavonoids may "spare" vitamin $\mathrm{C}$ and, thus, increase its bioavailability. Flavonoids have been shown to inhibit the in vitro oxidation of vitamin $\mathrm{C}$ [48-51], however, the in vivo relevance of metal-ion mediated oxidation of vitamin $\mathrm{C}$ is likely to be minimal as free metal ions are largely sequestered in the body [52]. Whether flavonoids can affect vitamin $\mathrm{C}$ uptake in vivo is uncertain due to the low plasma bioavailability of these compounds [53]. Thus, any interaction of flavonoids with vitamin $\mathrm{C}$ would be expected to occur primarily in the intestinal lumen prior to active uptake.

Of note, several in vitro studies have shown that various flavonoids can inhibit vitamin $\mathrm{C}$ and DHA uptake by their respective transporters. The flavonoid quercetin can reversibly inhibit SVCT1 expressed in Xenopus oocytes [54] and limited data from an animal model indicates that this may occur in vivo [54]. Quercetin and myricetin can inhibit the uptake of vitamin C and DHA into cultured monocytic (HL-60 and U937) and lymphocytic (Jurkat) cells via inhibition of GLUT1 and GLUT3 [55] and possibly also SVCT2, which is expressed in leukocytes [56]. Quercetin and phloretin can also inhibit the intestinal GLUT2 and GLUT8 transporters [19]. Thus, based on the above in vitro studies, it is unclear whether flavonoids will enhance in vivo vitamin $\mathrm{C}$ bioavailability through a sparing action, or decrease its bioavailability through inhibiting vitamin $\mathrm{C}$ transporters.

The effect of various purified flavonoids or flavonoid-rich fruits and vegetables on vitamin $\mathrm{C}$ bioavailability in different animal models and human subjects is discussed below. To test comparative vitamin $\mathrm{C}$ bioavailability, both steady-state and pharmacokinetic models have been used. The former monitors ascorbate levels in blood and/or urine following a number of weeks of supplementation, while the latter monitors transient changes in plasma levels and/or urinary excretion over the hours following ingestion of the vitamin C-containing test substance. The gold standard for analysis of vitamin C is HPLC with coulometric electrochemical detection due to its sensitivity and specificity [57]. Early studies, however, were limited primarily to colourimetric methods based on reduction of ferric iron compounds and are prone to interference by numerous other substances [57].

\section{Vitamin C Bioavailability Studies Using Animal Models}

There are a number of benefits to the use of animal models to investigate vitamin $\mathrm{C}$ bioavailability, particularly the ease of diet control and the ability to obtain tissues not normally accessible in human studies. However, results can vary widely depending on the animal model used and the different treatment and analytical methodologies employed. It should also be noted that not all of the animal models that have been used are naturally vitamin $\mathrm{C}$ deficient. The animal models of choice are the naturally vitamin $\mathrm{C}$ deficient guinea pig, and genetically scorbutic animal models, such as the Osteogenic Disorder Shionogi (ODS) rat [58], the L-gulono- $\gamma$-lactone oxidase $\left(\mathrm{Gulo}^{-/}\right)$knockout mouse [59], and the spontaneous bone fracture ( $s f x)$ mouse [60]. Although animal studies can provide useful information, translation of the findings to humans should always proceed with caution.

Studies investigating the comparative bioavailability of synthetic versus natural vitamin $\mathrm{C}$ in animal models are shown in Table 1. Studies carried out in guinea pigs showed enhanced uptake of vitamin $\mathrm{C}$ into specific organs (e.g., adrenals and spleen) in the presence of flavonoid-rich juices/extracts or purified plant flavonoids (e.g., hesperidin, rutin, and catechin) [42,61-64]. Vinson and Bose [65] 
carried out a pharmacokinetic study in guinea pigs and found a $148 \%$ increase in the area under the plasma ascorbate concentration-time curve when administered as citrus fruit media. They also noted that the citrus fruit group demonstrated delayed plasma vitamin $\mathrm{C}$ uptake compared with the synthetic vitamin C group [65]. Cotereau et al. [42] reported that animals given both vitmain $\mathrm{C}$ and catechin not only had four to eight-fold more vitamin $\mathrm{C}$ in the organs measured, but they were also the only group without scorbutic-type lesions. The latter finding was supported by a similar study showing fewer fresh hemorrhages in scorbutic guinea pigs receiving vitamin $\mathrm{C}$ with rutin or querceitin compared with vitamin $\mathrm{C}$ alone [66].

Several of the studies in Table 1, however, showed no differences in vitamin $\mathrm{C}$ accumulation in some organs (e.g., liver) [61-63,67]. Hughes et al. noted that the acerola cherry preparation they used was virtually flavonoid free due to dilution of the high vitamin $\mathrm{C}$ fruit extract, which they suggested may have accounted for its reduced efficacy compared with blackcurrant juice, which is flavonoid rich [64]. To account for the flavonoid-dependent differences in vitamin $\mathrm{C}$ uptake observed between the adrenals and livers of guinea pigs [62,63], Douglass and Kamp [62] noted that flavonols such as rutin are rapidly destroyed in liver tissue, but are relatively stable in adrenal homogenates. Papageorge et al. [63] also noted that when epinephrine oxidizes it can contribute to the destruction of vitamin $\mathrm{C}$ and thus the antioxidant effects of rutin may result in "sparing" of vitamin C in adrenals. A study by Levine's group [54] showed that the flavonoid quercetin can reversibly inhibit vitamin $\mathrm{C}$ intestinal transport and decrease plasma levels of the vitamin in the CD (Sprague-Dawley) rat, although it should be noted that this is not a vitamin C deficient animal model. Some of the variability observed in these different animal studies (Table 1) may be due to the varying ratios of flavonoid to vitamin $\mathrm{C}$ employed.

We recently carried out a comparative bioavailability study, using the Gulo mouse model, investigating the uptake of vitamin $\mathrm{C}$ from kiwifruit gel compared with synthetic vitamin $\mathrm{C}$ [68]. We found that the kiwifruit extract, which is rich in flavonoids $[69,70]$, provided significantly higher serum, leukocyte, heart, liver, and kidney levels of vitamin $C$ than the purified vitamin, suggesting some type of synergistic activity of the whole fruit in this model. As with Wilson et al. [61], we did not observe any difference between the two interventions with respect to vitamin $\mathrm{C}$ uptake into the brain. Indeed, there is significant retention of vitamin $\mathrm{C}$ in the brain during dietary depletion $[64,68]$, suggesting a vital role for vitamin $\mathrm{C}$ in the brain. Thus, a significant proportion of animal studies show enhanced circulating and organ levels of vitamin $\mathrm{C}$ in the presence of food-derived or purified flavonoids. 


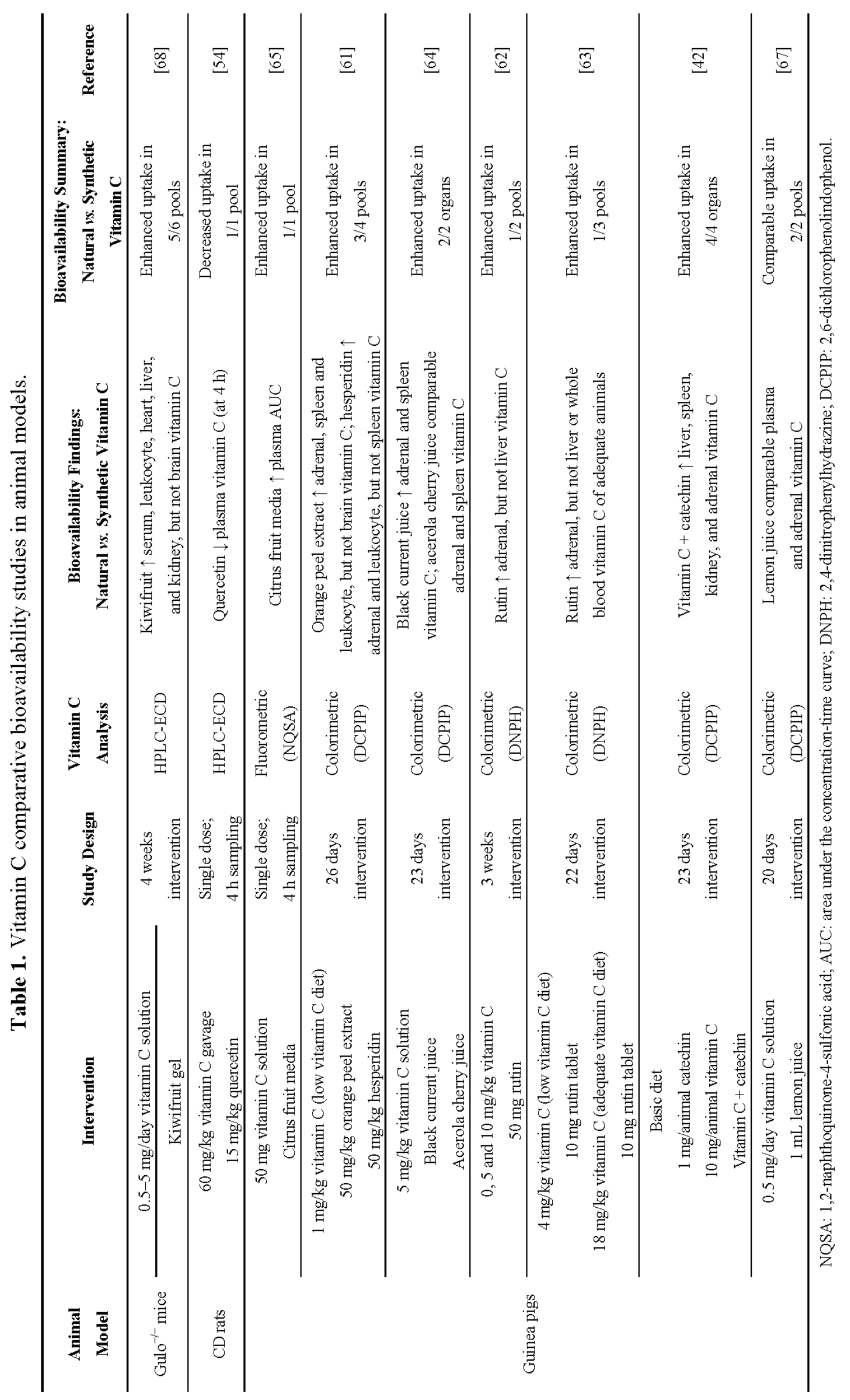




\section{Steady State Bioavailability Studies in Humans}

An early report of several patients with scurvy whose plasma vitamin C levels did not increase with synthetic vitamin $\mathrm{C}$ alone, but only in the form of lemon juice [45], initially leant support to the "vitamin P"/flavonoid theory. However, in contrast to the animal studies, all steady state human studies (summarized in Table 2) have shown little difference in plasma and/or urine bioavailability between synthetic vitamin $\mathrm{C}$ and that from different fruits, fruit juices, and vegetables [35,71-76]. Mangels et al. [35] did observe a 20\% lower plasma bioavailability of vitamin C from raw broccoli compared with cooked broccoli, however, this may have been due to differences in mechanical homogenization (chewing), a similar effect to that observed for carotenoid absorption from raw versus cooked carrots.

We recently carried out a steady state bioavailability study in young non-smoking men supplemented for six weeks with $50 \mathrm{mg}$ /day vitamin $\mathrm{C}$, in the form of a chewable vitamin $\mathrm{C}$ tablet or half a gold kiwifruit [77]. This dose was chosen as it lies on the steep part of the sigmoidal plasma uptake curve [78], thus enhancing the likelihood of detecting a difference between the two interventions. Although most steady state studies have used sequential or crossover study designs, we chose a randomized parallel arms design for a number of reasons. Block et al. [79] have previously observed a lower plasma vitamin $\mathrm{C}$ response to supplemental vitamin $\mathrm{C}$ in the second phase of a multiple depletion/repletion study. Furthermore, although washout of vitamin $\mathrm{C}$ could be monitored between the two phases of a cross-over study, it would not be possible to monitor washout of other kiwifruit-derived components, e.g., vitamin E, which may affect the second phase of a cross-over study due to potential in vivo interactions with the supplemental vitamin $\mathrm{C}$ [30].

Only one previous study has investigated the comparative bioavailability of synthetic versus natural vitamin $\mathrm{C}$ in leukocytes [71]. These investigators found no difference in leukocyte vitamin $\mathrm{C}$ uptake between synthetic vitamin $\mathrm{C}$ (in the presence or absence of rutin) and that in orange juice two hours after a single $75 \mathrm{mg}$ dose [71]. Therefore, in addition to plasma, urine, and semen samples, we also isolated peripheral blood mononuclear cells and neutrophils before and after intervention. Due to ease of accessibility and isolation, peripheral blood leukocytes are often used as a marker for tissue vitamin $\mathrm{C}$ status, however, whether they are a good model for all organs and tissues is uncertain. In support of this premise our animal study indicated that different organs exhibited maximal vitamin $\mathrm{C}$ uptake at varying doses of the vitamin [68] and we have recently shown that human skeletal muscle exhibits greater relative uptake of vitamin $C$ than leukocytes [80]. Therefore, we also carried out needle biopsies of skeletal muscle tissue (vastus lateralis), before and after intervention. In contrast to our earlier animal study [68], our human study clearly showed no differences in the steady-state bioavailability of kiwifruit-derived versus synthetic vitamin $C$ to plasma, semen, peripheral blood leukocytes, and skeletal muscle tissue [77]. Thus, other nutrients and phytochemicals present in kiwifruit are neither enhancing nor inhibiting the uptake of vitamin $\mathrm{C}$ from the whole fruit in humans. 


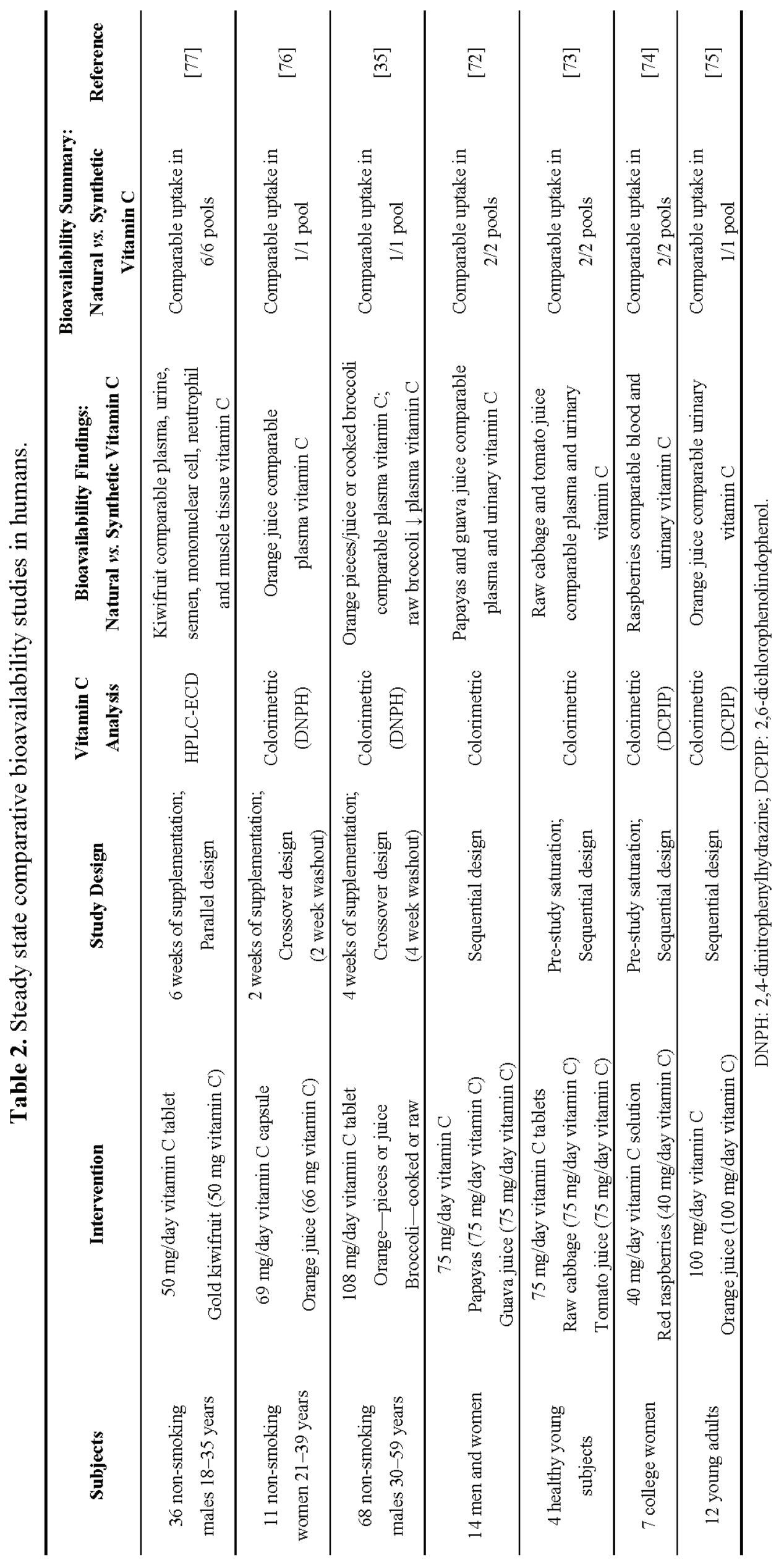




\section{Pharmacokinetic Bioavailability Studies in Humans}

Pharmacokinetic studies show transient changes in plasma vitamin $\mathrm{C}$ levels and urinary excretion over the hours following ingestion of the vitamin C-containing test substance (relevant studies are shown in Table 3). Supplemental vitamin C typically takes about two hours to reach maximal plasma levels following ingestion. An early animal study found that vitamin $\mathrm{C}$ provided in citrus fruit media took longer to reach peak plasma concentrations compared with a synthetic vitamin $\mathrm{C}$ solution and also provided a larger area under the plasma vitamin $\mathrm{C}$ concentration-time curve [65]. These same investigators observed a comparable trend in human subjects supplemented with $500 \mathrm{mg}$ vitamin $\mathrm{C}$ in the presence or absence of a citrus fruit extract [81]. The citrus fruit extract delayed maximal plasma levels by one hour and provided a 35\% increase in vitamin C bioavailability. Interestingly, the citrus fruit extract increased $24 \mathrm{~h}$ urinary vitamin $\mathrm{C}$ excretion in participants pre-saturated with vitamin $\mathrm{C}$, but decreased excretion in non-saturated participants compared with synthetic vitamin $\mathrm{C}$ alone. This suggests that the baseline vitamin $\mathrm{C}$ status of the individual may affect the comparative bioavailability of vitamin C. Although two other studies showed increased urinary excretion in vitamin $\mathrm{C}$ pre-saturated subjects in the presence of fruit juice $[71,82]$, another pre-saturation study showed comparable plasma levels and $24 \mathrm{~h}$ urinary excretion in the presence of mixed bioflavonoids [83]. It should be noted that doses of $500 \mathrm{mg}$ vitamin $\mathrm{C}$ have reduced intestinal bioavailability [78] and are significantly higher than would be obtained through a normal daily diet.

A number of pharmacokinetic studies have shown comparable bioavailability of vitamin $\mathrm{C}$ supplied in synthetic form or in the presence of foods or fruit juices [84-88]. Nelson et al. [88] used an intestinal triple lumen tube perfusion model to investigate the absorption of synthetic vitamin $\mathrm{C}$ and that from an orange juice solution. This method allows direct measurement of intraluminal events and showed no difference in the absorption of vitamin $\mathrm{C}$ from the two test solutions. A few pharmacokinetic studies have shown transient decreases in plasma vitamin C levels and/or urinary excretion at specific time points in the presence of food and fruit juices $[34,71,84,85]$. The physiological relevance of these transient differences is, however, likely minimal.

We recently carried out a pharmacokinetic bioavailability study of synthetic versus kiwifruit-derived vitamin $\mathrm{C}$ in nine non-smoking males (aged 18-35 years) who had "healthy" or "optimal" (i.e., $>50 \mu \mathrm{mol} / \mathrm{L}$ ) baseline levels of plasma vitamin C [89]. The participants received either a chewable tablet (200 mg vitamin C) or the equivalent dose from gold kiwifruit. Fasting blood and urine were collected half hourly to hourly over the eight hours following intervention. Plasma ascorbate levels increased from $0.5 \mathrm{~h}$ post intervention, although no significant differences in the plasma time-concentration curves were observed between the two interventions. An estimate of the total increase in plasma ascorbate indicated complete uptake of the ingested vitamin $\mathrm{C}$ tablet and kiwifruit-derived vitamin $\mathrm{C}$. There was an increase in urinary ascorbate excretion, relative to urinary creatinine, from two hours post intervention. There was also a significant difference between the two interventions, with enhanced ascorbate excretion observed in the kiwifruit group. Urinary excretion was calculated as $\sim 40 \%$ and $\sim 50 \%$ of the ingested dose from the vitamin $C$ tablet and kiwifruit arms, respectively. Overall, our pharmacokinetic study showed comparable relative bioavailability of kiwifruit-derived vitamin $\mathrm{C}$ and synthetic vitamin C [89]. 


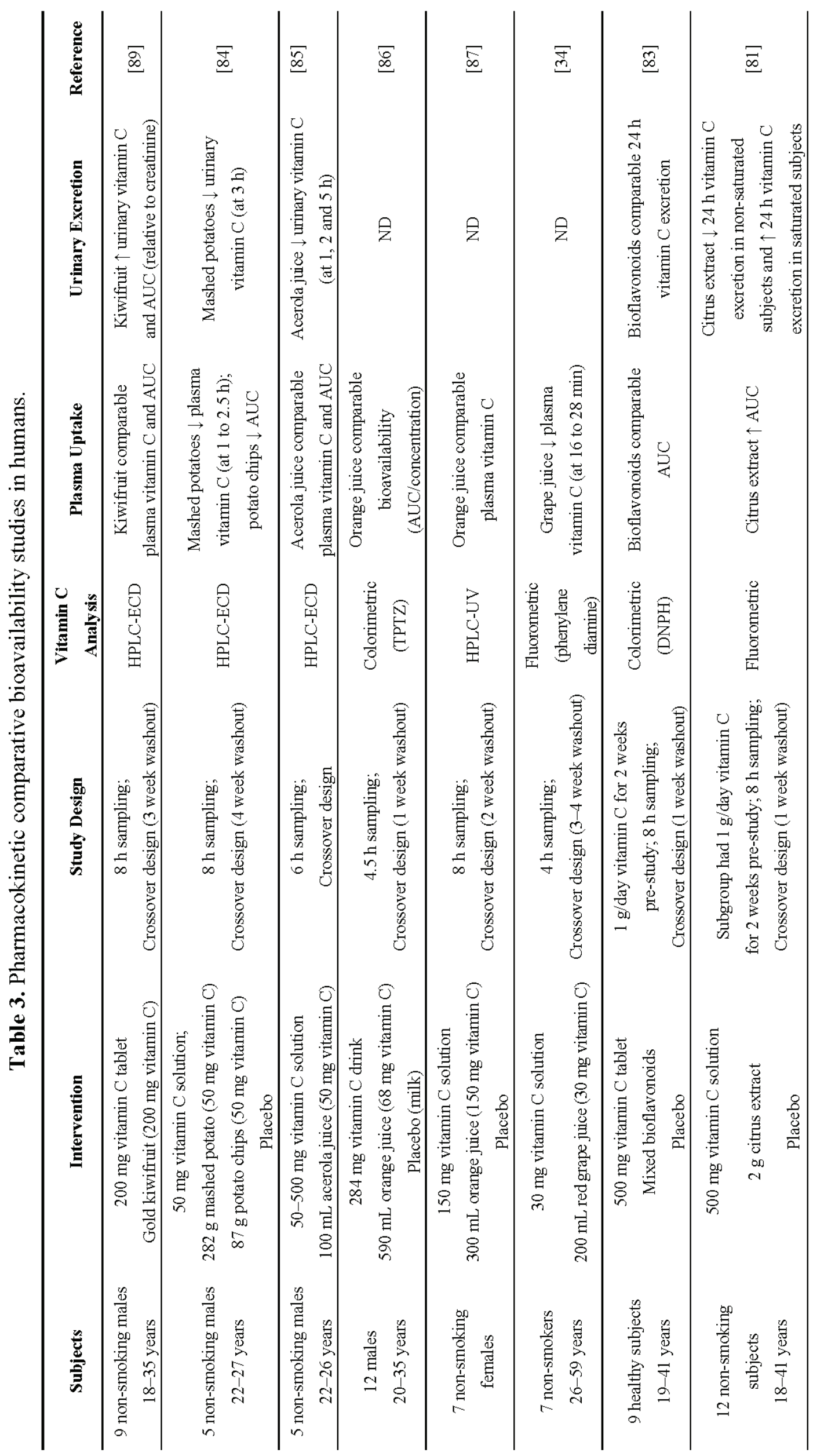




\section{Vitamin C Bioavailability from Different Tablet Formulations}

Doses of vitamin C up to $2000 \mathrm{mg}$ /day are considered safe for general consumption [90]. However, pharmacokinetic studies indicate that ingestion of single doses of vitamin $\mathrm{C}$ greater than $200 \mathrm{mg}$ have lower relative bioavailability [78], indicating that ingestion of several smaller doses each day is preferable to a single large dose. A number of studies have investigated the relative bioavailability of vitamin $\mathrm{C}$ from different tablet formulations and have shown that slow-release formulations provide superior vitamin bioavailability [91-94]. Salts of vitamin C, such as sodium and calcium ascorbate (Ester-C), have also been tested. Animal studies indicated that Ester-C (which contains calcium ascorbate, as well as DHA and calcium threonate) was absorbed more readily and excreted less rapidly than ascorbic acid [95] and had superior anti-scorbutic activity in ODS rats [96]. Johnston and Luo [83], however, found no significant differences between Ester-C and ascorbic acid bioavailability in humans. Nevertheless, Ester-C has been shown to be better tolerated in individuals sensitive to acidic foods [97].

\section{Conclusions}

Overall, a majority of animal studies have shown differences in the comparative bioavailability of synthetic versus food-derived vitamin $\mathrm{C}$, or vitamin $\mathrm{C}$ in the presence of isolated bioflavonoids, although the results varied depending on the animal model, study design and body compartments measured. In contrast, all steady state comparative bioavailability studies in humans have shown no differences between synthetic and natural vitamin $\mathrm{C}$, regardless of the subject population, study design or intervention used. Some pharmacokinetic studies in humans have shown transient and small comparative differences between synthetic and natural vitamin $\mathrm{C}$, although these differences are likely to have minimal physiological impact. Thus, not only do the reviewed studies reiterate the injunction that the findings of animal studies should not be directly translated to humans [98,99], but it is also apparent that additional comparative bioavailability studies in humans are unwarranted.

Although synthetic and food-derived vitamin $\mathrm{C}$ appear to be equally bioavailable in humans, ingesting vitamin $\mathrm{C}$ as part of a whole food is considered preferable because of the concomitant consumption of numerous other macro- and micronutrients and phytochemicals, which will confer additional health benefits. Numerous epidemiological studies have indicated that higher intakes of fruit and vegetables are associated with decreased incidence of stroke [100], coronary heart disease [101], and cancers at various sites [102,103]. Vitamin C status is one of the best markers for fruit and vegetable intake [104], and food-derived vitamin C is associated with decreased incidence of numerous chronic diseases [1], however, whether the observed health effects of fruit and vegetable ingestion are due to vitamin $\mathrm{C}$ and/or other plant-derived components is currently unknown. With respect to coronary heart disease, strong evidence exists for a protective effect of vegetables, moderate evidence for fruit and dietary vitamin $\mathrm{C}$ and insufficient evidence for supplemental vitamin C [105]. Some meta-analyses support the premise that dietary vitamin $\mathrm{C}$ is more protective than supplements [106], while others show reduced disease incidence with supplemental but not dietary vitamin C [107].

A major limitation with epidemiological studies is that they show only an association between dietary vitamin $\mathrm{C}$ intake and disease risk and cannot ascertain whether different sources of vitamin $\mathrm{C}$ 
(i.e., food-derived versus supplement) are a cause, consequence, or simply a correlate of the particular end-point measured. Interpretations can also vary significantly depending on the input of different confounders [108]. Furthermore, epidemiological studies rely predominantly on food frequency questionnaires $[109,110]$ and $24 \mathrm{~h}$ dietary recalls [111] to ascertain vitamin C intakes from foods and/or supplements [112]. This methodology has numerous limitations [113] and correlations with vitamin $\mathrm{C}$ status can vary depending on the methods employed as well as numerous other external factors [114]. Pooled or meta-analyses of epidemiological studies are particularly problematic due to the combining of variable study designs, cohorts and endpoints, often resulting in dilution or misinterpretation of study findings.

The gold standard for determining causality is the double-blind randomized placebo controlled clinical trial. Although this type of study design works well for comparing the effects of drugs against a placebo, it does not work for nutrients, such as vitamin $\mathrm{C}$, which are already in the food chain and are required for life, i.e., there is no true placebo. Numerous other methodological issues have been identified with the design of many clinical trials investigating the health effects of vitamin C [115]. For example, a major flaw with many vitamin $C$ intervention studies is the use of study populations with already adequate or even saturating vitamin $\mathrm{C}$ levels, which significantly decreases the likelihood of observing any effects of the intervention. Thus, it is recommended that study populations are comprised of individuals with sub-optimal vitamin $\mathrm{C}$ status (i.e., $<50 \mu \mathrm{mol} / \mathrm{L}$ plasma vitamin C) or that sub-group analysis is carried out on the low vitamin C sub-populations [116]. With pharmacokinetic studies, both unsaturated and saturated individuals can be used, but comparative bioavailability studies have shown that results may vary depending on the baseline vitamin $\mathrm{C}$ status of the study subjects. Furthermore, the vitamin $\mathrm{C}$ doses chosen for intervention are critical since doses above $200 \mathrm{mg}$ have decreased intestinal uptake [78], indicating that if higher doses are warranted then these should be provided as multiple doses of $\sim 200 \mathrm{mg}$ each to ensure complete bioavailability.

The comparative health effects of supplemental versus food-derived vitamin $\mathrm{C}$ will only be determined through the use of appropriate and well-designed studies. Determination of the physiological effects or health outcomes of intervention with synthetic versus natural vitamin $\mathrm{C}$ will depend largely on the endpoints measured. Only a handful of comparative intervention studies have been carried out to assess specific physiological or health endpoints. Guarnieri et al. [89] investigated potential protection of mononuclear leukocytes from supplemented individuals against ex vivo oxidative DNA damage. Although they found comparable vitamin C bioavailability between a single portion of orange juice (containing $150 \mathrm{mg}$ vitamin $\mathrm{C}$ ) and a synthetic vitamin $\mathrm{C}$ drink of the same dosage, they showed that only the orange juice protected the leukocytes from ex vivo oxidative DNA damage [89]. However, how closely ex vivo oxidation of DNA resembles events occurring in vivo is debatable and results could also vary significantly depending on the type of oxidative stress. Johnston et al. [76] compared plasma lipid peroxidation in individuals who had been supplemented with either orange juice or synthetic vitamin $C$ ( $\sim 70 \mathrm{mg} /$ day) for two weeks. They found comparable vitamin $\mathrm{C}$ bioavailability and a similar reduction in lipid peroxidation with both interventions [76]. Several studies have assessed the effects of synthetic and natural vitamin $\mathrm{C}$, or vitamin $\mathrm{C}$ in the presence of bioflavonoids, on the common cold. Two earlier studies showed a lack of an effect of vitamin C ( $200 \mathrm{mg} /$ day), with and without purified bioflavonoids, on the prevention 
and cure of the common cold [117,118]. Another study indicated that synthetic vitamin C ( $80 \mathrm{mg} / \mathrm{day})$ and orange juice both decreased the symptoms of the common cold compared with placebo, but there were no differences between the two interventions [119].

As alluded to in the introduction, vitamin $\mathrm{C}$ is known to enhance the bioavailability of other nutrients, such as vitamin $\mathrm{E}$ [30] and non-heme iron [31,32], which may enhance the health effects of vitamin C-containing foods. Bioflavonoids are also known to have numerous biological activities [120]. Recently vitamin $\mathrm{C}$ has been shown to modulate specific biological activities of quercetin and tea polyphenols [121,122]. Thus, future studies may elucidate the physiological relevance of these interactions.

\section{Conflicts of Interest}

The authors declare no conflict of interest.

\section{Acknowledgments}

Co-funding was provided by the New Zealand Ministry of Business, Innovation \& Employment and Zespri International Ltd., Mount Maunganui, New Zealand.

\section{References}

1. Carr, A.C.; Frei, B. Toward a new recommended dietary allowance for vitamin C based on antioxidant and health effects in humans. Am. J. Clin. Nutr. 1999, 69, 1086-1107.

2. Englard, S.; Seifter, S. The biochemical functions of ascorbic acid. Annu. Rev. Nutr. 1986, 6, 365-406.

3. Ozer, A.; Bruick, R.K. Non-heme dioxygenases: Cellular sensors and regulators jelly rolled into one? Nat. Chem. Biol. 2007, 3, 144-153.

4. Monfort, A.; Wutz, A. Breathing-in epigenetic change with vitamin C. EMBO Rep. 2013, 14, 337-346.

5. Hornig, D. Distribution of ascorbic acid, metabolites and analogues in man and animals. Ann. N. Y. Acad. Sci. 1975, 258, 103-118.

6. Omaye, S.T.; Schaus, E.E.; Kutnink, M.A.; Hawkes, W.C. Measurement of vitamin C in blood components by high-performance liquid chromatography. Implication in assessing vitamin $\mathrm{C}$ status. Ann. N. Y. Acad. Sci. 1987, 498, 389-401.

7. Tsao, C.S. An Overview of Ascorbic Acid Chemistry and Biochemistry. In Vitamin C in Health and Disease; Packer, L., Fuchs, J., Eds.; Marcel Dekker: New York, NY, USA, 1997; pp. 25-58.

8. Nishikimi, M.; Fukuyama, R.; Minoshima, S.; Shimizu, N.; Yagi, K. Cloning and chromosomal mapping of the human nonfunctional gene for L-gulono- $\gamma$-lactone oxidase, the enzyme for L-ascorbic acid biosynthesis missing in man. J. Biol. Chem. 1994, 269, 13685-13688.

9. Krebs, H.A. The Sheffield Experiment on the vitamin $\mathrm{C}$ requirement of human adults. Proc. Nutr. Soc. 1953, 12, 237-246. 
10. Sauberlich, H.E. A History of Scurvy and Vitamin C. In: Vitamin C in Health and Disease; Packer, L., Fuchs, J., Eds.; Marcel Dekker: New York, NY, USA, 1997; pp. 1-24.

11. Michels, A.J.; Hagen, T.M.; Frei, B. Human genetic variation influences vitamin C homeostasis by altering vitamin $\mathrm{C}$ transport and antioxidant enzyme function. Annu. Rev. Nutr. 2013, $33,45-70$.

12. Tsukaguchi, H.; Tokui, T.; Mackenzie, B.; Berger, U.V.; Chen, X.Z.; Wang, Y.; Brubaker, R.F.; Hediger, M.A. A family of mammalian $\mathrm{Na}^{+}$-dependent L-ascorbic acid transporters. Nature 1999, 399, 70-75.

13. Savini, I.; Rossi, A.; Pierro, C.; Avigliano, L.; Catani, M.V. SVCT1 and SVCT2: Key proteins for vitamin C uptake. Amino Acids 2008, 34, 347-355.

14. Rumsey, S.C.; Welch, R.W.; Garraffo, H.M.; Ge, P.; Lu, S.F.; Crossman, A.T.; Kirk, K.L.; Levine, M. Specificity of ascorbate analogs for ascorbate transport. Synthesis and detection of [(125)I]6-deoxy-6-iodo-L-ascorbic acid and characterization of its ascorbate-specific transport properties. J. Biol. Chem. 1999, 274, 23215-23222.

15. Goldman, H.M.; Gould, B.S.; Munro, H.N. The antiscorbutic action of L-ascorbic acid and D-isoascorbic acid (erythorbic acid) in the guinea pig. Am. J. Clin. Nutr. 1981, 34, 24-33.

16. Hughes, R.E.; Hurley, R.J. The uptake of D-araboascorbic acid (D-isoascorbic acid) by guinea-pig tissues. Br. J. Nutr. 1969, 23, 211-216.

17. Levine, M. Fruits and vegetables: There is no substitute. Am. J. Clin. Nutr. 1996, 64, 381-382.

18. Sauberlich, H.E.; Tamura, T.; Craig, C.B.; Freeberg, L.E.; Liu, T. Effects of erythorbic acid on vitamin C metabolism in young women. Am. J. Clin. Nutr. 1996, 64, 336-346.

19. Corpe, C.P.; Eck, P.; Wang, J.; Al-Hasani, H.; Levine, M. Intestinal dehydroascorbic acid (DHA) transport mediated by the facilitative sugar transporters, GLUT2 and GLUT8. J. Biol. Chem. 2013, 288, 9092-9101.

20. Rumsey, S.C.; Kwon, O.; Xu, G.W.; Burant, C.F.; Simpson, I.; Levine, M. Glucose transporter isoforms GLUT1 and GLUT3 transport dehydroascorbic acid. J. Biol. Chem. 1997, 272, 18982-18989.

21. Vera, J.C.; Rivas, C.I.; Fischbarg, J.; Golde, D.W. Mammalian facilitative hexose transporters mediate the transport of dehydroascorbic acid. Nature 1993, 364, 79-82.

22. Washko, P.W.; Wang, Y.; Levine, M. Ascorbic acid recycling in human neutrophils. J. Biol. Chem. 1993, 268, 15531-15535.

23. Corti, A.; Casini, A.F.; Pompella, A. Cellular pathways for transport and efflux of ascorbate and dehydroascorbate. Arch. Biochem. Biophys. 2010, 500, 107-115.

24. Nishiyama, I.; Yamashita, Y.; Yamanaka, M.; Shimohashi, A.; Fukuda, T.; Oota, T. Varietal difference in vitamin $\mathrm{C}$ content in the fruit of kiwifruit and other actinidia species. J. Agric. Food Chem. 2004, 52, 5472-5475.

25. Dhariwal, K.R.; Hartzell, W.O.; Levine, M. Ascorbic acid and dehydroascorbic acid measurements in human plasma and serum. Am. J. Clin. Nutr. 1991, 54, 712-716.

26. Ogiri, Y.; Sun, F.; Hayami, S.; Fujimura, A.; Yamamoto, K.; Yaita, M.; Kojo, S. Very low vitamin $\mathrm{C}$ activity of orally administered L-dehydroascorbic acid. J. Agric. Food Chem. 2002, $50,227-229$. 
27. Welch, R.W.; Wang, Y.; Crossman, A., Jr.; Park, J.B.; Kirk, K.L.; Levine, M. Accumulation of vitamin $\mathrm{C}$ (ascorbate) and its oxidized metabolite dehydroascorbic acid occurs by separate mechanisms. J. Biol. Chem. 1995, 270, 12584-12592.

28. Wang, Y.; Russo, T.A.; Kwon, O.; Chanock, S.; Rumsey, S.C.; Levine, M. Ascorbate recycling in human neutrophils: Induction by bacteria. Proc. Natl. Acad. Sci. USA 1997, 94, 13816-13819.

29. Packer, J.E.; Slater, T.F.; Willson, R.L. Direct observation of a free radical interaction between vitamin E and vitamin C. Nature 1979, 278, 737-738.

30. Tanaka, K.; Hashimoto, T.; Tokumaru, S.; Iguchi, H.; Kojo, S. Interactions between vitamin C and vitamin $\mathrm{E}$ are observed in tissues of inherently scorbutic rats. J. Nutr. 1997, 127, 2060-2064.

31. Beck, K.; Conlon, C.A.; Kruger, R.; Coad, J.; Stonehouse, W. Gold kiwifruit consumed with an iron-fortified breakfast cereal meal improves iron status in women with low iron stores: A 16-week randomised controlled trial. Br. J. Nutr. 2011, 105, 101-109.

32. Hallberg, L.; Brune, M.; Rossander, L. Effect of ascorbic acid on iron absorption from different types of meals. Studies with ascorbic-acid-rich foods and synthetic ascorbic acid given in different amounts with different meals. Hum. Nutr. Appl. Nutr. 1986, 40, 97-113.

33. Scheers, N.M.; Sandberg, A.S. Iron regulates the uptake of ascorbic acid and the expression of sodium-dependent vitamin C transporter 1 (SVCT1) in human intestinal Caco-2 cells. Br. J. Nutr. 2011, 105, 1734-1740.

34. Bates, C.J.; Jones, K.S.; Bluck, L.J. Stable isotope-labelled vitamin C as a probe for vitamin C absorption by human subjects. Br. J. Nutr. 2004, 91, 699-705.

35. Mangels, A.R.; Block, G.; Frey, C.M.; Patterson, B.H.; Taylor, P.R.; Norkus, E.P.; Levander, O.A. The bioavailability to humans of ascorbic acid from oranges, orange juice and cooked broccoli is similar to that of synthetic ascorbic acid. $J$. Nutr. 1993, 123, 1054-1061.

36. Keltz, F.R.; Kies, C.; Fox, H.M. Urinary ascorbic acid excretion in the human as affected by dietary fiber and zinc. Am. J. Clin. Nutr. 1978, 31, 1167-1171.

37. Rusznyak, S.; Szent-Gyorgyi, A. Vitamin P: Flavonols as vitamins. Nature 1936, 138, 27.

38. Bentsath, A.; Rusznyak, S.; Szent-Gyorgyi, A. Vitamin nature of flavones. Nature 1936, $138,798$.

39. Bentsath, A.; Rusznyak, S.; Szent-Gyorgyi, A. Vitamin P. Nature 1937, 139, 326-327.

40. Rusznyak, S.; Benko, A. Experimental vitamin P deficiency. Science 1941, 94, 25.

41. Zilva, S.S. Vitamin P. Biochem. J. 1937, 31, 915-919.

42. Cotereau, H.; Gabe, M.; Géro, E.; Parrot, J.-L. Influence of vitamin P (vitamin $\mathrm{C}_{2}$ ) upon the amount of ascorbic acid in the organs of the guinea pig. Nature 1948, 161, 557.

43. Scarborough, H. Deficiency of vitamin C and vitamin P in man. Lancet 1940, 236, 644-647.

44. Scarborough, H. Vitamin P. Biochem. J. 1939, 33, 1400-1407.

45. Elmby, A.; Warburg, E. The inadequacy of synthetic ascorbic acid as an antiscorbutic agent. Lancet 1937, 230, 1363-1365.

46. Ivanov, V.; Carr, A.C.; Frei, B. Red wine antioxidants bind to human lipoproteins and protect them from metal ion-dependent and -independent oxidation. J. Agric. Food Chem. 2001, 49, $4442-4449$. 
47. Bors, W.; Michel, C.; Saran, M. Flavonoid antioxidants: Rate constants for reactions with oxygen radicals. Methods Enzymol. 1994, 234, 420-429.

48. Beker, B.Y.; Sonmezoglu, I.; Imer, F.; Apak, R. Protection of ascorbic acid from copper(II)-catalyzed oxidative degradation in the presence of flavonoids: Quercetin, catechin and morin. Int. J. Food Sci. Nutr. 2011, 62, 504-512.

49. Clemetson, C.A.; Andersen, L. Plant polyphenols as antioxidants for ascorbic acid. Ann. N. Y. Acad Sci. 1966, 136, 341-376.

50. Harper, K.A.; Morton, A.D.; Rolfe, E.J. The phenolic compounds of blackcurrant juice and their protective effect on ascorbic acid III. The mechansim of ascorbic acid oxidation and its inhibition by flavonoids. Int. J. Food Sci. Technol. 1969, 4, 255-267.

51. Clegg, K.M.; Morton, A.D. The phenolic compounds of blackcurrant juice and their protective effect on ascorbic acid II. The stability of ascorbic acid in model systems containing some of the phenolic compounds associated with blackcurrant juice. Int. J. Food Sci. Technol. 1968, 3, 277-284.

52. Carr, A.; Frei, B. Does vitamin $\mathrm{C}$ act as a pro-oxidant under physiological conditions? FASEB J. 1999, 13, 1007-1024.

53. Lotito, S.B.; Frei, B. Consumption of flavonoid-rich foods and increased plasma antioxidant capacity in humans: Cause, consequence, or epiphenomenon? Free Radic. Biol. Med. 2006, 41, $1727-1746$.

54. Song, J.; Kwon, O.; Chen, S.; Daruwala, R.; Eck, P.; Park, J.B.; Levine, M. Flavonoid inhibition of sodium-dependent vitamin $\mathrm{C}$ transporter 1 (SVCT1) and glucose transporter isoform 2 (GLUT2), intestinal transporters for vitamin C and Glucose. J. Biol. Chem. 2002, 277, 15252-15260.

55. Park, J.B.; Levine, M. Intracellular accumulation of ascorbic acid is inhibited by flavonoids via blocking of dehydroascorbic acid and ascorbic acid uptakes in HL-60, U937 and Jurkat cells. J. Nutr. 2000, 130, 1297-1302.

56. Corpe, C.P.; Lee, J.H.; Kwon, O.; Eck, P.; Narayanan, J.; Kirk, K.L.; Levine, M. 6-Bromo-6-deoxy-L-ascorbic acid: An ascorbate analog specific for $\mathrm{Na}^{+}$-dependent vitamin $\mathrm{C}$ transporter but not glucose transporter pathways. J. Biol. Chem. 2005, 280, 5211-5220.

57. Washko, P.W.; Welch, R.W.; Dhariwal, K.R.; Wang, Y.; Levine, M. Ascorbic acid and dehydroascorbic acid analyses in biological samples. Anal. Biochem. 1992, 204, 1-14.

58. Mizushima, Y.; Harauchi, T.; Yoshizaki, T.; Makino, S. A rat mutant unable to synthesize vitamin C. Experientia 1984, 40, 359-361.

59. Maeda, N.; Hagihara, H.; Nakata, Y.; Hiller, S.; Wilder, J.; Reddick, R. Aortic wall damage in mice unable to synthesize ascorbic acid. Proc. Natl. Acad. Sci. USA 2000, 97, 841-846.

60. Mohan, S.; Kapoor, A.; Singgih, A.; Zhang, Z.; Taylor, T.; Yu, H.; Chadwick, R.B.; Chung, Y.S.; Donahue, L.R.; Rosen, C.; et al. Spontaneous fractures in the mouse mutant $s f x$ are caused by deletion of the gulonolactone oxidase gene, causing vitamin $\mathrm{C}$ deficiency. J. Bone Miner. Res. 2005, 20, 1597-1610.

61. Wilson, H.K.; Price-Jones, C.; Hughes, R.E. The influence of an extract of orange peel on the growth and ascorbic acid metabolism of young guinea-pigs. J. Sci. Food Agric. 1976, 27, 661-666. 
62. Douglass, C.D.; Kamp, G.H. The effect of orally administered rutin on the adrenal ascorbic acid level in guinea pigs. J. Nutr. 1959, 67, 531-536.

63. Papageorge, E.; Mitchell, G.L., Jr. The effect of oral administration of rutin on blood, liver and adrenal ascorbic acid and on liver and adrenal cholesterol in guinea pigs. J. Nutr. 1949, 37, 531-540.

64. Hughes, R.E.; Hurley, R.J.; Jones, P.R. The retention of ascorbic acid by guinea-pig tissues. Br. J. Nutr. 1971, 26, 433-438.

65. Vinson, J.A.; Bose, P. Comparative bioavailability of synthetic and natural vitamin C in guinea pigs. Nutr. Rep. Int. 1983, 27, 875-879.

66. Ambrose, A.M.; De, E.F. The value of rutin and quercetin in scurvy. J. Nutr. 1949, 38, 305-317.

67. Todhunter, E.N.; Robbins, R.C.; Ivey, G.; Brewer, W. A comparison of the utilization by guinea pigs of equivalent amounts of ascorbic acid (vitamin $\mathrm{C}$ ) in lemon juice and in crystalline form. J. Nutr. 1940, 19, 113-120.

68. Vissers, M.C.M.; Bozonet, S.M.; Pearson, J.F.; Braithwaite, L.J. Dietary ascorbate affects steady state tissue levels in vitamin $\mathrm{C}$-deficient mice: Tissue deficiency after sub-optimal intake and superior bioavailability from a food source (kiwifruit). Am. J. Clin. Nutr. 2011, 93, 292-301.

69. Latocha, P.; Krupa, T.; Wolosiak, R.; Worobiej, E.; Wilczak, J. Antioxidant activity and chemical difference in fruit of different Actinidia sp. Int J. Food Sci. Nutr. 2010, 61, 381-394.

70. Fiorentino, A.; D’Abrosca, B.; Pacifico, S.; Mastellone, C.; Scognamiglio, M.; Monaco, P. Identification and assessment of antioxidant capacity of phytochemicals from kiwi fruits. J. Agric. Food Chem. 2009, 57, 4148-4155.

71. Pelletier, O.; Keith, M.O. Bioavailability of synthetic and natural ascorbic acid. J. Am. Diet. Assoc. 1974, 64, 271-275.

72. Hartzler, E.R. The availability of ascorbic acid in papayas and guavas. J. Nutr. 1945, 30, 355-365.

73. Clayton, M.M.; Borden, R.A. The availability for human nutrition of the vitamin $\mathrm{C}$ in raw cabbage and home-canned tomato juice. J. Nutr. 1943, 25, 349-369.

74. Todhunter, E.N.; Fatzer, A.S. A comparison of the utilization by college women of equivalent amounts of ascorbic acid (vitamin C) in red raspberries and in crystalline form. J. Nutr. 1940, $19,121-130$.

75. Hawley, E.E.; Stephens, D.J.; Anderson, G. The excretion of vitamin C in normal individuals following a comparable quantitative administration in the form of orange juice, cevitamic acid by mouth and cevitamic acid intravenously. J. Nutr. 1936, 11, 135-145.

76. Johnston, C.S.; Dancho, C.L.; Strong, G.M. Orange juice ingestion and supplemental vitamin $\mathrm{C}$ are equally effective at reducing plasma lipid peroxidation in healthy adult women. J. Am. Coll. Nutr. 2003, 22, 519-523.

77. Carr, A.C.; Bozonet, S.M.; Pullar, J.M.; Simcock, J.W.; Vissers, M.C. A randomised steady-state bioavailability study of synthetic and natural (kiwifruit-derived) vitamin $\mathrm{C}$. Nutrients 2013, 5, 3684-3695. 
78. Levine, M.; Conry-Cantilena, C.; Wang, Y.; Welch, R.W.; Washko, P.W.; Dhariwal, K.R.; Park, J.B.; Lazarev, A.; Graumlich, J.F.; King, J.; et al. Vitamin C pharmacokinetics in healthy volunteers: Evidence for a recommended dietary allowance. Proc. Natl. Acad. Sci. USA 1996, 93, 3704-3709.

79. Block, G.; Mangels, A.R.; Patterson, B.H.; Levander, O.A.; Norkus, E.P.; Taylor, P.R. Body weight and prior depletion affect plasma ascorbate levels attained on identical vitamin $\mathrm{C}$ intake: A controlled-diet study. J. Am. Coll. Nutr. 1999, 18, 628-637.

80. Carr, A.C.; Bozonet, S.M.; Pullar, J.M.; Simcock, J.W.; Vissers, M.C. Human skeletal muscle ascorbate is highly responsive to changes in vitamin $\mathrm{C}$ intake and plasma concentrations. Am. J. Clin. Nutr. 2013, 97, 800-807.

81. Vinson, J.A.; Bose, P. Comparative bioavailability to humans of ascorbic acid alone or in a citrus extract. Am. J. Clin. Nutr. 1988, 48, 601-604.

82. Jones, E.; Hughes, R.E. The influence of bioflavonoids on the absorption of vitamin C. IRCS J. Med. Sci. 1984, 12, 320.

83. Johnston, C.S.; Luo, B. Comparison of the absorption and excretion of three commercially available sources of vitamin C. J. Am. Diet. Assoc. 1994, 94, 779-781.

84. Kondo, Y.; Higashi, C.; Iwama, M.; Ishihara, K.; Handa, S.; Mugita, H.; Maruyama, N.; Koga, H.; Ishigami, A. Bioavailability of vitamin $\mathrm{C}$ from mashed potatoes and potato chips after oral administration in healthy Japanese men. Br. J. Nutr. 2012, 107, 885-892.

85. Uchida, E.; Kondo, Y.; Amano, A.; Aizawa, S.; Hanamura, T.; Aoki, H.; Nagamine, K.; Koizumi, T.; Maruyama, N.; Ishigami, A. Absorption and excretion of ascorbic acid alone and in acerola (Malpighia emarginata) juice: Comparison in healthy japanese subjects. Biol. Pharm. Bull. 2011, 34, 1744-1747.

86. Carter, B.; Monsivais, P.; Drewnowski, A. Absorption of folic acid and ascorbic acid from nutrient comparable beverages. J. Food Sci. 2010, 75, H289-H293.

87. Guarnieri, S.; Riso, P.; Porrini, M. Orange juice vs vitamin C: Effect on hydrogen peroxide-induced DNA damage in mononuclear blood cells. Br. J. Nutr. 2007, 97, 639-643.

88. Nelson, E.W.; Streiff, R.R.; Cerda, J.J. Comparative bioavailability of folate and vitamin C from a synthetic and a natural source. Am. J. Clin. Nutr. 1975, 28, 1014-1019.

89. Carr, A.C.; Bozonet, S.M.; Vissers, M.C.M. A randomised cross-over pharmacokinetic bioavailabiltiy study of synthetic versus kiwifruit-derived vitamin C. Nutrients 2013, 5, 3684-3695.

90. Hathcock, J.N.; Azzi, A.; Blumberg, J.; Bray, T.; Dickinson, A.; Frei, B.; Jialal, I.; Johnston, C.S.; Kelly, F.J.; Kraemer, K.; et al. Vitamins E and C are safe across a broad range of intakes. Am. J. Clin. Nutr. 2005, 81, 736-745.

91. Yung, S.; Mayersohn, M.; Robinson, J.B. Ascorbic acid absorption in humans: A comparison among several dosage forms. J. Pharm. Sci. 1982, 71, 282-285.

92. Bhagavan, H.N.; Wolkoff, B.I. Correlation between the disintegration time and the bioavailability of vitamin C tablets. Pharm. Res. 1993, 10, 239-242.

93. Sacharin, R.; Taylor, T.; Chasseaud, L.F. Blood levels and bioavailability of ascorbic acid after administration of a sustained-release formulation to humans. Int. J. Vitam. Nutr. Res. 1977, 47, $68-74$. 
94. Nyyssonen, K.; Poulsen, H.E.; Hayn, M.; Agerbo, P.; Porkkala-Sarataho, E.; Kaikkonen, J.; Salonen, R.; Salonen, J.T. Effect of supplementation of smoking men with plain or slow release ascorbic acid on lipoprotein oxidation. Eur. J. Clin. Nutr. 1997, 51, 154-163.

95. Bush, M.J.; Verlangieri, A.J. An acute study on the relative gastro-intestinal absorption of a novel form of calcium ascorbate. Res. Commun. Chem. Pathol. Pharmacol. 1987, 57, 137-140.

96. Verlangieri, A.J.; Fay, M.J.; Bannon, A.W. Comparison of the anti-scorbutic activity of L-ascorbic acid and Ester $\mathrm{C}$ in the non-ascorbate synthesizing Osteogenic Disorder Shionogi (ODS) rat. Life Sci. 1991, 48, 2275-2281.

97. Gruenwald, J.; Graubaum, H.J.; Busch, R.; Bentley, C. Safety and tolerance of ester-C compared with regular ascorbic acid. Adv. Ther. 2006, 23, 171-178.

98. Knight, A. Systematic reviews of animal experiments demonstrate poor human clinical and toxicological utility. Altern. Lab. Anim. 2007, 35, 641-659.

99. Seok, J.; Warren, H.S.; Cuenca, A.G.; Mindrinos, M.N.; Baker, H.V.; Xu, W.; Richards, D.R.; McDonald-Smith, G.P.; Gao, H.; Hennessy, L.; et al. Genomic responses in mouse models poorly mimic human inflammatory diseases. Proc. Natl. Acad. Sci. USA 2013, 110, 3507-3512.

100. He, F.J.; Nowson, C.A.; MacGregor, G.A. Fruit and vegetable consumption and stroke: Meta-analysis of cohort studies. Lancet 2006, 367, 320-326.

101. Dauchet, L.; Amouyel, P.; Hercberg, S.; Dallongeville, J. Fruit and vegetable consumption and risk of coronary heart disease: A meta-analysis of cohort studies. J. Nutr. 2006, 136, 2588-2593.

102. Steinmetz, K.A.; Potter, J.D. Vegetables, fruit, and cancer prevention: A review. J. Am. Diet. Assoc. 1996, 96, 1027-1039.

103. Riboli, E.; Norat, T. Epidemiologic evidence of the protective effect of fruit and vegetables on cancer risk. Am. J. Clin. Nutr. 2003, 78, 559S-569S.

104. Block, G.; Norkus, E.; Hudes, M.; Mandel, S.; Helzlsouer, K. Which plasma antioxidants are most related to fruit and vegetable consumption? Am. J. Epidemiol. 2001, 154, 1113-1118.

105. Mente, A.; de Koning, L.; Shannon, H.S.; Anand, S.S. A systematic review of the evidence supporting a causal link between dietary factors and coronary heart disease. Arch. Intern. Med. 2009, 169, 659-669.

106. Ye, Z.; Song, H. Antioxidant vitamins intake and the risk of coronary heart disease: Meta-analysis of cohort studies. Eur. J. Cardiovasc. Prev. Rehabil. 2008, 15, 26-34.

107. Knekt, P.; Ritz, J.; Pereira, M.A.; O’Reilly, E.J.; Augustsson, K.; Fraser, G.E.; Goldbourt, U.; Heitmann, B.L.; Hallmans, G.; Liu, S.; et al. Antioxidant vitamins and coronary heart disease risk: A pooled analysis of 9 cohorts. Am. J. Clin. Nutr. 2004, 80, 1508-1520.

108. Lawlor, D.A.; Davey Smith, G.; Kundu, D.; Bruckdorfer, K.R.; Ebrahim, S. Those confounded vitamins: What can we learn from the differences between observational versus randomised trial evidence? Lancet 2004, 363, 1724-1727.

109. Maserejian, N.N.; Giovannucci, E.L.; McVary, K.T.; McKinlay, J.B. Dietary, but not supplemental, intakes of carotenoids and vitamin $\mathrm{C}$ are associated with decreased odds of lower urinary tract symptoms in men. J. Nutr. 2011, 141, 267-273. 
110. Osganian, S.K.; Stampfer, M.J.; Rimm, E.; Spiegelman, D.; Hu, F.B.; Manson, J.E.; Willett, W.C. Vitamin C and risk of coronary heart disease in women. J. Am. Coll. Cardiol. 2003, 42, 246-252.

111. Agarwal, M.; Mehta, P.K.; Dwyer, J.H.; Dwyer, K.M.; Shircore, A.M.; Nordstrom, C.K.; Sun, P.; Paul-Labrador, M.; Yang, Y.; Merz, C.N. Differing relations to early atherosclerosis between vitamin $\mathrm{C}$ from supplements vs. food in the Los Angeles atherosclerosis Study: A prospective cohort study. Open Cardiovasc. Med. J. 2012, 6, 113-121.

112. Henriquez-Sanchez, P.; Sanchez-Villegas, A.; Doreste-Alonso, J.; Ortiz-Andrellucchi, A.; Pfrimer, K.; Serra-Majem, L. Dietary assessment methods for micronutrient intake: A systematic review on vitamins. Br. J. Nutr. 2009, 102, S10-S37.

113. Thompson, F.E.; Byers, T. Dietary assessment resource manual. J. Nutr. 1994, 124, 2245S-2317S.

114. Dehghan, M.; Akhtar-Danesh, N.; McMillan, C.R.; Thabane, L. Is plasma vitamin C an appropriate biomarker of vitamin $\mathrm{C}$ intake? A systematic review and meta-analysis. Nutr. J. 2007, 6, 41 .

115. Lykkesfeldt, J.; Poulsen, H.E. Is vitamin C supplementation beneficial? Lessons learned from randomised controlled trials. Br. J. Nutr. 2010, 103, 1251-1259.

116. Carr, A.C.; Pullar, J.M.; Moran, S.; Vissers, M.C.M. Bioavailability of vitamin C from kiwifruit in non-smoking males: Determination of 'healthy' and 'optimal' intakes. J. Nutr. Sci. 2012, 1 , e14.

117. Franz, W.L.; Heyl, H.L.; Sands, G.W. Blood ascorbic acid level in bioflavonoid and ascorbic acid therapy of common cold. J. Am. Med. Assoc. 1956, 162, 1224-1226.

118. Arminio, J.J.; Johnston, J.H.; Tebrock, H.E. Usefulness of bioflavonoids and ascorbic acid in treatment of common cold. J. Am. Med. Assoc. 1956, 162, 1227-1233.

119. Baird, I.M.; Hughes, R.E.; Wilson, H.K.; Davies, J.E.; Howard, A.N. The effects of ascorbic acid and flavonoids on the occurrence of symptoms normally associated with the common cold. Am. J. Clin. Nutr. 1979, 32, 1686-1690.

120. Stevenson, D.E.; Hurst, R.D. Polyphenolic phytochemicals_-Just antioxidants or much more? Cell. Mol. Life Sci. 2007, 64, 2900-2916.

121. Gao, Y.; Li, W.; Jia, L.; Li, B.; Chen, Y.C.; Tu, Y. Enhancement of (-)-epigallocatechin-3-gallate and theaflavin-3-3'-digallate induced apoptosis by ascorbic acid in human lung adenocarcinoma SPC-A-1 cells and esophageal carcinoma Eca-109 cells via MAPK pathways. Biochem. Biophys. Res. Commun. 2013, 438, 370-374.

122. Calero, C.I.; Beltran Gonzalez, A.N.; Gasulla, J.; Alvarez, S.; Evelson, P.; Calvo, D.J. Quercetin antagonism of GAB receptors is prevented by ascorbic acid through a redox-independent mechanism. Eur. J. Pharmacol. 2013, doi:10.1016/j.ejphar.2013.07.044. 
Reprinted from Nutrients. Cite as: Carr, A.C.; Bozonet, S.M.; Pullar, J.M.; Simcock, J.W.; Vissers, M.C.M. A Randomized Steady-State Bioavailability Study of Synthetic versus Natural (Kiwifruit-Derived) Vitamin C. Nutrients 2013, 5, 3684-3695.

Article

\title{
A Randomized Steady-State Bioavailability Study of Synthetic versus Natural (Kiwifruit-Derived) Vitamin C
}

\author{
Anitra C. Carr ${ }^{1, *}$, Stephanie M. Bozonet ${ }^{1}$, Juliet M. Pullar ${ }^{1}$, Jeremy W. Simcock ${ }^{2}$ and \\ Margreet C. M. Vissers ${ }^{1}$
}

1 Centre for Free Radical Research, Department of Pathology \& Biomedical Science, University of Otago, Christchurch, PO Box 4345, Christchurch 8140, New Zealand; E-Mails: stephanie.bozonet@otago.ac.nz (S.M.B.); juliet.pullar@otago.ac.nz (J.M.P.); margreet.vissers@otag (M.C.M.V.)

2 Department of Plastic and Reconstructive Surgery, University of Otago, Christchurch, PO Box 4345, Christchurch 8140, New Zealand; E-Mail: jeremy.simcock@cdhb.health.nz

* Author to whom correspondence should be addressed; E-Mail: anitra.carr@otago.ac.nz; Tel.: +64-3-378-6498; Fax: +64-3-378-6540.

Received: 23 July 2013; in revised form: 15 August 2013 / Accepted: 26 August 2013 /

Published: 17 September 2013

\begin{abstract}
Whether vitamin $\mathrm{C}$ from wholefoods has equivalent bioavailability to a purified supplement remains unclear. We have previously showed that kiwifruit provided significantly higher serum and tissue ascorbate levels than synthetic vitamin $\mathrm{C}$ in a genetically vitamin $\mathrm{C}$-deficient mouse model, suggesting a synergistic activity of the whole fruit. To determine if these results are translatable to humans, we carried out a randomized human study comparing the bioavailability of vitamin $\mathrm{C}$ from kiwifruit with that of a vitamin $\mathrm{C}$ tablet of equivalent dosage. Thirty-six young non-smoking adult males were randomized to receive either half a gold kiwifruit (Actinidia Chinensis var. Hort 16A) per day or a comparable vitamin C dose $(50 \mathrm{mg})$ in a chewable tablet for six weeks. Ascorbate was monitored weekly in fasting venous blood and in urine, semen, leukocytes, and skeletal muscle (vastus lateralis) pre- and post-intervention. Dietary intake of vitamin $\mathrm{C}$ was monitored using seven day food and beverage records. Participant ascorbate levels increased in plasma $(P<0.001)$, urine $(P<0.05)$, mononuclear cells $(P<0.01)$, neutrophils $(P<0.01)$ and muscle tissue $(P<0.001)$ post intervention. There were no significant differences in vitamin $\mathrm{C}$ bioavailability between the two intervention groups in any of the fluid, cell or tissue samples tested. Overall, our study showed comparable bioavailability of synthetic and kiwifruit-derived vitamin C.
\end{abstract}


Keywords: ascorbate; ascorbic acid; human; plasma; urine; semen; leukocytes; skeletal muscle

\section{Introduction}

Vitamin C (ascorbate) is an essential water-soluble micronutrient that is obtained through the diet primarily from fruits and vegetables [1]. The bioavailability of dietary vitamin $\mathrm{C}$ represents the proportion absorbed by the intestines and available for metabolic processes within the body. Vitamin $\mathrm{C}$ is actively transported into the body via two sodium-dependent vitamin $\mathrm{C}$ transporters, SVCT1 and SVCT2, which exhibit different tissue distributions and uptake kinetics [2,3]. SVCT1 is expressed in epithelial tissue and is primarily responsible for intestinal uptake and renal reabsorption of vitamin $\mathrm{C}$, the latter maintaining whole body homeostasis [3]. SVCT2 is expressed in specialized and metabolically active tissues and is required for delivery of vitamin $\mathrm{C}$ to tissues with a high demand for the vitamin either for enzymatic reactions [4] and/or to help protect these tissues from oxidative stress [3].

Kiwifruit are rich in vitamin C [5] and we have previously used a genetically vitamin C-deficient mouse model (the Gulo mouse) to investigate the comparative bioavailability of synthetic versus kiwifruit-derived vitamin C [6]. Interestingly, we found that kiwifruit gel provided higher serum, leukocyte, heart, liver and kidney levels of ascorbate than equivalent amounts of purified vitamin $\mathrm{C}$, suggesting a synergistic activity of the whole fruit in this model. Although synthetic and food-derived vitamin $\mathrm{C}$ are chemically identical, the bioavailability of vitamin $\mathrm{C}$ could potentially be affected by the numerous micronutrients and phytochemicals with antioxidant properties that are present in fruits and vegetables [7,8]. For example, kiwifruit contain reasonable amounts of vitamin E [9], which has been shown to spare vitamin $C$ in an animal model [10]. Kiwifruit also contain numerous different flavonoids [9,11], some of which can inhibit the in vitro oxidation of vitamin $\mathrm{C}$ via direct scavenging of free radicals and/or chelation of redox-active metal ions $[7,8]$.

To determine if the results of our animal study [6] are translatable to humans, we carried out a randomized human study comparing the bioavailability of vitamin $\mathrm{C}$ from gold kiwifruit (Actinidia Chinensis var. Hort 16A) with a tablet of equivalent dosage. We have previously shown that consumption of half a gold kiwifruit per day results in a significant increase in plasma ascorbate in individuals with low initial levels $(<23 \mu \mathrm{mol} / \mathrm{L})$ [12]. For this study, we chose a dose of half a kiwifruit per day and the equivalent $50 \mathrm{mg}$ /day vitamin $\mathrm{C}$ since this dose lies on the steeply rising portion of the sigmoidal plasma bioavailability curve [13]. This enhances the likelihood of detecting a difference between the two interventions compared with doses $>100 \mathrm{mg} /$ day where plasma saturation is approached [13].

Several previous studies have tested the comparative bioavailability of synthetic versus food-derived vitamin C utilizing plasma and/or urine levels [14-18]. However, only one has investigated the comparative bioavailability of vitamin $C$ in leukocytes [19]. Therefore, in addition to plasma and urine, we have monitored the bioavailability of vitamin $\mathrm{C}$ in peripheral blood mononuclear cells and neutrophils, seminal fluid and skeletal muscle tissue [20] before and after the 
six week intervention. We also monitored the participants' dietary intake of vitamin $\mathrm{C}$ using seven day food and beverage records.

\section{Study Design and Methods}

\subsection{Participants}

This study was conducted according to the guidelines laid down in the Declaration of Helsinki and all procedures involving human participants were approved by the Upper South Regional Ethics Committee (\#URA/11/02/003). The study was registered with the Australian New Zealand Clinical Trials Registry (\#ACTRN12611000162910).

Non-smoking males aged 18-35 years from local tertiary institutes were screened to ascertain their eligibility for the study. Exclusion criteria included recent smoker (within previous year), allergy/intolerance to kiwifruit, taking vitamin C-containing supplements (within past three months), taking prescription medication (within past three months), excessive alcohol consumption ( $>21$ standard drinks/week), high fruit and vegetable consumption ( $>5$ servings per day), diabetes mellitus, bleeding disorders, and fainting due to fear of needles. Anthropometric measurements were carried out to determine body mass index (BMI) and a fasting venous blood sample was drawn to determine plasma ascorbate levels as described below.

Sample size calculations indicated that at $80 \%$ power and alpha $=0.05$, a sample size of 15 participants per intervention group would detect a minimum difference of $10 \mu \mathrm{mol} / \mathrm{L}$ ascorbate as determined using data derived from our previous vitamin $\mathrm{C}$ bioavailability study [12]. To allow for potential withdrawal during the study, 36 non-smoking participants (18 per group) with below average plasma ascorbate levels were enrolled for the study and provided written informed consent.

\subsection{Study Design}

The study employed a parallel arms design and the participants were randomized into a $50 \mathrm{mg}$ vitamin $\mathrm{C}$ per day group or a half kiwifruit per day group using a random numbers chart. A parallel arms rather than cross-over study design was chosen to avoid potential confounding by kiwifruit-derived constituents, e.g., vitamin E, which may not wash out prior to the vitamin $\mathrm{C}$ supplement phase of a cross-over study. A lead-in phase of five weeks allowed the participants time to control their dietary vitamin $\mathrm{C}$ intake by eliminating juice and substituting high vitamin $\mathrm{C}$ foods, e.g., citrus and kiwifruit, with low vitamin $\mathrm{C}$ foods, e.g., apples and bananas (guidelines were provided as to the vitamin $\mathrm{C}$ content of common foods). This was followed by an intervention phase of six weeks and a washout phase of four weeks (Figure 1). Fasting venous blood samples were drawn weekly to monitor plasma ascorbate levels. Twenty four hour urine, semen, and leukocyte samples were collected at week five (baseline), week 11 (post-intervention) and week 15 (post-washout). Muscle biopsies were carried out at baseline and post-intervention. Participants also completed four seven-day food and beverage records (at the beginning of the study, pre- and post-intervention and post-washout). 
Figure 1. Parallel groups study design. * Weekly plasma samples; ** urine, semen and leukocyte samples; *** urine, semen, leukocyte and skeletal muscle samples.
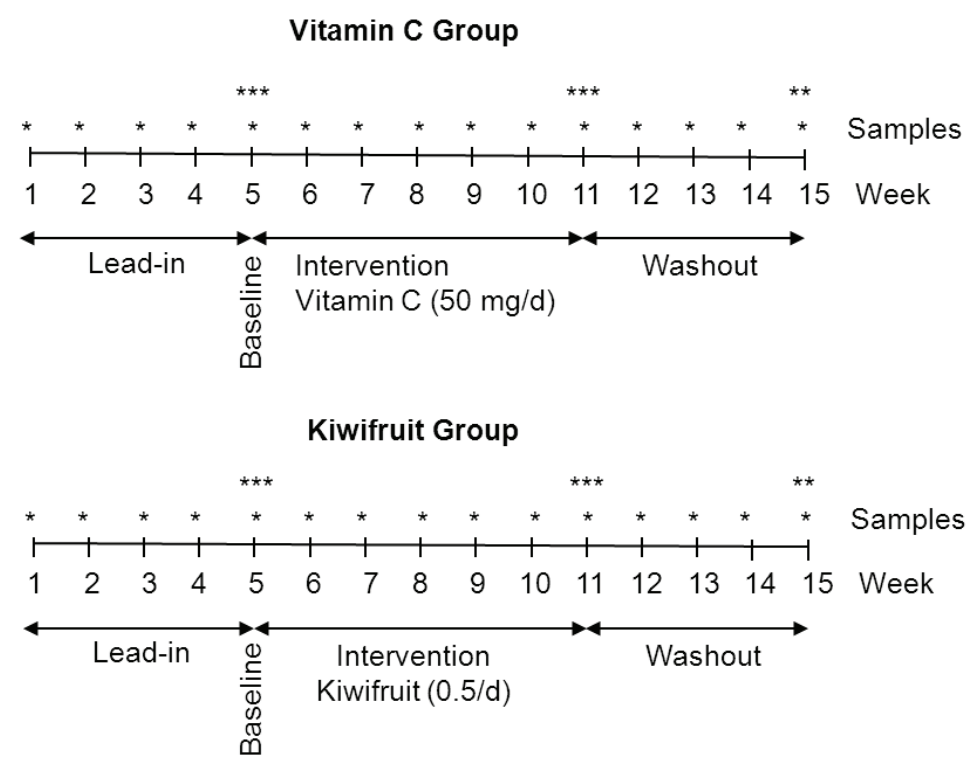

\subsection{Interventions}

Chewable orange-flavored vitamin $\mathrm{C}$ tablets were provided by Tishcon Corp., Westbury, NY, USA. Analysis of the tablets indicated that they contained $52 \mathrm{mg}$ of vitamin C per tablet. Participants in the vitamin $\mathrm{C}$ tablet arm of the study were asked to consume one tablet per day.

Gold kiwifruit (Actinidia chinensis var. Hort. 16A) were provided by Zespri International Ltd., Mount Maunganui, New Zealand, and were stored at $\leq 4{ }^{\circ} \mathrm{C}$. The vitamin $\mathrm{C}$ content of the kiwifruit was monitored by HPLC with electrochemical detection [12] and indicated that the kiwifruit flesh contained $116 \pm 10 \mathrm{mg}$ vitamin $\mathrm{C}$ per $100 \mathrm{~g}$ (mean $\pm \mathrm{SD}, n=5$ ); this value did not change during cold storage of the fruit. Participants in the kiwifruit arm were asked to consume half a fresh kiwifruit daily, not including the skin. Therefore, the actual amount of vitamin $\mathrm{C}$ consumed was estimated to be $\sim 53 \mathrm{mg}$ per half a kiwifruit.

\subsection{Sample Collection and Processing}

\subsubsection{Plasma, Urine and Semen}

Peripheral blood was collected into $5 \mathrm{~mL} \mathrm{~K}_{3}$-EDTA vacutainer tubes and plasma was isolated by centrifugation at $4{ }^{\circ} \mathrm{C}$. Urine was collected over $24 \mathrm{~h}$ into pre-weighed collection bottles containing $\mathrm{K}_{2}$-EDTA. Semen was collected into pre-weighed collection containers containing $\mathrm{K}_{2}$-EDTA and kept cold until processed. The plasma, urine and semen samples were extracted with perchloric acid containing DTPA, as described previously [12], prior to storage at $-80{ }^{\circ} \mathrm{C}$ until $\mathrm{HPLC}$ analysis.

\subsubsection{Mononuclear Leukocytes and Neutrophils}

White blood cells were purified from heparinized whole blood as described previously [20], the cell pellets counted and extracted with perchloric acid containing DTPA, and the supernatants stored at $-80{ }^{\circ} \mathrm{C}$ until HPLC analysis. 


\subsubsection{Skeletal Muscle Tissue}

A small piece of tissue ( $14 \mathrm{mg}$ ) was removed from the vastus lateralis using a Quick-Core biopsy needle (14 gauge, $6 \mathrm{~cm}$ long with a $20 \mathrm{~mm}$ throw, from Cook Medical Inc., Bloomington, IN, USA), and processed as described previously [20], extracted with perchloric acid containing DTPA, and stored at $-80{ }^{\circ} \mathrm{C}$ until HPLC analysis.

\subsection{Analysis of Vitamin C by HPLC}

The ascorbate content of the kiwifruit, plasma, urine, semen, leukocytes and muscle tissue was analyzed using reverse-phase HPLC with electrochemical detection as described previously [12]. Briefly, samples were separated on a Synergi $4 \mu$ Hydro-RP 80A $150 \times 4.6 \mathrm{~mm}$ column (Phenomenex NZ Ltd., Auckland, New Zealand) using a Waters 600 solvent delivery system with a Hitachi L-2200 refrigerated autosampler and an ESA Coulochem II electrochemical detector (+200 mV electrode potential and $20 \mu \mathrm{A}$ sensitivity). The mobile phase comprised $80 \mathrm{mM}$ sodium acetate buffer, $\mathrm{pH} 4.8$, containing DTPA $(0.54 \mathrm{mmol} / \mathrm{L})$ and freshly added paired-ion reagent $n$-octylamine $(1 \mu \mathrm{mol} / \mathrm{L})$, delivered at a flow rate of $1.2 \mathrm{~mL} / \mathrm{min}$. A standard curve of sodium-L-ascorbate, standardized spectrophotometrically, was freshly prepared for each HPLC run in $77 \mathrm{mmol} / \mathrm{L}$ HPLC-grade perchloric acid containing DTPA $(100 \mu \mathrm{mol} / \mathrm{L})$.

\subsection{Analysis of Food and Beverage Records}

The vitamin $\mathrm{C}$ content of the food and beverage dietary records was estimated using Diet Cruncher software [21], and the New Zealand FOODfiles Food Composition Database (2006) as described previously [12].

\subsection{Statistical Analysis}

Data is represented as mean $\pm \mathrm{SD}$ for group characteristics and mean $\pm \mathrm{SEM}$ for comparison of group means. The differences between paired and unpaired data were determined by two-tailed Students's $t$-tests and $P$ values $\leq 0.05$ were considered significant. Analysis of variance with Fisher pairwise multiple comparison procedure was carried out using SigmaStat software [22].

\section{Results}

\subsection{Participant Characteristics}

One hundred and thirty four non-smoking individuals were screened and their fasting plasma ascorbate concentrations determined. The average \pm SD plasma ascorbate concentration for these individuals was $48 \pm 16 \mu \mathrm{mol} / \mathrm{L}$ (Table 1). Thirty six of the individuals with below average plasma ascorbate levels, who also satisfied the other inclusion criteria, were enrolled in the study and randomized into the vitamin $\mathrm{C}$ tablet group or the half kiwifruit per day group. The average $\pm \mathrm{SD}$ fasting plasma ascorbate levels of the enrolled groups were $31 \pm 11 \mu \mathrm{M}$ and $34 \pm 10 \mu \mathrm{M}$, with a range of $3 \mu \mathrm{M}$ to $44 \mu \mathrm{M}$ (Table 1). There were no significant differences between the two groups. 
Table 1. Characteristics of individuals screened and enrolled in the study.

\begin{tabular}{cccc}
\hline & $\begin{array}{c}\text { Screened }^{\text {a }} \\
(\boldsymbol{n}=\mathbf{1 3 4})\end{array}$ & $\begin{array}{c}\text { Vitamin C Group }^{\text {a }} \\
(\boldsymbol{n}=\mathbf{1 8})\end{array}$ & $\begin{array}{c}\text { Kiwifruit Group }^{\text {a }} \\
(\boldsymbol{n}=\mathbf{1 8})\end{array}$ \\
\hline Age $($ years $)$ & $21 \pm 3$ & $21 \pm 3$ & $22 \pm 4$ \\
Weight $(\mathrm{kg})$ & $81 \pm 16$ & $84 \pm 19$ & $89 \pm 23$ \\
Height $(\mathrm{cm})$ & $182 \pm 7$ & $181 \pm 7$ & $181 \pm 7$ \\
BMI $\left(\mathrm{kg} / \mathrm{m}^{2}\right)$ & $24 \pm 4$ & $26 \pm 5$ & $27 \pm 6 *$ \\
Ascorbate $(\mu \mathrm{mol} / \mathrm{L})$ & $48 \pm 16$ & $31 \pm 11 * *$ & $34 \pm 10^{* *}$ \\
\hline
\end{tabular}

${ }^{a}$ Data represent mean $\pm \mathrm{SD} ; * P<0.05,{ }^{* *} P<0.001$ for unpaired $t$-test of intervention groups versus screened group. There were no significant differences between the intervention groups.

\subsection{Dietary Intake of Vitamin C}

Analysis of the food and beverage records indicated a vitamin $\mathrm{C}$ intake of $\sim 30 \mathrm{mg} /$ day at baseline (Table 2). Addition of the vitamin $\mathrm{C}$ tablet or half a kiwifruit per day to the daily diet of the two groups resulted in an increase in vitamin $\mathrm{C}$ intake, with both groups approaching $\sim 75 \mathrm{mg}$ (Table 2), and no difference between the two groups $(P=0.512)$. Following four weeks washout, the vitamin $\mathrm{C}$ intake had returned to baseline levels.

Table 2. Change in vitamin $\mathrm{C}$ intake and ascorbate concentration in body fluids, cells and tissue following supplementation with $50 \mathrm{mg}$ vitamin $\mathrm{C}$ or half a kiwifruit per day for six weeks.

\begin{tabular}{|c|c|c|c|c|c|c|c|}
\hline & \multicolumn{3}{|c|}{$\begin{array}{c}\text { Vitamin C group }{ }^{a} \\
\text { (50 mg/day) }\end{array}$} & \multicolumn{3}{|c|}{$\begin{array}{c}\text { Kiwifruit group a } \\
\text { (half/day) }\end{array}$} & \multirow{2}{*}{$\begin{array}{c}\text { Between } \\
\text { group } \\
\text { Intervention } \\
P_{\text {value }^{b}}\end{array}$} \\
\hline & Baseline & Intervention & Washout & Baseline & Intervention & Washout & \\
\hline Intake (mg/day) & $31.2 \pm 3.1$ & $76.7 \pm 2.6 * * *$ & $29.1 \pm 2.9$ & $28.6 \pm 3.1$ & $73.4 \pm 4.2 * * *$ & $31.4 \pm 5.1$ & 0.512 \\
\hline Plasma $(\mu \mathrm{mol} / \mathrm{L})$ & $23.5 \pm 2.5$ & $51.3 \pm 3.5 * * *$ & $34.3 \pm 4.4 *$ & $22.7 \pm 2.5$ & $45.5 \pm 2.5 * * *$ & $30.5 \pm 3.1$ & 0.860 \\
\hline Urine $(\mu \mathrm{mol} / 24 \mathrm{~h})$ & $42.8 \pm 9.2$ & $104.2 \pm 27.6 *$ & $59.5 \pm 19.9$ & $32.3 \pm 8.1$ & $70.5 \pm 17.0 *$ & $64.7 \pm 29.8$ & 0.503 \\
\hline Semen $(\mu \mathrm{mol} / \mathrm{L})$ & $284.8 \pm 27.0$ & $321.0 \pm 33.3$ & $264.4 \pm 31.1 *$ & $326.3 \pm 47.5$ & $378.8 \pm 42.7$ & $237.7 \pm 19.6 *$ & 0.676 \\
\hline $\begin{array}{l}\text { Mononuclear cells } \\
\left(\mathrm{nmol} / 10^{8} \text { cells }\right)\end{array}$ & $38.7 \pm 6.2$ & $84.5 \pm 6.3 * * *$ & $78.5 \pm 4.3 * *$ & $60.5 \pm 6.1$ & $90.9 \pm 6.1 * *$ & $75.2 \pm 5.2$ & 0.227 \\
\hline $\begin{array}{c}\text { Neutrophils } \\
\text { (nmol/10 } 10^{8} \text { cells) }\end{array}$ & $21.9 \pm 3.1$ & $39.6 \pm 3.8 * *$ & $24.9 \pm 2.1$ & $13.7 \pm 2.4$ & $30.4 \pm 2.5 * * *$ & $24.8 \pm 1.7 * * *$ & 0.798 \\
\hline $\begin{array}{l}\text { Skeletal muscle } \\
(\mathrm{nmol} / \mathrm{g})\end{array}$ & $14.5 \pm 2.0$ & $61.3 \pm 3.5 * * *$ & nd & $15.1 \pm 2.5$ & $52.8 \pm 5.0 * * *$ & nd & 0.429 \\
\hline
\end{tabular}

${ }^{\mathrm{a}}$ Data represent mean $\pm \mathrm{SEM} ;{ }^{\mathrm{b}} P$ values were determined by unpaired $t$-test of vitamin $\mathrm{C}$ group versus kiwifruit group post-intervention following subtraction of baseline values; $* P<0.05$ and $* * P<0.01$ and $* * * P<0.001$ for paired $t$-test of intervention versus baseline. $n d=$ not determined.

\subsection{Vitamin C Status of Plasma, Urine and Semen}

Plasma ascorbate levels increased significantly during the six week intervention phase, from $\sim 23 \mu \mathrm{mol} / \mathrm{L}$ to a maximum of $\sim 50 \mu \mathrm{mol} / \mathrm{L}$ (Table 2 ). There were no significant differences between the two groups during this period or during the four week washout phase (Figure 2). Similarly, there 
were no significant differences between the two treatment groups for either urinary ascorbate excretion $(P=0.503)$ or seminal ascorbate levels $(P=0.676)$ (Table 2$)$.

Figure 2. Plasma ascorbate concentrations in vitamin $C$ group $(50 \mathrm{mg} / \mathrm{day}, \bullet)$ and kiwifruit group $(0.5 /$ day, $\circ)$. Data represent mean \pm SEM. Two way analysis of variance with Fisher pairwise multiple comparison procedure indicated a significant increase in plasma ascorbate from one week post-intervention (week 6) onwards, but no significant difference between the two interventions.

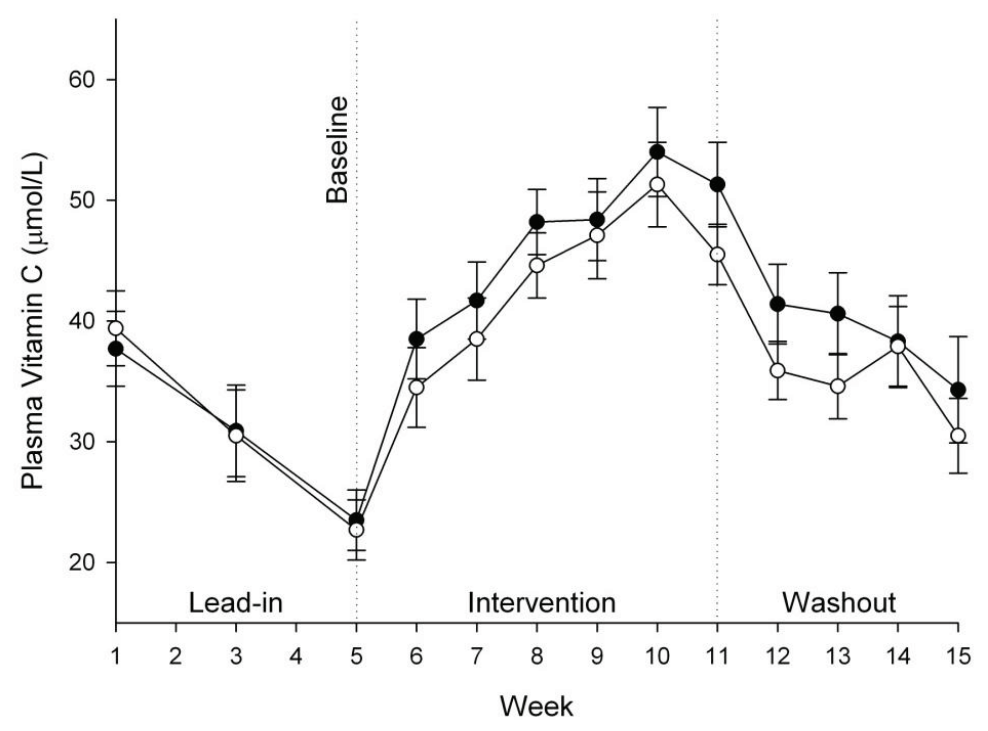

\subsection{Vitamin C Status of Leukocytes and Skeletal Muscle Tissue}

The ascorbate levels in mononuclear cells and neutrophils in both intervention groups increased following the six week intervention (Table 2), but there were no differences between the two interventions $(P=0.798)$. The baseline skeletal muscle tissue ascorbate levels were $\sim 15 \mathrm{nmoL} / \mathrm{g}$ wet weight, and following intervention there were 3.5- to 4-fold increases in tissue ascorbate levels in both treatment groups (Table 2). Once again, there were no significant differences between the two intervention groups $(P=0.429)$.

\section{Discussion}

In direct contrast to our previous Gulo knockout mouse study, which showed clear differences in comparative vitamin $\mathrm{C}$ bioavailability [6], this investigation has shown no differences in the steady-state bioavailability of synthetic versus kiwifruit-derived vitamin $\mathrm{C}$ in plasma, semen, peripheral blood leukocytes and skeletal muscle of humans. Other comparative bioavailability studies in guinea pigs have shown similar results to our animal study [23-27]. Enhanced uptake of vitamin C into specific organs of guinea pigs (e.g., adrenals and spleen) was observed when administered with flavonoid-rich juices/extracts or purified plant flavonoids [23-27], although comparable vitamin C accumulation was observed in some organs (e.g., liver) in several studies [23-25,28], suggesting either tissue specific differences or effects of differences in study design. 
In agreement with our current human study, others have shown little difference in steady-state plasma and/or urine bioavailability of synthetic vitamin $\mathrm{C}$ and that found in different fruits, fruit juices and vegetables [14-19]. Only one previous study has investigated the comparative bioavailability of synthetic versus natural vitamin C in leukocytes [19]. Although neutrophils express SVCT2 [29], when their respiratory burst is activated they primarily transport the oxidized form of ascorbate (dehydroascorbic acid) via the glucose transporters GLUT 1 and GLUT 3, followed by intracellular reduction [30]. In support of our observations with neutrophils and mononuclear cells, Pelletier et al. [19] found no difference in leukocyte ascorbate uptake between synthetic vitamin C (in the presence or absence of rutin) and that from orange juice. Interestingly, an in vitro study showed that the flavonoids myricetin and quercitin inhibited the uptake of both ascorbate and dehydroascorbic acid into monocytic (HL-60 and U937) and lymphocytic (Jurkat) cells [31]. Whether this occurs in vivo is, however, uncertain due to the low plasma bioavailability of flavonoids [32].

Although leukocyte ascorbate status is often used as an indicator of whole body status, whether this is an accurate model for other tissues and organs is uncertain. This premise is supported by our animal study which indicated that different organs exhibited maximal uptake at varying doses of vitamin C [6]. Therefore, we also investigated the previously unreported effects of synthetic versus natural vitamin $\mathrm{C}$ on skeletal muscle ascorbate status. Ascorbate is transported into muscle cells via SVCT2 $[33,34]$. In our study muscle tissue exhibited a greater relative uptake of ascorbate than leukocytes, however, there was again no difference in bioavailability between the synthetic and fruit-derived vitamin $\mathrm{C}$.

We also investigated seminal fluid ascorbate status. Although the baseline seminal fluid ascorbate was lower than vitamin $\mathrm{C}$ replete and healthy non-smoking men [35-37], little change was observed with the low dose vitamin $\mathrm{C}$ tablet or half kiwifruit per day dose. Early studies have shown that vitamin $\mathrm{C}$ intakes of up to $250 \mathrm{mg}$ /day are required to return depleted seminal ascorbate to normal levels [35,36,38], indicating that higher intakes of vitamin C, e.g., those that result in plasma saturation, are required to increase seminal fluid levels [39].

\section{Conclusions}

Numerous animal studies have shown differences between the tissue bioavailability of synthetic and natural or flavonoid-rich vitamin C. However, more evidence is accumulating to indicate that this is not the case in humans. Our current human study showed no difference in bioavailability to plasma, semen, peripheral blood leukocytes and skeletal muscle of kiwifruit-derived vitamin $\mathrm{C}$ compared with a chewable vitamin $\mathrm{C}$ tablet of equivalent dosage. Thus, other nutrients and phytochemicals present in kiwifruit appear to be neither enhancing nor inhibiting the uptake of vitamin C from the whole fruit. However, vitamin C is known to enhance the bioavailability of other nutrients, such as non-heme iron [40,41], and kiwifruit also contain numerous micronutrients and phytochemicals which will undoubtedly confer considerable health benefits in addition to the positive effects of the high levels of vitamin $\mathrm{C}$ also delivered by this fruit [42]. 


\section{Acknowledgments}

We are grateful to the young men who participated in this study, many of whom showed great dedication and perseverance. We acknowledge Maria Webb and Heather Webb for assistance with recruitment, Wathsala Kumarasinghe for the diet analysis, Jo Kepple for the use of Primorus Clinical Trials Unit, Lynley Drummond for consultation on study design, and John Pearson for consultation on statistical power. Role of each author: A.C.C. study design, recruitment, study co-ordination, sample processing, data analysis, writing manuscript; S.B. recruitment, sample processing and analysis, editing manuscript; J.P. editing manuscript; J.S. muscle biopsies, editing manuscript; M.C.M.V. study design, sample processing, editing manuscript. Sources of support included University of Otago, Dunedin, New Zealand and Zespri International Ltd., Mount Maunganui, New Zealand.

\section{Conflicts of Interest}

The authors declare no conflict of interest.

\section{References}

1. Carr, A.C.; Frei, B. Toward a new recommended dietary allowance for vitamin C based on antioxidant and health effects in humans. Am. J. Clin. Nutr. 1999, 69, 1086-1107.

2. Tsukaguchi, H.; Tokui, T.; Mackenzie, B.; Berger, U.V.; Chen, X.Z.; Wang, Y.; Brubaker, R.F.; Hediger, M.A. A family of mammalian $\mathrm{Na}^{+}$-dependent L-ascorbic acid transporters. Nature 1999, 399, 70-75.

3. Savini, I.; Rossi, A.; Pierro, C.; Avigliano, L.; Catani, M.V. SVCT1 and SVCT2: Key proteins for vitamin C uptake. Amino Acids 2008, 34, 347-355.

4. Englard, S.; Seifter, S. The biochemical functions of ascorbic acid. Annu. Rev. Nutr. 1986, 6, 365-406.

5. Nishiyama, I.; Yamashita, Y.; Yamanaka, M.; Shimohashi, A.; Fukuda, T.; Oota, T. Varietal difference in vitamin $\mathrm{C}$ content in the fruit of kiwifruit and other actinidia species. J. Agric. Food Chem. 2004, 52, 5472-5475.

6. Vissers, M.C.M.; Bozonet, S.M.; Pearson, J.F.; Braithwaite, L.J. Dietary ascorbate affects steady state tissue levels in vitamin C-deficient mice: Tissue deficiency after sub-optimal intake and superior bioavailability from a food source (kiwifruit). Am. J. Clin. Nutr. 2011, 93, 292-301.

7. Beker, B.Y.; Sonmezoglu, I.; Imer, F.; Apak, R. Protection of ascorbic acid from copper(II)-catalyzed oxidative degradation in the presence of flavonoids: Quercetin, catechin and morin. Int. J. Food Sci. Nutr. 2011, 62, 504-512.

8. Clemetson, C.A.; Andersen, L. Plant polyphenols as antioxidants for ascorbic acid. Ann. N. Y. Acad. Sci. 1966, 136, 341-476.

9. Fiorentino, A.; D’Abrosca, B.; Pacifico, S.; Mastellone, C.; Scognamiglio, M.; Monaco, P. Identification and assessment of antioxidant capacity of phytochemicals from kiwi fruits. J. Agric. Food Chem. 2009, 57, 4148-4155. 
10. Tanaka, K.; Hashimoto, T.; Tokumaru, S.; Iguchi, H.; Kojo, S. Interactions between vitamin C and vitamin $\mathrm{E}$ are observed in tissues of inherently scorbutic rats. J. Nutr. 1997, 127, 2060-2064.

11. Latocha, P.; Krupa, T.; Wolosiak, R.; Worobiej, E.; Wilczak, J. Antioxidant activity and chemical difference in fruit of different Actinidia sp. Int. J. Food Sci. Nutr. 2010, 61, 381-394.

12. Carr, A.C.; Pullar, J.M.; Moran, S.; Vissers, M.C.M. Bioavailability of vitamin C from kiwifruit in non-smoking males: Determination of "healthy" and "optimal" intakes. J. Nutr. Sci. 2012, $1, \mathrm{e} 14$.

13. Levine, M.; Conry-Cantilena, C.; Wang, Y.; Welch, R.W.; Washko, P.W.; Dhariwal, K.R.; Park, J.B.; Lazarev, A.; Graumlich, J.F.; King, J.; et al. Vitamin C pharmacokinetics in healthy volunteers: Evidence for a recommended dietary allowance. Proc. Natl. Acad. Sci. USA 1996, 93, 3704-3709.

14. Mangels, A.R.; Block, G.; Frey, C.M.; Patterson, B.H.; Taylor, P.R.; Norkus, E.P.; Levander, O.A. The bioavailability to humans of ascorbic acid from oranges, orange juice and cooked broccoli is similar to that of synthetic ascorbic acid. J. Nutr. 1993, 123, 1054-1061.

15. Hartzler, E.R. The availability of ascorbic acid in papayas and guavas. J. Nutr. 1945, 30, 355-365.

16. Clayton, M.M.; Borden, R.A. The availability for human nutrition of the vitamin $\mathrm{C}$ in raw cabbage and home-canned tomato juice. J. Nutr. 1943, 25, 349-369.

17. Todhunter, E.N.; Fatzer, A.S. A comparison of the utilization by college women of equivalent amounts of ascorbic acid (vitamin C) in red raspberries and in crystalline form. J. Nutr. 1940, $19,121-130$.

18. Hawley, E.E.; Stephens, D.J.; Anderson, G. The excretion of vitamin C in normal individuals following a comparable quantitative administration in the form of orange juice, cevitamic acid by mouth and cevitamic acid intravenously. J. Nutr. 1936, 11, 135-145.

19. Pelletier, O.; Keith, M.O. Bioavailability of synthetic and natural ascorbic acid. J. Am. Diet. Assoc. 1974, 64, 271-275.

20. Carr, A.C.; Bozonet, S.M.; Pullar, J.M.; Simcock, J.W.; Vissers, M.C. Human skeletal muscle ascorbate is highly responsive to changes in vitamin $\mathrm{C}$ intake and plasma concentrations. Am.J. Clin. Nutr. 2013, 97, 800-807.

21. Diet Cruncher, version 1.6; Nutricomp: Dunedin, New Zealand, 2003.

22. SigmaStat, version 11; Systat Software Inc.: San Jose, CA, USA, 2009.

23. Wilson, H.K.; Price-Jones, C.; Hughes, R.E. The influence of an extract of orange peel on the growth and ascorbic acid metabolism of young guinea-pigs. J. Sci. Food Agric. 1976, 27, 661-666.

24. Douglass, C.D.; Kamp, G.H. The effect of orally administered rutin on the adrenal ascorbic acid level in guinea pigs. J. Nutr. 1959, 67, 531-536.

25. Papageorge, E.; Mitchell, G.L., Jr.; The effect of oral administration of rutin on blood, liver and adrenal ascorbic acid and on liver and adrenal cholesterol in guinea pigs. J. Nutr. 1949, 37, 531-540.

26. Cotereau, H.; Gabe, M.; Gero, E.; Parrot, J.L. Influence of vitamin P (vitamin C2) upon the amount of ascorbic acid in the organs of the guinea pig. Nature 1948, 161, 557-558. 
27. Hughes, R.E.; Hurley, R.J.; Jones, P.R. The retention of ascorbic acid by guinea-pig tissues. Br. J. Nutr. 1971, 26, 433-438.

28. Todhunter, E.N.; Robbins, R.C.; Ivey, G.; Brewer, W. A comparison of the utilization by guinea pigs of equivalent amounts of ascorbic acid (vitamin $\mathrm{C}$ ) in lemon juice and in crystalline form. J. Nutr. 1940, 19, 113-120.

29. Corpe, C.P.; Lee, J.H.; Kwon, O.; Eck, P.; Narayanan, J.; Kirk, K.L.; Levine, M. 6-Bromo-6-deoxy-L-ascorbic acid: An ascorbate analog specific for $\mathrm{Na}^{+}$-dependent vitamin $\mathrm{C}$ transporter but not glucose transporter pathways. J. Biol. Chem. 2005, 280, 5211-5220.

30. Washko, P.W.; Wang, Y.; Levine, M. Ascorbic acid recycling in human neutrophils. J. Biol. Chem. 1993, 268, 15531-15535.

31. Park, J.B.; Levine, M. Intracellular accumulation of ascorbic acid is inhibited by flavonoids via blocking of dehydroascorbic acid and ascorbic acid uptakes in HL-60, U937 and Jurkat cells. J. Nutr. 2000, 130, 1297-1302.

32. Lotito, S.B.; Frei, B. Consumption of flavonoid-rich foods and increased plasma antioxidant capacity in humans: Cause, consequence, or epiphenomenon? Free Radic. Biol. Med. 2006, 41, 1727-1746.

33. Savini, I.; Catani, M.V.; Duranti, G.; Ceci, R.; Sabatini, S.; Avigliano, L. Vitamin C homeostasis in skeletal muscle cells. Free Radic. Biol. Med. 2005, 38, 898-907.

34. Low, M.L.; Sandoval, D.; Aviles, E.; Perez, F.; Nualart, F.; Henriquez, J.P. The ascorbic acid transporter SVCT2 is expressed in slow-twitch skeletal muscle fibres. Histochem. Cell. Biol. 2009, 131, 565-574.

35. Jacob, R.A.; Pianalto, F.S.; Agee, R.E. Cellular ascorbate depletion in healthy men. J. Nutr. 1992, 122, 1111-1118.

36. Fraga, C.G.; Motchnik, P.A.; Shigenaga, M.K.; Helbock, H.J.; Jacob, R.A.; Ames, B.N. Ascorbic acid protects against endogenous oxidative DNA damage in human sperm. Proc. Natl. Acad. Sci. USA 1991, 88, 11003-11006.

37. Kandar, R.; Drabkova, P.; Hampl, R. The determination of ascorbic acid and uric acid in human seminal plasma using an HPLC with UV detection. J. Chromatogr. B 2011, 879, 2834-2839.

38. Jacob, R.A.; Kelley, D.S.; Pianalto, F.S.; Swendseid, M.E.; Henning, S.M.; Zhang, J.Z.; Ames, B.N.; Fraga, C.G.; Peters, J.H. Immunocompetence and oxidant defense during ascorbate depletion of healthy men. Am. J. Clin. Nutr. 1991, 54, 1302S-1309S.

39. Dawson, E.B.; Harris, W.A.; Rankin, W.E.; Charpentier, L.A.; McGanity, W.J. Effect of ascorbic acid on male fertility. Ann. N. Y. Acad. Sci. 1987, 498, 312-323.

40. Beck, K.; Conlon, C.A.; Kruger, R.; Coad, J.; Stonehouse, W. Gold kiwifruit consumed with an iron-fortified breakfast cereal meal improves iron status in women with low iron stores: A 16-week randomised controlled trial. Br. J. Nutr. 2011, 105, 101-109.

41. Hallberg, L.; Brune, M.; Rossander, L. Effect of ascorbic acid on iron absorption from different types of meals. Studies with ascorbic-acid-rich foods and synthetic ascorbic acid given in different amounts with different meals. Hum. Nutr. Appl. Nutr. 1986, 40, 97-113.

42. Stonehouse, W.; Gammon, C.S.; Beck, K.L.; Conlon, C.A.; von Hurst, P.R.; Kruger, R. Kiwifruit: Our daily prescription for health. Can. J. Physiol. Pharmacol. 2013, 91, 442-447. 
Reprinted from Nutrients. Cite as: Carr, A.C.; Bozonet, S.M.; Vissers, M.C.M. A Randomised Cross-Over Pharmacokinetic Bioavailability Study of Synthetic versus Kiwifruit-Derived Vitamin C. Nutrients 2013, 5, 4451-4461.

Article

\title{
A Randomised Cross-Over Pharmacokinetic Bioavailability Study of Synthetic versus Kiwifruit-Derived Vitamin C
}

\author{
Anitra C. Carr *, Stephanie M. Bozonet and Margreet C. M. Vissers
}

Centre for Free Radical Research, Department of Pathology \& Biomedical Science, University of Otago, Christchurch, P.O. Box 4345, Christchurch 8140, New Zealand; E-Mails: stephanie.bozonet@otago.ac.nz (S.M.B.); margreet.vissers@otago.ac.nz (M.C.M.V.)

* Author to whom correspondence should be addressed; E-Mail: anitra.carr@otago.ac.nz; Tel.: +64-3-378-6498; Fax: +64-3-378-6540.

Received: 30 August 2013; in revised form: 8 October 2013 / Accepted: 24 October 2013 / Published: 11 November 2013

\begin{abstract}
Kiwifruit are a rich source of vitamin $\mathrm{C}$ and also contain numerous phytochemicals, such as flavonoids, which may influence the bioavailability of kiwifruit-derived vitamin $\mathrm{C}$. The aim of this study was to compare the relative bioavailability of synthetic versus kiwifruit-derived vitamin $\mathrm{C}$ using a randomised cross-over pharmacokinetic study design. Nine non-smoking males (aged 18-35 years) received either a chewable tablet (200 $\mathrm{mg}$ vitamin C) or the equivalent dose from gold kiwifruit (Actinidia chinensis var. Sungold). Fasting blood and urine were collected half hourly to hourly over the eight hours following intervention. The ascorbate content of the plasma and urine was determined using HPLC with electrochemical detection. Plasma ascorbate levels increased from $0.5 \mathrm{~h}$ after the intervention $(P=0.008)$. No significant differences in the plasma time-concentration curves were observed between the two interventions $(P=0.645)$. An estimate of the total increase in plasma ascorbate indicated complete uptake of the ingested vitamin $\mathrm{C}$ tablet and kiwifruit-derived vitamin $\mathrm{C}$. There was an increase in urinary ascorbate excretion, relative to urinary creatinine, from two hours post intervention $(P<0.001)$. There was also a significant difference between the two interventions, with enhanced ascorbate excretion observed in the kiwifruit group $(P=0.016)$. Urinary excretion was calculated as $\sim 40 \%$ and $\sim 50 \%$ of the ingested dose from the vitamin $\mathrm{C}$ tablet and kiwifruit arms, respectively. Overall, our pharmacokinetic study has shown comparable relative bioavailability of kiwifruit-derived vitamin $\mathrm{C}$ and synthetic vitamin C.
\end{abstract}


Keywords: human; ascorbic acid; ascorbate; plasma; urine; kiwi fruit

\section{Introduction}

In contrast to most mammals, humans cannot synthesis vitamin $\mathrm{C}$ (ascorbate) due to mutation of the terminal biosynthetic enzyme [1]. Thus, the micronutrient must be obtained from dietary sources in order to prevent hypovitaminosis $\mathrm{C}$ and the potentially fatal deficiency disease scurvy [1]. Vitamin C was first isolated from fruit and vegetables and the adrenal glands of animals in the early 1930s and was chemically synthesized in 1933 [2]. Although synthetic and food-derived vitamin C is chemically identical, fruit and vegetables contain numerous nutrients and phytochemicals, e.g., flavonoids, which may affect the bioavailability of food-derived vitamin C. Flavonoids can act as antioxidants via direct scavenging of free radicals [3] and/or chelation of redox-active metal ions $[4,5]$. Thus, it has been proposed that food-derived flavonoids may "spare" vitamin $\mathrm{C}$ and thus increase its bioavailability.

Due to the low bioavailability of flavonoids [6] and tight sequestration of metal ions in vivo [7], this vitamin C "sparing" mechanism may be expected to occur primarily in the intestinal lumen. Vitamin $\mathrm{C}$ is actively transported through the intestinal epithelium via the sodium-dependent vitamin $\mathrm{C}$ transporter 1 (SVCT1) [8]. This transporter is also responsible for renal reabsorption of vitamin C, which helps to maintain whole body homeostasis [9]. SVCT1 has a higher capacity, but lower affinity, for vitamin $\mathrm{C}$ than the SCVT2 isoform, which is found in most other metabolically active cells and tissues [9].

Although food matrix interactions can influence the bioavailability of some nutrients, such as carotenoids [10], the bioavailability of vitamin $\mathrm{C}$ does not appear to be influenced by the food matrix. Kamp et al. [11] found no difference in vitamin C bioavailability from a micronutrient supplement administered in the absence or presence of a corn-based porridge. Mangels et al. [12] also found no difference between vitamin $\mathrm{C}$ bioavailability from oranges compared with orange juice, and although there was a difference in bioavailability between raw and cooked broccoli, this was likely due to differences in mechanical homogenization (chewing), a similar effect to that observed for carotenoid absorption from raw versus cooked carrots.

Vitamin C bioavailability can be determined using either steady-state or pharmacokinetic study designs. The former monitors ascorbate levels in blood, cells, tissues and/or urine following a number of weeks of supplementation, while the latter monitors transient changes in plasma levels and/or urinary excretion over the hours following ingestion of the test substance. We have carried out a steady state bioavailability study comparing synthetic with kiwifruit-derived vitamin $\mathrm{C}$ in healthy non-smoking males supplemented with a vitamin $\mathrm{C}$ tablet or the equivalent dose of vitamin $\mathrm{C}$ from gold kiwifruit [13]. No differences in steady state bioavailability were observed in plasma, urine, semen, leukocytes, or muscle tissue following six weeks of supplementation, despite significant differences being observed in our earlier animal model study [14].

Transient differences between synthetic vitamin $\mathrm{C}$ and that from different fruit juices have been observed using pharmacokinetic models [15-18]. Therefore, the aim of the current study was to compare the relative bioavailability of synthetic versus kiwifruit-derived vitamin $\mathrm{C}$ using a 
randomised cross-over pharmacokinetic study design to determine whether there are any transient differences between the two interventions. Uptake of vitamin $\mathrm{C}$ exhibits sigmoidal steady state kinetics between doses of 30-400 mg/day, with plasma uptake decreasing at doses of $>200 \mathrm{mg} / \mathrm{day}$ and urinary excretion increasing at doses $\geq 100 \mathrm{mg}$ /day [19]. Therefore, we chose a dose of $200 \mathrm{mg}$ vitamin $\mathrm{C}$ and the comparable dose derived from gold kiwifruit (Actinidia chinensis var. Sungold). Our study participants were young non-smoking men with "healthy" (i.e., $>50 \mu \mathrm{mol} / \mathrm{L}$ ) baseline levels of plasma vitamin $\mathrm{C}$.

\section{Study Design and Methods}

\subsection{Participants}

All procedures involving human participants were approved by the New Zealand Health and Disability Ethics Committee (\#URA/11/02/003). Non-smoking males aged 18-35 years were recruited from our previous vitamin $\mathrm{C}$ study databases, and were primarily from local tertiary institutes. Exclusion criteria included: recent smoker (within previous year), low plasma vitamin $\mathrm{C}$ $(<50 \mu \mathrm{mol} / \mathrm{L})$, allergy/intolerance to kiwifruit, taking prescription medication (within past three months), and fainting due to fear of needles. Anthropometric measurements were carried out to determine body mass index (BMI) and a fasting venous blood sample was drawn to determine plasma ascorbate levels as described below. Nine participants with above average plasma ascorbate levels were enrolled for the study and provided written informed consent.

Figure 1. Pharmacokinetic study design. Participants received either 1.5 Sungold kiwifruit (containing $200 \mathrm{mg}$ vitamin C) or chewable vitamin C tablets (total of $200 \mathrm{mg}$ vitamin $\mathrm{C}$ ) in a randomised cross-over design with a three week washout period between the two clinic days.

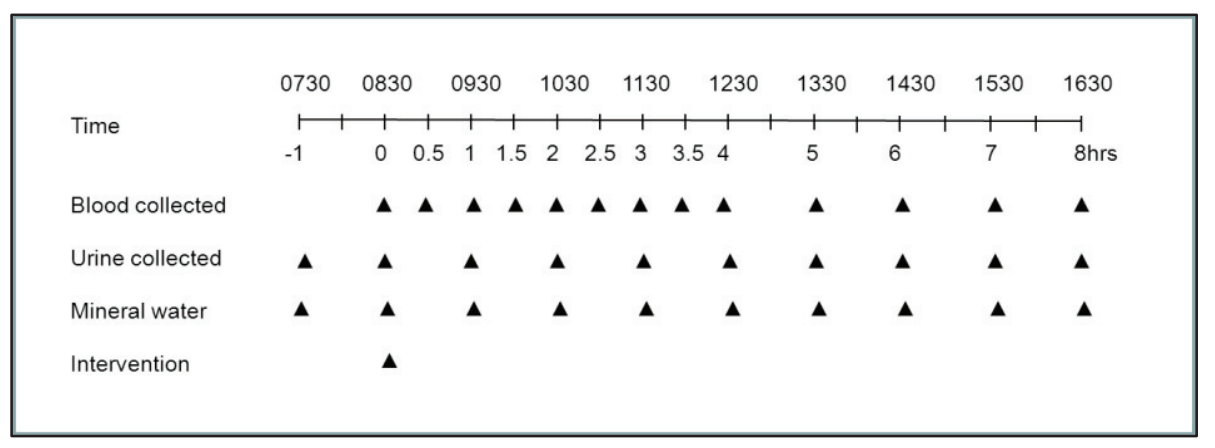

\subsection{Study Design}

The randomised cross-over pharmacokinetic study design comprised eight hours of sampling after a single dose of 1.5 Sungold kiwifruit (containing $200 \mathrm{mg}$ vitamin C) or chewable vitamin C tablets (total of $200 \mathrm{mg}$ vitamin C) with a three-week washout period between the two clinic days (Figure 1) [20]. Following an overnight fast, the participants had a venous cannula inserted and blood samples were collected at baseline, and every $30 \mathrm{~min}$ post-intervention for the first four hours, then every hour for the next four hours. The cannulae were flushed with up to $10 \mathrm{~mL}$ sterile saline after each sampling. Urine was collected at baseline, after emptying the bladder an hour earlier, and then 
every hour post-intervention for the next eight hours. Mineral water $(75 \mathrm{~mL})$ was provided every hour and a cheese sandwich at four hours post-intervention. One of the nine participants did not carry out the vitamin $\mathrm{C}$ tablet supplementation arm of the study.

\subsection{Interventions}

Chewable orange-flavoured vitamin $\mathrm{C}$ tablets were provided by Tishcon Corp., Westbury, NY, USA. Analysis of the tablets indicated that they contained $49 \mathrm{mg}$ of vitamin C per tablet. Participants received a single dose of four tablets, equivalent to $\sim 196 \mathrm{mg}$ vitamin $\mathrm{C}$.

Gold kiwifruit (Actinidia chinensis var. Sungold) were provided by Zespri International Ltd., Mount Maunganui, New Zealand, and were stored at $\leq 4{ }^{\circ} \mathrm{C}$. The vitamin $\mathrm{C}$ content of the kiwifruit was monitored by HPLC with electrochemical detection [21]. HPLC analysis indicated there was $162 \pm 18 \mathrm{mg}$ vitamin $\mathrm{C}$ per $100 \mathrm{~g}$ fruit $(n=5)$. Participants consumed 1.5 kiwifruit, without the skin (i.e., $78 \%$ of $103 \pm 8.4 \mathrm{~g}$ fruit); therefore the actual amount of vitamin $\mathrm{C}$ consumed was estimated to be $\sim 194 \mathrm{mg}$ per 1.5 kiwifruit.

\subsection{Sample Collection and Processing}

Plasma. Peripheral blood was collected into $4 \mathrm{~mL} \mathrm{~K}$-EDTA vacutainer tubes, which were kept on ice at all times. Samples were centrifuged at $4{ }^{\circ} \mathrm{C}$ to pellet cells and the plasma was collected and kept on ice for extraction of ascorbate. Plasma samples were treated with an equal volume of ice-cold $0.54 \mathrm{M}$ perchloric acid containing the metal chelator DTPA $(100 \mu \mathrm{mol} / \mathrm{L})$ to precipitate protein [21]. The de-proteinated supernatants were stored at $-80{ }^{\circ} \mathrm{C}$ until HPLC analysis.

Urine. Urine was collected into pre-weighed $50 \mathrm{~mL}$ sample containers and an aliquot was removed for urinary creatinine determinations. Urine samples were treated with an equal volume of ice-cold $0.54 \mathrm{M}$ perchloric acid containing the metal chelator DTPA $(100 \mu \mathrm{mol} / \mathrm{L})$ to precipitate protein [21]. The de-proteinated supernatants were stored at $-80{ }^{\circ} \mathrm{C}$ until HPLC analysis.

\subsection{Plasma and Urine Analysis}

Creatinine. Urinary creatinine was determined by the Jaffe reaction using an Abbot c8000 analyser (Canterbury Health Laboratories, Christchurch, New Zealand).

Ascorbate. The ascorbate content of the plasma and urine was analysed using reverse-phase HPLC, with a Synergi $4 \mu$ Hydro-RP 80A column and an ESA Coulochem II electrochemical detector, as previously described in detail [13]. In order to measure total ascorbate (reduced and oxidised), duplicate samples were reduced with tris(2-carboxyethyl)phosphine hydrochloride as described previously [22].

\subsection{Data and Statistical Analysis}

Area under the plasma and urinary ascorbate time-concentration curve calculations were determined using the trapezoidal rule as described previously [23]. The increase in circulating ascorbate (by weight) was calculated using Nadler's formula for estimating total blood volume [24] and the increase in urinary ascorbate (by weight) was calculated from the total volume of urine 
collected. Data are represented as mean $\pm \mathrm{SD}$ for group characteristics or mean $\pm \mathrm{SEM}$ for comparisons between group means. Analysis of paired data was determined using two-tailed Students $t$-test with $P<0.05$ indicating statistical difference. Two way analysis of variance with Fisher pairwise multiple comparison procedure was carried out using SigmaStat software (version 11, Systat Software Inc., San Jose, CA, USA).

\section{Results}

\subsection{Participant Characteristics}

At screening, the participants' mean \pm SD age was $24 \pm 5$ years, weight was $78 \pm 10 \mathrm{~kg}$, height was $180 \pm 8 \mathrm{~cm}$, and BMI was $24 \pm 2 \mathrm{~kg} / \mathrm{m}^{2}$. The participants selected for this study had "healthy" (i.e., $>50 \mu \mathrm{mol} / \mathrm{L}$ ) fasting plasma ascorbate concentrations, their mean $\pm \mathrm{SD}$ at screening being $67 \pm 17 \mu \mathrm{mol} / \mathrm{L}(n=9)$. This was to avoid the potentially confounding effects of preferential tissue uptake in individuals with suboptimal ascorbate status at baseline, which could affect the comparative ascorbate levels observed in plasma and urine. Urinary ascorbate levels were detectible in the subjects at baseline, further indicating that plasma ascorbate levels were at or above the renal threshold.

Figure 2. Change in plasma ascorbate uptake following ingestion of $200 \mathrm{mg}$ vitamin $\mathrm{C}$ $(\bullet)$ or 1.5 Sungold kiwifruit (o). Data represent mean $\pm \operatorname{SEM}(n=9)$. Baseline plasma ascorbate concentrations were $61 \pm 6 \mu \mathrm{mol} / \mathrm{L}$ and $66 \pm 6 \mu \mathrm{mol} / \mathrm{L}$ for the vitamin $\mathrm{C}$ and kiwifruit groups, respectively. Two way analysis of variance with Fisher pairwise multiple comparison procedure indicated a significant increase in plasma ascorbate from $0.5 \mathrm{~h}$ post intervention $(P=0.008)$, but no significant difference between the two interventions $(P=0.645)$.

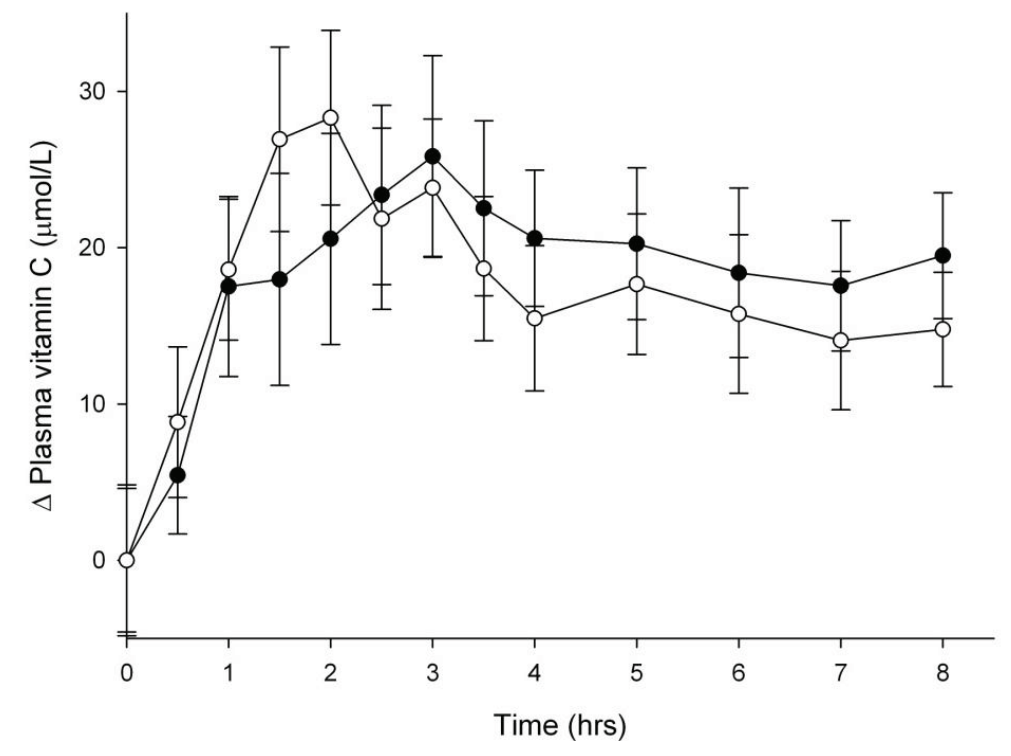

\subsection{Vitamin C Uptake into Plasma}

Plasma ascorbate levels, following ingestion of either $200 \mathrm{mg}$ vitamin C tablets or 1.5 Sungold kiwifruit (providing an equivalent dose of $\sim 200 \mathrm{mg}$ vitamin C), are shown in Figure 2. A statistically 
significant increase in plasma ascorbate was observed as early as $0.5 \mathrm{~h}$ post intervention $(P=0.008)$. No significant differences in the plasma time-concentration curves were observed between the two interventions $(P=0.645)$. Area under the ascorbate time-concentration curves also indicated no difference between the two interventions (Table 1). An estimate of the total increase in plasma ascorbate indicated that all of the ingested vitamin $\mathrm{C}$ tablet and kiwifruit-derived vitamin $\mathrm{C}$ accumulated in plasma over the eight-hour time course (Table 1).

Table 1. Area under the plasma and urinary ascorbate time-concentration curves (AUC) and total increase in plasma and urinary ascorbate. Subjects were supplemented with $200 \mathrm{mg}$ vitamin C or 1.5 Sungold kiwifruit and ascorbate concentrations in plasma and urine were determined over the eight hours post intervention.

\begin{tabular}{cccc}
\hline & Vitamin C (200 mg) $^{\mathbf{a}}$ & Kiwifruit (1.5 Sungold) $^{\mathbf{a}}$ & $\boldsymbol{P}^{\text {P value }}{ }^{\mathbf{b}}$ \\
\hline Plasma AUC $(\mathrm{h} \times \mu \mathrm{mol} / \mathrm{L})$ & $220 \pm 23$ & $237 \pm 13$ & 0.483 \\
Plasma ascorbate $(\mathrm{mg})^{\mathrm{c}}$ & $211 \pm 18$ & $227 \pm 16$ & 0.496 \\
Urinary AUC $(\mathrm{h} \times \mu \mathrm{mol} / \mathrm{mmol}$ creatinine) & $618 \pm 133$ & $856 \pm 118$ & 0.004 \\
Urinary ascorbate $(\mathrm{mg})$ & $74 \pm 14$ & $101 \pm 10$ & 0.033 \\
\hline
\end{tabular}

${ }^{\mathrm{a}}$ Data represent mean $\pm \mathrm{SEM} ;{ }^{\mathrm{b}} P$ values were determined using paired two-tailed Students $t$-test. ${ }^{\mathrm{c}}$ Total blood volumes were estimated using Nadler's formula [24].

Figure 3. Change in urinary ascorbate excretion following ingestion of $200 \mathrm{mg}$ vitamin $\mathrm{C}(\bullet)$ or 1.5 Sungold kiwifruit $(\circ)$. Data represent mean $\pm \operatorname{SEM}(n=9)$. Baseline urinary ascorbate concentrations were $10 \pm 4 \mu \mathrm{mol} / \mathrm{mmol}$ creatinine and $14 \pm 3 \mu \mathrm{mol} / \mathrm{mmol}$ creatinine for the vitamin $\mathrm{C}$ and kiwifruit groups, respectively. Two way analysis of variance with Fisher pairwise multiple comparison procedure indicated a significant increase in plasma vitamin $\mathrm{C}$ from two hours post intervention $(P<0.001)$, as well as a significant difference between the two interventions $(P=0.016)$.

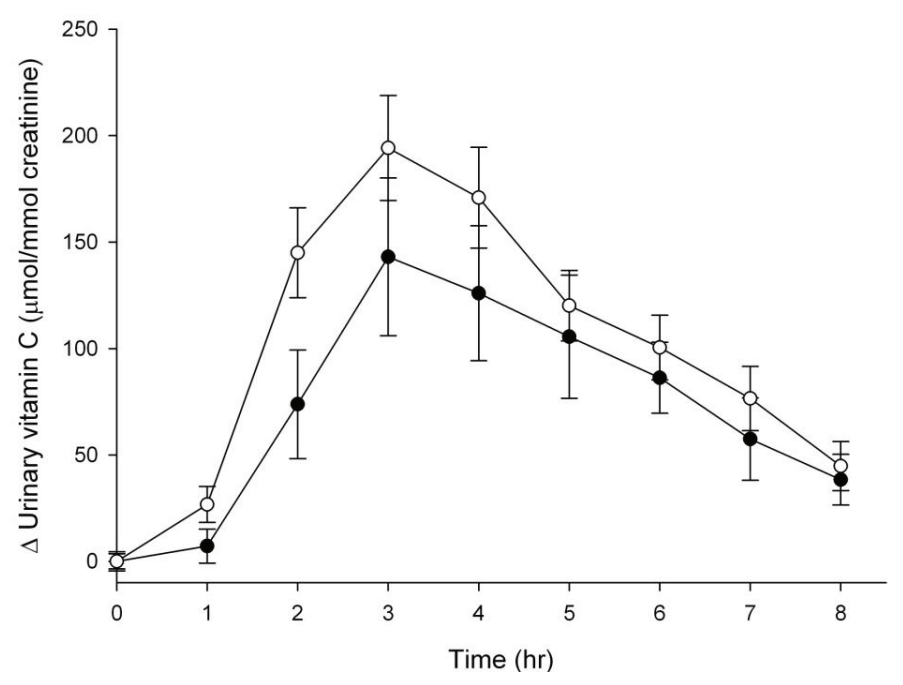

\subsection{Vitamin C Excretion in Urine}

Urinary ascorbate excretion was monitored over the eight hours following intervention with vitamin $\mathrm{C}$ tablets or Sungold kiwifruit and standardised to urinary creatinine concentrations (Figure 3). There was a significant increase in ascorbate excretion from two hours post intervention 
$(P<0.001)$ and also a significant difference between the two interventions, with enhanced ascorbate excretion observed in the kiwifruit group $(P=0.016$, Figure 3$)$. This difference was confirmed with ascorbate area under the time-concentration curves (Table 1). The total increase in ascorbate excretion over the eight hours indicated $\sim 40 \%$ and $\sim 50 \%$ excretion of the ingested dose from the supplement and kiwifruit arms, respectively (Table 1).

\section{Discussion}

Our pharmacokinetic study has shown comparable plasma uptake of synthetic and kiwifruit-derived vitamin $\mathrm{C}$ in subjects with "healthy" (i.e., $>50 \mu \mathrm{mol} / \mathrm{L}$ ) baseline ascorbate status. Several other pharmacokinetic studies have shown comparable plasma uptake of vitamin C supplied in synthetic form versus that in fruit juices [15,23,25]. Nelson et al. [26] used an intestinal triple lumen tube perfusion model to investigate the absorption of synthetic vitamin $\mathrm{C}$ and that from an orange juice solution. This method allowed direct measurement of intraluminal events and showed no difference in the absorption of vitamin $\mathrm{C}$ from the two test solutions. Some pharmacokinetic studies have shown small or transient decreases in plasma ascorbate levels in the presence of fruit juices $[16,18]$. The physiological relevance of these small or transient differences is, however, likely minimal.

Vinson and Bose are the only investigators to have shown increased uptake of vitamin $\mathrm{C}$, in the presence of citrus fruit extract, using a pharmacokinetic study design [27,28]. Their initial study, carried out in guinea pigs, indicated that vitamin $\mathrm{C}$ provided in a citrus fruit medium took longer to reach peak plasma concentrations compared with a synthetic vitamin $\mathrm{C}$ solution and also provided a larger area under the plasma ascorbate concentration-time curve [27]. These investigators also observed a comparable trend in human subjects supplemented with $500 \mathrm{mg}$ vitamin $\mathrm{C}$ in the presence or absence of a citrus fruit extract [28]. The citrus extract delayed maximal plasma levels by one hour and provided a $35 \%$ increase in vitamin C levels.

The different outcomes observed in the pharmacokinetic studies discussed above could be explained by differences in study design and subjects. For example, the doses of vitamin $\mathrm{C}$ used varied by more than 10-fold, from $30 \mathrm{mg}$ to $500 \mathrm{mg}$. Vitamin C bioavailability is non-linear [29] and although $\sim 100 \%$ bioavailability is observed at a vitamin $\mathrm{C}$ dose of $200 \mathrm{mg}$, doses exceeding this exhibit decreased intestinal uptake [19]. Doses of $500 \mathrm{mg}$ vitamin $\mathrm{C}$ are also significantly higher than would be obtained through a normal daily diet. Furthermore, the participants in these pharmacokinetic studies exhibited varying baseline plasma ascorbate levels, from $35 \mu \mathrm{mol} / \mathrm{L}$ to $75 \mu \mathrm{mol} / \mathrm{L}$, the former being non-saturating and the latter being saturating levels of ascorbate [21]. These different baseline levels may affect vitamin $\mathrm{C}$ uptake and clearance kinetics as it is likely that there will be preferential uptake into cells and tissues in individuals with suboptimal vitamin $\mathrm{C}$ status [21,30]. Thus, it is possible that kiwifruit-derived vitamin $\mathrm{C}$ may exhibit different uptake kinetics in individuals with sub-optimal ascorbate status at baseline.

We observed a transient increase in urinary excretion when ascorbate was supplied as kiwifruit compared with tablets. Vinson and Bose [28] observed increased excretion of ascorbate when given in the presence of citrus fruit extract, but only in individuals who had been saturated with vitamin $\mathrm{C}$ prior to beginning the study. Similarly, others have shown small or transient increases in ascorbate excretion in the presence of fruit juice in pre-saturated subjects [17,18]. In contrast, Uchida et al. [15] 
recently reported decreased ascorbate excretion when given in the presence of acerola juice. Decreased excretion of fruit-derived vitamin $\mathrm{C}$ was observed in subjects with low baseline ascorbate status [15,28], supporting the premise that baseline ascorbate status may affect comparative vitamin $\mathrm{C}$ bioavailability.

The mechanism whereby kiwifruit enhances urinary excretion of ascorbate without affecting plasma levels is unkown. Certain dietary fibres, such as hemicellulose, which is present in kiwifruit [31], have been shown to increase the excretion of ascorbate [32]. The flavonoid quercetin, found in kiwifruit [33], is a reversible, non-competitive inhibitor of ascorbate transport by SVCT1 [34]. Due to the low intestinal bioavailabiltiy of flavonoids [6], this mechanism would be expected to occur primarily in the intestinal lumen. Although we did not observe an effect of kiwifruit on plasma uptake of ascorbate, alternative mechanisms for intestinal uptake have been implicated using SVCT1 knockout mice [35]. An alternative, hypothetical mechanism may involve a kiwifruit-derived metabolite, which is excreted into urine and thus selectively inhibits ascorbate reabsorption via SVCT1 in the kidney tubules. Urinary excretion of ascorbate may be advantageous with respect to diseases or infections of the urinary tract $[36,37]$.

\section{Conclusions}

Our pharmacokinetic study has shown comparable plasma uptake of synthetic and kiwifruit-derived vitamin $\mathrm{C}$ and enhanced urinary excretion of kiwifruit-derived vitamin $\mathrm{C}$, although the $\sim 10 \%$ increase in total excretion is unlikely to be physiologically significant. Of interest, in our subjects with $>50 \mu \mathrm{mol} / \mathrm{L}$ to saturating baseline plasma ascorbate, $\sim 50 \%-60 \%$ of the ingested ascorbate was unaccounted for by urinary excretion, despite complete plasma uptake. This indicates possible tissue uptake even in individuals with supposedly "healthy" or "optimal" plasma ascorbate status.

\section{Acknowledgments}

We thank the young men who participated in this study, Jo Kepple and her staff at Primorus Clinical Trials Unit, Lynley Drummond for consultation on study design, Heather Webb for technical assistance, and Juliet Pullar for editing the manuscript. Sources of Support: Co-funding was provided by the New Zealand Ministry of Business, Innovation \& Employment and Zespri International Ltd., Mount Maunganui, New Zealand.

\section{Conflicts of Interest}

The authors declare no conflict of interest.

\section{References}

1. Tsao, C.S. An Overview of Ascorbic Acid Chemistry and Biochemistry. In Vitamin C in Health and Disease; Packer, L., Fuchs, J., Eds.; Marcel Dekker: New York, NY, USA, 1997; pp. 25-58.

2. Sauberlich, H.E. A History of Scurvy and Vitamin C. In Vitamin C in Health and Disease; Packer, L., Fuchs, J., Eds.; Marcel Dekker: New York, NY, USA, 1997; pp. 1-24. 
3. Ivanov, V.; Carr, A.C.; Frei, B. Red wine antioxidants bind to human lipoproteins and protect them from metal ion-dependent and -independent oxidation. J. Agric. Food Chem. 2001, 49, $4442-4449$.

4. Beker, B.Y.; Sonmezoglu, I.; Imer, F.; Apak, R. Protection of ascorbic acid from copper(II)-catalyzed oxidative degradation in the presence of flavonoids: Quercetin, catechin and morin. Int. J. Food Sci. Nutr. 2011, 62, 504-512.

5. Clemetson, C.A.; Andersen, L. Plant polyphenols as antioxidants for ascorbic acid. Ann. N. Y. Acad. Sci. 1966, 136, 341-376.

6. Lotito, S.B.; Frei, B. Consumption of flavonoid-rich foods and increased plasma antioxidant capacity in humans: Cause, consequence, or epiphenomenon? Free Radic. Biol. Med. 2006, 41, $1727-1746$.

7. Carr, A.; Frei, B. Does vitamin C act as a pro-oxidant under physiological conditions? FASEB J. 1999, 13, 1007-1024.

8. Tsukaguchi, H.; Tokui, T.; Mackenzie, B.; Berger, U.V.; Chen, X.Z.; Wang, Y.; Brubaker, R.F.; Hediger, M.A. A family of mammalian $\mathrm{Na}^{+}$-dependent L-ascorbic acid transporters. Nature 1999, 399, 70-75.

9. Savini, I.; Rossi, A.; Pierro, C.; Avigliano, L.; Catani, M.V. SVCT1 and SVCT2: Key proteins for vitamin C uptake. Amino Acids 2008, 34, 347-355.

10. Parada, J.; Aguilera, J.M. Food microstructure affects the bioavailability of several nutrients. J. Food Sci. 2007, 72, R21-R32.

11. Kamp, F.; Jandel, D.; Hoenicke, I.; Pietrzk, K.; Gross, R.; Trugo, N.M.; Donangelo, C.M. Bioavailability of iron, zinc, folate, and vitamin $\mathrm{C}$ in the IRIS multi-micronutrient supplement: Effect of combination with a milk-based cornstarch porridge. Food Nutr. Bull. 2003, 24 (3 Suppl.), $20-26$.

12. Mangels, A.R.; Block, G.; Frey, C.M.; Patterson, B.H.; Taylor, P.R.; Norkus, E.P.; Levander, O.A. The bioavailability to humans of ascorbic acid from oranges, orange juice and cooked broccoli is similar to that of synthetic ascorbic acid. J. Nutr. 1993, 123, 1054-1061.

13. Carr, A.C.; Bozonet, S.M.; Pullar, J.M.; Simcock, J.W.; Vissers, M.C. A randomised steady-state bioavailability study of synthetic and natural (kiwifruit-derived) vitamin C. Nutrients 2013, 5, 3684-3695.

14. Vissers, M.C.M.; Bozonet, S.M.; Pearson, J.F.; Braithwaite, L.J. Dietary ascorbate affects steady state tissue levels in vitamin C-deficient mice: Tissue deficiency after sub-optimal intake and superior bioavailability from a food source (kiwifruit). Am. J. Clin. Nutr. 2011, 93, 292-301.

15. Uchida, E.; Kondo, Y.; Amano, A.; Aizawa, S.; Hanamura, T.; Aoki, H.; Nagamine, K; Koizumi, T.; Maruyama, N.; Ishigami, A. Absorption and excretion of ascorbic acid alone and in acerola (Malpighia emarginata) juice: Comparison in healthy Japanese subjects. Biol. Pharm. Bull. 2011, 34, 1744-1747.

16. Bates, C.J.; Jones, K.S.; Bluck, L.J. Stable isotope-labelled vitamin C as a probe for vitamin C absorption by human subjects. Br. J. Nutr. 2004, 91, 699-705.

17. Jones, E.; Hughes, R.E. The influence of bioflavonoids on the absorption of vitamin C. IRCS Med. Sci. 1984, 12, 320. 
18. Pelletier, O.; Keith, M.O. Bioavailability of synthetic and natural ascorbic acid. J. Am. Diet. Assoc. 1974, 64, 271-275.

19. Levine, M.; Conry-Cantilena, C.; Wang, Y.; Welch, R.W.; Washko, P.W.; Dhariwal, K.R.; Park, J.B.; Lazarev, A.; Graumlich, J.K.; King, J.; et al. Vitamin C pharmacokinetics in healthy volunteers: Evidence for a recommended dietary allowance. Proc. Natl. Acad. Sci. USA 1996, 93, 3704-3709.

20. Kondo, Y.; Higashi, C.; Iwama, M.; Ishihara, K.; Handa, S.; Mugita, H.; Maruyama, N.; Koga, H.; Ishigami, A. Bioavailability of vitamin $\mathrm{C}$ from mashed potatoes and potato chips after oral administration in healthy Japanese men. Br. J. Nutr. 2012, 107, 885-892.

21. Carr, A.C.; Pullar, J.M.; Moran, S.; Vissers, M.C.M. Bioavailability of vitamin C from kiwifruit in non-smoking males: Determination of 'healthy' and 'optimal' intakes. J. Nutr. Sci. 2012, 1, e14. doi:10.1017/jns.2012.15.

22. Sato, Y.; Uchiki, T.; Iwama, M.; Kishimoto, Y.; Takahashi, R.; Ishigami, A. Determination of dehydroascorbic acid in mouse tissues and plasma by using tris(2-carboxyethyl)phosphine hydrochloride as reductant in metaphosphoric acid/ethylenediaminetetraacetic acid solution. Biol. Pharm. Bull. 2010, 33, 364-369.

23. Carter, B.; Monsivais, P.; Drewnowski, A. Absorption of folic acid and ascorbic acid from nutrient comparable beverages. J. Food Sci. 2010, 75, H289-H293.

24. Nadler, S.B.; Hidalgo, J.H.; Bloch, T. Prediction of blood volume in normal human adults. Surgery 1962, 51, 224-232.

25. Guarnieri, S.; Riso, P.; Porrini, M. Orange juice vs. vitamin C: Effect on hydrogen peroxide-induced DNA damage in mononuclear blood cells. Br. J. Nutr. 2007, 97, 639-643.

26. Nelson, E.W.; Streiff, R.R.; Cerda, J.J. Comparative bioavailability of folate and vitamin C from a synthetic and a natural source. Am. J. Clin. Nutr. 1975, 28, 1014-1019.

27. Vinson, J.A.; Bose, P. Comparative bioavailability of synthetic and natural vitamin $\mathrm{C}$ in guinea pigs. Nutr. Rep. Int. 1983, 27, 875-879.

28. Vinson, J.A.; Bose, P. Comparative bioavailability to humans of ascorbic acid alone or in a citrus extract. Am. J. Clin. Nutr. 1988, 48, 601-604.

29. Graumlich, J.F.; Ludden, T.M.; Conry-Cantilena, C.; Cantilena, L.R., Jr.; Wang, Y.; Levine, M. Pharmacokinetic model of ascorbic acid in healthy male volunteers during depletion and repletion. Pharm. Res. 1997, 14, 1133-1139.

30. Carr, A.C.; Bozonet, S.M.; Pullar, J.M.; Simcock, J.W.; Vissers, M.C. Human skeletal muscle ascorbate is highly responsive to changes in vitamin $\mathrm{C}$ intake and plasma concentrations. Am. J. Clin. Nutr. 2013, 97, 800-807.

31. Sims, I.M.; Monro, J.A. Fiber: Composition, structures, and functional properties. Adv. Food Nutr. Res. 2013, 68, 81-99.

32. Keltz, F.R.; Kies, C.; Fox, H.M. Urinary ascorbic acid excretion in the human as affected by dietary fiber and zinc. Am. J. Clin. Nutr. 1978, 31, 1167-1171.

33. Lee, D.E.; Shin, B.J.; Hur, H.J.; Kim, J.H.; Kim, J.; Kang, N.J.; Kim, D.O.; Lee, C.Y.; Lee, K.W.; Lee, H.J. Quercetin, the active phenolic component in kiwifruit, prevents hydrogen peroxide-induced inhibition of gap-junction intercellular communication. Br. J. Nutr. 2010, 104, 164-170. 
34. Song, J.; Kwon, O.; Chen, S.; Daruwala, R.; Eck, P.; Park, J.B.; Levine, M. Flavonoid inhibition of sodium-dependent vitamin $\mathrm{C}$ transporter 1 (SVCT1) and glucose transporter isoform 2 (GLUT2), intestinal transporters for vitamin C and glucose. J. Biol. Chem. 2002, 277, $15252-15260$.

35. Corpe, C.P.; Tu, H.; Eck, P.; Wang, J.; Faulhaber-Walter, R.; Schnermann, J.; Margolis, S.; Padayatty, S.; Sun, H.; Wang, Y.; Nussbaum, R.L.; Espey, M.G.; Leine, M. Vitamin C transporter Slc23a1 links renal reabsorption, vitamin $\mathrm{C}$ tissue accumulation, and perinatal survival in mice. J. Clin. Investig. 2010, 120, 1069-1083.

36. Yalcin, O.; Karatas, F.; Erulas, F.A.; Ozdemir, E. The levels of glutathione peroxidase, vitamin $\mathrm{A}, \mathrm{E}, \mathrm{C}$ and lipid peroxidation in patients with transitional cell carcinoma of the bladder. BJU Int. 2004, 93, 863-866.

37. Ochoa-Brust, G.J.; Fernandez, A.R.; Villanueva-Ruiz, G.J.; Velasco, R.; Trujillo-Hernandez, B.; Vasquez, C. Daily intake of $100 \mathrm{mg}$ ascorbic acid as urinary tract infection prophylactic agent during pregnancy. Acta Obstet. Gynecol. Scand. 2007, 86, 783-787. 
Reprinted from Nutrients. Cite as: Delanghe, J.R.; De Buyzere, M.L.; Speeckaert, M.M.; Langlois, M.R. Genetic Aspects of Scurvy and the European Famine of 1845-1848. Nutrients 2013, 5, 3582-3588.

Article

\title{
Genetic Aspects of Scurvy and the European Famine of 1845-1848
}

Joris R. Delanghe ${ }^{1, *}$, Marc L. De Buyzere ${ }^{2}$, Marijn M. Speeckaert ${ }^{3}$ and Michel R. Langlois ${ }^{4}$

1 Department of Laboratory Medicine, Ghent University Hospital, B 9000 Ghent, Belgium

2 Department of Cardiology, Ghent University Hospital, B 9000 Ghent, Belgium;

E-Mail: marc.debuyzere@ugent.be

3 Department of Nephrology, Ghent University Hospital, B 9000 Ghent, Belgium;

E-Mail: marijn.speeckaert@ugent.be

4 Department of Laboratory Medicine, AZ Sint Jan Hospital, B 8000 Bruges, Belgium;

E-Mail: Michel.Langlois@azsintjan.be

* Author to whom correspondence should be addressed; E-Mail: joris.delanghe@ugent.be; Tel.: +32-9-332-29-56; Fax: +32-9-332-36-59.

Received: 17 July 2013; in revised form: 13 August 2013 / Accepted: 22 August 2013 /

Published: 12 September 2013

\begin{abstract}
The view of scurvy being exclusively a nutritional disorder needs to be updated. Genetic polymorphisms of HFE and haptoglobin (Hp) may explain the geographic variability of mortality caused by the European famine of the mid-19th century. In this period, potatoes had fallen victim to the potato blight and Ireland was more severely hit than continental Europe. Hereditary hemochromatosis is a genetic disorder with mutations in the HFE gene, characterized by iron overload (with a reduced vitamin $\mathrm{C}$ stability) and with a predominance of affected men. The Irish have the world's highest frequency of the C282Y mutation and the particular iron metabolism of the Irish helps to understand the size of the catastrophe and the observed overrepresentation of male skeletons showing scurvy. $\mathrm{Hp}$ is a plasma $\alpha_{2}$-glycoprotein characterized by 3 common phenotypes (Hp 1-1, Hp 2-1 and Hp 2-2). When the antioxidant capacity of Hp is insufficient, its role is taken over by hemopexin and vitamin $C$. The relative number of scurvy victims corresponds with the Hp 2-2 frequency, which is associated with iron conservation and has an impact on vitamin $\mathrm{C}$ stability. As iron is more abundant in males, males are overrepresented in the group of skeletons showing scurvy signs.
\end{abstract}

Keywords: haptoglobin; iron; polymorphism; scurvy; vitamin C 


\section{Introduction}

In the mid-19th century, Ireland's potatoes had fallen victim to the potato blight (phytophthora infestans). However, the epidemics of the potato late blight in this period were not restricted to Ireland. Also in continental Europe, the potato blight caused the Continental Famine (1844-1846) and - in the longer term — contributed to the European revolutions of 1848. However, it cannot be denied that Ireland was more severely hit than nations of continental Europe [1]. Since potatoes were the main source of vitamin $\mathrm{C}$ in the diet, scurvy is generally explained by a shortage of potatoes. However, the relative decline in potato harvest in 1845 in continental Europe was higher than the decline in Ireland [2]. On average, the human body loses $\pm 3 \%$ of its vitamin $\mathrm{C}$ content per day, which severely limits the disease-free and survival time when subjects are on a diet poor in vitamin C [3]. Although scurvy is classified as a nutritional disorder or avitaminosis, only about $17 \%$ of the variance of the serum vitamin $\mathrm{C}$ concentration in humans can be explained by vitamin $\mathrm{C}$ intake [4]. The vitamin $\mathrm{C}$ status is not only determined by diet, but also by environment, lifestyle, biological and pathological conditions [5,6]. The present paper wants to highlight the impact of the particular genetic effects, which helps to understand the geographical distribution of the European mid-19th century famine.

\section{Why Was Ireland Struck More than the Continent?}

Geber and Murphy reported on the pronounced gender difference during the mid-19th century scurvy epidemic [7]. This finding is remarkable since a shared vitamin $\mathrm{C}$ poor diet is assumed. In order to better understand the severity of the scurvy outbreak, one should take into account the particular population genetics of the Irish. Hemochromatosis is a disease characterized by iron overload which has been associated with the C282Y (and H63D) mutation. This mutation has probably arisen in either a Celtic or a Viking ancestor some 60 generations ago [8,9]. Clinical symptoms of hemochromatosis show a marked gender difference predominantly affecting men. Moreover, clinical manifestations occur at an earlier age in men. While the Scandinavians have a high C282Y mutation frequency, the Irish have the highest frequency (around 11\%) of the C282Y mutation in the world. The hemochromatosis associated iron overload reduces vitamin $\mathrm{C}$ stability in vivo, which explains the Irish population to be more prone to scurvy than other Europeans in times of reduced vitamin C supply. The particular iron metabolism of the Irish helps to understand the size of the scurvy catastrophe and the observed overrepresentation of male skeletons showing scurvy lesions. However, analyzing those data, we have to take into account that diagnostic traits of disease in the human skeleton normally only occur in its chronic and advanced stage. The prevalence of skeletal indicators of disease is therefore not equal to the true prevalence of the disease, as deceased individuals without any evidence of disease may very well have suffered and died as a consequence of it before it manifested itself skeletally. This fundamental understanding of the representation of disease in skeletal populations is usually referred to as "the osteological paradox" [10]: the healthiest and strongest individuals in a population are likely to be those that developed the worst and most substantial degree of pathological lesions, as they were strong enough to survive long enough for it to be manifested in their skeletal tissues. This is a limitation of using palaeopathological data from archaeological populations in direct analogy with modern clinical data. 
The proportion of observed scurvy cases in mid-19th century Ireland is higher in comparison with some well-known documented historical scurvy outbreaks among Europeans. Generally, scurvy outbreaks affect $15 \%-30 \%$ of the European population: the French expedition of Cartier in 1536 counted 25 victims (a rate of $\pm 30 \%$ ). For the Dutch East Indies Company, typical crew losses for a one-way trip between Europe and the Indonesian archipelago in the 17 th century were $\pm 20 \%$. The Dutch expedition to Novaya Zemlya in 1596 counted only 2 scurvy victims among 17 crew members. In the Crimean War (1854-1856), the French Navy counted $\pm 30 \%$ of scurvy cases. In Perth general prison in Scotland, scurvy occurred at a rate of 50 of 330 inmates during 1845-1846. In 1819, 160 scurvy victims were counted of 800 soldiers in the US Army outpost at Fort Atkinson (Nebraska) [5,11]. Despite the fact that conditions are not completely comparable, these data are striking because they were recorded in relative homogeneous populations sharing a uniform diet. The prevalence of the haptoglobin $(\mathrm{Hp})$ 2-2 phenotype may partly explain the findings described above, as the relative number of scurvy victims roughly corresponds with the relative frequency of the Hp 2-2 phenotype [12].

Human Hp is a plasma $\alpha_{2}$-glycoprotein characterized by 3 common phenotypes (Hp 1-1, Hp 2-1 and

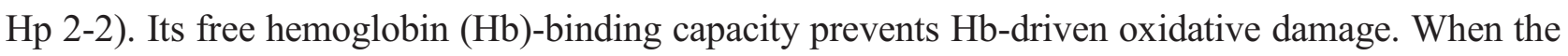
antioxidant capacity of $\mathrm{Hp}$ is insufficient, its role is taken over by hemopexin (heme-binding protein) and by vitamin $\mathrm{C}$ (free radical scavenger) [13]. Hp phenotypes show important structural and functional differences, which offer a plausible explanation how during the course of human history, some populations characterized by a high $\mathrm{Hp} 1$ allele frequency have been able to survive on a vitamin $\mathrm{C}$ poor diet [6]. In comparison with the other Hp phenotypes, Hp 2-2 is associated with a better iron conservation (characterized by a higher serum iron concentration, higher serum ferritin concentrations and increased transferrin saturation levels) which may act synergistic in presence of hemochromatosis $[14,15]$. However, the Hp 2-2 phenotype has also a major impact on vitamin $\mathrm{C}$ stability in vivo [13]. Hp 2-2 subjects are less efficient in removing free $\mathrm{Hb}$ from plasma, which may favour an iron-mediated vitamin $\mathrm{C}$ depletion [13,16]. Iron retention in scurvy-prone $\mathrm{Hp} \mathrm{2-2}$ individuals results in iron-driven oxidative stress, which is reflected by lower serum vitamin $\mathrm{C}$ concentrations in healthy Hp 2-2 subjects [3].

Furthermore, the iron delocalization pathway, selectively occurring in Hp 2-2 subjects has consequences in infections. Iron withholding is an important example of nutritional immunity in the defense against infectious diseases [17]. Hp acts as a natural bacteriostat by preventing the utilization of $\mathrm{Hb}$ by pathogenic bacteria which require iron for their growth. Hp polymorphism plays a role in a number of bacterial and viral infections [18]. As Hp 2-2 subjects are more prone to e.g., tuberculosis, a relationship between susceptibility for scurvy and the presence of life threatening infections may be postulated. In an Irish population, prevalence of the Hp 2-2 phenotype is $\pm 41 \%$ [19], which roughly corresponds to the proportion of skeletons showing scurvy signs [7]. As iron is more abundant in males than in females, it is not surprising that males are overrepresented in the group of skeletons showing scurvy signs.

\section{The Situation on the European Continent}

Despite the fact that the potato blight also struck continental Europe and the unfavourable climatological circumstances (temperature, humidity) between 1845 and 1847 were comparable 
among Western European countries, continental Europe was less affected than Ireland. When plotting the $\mathrm{C} 282 \mathrm{Y}$ allele frequencies of the various European countries (Belgium, The Netherlands, France, Switzerland, Germany) against the excess mortality of the years 1845-1847 and the decline in potato yields, a good correlation $(y$ (excess mortality per million $)=-32.42+9.22 x(\mathrm{C} 282 \mathrm{Y}$ allele frequency, \%), $r^{2}=0.98$ ) was obtained (Table 1). As the Hp phenotype frequency shows more limited differences among European countries [11], no significant correlation between the Hp 2 allele frequency and the excess in mortality was found.

Also in France, a country with important Celtic influences, a marked correlation between the $\mathrm{C} 282 \mathrm{Y}$ mutation frequency and mortality was observed for the various departments. When comparing the 1846 mortality among the population aged 30-35 years with the C282Y allele frequency for the various departments (Figure 1), a marked correlation was found, which resembles the one found for Western Europe. Higher C282Y frequencies were associated with higher mortality rates. Both in the 1841 and 1851 French census [20], the effect of the C282Y allele frequency on mortality of 30 year old subjects was greatly reduced, as evidenced by the regression coefficients (1841:0.016; 1846:0.024; 1851:0.016).

Figure 1. Mortality in 30-35 year old subjects in 1846 according to C282Y allele frequencies in a number of French departments. The $Y$-axis depicts the death probabilities q $(x)$ for every age $(x)$ computed for that period. The $X$-axis represents the $\mathrm{C} 282 \mathrm{Y}$ allele frequency in the different departments.

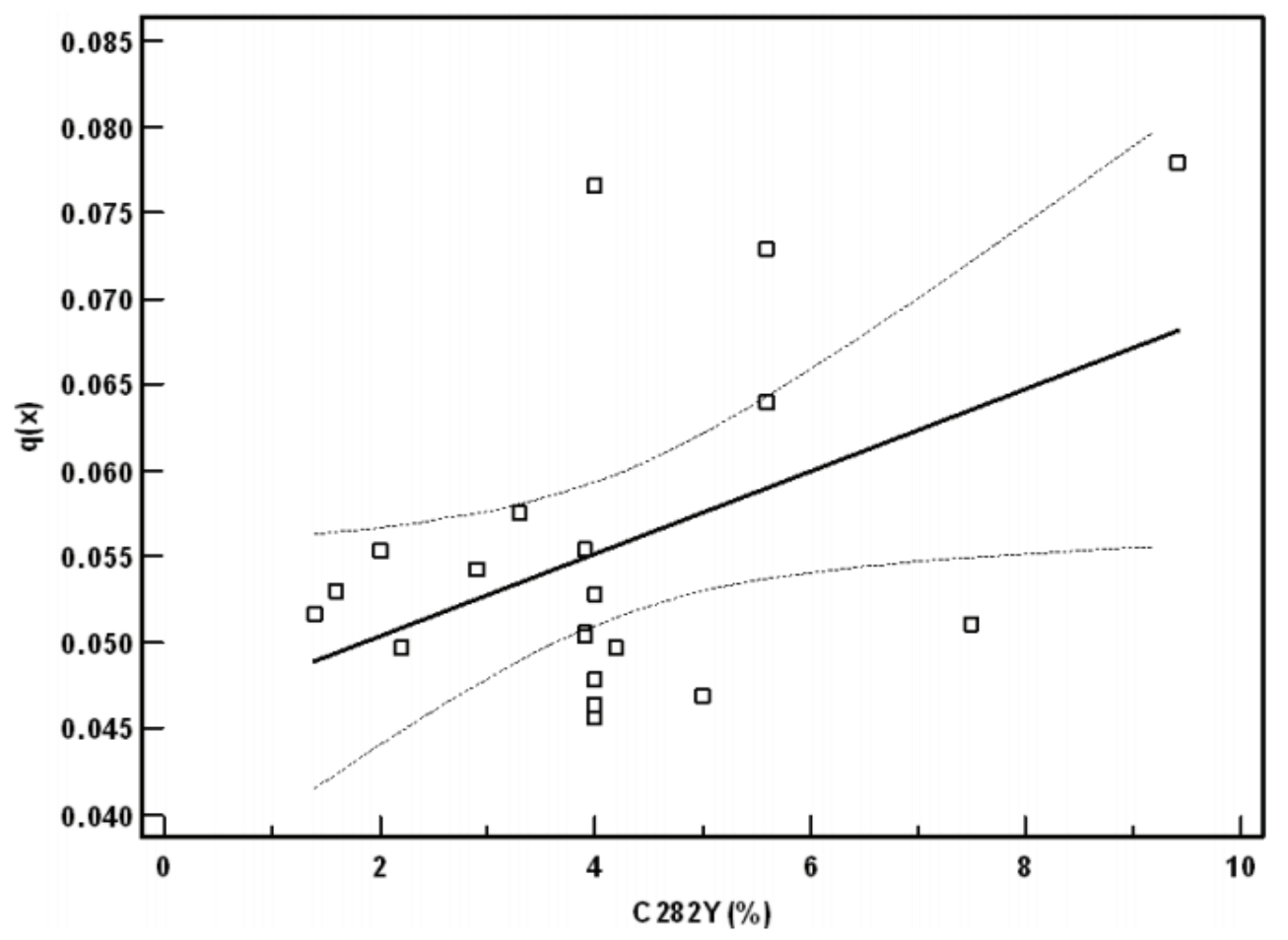


Table 1. Overview of annual excess mortality, decline in potato yields and C282Y mutation frequency in various European countries.

\begin{tabular}{cccc}
\hline Country & $\begin{array}{c}\text { Annual Excess } \\
\text { Mortality [1846-1848 } \\
\text { (per million)] [1] }\end{array}$ & $\begin{array}{c}\text { Decline in Potato Yields } \\
\mathbf{( 1 8 4 5 )}[2]\end{array}$ & $\begin{array}{c}\text { C282Y Mutation } \\
\text { Frequency [9] }\end{array}$ \\
\hline Austria & 7 & & $4.1 \%$ \\
Belgium & 3 & $-87 \%$ & $4.1 \%$ \\
France & 1 & $-20 \%$ & $4 \%$ \\
Germany & 2 & $-55 \%$ & $2.6 \%$ \\
Ireland & 100 & $-30 \%$ & $14.2 \%$ \\
The Netherlands & 3 & $-71 \%$ & $4.1 \%$ \\
Switzerland & 0 & & $4.1 \%$ \\
\hline
\end{tabular}

\section{Conclusions}

In conclusion, the classical view of vitamin $\mathrm{C}$ deficiency and scurvy being exclusively nutritional disorders needs to be updated. The genetic polymorphism of HFE and (to a lesser extent) Hp may have played an important role in explaining the remarkable geographic variability of excess in mortality during famine and scurvy episodes in recent European history. The data confirm the importance of the interplay between the iron status and vitamin $\mathrm{C}$ requirements. In order to further elucidate the mechanisms of scurvy, $H F E$ and Hp genotyping of skeletons of scurvy victims could be considered in the future. Besides the differential genetic background, cultural, social and economic aspects relating to access to food and differential diets in the 19th century should be taken into account when interpreting those results.

\section{Acknowledgments}

The authors wish to thank Eric Vanhaute (Department of History, Ghent University, Ghent, Belgium) and Emeritus Nadine Vivier (UFR Lettres, Langues et Sciences Humaines Université du Maine-Le Mans, Le Mans CEDEX, France) for their historical advice.

\section{Conflicts of Interest}

The authors declare no conflict of interest.

\section{References}

1. Zadoks, J.C. The potato murrain on the European continent and the revolutions of 1848 . Potato Res. 2008, 51, 5-45.

2. Vanhaute, E.; Paping, R.; Ó Gràda, C. The European Subsistence Crisis of 1845-1850: A Comparative Perspective; University College Dublin, Department of Economics: Dublin, Ireland, 2006; pp. 1-30.

3. Langlois, M.R.; Delanghe, J.R.; de Buyzere, M.L.; Bernard, D.R.; Ouyang, J. Effect of haptoglobin on the metabolism of vitamin C. Am. J. Clin. Nutr. 1997, 66, 606-610. 
4. Hampl, J.S.; Taylor, C.A.; Johnston, C.S. Vitamin C deficiency and depletion in the United States: The Third National Health and Nutrition Examination Survey, 1988 to 1994. Am. J. Public Health 2004, 94, 870-875.

5. Delanghe, J.; Langlois, M.; de Buyzere, M.; Torck, M. Vitamin C deficiency and scurvy are not only a dietary problem but are codetermined by the haptoglobin polymorphism. Clin. Chem. 2007, 53, 1397-1400.

6. Delanghe, J.R.; Langlois, M.R.; de Buyzere, M.L.; Na, N.; Ouyang, J.; Speeckaert, M.M.; Torck, M.A. Vitamin C deficiency: More than just a nutritional disorder. Genes Nutr. 2011, 6, 341-346.

7. Geber, J.; Murphy, E. Scurvy in the Great Irish Famine: Evidence of vitamin C deficiency from a mid-19th century skeletal population. Am. J. Phys. Anthropol. 2012, 148, 512-524.

8. Lucotte, G.; Dieterlen, F. A European allele map of the C282Y mutation of hemochromatosis: Celtic versus Viking origin of the mutation? Blood Cell. Mol. Dis. 2003, 31, 262-267.

9. Whittington, C.A. Was the $\mathrm{C} 282 \mathrm{Y}$ mutation an Irish Gaelic mutation that the Vikings help disseminate? Med. Hypotheses 2006, 67, 1270-1273.

10. Wood, J.W.; Milner, G.R.; Harpending, H.C.; Weiss, K.M. The osteological paradox: Problems of inferring prehistoric health from skeletal samples. Curr. Anthropol. 1992, 33, 343-370.

11. Delanghe, J.R.; Langlois, M.R. Haptoglobin polymorphism and body iron stores. Clin. Chem. Lab. Med. 2002, 40, 212-216.

12. Cavalli-Sforza, L.L.; Menozzi, P.; Piazza, A. The History and Geography of Human Genes; Princeton University Press: Princeton, NJ, USA, 1994; pp. 1-1088.

13. Langlois, M.; Delanghe, J. Biological and clinical significance of haptoglobin polymorphism in humans. Clin. Chem. 1996, 42, 1589-1600.

14. Langlois, M.R.; Martin, M.E.; Boelaert, J.R.; Beaumont, C.; Taes, Y.E.; de Buyzere, M.L.; Bernard, D.R.; Neels, H.M.; Delanghe, J.R. The haptoglobin 2-2 phenotype affects serum markers of iron status in healthy males. Clin. Chem. 2000, 46, 1619-1625.

15. Van Vlierberghe, H.; Langlois, M.; Delanghe, J.; Horsmans, Y.; Michielsen, P.; Henrion, J.; Cartuyvels, R.; Billiet, J.; de Vos, M.; Leroux-Roels, G. Haptoglobin phenotype 2-2 overrepresentation in Cys282Tyr hemochromatotic patients. J. Hepatol. 2001, 35, 707-711.

16. Na, N.; Delanghe, J.R.; Taes, Y.E.; Torck, M.; Baeyens, W.R.; Ouyang, J. Serum vitamin C concentration is influenced by haptoglobin polymorphism and iron status in Chinese. Clin. Chim. Acta 2006, 365, 319-324.

17. Weinberg, E.D. Iron withholding: A defense against infection and neoplasia. Physiol. Rev. 1984, 64, 65-102.

18. Kasvosve, I.; Speeckaert, M.M.; Speeckaert, R.; Masukume, G.; Delanghe, J.R. Haptoglobin polymorphism and infection. Adv. Clin. Chem. 2010, 50, 23-46.

19. Conway, B.R.; Savage, D.A.; Brady, H.R.; Maxwell, A.P. Association between haptoglobin gene variants and diabetic nephropathy: Haptoglobin polymorphism in nephropathy susceptibility. Nephron Exp. Nephrol. 2007, 105, e75-e79.

20. Life Table for the Whole of France and by Department and Tables of Net Migration by Department, 1806-1906. Available online: http://table_mortalite_bonneuil.site.ined.fr/fr/ presentation/ (accessed on 15 February2013). 
Reprinted from Nutrients. Cite as: Lindblad, M.; Tveden-Nyborg, P.; Lykkesfeldt, J. Regulation of Vitamin C Homeostasis during Deficiency. Nutrients 2013, 5, 2860-2879.

Review

\title{
Regulation of Vitamin C Homeostasis during Deficiency
}

\section{Maiken Lindblad, Pernille Tveden-Nyborg and Jens Lykkesfeldt *}

Section of Experimental Animal Models, Department of Veterinary Disease Biology, Faculty of Health and Medical Sciences, University of Copenhagen, Ridebanevej 9, Frederiksberg C 1870, Denmark; E-Mails: mali@sund.ku.dk (M.L.); ptn@sund.ku.dk (P.T.-N.)

* Author to whom correspondence should be addressed; E-Mail: jopl@sund.ku.dk; Tel.: +45-353-33163; Fax: +45-353-53514.

Received: 3 June 2013; in revised form: 15 July 2013 / Accepted: 18 July 2013 /

Published: 25 July 2013

\begin{abstract}
Large cross-sectional population studies confirm that vitamin $\mathrm{C}$ deficiency is common in humans, affecting 5\%-10\% of adults in the industrialized world. Moreover, significant associations between poor vitamin $\mathrm{C}$ status and increased morbidity and mortality have consistently been observed. However, the absorption, distribution and elimination kinetics of vitamin $\mathrm{C}$ in vivo are highly complex, due to dose-dependent non-linearity, and the specific regulatory mechanisms are not fully understood. Particularly, little is known about how adaptive mechanisms during states of deficiency affect the overall regulation of vitamin $\mathrm{C}$ transport in the body. This review discusses mechanisms of vitamin $\mathrm{C}$ transport and potential means of regulation with special emphasis on capacity and functional properties, such as differences in the $K_{\mathrm{m}}$ of vitamin $\mathrm{C}$ transporters in different target tissues, in some instances demonstrating a tissue-specific distribution.
\end{abstract}

Keywords: vitamin C transport; sodium-dependent vitamin C transporters, SVCT1 and SVCT2; glucose transporters (GLUTs); regulation of transport

\section{Introduction}

Several reports have pointed towards a putative link between vitamin C (vitC) deficiency and lifestyle-associated disease [1-6]. Moreover, vitC deficiency has also been associated with impaired brain development and reduced hippocampal function in experimental animal studies [7-10]. Scurvy is the terminal outcome of a prolonged period of severe vitC deficiency (a plasma concentration $<11 \mu \mathrm{M}[11]$ ) and is rarely encountered, as it can be prevented with as little as $10 \mathrm{mg}$ vitC/day. 
In contrast, marginal vitamin deficiency or hypovitaminosis $\mathrm{C}$, defined as a plasma concentration below $23 \mu \mathrm{M}$ [11], has been estimated to affect as much as $10 \%$ of adults in the industrialized world [12-14], with subgroups, such as smokers and families with a low socio-economic status, displaying an even higher prevalence [15-19]. Adverse effects of marginal vitC deficiency could, therefore, potentially affect a substantial amount of people who, unknowingly and devoid of known clinical symptoms, are at risk of experiencing negative long-term effects of vitC deficiency, including increased mortality associated with a variety of disease complexes [20].

The pharmacokinetics of vitC, i.e., the absorption, distribution, metabolism and elimination, is quite complex and involves several different active and passive transport mechanisms, as well as intracellular reduction permitting the recycling of vitC within specific tissues [18,21]. Furthermore, vitC is differentially distributed between tissues, with brain and neurons, in particular, upholding a much higher concentration than most other organs [22-24]. However, whereas vitC homeostasis has been studied in detail during sufficiency [25-28], less is known about the potential adaptive mechanisms during deficiency. Thus, for example, studies of vitC transport activity during deficiency have not provided evidence supporting a direct relationship between local tissue-concentration and the expression of vitC specific transporters [29-31], prompting suggestions of possible alternative transport mechanisms [29]. The present review elaborates on the mechanisms currently known to be involved in the regulation of vitC transport and the potential effects during states of deficiency.

\section{Vitamin C Transport}

Most mammals synthesize vitC in the liver by enzymatic conversion of glucose; however, a few species, including humans, guinea pigs and bats, lack a functional L-gulono-lactone oxidase enzyme catalyzing the final step in the biosynthesis and, therefore, rely completely on a dietary supply of vitC [32-34]. After consumption, vitC is absorbed from the intestinal lumen and released into the bloodstream. The dose-to-plasma concentration relationship is reflected in a saturation curve, attaining an initial steep and non-linear course, until steady-state is reached, defining plasma saturation at around $70 \mu \mathrm{M}$ in humans [25]. At doses above saturation, urinary excretion is increased and oral bioavailability decreased, thereby sustaining steady-state equilibrium [25]. In the gastro-intestinal tract, the ionized form of vitC, ascorbate (ASC), and its oxidized counterpart, dehydroascorbic acid (DHA), are absorbed through different transporter systems with an increased affinity for ASC $\left(K_{\mathrm{m}}\right.$ of $0.2 \mathrm{mM})$ compared to DHA $\left(K_{\mathrm{m}} 0.8 \mathrm{mM}\right)$ and following a Michaelis-Menten absorption rate, where saturation reflects increases in substrate concentration [21,35]. The distribution from plasma to tissues is differentially regulated, and organs and tissues vary considerably in vitC content, as depicted in Figure 1. Within the body, the brain has a uniquely high vitC level $[22,36]$ and is able to maintain a superior concentration relative to most other organs during periods of vitC deficiency [37-39], placing the brain as an organ of particular interest when assessing the effects of vitC deficiency.

The tight regulation of vitC homeostasis is primarily controlled by four regulatory systems: (i) intestinal uptake (bioavailability); (ii) tissue accumulation and distribution; (iii) rate of utilization and recycling; and (iv) renal excretion and reabsorption [26] (Figure 1). This is achieved by different mechanisms, including passive diffusion, facilitated diffusion, active transport and recycling [40]. 
Figure 1. Distribution of vitamin $C$ in the body. Vitamin $C$ is ingested, absorbed from the intestinal lumen and transported to various peripheral organs with the blood. Finally, vitamin $\mathrm{C}$ is excreted in the renal glomeruli and reabsorbed through the tubular systems. Tissue concentrations are dependent on all of these processes.

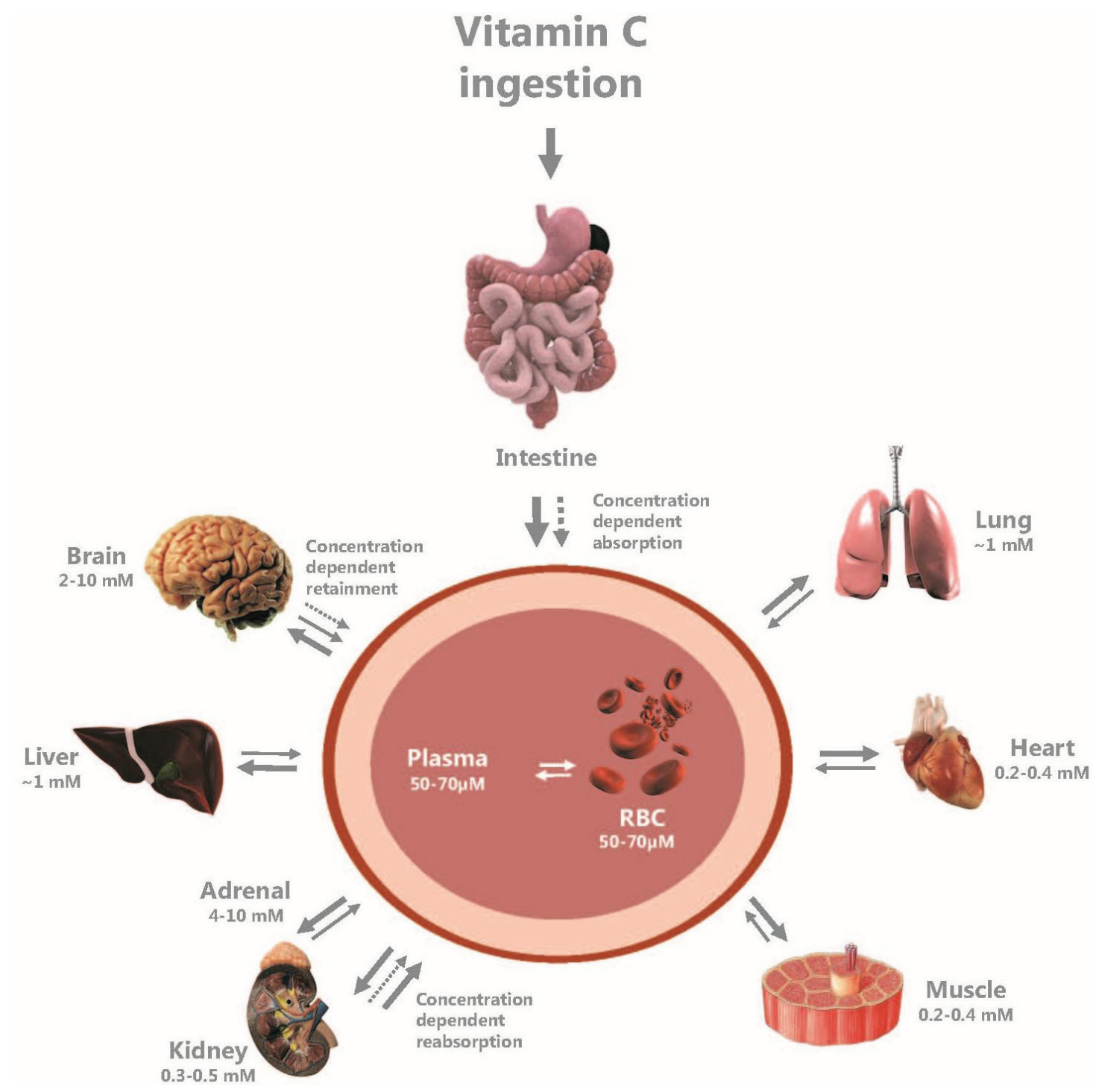

\subsection{Passive Diffusion}

In solution at physiological $\mathrm{pH}$, vitC predominantly attains its ionized form, ASC. Because of a relatively low hydrophobicity, ASC and DHA do not easily cross biological membranes and primarily rely on the interaction with transporter molecules anchored in the cell membrane $[41,42]$. However, both ASC and DHA can, to a small extent, move by passive diffusion, and DHA diffuses through cellular membranes more willingly than ASC [43]. It has also been suggested that during intestinal uptake and in renal reabsorption, ASC leaves the epithelial cells from the basolateral membranes down a concentration gradient by passive diffusion [44]. Moreover, being a week acid with a pKa of 4.2 [21], the theoretical equilibrium between blood and tissue favors plasma with a ratio of 2.5 to one. Regardless, the fraction by which passive diffusion contributes to the overall regulation of vitC homeostasis at physiological conditions is believed to be of minor importance [40]. 


\subsection{Facilitated Diffusion}

Transport of DHA occurs by facilitated diffusion, enabling transport along a concentration gradient. This gradient is maintained as DHA is reduced to ASC immediately after crossing the membrane [21,45-47]. DHA, but not ASC [48,49], is transported by facilitated diffusion through four of the 14 glucose transporters (GLUT 1-4), although with varying affinities and efficiencies [47-50]. In general, these transporters have 12 transmembrane domains and are approximately 500 amino acids-long [51]. The GLUT-transport of DHA is competitively inhibited by glucose, e.g., excess glucose in plasma or intestine will block the receptor-binding site and, subsequently, decrease GLUT-facilitated DHA transport $[21,47,48]$. This association is also seen for DHA absorption to some specific cell types, whereas in others, glucose has less or even no significant effect on DHA absorption (i.e., the luminal surface of absorptive gut epithelium and in tubular cells in the kidney) [21,35,47,52,53].

The distribution and transport properties vary among the different GLUTs. Thus, GLUT1 is expressed in a broad variety of cells throughout the body [48,51]; GLUT2 is primarily expressed in liver, spleen and the basolateral membrane of intestinal and renal epithelial cells [50,51]; GLUT3 is found particularly in the brain and in neurons [48,51] and GLUT4 in skeletal and cardiac muscle cells, as well as in adipose tissues [47,51].

\subsection{Recycling}

Following uptake across the intestinal epithelium, vitC is released into the bloodstream as ASC ( $>95 \%$ of vitC in human plasma is in the form of ASC [52,54]). Here, ASC is easily oxidized and the produced DHA rapidly taken up through GLUT1 transporters on the erythrocytes [55-57]. May and co-workers have demonstrated that DHA is rapidly recycled in erythrocytes predominantly via glutathione-dependent DHA reductases and with small contributions from reduced nicotinamide adenine dinucleotide phosphate (NADPH)-dependent DHA reductases, such as thioredoxin reductase [58-66]. The resulting ASC may subsequently be released to the bloodstream [65]. During oxidizing conditions in the extracellular space, the generated DHA is absorbed by the surrounding cells and immediately reduced [45,55]. This reduction of DHA to ASC is either by chemical reduction by glutathione (GSH) [67] or by enzymatic reduction by glutaredoxin [68-70], protein disulfide isomerase (PDI) $[69,70]$ or 3- $\alpha$-hydroxysteroid dehydrogenase [71]. The mechanism by which DHA is reduced to ASC is a continuous process within the cellular cytoplasm, sustaining adequate levels of ASC to quench free radicals, such as superoxide, and/or reduce other antioxidants, such as vitamin E, maintaining redox homeostasis [56,72,73]. The rapid intracellular recycling of DHA also drives the efficient facilitated diffusion of DHA through the GLUTs, as the intracellular concentration is kept sufficiently low to allow DHA to move along a concentration gradient.

\subsection{Active Transport of ASC}

The existence of specific vitC transporters were proposed long before the actual transporters could be identified [42,74-82]. The transport was found to be concentration-, energy-, temperatureand sodium-dependent, satiable and mediated by two different components [42,74-82]. Two specific 
transporters were defined by Tsukaguchi and co-workers as sodium-dependent vitamin $\mathrm{C}$ transporter (SVCT) 1 and SVCT2 [83]. These enable the active transport of ASC against a concentration gradient, allowing an accumulation in cells of concentrations more than 50-fold that of extracellular fluids [76,77]. The sodium-dependency of the transport has been shown to have a stoichiometry of two $\mathrm{Na}^{+}$-ions to one ASC anion [84-86], demonstrating the transport as a secondary active transport, with the sodium gradient driving the transport of ASC, which, in turn, is maintained by the sodium/potassium-ATPase.

In humans, SVCT1 is a 598 amino acid protein, while SVCT2 measures 650 amino acids [87]. The proteins are encoded by the genes, SLC23A1 (located at chromosome 5q31.2-31.3) and SLC23A2 (at 20p12.2-12.3), respectively [88-91]. The transporters share 65\% identity [83] and are differentially distributed within the body. The SVCT1 is primarily expressed in epithelial cells $[90,92,93]$. It has a relatively high $K_{\mathrm{m}}$ of $65-252 \mu \mathrm{M}[52,87,94-97]$ and a $V_{\max }$ of approximately $15 \mathrm{pmol} / \mathrm{min} /$ cell [87,90], establishing SVCT1 as a low affinity/high capacity transporter and corresponding well to its function believed to be maintaining whole-body homeostasis [52,87,96-98]. The SVCT2 is expressed in various organ systems [83]. SVCT2 has high affinity $\left(K_{\mathrm{m}}\right.$-values of 8-69 $\mu \mathrm{M}[52,87,94-97]$ ), but low capacity (approximately $1 \mathrm{pmol} / \mathrm{min} / \mathrm{cell}[87,90]$ ) for vitC transport $[52,87,96,98]$, enabling cells of peripheral organs to take up ASC from the extracellular fluid $[40,97]$. The SVCT2 transporter is believed to be the primary transporter of ASC to the brain, enabling this organ to obtain and preserve a strikingly high vitC concentration, even during states of (severe) deficiency [37-39]. The distribution of the two transporters taken together with their kinetic properties suggests a distinct polarity of expression with SVCT1 expressed on the apical side of the epithelial cell membrane, while SVCT2 is suggested to be located in the basolateral membrane [52]. This has been confirmed by in vitro studies displaying apical SVCT1 expression and SVCT2 expression in the basolateral membrane of enterocytes and renal tubule cells [99,100], supporting the distinctive roles of the two transporters in regulating overall vitC concentration; SVCT1 adhering to luminal surfaces in the intestinal tract and kidney and SVCT2 being associated with the further distribution and/or re-uptake of ASC [97,101]. Findings in Caco-2 cells, an in vitro model of human enterocytes, suggest that the basolateral SVCT2 primarily transports ASC into the enterocytes from the blood stream, while not contributing to the absorption of dietary vitC as such [99].

Importantly, recent knowledge of human genetic variation in the SVCTs and its impact on vitC homeostasis has complicated the interpretation of the existing clinical literature (for a comprehensive review, see [102]. Using pooled human genotype data, Corpe and coworkers calculated average population prevalences of SLC23A1 polymorphisms, and following an extensive experimental in vivo study in mice, they modeled dose vs. plasma concentrations in an attempt to predict human steady-state levels as a function of known allelic genotypes [103]. Their results surprisingly suggest that, e.g., the $\mathrm{A} 772 \mathrm{G}$ allele results in a vitC deficient phenotype regardless of the vitC intake (up to $2500 \mathrm{mg}$ /day). Genetic variation is also well known in SLC23A2, with about 2200 identified single-nucleotide polymorphisms (SNPs) [102]. However, changes in SLC23A2 can be expected to primarily influence tissue rather than plasma levels of ASC, and indeed, little is known about their impact on vitC homeostasis in general. Future human studies of vitC homeostasis should preferably include genotyping, as well as assessment of tissue/cellular concentrations through biopsy or leukocyte analysis, in addition to that of plasma. 


\subsection{Vitamin C Distribution}

The absorption of both ASC and DHA takes place in all segments of the small intestine (duodenum, jejunum and ileum) [35,82,104]. The release of vitC from intestinal and renal epithelial cells after luminal absorption has not yet been conclusively determined, although both passive diffusion and facilitated diffusion through volume-sensitive anion-channels has been suggested [44]. Enterocytes express GLUT1, GLUT2 and GLUT3, although they have distinct cellular locations [105-107]. GLUT3 is found on the apical brush-border membrane, while GLUT1 is found on the basolateral membrane, and GLUT2 has been localized on both sides of the membrane. It is possible that vitC enters the blood stream by diffusion through discontinuities in the endothelial wall and circulates in the body with the blood, where it is primarily found as the ASC anion [40] (Figure 2).

The entry of ASC into the brain is hampered by the blood-brain barrier, which is impermeable to ASC and lacks the expression of SVCT2 [108-110]. Instead, ASC is thought to enter the cerebrospinal fluid of the brain through SVCT2 transporters in the choroid plexus [110,111]. DHA, on the other hand, readily crosses the barrier, due to the expression of GLUT1 [108,112]. However, due to the limited amounts of circulating DHA compared to the very high ASC concentrations maintained by the brain, this mechanism is generally believed to be of little significance $[25,54]$. From the extracellular space in the brain, ASC is taken up by neurons through SVCT2 [83,89,113,114]. Inside the neurons, ASC is oxidized to DHA, which is then recycled to ASC or transported out of the neurons as DHA by GLUT3 within the neuronal membrane [113]. The DHA now present in the extracellular space can then be transported to the blood stream by GLUT1 in the blood-brain barrier or taken up by astrocytes expressing GLUT1, recycled back to ASC and, concomitantly, released to the extracellular space by a yet undisclosed mechanism or possibly by diffusion $[36,113]$.

In the renal glomeruli, ASC in the blood is filtered into the urine [115]. However, depending on the vitC status of the individual, a large proportion is reabsorbed along the proximal tubules [116,117]. This reabsorption primarily takes place at the apical side of the epithelial membrane through SVCT1 [40,118-120]. It is assumed that DHA is also reabsorbed from the glomerular filtrate, although it has not been confirmed in vivo, most likely due to the negligible amounts of DHA commonly found in plasma [55]. In early in vitro studies, DHA transport in kidney cells was found to have all the characteristics of the facilitated diffusion through the GLUTs [121,122]. In spite of this, the transport of DHA seems to have an insignificant role in the renal reabsorption, as Slc23al ${ }^{-/}$ mice, unable to reabsorb ASC from the urine, showed an 18-fold increased fractional excretion of ASC that could not be replaced by the uptake of DHA [103].

A recent study in mice further revealed SVCT2 as the functional transporter in the kidney during fetal development, whereas SVCT1 expression rapidly increased in early postnatal life to become the dominant transporter in adult life [123]. The results were supported by findings of SVCT2, but not SVCT1, being expressed in human embryonic kidney cells [123]. These findings demonstrate a developmental shift in both expression and cellular localization of the SVCT transporters, with SVCT2 primarily located at the apical membrane during embryonic development, only to be translocated to the cytoplasm and the basolateral membrane within the first days of postnatal life, following which, the SVCT2 was cleared and replaced by SVCT1 [123]. A shift in SVCT-expression patterns has also been shown for other tissues. In an in vivo study in mice, an altered expression of 
SVCT2 during fetal and postnatal development was found [29]. A marked increase in SVCT2 mRNA and protein occurred from embryonic day E15 to postnatal day P32 in both cortex and cerebellum. In liver, the increase occurred already at P1 for mRNA and P10 for protein. The changes in SVCT2 expression appeared to be directly related to the tissue content of ASC in liver, but inversely related in brain [29].

Figure 2. Transport mechanisms between intestines, blood and kidney. Ingested vitC is absorbed across the intestinal epithelium primarily by membrane transporters in the apical brush border membrane, either as ascorbate by sodium-coupled active transport via the sodium-dependent vitamin $\mathrm{C}$ transporter (SVCT) 1 transporter or as dehydroascorbic acid (DHA) through facilitated diffusion via glucose transporter (GLUT) 2 or GLUT3 transporters. Once inside the cell, DHA is efficiently converted to ascorbate (ASC) or transported to the blood-stream by GLUT1 and GLUT2 in the basolateral membrane, thereby maintaining a low intracellular concentration and facilitating further DHA uptake. ASC is conveyed to plasma by diffusion, possibly also by facilitated diffusion through volume-sensitive anion channels. SVCT2 located in the basolateral membrane enables re-uptake of ASC from plasma to the intestinal epithelium. In the kidney, ASC is excreted by glomerular filtration to the renal tubule lumen. Reabsorption is primarily achieved by SVCT1 transporters in the apical membrane, although diffusion from the luminal surface may also contribute to the overall uptake. While not confirmed in vivo, DHA is presumably re-absorbed in the renal tubule cells; however, the availability of DHA for re-absorption is thought to be negligible, due to the very low DHA concentrations in plasma. As in the intestinal epithelium, ASC can be released to the blood-stream through both passive and facilitated diffusion. GLUT2 transporters are located in the basolateral membrane, enabling transport of DHA to plasma.

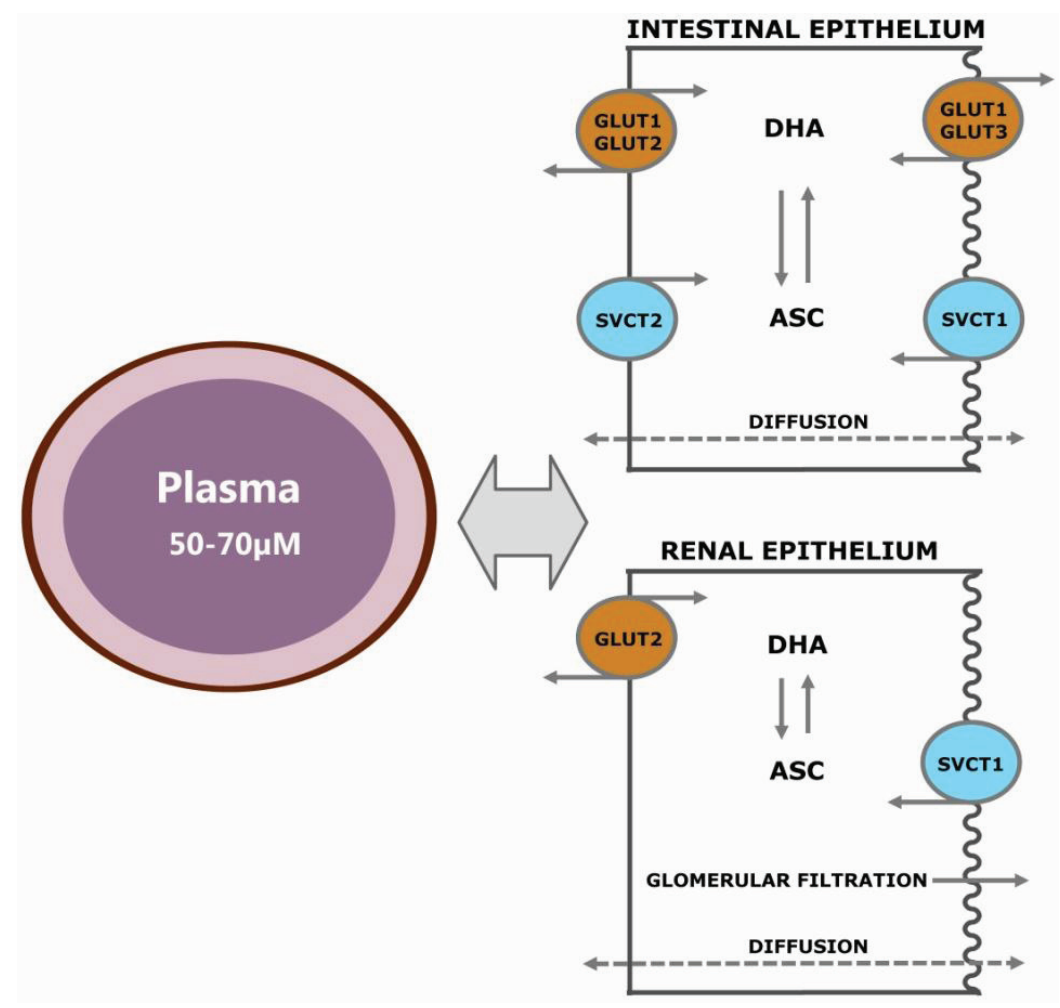




\section{Regulation of VitC Transport by Substrate Concentrations}

VitC transport can potentially be regulated by the amount of the various transporters or by their state of activity, thus relying on the rate of de novo synthesis (and degradation) and on activation of non-functional transporters, putatively also involving cellular translocation. Translational regulation of SVCT2 has been demonstrated in vitro in human platelets [124], and both SVCT1 and SVCT2 contain potential sites for glycosylation and phosphorylation as putative targets for post-translational regulation (Table 1). $\mathrm{N}$-linked glycosylation sites are located in the second and third extracellular loop, and a single protein kinase A-dependent and several protein kinase $\mathrm{C}$-dependent phosphorylation sites have been described in the amino acid sequence [52]. In humans, enzymatic activity of protein kinase C elicits different responses of SVCT1 and SVCT2. For SVCT1, the cytoplasm-to-membrane translocation is reduced, whereas the phosphorylation elicits a conformational alteration of the SVCT2 [97]. An association between the availability of vitC and an effect on transport mechanisms has been suggested, displaying the characteristics of a substrate-dependent regulation [124-130].

Table 1. Overview of in vitro studies regarding the regulation of the transport of vitamin $\mathrm{C}$ (vitC) during deficiency.

\begin{tabular}{|c|c|c|}
\hline Cell Line & Deficiency Regimen & Principal Findings \\
\hline $\begin{array}{l}\text { Human intestinal } \\
\text { cell line (Caco-2 } \\
\text { TC7) }[125]\end{array}$ & $\begin{array}{l}\text { Culture medium was } \\
\text { supplemented with ASC at } \\
\text { concentrations of } 45 \mu \mathrm{g} / \mathrm{mL} \text {, } \\
450 \mu \mathrm{g} / \mathrm{mL} \text { or } 4.5 \mathrm{mg} / \mathrm{mL} \text {. }\end{array}$ & $\begin{array}{l}\text { Exposure to } 4.5 \mathrm{mg} / \mathrm{mL} \text { ASC significantly reduced the ASC } \\
\text { uptake by } 50 \% \text { and expression of SVCT } 1 \mathrm{mRNA} \text { by } 77 \% \\
\text { compared to control conditions. }\end{array}$ \\
\hline $\begin{array}{l}\text { Primary human } \\
\text { platelets [124] }\end{array}$ & $\begin{array}{l}\text { Culture medium's ASC } \\
\text { concentration was reduced to } \\
30 \% \text { of standard levels. }\end{array}$ & $\begin{array}{l}V_{\max } \text { increased by } 240 \% \text { in response to the reduction of ASC } \\
\text { concentration. A subsequent increase in SVCT } 2 \text { protein } \\
\text { level was reported. An ASC supplement of } 500 \mu \mathrm{M} \text { only } \\
\text { slightly decreased SVCT2 levels. }\end{array}$ \\
\hline $\begin{array}{l}\text { Human hepatic } \\
\text { cell line } \\
(\text { HepG2) [127] }\end{array}$ & $\begin{array}{l}\text { Cells were incubated with } 10 \% \\
\text { fetal bovine serum containing } \\
10 \mathrm{mM} \text { ASC (supplemented), } \\
0.7 \mu \mathrm{M} \text { ASC (control), } \\
0 \mu \mathrm{M} \text { ASC (depleted). }\end{array}$ & $\begin{array}{l}\text { ASC-supplemented cells responded with a reduced } \\
\text { transport of ASC and a coherent reduced SVCT1 } \\
\text { expression (mRNA and protein). Depleted cells displayed } \\
\text { increased ASC transport and increased SVCT1 expression. } \\
\text { No changes were found for SVCT2. }\end{array}$ \\
\hline $\begin{array}{l}\text { Primary rat } \\
\text { astrocytes [131] }\end{array}$ & $\begin{array}{l}\text { Astrocytes were incubated with } \\
\text { ASC (from } 0 \text { to } 300 \mu \mathrm{M} \text { ) in } \\
\text { culture medium prior to } \\
\text { measurements of uptake rates. }\end{array}$ & $\begin{array}{l}\text { ASC depletion of culture medium increased the } V_{\max } \text { by } \\
15 \% \text { after one hour and } 20 \% \text { after } 6 \mathrm{~h} \text {. ASC repletion } \\
\text { resulted in a } 20 \% \text { decrease after one hour and } 30 \% \\
\text { after } 18 \mathrm{~h} \text {. }\end{array}$ \\
\hline $\begin{array}{l}\text { Rat osteosarcoma } \\
\text { cell line (ROS } \\
17 / 2.8)[132]\end{array}$ & $\begin{array}{l}\text { Cells were incubated with ASC } \\
\text { (from } 0 \text { to } 300 \mu \mathrm{M} \text { ) in culture } \\
\text { medium prior to measurements } \\
\text { of uptake rates. }\end{array}$ & $\begin{array}{l}\text { ASC depletion of culture medium increased the } V_{\max } \text { by } \\
41 \% \text { after six hours. ASC repletion resulted in a } 40 \% \\
\text { decrease after six hours. }\end{array}$ \\
\hline $\begin{array}{l}\text { Porcine proximal } \\
\text { tubule cell line } \\
(\text { LLC-PK1) }[128]\end{array}$ & $\begin{array}{l}\text { Cells were incubated with } \\
\text { increasing concentrations of } \\
\text { ASC in culture medium } \\
(10,25,50 \text { and } 100 \mu \mathrm{M}) \text {. }\end{array}$ & $\begin{array}{l}\text { Increasing concentrations in ASC reduced apical SVCT1 } \\
\text { expression and induced translocation of SVCT1 to the } \\
\text { cytoplasm before the signal was diminished. }\end{array}$ \\
\hline
\end{tabular}

Findings in vitro have shown a significant decrease in ASC uptake and concurrent mRNA expression-reduction following high-dose ASC culture, suggesting a putative feed-back mechanism and the association of transcriptional repressor elements in the regulation of ASC uptake $[125,126]$ 
(Table 1). This has been further supported by studies in human hepatic cells, in which ASC depletion significantly increased SVCT1 expression [127]. The involvement of a transcriptional regulatory mechanism was confirmed by the finding of an altered promoter activity associated to the Hepatic Nuclear Factor 1 (HNF-1) binding sites on the SVCT1 promoter region [127]. The involvement of translational regulation has been shown for the SVCT2 transporter in human platelets [124]. A reduction of the ASC concentration in the surrounding medium to $30 \%$ increased the $V_{\text {max }}$-value by almost $240 \%$, whereas the $K_{\mathrm{m}}$-value remained unchanged, indicating a responsive increased transport rate [124]. This was later confirmed by a marked increase in SVCT2 protein expression compared to platelets prior to deprivation of ASC [124]. In renal proximal tubule cells, increasing concentrations of ASC led to a signal translocation from the apical membrane to the cytoplasm before the signal was diminished [128]. The effect was a 50\% reduction in transport by cells pretreated with amounts of ASC corresponding to plasma saturation (50-100 $\mu \mathrm{M})$, suggesting that apical levels play a pivotal role in the regulation of ASC uptake in vitro.

A substrate-dependent differential regulation has also been supported by in vivo studies (Table 2). Almost four decades ago, Rose and Nahrwold showed that guinea pigs receiving diets containing five or 25 times the vitC of a standard diet reduced the rate of ASC uptake by $32 \%-52 \%$ compared to control animals [129]. Daily intramuscular injections of $300 \mathrm{mg}$ ASC reduced ASC influx into the intestinal mucosa by $16 \%$, suggesting that high circulating ASC levels may inhibit transport across the intestinal epithelium, but not ruling out that ASC concentrations in the intestinal lumen or cytoplasm of epithelial cells contribute to the regulation [129]. In guinea pigs, a diet containing high amounts of vitC ( $5000 \mathrm{mg} / \mathrm{kg}$ feed) reduced ASC uptake across isolated ileac mucosa by $25 \%-50 \%$ in both adult male, lactating female and juvenile animals compared to controls $(200 \mathrm{mg} \mathrm{vitC} / \mathrm{kg}$ feed) [130]. The observed decrease in uptake did not result in alterations of $K_{\mathrm{m}}$-values, indicating that the reduced absorption rate was due to either reduced abundance or increased degradation of the transporting molecules [130]. However, guinea pig counterparts receiving low levels of vitC (3.5 mg/week) did not show an increased rate of ASC uptake in response to deficiency [130]. The authors suggested that transporters have a nearly complete extraction efficiency, even under standard conditions (control animals) [130]. Likewise, an absence of up-regulation of SVCT-transporters following long-term vitC deficiency (100 $\mathrm{mg}$ vitC/ $\mathrm{kg}$ feed) in guinea pigs has been shown [31].

In humans, plasma concentrations following oral dosing of vitC are tightly regulated with peak plasma levels of around $200 \mu \mathrm{mol} / \mathrm{L}$ and a steady-state of $70-85 \mu \mathrm{mol} / \mathrm{L}$, even when excessive amounts (3 g) vitC are ingested [25,133]. A steep decline in ASC bioavailability following increased oral doses suggests that intestinal transport is a key factor in maintaining whole body vitC homeostasis. This regulation may, however, be bypassed by intravenous dosage, achieving plasma concentrations well beyond the threshold of oral administration levels [26,133]. Concentrations in plasma have been shown to pose a direct effect on the tissue accumulation of ASC. Oral supplementation of mice unable to synthesize vitC due to a mutation in the L-gulono- $\gamma$-lactone gene $\left(\right.$ gulo $\left.^{-/}\right)$has shown that increased plasma levels are required to achieve optimal concentration in several tissues (liver, heart and kidney) compared to levels necessary to obtain saturation in the brain [134]. This confirms the brain as being particularly efficient in retaining ASC, but also points towards a direct association between differences in plasma concentration and tissue-specific uptake [134]. In a recent intervention study in humans, the bioavailability of vitC in skeletal muscle 
relative to dietary intake was shown to correspond to plasma concentrations, whereas the same close correlation could not be found in leukocytes [135]. This could indicate an increased sensitivity in muscle towards alterations in plasma concentration or/and a differential uptake in leukocyte cells, possibly linked to differences in SVCT2 expression between the two cell types [135].

Table 2. Overview of in vivo studies regarding the regulation of vitC transport in response to vitC levels.

\begin{tabular}{|c|c|c|}
\hline Anin & Vitamin C regimen & Principal findings \\
\hline Guinea pig [129] & $\begin{array}{l}\text { ASC content in diet was } \\
\text { increased by five- and } \\
\text { 25-times compared to } \\
\text { standard diets. }\end{array}$ & $\begin{array}{l}\text { A reduction in ASC influx across the ileum by } \\
32 \%-52 \% \text { in animals fed high ASC diet compared } \\
\text { to controls (standard). }\end{array}$ \\
\hline Guinea pig [130] & $\begin{array}{l}\text { Animals received either high } \\
(5000 \mathrm{mg} / \mathrm{kg} \text { diet }), \text { low } \\
(0 \mathrm{mg} / \mathrm{kg} \text { diet }) \text { or control } \\
\text { (maintenance) }(200 \mathrm{mg} / \mathrm{kg} \\
\text { diet) levels of vitC. }\end{array}$ & $\begin{array}{l}\text { A high vitC level (hypervitaminosis) reduced the ASC } \\
\text { rate of uptake across the intestinal brush border by } \\
25 \%-50 \% \text { compared to controls. Hypovitaminotic } \\
\text { animals were not found to be different from controls. }\end{array}$ \\
\hline Guinea pig [31] & $\begin{array}{l}\text { Young and old animals, } \\
\text { long-term on either control } \\
(325 \mathrm{mg} \text { vitC } / \mathrm{kg}) \text { or deficient } \\
(100 \mathrm{mg} \text { vitC } / \mathrm{kg}) \text { diets. }\end{array}$ & $\begin{array}{l}\text { No effect of dietary vitC regimen on the expression of } \\
\text { SVCT1 or SVCT2 mRNA in liver or brain. }\end{array}$ \\
\hline $\begin{array}{l}\text { Knockout mice } \\
\left(\operatorname{smp} 30 / \mathrm{gnl}^{-/}\right) \\
{[30]}\end{array}$ & $\begin{array}{l}\text { Effects of vitC depletion } v s \text {. } \\
\text { control }(1.5 \mathrm{~g} \text { vitC/L water) } \\
\text { in wild-type (WT) and } \\
\text { knockout }(\mathrm{KO}) \text { mice. }\end{array}$ & $\begin{array}{l}\text { In KO, mice vitC depletion increased SVCT } 1 \text { and } \\
\text { SVCT } 2 \text { mRNA expression in the liver (by } 21 \text { and } 55 \% \text {, } \\
\text { respectively) and increased SVCT1 by } 55 \% \text { in the } \\
\text { small intestine compared to control counterparts. } \\
\text { No changes were found in the kidney or cerebellum. } \\
\text { In WT-mice, depletion increased SVCT } 2 \text { expression } \\
\text { in the small intestine by } 43 \% \text {. }\end{array}$ \\
\hline $\begin{array}{l}\text { Knockout mice } \\
\left(\text { gulo }^{-1-}\right)[29]\end{array}$ & $\begin{array}{l}\text { Gulo }^{-/-} \text {mice exposed to } \\
\text { different ASC levels (drinking } \\
\text { water): high } 3.33 \mathrm{~g} / \mathrm{L} ; \\
\text { standard } 0.33 \mathrm{~g} / \mathrm{L} ; \text { low } \\
0.033 \mathrm{~g} / \mathrm{L} \text { and depletion } \\
0 \mathrm{~g} / \mathrm{L} . \text { WT mice were } \\
\text { included as controls. }\end{array}$ & $\begin{array}{l}\text { Depletion resulted in an increased mRNA expression } \\
\text { of SVCT2 in the liver compared to WT controls. } \\
\text { A trend towards increased protein levels of SVCT2 in } \\
\text { liver and cerebellum was reported, although it did not } \\
\text { reach a statistical level of significance. }\end{array}$ \\
\hline
\end{tabular}

An increase in SVCT-expression as a response to a reduction in ASC, followed by a decrease in SVCT mRNA when ASC levels increased, has been reported in developing teleost fish, which naturally are unable to synthesize vitC [136]. Although this could be part of a normal developmental sequence of events, it may also be a substrate-dependent response on SVCT-expression by which ASC uptake is regulated [136]. Studies of senescence marker protein-30 (SMP30)/gluconolactonase (GNL) knockout mice has revealed that vitC depletion increases SVCT1 mRNA levels in liver and small intestine and SVCT2 mRNA in liver [30]. VitC sufficient wild-type (WT) mice had 43\% less SVCT2 mRNA compared to ASC-depleted WT mice, suggesting a deficiency associated mRNA upregulation. No changes were found in kidney or cerebellum of either WT or smp30/gnl ${ }^{-/-}$ groups [30]. The study did not find any significant changes in the expression of GLUT-transporters (1, 3 and 4, respectively) [30]. In another study, Meredith et al. reported a significant increase in liver 
SVCT2 mRNA during vitC deficiency in the gulo $^{-/-}$mice, whereas no effect was found in either cortex or cerebellum of the brain [29]. These findings may indicate the existence of an alternative transport mechanism involved in the regulation of vitC during deficiency [29]. In a study of fetal vs. maternal vitC status in guinea pigs, it was recently found that an inadequate maternal vitC intake during pregnancy resulted in reduced plasma vitC in newborn deficient pups, compared with their mothers [137]. The authors interpreted their findings as an indication that preferential transport of vitC from the mother to the fetus is overridden during a prolonged maternal vitC deficiency, thereby maintaining a basal maternal vitC concentration at the expense of the offspring [137]. Thus, several studies suggest that the exact mechanisms controlling the substrate-mediated regulation of vitC homeostasis in the body remains to be fully disclosed, but it appears likely that there are different mechanisms involved and that regulatory control may vary within specific tissues. Other regulatory mechanisms have also been suggested to contribute to vitC regulation, i.e., feed-back sensing and hormonal regulation [26].

\section{Concluding Remarks}

VitC homeostasis is tightly regulated by a variety of more or less specific transport mechanisms, some of which may remain to be established. Several in vitro and in vivo studies have reported dose- and concentration-dependent rates of transport, both during ASC depletion and following supplementation, and these changes in transport activity apparently occur without change in affinity for the substrate. However, conflicting evidence has also been put forward in which the resulting changes in tissue compartment concentrations cannot be explained by alterations in the abundance of known vitC transporters. Moreover, as genetic variation has been shown to significantly influence vitC homeostasis per se, such information needs to be taken into consideration in future studies. Thus, continued efforts are required to establish the mechanisms by which the body efficiently adapts to declining vitC intakes.

\section{Acknowledgments}

The authors are supported by the Danish Research Councils and The LifePharm Centre for in vivo pharmacology.

\section{Conflicts of Interest}

The authors declare no conflict of interest.

\section{References}

1. Frei, B.; Birlouez-Aragon, I.; Lykkesfeldt, J. Authors' perspective: What is the optimum intake of vitamin C in humans? Crit. Rev. Food Sci. Nutr. 2012, 52, 815-829.

2. Agarwal, M.; Mehta, P.K.; Dwyer, J.H.; Dwyer, K.M.; Shircore, A.M.; Nordstrom, C.K.; Sun, P.; Paul-Labrador, M.; Yang, Y.; Merz, C.N.B. Differing relations to early atherosclerosis between vitamin $\mathrm{C}$ from supplements $v$. FOOD in the Los Angeles atherosclerosis study: A prospective cohort study. Open Cardiovasc. Med. J. 2012, 6, 113-121. 
3. Langlois, M.; Duprez, D.; Delanghe, J.; de Buyzere, M.; Clement, D.L. Serum vitamin C concentration is low in peripheral arterial disease and is associated with inflammation and severity of atherosclerosis. Circulation 2001, 103, 1863-1868.

4. Myint, P.K.; Luben, R.N.; Welch, A.A.; Bingham, S.A.; Wareham, N.J.; Khaw, K.-T. Plasma vitamin $\mathrm{C}$ concentrations predict risk of incident stroke over $10 \mathrm{y}$ in 20,649 participants of the European Prospective Investigation into Cancer-Norfolk prospective population study. Am. J. Clin. Nutr. 2008, 87, 64-69.

5. Simon, J.A.; Hudes, E.S.; Browner, W.S. Serum ascorbic acid and cardiovascular disease prevalence in US adults. Epidemiology 1998, 9, 316-321.

6. Tveden-Nyborg, P.; Lykkesfeldt, J. Does vitamin C deficiency increase lifestyle-associated vascular disease progression? Evidence based on experimental and clinical studies. Antioxid. Redox Signal. 2013, in press.

7. Tveden-Nyborg, P.; Johansen, L.K.; Raida, Z.; Villumsen, C.K.; Larsen, J.O.; Lykkesfeldt, J. Vitamin $\mathrm{C}$ deficiency in early postnatal life impairs spatial memory and reduces the number of hippocampal neurons in guinea pigs. Am. J. Clin. Nutr. 2009, 90, 540-546.

8. $\quad$ Tveden-Nyborg, P.; Vogt, L.; Schjoldager, J.G.; Jeannet, N.; Hasselholt, S.; Paidi, M.D.; Christen, S.; Lykkesfeldt, J. Maternal vitamin C deficiency during pregnancy persistently impairs hippocampal neurogenesis in offspring of guinea pigs. PLoS One 2012, 7, e48488.

9. Harrison, F.E.; Dawes, S.M.; Meredith, M.E.; Babaev, V.R.; Li, L.; May, J.M. Low vitamin $\mathrm{C}$ and increased oxidative stress and cell death in mice that lack the sodium-dependent vitamin C transporter SVCT2. Free Radic. Biol. Med. 2010, 49, 821-829.

10. Sotiriou, S.; Gispert, S.; Cheng, J.; Wang, Y.; Chen, A.; Hoogstraten-Miller, S.; Miller, G.F.; Kwon, O.; Levine, M.; Guttentag, S.H.; et al. Ascorbic-acid transporter Slc23al is essential for vitamin $\mathrm{C}$ transport into the brain and for perinatal survival. Nat. Med. 2002, 8, 514-517.

11. Smith, J.L.; Hodges, R.E. Serum levels of vitamin C in relation to dietary and supplemental intake of vitamin C in smokers and nonsmokers. Ann. N. Y. Acad. Sci. 1987, 498, 144-152.

12. Touvier, M.; Lioret, S.; Vanrullen, I.; Bocle, J.-C.; Boutron-Ruault, M.-C.; Berta, J.-L.; Volatier, J.-L. Vitamin and mineral inadequacy in the French population: Estimation and application for the optimization of food fortification. Int. J. Vitam. Nutr. Res. 2006, 76, 343-351.

13. Schleicher, R.L.; Carroll, M.D.; Ford, E.S.; Lacher, D.A. Serum vitamin C and the prevalence of vitamin C deficiency in the United States: 2003-2004 National Health and Nutrition Examination Survey (NHANES). Am. J. Clin. Nutr. 2009, 90, 1252-1263.

14. Simon, J.A.; Hudes, E.S. Serum ascorbic acid and cardiovascular disease prevalence in US adults: The Third National Health and Nutrition Examination Survey (NHANES III). Ann. Epidemiol. 1999, 9, 358-365.

15. Lykkesfeldt, J.; Priemé, H.; Loft, S.; Poulsen, H.E. Effect of smoking cessation on plasma ascorbic acid concentration. BMJ 1996, 313, 91.

16. Lykkesfeldt, J. Smoking depletes vitamin C: Should smokers be recommended to take supplements? Cigar. Smoke Oxid. Stress 2006, 237-260, doi:10.1007/3-540-32232-9_9.

17. Wei, W.; Kim, Y.; Boudreau, N. Association of smoking with serum and dietary levels of antioxidants in adults: NHANES III, 1988-1994. Am. J. Public Health 2001, 91, 258-264. 
18. Frikke-Schmidt, H.; Tveden-Nyborg, P.; Lykkesfeldt, J. Vitamin C in Human Nutrition. In Vitamins in the Prevention of Human Diseases; Herrmann, W., Obeid, R., Eds.; de Gruyter, Walter: Berlin, Germany, 2011.

19. Lykkesfeldt, J.; Loft, S.; Nielsen, J.B.; Poulsen, H.E. Ascorbic acid and dehydroascorbic acid as biomarkers of oxidative stress caused by smoking. Am. J. Clin. Nutr. 1997, 65, 959-963.

20. Lykkesfeldt, J.; Poulsen, H.E. Is vitamin C supplementation beneficial? Lessons learned from randomised controlled trials. Br. J. Nutr. 2010, 103, 1251-1259.

21. Wilson, J.X. Vitamin C Transport in Animals and Plants. In Vitamin C-Functions and Biochemistry in Animals and Plants; Asard, H., May, J.M., Smirnoff, N., Eds.; BIOS Scientific Publishers: London, UK, 2004.

22. Rice, M.E. Ascorbate regulation and its neuroprotective role in the brain. Trends Neurosci. 2000, 23, 209-216.

23. Rice, M.E.; Russo-Menna, I. Differential compartmentalization of brain ascorbate and glutathione between neurons and glia. Neuroscience 1997, 82, 1213-1223.

24. Frikke-Schmidt, H.; Tveden-Nyborg, P.; Birck, M.M.; Lykkesfeldt, J. High dietary fat and cholesterol exacerbates chronic vitamin $\mathrm{C}$ deficiency in guinea pigs. Br. J. Nutr. 2011, 105, $54-61$.

25. Levine, M.; Conry-Cantilena, C.; Wang, Y.; Welch, R.W.; Washko, P.W.; Dhariwal, K.R.; Park, J.B.; Lazarev, A.; Graumlich, J.F.; King, J.; et al. Vitamin C pharmacokinetics in healthy volunteers: Evidence for a recommended dietary allowance. Proc. Natl. Acad. Sci. USA 1996, 93, 3704-3709.

26. Levine, M.; Padayatty, S.J.; Espey, M.G. Vitamin C: A concentration-function approach yields pharmacology and therapeutic discoveries. Adv. Nutr. 2011, 2, 78-88.

27. Levine, M.; Rumsey, S.C.; Daruwala, R.; Park, J.B.; Wang, Y. Criteria and recommendations for vitamin C intake. JAMA 1999, 281, 1415-1423.

28. Harris, L.J.; Ray, S.N.; Ward, A. The excretion of vitamin C in human urine and its dependence on the dietary intake. Biochem. J. 1933, 27, 2011-2015.

29. Meredith, M.E.; Harrison, F.E.; May, J.M. Differential regulation of the ascorbic acid transporter SVCT2 during development and in response to ascorbic acid depletion. Biochem. Biophys. Res. Commun. 2011, 414, 737-742.

30. Amano, A.; Aigaki, T.; Maruyama, N.; Ishigami, A. Ascorbic acid depletion enhances expression of the sodium-dependent vitamin C transporters, SVCT1 and SVCT2, and uptake of ascorbic acid in livers of SMP30/GNL knockout mice. Arch. Biochem. Biophys. 2010, 496, $38-44$.

31. Tveden-Nyborg, P.; Hasselholt, S.; Miyashita, N.; Moos, T.; Poulsen, H.E.; Lykkesfeldt, J. Chronic vitamin $\mathrm{C}$ deficiency does not accelerate oxidative stress in ageing brains of guinea pigs. Basic Clin. Pharmacol. Toxicol. 2012, 110, 524-529.

32. Nishikimi, M.; Fukuyama, R.; Minoshima, S.; Shimizu, N.; Yagi, K. Cloning and chromosomal mapping of the human nonfunctional gene for L-gulono-gamma-lactone oxidase, the enzyme for L-ascorbic acid biosynthesis missing in man. J. Biol. Chem. 1994, 269, 13685-13688.

33. Chatterjee, I. Evolution and the biosynthesis of ascorbic acid. Science 1973, 182, 1271-1272. 
34. Nandi, A.; Mukhopadhyay, C.K.; Ghosh, M.K.; Chattopadhyay, D.J.; Chatterjee, I.B. Evolutionary significance of vitamin $\mathrm{C}$ biosynthesis in terrestrial vertebrates. Free Radic. Biol. Med. 1997, 22, 1047-1054.

35. Malo, C.; Wilson, J.X. Glucose modulates vitamin C transport in adult human small intestinal brush border membrane vesicles. J. Nutr. 2000, 130, 63-69.

36. Harrison, F.E.; May, J.M. Vitamin C function in the brain: Vital role of the ascorbate transporter SVCT2. Free Radic. Biol. Med. 2009, 46, 719-730.

37. Lykkesfeldt, J.; Trueba, G.P.; Poulsen, H.E.; Christen, S. Vitamin C deficiency in weanling guinea pigs: Differential expression of oxidative stress and DNA repair in liver and brain. Br. J. Nutr. 2007, 98, 1116-1119.

38. Hughes, R.E.; Hurley, R.J.; Jones, P.R. The retention of ascorbic acid by guinea-pig tissues. Br. J. Nutr. 1971, 26, 433-438.

39. Kuo, C.-H.; Yonehara, N.; Yoshida, H. Subcellular ascorbic acid in scorbutic guinea pig brain. J. Nutr. Sci. Vitaminol. 1979, 25, 9-13.

40. Wilson, J.X. Regulation of vitamin C transport. Annu. Rev. Nutr. 2005, 25, 105-125.

41. Rose, R.C. Solubility properties of reduced and oxidized ascorbate as determinants of membrane permeation. Biochim. Biophys. Acta 1987, 924, 254-256.

42. Wilson, J.X.; Dixon, S.J. High-affinity sodium-dependent uptake of ascorbic acid by rat osteoblasts. J. Membr. Biol. 1989, 111, 83-91.

43. Wagner, E.S.; White, W.; Jennings, M.; Bennett, K. The entrapment of $\left[{ }^{14} \mathrm{C}\right]$ ascorbic acid in human erythrocytes. Biochim. Biophys. Acta 1987, 902, 133-136.

44. Goldenberg, H.; Schweinzer, E. Transport of vitamin $\mathrm{C}$ in animal and human cells. J. Bioenerg. Biomembr. 1994, 26, 359-367.

45. Washko, P.W.; Wang, Y.; Levine, M. Ascorbic acid recycling in human neutrophils. J. Biol. Chem. 1993, 268, 15531-15535.

46. Hughes, R.; Maton, S. The passage of vitamin C across the erythrocyte membrane. Br. J. Haematol. 1968, 14, 247-253.

47. Rumsey, S.C.; Daruwala, R.; Al-Hasani, H.; Zarnowski, M.J.; Simpson, I.A.; Levine, M. Dehydroascorbic acid transport by GLUT4 in Xenopus Oocytes and isolated rat adipocytes. J. Biol. Chem. 2000, 275, 28246-28253.

48. Rumsey, S.C.; Kwon, O.; Xu, G.W.; Burant, C.F.; Simpson, I.; Levine, M. Glucose transporter isoforms GLUT1 and GLUT3 transport dehydroascorbic acid. J. Biol. Chem. 1997, 272, 18982-18989.

49. Vera, J.C.; Rivas, C.I.; Fischbarg, J.; Golde, D.W. Mammalian facilitative hexose transporters mediate the transport of dehydroascorbic acid. Nature 1993, 364, 79-82.

50. Mardones, L.; Ormazabal, V.; Romo, X.; Jaña, C.; Binder, P.; Peña, E.; Vergara, M.; Zúñiga, F.A. The glucose transporter-2 (GLUT2) is a low affinity dehydroascorbic acid transporter. Biochem. Biophys. Res. Commun. 2011, 410, 7-12.

51. Zhao, F.-Q.; Keating, A.F. Functional properties and genomics of glucose transporters. Curr. Genomics 2007, 8, 113-128.

52. Liang, W.-J.; Johnson, D.; Jarvis, S.M. Vitamin C transport systems of mammalian cells. Mol. Membr. Biol. 2001, 18, 87-95. 
53. Vera, J.C.; Rivas, C.I.; Velásquez, F.V.; Zhang, R.H.; Concha, I.I.; Golde, D.W. Resolution of the facilitated transport of dehydroascorbic acid from its intracellular accumulation as ascorbic acid. J. Biol. Chem. 1995, 270, 23706-23712.

54. Dhariwal, K.R.; Hartzell, W.O.; Levine, M. Ascorbic acid and dehydroascorbic acid measurements in human plasma and serum. Am. J. Clin. Nutr. 1991, 54, 712-716.

55. Rumsey, S.C.; Levine, M. Absorption, transport, and disposition of ascorbic acid in humans. J. Nutr. Biochem. 1998, 9, 116-130.

56. Lykkesfeldt, J. Increased oxidative damage in vitamin $\mathrm{C}$ deficiency is accompanied by induction of ascorbic acid recycling capacity in young but not mature guinea pigs. Free Radic. Res. 2002, 36, 567-574.

57. Lykkesfeldt, J.; Viscovich, M.; Poulsen, H.E. Ascorbic acid recycling in human erythrocytes is induced by smoking in vivo. Free Radic. Biol. Med. 2003, 35, 1439-1447.

58. May, J.M.; Qu, Z.-C.; Whitesell, R.R.; Cobb, C.E. Ascorbate recycling in human erythrocytes: Role of GSH in reducing dehydroascorbate. Free Radic. Biol. Med. 1996, 20, 543-551.

59. May, J.M.; Mendiratta, S.; Hill, K.E.; Burk, R.F. Reduction of dehydroascorbate to ascorbate by the selenoenzyme thioredoxin reductase. J. Biol. Chem. 1997, 272, 22607-22610.

60. May, J.M.; Qu, Z.-C.; Mendiratta, S. Protection and recycling of $\alpha$-tocopherol in human erythrocytes by intracellular ascorbic acid. Arch. Biochem. Biophys. 1998, 349, 281-289.

61. May, J.M.; Qu, Z.-C.; Morrow, J.D.; Cobb, C.E. Ascorbate-dependent protection of human erythrocytes against oxidant stress generated by extracellular diazobenzene sulfonate. Biochem. Pharmacol. 2000, 60, 47-53.

62. May, J.M.; Qu, Z.-C.; Cobb, C.E. Extracellular reduction of the ascorbate free radical by human erythrocytes. Biochem. Biophys. Res. Commun. 2000, 267, 118-123.

63. May, J.M.; Qu, Z.-C.; Morrow, J.D. Mechanisms of ascorbic acid recycling in human erythrocytes. Biochim. Biophys. Acta 2001, 1528, 159-166.

64. May, J.M.; Qu, Z.-C.; Cobb, C.E. Recycling of the ascorbate free radical by human erythrocyte membranes. Free Radic. Biol. Med. 2001, 31, 117-124.

65. Mendiratta, S.; Qu, Z.-C.; May, J.M. Erythrocyte ascorbate recycling: Antioxidant effects in blood. Free Radic. Biol. Med. 1998, 24, 789-797.

66. Mendiratta, S.; Qu, Z.-C.; May, J.M. Enzyme-dependent ascorbate recycling in human erythrocytes: Role of thioredoxin reductase. Free Radic. Biol. Med. 1998, 25, 221-228.

67. Winkler, B.S.; Orselli, S.M.; Rex, T.S. The redox couple between glutathione and ascorbic acid: A chemical and physiological perspective. Free Radic. Biol. Med. 1994, 17, 333-349.

68. Park, J.B.; Levine, M. Purification, cloning and expression of dehydroascorbic acid-reducing activity from human neutrophils: Identification as glutaredoxin. Biochem. J. 1996, 315, 931-938.

69. Wells, W.W.; Xu, D.P. Dehydroascorbate reduction. J. Bioenerg. Biomembr. 1994, 26, 369-377.

70. Wells, W.W.; Xu, D.P.; Yang, Y.; Rocque, P.A. Mammalian thioltransferase (glutaredoxin) and protein disulfide isomerase have dehydroascorbate reductase activity. J. Biol. Chem. 1990, 265, 15361-15364. 
71. Del Bello, B.; Maellaro, E.; Sugherini, L.; Santucci, A.; Comporti, M.; Casini, A.F. Purification of NADPH-dependent dehydroascorbate reductase from rat liver and its identification with 3 alpha-hydroxysteroid dehydrogenase. Biochem. J. 1994, 304, 385-390.

72. Buettner, G. The pecking order of free radicals and antioxidants: Lipid peroxidation, $\alpha$-tocopherol, and ascorbate. Arch. Biochem. Biophys. 1993, 300, 535-543.

73. Linster, C.L.; van Schaftingen, E. Vitamin C. FEBS J. 2006, 274, 1-22.

74. Diliberto, E.J., Jr.; Heckman, G.D.; Daniels, A.J. Characterization of ascorbic acid transport by adrenomedullary chromaffin cells. Evidence for $\mathrm{Na}^{+}$-dependent co-transport. J. Biol. Chem. 1983, 258, 12886-12894.

75. Welch, R.W.; Wang, Y.; Crossman, A., Jr.; Park, J.B.; Kirk, K.L.; Levine, M. Accumulation of vitamin $\mathrm{C}$ (ascorbate) and its oxidized metabolite dehydroascorbic acid occurs by separate mechanisms. J. Biol. Chem. 1995, 270, 12584-12592.

76. Welch, R.W.; Bergsten, P.; Butler, J.D.; Levine, M. Ascorbic acid accumulation and transport in human fibroblasts. Biochem. J. 1993, 294, 505-510.

77. Washko, P.; Rotrosen, D.; Levine, M. Ascorbic acid transport and accumulation in human neutrophils. J. Biol. Chem. 1989, 264, 18996-19002.

78. Padh, H.; Aleo, J.J. Characterization of the ascorbic acid transport by 3 T6 fibroblasts. Biochim. Biophys. Acta 1987, 901, 283-290.

79. Zhou, A.; Nielsen, J.H.; Farver, O.; Thorn, N.A. Transport of ascorbic acid and dehydroascorbic acid by pancreatic islet cells from neonatal rats. Biochem. J. 1991, 274, 739-744.

80. Bergsten, P.; Yu, R.; Kehrl, J.; Levine, M. Ascorbic acid transport and distribution in human B lymphocytes. Arch. Biochem. Biophys. 1995, 317, 208-214.

81. Wright, J.; Castranova, V.; Colby, H.; Miles, P. Ascorbate uptake by isolated rat lung cells. J. Appl. Physiol. 1981, 51, 1477-1483.

82. Stevenson, N.R. Active transport of L-ascorbic acid in the human ileum. Gastroenterology 1974, 67, 952-956.

83. Tsukaguchi, H.; Tokui, T.; Mackenzie, B.; Berger, U.V.; Chen, X.-Z.; Wang, Y.; Brubaker, R.F.; Hediger, M.A. A family of mammalian $\mathrm{Na}^{+}$-dependent L-ascorbic acid transporters. Nature 1999, 399, 70-75.

84. Wilson, J.X.; Jaworski, E.M.; Dixon, S.J. Evidence for electrogenic sodium-dependent ascorbate transport in rat astroglia. Neurochem. Res. 1991, 16, 73-78.

85. Maffia, M.; Ahearn, G.; Vilella, S.; Zonno, V.; Storelli, C. Ascorbic acid transport by intestinal brush-border membrane vesicles of the teleost Anguilla anguilla. Am. J. Physiol. Regul. Integr. Comp. Physiol. 1993, 264, R1248-R1253.

86. Helbig, H.; Korbmacher, C.; Wohlfarth, J.; Berweck, S.; Kuhner, D.; Wiederholt, M. Electrogenic $\mathrm{Na}+$-ascorbate cotransport in cultured bovine pigmented ciliary epithelial cells. Am. J. Physiol. Cell Physiol. 1989, 256, C44-C49.

87. Daruwala, R.; Song, J.; Koh, W.S.; Rumsey, S.C.; Levine, M. Cloning and functional characterization of the human sodium-dependent vitamin $\mathrm{C}$ transporters hSVCT1 and hSVCT2. FEBS Lett. 1999, 460, 480-484. 
88. Eck, P.; Erichsen, H.C.; Taylor, J.G.; Yeager, M.; Hughes, A.L.; Levine, M.; Chanock, S.J. Comparison of the genomic structure and variation in the two human sodium-dependent vitamin C transporters, SLC23A1 and SLC23A2. Hum. Genet. 2004, 115, 285-294.

89. May, J.M. The SLC23 family of ascorbate transporters: Ensuring that you get and keep your daily dose of vitamin C. Br. J. Pharmacol. 2011, 164, 1793-1801.

90. Wang, Y.; Mackenzie, B.; Tsukaguchi, H.; Weremowicz, S.; Morton, C.C.; Hediger, M.A. Human vitamin C (L-ascorbic acid) transporter SVCT1. Biochem. Biophys. Res. Commun. 2000, 267, 488-494.

91. Takanaga, H.; Mackenzie, B.; Hediger, M.A. Sodium-dependent ascorbic acid transporter family SLC23. Pflüg. Arch. Eur. J. Physiol. 2004, 447, 677-682.

92. Wang, H.; Dutta, B.; Huang, W.; Devoe, L.D.; Leibach, F.H.; Ganapathy, V.; Prasad, P.D. Human Na+-dependent vitamin C transporter 1 (hSVCT1): Primary structure, functional characteristics and evidence for a non-functional splice variant. Biochim. Biophys. Acta 1999, $1461,1-9$.

93. Kuo, S.-M.; MacLean, M.E.; McCormick, K.; Wilson, J.X. Gender and sodium-ascorbate transporter isoforms determine ascorbate concentrations in mice. J. Nutr. 2004, 134, 2216-2221.

94. Liang, W.-J.; Johnson, D.; Ma, L.-S.; Jarvis, S.M. Regulation of the human vitamin C transporters expressed in COS-1 cells by protein kinase C. Am. J. Physiol. Cell Physiol. 2002, 283, C1696-C1704.

95. Maulén, N.P.; Henríquez, E.A.; Kempe, S.; Cárcamo, J.G.; Schmid-Kotsas, A.; Bachem, M.; Grünert, A.; Bustamante, M.E.; Nualart, F.; Vera, J.C. Up-regulation and polarized expression of the sodium-ascorbic acid transporter SVCT1 in post-confluent differentiated CaCo-2 cells. J. Biol. Chem. 2003, 278, 9035-9041.

96. Godoy, A.; Ormazabal, V.; Moraga-Cid, G.; Zúñiga, F.A.; Sotomayor, P.; Barra, V.; Vasquez, O.; Montecinos, V.; Mardones, L.; Guzmán, C. Mechanistic insights and functional determinants of the transport cycle of the ascorbic acid transporter SVCT2-Activation by sodium and absolute dependence on bivalent cations. J. Biol. Chem. 2007, 282, 615-624.

97. Savini, I.; Rossi, A.; Pierro, C.; Avigliano, L.; Catani, M.V. SVCT1 and SVCT2: Key proteins for vitamin C uptake. Amino Acids 2008, 34, 347-355.

98. Rivas, C.I.; Zuniga, F.A.; Salas-Burgos, A.; Mardones, L.; Ormazabal, V.; Vera, J.C. Vitamin C transporters. J. Physiol. Biochem. 2008, 64, 357-375.

99. Boyer, J.C.; Campbell, C.E.; Sigurdson, W.J.; Kuo, S.M. Polarized localization of vitamin C transporters, SVCT1 and SVCT2, in epithelial cells. Biochem. Biophys. Res. Commun. 2005, $334,150-156$.

100. Varma, S.; Sobey, K.; Campbell, C.E.; Kuo, S.-M. Hierarchal contribution of $N$-and $C$-terminal sequences to the differential localization of homologous sodium-dependent vitamin C transporters, SVCT1 and SVCT2, in Epithelial Cells. Biochemistry 2009, 48, 2969-2980.

101. Subramanian, V.S.; Subramanya, S.B.; Ghosal, A.; Marchant, J.S.; Harada, A.; Said, H.M. Modulation of function of sodium-dependent vitamin C transporter 1 (SVCT1) by Rab8a in intestinal epithelial cells: Studies utilizing Caco-2 cells and Rab8a knockout mice. Dig. Dis. Sci. 2012, 58, 641-649. 
102. Michels, A.J.; Hagen, T.M.; Frei, B. Human genetic variation influences vitamin C homeostasis by altering vitamin $\mathrm{C}$ transport and antioxidant enzyme function. Ann. Rev. Nutr. 2013, 33, 45-70.

103. Corpe, C.P.; Tu, H.; Eck, P.; Wang, J.; Faulhaber-Walter, R.; Schnermann, J.; Margolis, S.; Padayatty, S.; Sun, H.; Wang, Y.; et al. Vitamin C transporter Slc23a1 links renal reabsorption, vitamin $\mathrm{C}$ tissue accumulation, and perinatal survival in mice. J. Clin. Investig. 2010, 120, 1069-1083.

104. Mellors, A.J.; Nahrwold, D.L.; Rose, R.C. Ascorbic acid flux across mucosal border of guinea pig and human ileum. Am. J. Physiol. 1977, 233, E374-E379.

105. Harris, D.S.; Slot, J.W.; Geuze, H.J.; James, D.E. Polarized distribution of glucose transporter isoforms in Caco-2 cells. Proc. Natl. Acad. Sci. USA 1992, 89, 7556-7560.

106. Helliwell, P.A.; Richardson, M.; Affleck, J.; Kellett, G.L. Stimulation of fructose transport across the intestinal brush-border membrane by PMA is mediated by GLUT2 and dynamically regulated by protein kinase C. Biochem. J. 2000, 350, 149-154.

107. Mesonero, J.; Mahraoui, L.; Matosin, M.; Rodolosse, A.; Rousset, M.; Brot-Laroche, E. Expression of the hexose transporters GLUTI-GLUT5 and SGLTI in clones of Caco-2 cells. Biochem. Soc. Trans. 1994, 22, 681-684.

108. Agus, D.B.; Gambhir, S.S.; Pardridge, W.M.; Spielholz, C.; Baselga, J.; Vera, J.C.; Golde, D.W. Vitamin C crosses the blood-brain barrier in the oxidized form through the glucose transporters. J. Clin. Investig. 1997, 100, 2842-2848.

109. Qiao, H.; May, J.M. Development of ascorbate transporters in brain cortical capillary endothelial cells in culture. Brain Res. 2008, 1208, 79-86.

110. García, M.D.L.A.; Salazar, K.; Millán, C.; Rodríguez, F.; Montecinos, H.; Caprile, T.; Silva, C.; Cortes, C.; Reinicke, K.; Vera, J.C. Sodium vitamin C cotransporter SVCT2 is expressed in hypothalamic glial cells. Glia 2005, 50, 32-47.

111. Mun, G.H.; Kim, M.J.; Lee, J.H.; Kim, H.J.; Chung, Y.H.; Chung, Y.B.; Kang, J.S.; Hwang, Y.I.; Oh, S.H.; Kim, J.-G.; et al. Immunohistochemical study of the distribution of sodium-dependent vitamin $\mathrm{C}$ transporters in adult rat brain. J. Neurosci. Res. 2006, 83, 919-928.

112. Farrell, C.L.; Yang, J.; Pardridge, W.M. GLUT-1 glucose transporter is present within apical and basolateral membranes of brain epithelial interfaces and in microvascular endothelia with and without tight junctions. J. Histochem. Cytochem. 1992, 40, 193-199.

113. Hediger, M.A. New view at C. Nat. Med. 2002, 8, 445-446.

114. Berger, U.V.; Hediger, M.A. The vitamin C transporter SVCT2 is expressed by astrocytes in culture but not in situ. Neuroreport 2000, 11, 1395-1399.

115. Ralli, E.P.; Friedman, G.J.; Rubin, S.H. The mechanism of the excretion of vitamin C by the human kidney. J. Clin. Investig. 1938, 17, 765-770.

116. Rose, R.C. Ascorbic acid transport in mammalian kidney. Am. J. Physiol. 1986, 250, F627-F632.

117. Bowers-Komro, D.M.; McCormick, D.B. Characterization of ascorbic acid uptake by isolated rat kidney cells. J. Nutr. 1991, 121, 57-64. 
118. Subramanian, V.S.; Marchant, J.S.; Boulware, M.J.; Said, H.M. A C-terminal region dictates the apical plasma membrane targeting of the human sodium-dependent vitamin $\mathrm{C}$ transporter-1 in polarized epithelia. J. Biol. Chem. 2004, 279, 27719-27728.

119. Lee, J.H.; Oh, C.S.; Mun, G.H.; Kim, J.H.; Chung, Y.H.; Hwang, Y.I.; Shin, D.H.; Lee, W.J. Immunohistochemical localization of sodium-dependent L-ascorbic acid transporter 1 protein in rat kidney. Histochem. Cell Biol. 2006, 126, 491-494.

120. Martin, M.; Ferrier, B.; Roch-Ramel, F. Renal excretion of ascorbic acid in the rat: A micropuncture study. Am. J. Physiol. 1983, 244, F335-F341.

121. Bianchi, J.; Rose, R.C. Transport of L-ascorbic acid and dehydro-L-ascorbic acid across renal cortical basolateral membrane vesicles. Biochim. Biophys. Acta 1985, 820, 265-273.

122. Bianchi, J.; Rose, R.C. $\mathrm{Na}^{+}$-independent dehydro-L-ascorbic acid uptake in renal brush-border membrane vesicles. Biochim. Biophys. Acta 1985, 819, 75-82.

123. Nualart, F.; Castro, T.; Low, M.; Henriquez, J.P.; Oyarce, K.; Cisternas, P.; Garcia, A.; Yanez, A.J.; Bertinat, R.; Montecinos, V.P.; et al. Dynamic expression of the sodium-vitamin C co-transporters, SVCT1 and SVCT2, during perinatal kidney development. Histochem. Cell Biol. 2013, 139, 233-247.

124. Savini, I.; Catani, M.V.; Arnone, R.; Rossi, A.; Frega, G.; del Principe, D.; Avigliano, L. Translational control of the ascorbic acid transporter SVCT2 in human platelets. Free Radic. Biol. Med. 2007, 42, 608-616.

125. MacDonald, L.; Thumser, A.E.; Sharp, P. Decreased expression of the vitamin C transporter SVCT1 by ascorbic acid in a human intestinal epithelial cell line. Br. J. Nutr. 2002, 87, 97-100.

126. Karaczyn, A.; Ivanov, S.; Reynolds, M.; Zhitkovich, A.; Kasprzak, K.S.; Salnikow, K. Ascorbate depletion mediates up-regulation of hypoxia-associated proteins by cell density and nickel. J. Cell. Biochem. 2006, 97, 1025-1035.

127. Reidling, J.C.; Rubin, S.A. Promoter analysis of the human ascorbic acid transporters SVCT1 and 2: Mechanisms of adaptive regulation in liver epithelial cells. J. Nutr. Biochem. 2011, 22, 344-350.

128. Castro, T.; Low, M.; Salazar, K.; Montecinos, H.; Cifuentes, M.; Yáñez, A.J.; Slebe, J.C.; Figueroa, C.D.; Reinicke, K.; de los Angeles García, M. Differential distribution of the Sodium-vitamin C cotransporter-1 along the proximal tubule of the mouse and human kidney. Kidney Int. 2008, 74, 1278-1286.

129. Rose, R.C.; Nahrwold, D.L. Intestinal ascorbic acid transport following diets of high or low ascorbic acid content. Int. J. Vitam. Nutr. Res. 1978, 48, 382-386.

130. Karasov, W.H.; Darken, B.W.; Bottum, M.C. Dietary regulation of intestinal ascorbate uptake in guinea pigs. Am. J. Physiol. 1991, 260, G108-G118.

131. Schjoldager, J.G.; Tveden-Nyborg, P.; Lykkesfeldt, J. Prolonged maternal vitamin C deficiency overrides preferential fetal ascorbate transport but does not influence perinatal survival in guinea pigs. Br. J. Nutr. 2013, in press.

132. Wilson, J.X.; Jaworski, E.M.; Kulaga, A.; Dixon, S.J. Substrate regulation of ascorbate transport activity in astrocytes. Neurochem. Res. 1990, 15, 1037-1043. 
133. Padayatty, S.J.; Sun, H.; Wang, Y.; Riordan, H.D.; Hewitt, S.M.; Katz, A.; Wesley, R.A.; Levine, M. Vitamin C pharmacokinetics: Implications for oral and intravenous use. Ann. Intern. Med. 2004, 140, 533-537.

134. Vissers, M.C.M.; Bozonet, S.M.; Pearson, J.F.; Braithwaite, L.J. Dietary ascorbate intake affects steady state tissue concentrations in vitamin C-deficient mice: Tissue deficiency after suboptimal intake and superior bioavailability from a food source (kiwifruit). Am. J. Clin. Nutr. 2011, 93, 292-301.

135. Carr, A.C.; Bozonet, S.M.; Pullar, J.M.; Simcock, J.W.; Vissers, M.C. Human skeletal muscle ascorbate is highly responsive to changes in vitamin $\mathrm{C}$ intake and plasma concentrations. Am. J. Clin. Nutr. 2013, 97, 800-807.

136. Jiménez-Fernández, E.; Ponce, M.; Zuasti, E.; Fernández-Díaz, C.; Manchado, M.; Infante, C. Molecular characterization and transcriptional regulation of the sodium-dependent vitamin $\mathrm{C}$ transporter genes (slc23a1 and slc23a2) in a teleost fish, the Senegalese sole (Solea senegalensis). Comp. Biochem. Physiol. B 2012, 161, 208-218.

137. Dixon, S.J.; Wilson, J.X. Adaptive regulation of ascorbate transport in osteoblastic cells. J. Bone Miner. Res. 1992, 7, 675-681. 
Reprinted from Nutrients. Cite as: Gallie, D.R. Increasing Vitamin C Content in Plant Foods to Improve Their Nutritional Value-Successes and Challenges. Nutrients 2013, 5, 3424-3446.

Review

\title{
Increasing Vitamin C Content in Plant Foods to Improve Their Nutritional Value-Successes and Challenges
}

\section{Daniel R. Gallie}

Department of Biochemistry, University of California, Riverside, CA 92521-0129, USA;

E-Mail: drgallie@citrus.ucr.edu; Tel.: +1-951-827-7298; Fax: +1-951-827-4434.

Received: 19 July 2013; in revised form: 16 August 2013 / Accepted: 21 August 2013 /

Published: 30 August 2013

\begin{abstract}
Vitamin C serves as a cofactor in the synthesis of collagen needed to support cardiovascular function, maintenance of cartilage, bones, and teeth, as well as being required in wound healing. Although vitamin $C$ is essential, humans are one of the few mammalian species unable to synthesize the vitamin and must obtain it through dietary sources. Only low levels of the vitamin are required to prevent scurvy but subclinical vitamin $\mathrm{C}$ deficiency can cause less obvious symptoms such as cardiovascular impairment. Up to a third of the adult population in the U.S. obtains less than the recommended amount of vitamin $\mathrm{C}$ from dietary sources of which plant-based foods constitute the major source. Consequently, strategies to increase vitamin $\mathrm{C}$ content in plants have been developed over the last decade and include increasing its synthesis as well as its recycling, i.e., the reduction of the oxidized form of ascorbic acid that is produced in reactions back into its reduced form. Increasing vitamin C levels in plants, however, is not without consequences. This review provides an overview of the approaches used to increase vitamin $\mathrm{C}$ content in plants and the successes achieved. Also discussed are some of the potential limitations of increasing vitamin $\mathrm{C}$ and how these may be overcome.
\end{abstract}

Keywords: L-ascorbic acid; ascorbate; photosynthesis; DHAR; MDAR; reactive oxygen species

\section{Abbreviations:}

ABA, abscisic acid;

AO, ascorbate oxidase;

APX, ascorbate peroxidase; 
Asc, ascorbate;

CaMV, cauliflower mosaic virus;

CAT, catalase;

chl, chlorophyll;

DHA, dehydroascorbate;

DHAR, dehydroascorbate reductase;

ETR, electron transport rate;

$\mathrm{Fd}$, ferredoxin;

GalLDH, L-galactono-1,4-lactone dehydrogenase;

GulLO, L-gulono-1,4-lactone oxidase;

GalUR, D-galacturonic acid reductase;

GR, glutathione reductase;

GSH, glutathione;

GST, glutathione-S-transferase;

MDA, monodehydroascorbate reductase;

MDAR, monodehydroascorbate reductase;

NPQ, non-photochemical quenching;

$\varphi$ PSII, quantum yield of PSII;

qE, energy-dependent NPQ;

qI, photoinhibition;

RbcL, ribulose bisphosphate carboxylase/oxygenase large subunit;

QC, quiescent center;

PSI, photosystem I;

PSII, photosystem II;

ROS, reactive oxygen species;

SOD, superoxide dismutase;

TBARS, thiobarbituric acid reactive substance;

VDE, violaxanthin de-epoxidase

\section{Introduction}

In addition to its roles in cardiovascular function, immune cell development, and iron utilization, vitamin C (L-ascorbic acid) serves as a water-soluble antioxidant in animals [1-3]. Despite the fact that most mammals can synthesize ascorbic acid (Asc), humans are an exception as a result of a mutation to L-gulono-1,4-lactone oxidase, the last enzyme in the animal Asc biosynthetic pathway [4]. Because Asc is water-soluble, it is not stored and is readily excreted from the body. Therefore, Asc must be obtained regularly from dietary sources. The National Academy of Sciences has recommend $90 \mathrm{mg}$ /day of the vitamin for adult males and $75 \mathrm{mg} /$ day for adult females. Although vitamin $\mathrm{C}$ can be obtained from the consumption of fresh meat, it is destroyed by heating and is more typically obtained from plant sources. Asc is present in high amounts generally in fruits and leafy vegetables whereas grains typically have much lower levels of the vitamin, particularly in dried grain. Moreover, the diet of a significant portion of the global population consists largely of plant-based foods. Although post-harvest reductions in Asc can occur, particularly in leafy vegetables, 
increasing Asc content would help to preserve the nutritional quality of stored foods. As a result, much research has focused on developing strategies to increase vitamin $\mathrm{C}$ content in plant foods to improve their nutritional value including strategies to increase the biosynthetic capacity of plants and to increase the recycling of Asc once it has been used in a reaction [5-7].

Figure 1. Plants and animals employ distinct pathways for the synthesis of L-ascorbic acid. The pathway in animals is represented by reactions 1-8 whereas the pathways in plants are represented by reactions 9-24. Enzymes catalyzing the reactions are: 1, phosphoglucomutase; 2, UDP-glucose pyrophosphorylase; 3, UDP-glucose dehydrogenase; 4, glucuronate-1-phosphate uridylyltransferase; 5, glucuronate 1-kinase; 6, glucuronate reductase; 7, aldonolactonase (gluconolactonase); 8, gulono-1,4-lactone oxidase or dehydrogenase; 9, glucose-6-phosphate isomerase; 10, mannose-6-phosphate isomerase; 11, phosphomannose mutase; 12, GDP-mannose pyrophosphorylase (mannose-1-phosphate guanylyltransferase) (VTC1); 13, GDP-mannose-3',5'-epimerase; 14, GDP-L-galactose phosphorylase (VTC2 and VTC5); 15, L-galactose-1-phosphate phosphatase (VTC4); 16, L-galactose dehydrogenase; 17, L-galactono-1,4-lactone dehydrogenase; 18, methylesterase; 19, D-galacturonate reductase; 20, aldonolactonase; 21, phosphodiesterase; 22, sugar phosphatase; 23, L-gulose dehydrogenase; 24, myo-inositol oxygenase.

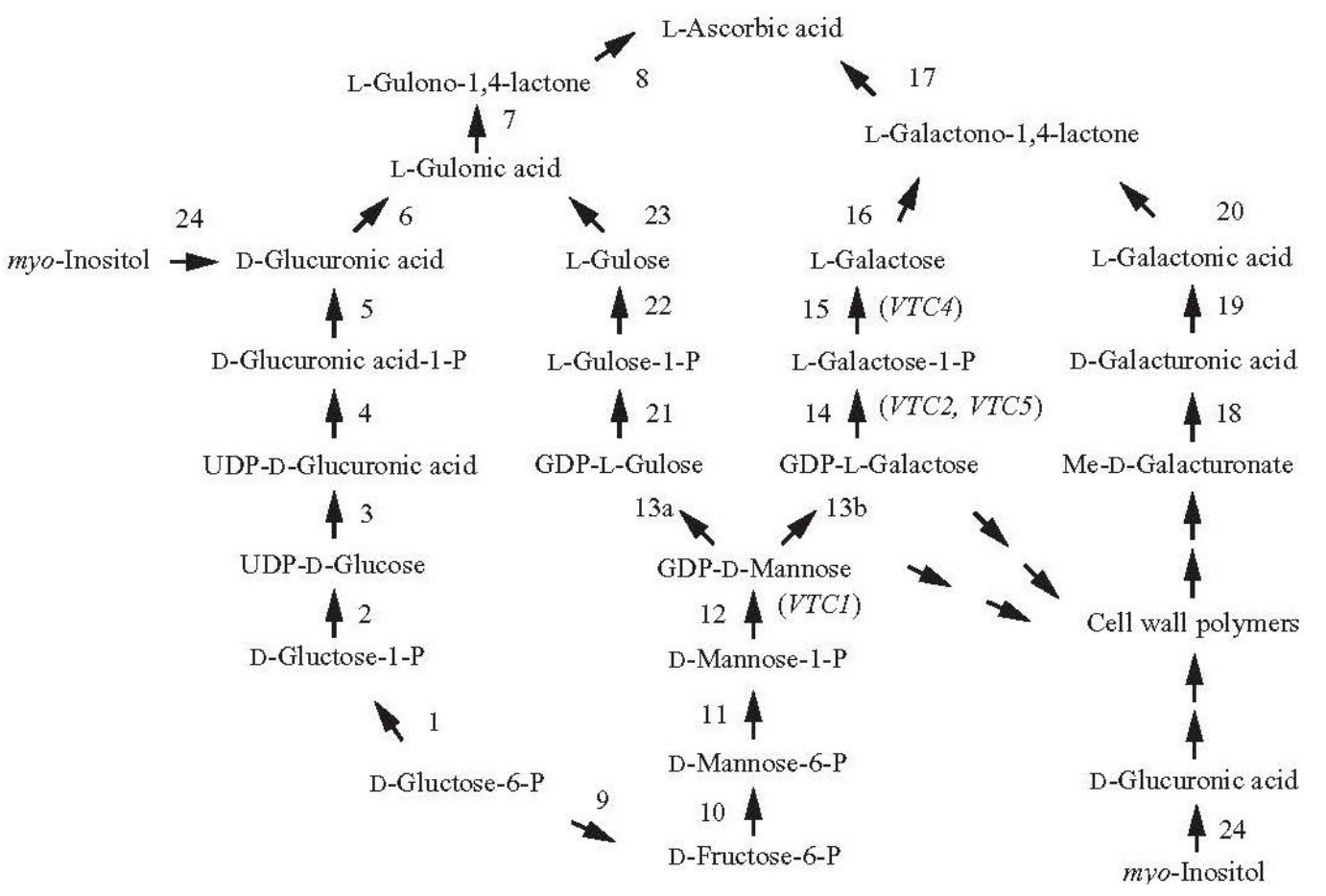

\section{Increasing Vitamin C Content through Improved Biosynthesis}

The pathway of vitamin $\mathrm{C}$ synthesis in mammals begins with D-glucose and proceeds through D-glucose-1-P, UDP-glucose, UDP-D-glucuronic acid, UDP-D-glucuronic acid-1-P, D-glucuronic acid, L-gulonic acid, and finally gulono-1,4-lactone (Figure 1). Gulono-1,4-lactone oxidase then converts gulono-1,4-lactone into 2-keto-gulono- $\gamma$-lactone which spontaneously converts to L-ascorbic acid [8]. 
In contrast to this single pathway, there are at least four biosynthetic pathways suggested to date in plants. The first discovered was the Smirnoff-Wheeler pathway in which Asc synthesis originates with L-galactose [9] (Figure 1). L-Galactose is produced from mannose-1-phosphate through the intermediates guanosine diphosphate (GDP)-mannose and GDP-L-galactose [10]. L-Galactose then undergoes oxidation to L-galactono-1,4-lactone catalyzed by the NAD-dependent L-galactose dehydrogenase followed by oxidation to L-ascorbic acid by the mitochondrial-localized L-galactono-1,4-lactone dehydrogenase [11,12].

Feeding experiments provided support for the Smirnoff-Wheeler pathway. For example, feeding leaf tissue with the Asc precursors L-galactose or L-galactono-1,4-lactone resulted in their conversion to Asc and therefore increased Asc content $[9,13,14]$. In another study, exogenous application of L-galactono-1,4-lactone to Arabidopsis or Medicago sativa leaves increased foliar Asc content up to 8-fold and was proportional to the amount applied [15]. Application of L-galactono-1,4-lactone or L-galactose to source potato leaves also increased the Asc content of these leaves as well as in sink organs, e.g., flowers and developing tubers [16].

Arabidopsis mutants affected at different steps in the Smirnoff-Wheeler pathway resulted in substantial reductions in Asc content, supporting the conclusion that this pathway is responsible for much of the Asc biosynthetic capacity in this species. For example, the vtcl mutant lacks GDP-mannose pyrophosphorylase expression whereas the $v t c 2$ and $v t c 5$ mutants lack GDP-L-galactose phosphorylase expression. The $v t c 1$ mutant exhibits a 70\%-75\% reduction in Asc content while the $v t c 2$ mutant contains just $10 \%-20 \%$ of the wild-type level of Asc, $v t c 5$ contains $80 \%$ of the wild-type level, and the vtc2/vtc5 double mutant bleaches in the absence of exogenous Asc or L-galactose which overcomes the block in the pathway $[13,17,18]$. The $v t c 4$ mutant results from a mutation in L-galactose-1-P phosphatase [19,20].

Attempts to increase Asc content through increasing its biosynthesis have achieved some success. Overexpression of GDP-L-galactose phosphorylase from kiwifruit (Actinidia chinensis) increased Asc content in tobacco leaves by more than 3-fold with an accompanying 50-fold increase in enzyme activity [21]. Although the agroinfection approach employed resulted in only a transient increase in enzyme expression, up to a 4-fold increase in Asc content was observed in stably-transformed Arabidopsis where the enzyme was overexpressed [21,22]. Stable transformation of GDP-L-galactose phosphorylase into potato, tomato, and strawberry resulted in up to a 3,6, and 2-fold increase in Asc, respectively, in tubers and fruits, although some loss of seed and the jelly of locular tissue surrounding the seed were observed in tomato and an increase in polyphenolic content was observed in strawberry and tomato [23]. A combinatorial approach in which kiwifruit GDP-L-galactose phosphorylase and GDP-mannose-3',5'-epimerase were transiently overexpressed in agroinfected tobacco leaves increased Asc content up to 7-fold [22]. Overexpression of L-galactose dehydrogenase, which catalyzes the conversion of L-galactose to L-galactono-1,4-lactone (Figure 1), however, failed to increase foliar Asc content in tobacco despite a 3.5-fold increase in the activity of the enzyme [24], suggesting that the endogenous level of L-galactose dehydrogenase is not rate-limiting. Transformation of Arabidopsis with GDP-galactose guanylyltransferase resulted in a 2.9-fold increase in Asc and co-transformation with either L-galactose-1-phosphate phosphatase or L-galactono-1,4-lactone dehydrogenase increased Asc content up to 4.1-fold [25]. Overexpressing multiple enzymes within the Smirnoff-Wheeler pathway, particularly those whose endogenous level 
is closest to being rate-limiting, may offer more promise to achieving substantial increases in Asc rather than the overexpression of any one enzyme. The choice of which enzymes to overexpress may be species-dependent as the level of expression for each enzyme in the pathway may differ among species.

Evidence for other biosynthetic pathways has suggested three alternative routes for the synthesis of Asc. In the first of these alternative pathways, D-galacturonic acid, generated from the breakdown of pectin during fruit ripening, serves as the starting point for Asc synthesis and is reduced to L-galactonic acid as catalyzed by the NADPH-dependent D-galacturonic acid reductase (GalUR) [26] (Figure 1). L-Galactonic acid spontaneously converts to L-galactono-1,4 lactone which L-galactono-1,4-lactone dehydrogenase converts to Asc [26]. Early support for this pathway came from the observation that D-galacturonic acid- $1-{ }^{14} \mathrm{C}$ was metabolized to L-ascorbic acid-6- ${ }^{14} \mathrm{C}$ through an inversion pathway in detached ripening strawberry fruit [27]. Supplying a methyl ester of D-galacturonic acid to cress seedlings and Arabidopsis cell cultures also increased Asc [28,29], suggesting that GalUR expression was not confined to fruits. Expression of the GalUR gene from strawberry increased whole-plant Asc content 2- to 3-fold in Arabidopsis [30], supporting the existence of this pathway. Demonstration that GalUR can increase foliar Asc biosynthesis through D-galactonic acid and D-galacturonic acid suggests that the substrates for this pathway are present in leaves. The potential for manipulating this pathway to achieve increased Asc content will depend on whether the enzymes of the pathway are expressed and whether D-galacturonic acid is present, e.g., following pectin degradation.

An example of the contingent basis of this pathway was observed in developing tomato fruit. Feeding tomato plants with D-galacturonate failed to increase Asc content in immature green tomato fruit while feeding with L-galactose, representing the D-mannose/L-galactose (or Smirnoff-Wheeler) pathway, did increase Asc content [31]. In contrast, feeding of either precursor increased Asc content of red ripened fruits, correlating with the increase in activity of D-galacturonate reductase and aldonolactonase, the last two enzymes of the D-galacturonate pathway in ripe fruits [31]. These observations suggest that the D-galacturonate pathway is not operative prior to ripening during which pectin is degraded. Thus, the contribution that the D-galacturonate pathway makes to Asc biosynthesis in tomato fruit may be limited to the ripening stage while the Smirnoff-Wheeler pathway is operative throughout fruit development (e.g., [23]). In addition, tracer studies have suggested that the D-galacturonate pathway may contribute only moderately to fruit Asc content [32] while its contribution in other organs has not been examined. In the second alternative pathway, GDP-mannose 3',5'-epimerase, which catalyzes conversion of GDP-D-mannose to GDP-L-galactose in the L-galactose pathway [10], also catalyzes the 5'-epimerization of GDP-D-mannose to produce GDP-L-gulose [33] (Figure 1). Conversion of GDP-L-gulose to L-gulonic acid allows Asc synthesis essentially as described in the animal pathway although evidence for this is still lacking. The presence of L-gulonic acid and L-gulono-1,4-lactone dehydrogenase activity [33,34] supports the existence of this pathway in plants. The expression of L-gulono-1,4-lactone oxidase (GulLO) from rat in lettuce and tobacco increased Asc content up to 7-fold [35] and reversed the reduction in Asc content in Arabidopsis mutants affected in the Smirnoff-Wheeler pathway [36] although it is not known whether L-gulono-1,4-lactone or L-galactono-1,4-lactone served as the substrate as the possibility that L-galactono-1,4-lactone served as the substrate was not examined. Although feeding 
with L-gulono-1,4-lactone did not increase the Asc content of tomato fruit at any developmental stage [31], its conversion to Asc has been reported for several plant species [28,37,38], supporting the presence of this pathway in plants. Expression of a foreign gene, however, can result in the ectopic expression of a pathway or the introduction of a novel pathway. Therefore, the degree to which this pathway functions in plants remains to be determined. Demonstrating that a labeled precursor directly labels Asc or that mutating a specific enzyme decreases Asc would provide more compelling evidence for such pathways.

The third alternative pathway involves D-glucuronic acid, an intermediate of the animal pathway which in plants can be generated by myo-inositol oxygenase (Figure 1). Support for this pathway in plants comes from the observation that overexpressing an Arabidopsis gene having homology to a porcine myo-inositol oxygenase increased Asc content [39]. As myo-inositol does not function as a precursor of Asc in strawberry fruit or in parsley leaves [32], this raises the question of the extent to which this pathway contributes to Asc content in plants. Nevertheless, the ability to increase Asc through the overexpression of this putative myo-inositol oxygenase gene may provide another strategy for increasing Asc biosynthesis.

Although multiple Asc biosynthetic pathways may exist in plants, the observation that mutants affected in the Smirnoff-Wheeler pathway result in substantial reductions in Asc content does indicate that the alternative pathways are unable to compensate for the loss in Asc biosynthetic capacity in Smirnoff-Wheeler pathway mutants. Thus, these alternative pathways may make only minor contributions to Asc biosynthesis and strategies focusing on these other pathways may be limited to increasing Asc in specific organs or at specific developmental stages.

\section{Increasing Vitamin C Content through Improved Asc Recycling}

\subsection{Targeting MDAR Expression to Increase Ascorbic Acid}

Once used in enzymatic or non-enzymatic reactions, Asc is oxidized to monodehydroascorbate (MDHA). Asc can be regenerated from MDHA through reduction by several means. If MDHA is produced in the chloroplast stroma, it can be recycled to Asc by ferredoxin (Fd), which is part of the photosynthetic electron transport chain, or by monodehydroascorbate reductase (MDAR) in the stroma [40]. Other MDAR isoforms are present in the cytosol, peroxisome, and mitochondria which reduce MDHA produced in those compartments. As no MDAR isoform is in the thylakoid lumen, MDHA cannot be reduced by MDAR or by Fd, which lies on the stromal side of the thylakoid membrane. As a result, this short-lived radical spontaneously disproportionates rapidly to Asc and DHA, particularly when the $\mathrm{pH}$ of the thylakoid lumen is low which occurs during the light driven transport of protons across the thylakoid membrane from the stroma into the lumen [40,41]. Under these conditions, the high $\mathrm{pH}$ of the stroma slows the disproportionation of MDHA and it undergoes reduction primarily through Fd or MDAR. Once photoreduced by PsaC in the PSI complex, Fd reduces MDHA directly or alternatively reduces $\mathrm{NADP}^{+}$to NADPH as catalyzed by Fd-NADP ${ }^{+}$ reductase (FNR) which MDAR uses (or NADH instead of NADPH) to reduce MDHA to Asc [42,43]. Fd reduces MDHA at a rate that is 34-fold greater than the rate of photoreduction of $\mathrm{NADP}^{+}$so that MDHA is likely reduced through Fd as part of the thylakoid scavenging system rather than by stromal MDAR when it is produced proximal to the thylakoid membrane [42]. MDAR, however, is 
available to reduce any stromal MDHA produced distal to the thylakoid membrane as part of the stromal scavenging system.

The multiple isoforms of MDAR are encoded by a five member nuclear gene family in Arabidopsis (referred to as AtMDAR1 through AtMDAR5) that are targeted to the cytosol, chloroplast, mitochondria, and peroxisomes [44]. Dual targeting of MDAR to chloroplasts and mitochondria results from the use of at least two transcription start sites which produce a seven amino acid extension in the mitochondrial-targeted form of the protein [45]. The 47-kDa AtMDAR1 and 54-kDa AtMDAR4 isoforms contain a $C$-terminal sequence that targets them to the peroxisomal matrix (PTS1) and peroxisomal membrane, respectively [46]. MDAR isoforms targeted to peroxisomes, chloroplasts, or mitochondria typically function together with ascorbate peroxidase (APX) to scavenge $\mathrm{H}_{2} \mathrm{O}_{2}$ [47] through the transfer of electrons from two molecules of Asc to $\mathrm{H}_{2} \mathrm{O}_{2}$ to form water and two molecules of MDHA. Disproportionation of $\mathrm{H}_{2} \mathrm{O}_{2}$ is also catalyzed by catalase (CAT) when present, e.g., in the peroxisome.

Increasing Asc content by targeting MDAR expression has achieved only limited success. Expression of a cytosolic tomato MDAR from a constitutive promoter in tomato (var. Micro-Tom) resulted in a reduction in Asc in mature green tomato fruits but unaltered foliar Asc content [48] although it may improve the chilling tolerance of fruit [49]. Improved tolerance against salt and osmotic stresses was also observed following an increase in MDAR expression in tobacco [50]. Increasing expression of a tomato chloroplast-targeted MDAR in tomato increased Asc marginally (1.2-fold) but was accompanied by a decrease in DHA, resulting in an approximate doubling of the Asc redox state [51]. Similar results were obtained following the expression of an Arabidopsis cytosolic MDAR in tobacco [52]. The little work that has been reported to date suggests that increasing MDAR expression may achieve only minor increases in Asc content.

\subsection{Targeting DHAR Expression to Increase Ascorbic Acid}

If MDHA is not enzymatically reduced by $\mathrm{Fd}$ or MDAR, it will undergo spontaneous disproportionation to Asc and DHA, the rate of which is dependent on the $\mathrm{pH}$, such as in the thylakoid lumen where $\mathrm{Fd}$ and MDAR are absent and the $\mathrm{pH}$ is low during light exposure. Disproportionation of MDHA can also occur in other cellular compartments if not enzymatically reduced. The DHA produced can be reduced to Asc by dehydroascorbate reductase (DHAR) using glutathione (GSH) as the reductant [53,54] (Figure 2). If it is not rapidly reduced, DHA undergoes irreversible hydrolysis to 2,3-diketogulonic acid and, as this is unable to be converted to Asc, it is lost to the Asc pool. Increasing the level of DHAR activity, therefore, limits DHA degradation by improving its recycling back into Asc before it is lost. As DHAR activity determines the relative levels of DHA and Asc and the enzyme is expressed in rate-limiting amounts in plants, it serves as a major regulator of the Asc redox state [5,55-57]. 
Figure 2. L-Ascorbic acid recycling through DHAR and MDAR. Following Asc synthesis from L-galactono-1,4-lactone by L-galactono-1,4-lactone dehydrogenase (GLDH) and oxidization to monodehydroascorbate (MDHA), monodehydroascorbate reductase (MDAR) can reduce MDHA to Asc. Alternatively, two MDHA molecules can disproportionate non-enzymatically to Asc and dehydroascorbate (DHA). Dehydroascorbate reductase (DHAR) can reduce DHA to Asc using glutathione (GSH) as the reductant. Oxidized glutathione (GSSG) is reduced by glutathione reductase (GR) to GSH using NADPH as the reductant. DHA will spontaneously hydrolyze to 2,3-diketogulonic acid if not reduced by DHAR.

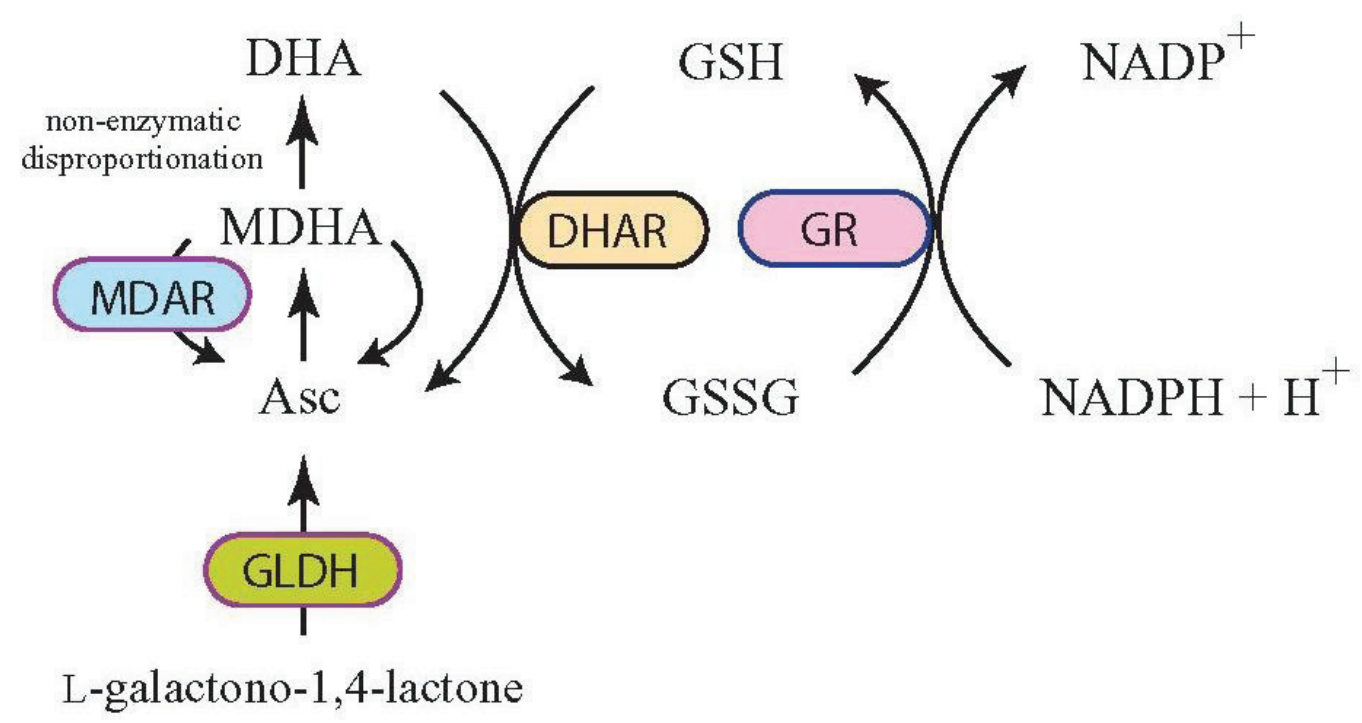

DHAR is encoded by three gene members in Arabidopsis: (AtDHAR1; At5g16710), (AtDHAR3; Atlg75270), and (AtDHAR5; Atlg19570) [44]. Microarray expression analysis suggests another gene, At5g36270 (AtDHAR2), is likely a pseudogene as it may not be expressed [44]. A fifth gene, Atlg19550 (AtDHAR4), is smaller than other DHAR paralogs due to multiple deletions throughout the polypeptide. AtDHAR3 is likely cytoplasmic while AtDHAR5 and AtDHAR1 are targeted to the mitochondria and chloroplast, respectively [58]. No DHAR isoform is transported to the apoplast. Consequently, any Asc transported to the apoplast is quickly oxidized, disproportionates, and the resulting DHA is either degraded or transported to the cytoplasm for recycling into Asc.

Because DHAR is a major recycler of Asc, a number of studies have focused on increasing the expression of this enzyme as a means to increase Asc content in plants, which has achieved success in several species. Although ectopic expression of a human DHAR in tobacco chloroplasts failed to increase Asc despite a 2-fold increase in DHAR activity [59,60], expression of a cytosolic wheat DHAR in tobacco did increase Asc content up to 4-fold as well as the redox state (i.e., an increase in the Asc to DHA ratio) with a simultaneous increase in Asc and a decrease in DHA [5]. Similar results were obtained when this cytosolic wheat DHAR was expressed in leaves and developing kernels of maize [5], demonstrating that changes in Asc can be made in photosynthetic and non-photosynthetic organs. Because Asc is transported from the cytoplasm to the apoplast and apoplastic DHA is transported back to the cytoplasm, expression of the cytosolic wheat DHAR not only increased the Asc content of the cytosol but the apoplast as well when measured from the apoplastic fluid [55], demonstrating that cytosolic DHAR regulates the symplastic and apoplastic Asc pool size and redox 
state. No change in Asc biosynthesis was observed following the increase in DHAR expression indicating that the synthesis of Asc and its recycling are independently controlled. The increase in DHAR expression and Asc recycling was accompanied by an increase in the GSH pool size and redox state [5]. As GSH is used by DHAR as the reductant, this suggests that the GSH pool size is affected by changes in DHAR activity. The extent to which the increase in GSH contributed to any physiological changes in these plants was not examined.

As the Asc pool size is determined by the rate of its synthesis and decay, the ability of DHAR to increase Asc content is a consequence of its recycling function that reduces DHA before it is lost through decay. As increasing DHAR expression results in improved Asc recycling and higher Asc levels, the endogenous level of DHAR is likely rate-limiting. Whether this is generally true throughout plant species is unknown. Consequently, the potential to increase Asc through increased DHAR expression will be greatest for species in which DHAR expression is rate-limiting. The strategy of increasing Asc content through increased DHAR expression, however, has been validated by subsequent studies that increased DHAR expression in the cytosol or in the chloroplast of a variety of species. Two studies that expressed a cytosolic DHAR from Arabidopsis in tobacco reported increases in Asc content by nearly 2-fold [52,61] whereas expression of an Arabidopsis cytosolic DHAR in Arabidopsis increased foliar Asc by 2 to 4.25-fold [62]. Expression of a rice cytosolic DHAR in Arabidopsis resulted in a slight increase in Asc content [63] as did expression of a rice DHAR in transformed tobacco chloroplasts [64]. Further increases in Asc content were observed when chloroplasts were transformed with glutathione reductase (GR) and DHAR [64].

Grains represent the most important food group supporting the global population either directly or indirectly as use in animal feed. Improving the nutritional value of grains offers the greatest potential for improving the diet of many and recent research has focused on engineering increasing multiple vitamins and micronutrients in grains as an efficient delivery mechanism for those whose diets are deficient in several vitamins. Although Asc content in grains is typically low, it is present during grain development but undergoes progressive oxidation during late development and is largely present as DHA by maturity [65]. The relationship between increased DHAR expression and increased Asc content in cereals was first shown in developing maize grain [5]. This was followed by a combinatorial approach to increase the levels of Asc, folate and $\beta$-carotene in maize grain using a barley D-hordein promoter to drive expression of a rice DHAR and an E. coli GTP cyclohydrolase $(f o l E)$ to increase the level of Asc and folate, respectively, and a wheat LMW glutenin promoter to drive expression of maize phytoene synthase ( $p s y l)$ and the D-hordein promoter to drive expression of Pantoea ananatis carotene desaturase (crtl) in order to increase $\beta$-carotene content [7]. These transgenes resulted in a 6-fold increase in Asc, a 2-fold increase in folate, and a 169-fold increase in $\beta$-carotene [7], demonstrating that an increase in Asc content can be combined with increases in the level of other vitamins to improve substantially the nutritional value of a fundamentally important staple. Increasing Asc content in other important, non-grain foods has been reported. Expression of a potato cytosolic DHAR from the CaMV 35S promoter increased foliar Asc content by more than 1.6-fold and in tubers by more than 1.2-fold which correlated with its expression where it is expressed higher in tubers than in leaves [66]. Expression of a chloroplast-localized potato DHAR increased foliar Asc content up to 1.5-fold but not in tubers which also correlated with its expression in leaves but a lack of expression in tubers [66]. Therefore, the strategy of increasing Asc content 
through increased Asc recycling through chloroplast-targeting of DHAR expression is likely to be limited to photosynthetically active tissues whereas increasing Asc content in non-photosynthetic organs will likely require expression of a cytosolic isoform of DHAR. Supporting this conclusion were results from the expression of a sesame DHAR under the control of a patatin promoter in potato. Just as the patatin promoter is active in tubers but not in leaves, expression of the sesame DHAR increased Asc 1.1 to 1.3-fold in tubers but no increase was observed in leaves. In contrast, expression of sesame DHAR under the constitutively active CaMV 35S promoter increased Asc content in leaves by 1.5-fold and 1.6-fold in tubers [67]. Overexpressing a cytosolic tomato DHAR from a constitutive promoter in tomato (var. Micro-Tom) increased Asc content in mature green and red ripe fruit by 1.6-fold in plants grown under low light [48]. In this example, however, no increase in foliar Asc was observed. The increase in Asc and GSH observed during the initial phases of embryogeny in Norway spruce following overexpression of the class I homeobox of knox 3 gene, HBK3, was attributed to increased activities of DHAR, GR, and ascorbate free radical reductase [68], suggesting that DHAR may also contribute to regulating Asc content in gymnosperm species.

\section{Consequences of Increasing Asc Content in Plants}

\subsection{Effects on Other Antioxidants and ROS-Detoxifying Enzymes}

As a major antioxidant in plants, changes in Asc content may well affect other antioxidant pools. Moreover, different approaches used for increasing Asc might be expected to affect specific antioxidants disproportionately. For example, increasing Asc by increasing biosynthetic activity would impact different antioxidant pools than would increasing Asc through improved Asc recycling which requires GSH and NADPH (or NADH) for the reduction of DHA or MDHA by DHAR or MDAR, respectively. Although most reports have observed increases in Asc content and/or in the Asc redox state following an increase in DHAR expression, the impact of this increase of DHAR activity on other antioxidants is less clear. Increases in GSH were observed in tobacco and maize expressing wheat DHAR with no change in glutathione reductase (GR), superoxide dismutase (SOD), APX, or CAT activities [5], suggesting coordinate regulation between DHAR and GSH. A similar increase in GSH content was reported for Arabidopsis overexpressing DHAR [62]. In contrast, expression of human DHAR in tobacco chloroplasts resulted in a reduction in GSH that was accompanied by a 1.43 -fold increase in GR activity [59]. Whether this was a consequence of expression of DHAR in chloroplasts remains to be determined.

\subsection{Increasing Ascorbic Acid Improves Tolerance to Many Environmental Stresses}

Although oxygen is essential to plants, it can be highly damaging, particularly as singlet oxygen $\left({ }^{1} \mathrm{O}_{2}\right)$ or in its reactive forms such as the superoxide anion $\left(\mathrm{O}_{2}{ }^{-}\right)$, hydroxyl radical $\left({ }^{\circ} \mathrm{OH}\right)$, or hydrogen peroxide $\left(\mathrm{H}_{2} \mathrm{O}_{2}\right)$. ROS are detoxified through the action of antioxidants such as Asc and GSH either directly or in reactions catalyzed by SOD, APX, and CAT [69,70]. Under conditions of excess light, $\mathrm{O}_{2}{ }^{--}$is produced during photosynthesis and is converted by SOD to $\mathrm{H}_{2} \mathrm{O}_{2}$ which is reduced to $\mathrm{H}_{2} \mathrm{O}$ by APX as one means to maintain electron flow through the photosystems [71]. Abiotic stresses such as cold, drought, or high light increase ROS production by creating conditions 
of light stress at lower light levels. $\mathrm{H}_{2} \mathrm{O}_{2}$ rapidly inactivates APX if Asc is limiting [72] and inhibits $\mathrm{CO}_{2}$ assimilation by inhibiting several Calvin cycle enzymes [40]. ROS can invade a plant in the form of environmental pollutants, e.g., ozone [73,74], which damages cell membranes or induces programmed cell death [75-77]. As a defense mechanism, $\mathrm{H}_{2} \mathrm{O}_{2}$ produced from ozone functions as a signaling intermediate in guard cells to promote stomatal closure thus limiting ozone entry into the leaf interior [78,79].

As an antioxidant, Asc would be expected to affect tolerance to environmental stress. This was first demonstrated using vtc mutants of Arabidopsis in which their reduced Asc content correlated with a reduction in tolerance to environmental ROS. With $70 \%-75 \%$ less Asc, the vtcl mutant is hypersensitive to ozone and sulfur dioxide $[13,19,80]$ and contains a higher oxidative load relative to wild-type plants when exposed to stress conditions such as salt despite its increased GSH content [81]. The expression level of regulators of Asc biosynthesis can also affect the degree of ozone tolerance. Knockout mutants of AMR1 (for ascorbic acid mannose pathway regulator 1) resulted in up to 3-fold greater foliar Asc content in Arabidopsis and increased ozone tolerance [82]. In contrast, plants with increased expression of AMR1 through activation-tagging exhibited a 60\% reduction in Asc and greater ozone sensitivity [82]. As AMR1 coordinately regulates transcript expression of six Smirnoff-Wheeler pathway enzyme genes to negatively regulate Asc biosynthesis, targeting regulators of biosynthetic pathways offers yet another promising approach to alter Asc content. In a second study, overexpression of the Arabidopsis ethylene response factor gene AtERF98 increased Asc content up to approximately 1.6-fold which was attributed primarily to an increase in the expression of genes in the Smirnoff-Wheeler pathway [83]. As AtERF98 binds to the promoter of VTC1, AtERF98 likely functions as a transcriptional activator of one or more genes in the Smirnoff-Wheeler pathway [83]. Increasing AtERF98 expression resulted in enhanced salt tolerance, demonstrating that increasing Asc biosynthesis improves tolerance to this abiotic stress [83].

That the endogenous level of apoplastic Asc is important in detoxifying ozone was shown in tobacco in which the level of apoplastic Asc was specifically altered [84]. Overexpressing an apoplastic-localized cucumber ascorbate oxidase (AO), which oxidizes apoplastic Asc, increased the ozone sensitivity of transgenic tobacco, correlating with the conversion of virtually all apoplastic Asc to DHA and depriving the apoplast of its ability to detoxify ozone entering the leaf interior [84]. A decrease in the cytosolic Asc redox state was also observed which would compromise the ability of a cell to detoxify ozone entering the cytosol.

Increased sensitivity to ozone following a reduction in Asc recycling was observed following loss of cytosolic DHAR expression in the Arabidopsis AtDHAR3 mutant [58]. The lower redox state but not pool size of Asc in this mutant indicates that Asc recycling is important in preventing oxidative damage. Consistent with its role in ozone tolerance, AtDHAR3 expression is induced by ozone [58].

If decreasing Asc content reduces tolerance to environmental ROS, increasing Asc content would be predicted to have the opposite effect, a notion supported by several studies published to date. Increasing Asc content in tobacco by increasing DHAR expression increased the Asc content of the apoplast and symplast and thus increased tolerance to ozone by reducing the oxidative load of the plant (i.e., a lower level of foliar and apoplastic $\mathrm{H}_{2} \mathrm{O}_{2}$ ) which was accompanied by a lower induction of antioxidant-related enzyme activities, more chlorophyll, and a higher level of photosynthetic activity following ozone exposure [56]. This increase in tolerance occurred despite the guard cells 
being less responsive to ozone as a consequence of their higher Asc content which reduces $\mathrm{H}_{2} \mathrm{O}_{2}$ levels $[73,85]$. Thus, increasing Asc content throughout a plant reduces guard cell responsiveness which permits more ozone to enter the leaf interior. The increased ozone tolerance can be understood, however, by the increased ability of every cell to detoxify ozone invading the leaf interior. Consistent with these findings, increasing Asc content 2-fold in tobacco through the expression of a cytosolic Arabidopsis DHAR enhanced its tolerance to ozone as well as drought, salt, or polyethylene glycol [61].

Conversely, a reduction in Asc recycling through the suppression of DHAR expression increased the responsiveness of guard cells to ozone thereby limiting ozone diffusion into the leaf interior [56]. At the same time, however, the decrease in DHAR activity lowered the Asc content of leaf cells and thus reduced their ability to detoxify any ozone that did invade [56]. Thus, increasing Asc content provides greater protection against environmental oxidative damage without compromising photosynthetic activity than does increasing guard cell responsiveness through decreasing Asc which reduces ozone entry but also reduces photosynthetic activity.

In addition to ozone, increasing Asc content provides greater tolerance to other environmental stresses. Arabidopsis with increased Asc content and redox state resulting from an increase in DHAR expression retained more Asc and chlorophyll with less membrane damage following exposure to high light and temperature or following treatment with paraquat [62]. Arabidopsis expressing a rice DHAR had greater tolerance to salt stress despite the small increases in DHAR activity and Asc achieved although no difference in cold tolerance was observed [63]. Although tobacco expressing a chloroplast-targeted human DHAR failed to increase Asc, it did increase the Asc redox state and the plants experienced less membrane damage following exposure to methyl viologen or $\mathrm{H}_{2} \mathrm{O}_{2}$ and had improved tolerance to low temperature and salt [60]. Combining expression of a chloroplast-localized DHAR with the expression of a chloroplast-localized CuZnSOD and APX increased the Asc and GSH redox states and the plants exhibited greater tolerance to paraquat and salt [86]. Greater tolerance to salt and cold was also observed in tobacco following the simultaneous expression of two pairs of chloroplast-localized enzymes, i.e., an E. coli GR with either an E. coli glutathione-S-transferase (GST) or a rice DHAR, that increased Asc and GSH content and their redox states [64].

Because fewer studies on increasing Asc through MDAR expression have been reported and those that have been carried out have observed smaller increases in Asc content, much less is known about the effects of MDAR-mediated increases in Asc on plant growth and plant responses. However, the results to date suggest that increasing Asc through MDAR expression has similar effects to those following an increase in DHAR expression. The slight increase in Asc content and decrease in DHA content that resulted in an approximate doubling of the Asc redox state in tomato seedlings overexpressing a chloroplast-targeted tomato MDAR resulted in a reduced oxidative load (as measured by $\mathrm{H}_{2} \mathrm{O}_{2}$ ), lower thiobarbituric acid reactive substance (TBARS) content (a measure of membrane damage), a higher net photosynthetic rate, higher maximal photochemical efficiency of PSII and greater fresh weight when subjected to low or high temperature stress [51]. Reducing Asc and its redox state through the suppression of MDAR expression resulted in largely opposite phenotypes [51]. In agreement with these results, greater tolerance to ozone, reduced $\mathrm{H}_{2} \mathrm{O}_{2}$ levels, and increased photosynthetic activity were observed in tobacco expressing an Arabidopsis MDAR following salt stress [50]. 
ROS can also be generated during development. For example, $\mathrm{H}_{2} \mathrm{O}_{2}$ is produced in the peroxisome of oilseeds as a by-product of fatty acid $\beta$-oxidation during lipid catabolism that accompanies seedling growth $[87,88]$. Catalase in the peroxisomal matrix detoxifies $\mathrm{H}_{2} \mathrm{O}_{2}$ and a membrane-bound APX3 and MDAR4, encoded by SUGAR-DEPENDENT2 (SDP2), together detoxify $\mathrm{H}_{2} \mathrm{O}_{2}$ using Asc [87,89-91]. Loss of MDAR4 expression in the Arabidopsis sdp2 mutant is conditionally seedling-lethal as MDAR activity is needed to reduce leakage of $\mathrm{H}_{2} \mathrm{O}_{2}$ from peroxisomes that protects $S D P 1$-encoded triacylglycerol (TAG) lipase activity and storage oil hydrolysis in the closely associated oil bodies during seedling growth [92]. Loss of MDAR4 activity results in inactivation of TAG lipase by $\mathrm{H}_{2} \mathrm{O}_{2}$ and a reduced ability to catabolize storage oil needed to support seedling growth [92]. Whether increasing Asc through increasing MDAR4 expression might improve seedling growth has not been examined. However, increasing APX3 expression increases tolerance against oxidative stress [93], suggesting an increase in Asc and the peroxisomal-associated APX3 and MDAR4 that use and recycle Asc may improve seedling tolerance against oxidative stress.

\subsection{Increasing Ascorbic Acid Improves Tolerance to High Light}

As mentioned above, in addition to environmental sources, ROS is generated during exposure to high light. Excess light energy can generate triplet state chlorophyll $\left({ }^{3} \mathrm{Chl}\right)$ which transfers its energy to ground-state $\mathrm{O}_{2}$ to produce ${ }^{1} \mathrm{O}_{2}$. Photosystem over reduction also produces $\mathrm{ROS}$ such as $\mathrm{O}_{2}^{\cdot-}$ and $\mathrm{H}_{2} \mathrm{O}_{2}$ [94] which can damage proteins, membranes, and pigments of photosystem I (PSI) and photosystem II (PSII), resulting in the inactivation of reaction centers as well as compromise their repair $[95,96]$. An increase in DHAR expression in tobacco resulted in less photoinhibition following exposure to high light that was likely due to an increase in the foliar levels of xanthophyll pigments and chlorophyll as well as in the electron transport rate (ETR) and $\mathrm{CO}_{2}$ assimilation, particularly at high light intensities, while ROS were reduced [97]. Thus, an increase in Asc maintains photosynthetic functioning by limiting ROS-mediated damage. Conversely, reducing Asc through suppression of DHAR results in elevated ROS and photoinhibition that is accompanied by reductions in the quantum yield of PSII and ETR [97].

\subsection{Increasing Ascorbic Acid Decreases Tolerance to Drought Stress}

While ROS are generally detrimental they also serve as important signaling cues about the external environment, e.g., the role of $\mathrm{H}_{2} \mathrm{O}_{2}$ in guard cells in regulating gas exchange and transpiration in response to water availability [98]. Abscisic acid (ABA) can promote $\mathrm{H}_{2} \mathrm{O}_{2}$ production during periods of water limitation which signals for stomatal closure [99]. Although tobacco overexpressing DHAR grew normally under well-watered conditions, the higher Asc content in guard cells not only reduced their responsiveness to ozone but also their responsiveness to the onset of water stress which normally triggers stomatal closure to prevent further water loss [55]. The reduction in responsiveness can be understood through the role of Asc as a scavenger of $\mathrm{H}_{2} \mathrm{O}_{2}$ and the balance between $\mathrm{H}_{2} \mathrm{O}_{2}$ production and Asc establishes whether $\mathrm{H}_{2} \mathrm{O}_{2}$ rises to a level that triggers stomatal closure. As a consequence, increasing Asc in DHAR-overexpressing tobacco maintains $\mathrm{H}_{2} \mathrm{O}_{2}$ at a lower level which delays stomatal closure upon onset of water stress, resulting in greater open stomatal area, increased transpiration and water loss, and ultimately decreased tolerance 
to water stress [55]. Reducing Asc content through suppressing DHAR expression results in an elevated accumulation of $\mathrm{H}_{2} \mathrm{O}_{2}$ in guard cells and a greater degree of stomatal closure even under non-stress conditions [55]. This hyperresponsiveness enables such plants to reduce transpiration during drought conditions resulting in up to 30\% less water loss [55]. Thus, increasing Asc content throughout a plant confers protection against environmental ROS while reducing drought tolerance whereas reducing Asc content reduces $\mathrm{CO}_{2}$ assimilation under normal growth conditions as a consequence of the reduction in the open stomatal area but also reduces water loss resulting in improved drought tolerance. A strategy to increase foliar Asc content while maintaining normal levels of Asc in guard cells may improve nutritional value and tolerance to environmental ROS without increasing sensitivity to drought conditions.

\subsection{Increasing Ascorbic Acid Prolongs Leaf Function}

Increasing Asc through increasing DHAR expression resulted in higher levels of ribulose bisphosphate carboxylase/oxygenase large subunit ( $\mathrm{RbcL}$ ), chlorophyll, and $\mathrm{CO}_{2}$ assimilation but this had no effect on plant growth under normal conditions [57]. In contrast, reducing Asc content through reduced biosynthesis resulted in slower shoot growth, smaller leaves, and reduced shoot fresh weight and dry weight [80] while plants with lower Asc content following suppression of DHAR expression exhibited a slower rate of leaf expansion, slower shoot growth, delayed flowering time, and reduced foliar dry weight [57]. These phenotypes correlated with reduced leaf function as measured by a disproportionate loss in chlorophyll a, a reduction in $\mathrm{RbcL}$, and a lower rate of $\mathrm{CO}_{2}$ assimilation [57]. The lower rate of $\mathrm{CO}_{2}$ assimilation was not due to a limitation in $\mathrm{CO}_{2}$ diffusion into DHAR-suppressed leaves as the sub-stomatal $\mathrm{CO}_{2}$ concentration was actually higher [57]. Rather, the reduced growth likely resulted from a premature loss of leaf function and early onset of senescence in mature leaves that may have reduced photosynthate available to young leaves.

\subsection{Increasing Ascorbic Acid Can Alter Pathogen Defense Responses}

The role of Asc in pathogen defense has received only limited attention. In an early study, the reduced Asc content of Arabidopsis vtc1 or vtc2 mutants resulted in reduced growth of the bacterial pathogen Pseudomonas syringae pv maculicola ES4326 and hyphal growth of the fungal pathogen Peronospora parasitica pv Noco [100]. The reduction in growth of P. syringae in vtcl plants correlated with a greater induction of the pathogenesis-related proteins PR-1 and PR-5, increased expression from some senescence-associated gene (SAG) genes and higher levels of salicylic acid. The reduced Asc content in these mutants resulted in the premature senescence of uninfected plants with an accompanying increase in salicylic acid [100]. These observations suggest that reducing Asc content predisposed Arabidopsis to induce defense responses faster upon pathogen attack. Whether an increase in Asc content would have had the opposite effect on these pathogens was not examined in this study. Quite different results were observed in a more recent study that also employed the same mutants. In this study, Arabidopsis vtcl and vtc2 were more susceptible to the pathogenic ascomycete Alternaria brassicicola and Asc strongly inhibited growth of fungal cultures [101]. Asc levels decreased following $A$. brassicicola infection with an increase in DHA, suggesting that Asc is being consumed during infection [101]. Given the limited number of studies focusing on the 
relationship between Asc content and pathogen defense, it is not possible at this point to conclude how increasing Asc content will affect defense responses. These two studies do suggest, however, that changes in Asc content may affect defense responses in a very pathogen-specific manner.

\subsection{Increasing Ascorbic Acid Induces Twinning}

In addition to being an antioxidant, Asc regulates the cell cycle by promoting $\mathrm{G}_{1}$ to $\mathrm{S}$ progression of cells, e.g., in the quiescent center of onion roots [102-106]. Repression of L-galactono-1,4-lactone dehydrogenase (GalLDH) expression in tobacco BY-2 cell lines resulted in 30\% less Asc and a reduction in the rate of cell division and growth [107]. The ability of Asc to promote cell division had dramatic consequences when its level was elevated during early embryo development. Embryo development initiates with a transverse zygotic division to produce an apical, proembryo cell and a basal cell that gives rise to the suspensor and in most species, a single embryo develops in each seed. Increasing Asc content in tobacco by increasing DHAR expression, however, resulted in monozygotic twinning and polycotyly [108]. The twin zygotes resulted from a longitudinal instead of transverse cell division and these twin zygotes developed into embryos of equal size. Direct injection of Asc into tobacco ovaries was sufficient to induce twinning but only if delivered within the first two days after pollination during which the zygote undergoes its first division. The twinning can be understood as an Asc-induced alteration in the normal transverse division of the zygote that results in a loss of the positional cues needed for the normal differentiation of the apical cell into the embryo and the basal cell into the suspensor.

Polycotyly (i.e., the development of more than two cotelydons) was also induced by Asc, either following an increase in DHAR expression or when Asc was injected at the globular stage of embryo development prior to the initiation of cotyledon development [108]. As in zygotic division, an Asc-induced alteration in cell division during the specification of cotyledon-forming fields likely is responsible for the observed polycotyly. Although Asc likely affects cell division in other tissues [102-106], the lack of a readily observable phenotype may make the effect of increased Asc content in other aspects of plant development less apparent.

\section{Conclusions}

From its role as an antioxidant essential for photosynthesis and for detoxifying ROS from endogenous and exogenous sources, to its role in regulating cell division and flowering, to its function as a co-factor in multiple enzymatic reactions, ascorbic acid has fundamentally enabled the colonization of land by plant species. This is likely due to the challenge that the rise in atmospheric oxygen during Earth's past presented to multicellular organisms, which required limiting the harmful consequences of increased exposure to oxygen that a land-based existence entails. Vitamin $\mathrm{C}$ is critical to plants as it is unlikely they could tolerate a single day of exposure to sunlight without ascorbic acid detoxifying the ROS generated by photosynthetic activity. In contrast, animals unable to synthesize ascorbic acid, such as humans, can survive the absence of the vitamin for weeks or even months before succumbing to disease and death. Despite the importance of its role in detoxifying ROS, ascorbic acid's functions are now so integrated into plant growth and development that its importance cannot be underestimated. Because of the complexity of its many roles, any attempts to 
engineer changes in ascorbic acid content in plants that improves one aspect, such as nutritional content, will require close examination of how such changes impact the overall health and performance of the plant under field conditions. In addition to the engineering approaches described above, genetic diversity within plants offers another means to increase Asc content through standard breeding approaches [109], although whether changes in Asc content through these means may limit any deleterious effects on plant growth and development is unknown at this time. The most successful strategies will undoubtedly involve highly targeted approaches to alter ascorbic acid content in specific cell types or tissues to achieve a desired end while limiting possible unintended consequences in other aspects of growth, development, and responses to biotic and abiotic stresses.

\section{Acknowledgments}

The author thanks the United States Department of Agriculture (NRICGP 02-35100-12469) and the University of California Agricultural Experiment Station for support of his work cited.

\section{Conflicts of Interest}

The author declares no conflict of interest.

\section{References}

1. Sies, H.; Stahl, W. Vitamins E and C, $\beta$-carotene, and other carotenoids as antioxidants. Am. J. Clin. Nutr. 1995, 62, 1315S-1321S.

2. Levine, M. New concepts in the biology and biochemisty of ascorbic acid. N. Engl. J. Med. 1986, 314, 892-902.

3. Levine, M.; Cantilena, C.C.; Dhariwal, K.R. Determination of optimal vitamin C requirements in humans. Am. J. Clin. Nutr. 1995, 62, 1347S-1356S.

4. Chatterjee, I.B. Evolution and the biosynthesis of ascorbic acid. Science 1973, 182, 1271-1272.

5. Chen, Z.; Young, T.E.; Ling, J.; Chang, S.-C.; Gallie, D.R. Increasing vitamin C content of plants through enhanced ascorbate recycling. Proc. Natl. Acad. Sci. USA 2003, 100, 3525-3530.

6. Hancock, R.D.; Viola, R. Improving the nutritional value of crops through enhancement of L-ascorbic acid (vitamin C) content: Rationale and biotechnological opportunities. J. Agric. Food Chem. 2005, 53, 5248-5257.

7. Naqvi, S.; Zhu, C.; Farre, G.; Ramessar, K.; Bassie, L.; Breitenbach, J.; Perez Conesa, D.; Ros, G.; Sandmann, G.; Capell, T.; et al. Transgenic multivitamin corn through biofortification of endosperm with three vitamins representing three distinct metabolic pathways. Proc. Natl. Acad. Sci. USA 2009, 106, 7762-7767.

8. Burns, J.J. Ascorbic Acid. In Metabolic Pathways, 3rd ed.; Greenberg, D.M., Ed.; Academic Press: New York, NY, USA, 1967; pp. 394-411.

9. Wheeler, G.L.; Jones, M.A.; Smirnoff, N. The biosynthetic pathway of vitamin C in higher plants. Nature 1998, 393, 365-369. 
10. Wolucka, B.A.; Persiau, G.; van Doorsselaere, J.; Davey, M.W.; Demol, H.; Vandekerckhove, J.; van Montagu, M.; Zabeau, M.; Boerjan, W. Partial purification and identification of GDP-mannose 3',5'-epimerase of Arabidopsis thaliana, a key enzyme of the plant vitamin C pathway. Proc. Natl. Acad. Sci. USA 2001, 98, 14843-14848.

11. Siendones, E.; González-Reyes, J.A.; Santos-Ocaña Navas, P.; Córdoba, F. Biosynthesis of ascorbic acid in kidney bean. L-Galactono- $\gamma$-lactone dehydrogenase is an intrinsic protein located at the mitochondrial inner membrane. Plant Physiol. 1999, 120, 907-912.

12. Bartoli, C.G.; Pastori, G.M.; Foyer, C.H. Ascorbate biosynthesis in mitochondria is linked to the electron transport chain between complexes III and IV. Plant Physiol. 2000, 123, 335-343.

13. Conklin, P.L.; Williams, E.H.; Last, R.L. Environmental stress sensitivity of an ascorbic acid-deficient Arabidopsis mutant. Proc. Natl. Acad. Sci. USA 1996, 93, 9970-9974.

14. Conklin, P.L.; Pallanca, J.E.; Last, R.L.; Smirnoff, N. L-Ascorbic acid metabolism in the ascorbate-deficient Arabidopsis mutant vtc1. Plant Physiol. 1997, 115, 1277-1285.

15. Franceschi, V.R.; Tarlyn, N.N. L-Ascorbic acid is accumulated in source leaf phloem and transported to sink tissues in plants. Plant Physiol. 2002, 130, 649-656.

16. Tedone, L.; Hancock, R.D.; Alberino, S.; Haupt, S.; Viola, R. Long-distance transport of L-ascorbic acid in potato. BMC Plant Biol. 2004, 4, 16.

17. Conklin, P.L.; Saracco, S.A.; Norrism, S.R.; Last, R.L. Identification of ascorbic acid-deficient Arabidopsis thaliana mutants. Genetics 2000, 154, 847-856.

18. Dowdle, J.; Ishikawa, T.; Gatzek, S.; Rolinski, S.; Smirnoff, N. Two genes in Arabidopsis thaliana encoding GDP-L-galactose phosphorylase are required for ascorbate biosynthesis and seedling viability. Plant J. 2007, 52, 673-689.

19. Conklin, P.L.; Norris, S.R.; Wheeler, G.L.; Williams, E.H.; Smirnoff, N.; Last, R.L. Genetic evidence for the role of GDP-mannose in plant ascorbic acid (vitamin C) biosynthesis. Proc. Natl. Acad. Sci. USA 1999, 96, 4198-4203.

20. Conklin, P.L.; Gatzek, S.; Wheeler, G.L; Dowdle, J.; Raymond, M.J.; Rolinski, S.; Isupov, M.; Littlechild, J.A.; Smirnoff, N. Arabidopsis thaliana VTC4 encodes L-galactose-1-P phosphatase, a plant ascorbic acid biosynthetic enzyme. J. Biol. Chem. 2006, 281, 15662-15670.

21. Laing, W.A.; Wright, M.A.; Cooney, J.; Bulley, S.M. The missing step of the L-galactose pathway of ascorbate biosynthesis in plants, an L-galactose guanyltransferase, increases leaf ascorbate content. Proc. Natl. Acad. Sci. USA 2007, 104, 9534-9539.

22. Bulley, S.M.; Rassam, M.; Hoser, D.; Otto, W.; Schünemann, N.; Wright, M.; MacRae, E.; Gleave, A.; Laing, W. Gene expression studies in kiwifruit and gene over-expression in Arabidopsis indicates that GDP-L-galactose guanyltransferase is a major control point of vitamin C biosynthesis. J. Exp. Bot. 2009, 60, 765-778.

23. Bulley, S.; Wright, M.; Rommens, C.; Yan, H.; Rassam, M.; Lin-Wang, K.; Andre, C.; Brewster, D.; Karunairetnam, S.; Allan, A.C.; et al. Enhancing ascorbate in fruits and tubers through over-expression of the L-galactose pathway gene GDP-L-galactose phosphorylase. Plant Biotechnol. J. 2012, 10, 390-397.

24. Gatzek, S.; Wheeler, G.L.; Smirnoff, N. Antisense suppression of L-galactose dehydrogenase in Arabidopsis thaliana provides evidence for its role in ascorbate synthesis and reveals light modulated L-galactose synthesis. Plant J. 2002, 30, 541-553. 
25. Zhou, Y.; Tao, Q.C.; Wang, Z.N.; Fan, R.; Li, Y.; Sun, X.F.; Tang, K.X. Engineering ascorbic acid biosynthetic pathway in Arabidopsis leaves by single and double gene transformation. Biol. Plant. 2012, 56, 451-457.

26. Valpuesta, V.; Botella, M.A. Biosynthesis of L-ascorbic acid in plants: New pathways for an old antioxidant. Trends Plant Sci. 2004, 9, 573-577.

27. Loewus, F.A.; Kelly, S. The metabolism of D-galacturonic acid and its methyl ester in the detached ripening strawberry. Arch. Biochem. Biophys. 1961, 95, 483-493.

28. Isherwood, F.A.; Chen, Y.T.; Mapson, L.W. Synthesis of L-ascorbic acid in plants and animals. Biochem. J. 1954, 56, 1-15.

29. Davey, M.W.; Gilot, C.; Persiau, G.; Østergaard, J.; Han, Y.; Bauw, G.C.; van Montagu, M.C. Ascorbate biosynthesis in Arabidopsis cell suspension culture. Plant Physiol. 1999, 121, 535-543.

30. Agius, F.; Gonzalez-Lamothe, R.; Caballero, J.L.; Munoz-Blanco, J.; Botella, M.A.; Valpuesta, $\mathrm{V}$. Engineering increased vitamin C levels in plants by overexpression of a D-galacturonic acid reductase. Nat. Biotechnol. 2003, 21, 177-181.

31. Badejo, A.A.; Wada, K.; Gao, Y.; Maruta, T.; Sawa, Y.; Shigeoka, S.; Ishikawa, T. Translocation and the alternative D-galacturonate pathway contribute to increasing the ascorbate level in ripening tomato fruits together with the D-mannose/L-galactose pathway. J. Exp. Bot. 2012, 63, 229-239.

32. Loewus, F.A. Tracer studies on ascorbic acid formation in plants. Phytochemistry 1963, 2, 109-128.

33. Wolucka, B.A.; van Montagu, M. GDP-mannose 3',5'-epimerase forms GDP-L-gulose, a putative intermediate for the de novo biosynthesis of vitamin $\mathrm{C}$ in plants. J. Biol. Chem. 2003, 278, 47483-47490.

34. Wagner, C.; Sefkow, M.; Kopka, J. Construction and application of a mass spectral and retention time index database generated from plant GC/EI-TOF-MS metabolite profile. Phytochemistry 2003, 62, 887-900.

35. Jain, A.K.; Nessler, C.L. Metabolic engineering of an alternative pathway for ascorbic acid biosynthesis in plants. Mol. Breed. 2000, 6, 73-78.

36. Radzio, J.A.; Lorence, A.; Chevone, B.I.; Nessler, C.L. L-Gulono-1,4-lactone oxidase expression rescues vitamin C-deficient Arabidopsis (vtc) mutants. Plant Mol. Biol. 2004, 53, 837-844.

37. Baig, M.M.; Kelly, S.; Loewus, F. L-Ascorbic acid biosynthesis in higher plants from L-gulono-1,4-lactone and L-galactono-1,4-lactone. Plant Physiol. 1970, 46, 277-280.

38. Oba, K.; Fukui, M.; Imai, Y.; Iriyama, S.; Nogami, K. L-Galactono- $\gamma$-lactone dehydrogenase: Partial characterization, induction of activity and role in the synthesis of ascorbic acid in wounded white potato tuber tissue. Plant Cell Physiol. 1994, 35, 473-478.

39. Lorence, A.; Chevone, B.I.; Mendes, P.; Nessler, C.L. myo-Inositol oxygenase offers a possible entry point into plant ascorbate biosynthesis. Plant Physiol. 2004, 134, 1200-1205.

40. Asada, K. The water-water cycle in chloroplasts: Scavenging of active oxygens and dissipation of excess photons. Annu. Rev. Plant Physiol. Plant Mol. Biol. 1999, 50, 601-639. 
41. Mano, J.; Hideg, E.; Asada, K. Ascorbate in thylakoid lumen functions as an alternative electron donor to photosystem II and photosystem I. Arch. Biochem. Biophys. 2004, 429, 71-80.

42. Miyake, C.; Asada, K. Ferredoxin dependent photoreduction of monodehydroascorbate radical in spinach thylakoids. Plant Cell Physiol. 1994, 34, 539-549.

43. Sano, S.; Miyake, C.; Mikami, B.; Asada, K. Molecular characterization of monodehydroascorbate radical reductase from cucumber overproduced in Escherichia coli. J. Biol. Chem. 1995, 270, 21354-21361.

44. Mittler, R.; Vanderauwera, S.; Gollery, M.; van Breusegem, F. Reactive oxygen gene network of plants. Trends Plant Sci. 2004, 9, 490-498.

45. Obara, K.; Sumi, K.; Fukuda, H. The use of multiple transcription starts causes the dual targeting of Arabidopsis putative monodehydroascorbate reductase to both mitochondria and chloroplasts. Plant Cell Physiol. 2002, 43, 697-705.

46. Lisenbee, C.S.; Lingard, M.J.; Trelease, R.N. Arabidopsis peroxisomes possess functionally redundant membrane and matrix isoforms of monodehydroascorbate reductase. Plant J. 2005, 43, 900-914.

47. Jimenez, A.; Hernandez, J.A.; del Reo, L.A.; Sevilla, F. Evidence for the presence of the ascorbate-glutathione cycle in mitochondria and peroxisomes of pea leaves. Plant Physiol. 1997, 114, 272-284.

48. Haroldsen, V.M.; Chi-Ham, C.L.; Kulkarni, S.; Lorence, A.; Bennett, A.B. Constitutively expressed DHAR and MDHAR influence fruit, but not foliar ascorbate levels in tomato. Plant Physiol. Biochem. 2011, 49, 1244-1249.

49. Stevens, R.; Page, D.; Gouble, B.; Garchery, C.; Zamir, D.; Causse, M. Tomato fruit ascorbic acid content is linked with monodehydroascorbate reductase activity and tolerance to chilling stress. Plant Cell Environ. 2008, 31, 1086-1096.

50. Eltayeb, A.E.; Kawano, N.; Badawi, G.H.; Kaminaka, H.; Sanekata, T.; Shibahara, T.; Inanaga, S.; Tanaka, K. Overexpression of monodehydroascorbate reductase in transgenic tobacco confers enhanced tolerance to ozone, salt and polyethylene glycol stresses. Planta 2007, 225, 1255-1264.

51. Li, F.; Wu, Q.Y.; Sun, Y.L.; Wang, L.Y.; Yang, X.H.; Meng, Q.W. Overexpression of chloroplastic monodehydroascorbate reductase enhanced tolerance to temperature and methyl viologen-mediated oxidative stresses. Physiol. Plant 2010, 139, 421-434.

52. Yin, L.; Wang, S.; Eltayeb, A.E.; Uddin, M.I.; Yamamoto, Y.; Tsuji, W.; Takeuchi, Y.; Tanaka, K. Overexpression of dehydroascorbate reductase, but not monodehydroascorbate reductase, confers tolerance to aluminum stress in transgenic tobacco. Planta 2010, 231, 609-621.

53. Smirnoff, N.; Conklin, P.L.; Loewus, F.A. Biosynthesis of ascorbic acid in plants: A Renaissance. Annu. Rev. Plant Physiol. Plant Mol. Biol. 2001, 52, 437-467.

54. Noctor, G.; Foyer, C.H. Ascorbate and glutathione: Keeping active oxygen under control. Ann. Rev. Plant Physiol. Plant Mol. Biol. 1998, 49, 249-279.

55. Chen, Z.; Gallie, D.R. The ascorbic acid redox state controls guard cell signaling and stomatal movement. Plant Cell 2004, 16, 1143-1162. 
56. Chen, Z.; Gallie, D.R. Increasing tolerance to ozone by elevating foliar ascorbic acid confers greater protection against ozone than increasing avoidance. Plant Physiol. 2005, 138, 1673-1689.

57. Chen, Z.; Gallie, D.R. Dehydroascorbate reductase affects leaf growth, development, and function. Plant Physiol. 2006, 142, 775-787.

58. Yoshida, S.; Tamaoki, M.; Shikano, T.; Nakajima, N.; Ogawa, D.; Ioki, M.; Aono, M.; Kubo, A.; Kamada, H.; Inoue, Y.; et al. Cytosolic dehydroascorbate reductase is important for ozone tolerance in Arabidopsis thaliana. Plant Cell Physiol. 2006, 47, 304-308.

59. Kwon, S.Y.; Ahn, Y.O.; Lee, H.S.; Kwak, S.S. Biochemical characterization of transgenic tobacco plants expressing a human dehydroascorbate reductase gene. J. Biochem. Mol. Biol. 2001, 34, 316-321.

60. Kwon, S.Y.; Choi ,S.M.; Ahn, Y.O.; Lee, H.S.; Lee, H.B.; Park, Y.M.; Kwak, S.S. Enhanced stress-tolerance of transgenic tobacco plants expressing a human dehydroascorbate reductase gene. J. Plant Physiol. 2003, 160, 347-353.

61. Eltayeb, A.E.; Kawano, N.; Badawi, G.H.; Kaminaka, H.; Sanekata, T.; Morishima, I.; Shibahara, T.; Inanaga, S.; Tanaka, K. Enhanced tolerance to ozone and drought stresses in transgenic tobacco overexpressing dehydroascorbate reductase in cytosol. Physiol. Plant 2006, $127,57-65$.

62. Wang, Z.; Xiao, Y.; Chen, W.; Tang, K.; Zhang, L. Increased vitamin C content accompanied by an enhanced recycling pathway confers oxidative stress tolerance in Arabidopsis. J. Integr. Plant Biol. 2010, 52, 400-409.

63. Ushimaru, T.; Nakagawa, T.; Fujioka, Y.; Daicho, K.; Naito, M.; Yamauchi, Y.; Nonaka, H.; Amako, K.; Yamawaki, K.; Murata, N. Transgenic Arabidopsis plants expressing the rice dehydroascorbate reductase gene are resistant to salt stress. J. Plant Physiol. 2006, 163, 1179-1184.

64. Le Martret, B.; Poage, M.; Shiel, K.; Nugent, G.D.; Dix, P.J. Tobacco chloroplast transformants expressing genes encoding dehydroascorbate reductase, glutathione reductase, and glutathione-S-transferase, exhibit altered anti-oxidant metabolism and improved abiotic stress tolerance. Plant Biotechnol. J. 2011, 9, 661-673.

65. Arrigoni, O.; De Gara, L.; Tommasi, F.; Liso, R. Changes in the ascorbate system during seed development of Vicia faba L. Plant Physiol. 1992, 99, 235-238.

66. Qin, A.; Shi, Q.; Yu, X. Ascorbic acid contents in transgenic potato plants overexpressing two dehydroascorbate reductase genes. Mol. Biol. Rep. 2011, 38, 1557-1566.

67. Goo, Y.M.; Chun, H.; Kim, T.W.; Lee, C.H.; Ahn, M.J.; Bae, S.C.; Cho, K.J.; Chun, J.A.; Chung, C.H.; Lee, S.W. Expressional characterization of dehydroascorbate reductase cDNA in transgenic potato plants. J. Plant Biol. 2008, 51, 35-41.

68. Belmonte, M.F.; Stasolla, C. Altered HBK3 expression affects glutathione and ascorbate metabolism during the early phases of Norway spruce (Picea abies) somatic embryogenesis. Plant Physiol. Biochem. 2009, 47, 904-911.

69. Halliwell, B.; Gutteridge, J.M.C. Free Radicals in Biology and Medicine; Oxford University Press: New York, NY, USA, 2000. 
70. Foyer, C.H. Oxygen Metabolism and Electron Transport in Photosynthesis. In Oxidative Stress and the Molecular Biology of Antioxidant Defenses; Scandalios, J.G., Ed.; Cold Spring Harbor Laboratory Press: Cold Spring Harbor, NY, USA, 1997; pp. 587-621.

71. Asada, K. The water-water cycle as alternative photon and electron sinks. Philos. Trans. R. Soc. Lond. B 2000, 355, 1419-1431.

72. Nakano, Y.; Asada, K. Spinach chloroplasts scavenge hydrogen peroxide on illumination. Plant Cell Physiol. 1980, 21, 1295-1307.

73. Mudd, J.B. Biochemical Basis for the Toxicity of Ozone. In Plant Response to Air Pollution; Yunus, M., Iqba, M., Eds.; Wiley \& Sons: New York, NY, USA, 1997; pp. 267-284.

74. Schraudner, M.; Moeder, W.; Wiese, C.; van Camp, W.; Inze, D.; Langebartels, C.; Sandermann, H., Jr. Ozone-induced oxidative burst in the ozone biomonitor plant, tobacco Bel W3. Plant J. 1998, 16, 235-245.

75. Rao, M.V.; Koch, J.R.; Davis, K.R. Ozone: A tool for probing programmed cell death in plants. Plant Mol. Biol. 2000, 44, 345-358.

76. Koch, J.R.; Creelman, R.A.; Eshita, S.M.; Seskar, M.; Mullet, J.E.; Davis, K.R. Ozone sensitivity in hybrid poplar correlates with insensitivity to both salicylic acid and jasmonic acid. The role of programmed cell death in lesion formation. Plant Physiol. 2000, 123, 487-496.

77. Pasqualini, S.; Piccioni, C.; Reale, L.; Ederli, L.; Della Torre, G.; Ferranti, F. Ozone-induced cell death in tobacco cultivar bel w3 plants. The role of programmed cell death in lesion formation. Plant Physiol. 2003, 133, 1122-1134.

78. Pei, Z.M.; Murata, Y.; Benning, G.; Thomine, S.; Klusener, B.; Allen, G.J.; Grill, E.; Schroeder, J.I. Calcium channels activated by hydrogen peroxide mediate abscisic acid signaling in guard cells. Nature 2000, 406, 731-734.

79. Zhang, X.; Zhang, L.; Dong, F.; Gao, J.; Galbraith, D.W.; Song, C.P. Hydrogen peroxide is involved in abscisic acid-induced stomatal closure in vicia faba. Plant Physiol. 2001, 126, 1438-1448.

80. Veljovic-Jovanovic, S.D.; Pignocchi, C.; Noctor, G.; Foyer, C.H. Low ascorbic acid in the $v t c-1$ mutant of Arabidopsis is associated with decreased growth and intracellular redistribution of the antioxidant system. Plant Physiol. 2001, 127, 426-435.

81. Huang, C.; He, W.; Guo, J.; Chang, X.; Su, P.; Zhang, L. Increased sensitivity to salt stress in an ascorbate-deficient Arabidopsis mutant. J. Exp. Bot. 2005, 56, 3041-3049.

82. Zhang, W.; Lorence, A.; Gruszewski, H.A.; Chevone, B.I.; Nessler, C.L. AMR1, an Arabidopsis gene that coordinately and negatively regulates the mannose/L-galactose ascorbic acid biosynthetic pathway. Plant Physiol. 2009, 150, 942-950.

83. Zhang, Z.; Wang, J.; Zhang, R.; Huang, R. The ethylene response factor Aterf98 enhances tolerance to salt through the transcriptional activation of ascorbic acid synthesis in Arabidopsis. Plant J. 2012, 71, 273-287.

84. Sanmartin, M.; Drogoudi, P.A.; Lyons, T.; Pateraki, I.; Barnes, J.; Kanellis, A.K. Over-expression of ascorbate oxidase in the apoplast of transgenic tobacco results in altered ascorbate and glutathione redox states and increased sensitivity to ozone. Planta 2003, 216, 918-928. 
85. Grimes, H.D.; Perkins, K.K.; Boss, W.F. Ozone degrades into hydroxyl radical under physiological conditions: A spin trapping study. Plant Physiol. 1983, 72, 1016-1020.

86. Lee, Y.P.; Kim, S.H.; Bang, J.W.; Lee, H.S.; Kwak, S.S.; Kwon, S.Y. Enhanced tolerance to oxidative stress in transgenic tobacco plants expressing three antioxidant enzymes in chloroplasts. Plant Cell Rep. 2007, 26, 591-598.

87. Mullen, R.T.; Trelease, R.N. Biogenesis and membrane properties of peroxisomes: Does the boundary membrane serve and protect? Trends Plant Sci. 1996, 1, 389-394.

88. Graham, I.A.; Eastmond, P.J. Pathways of straight and branched chain fatty acid catabolism in higher plants. Prog. Lipid Res. 2002, 41, 156-181.

89. Yamaguchi, K.; Mori, H.; Nishimura, M. A novel isoenzyme of ascorbate peroxidase localized on glyoxysomal and leaf peroxisomal membranes in pumpkin. Plant Cell Physiol. 1995, 36, 1157-1162.

90. Bunkelmann, J.R.; Trelease, R.N. Ascorbate peroxidase: A promenent membrane protein in oilseed glyoxysomes. Plant Physiol. 1996, 110, 589-598.

91. Karyotou, K.; Donaldson, R.P. Ascorbate peroxidase, a scavenger of hydrogen peroxide in glyoxysomal membranes. Arch. Biochem. Biophys. 2005, 434, 248-257.

92. Eastmond, P.J. MONODEHYROASCORBATE REDUCTASE4 is required for seed storage oil hydrolysis and postgerminative growth in Arabidopsis. Plant Cell 2007, 19, 1376-1387.

93. Wang, J.; Zhang, H.; Allen, R.D. Overexpression of an Arabidopsis peroxisomal ascorbate peroxidase gene in tobacco increases protection against oxidative stress. Plant Cell Physiol. 1999, 40, 725-732.

94. Asada, K.; Takahashi, M. Production and Scavenging of Active Oxygen in Photosynthesis. In Photoinhibition; Kyle, D.J., Osmond, C.B., Arntzen, C.J., Eds.; Elsevier: Amsterdam, The Netherlands, 1987; pp. 227-287.

95. Aro, E.M.; Virgin, I.; Andersson, B. Photoinhibition of photosystem II. Inactivation, protein damage and turnover. Biochim. Biophys. Acta 1993, 1143, 113-134.

96. Nishiyama, Y.; Allakhverdiev, S.I.; Murata, N. A new paradigm for the action of reactive oxygen species in the photoinhibition of photosystem II. Biochim. Biophys. Acta 2006, 7157, $742-749$.

97. Chen, Z.; Gallie, D.R. Dehydroascorbate reductase affects non-photochemical quenching and photosynthetic performance. J. Biol. Chem. 2008, 283, 21347-21361.

98. Assmann, S.M.; Wang, X.Q. From milliseconds to millions of years: Guard cells and environmental responses. Curr. Opin. Plant Biol. 2001, 4, 421-428.

99. Schroeder, J.I.; Allen, G.J.; Hugouvieux, V.; Kwak, J.M.; Waner, D. Guard cell signal transduction. Annu. Rev. Plant Physiol. Plant Mol. Biol. 2001, 52, 627-658.

100. Barth, C.; Moeder, W.; Klessig, D.F.; Conklin, P.L. The timing of senescence and response to pathogens is altered in the ascorbate-deficient Arabidopsis mutant vitamin c-1. Plant Physiol. 2004, 134, 1784-1792.

101. Botanga, C.J.; Bethke, G.; Chen, Z.; Gallie, D.R.; Fiehn, O.; Glazebrook, J. Metabolite profiling of Arabidopsis inoculated with Alternaria brassicicola reveals that ascorbate reduces disease severity. Mol. Plant Microbe Interact. 2012, 25, 1628-1638. 
102. Liso, R.; Calabrese, G.; Bitonti, M.B.; Arrigoni, O. Relationship between ascorbic acid and cell division. Exp. Cell Res. 1984, 150, 314-320.

103. Arrigoni, O.; Bitonti, M.B.; Cozza, R.; Innocenti, A.M.; Liso, R.; Veltri, R. Ascorbic acid effect on pericycle cell line in Allium cepa root. Caryologia 1989, 42, 213-216.

104. Innocenti, A.M.; Bitonti, M.B.; Arrigoni, O.; Liso, R. The size of quiescent centre in roots of Allium Cepa L. grown with ascorbic acid. New Phytol. 1990, 110, 507-509.

105. Arrigoni, O. Ascorbate system in plant development. J. Bioenerg. Biomembr. 1994, 26, 407-419.

106. Citterio, S.; Sgorbati, S.; Scippa, S.; Sparvoli, E. Ascorbic acid effect on the onset of cell proliferation in pea root. Physiol. Plant 1994, 92, 601-607.

107. Tabata, K.; Oba, K.; Suzuki, K.; Esaka, M. Generation and properties of ascorbic acid-deficient transgenic tobacco cells expressing antisense RNA for L-galactono-1,4-lactone dehydrogenase. Plant J. 2001, 27, 139-148.

108. Chen, Z.; Gallie, D.R. Induction of monozygotic twinning by ascorbic acid in tobacco. PLoS One 2012, 7, e39147.

109. Gest, N.; Gautier, H.; Stevens, R. Ascorbate as seen through plant evolution: The rise of a successful molecule? J. Exp. Bot. 2013, 64, 33-53. 
MDPI AG

Klybeckstrasse 64

4057 Basel, Switzerland

Tel. +41616837734

Fax +41613028918

http://www.mdpi.com/

Nutrients Editorial Office

E-mail: nutrients@mdpi.com

http://www.mdpi.com/journal/nutrients 

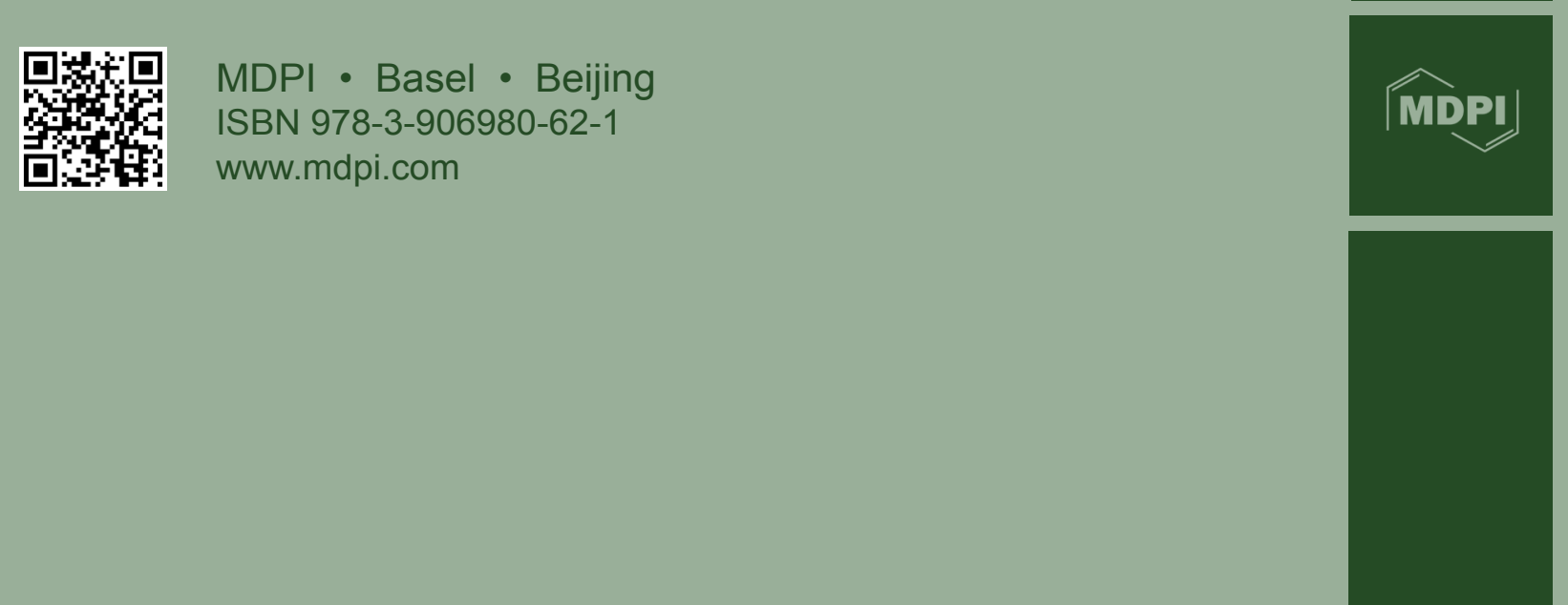